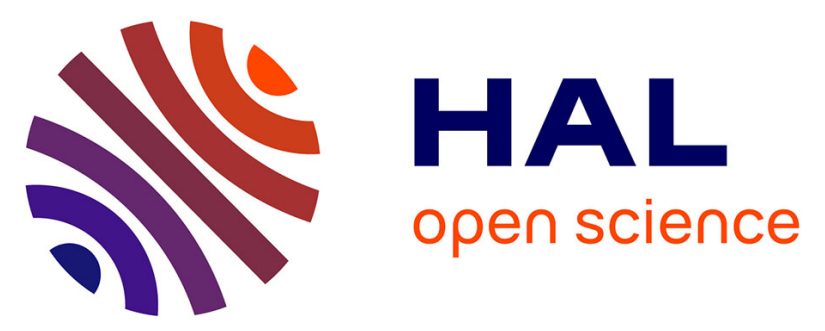

\title{
Detrital zircon age distribution from Devonian and Carboniferous sandstone in the Southern Variscan Fold-and-Thrust belt (Montagne Noire, French Massif Central), and their bearings on the Variscan belt evolution
}

Wei Lin, Michel Faure, Xian-Hua Li, Yang Chu, Wenbin Ji, Zhenhua Xue

\section{To cite this version:}

Wei Lin, Michel Faure, Xian-Hua Li, Yang Chu, Wenbin Ji, et al.. Detrital zircon age distribution from Devonian and Carboniferous sandstone in the Southern Variscan Fold-and-Thrust belt (Montagne Noire, French Massif Central), and their bearings on the Variscan belt evolution. Tectonophysics, 2016, 677-678, pp.1-33. 10.1016/j.tecto.2016.03.032 . insu-01303804

HAL Id: insu-01303804

https://hal-insu.archives-ouvertes.fr/insu-01303804

Submitted on 18 Apr 2016

HAL is a multi-disciplinary open access archive for the deposit and dissemination of scientific research documents, whether they are published or not. The documents may come from teaching and research institutions in France or abroad, or from public or private research centers.
L'archive ouverte pluridisciplinaire HAL, est destinée au dépôt et à la diffusion de documents scientifiques de niveau recherche, publiés ou non, émanant des établissements d'enseignement et de recherche français ou étrangers, des laboratoires publics ou privés. 


\section{Accepted Manuscript}

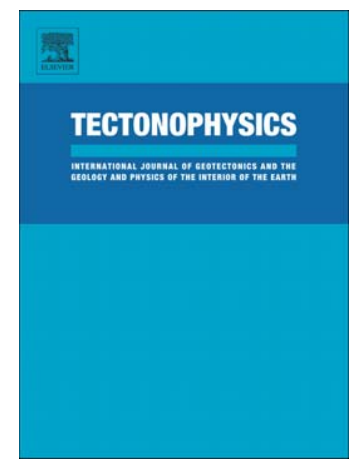

Detrital zircon age distribution from Devonian and Carboniferous sandstone in the Southern Variscan Fold-and-Thrust belt (Montagne Noire, French Massif Central), and their bearings on the Variscan belt evolution

Wei Lin, Michel Faure, Xian-hua Li, Yang Chu, Wenbin Ji, Zhenhua Xue

PII: $\quad$ S0040-1951(16)30027-0

DOI: $\quad$ doi: $10.1016 / j$. tecto.2016.03.032

Reference: $\quad$ TECTO 127024

To appear in: $\quad$ Tectonophysics

Received date: 19 January 2016

Revised date: $\quad 21$ March 2016

Accepted date: 23 March 2016

Please cite this article as: Lin, Wei, Faure, Michel, Li, Xian-hua, Chu, Yang, Ji, Wenbin, Xue, Zhenhua, Detrital zircon age distribution from Devonian and Carboniferous sandstone in the Southern Variscan Fold-and-Thrust belt (Montagne Noire, French Massif Central), and their bearings on the Variscan belt evolution, Tectonophysics (2016), doi: 10.1016/j.tecto.2016.03.032

This is a PDF file of an unedited manuscript that has been accepted for publication. As a service to our customers we are providing this early version of the manuscript. The manuscript will undergo copyediting, typesetting, and review of the resulting proof before it is published in its final form. Please note that during the production process errors may be discovered which could affect the content, and all legal disclaimers that apply to the journal pertain. 
Detrital zircon age distribution from Devonian and Carboniferous sandstone in the Southern Variscan Fold-and-Thrust belt (Montagne Noire, French Massif Central), and their bearings on the Variscan belt evolution

Wei Lin ${ }^{\mathrm{a}^{*}}$, Michel Faure ${ }^{\mathrm{b}}$, Xian-hua $\mathrm{Li}^{\mathrm{a}}$, Yang Chu ${ }^{\mathrm{a}}$, Wenbin $\mathrm{Ji}^{\mathrm{a}}$, Zhenhua Xue ${ }^{\mathrm{a}}$

${ }^{\text {a }}$ State Key Laboratory of Lithospheric Evolution, Institute of Geology and Geophysics, Chinese Academy of Sciences, Beijing 100029, China

${ }^{\mathrm{b}}$ Université d'Orléans-CNRS/INSU, Institut des Sciences de la Terre d'Orléans (ISTO), Campus Géosciences, 1A Rue de la Férollerie 45071 Orléans Cedex 2, France

Corresponding author. Tel.: +861082 998546; fax: +861062010846.

E-mail address: linwei@mail.iggcas.ac.cn (W. Lin).

\section{Abstract}

In the Southern French Massif Central, the Late Paleozoic sedimentary sequences of the Montagne Noire area provide clues to decipher the successive tectonic events that occurred during the evolution of the Variscan belt. Previous sedimentological studies already demonstrated that the siliciclastic deposits were supplied from the northern part of the Massif Central. In this study, detrital zircon provenance analysis has been investigated in Early Devonian (Lochkovian) conglomerate and sandstone, and in Carboniferous (Visean to Early Serpukhovian) sandstone from the recumbent folds and the foreland basin of the Variscan Southern Massif Central in Montagne Noire. The zircon grains from all of the samples yielded $\mathrm{U}-\mathrm{Pb}$ age spectra ranging from Neoarchean to Late Paleozoic with 
several age population peaks at $2700 \mathrm{Ma}, 2000 \mathrm{Ma}, 980 \mathrm{Ma}, 750 \mathrm{Ma}, 620 \mathrm{Ma}, 590 \mathrm{Ma}, 560 \mathrm{Ma}, 480$ Ma, $450 \mathrm{Ma}$, and $350 \mathrm{Ma}$. The dominant age populations concentrate on the Neoproterozoic and Paleozoic. The dominant concordant detrital zircon age populations in the Lochkovian samples, the 480-445 Ma with a statistical peak around $450 \mathrm{Ma}$, are interpreted as reflecting the rifting event that separated several continental stripes, such as Armorica, Mid-German Crystalline Rise, and Avalonia from the northern part of Gondwana. However, Ediacaran and Cambrian secondary peaks are also observed. The detrital zircons with ages at 352-340 Ma, with a statistical peak around $350 \mathrm{Ma}$, came from the Early Carboniferous volcanic and plutonic rocks similar to those exposed in the NE part of the French Massif Central. Moreover, some Precambrian grains recorded a more complex itinerary and may have experienced a multi-recycling history: the Archean and Proterozoic grains been firstly deposited in Cambrian or Ordovician terrigenous rocks, and secondly re-sedimented in Devonian and/or Carboniferous formations. Another possibility is that ancient grains would be inherited grains, scavenged from an underlying but not exposed Precambrian basement.

Keywords: Detrital zircon provenance analysis; Foreland basin; Fold-and-Thrust belt; Variscan Belt; Montagne Noire

\section{Introduction}

Sedimentological and provenance studies of the terrigenous detritus found in the foreland basins are used since a long time to analyze the geomorphologic and tectonic evolution of orogens, particularly the uplift and erosion of the inner zones of mountain belts. More recently, age distribution probability of detrital zircon enclosed in terrigeneous unmetamorphosed or low-grade metasedimentary rocks became a popular method to infer the possible source area of the sediments (e.g. Dickinson and Gerhels, 2009; Fedo et al., 2003). This approach has been applied all along the Paleozoic Variscan belt from Iberia to Turkey, through Central Europe (e.g. Fernández-Suárez et al., 
2002, 2003; Martínez Catalán et al., 2004, 2008, 2016; Linnemann et al., 2004; Okay et al., 2011;

Dinis et al., 2012). Nevertheless, such inherited detrital zircon provenance analyses are rare in the French Variscan Belt. Studies are limited to the Southern Massif Armoricain (Ducassou et al., 2014) or Massif Central (Melleton et al., 2010). This last work dealt with magmatic and metamorphic rocks: 7 orthogneiss, one metarhyolite, and 6 paragneiss sampled in the western part (Limousin area) of the French Massif Central. Only one Cambrian metasandstone was investigated. Provenance studies from detrital zircon have never been carried out in the southern foreland basin that crops out in the southern part of the French Massif Central. This study focuses on the detrital zircon age distribution of the Late Visean-Early Serpukhovian turbiditic sandstone, and also on Early Devonian (Lochkovian) detrital rocks that unconformably overlie the Ordovician sandstone involved in the recumbent folds of the southern flank of the Montagne Noire (Fig. 1). The significance of the statistical distribution of zircon ages for the tectonic evolution of the French Massif Central is discussed.

\section{The Variscan French Massif Central}

The Variscan orogen is a complex belt built up by multiple collisions of continents and microcontinents that develops from SW Iberia to Poland over ca $5000 \mathrm{~km}$ along strike and $1000 \mathrm{~km}$ in width. A general zonation, and several geodynamic evolution models have been proposed (e.g. Matte, 1986, 2001; Cocks, 2000; Franke, 2000, 2014; Martínez Catalán et al., 2009; Faure et al., 2005, 2009; Ballèvre et al., 2009; Schulmann et al., 2009; Lardeaux et al., 2014).

The French Massif Central (FMC; Fig. 1) is one of the largest Variscan massifs. It consists of a stack of nappes developed at the expense of sedimentary and magmatic rocks belonging to the southern margin of Gondwana (e.g. Faure et al., 2009 and enclosed references). From top to bottom, and globally from North to South, the following units are recognized. The Upper Gneiss Unit (UGU) is formed by a bimodal magmatic association (termed the "leptynite-amphibolite complex") with acidic and mafic rocks, and paragneiss. This unit contains high-pressure rocks (eclogites and 
granulites) dated at ca 420-400 Ma (Pin and Peucat, 1986). The upper part of the UGU consists of migmatites yielding zircon U-Pb ages around 385-380 Ma. The Lower Gneiss Unit (LGU) is composed of metagreywacke, metapelite, metarhyolite, and a small amount of mafic rocks, metamorphosed into amphibolites. Cambrian and Ordovician alkaline porphyritic granites, now transformed into augen orthogneiss, intruded the sedimentary-volcanic series. Like the UGU, the LGU experienced a Late Devonian crustal melting dated around 375-370 Ma. The Para-autochthonous Unit consists of a metapelite-metagreywacke series with rare mafic lava, and intruded by rare orthogneiss. Zircon from the magmatic rocks indicates Ordovician ages. The southernmost part of the FMC contains clastic sediments of Late Viséan to Serpukhovian age, which were incorporated into the grossly southward propagating fold and thrust belt. The southern foreland basin consists of a Middle Visean basin. Since the last two units are well developed in the Montagne Noire that constitutes the topic of this paper, stratigraphic and structural details will be provided in the following section.

Other lithotectonic and magmatic units of the FMC are exposed in limited areas. Devonian rocks crop out in the NE part (Montagne Bourbonnaise and Morvan area, Fig. 1). The Brévenne ophiolitic Unit, exposed west of Lyon, consists of mafic rocks (gabbro, diabase, pillow lava, and volcano-clastites), serpentinites, and siliceous sedimentary rocks (e.g. Pin and Paquette, 1998; Leloix et al., 1999). To the North, the Somme series consists of acidic to intermediate volcanic rocks (lava flows, pyroclastites) interlayered with sandstones, grauwackes, conglomerates and limestones (Delfour, 1989; Schneider et al., 1989). The Somme series and Brévenne ophiolites are interpreted as a magmatic arc and a back arc basin, respectively. This system developed in the upper plate of a Southdirected subduction (Faure et al., 2009). The low metamorphic grade Upper Units that develop from the southern margin of Limousin to the Albigeois area, North of the Montagne Noire, consist of Cambrian-Ordovician sedimentary and volcanic formations.

Furthermore, in the northern part of the FMC, the Late Visean "Tufs anthracifères" series formed by conglomerates, sandstone, mudstone with coal measures and rhyolitic to dacitic volcanites and volcano-sedimentary rocks represent an important time marker, as this series unconformably covers the tectono-metamorphic stack of nappes of northern Massif Central whereas, at the same time, 
in the south, the Fold-and-Thrust belt is still developing. The Variscan magmatism is also well developed in the FMC. After an Early Carboniferous event represented by the biotite ( \pm cordierite) Guéret massif, the Visean magmatism, represented by "red granites", microgranites, and dykes coeval with the "Tufs anthracifères" series, is dominant in the NE Massif Central, in Morvan and Montagne Bourbonnaise (e.g. Duthou et al., 1984; Leistel and Gagny, 1984; Binon and Pin, 1989; Pin and Duthou, 1990; Pin, 1991). Lastly, Serpukhovian to Baskhirian (or Namurian to Westphalian in the W. European stratigraphic scale) plutons, represented by two-mica peraluminous and biotite-K feldspar megacrysts monzogranites, are widespread all over the FMC.

This stack of nappes was built up throughout several tectonic-metamorphic events. A polyorogenic evolution accounts well for the present architecture (e.g. Faure et al., 2009; Fig. 1). In this view, a Silurian-Devonian eo-Variscan cycle was responsible for the development of the high pressure-medium temperature $\left(\mathrm{D}_{0}\right.$ event), followed by the crustal melting ( $\mathrm{D}_{1}$ event) observed in the UGU and LGU. The Variscan orogenic cycle developed during the Latest Devonian (Famennian) and Carboniferous. The major tectono-metamorphic event $\left(\mathrm{D}_{2}\right)$, coeval with a medium pressure-medium temperature metamorphism, is characterized by a top-to-the-NW ductile shearing. This $\mathrm{D}_{2}$ event was dated at ca 365-360 Ma (Melleton et al., 2009; Do Couto et al., 2015). The next $\mathrm{D}_{3}$ event developed in the southern MCF with a clear geographic and chronological evolution. From North-South, the UGU and LGU were reactivated, and transported upon the Para-autochthonous Unit, itself emplaced upon the Fold-and-Thrust Belt, and then in the foreland basin. The consistent top-to-the-South shearing and thrusting became younger and younger from North to South, namely from Early Visean (ca $345 \mathrm{Ma}$ ) in the Margeride up to Late Visean- Early Serpukhovian (ca 330-325 Ma) in the Montagne Noire.

At the scale of the entire Massif Central, the Late Visean period represents a turning point since the $\mathrm{D}_{3}$ nappe stacking active in the southern part was coeval with the onset of orogenic extension in the northern part of the massif. The emplacement of the Late Visean (ca $330 \mathrm{Ma}$ ) dyke swarm belonging to the "Tufs Anthracifères" series was controlled by a NW-SE crustal stretching. Nevertheless, the main time for crustal extension, as represented by the syntectonic emplacement of peraluminous granites and K-feldspar porphyritic monzogranites, took place in Late Serpukhovian to 
Bashkirian (ca 320-315 Ma; Fig. 1). This tectono-magmatic phase will not be presented here as this event post-dates the deposition of the sedimentary rocks in the foreland basin. On the contrary, the Tournaisian to Middle Visean magmatism observed in the northern FMC will be presented in the discussion section as it might be interpreted as a possible source for the detrital zircons deposited in the Late Visean-early Serpukhovian foreland basin.

\section{The Montagne Noire}

Since early works (e.g. Gèze, 1949; Arthaud, 1970), the Montagne Noire, in the southernmost part of the FMC, is subdivided into a northern flank and a southern flank formed by folded and thrust sedimentary formations, separated by a metamorphic, granitic, and migmatitic Axial zone (Fig. 2).

Due to the low metamorphic grade experienced by the sedimentary series, the southern flank is a wellknown area for Paleozoic biostratigraphy (e.g. Alabouvette et al., 2003). In the following, only a brief lithostratigraphic outline is provided here; details can be found in numerous papers (e.g. Engel et al., 1980-1981; Feist, 1985; Feist and Galtier, 1985; Alvaro and Vizcaïno, 1998; Vizcaïno and Alvaro, 2001; Poty et al., 2002; Vachard and Aretz, 2004).

The Early Ordovician rocks (Fig. 3) conformably overlie Early Cambrian green sandstone, grauwacke and limestone. A ca 15-20m thick white massive quartzite covers a 500 to $800 \mathrm{~m}$ thick alternation of sandstone, siltstone and mudstone of Tremadocian age. These turbidites are interpreted as contourites (e.g. Alabouvette et al., 2003) deposited along a passive continental margin during the rifting event that separated the Armorica microcontinent from the main part of Gondwana (Matte, 2001; Faure et al., 2009). In the Montagne Noire, the upper Ordovician and Silurian deposits are generally missing, except in the olistoliths known as the "Ecailles de Cabrières" (or Cabrières Schuppen, Fig. 2). There, they consist of mudstone, sandstone, black shale and rare limestone. The absence of the late Ordovician and Silurian rocks is interpreted as a consequence of the emersion and erosion of rift shoulders that followed the early Ordovician rifting (Alabouvette et al., 2003). Under 
tropical climatic conditions, a Fe-rich laterite developed. The Devonian formations are known only in the southern flank of the Montagne Noire. The Early Devonian (Lochkhovian, ca 416-411 Ma), deposit unconformably overlies the Early Ordovician sandstone (Fig. 4a). The basal conglomerate reworks lithic fragments, laterite, andesites, silcrete, phosphatic clasts, micaschists, volcanic quartz grains, and magmatic or metamorphic minerals such as garnet, zircon, monazite, rutile, tourmaline, allanite (Feist, 1985; Quémart et al., 1993). Sedimentological studies indicate that this material was supplied from a northern source (Feist and Schönlaub, 1973, Feist, 1985, Quémart et al., 1993). The sedimentary series continues with iron rich sandstone, oolithic ferruginous sandstone, and dolomite. This ca $15 \mathrm{~m}$ thick basal sequence is overlain by white massive sandstone with quartz arenite called "mur quartzeux" (quartzose wall) reworking abundant zircon, rutile, and tourmaline (Figs 3, 4b).

The Middle and Upper Devonian rocks consist of shallow water limestone and dolomite. The carbonate platform develops widely in the North Gondwana margin in Pyrenees, Mediterranean Variscan massifs, (SE Spain, Calabria, Kabylia, Sardinia, Sicily), and farther East in Middle East, up to SE Asia. The Late Devonian (Famennian) carbonates are represented by typical red nodular limestone known as "griotte facies" (Fig. 3) that denotes a high- energy slope environment indicating the onset of the drawning carbonate platform. The Early Carboniferous (Tournaisian) is represented by a ca $30 \mathrm{~m}$ thick black radiolarian chert (lydite), and a ca $20 \mathrm{~m}$ thick succession of siliceous limestone, clayed and bioclastic limestone, argillite, breccia and limy turbidite locally known as "calcaire de Faugères". The Devonian carbonate platform that progressively subsided during the Tournaisian was replaced by silico-clastic sedimentation during the Late Visean. The $\mathrm{cm}$-scale alternations of siltstone and sandstone representative of distal turbidite were followed by more proximal deposits characterized by 1 to $5 \mathrm{~m}$ thick coarse-grained sandstone with intraformational conglomerate, disrupted beds, and slumps, Fig. 4c, d, e). Lastly, the chaotic sedimentation gave rise to an olistostrome with $\mathrm{m}$ - to kmsized olistoliths composed by Visean or Devonian limestone, and rare Silurian and Ordovician sandstone and lava. The lower part of this turbiditic series yielded late Visean goniatite (Bohm, 1935; Engel et al., 1980-81). Early Serpukhovian (Namurian A) plants have been recovered from turbiditic mudstone (Feist and Galtier, 1985). More recently, corals, foraminifera, and algae indicating a Late 
Visean to Early Serpukhovian age for some of the limestone blocks have been documented (Poty et al., 2002; Vachard and Aretz, 2004). Thus in the present state of knowledge, a Serpukhovian age (ca $325 \mathrm{Ma})$ is inferred for the olistostrome.

From the structural point of view, the Montagne Noire is well known for km-scale, southverging recumbent folds (Arthaud, 1970). The uppermost recumbent fold, called the Pardailhan nappe, consists of an inverted series of Cambrian, Ordovician and Devonian sedimentary rocks tectonically overlying another inverted series of Ordovician, Devonian, and Carboniferous series, called the MtPeyroux nappe. This lower recumbent fold tectonically overlies a series of autochthonous and paraautochthonous tectonic units emplaced within the Late Visean-Serpukhovian foreland basin that can be considered as a flexural basin (Fig. 5). Whatever the detail of this complex fold-and-thrust belt, the mechanism of which remains poorly understood (e.g. Perrin et al., 2013), there is a general agreement to acknowledge both a northern origin of the detritus reworked in the foreland basin, and also a bulk southward direction of the tectonic transport responsible for the emplacement of the recumbent folds and the formation of the flexural basin. Although the root zone of the recumbent folds is not precisely settled, on the basis of stratigraphy and facies correlations, it should be located in the southern part of the Montagne Noire north flank, and partly in the northernmost part of the Axial zone (Arthaud, 1970; Demange, 1993; Alabouvette et al., 2003). It is worth to note that the Axial Zone does not represent a pre-Variscan basement since most of the orthogneiss exposed there are Early Paleozoic granitoids (Roger et al., 2004; Faure et al., 2010). Instead, the Axial Zone was involved in the south-directed tectonics responsible for the recumbent folds (e.g. Alabouvette et al., 2003; Faure et al., 2014, and enclosed references). The metamorphic Axial zone, and the recumbent folds represent the infrastructure and suprastructure of the same stack of nappes, respectively (Fig. 5).

\section{Sample description}


In this study, samples for detrital zircon analysis come from two parts of the stratigraphic column

(Fig 3; Table 1). The basal Devonian (Lochkhovian) black microconglomerate (MO 23, 24) crops out in the northern subunit of the upper recumbent fold (Malviès synform). The overlying white quartzite was sampled in the lower recumbent fold, near the Lower Landeyran bridge (MO 18); and in La Guette, west of Roquebrun (MO 22A).

The analyzed black microconglomerate samples (MO 23, 24) consist essentially of $1 \mathrm{~mm}$ to $1 \mathrm{~cm}$-sized clasts of quartz and subordinate feldspar and lithic elements enclosed in a black matrix (Fig 6d). The quartz gains exhibit deformation microstructures such as undulose extinction, subgrain boundaries, and more rarely dynamic recrystallization with core and mantle structure.

The white quartzite (MO 18, MO 22A) is formed by well rounded quartz grains. Heavy minerals such as zircon, monazite, rutile, and tourmaline can be sometimes observed under the microscope (Fig 6c).

The Late Visean-Serpukhovian terrigenous rocks belong either to the lower recumbent fold (MO 17, 13 FR 52) or to the autochthonous foreland basin (MO 15, MO 16, and MO 26). Whatever their structural position, the analyzed samples are mature sandstone with mm-sized quartz grains. Detrital muscovite is commonly observed (Figs. 6 a, b).

\section{LA-ICP-MS zircon U-Pb analytical procedure}

Standard heavy liquid and magnetic separation techniques have been used to separate zircons from samples. After handpicking, zircon grains were mounted in epoxy resin and then polished to section the crystals for analysis. Before experiments, all zircons were photographed in both transmitted and reflected light under a microscope, and cathodoluminescence (CL) images were obtained by a CAMECA electron microscope. Based on these photographs, internal structures of zircons have been carefully examined. 
Laser ablation ICP-MS zircon U-Pb analyses were conducted on an Agilent 7500a ICP-MS with a $193 \mathrm{~nm}$ laser at the MC-ICP-MS laboratory, Institute of Geology and Geophysics, Chinese Academy of Sciences in Beijing. U-Th- $\mathrm{Pb}$ ratios and absolute abundances were determined relative to the standard zircon. Detailed analytical procedures are described by Xie et al. (2008). The spot diameter is $44 \mu \mathrm{m}$ or $60 \mu \mathrm{m}$ in size. Correction of common lead was applied following the method described by Andersen (2002). The GLITTER program was used for data processing (van Achterbergh et al., 2001). Uncertainties on individual analyses in data tables are reported at a $1 \sigma$ level. Age diagrams of samples plotted using the Isoplot program (Ludwig, 2003). Zircon ages younger than 1000 Ma are based on ${ }^{206} \mathrm{~Pb} /{ }^{238} \mathrm{U}$ ratios whereas ages older than $1000 \mathrm{Ma}$ are based on ${ }^{207} \mathrm{~Pb} /{ }^{206} \mathrm{~Pb}$ ratios. In this study, we excluded zircon age analyses with $>10 \%$ discordance.

\section{Description of the zircon U-Pb analytical results}

All analyzed samples, yield zircon detrital grains with a wide range in size from $50 \mu \mathrm{m}$ to $200 \mu \mathrm{m}$ (Fig. 7). Except several prismatic, most of the grains have a rounded shape with abraded crystallographic faces indicating important fluvial transportation. Most of the grains exhibit a zonal structure with an inherited core surrounded by recrystallization rims with clear oscillatory zoning. Due to analytic constraints on the beam size, only the large grains have been dated.

\subsection{Detrital zircons from the Lower Devonian (Lochkhovian) rocks}

Two samples were collected on the basal Devonian (Lochkhovian) black microconglomerate (Fig. 6d) situated in the northern subunit of the upper recumbent fold (also called Pardailhan nappe).

MO 23

Among the 100 analyses on 100 zircon grains, 4 are discordant (Table 2). Except seven zircon grains that yield $\mathrm{Th} / \mathrm{U}$ ratio lower than 0.1 , most analytical results have higher $\mathrm{Th} / \mathrm{U}$ ratio. Combined with the CL images, these features of the zircons indicate a magmatic origin for these grains (Fig. 7). Most of the zircon ages cluster between $850 \mathrm{Ma}$ and $436 \mathrm{Ma}$, with several ages older than $1500 \mathrm{Ma}$. 
The diagram shows one pronounced age peak around $450 \mathrm{Ma}$, and three subordinate peaks around 590 Ma, $620 \mathrm{Ma}$, and $750 \mathrm{Ma}$ (Fig. 8).

MO 24

Among the 100 analyses conducted on 100 zircons for sample MO24, 98 are concordant within uncertainties (Table 2). All zircons have Th/U ratios varying from 0.10 to 2.37 (Table 2). Similarly with the MO 23 sample, most of the concordant ages range from $850 \mathrm{Ma}$ to $436 \mathrm{Ma}$, with two dominant peaks at $620 \mathrm{Ma}$ and $450 \mathrm{Ma}$, and three subordinate peaks around $590 \mathrm{Ma}, 620 \mathrm{Ma}$, and 750 Ma (Fig. 8). Several grains indicate a Paleoproterozoic source around 2000 Ma (Fig. 8).

In this work, two samples of Early Devonian white quartzite were collected in the lower recumbent fold (or Mt-Peyroux nappe). As these rocks consist of well sorted, nearly pure quartz, deposited in a more distal part of the basin than the previous samples (MO23 and MO 24), they will allow us to compare detrital zircons in a sedimentary setting different from that of the microconglomerate.

MO 18

One hundred analyses of 100 zircons were made (Table 2). Among these analyses, 91 are concordant within uncertainties. These ages range from $3249 \mathrm{Ma}$ to $234 \mathrm{Ma}$. Th/U ratios of all zircons but two are higher than 0.1 . Almost all ages vary from $1000 \mathrm{Ma}$ to $416 \mathrm{Ma}$, with 6 grains older than $1800 \mathrm{Ma}$ (Fig. 8). Two major peaks at $630 \mathrm{Ma}$ and $460 \mathrm{Ma}$, and two subordinate peaks around $970 \mathrm{Ma}$ and $750 \mathrm{Ma}$ are identified (Fig. 8). It is worth to note that this sample shows a minor difference with the others as five grains (analytical points $25,51,65,68,72$ ) are younger (such as $234 \mathrm{Ma}$ ) than the Early Devonian sedimentary age of the rock, and four grains yield ages comprised between $384 \mathrm{Ma}$ and $332 \mathrm{Ma}$ (Table 2). The CL images of these abnormal grains reveal that analytical spots involved cracks or inclusions, leading to $\mathrm{Pb}$ loss and unreliable ages. Thus they have been discarded from the age spectra distribution. 
MO $22 A$

In this sample, 100 zircon grains were selected to conduct 100 analyses and obtain 91 concordant ages. Zircon ages range from $2748 \mathrm{Ma}$ and $435 \mathrm{Ma}$ with 11 zircons older than $1800 \mathrm{Ma}$, and $\mathrm{Th} / \mathrm{U}$ ratios vary from 0.04 to 2.90 (Table 2). Except 3 grains, the remaining ones correspond to magmatic zircons. The diagram shows one major group at 1000-435 Ma with two age peaks at $457 \mathrm{Ma}$ and 618 Ma; one subordinate peak around $980 \mathrm{Ma}$ is identified; an unseparated cluster between $795 \mathrm{Ma}$ and $700 \mathrm{Ma}$ is also fixed (Fig. 8). Ages older than $1000 \mathrm{Ma}$ are concentrated around $1980 \mathrm{Ma}$.

\subsection{Detrital zircons from the Carboniferous (Late Visean-Serpukhovian) rocks}

Two mature sandstone, with mm-sized quartz grains, were collected on the lower recumbent fold (Fig. 6a)

MO 17

A total of 100 analyses of 100 grains were undertaken and 2 are discordant (Table 3). Ninetyeight zircons yield ages ranging from $2879 \mathrm{Ma}$ to $328 \mathrm{Ma}$, with $\mathrm{Th} / \mathrm{U}$ ratios from 0.12 to 1.44 , except 3 between 0.02 and 0.06 . More than $80 \%$ analytical results are situated between 760-330 Ma with four major groups of Phanerozoic ages: 350 Ma, 470 Ma, 580 Ma, and 630 Ma (Fig. 9). In this sample, 14 zircon grains are older than 1500 Ma with a cluster around 2700 Ma.

\section{FR 52}

One hundred analyses of 100 zircon grains have been conducted, and only 3 zircon ages are discordant (Table 3). All but one zircon (13FR52-02) show magmatic features with $\mathrm{Th} / \mathrm{U}$ ratio $>0.1$. The concordant ages range from 2682 Ma to $319 \mathrm{Ma}$; around 15\% analytical result indicated the ages more than 1000 Ma with a small peak around 2100 Ma (Fig. 9). In fact, most of concordant ages range from $680 \mathrm{Ma}$ and $320 \mathrm{Ma}$ with two dominant peaks at $490 \mathrm{Ma}$ and $350 \mathrm{Ma}$, and two subordinate peaks around $540 \mathrm{Ma}$ and $610 \mathrm{Ma}$ (Fig. 9). 
Three mature sandstones were analyzed from the autochthonous foreland basin (Fig. 6b).

MO 15

In this sample, we analyzed 100 spots of 100 zircon grains, among which 6 ages are discordant (Table 3). Except 3 grains, Th/U ratios of all zircons are higher than 0.1. Similar to the 13FR52, 16 zircons are older than $1500 \mathrm{Ma}$ with a cluster around $2100 \mathrm{Ma}$. Most zircons yield ages range from $670 \mathrm{Ma}$ and $320 \mathrm{Ma}$ with three dominant peaks at $542 \mathrm{Ma}, 489 \mathrm{Ma}$, and $340 \mathrm{Ma}$ (Fig. 9). The youngest zircon age is $315 \mathrm{Ma}$.

MO 16

Among the 100 analyses on 100 zircon grains, 90 are concordant ages within uncertainties (Table 3). Two grains yield $\mathrm{Th} / \mathrm{U}$ ratio lower than 0.1. 67 zircons are grouped between $680 \mathrm{Ma}$ and $330 \mathrm{Ma}$, and a small group at 2150-1800 Ma with two peaks around $1890 \mathrm{Ma}$ and $2140 \mathrm{Ma}$ also exists (Table 3 and Fig. 9). The diagram shows one conspicuous age peak at $452 \mathrm{Ma}$, and four subordinate peaks at $352 \mathrm{Ma}, 504 \mathrm{Ma}, 571 \mathrm{Ma}$, and $616 \mathrm{Ma}$, respectively (Fig. 9).

MO 26

One hundred spots on 100 zircons were analyzed, and 89 yield concordant ages within uncertainties. Th/U ratios of all zircons but one are higher than 0.1 (Table 3). The concordant ages range from $2966 \mathrm{Ma}$ to $325 \mathrm{Ma}$; nearly $30 \%$ of the analytical result indicated ages older than $1000 \mathrm{Ma}$, and a cluster at 2060-1940 Ma with a peak around 2053 Ma. This sample contains the largest number of Archean or Proterozoic ages (Table 3 and Fig. 9). In fact, most zircons yield ages <700 Ma with two dominant peaks at $496 \mathrm{Ma}$ and $352 \mathrm{Ma}$, and two subordinate peaks around $559 \mathrm{Ma}$ and $586 \mathrm{Ma}$ (Fig. 9). 
The four Devonian samples yield similar age distribution spectra (Fig. 8). The main peak of inherited grains ranges between 460 and $445 \mathrm{Ma}$ (Ordovician) with a peak around $450 \mathrm{Ma}$ (Fig. 10a). A secondary maximum appears at 628-551 Ma (Cryogenian-Ediacaran) with a peak around $620 \mathrm{Ma}$ (Fig. 10a). Furthermore, several minor secondary peaks represented by a few grains indicate a Paleoproterozoic to Neoproterozoic cluster between $2000 \mathrm{Ma}$ (samples MO 18 and MO 24), $1980 \mathrm{Ma}$ (sample MO 22A and MO 23), and $750 \mathrm{Ma}$ (Fig. 10a). Finally, single isolated grains yield Neoarchean ages (ca 2500 Ma to $3200 \mathrm{Ma}$ ) with a small peak around $2700 \mathrm{Ma}$ (sample MO 18; Fig. 10a). It is worth to note that eo-Variscan zircon grains, i.e. slightly younger than $415 \mathrm{Ma}$, are relatively rare. This can be explained by the fact that analytic spots focus on zircon core rather than rims.

The five Late Visean to Early Serpukhovian sandstone samples exhibit comparable detrital zircon age distribution patterns whatever their structural position whether in the foreland basin or in the recumbent fold (Fig. 11). A significant peak corresponds to Early Carboniferous ages around $352 \mathrm{Ma}$ to $340 \mathrm{Ma}$ (Fig. 9) with a maximum around $350 \mathrm{Ma}$ (Fig. 10b). All these 5 samples even have ages as young as Visean at 340 Ma. The main peak yields Early Paleozoic ages at 490 to $452 \mathrm{Ma}$ with a statistical peak around $485 \mathrm{Ma}$ (Fig. 10b). Three Neoproterozoic peaks around $620 \mathrm{Ma}, 580 \mathrm{Ma}$, and $560 \mathrm{Ma}$ are also represented. On the contrary, Paleoproterozoic ages cluster around $2000 \mathrm{Ma}$ (Fig. 10b). Similar with the analytical results of Devonian samples, single isolated grains yield Neoarchean ages (ca 2500 Ma to $3200 \mathrm{Ma}$ ) with a small statistical peak around $2700 \mathrm{Ma}$ (Fig. 10b). Furthermore, Middle Devonian to Silurian ages, i.e. $440 \mathrm{Ma}$ to $380 \mathrm{Ma}$, are quite rare: 2 grains in sample MO 15, 1 grain in sample MO 16, 2 grains in sample MO 17, and 3 grains in sample MO 26.

Whatever their structural position, all the samples show two significant peaks around $350 \mathrm{Ma}$ and $450 \mathrm{Ma}$ or $480 \mathrm{Ma}$ (Fig. 11). Concerning the Ediacaran ages, the samples (MO 15, MO 16, MO 26) from the foreland basin show a peak around $559 \mathrm{Ma}$ that is shifted to $585 \mathrm{Ma}$ in the recumbent fold (MO 17, 13 Fr 52, Fig. 11). The Paleoproterozoic to Archean zircons recovered from the foreland basin define a significant cluster around $2000 \mathrm{Ma}$; on the contrary, a small peak around $2700 \mathrm{Ma}$ is shown in the population from the recumbent fold. The difference in the Precambrian age results suggests that the source that supplied the zircons may have changed slightly from the foreland basin to 
the recumbent fold. Furthermore, it is worth to note that such Precambrian rocks are presently not exposed in the Massif Central.

\section{Discussion}

The zircon age distribution spectra displayed by the Early Devonian and Late ViseanSerpukhovian terrigenous rocks of the Montagne Noire recumbent folds and foreland basin are similar (Fig. 11). According to the sedimentological data suggest a northern source, i.e. from the already deformed and metamorphosed part of the inner part of the Massif Central. In the following, the possible provenance for the pre-Devonian zircons will be discussed together for Devonian and Carboniferous rocks. Then the case of the Late Devonian to Early Carboniferous zircons will be examined separately.

\subsection{Ordovician ages}

All the analyzed samples dominantly rework Ordovician zircons ranging between 490 and 445 Ma with statistical peaks around $450 \mathrm{Ma}$ and $480 \mathrm{Ma}$ (Fig. 10). The Ordovician magmatism is well documented in the Variscan belt, where it is interpreted as a consequence of the rifting event that separated several continental stripes, such as Armorica, Mid-German Crystalline Rise, and Avalonia from the northern part of Gondwana (e.g. Matte, 2001; Martínez Catalán et al., 2004, 2009; Faure et al., 2005; Ballèvre et al., 2009). In the French Massif Central, the Ordovician magmatism is mainly represented by alkaline porphyritic granite now transformed into augen orthogneiss. These rocks were probably still buried when the Early Devonian and Carboniferous detritus were deposited in the southern part of the Montagne Noire, however, Ordovician lava and volcanic-sedimentary rocks crop out in the Para-autochthonous Unit, Lower Gneiss Unit and Upper Gneiss Unit (e.g. Pin and Marini, 1993; Alabouvette et al., 2003; Faure et al., 2009). These formations are the most likely potential source rocks that supplied the detrital zircons. A small difference appears between the microconglomerate (MO 23, 24), and the black sandstone (MO 18 and MO 22A) samples. The older 
has a zircon peak around $450 \mathrm{Ma}$, and the younger around $460 \mathrm{Ma}$ (Fig. 8). However, given the error bars of the analysis, the difference between these two peaks is not significant. Moreover, the Late Visean-Serpukhovian samples exhibit a prominent statistical peak around $480 \mathrm{Ma}$ (Fig. 9 and 10). The reasons for these differences might be due to a change in the zircon source between the Devonian and the Carboniferous.

\subsection{Neoproterozoic-Early Cambrian ages}

Most of the samples yield Neoproterozoic ages with population peaks at $620 \mathrm{Ma}, 590 \mathrm{Ma}$ and $560 \mathrm{Ma}$ (Figs. 8, 9, and 10), and the early Devonian sedimentary rocks show a smaller peak around $980 \mathrm{Ma}$, and $750 \mathrm{Ma}$ (Fig. 10). It is often argued that the magmatic rocks emplaced during the Ediacaran Cadomian orogeny (650 - 550 Ma), developed along on the margin of the Gondwana continent, and supplied the late Precambrian zircons. Such a possibility cannot be ruled out (Fig. 12). However, it is worth to note that in contrast to the Massif Armoricain, in the FMC, evidence for an Early Cambrian unconformity supporting a Cadomian orogeny is absent, although Neoproterozoic sedimentary and magmatic rocks do exist in the FMC. Indeed, NeoproterozoicCambrian magmatic rocks are recognized in most of the litho-tectonic units of the FMC. For instance, Early Cambrian acidic volcanites, known as "porphyroids" crop out in the Cévennes, Albigeois, Rouergue, Limousin. Also, ca 560 Ma diorite and quartz diorite plutons intrude the grauwacke series belonging to the Lower Gneiss Unit in the Lot series (Fig 1; Pin and Lancelot, 1978), the Cévennes Para-autochthonous Unit (Caron, 1994) or the UGU and LGU in the Rouergue area (Lafon, 1986; Lévêque, 1990). Thus several source rocks for the Tonian-Cryogenian-Edicaran-Early Cambrian detrital zircons can be speculated in the litho-tectonic units exposed north of the Montagne Noire. Presently there are no exposures of $980 \mathrm{Ma}, 750 \mathrm{Ma}$ and $620 \mathrm{Ma}$ rocks in the FMC. One possibility is that these zircons were already present in Cambrian or Ordovician detrital rocks that form the hostrocks of the magmatic rocks. In such a case, the primary source for the Neoproterozoic-Early 
Cambrian ages would be located in the Gondwana, more to the South than the MCF, and transported to the North by the river drainage network (Fig 12).

\subsection{Paleoproterozoic and Neoarchean}

In spite of absence of outcrop exposing such old rocks in the FMC, Precambrian inherited zircons are frequently found, but always with a small number of grains, as xenocrysts, in Early Paleozoic magmatic rocks (Cocherie et al., 2005; Lafon, 1986; Alexandrov, 2000; Melleton et al., 2010). A 2000 Ma age cluster is indicated in all the samples (Figs 8, 9). On the contrary, a small peak around $2700 \mathrm{Ma}$ is exhibited in the Late Visean-Serpukhovian samples. Thus, in the Montagne Noire terrigenous rocks, these grains were possibly supplied by the erosion of Early Paleozoic magmatic rocks or sedimentary formations that already contained different Paleoproterozoic and Neoarchean age populations. If those zircons were supplied by the erosion of Neoproterozoic, Cambrian or Ordovician magmatic rocks, the Paleoproterozoic and Neoarchean zircon record is an indirect evidence for a Paleoproterozoic, and even Archean, basement underneath the Variscan belt.

\subsection{Late Devonian to Early Carboniferous}

The Late Visean-Serpukhovian turbidite yields also Famennian-Tournaisian detrital zircons. Metamorphic and magmatic rocks of this age are well known in the Lower Gneiss Unit. Biotitegarnet-staurolite gneiss are well developed in the Lower Gneiss Unit where they yield monazite U-Th$\mathrm{Pb}$ ages around 365-350 Ma (Melleton et al., 2009; Do Couto et al., 2015), but metamorphic zircon ages have not been measured in these rocks. 355-350 Ma magmatic rocks, well exposed in the NW part of the FMC, correspond to the emplacement age of the biotite \pm cordierite Guéret granite (Fig 1; Cartannaz et al., 2007). These rocks are suitable sources for the Montagne Noire detrital zircons since they were already exhumed in Tournaisian-Early Visean, as documented by the fossiliferous sandstone 
and limestone series that overlies the Guéret massif (Mamet et al., 1970). Furthermore, mafic and felsic rocks belonging to the Brévenne ophiolitic series of Eastern Massif Central yielding ca $366 \pm 5$ Ma ages are also possible source rocks (Fig. 1; Pin and Paquette, 1998).

Lastly, 345 to $335 \mathrm{Ma}$ (Early and Middle Visean) magmatic rocks crop out in the NE part of the FMC, namely Montagne Bourbonnaise and Morvan areas (Fig. 1). There, several sedimentary and volcanic series dated of Tournaisian, Early and Middle Devonian by foraminifera and corals in limestone, and plants debris in terrigenous formations, are covered by the Late Visean "Tufs Anthracifères" (Peyrel and Didier, 1983; Duthou et al., 1984; Leistel and Gagny, 1984; Binon and Pin, 1989; Delfour, 1989; Pin and Duthou, 1990; Pin, 1991; Leloix et al., 1999; Faure et al., 2002; Fig 13). The various volcanic rocks, such as rhyolite, dacite, trachyte, and basaltic lava flows, pyroclastites, volcanic breccias and grauwackes are potential sources for the detrital zircons recovered in the Montagne Noire turbidite.

The NE Massif Central and Montagne Noire are presently separated by ca $250 \mathrm{~km}$ where Tournaisan-Visean rocks are presently lacking. However such a rocks might have been exposed there and eroded to supply the material recovered in the Montagne Noire foreland basin. Contemporaneous plutonic rocks are also reported in the Montagne Bourbonnaise (Fig. 13), particularly the biotite-Kfeldspar ( \pm hornblende) Bois Noirs and Mayet-de-Montagne massifs dated at 328 \pm 6 , and $328 \pm 4$ Ma, respectively (Binon and Pin, 1989). Nevertheless, it is not settled yet if plutons contemporaneous to these ones were already exposed in Late Visean.

Furthermore, the eo-Variscan rocks, either HP/LT metamorphic rocks or HT/LP gneiss and migmatites with ages ranging from 440 to $400 \mathrm{Ma}$, and 385-375 Ma, respectively are rare in the sedimentary record, both in Early Devonian and Carboniferous terrigenous rocks. A possible interpretation of this phenomenon is that the eo-Variscan deep seated metamorphic rocks were not already exposed to the surface in the Devonian or Late Variscan to Early Serpukhovian. Moreover, this scarcity may also result of selective sampling of the analyzed grains as large magmatic zircons were preferentially chosen instead of the small metamorphic rims. In a future work, detail analyses 
should be done to investigate the recrystallization rims around zircon in order to reveal the eoVariscan magmatic and metamorphic events.

On the basis on our results, a Visean reconstruction of the paleotopography of the Massif Central, showing the possible sources and drainage patterns, is proposed in Fig. 14. Moreover, as discussed above, it must not be forgotten that a part of the detrital zircon grains enclosed in the Devonian and Carboniferous sandstones may have experienced multiple reworking and recycling, as already documented for the magmatic rocks (Alexandrov, 2000; Cocherie et al., 2005; Ducassou et al., 2014; Faure et al., 2010; Melleton et al., 2010). Thus, the search for a source to the North of the Massif Central is not the only possibility. Particularly, the Proterozoic and Archean grains might have been eroded and deposited several times in the Early Paleozoic sedimentary rocks or scavenged by the magmas before their final depositions.

\section{Conclusion}

This first study of the detrital zircons deposited in the Early Devonian and the ViseanSerpukhovian detrital rocks of the Montagne Noire area shows that a wide range of detrital zircons were supplied from the northern inner part of the Variscan belt of the FMC. The zircon grains from all of the samples yielded $\mathrm{U}-\mathrm{Pb}$ age spectra ranging from Neoarchean to Late Paleozoic with several age population peaks at $2700 \mathrm{Ma}, 2000 \mathrm{Ma}, 980 \mathrm{Ma}, 750 \mathrm{Ma}, 620 \mathrm{Ma}, 590 \mathrm{Ma}, 560 \mathrm{Ma}, 480 \mathrm{Ma}, 450 \mathrm{Ma}$, and $350 \mathrm{Ma}$. The Precambrian grains recorded more complex itinerary and may be experienced a multi-recycling history. The Ordovician magmatism (around $450 \mathrm{Ma}$ ) appears as the main component of detrital zircons.

The subordinate ages of detrital zircons were 352-340 Ma with a statistical peak around 350 Ma in the Late Visean-Early Serpukhovian turbidite complies with the Tournaisian Guéret type granites emplaced at the end of the main Variscan phase, and exhumed in Early Carboniferous. Since Tournaisian magmatic rocks crop out only in the northern part of the FMC, this result complies with 
the previous conclusion inferred from sedimentological studies that the drainage pattern was southward directed (Fig. 14). Concerning the provenance of the Early to Middle Visean detritus, a northern source is also inferred on the basis of sedimentology. Presently, magmatic rocks of this age crop out only in the Montagne Bourbonnaise-Morvan area, ca $250 \mathrm{~km}$ to the North of the deposition area, suggesting also a south-directed drainage towards the Late Visean-Serpukhovian Variscan foreland basin. Nevertheless, a presently eroded, closer source cannot be ruled out.

\section{Acknowledgements}

This work is supported by the State Key Laboratory of Lithospheric Evolution, IGG-CAS, “ Strategic Priority Research Program” of the Chinese Academy of Science (XDB03010802), and the National Natural Science foundation of China (grant 41273070, 41225009, 41202079). A part of the expenses for M. F. has been also provided by UMR 7327 CNRS-Université d'Orléans. Constructive comments and suggestions by W. Franke and J.R. Martínez Catalán to improve the manuscript are deeply acknowledged.

\section{References}

Andersen, T., 2002. Correction of common lead in U-Pb analyses that do not report ${ }^{204} \mathrm{~Pb}$. Chem. Geol. 192, 59-79.

Alabouvette, B., Demange, M., Guérangé-Lozes, J., Ambert, P., 2003. Notice explicative de la carte géologique au 1:250000 de Montpellier, Bureau de Recherches Géologiques et Minières, Orléans, France.

Alexandrov, P., 2000. Géochronologie U/Pb et ${ }^{40} \mathrm{Ar} /{ }^{39} \mathrm{Ar}$ de deux segments de la chaîne varisque: le haut Limousin et les Pyrénées orientales. Ph.D. thesis, Institut National Polytechnique de Lorraine, Univ. Nancy.

Alvaro, J.J., Vizcaïno, D., 1998. Revision biostratigraphique du Cambrien moyen du versant méridional de la Montagne Noire (Languedoc, France). Bull. Soc. Géol. Fr. 169, 233-242. 
Arthaud, F., 1970. Etude tectonique et microtectonique comparée de deux domaines hercyniens: les nappes de la Montagne Noire (France) et l'anticlinorium de l'Iglesiente (Sardaigne). Ph.D. thesis, Université de Montpellier.

Ballèvre, M., Bosse, V., Ducassou, C., Pitra, P., 2009. Palaeozoic history of the Armorican Massif: models for the tectonic evolution of the suture zones. CR Géosci. 341, 174-201.

Binon, M., Pin, C., 1989. Géochronologie Rb-Sr et U-Pb des granites du Mayet-de-Montagne et des Bois Noirs, Montagne Bourbonnaise (Massif Central). Bull. Soc. Géol. Fr. 5, 695-703.

Böhm, R., 1935. Etude sur les faunes du Dévonien supérieur et du Carbonifère de la Montagne Noire. Thèse, Université de Montpellier.

Caron, C., 1994. Les minéralisations Pb-Zn associées au Paléozoïque inférieur d'Europe méridionale. Traçage isotopique $\mathrm{Pb}-\mathrm{Pb}$ des gîtes de l'Iglesiente ( $\mathrm{SW}$ Sardaigne) et des Cévennes et évolution du socle encaissant par la géochronologie U-Pb, ${ }^{40} \mathrm{Ar}-{ }^{39} \mathrm{Ar}$ et K-Ar. Université de Montpellier.

Cartannaz, C., Rolin, P., Cocherie, A., Marquer, D., Legendre, O., Fanning, C.M., Rossi, P., 2007. Characterization of wrench tectonics from dating of syn- to post-magmatism in the northwestern French Massif Central. Int. J. Earth Sci. 96, 271-287.

Cocherie, A., Boudin, T., Autran, A., Guerrot, C., Fanning, M., Laumonier, B., 2005. U-Pb zircon (ID-TIMS and SHRIMP) evidence for the early Ordovician intrusion of metagranites in the late Proterozoic Canaveilles Group of the Pyrenees and the Montagne Noire (France). B. Soc. Géol. Fr. 176, 269-282.

Cocks, L.R.M., 2000. The Early Paleozoic geography of Europe. J. Geol. Soc. London 157, 1-10.

Delfour, J., 1989. Données lithostratigraphiques et géochimiques sur le Dévono-Dinantien de la partie sud du faisceau du Morvan (nord-est du Massif central français). Géol. de la Fr. 4, 49-77.

Demange, M., 1993. Que signifie la faille des Monts de Lacaune (Montagne Noire, France)? Implications quant au problème de la patrie des nappes. CR Acad. Sci. 317, 411-418.

Dickinson, W., Gehrels, G., 2009. Use of U-Pb ages of detrital zircons to infer maximum depositional ages of strata: A test against a Colorado Plateau Mesozoic database. Earth Planet. Sci. Lett. 288, $115-125$. 
Dinis, P., Andersen, T., Machado, G., Guimarães, F., 2012. Detrital zircon U-Pb ages of a lateVariscan Carboniferous succession associated with the Porto-Tomar shear zone (West Portugal): Provenance implications. Sediment. Geol. 273-274, 19-29.

Do Couto, D., Faure, M., Augier, R., Cocherie, A., Rossi, P., Li, X-H., Lin, W., 2015. Monazite U-Th$\mathrm{Pb}$ EPMA and zircon U-Pb SIMS chronological constraints on the tectonic, metamorphic, and thermal events in the inner part of the Variscan orogen, example from the Sioule series, French Massif Central. Int. J. Earth Sci. DOI 10.1007/s00531-015-1184-0.

Ducassou, C., Poujol, M., Rufet, G., Bruguier, O., Ballèvre, M., 2014. Relief variation and erosion of the Variscan belt: detrital geochronology of the Palaeozoic sediments from the Mauges Unit (Armorican Massif, France), in: Schulmann, K., Martínez Catalán, J.R., Lardeaux, J.M., Janoušek, V., Oggiano, G. (Eds), The Variscan Orogeny: extent, timescale and the formation of the European crust. Geol. Soc. London, Spec. Publ., 405, pp. 137-167.

Duthou, J.L., Cantagrel, J.M., Didier, J., Vialette, Y., 1984. Paleozoic granitoids from the French Massif Central: age and origin studied by Rb-Sr system. Phys. Earth Planet. Int. 35, 131-144.

Engel, W., Feist, R., Franke, W., 1980-1981. Le Carbonifère anté-stéphanien de la Montagne Noire: rapports entre mise en place des nappes et sédimentation. Bulletin du BRGM. 2, 341-389.

Faure, M., Monié, P., Maluski, H., Pin, C., Leloix, C., 2002. Late Visean thermal event in the northern part of the French Massif Central. New ${ }^{40} \mathrm{Ar} /{ }^{39} \mathrm{Ar}$ and $\mathrm{Rb}-\mathrm{Sr}$ isotopic constraints on the Hercynian syn-orogenic extension. Int. J. Earth Sci. 91, 53-75.

Faure, M., Bé Mézème, E., Duguet, M., Cartier, C., Talbot, J.Y., 2005. Paleozoic tectonic evolution of Medio-Europa from the example of the French Massif Central and Massif Armoricain. J. of Virtual Explorer Electr. edition ISSN 1441-8142, 19 paper 5, 1-21.

Faure, M., Lardeaux, J.M., Ledru, P., 2009. A review of the pre-Permian geology of the French Massif Central. CR Géosci. 341, 202-213. doi:10.1016/j.crte. 2008.12. 001.

Faure, M., Cocheri,e A., Bé Mézème, E., Charles, N., Rossi, P., 2010. Middle Carboniferous crustal melting in the Variscan Belt: New insights from U-Th- $\mathrm{Pb}$ tot monazite and U-PB zircon ages of the Montagne Noire Axial Zone (southern French Massif Central). Gondwana Res. 18, 653-673.

Faure, M., Cocherie, A., Gaché, J., Esnault, C., Guerrot, C., Rossi, P., Lin, W., Li, Q., 2014. Middle Carboniferous intracontinental subduction in the Outer Zone of the Variscan Belt (Montagne Noire Axial Zone, French Massif Central): multimethod geochronological approach of 
polyphase metamorphism, in: Schulmann, K., Martínez Catalán, J.R., Lardeaux, J.M., Janoušek, V., Oggiano, G. (Eds), The Variscan Orogeny: extent, timescale and the formation of the European crust. Geol. Soc. London, Spec. Publ., 405, pp. 289-311. Doi:10.1144/SP405.2.

Fedo, C.M., Sircombe, K.N., Rainbird, R.H., 2003. Detrital zircon analysis of the sedimentary record. Rev. Mineral. Geochem. 53, 277-303.

Feist, R., 1985. Devonian stratigraphy of the southeastern Montagne Noire (France), Cour. Forsch. Inst. Senckenberg, Frankfurt, 75, pp. 331-352.

Feist, R., Schönlaub, H.P., 1973. Le passage siluro-dévonien dans la Montagne Noire orientale. CR Acad. Sci. 276, 1276-1270.

Feist, R., Galtier, J., 1985. Découverte de flores d'âge namurien probable dans le flysch à olistolithes de Cabrières (Hérault). Implications sur la durée de la sédimentation synorogénique dans la Montagne Noire (France Méridionale). CR Acad. Sci. 300, 207-212.

Fernández-Suárez, J., Gutiérrez-Alonso, G., Jeffries, T.E., 2002. The importance of along-margin terrane transport in northern Gondwana: insights from detrital zircon parentage in Neoproterozoic rocks from Iberia and Brittany. Earth Planet. Sci. Lett. 204, 75-88.

Fernández-Suárez, J., Díaz-García, F., Jeffries, T.E., Arenas, R., Abati, J., 2003. Constraints on the provenance of the uppermost allochtonous terrane of the NW Iberian Massif: inferences from detrital zircon U-Pb age. Terra Nova 15, 138-144.

Franke, W., 2000. The mid-European segment of the Variscides: tectonostratigraphic units, terrane boundaries and plate tectonic evolution, in: Franke, W., Haak, V., Oncken, O., Tanner, D. (Eds.), Orogenic Processes: Quantification and Modeling in the Variscan Belt. Geol. Soc. London, Spec. Publ., 179, pp. 35-61.

Franke, W., 2014. Topography of the Variscan orogen in Europe: failed-not collapsed. Int. J. Earth Sci. 103, 1471-1499.

Gèze, B., 1949. Etude géologique de la Montagne Noire et des Cévennes méridionales. Mém. Soc. Géol. Fr. 62, 1-215.

Lafon, J.M., 1986. Géochronologie U-Pb appliquée à deux segments du Massif central français: le Rouergue oriental et le Limousin central. Ph.D. thesis, Université de Montpellier. 
Lardeaux, J.M., Schulmann, K., Faure, M., Janousek, V., Lexa, O., Skrzypek, E., Edel, J-B., Stipska, P., 2014. The Moldanubian Zone in French Massif Central, Vosges/Schwarzwald and Bohemian Massif revisited: differences and similarities, in: Schulmann, K., Martínez Catalán, J.R.,

Lardeaux, J.M., Janoušek, V., Oggiano, G. (Eds), The Variscan Orogeny: extent, timescale and the formation of the European crust. Geol. Soc. London Spec. Publ., 405, pp. 7 44. doi:10.1144/SP405.14.

Leistel, J.M., Gagny, C., 1984. Mise en evidence d'une fosse volcano-tectonique au Viséen supérieur dans le nord-est du Massif central français. Rev. Géol. Dynamique et Géographie Physique 25, 19-31.

Leloix, C., Faure, M., Feybesse, J.L., 1999. Hercynian polyphase tectonics in the northeast French Massif Central: the closure of the Brévenne Devonian-Dinantian rift. Int. J. Earth Sci. 88, 409421.

Lévêque, M.H., 1990. Contribution de la geochronology U/Pb à la caractérisation du Magmatisme cadomien de la partie sud-est du Massif central. Ph.D. thesis, Université de Montpellier.

Linnemann, U., McNaughton, N.J., Romer, R.L., Gehmlich, M., Drost, K.. Tonk, C., 2004. West African provenance for Saxo-Thuringian (Bohemian Massif): did Armorica ever leave prePangean Gondwana? U/Pb-SHRIMP zircon evidence and the Nd-isotopic record. Int. J. Earth Sci. 93, 683-705.

Ludwig, K.R., 2003. ISOPLOT 3.0: a geochronological toolkit for Microsoft Excel, Berkeley Geochronology Center. Special Publication vol. 4.

Mamet, B., 1970. Sur une microfaune tournaisienne du Massif central (moulin du Chât-Cros, Creuse). Comp. Rend. sommaire Soc. Géol. Fr., 110-111.

Martínez Catalán, J.R.,, Fernández-Suárez, J., Jenner, G.A., Belousova, E., Díez Montes, A., 2004. Provenance constraints from detrital zircon U-Pb ages in the NW Iberian Massif: implications from Palaeozoic plate configuration and Variscan evolution. J. Geol. Soc. London 161, 463-476.

Martínez Catalán, J.R., Fernandez-Suárez, J., Meireles, C., González-Clavijo, E, Belousova, E., Saeed, A., 2008. U-Pb detrital zircon ages in synorogenic deposits of the NW Iberian Massif (Variscan belt): interplay of Devonian-Carboniferous sedimentation and thrust tectonics. Int. J. Earth Sci. $165,687-698$. 
Martínez Catalán, J.R., Arenas, R., Abati, J., Sánchez Martínez, S., Díaz García, F., Fernández

Suárez, J., González Cuadra, P., Castiñeiras, P., Gómez Barreiro, J., Díez Montes, A., González

Clavijo, E., Rubio Pascual, F.J., Andonaegui, P., Jeffries, T.E., Alcock, J.E., Díez Fernández, R., López Carmona, A., 2009. A rootless suture and the loss of the roots of a mountain chain: the Variscan belt of NW Iberia. CR Géosci. 341, 114-126.

Martínez Catalán, J.R., González Clavijo, E., Meireles, C., Díez Fernández, R., Bevis, J., 2016.

Relationships between syn-orogenic sedimentation and nappe emplacement in the hinterland of the Variscan belt in NW Iberia deduced from detrital zircons. Geol. Mag. 153, 38-60.

Matte, P., 1986. Tectonics and plate tectonics model for the Variscan belt of Europe. Tectonophysics $126,329-374$.

Matte, P., 2001. The Variscan collage and orogeny (480-290 Ma) and the tectonic definition of the Armorica microplate: a review. Terra Nova 13, 122-128.

Melleton J, Faure M, Cocherie A., 2009. Monazite U-Th/Pb chemical dating of the Early Carboniferous syn-kinematic MP/MT metamorphism in the Variscan French Massif Central. B. Soc. Géol. Fr. 180, 283-292.

Melleton, J., Cocherie, A., Faure, M., Rossi, P., 2010. Precambrian protoliths and Early Paleozoic magmatism in the French Massif Central: U-Pb data and the North Gondwana connection in the west European Variscan belt. Gondwana Res. 17, 13-25.

Okay, N., Zack, T., Okay, A., Barth, M., 2011. Sinistral transport along the Trans-European Suture Zone: detrital zircon-rutile geochronology and sandstone petrography from the Carboniferous flysch of the Pontides. Geol. Mag. 148, 380-403.

Perrin, C., Clemenzi, L., Malavieille, J., Molli, G., Taboada, A., Dominguez, S., 2013. Impact of erosion and décollements on large-scale faulting and folding in orogenic wedges: analogue models and case studies. J. Geol. Soc. London 170, 893-904.

Peyrel, J.Y., Didier, J., 1983. La famille des granites rouges du nord-est du Massif Central. Géologie de la France 2, 73-80.

Pin, C., 1991. Sr-Nd isotopic study of igneous and metasedimentary enclaves in some hercynian granitoids from the Massif Central France, in: Didier, J., Barbarin, B. (Eds), Developments in petrology vol. 13. Enclaves and granite petrology. Elsevier, Amsterdam, pp. 333-343. 
Pin, C., Lancelot, J., 1978. Un exemple de magmatisme cambrien dans le Massif Central: les métadiorites quartzitiques intrusives dans la série du Lot. B. Soc. Géol. Fr. 149, 203-208.

Pin, C., Peucat, J-J., 1986. Ages des épisodes de métamorphisme paléozoïques dans le Massif central et le Massif Armoricain. B. Soc. Géol. Fr. 8, 461-469.

Pin, C., Marini, F., 1993. Early Ordovician continental break-up in Variscan Europe: Nd-Sr isotope and trace element evidence for bimodal igneous associations of the southern Massif Central, France. Lithos 29, 177-196.

Pin, C., Paquette, J.L., 1998. A mantle-derived bimodal suite in the Hercynian Belt: Nd isotope and trace element evidence for a subduction-related rift origin of the Late Devonian Brévenne metavolcanics, Massif Central (France). Contrib. Mineral. Petr. 129, 222-238.

Pin, C., Duthou, J.L., 1990. Sources of Hercynian granitoids fromthe FrenchMassif Central: inferences from $\mathrm{Nd}$ isotopes and consequences for crustal evolution. Chem. Geol. 83, 281-296.

Poty, E., Aretz, M., Barchy, L., 2002. Stratigraphie et sédimentologie des calcaires à Productusdu Carbonifère inférieur de la Montagne Noire (Massif central, France). CR Acad. Sci. 334, 843848.

Quémart, P., Dabard, M.P., Chauvel, J.J., Feist, R., 1993. La transgression éo-dévonienne sur le Paléozoïque ancien dans la nappe du Mont Peyroux (Montagne Noire, Hérault): signature pétrographique et implications géodynamiques. CR Acad. Sci. 317, 655-661.

Roger, F., Respaut, J.P., Brunel, M., Matte, P., Paquette, J.L., 2004. Première datation U- Pb des orthogneiss oeillés de la zone axiale de la Montagne Noire (sud du Massif Central): nouveaux témoins du magmatisme ordovicien dans la chaîne Varisque. CR Géosci. 336, 19-28.

Schneider, J.L., Maas, R., Gall, J.C., Duringer, P., 1989. L'événement intraviséen dans la zone moldanubienne de la chaîne varisque d'Europe: les données des formations volcanosédimentaires dévono-dinantiennes du Massif Central français, des Vosges du Sud et de la Forêt Noire (RFA). Geol. Rundsch. 78, 555-570.

Schulmann, K., Konopásek, J., Janoušek, V., Lexa, O., Lardeaux, J.M., Edel, J.B., Stipská, P., Ulrich, S., 2009. An andean type Palaeozoic convergence in the Bohemian Massif. CR Géosci. 341, 266-286. doi :10.1016/j.crte.2008.12. 006. 
van Achterbergh, E., Ryan, C., Jackson, S., Griffin, W.L., 2001. Appendix 3 data reduction software for LA-ICP-MS, in: Sylvester, P. (Eds.), Laser-Ablation-ICPMS in the Earth Sciences. Mineralogical Association of Canada, Short Courses 29, pp239-243.

Vachard, D., Aretz, M., 2004. Biostratigraphical precision on on the early Serpukhovian (Late Mississipian) by means of carbonate algal microflora (cyanobacteria, algae and pseudo-algae) from La Serre (Montagne Noire, France). Geobios 37, 643-666.

Vizcaïno, D., Alvaro, J.J., 2001. The Cambrian ad Lower Ordovician of the southern Montagne Noire: a synthesis for the beginning of the new century. Ann. Soc. Géol. Nord 8, 185-242.

Xie, L.W., Zhang, Y.B., Zhang, H.H., Sun, J.F., Wu, F.Y., 2008. In situ simultaneous determination of trace elements, U-Pb and Lu-Hf isotopes in zircon and baddeleyite. Chin. Sci. Bull. 53, 15651573.

Figure Captions

Fig. 1. Structural map of the Variscan French Massif Central (modified from Faure et al., 2009).

Fig. 2. Structural map of the Montagne Noire with location of the dated detrital zircons samples.

Fig. 3. Lithostratigraphic log of the Paleozoic series cropping out in the Montagne Noire (modified from Arthaud, 1970; Alvaro and Vizcaïno, 1998; and Vizcaïno and Alvaro, 2001).

Fig. 4. Field picture of Devonian quartzite and Carboniferous turbidite. (a). Devonian unconformity overlying Early Ordovician sandstone (due to the Carboniferous tectonics, the unconformity is upside down); (b). Early Devonian massive white quartzite (Mur quartzeux), sample MO 18; (c).Visean-Serpukhovian turbidite with intraformational matrix-supported conglomerate; (d). General view of the Visean-Serpukhovian turbidite; (e). Close-up of D showing intraformational conglomerate with white quartz, radiolarian chert, and black siltite clasts (sample MO 17).

Fig. 5. Schematic cross section of Montagne Noire with sample location; italics: Devonian sandstone, plain: Visean-Serpukhovian sandstone. (Modified from Faure et al., 2014). 
Fig. 6. Microscope thin sections representative of dated rocks. Late Visean-Serpukhovian rocks, (a). Fine grained sandstone (13FR 52), (b). Coarse grained sandstone (MO 15) with detrital muscovite. Early Devonian rocks, (c). White quartzite with detrital zircon (MO 18), (d). Black microconglomerate (MO 24).

Fig. 7. Representative cathodoluminescence (CL) images of selected detrital zircons with a wide range in size and morphology. Most of the analyzed grains exhibit an inherited core surrounded by several recrystallization rims. The circles represent U-Pb analytical sites; Analytical numbers and ages presented below; the scale length is $100 \mu \mathrm{m}$.

Fig. 8. Cumulative probability plots of detrital zircon U-Pb ages in Early Devonian sandstone, see Figs 2 and 5, and Table 1 for location.

Fig. 9. Cumulative probability plots of detrital zircon U-Pb ages in Late Visean-Early Serpukhovian sandstone, see Figs 2 and 5, and Table 1 for location.

Fig. 10 Synthetic and comparison of the cumulative probability plots of detrital zircon $\mathrm{U}-\mathrm{Pb}$ ages from the (a). Early Devonian sandstone, (b). Late Visean-Early Serpukhovian sandstone, and (c). Histograms of all the concordant detrital zircon ages obtained in this study.

Fig. 11. Synthetic cumulative probability plots of detrital zircon U-Pb ages from the Carboniferous autochthonous foreland basin and the Carboniferous and Devonian from the recumbent folds.

Fig. 12. Schematic paleogeographic map of the main continents at $550 \mathrm{Ma}-490 \mathrm{Ma}$ showing the situation of the French Massif Central (FMC) in the northern margin of Gondwana, and the Avalonia, Mid-German Crystalline Rise (MGCR) and Armorica microcontinents separated from Gondwana in Early Ordovician. Arrows indicate the possible source areas for the detrital zircons. In the Neoproterozoic-Early Cambrian, both northern (from the Cadomian belt), and southern (from the West African craton) sources are possible. In Ordovician, due to the rifting, only a southern (i.e. 
Gondwanian) source was allowed. Note that after the Eovariscan tectonics, responsible for the rewelding of Armorica with Gondwana, the northern source was again possible (modified from Matte, 2001).

Fig. 13. Schematic geological map and lithostratigraphic logs of Devonian-Visean series recognized in NE Massif Central (modified from Leistel and Gagny, 1984; Binon and Pin, 1989; Delfour, 1989; Leloix et al., 1999). Ages of Visean plutons are from Binon and Pin (1989) for zircon ages, and A. Cocherie (personal communication) for monazite. Zr: zircon, mz: monazite. In the map, due to their limited extension, the middle Visean series (V2) have been grouped with the early Visean (V1) ones. The late Visean (V3) "Tufs Anthracifères" series unconformably covers all previous series.

Fig. 14. Schematic topographic reconstruction of the French Massif Central during the Visean and the possible sources for the detrital zircons of the Foreland basin.

Table 1. Summary of samples from the foreland basin of the Variscan Southern Massif Central in Montagne Noire

Table 2. Analytical data for the dated Devonian zircons: MO 18, MO 22A, MO 23, MO 24.

Table 3. Analytical data for the dated Carboniferous zircons: 13 FR 52, MO 15, MO 16, MO 17, MO 26. 


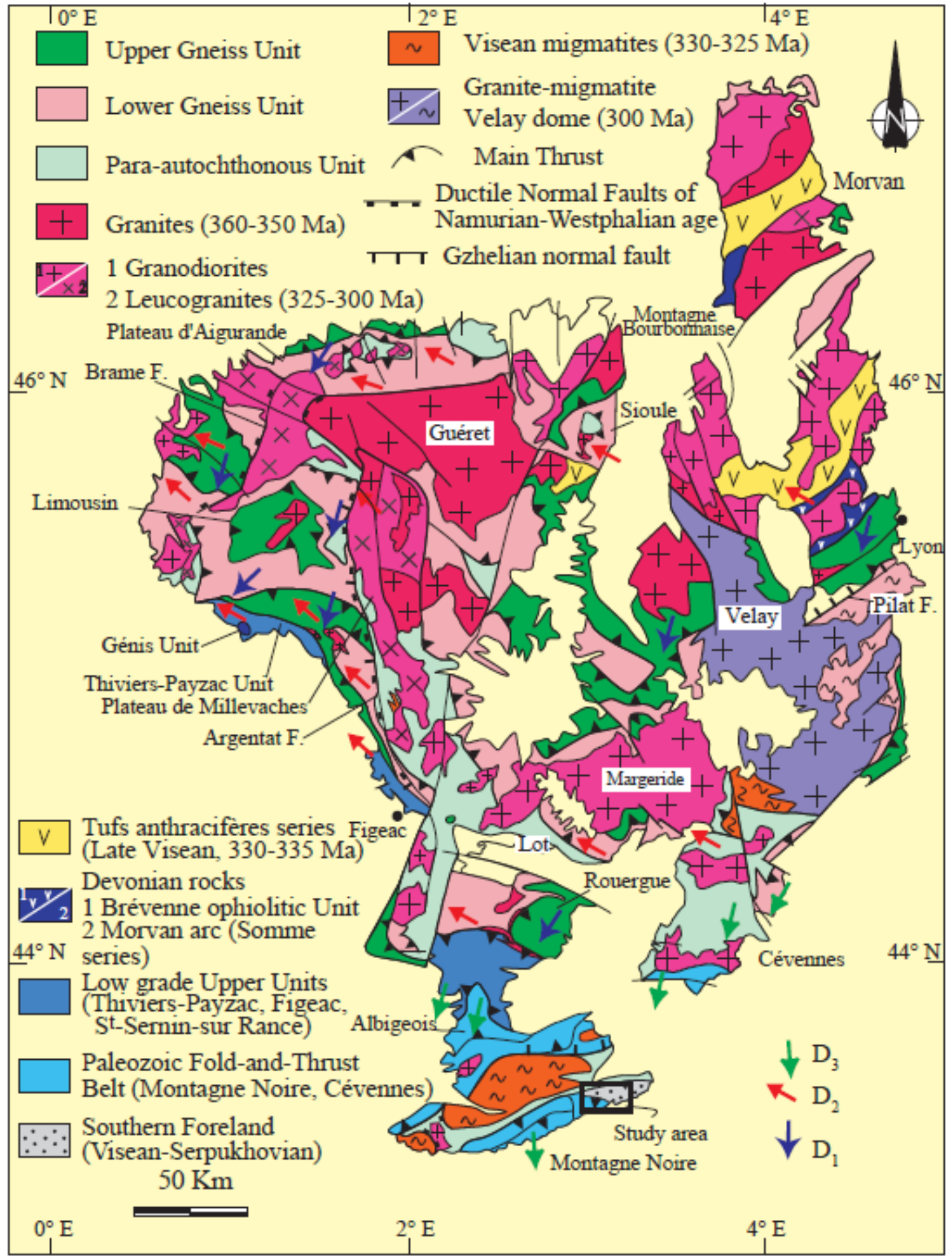

Fig. 1. Structural map of the Variscan French Massif Central (modified from Faure et al., 2009). 


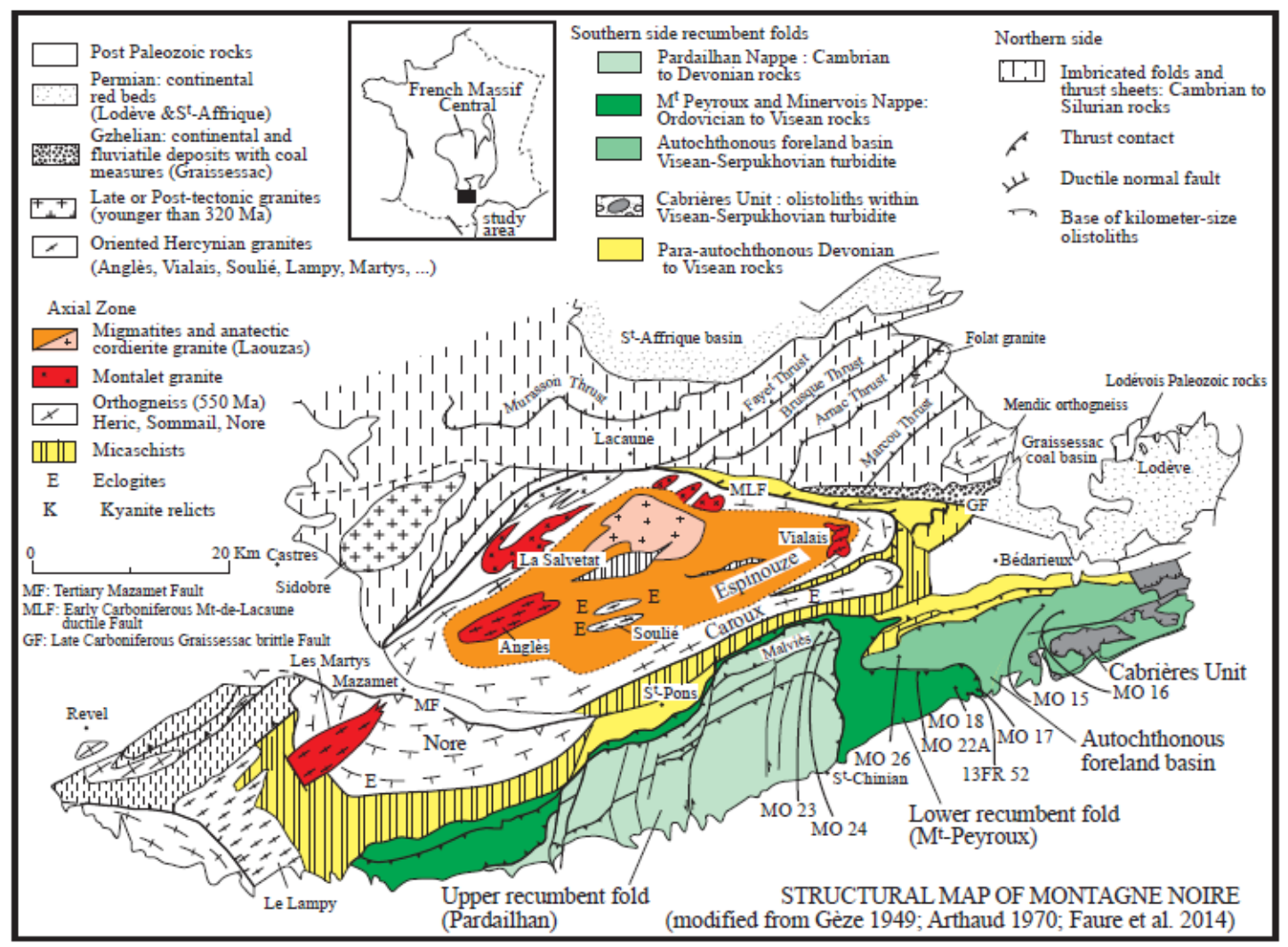

Fig. 2. Structural map of the Montagne Noire with location of the dated detrital zircons samples. 


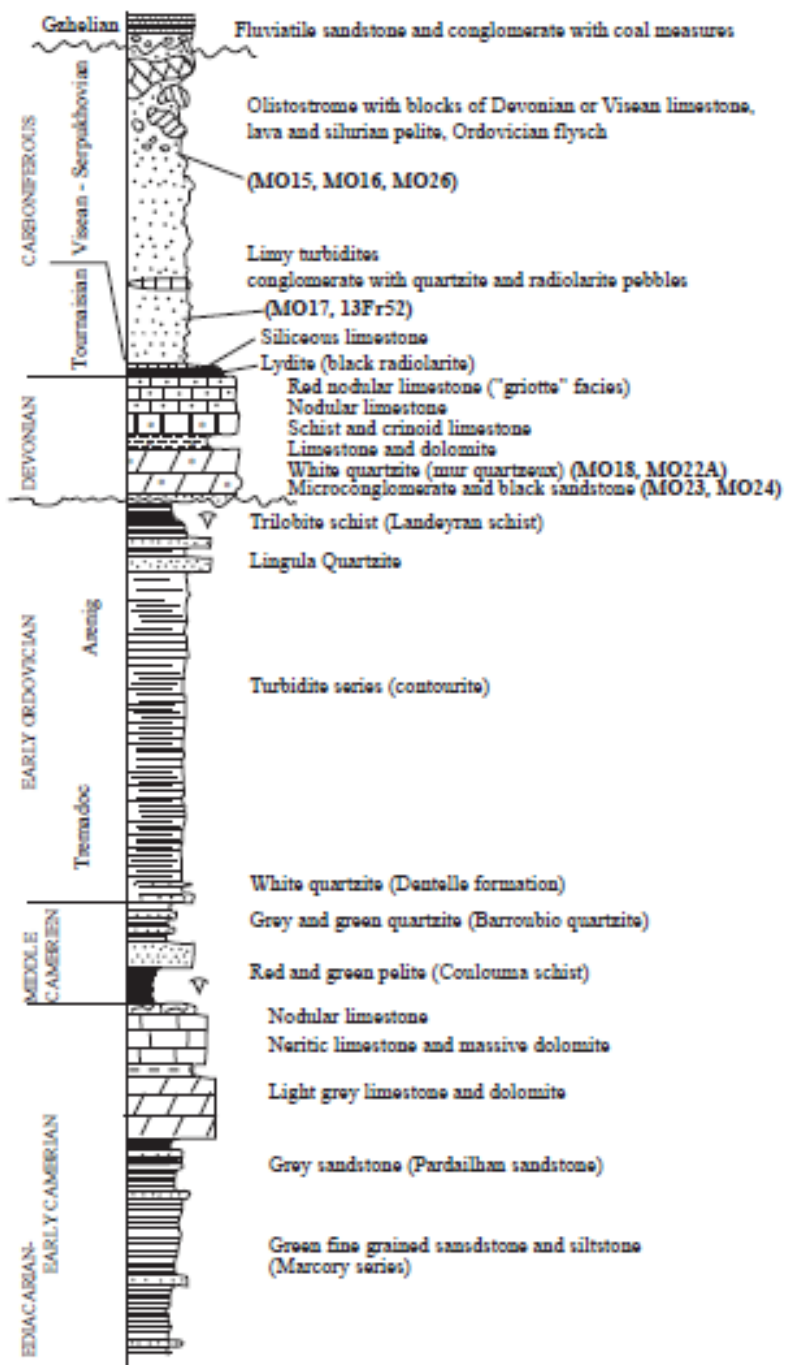

Fig. 3. Lithostratipraphic log of the Paleozoic series cropping out in the Montagne Noire (modified from Arthaud, 1970; Alvaro and Vizcaino, 1998; and Vizcaino and Alvaro, 2001). 


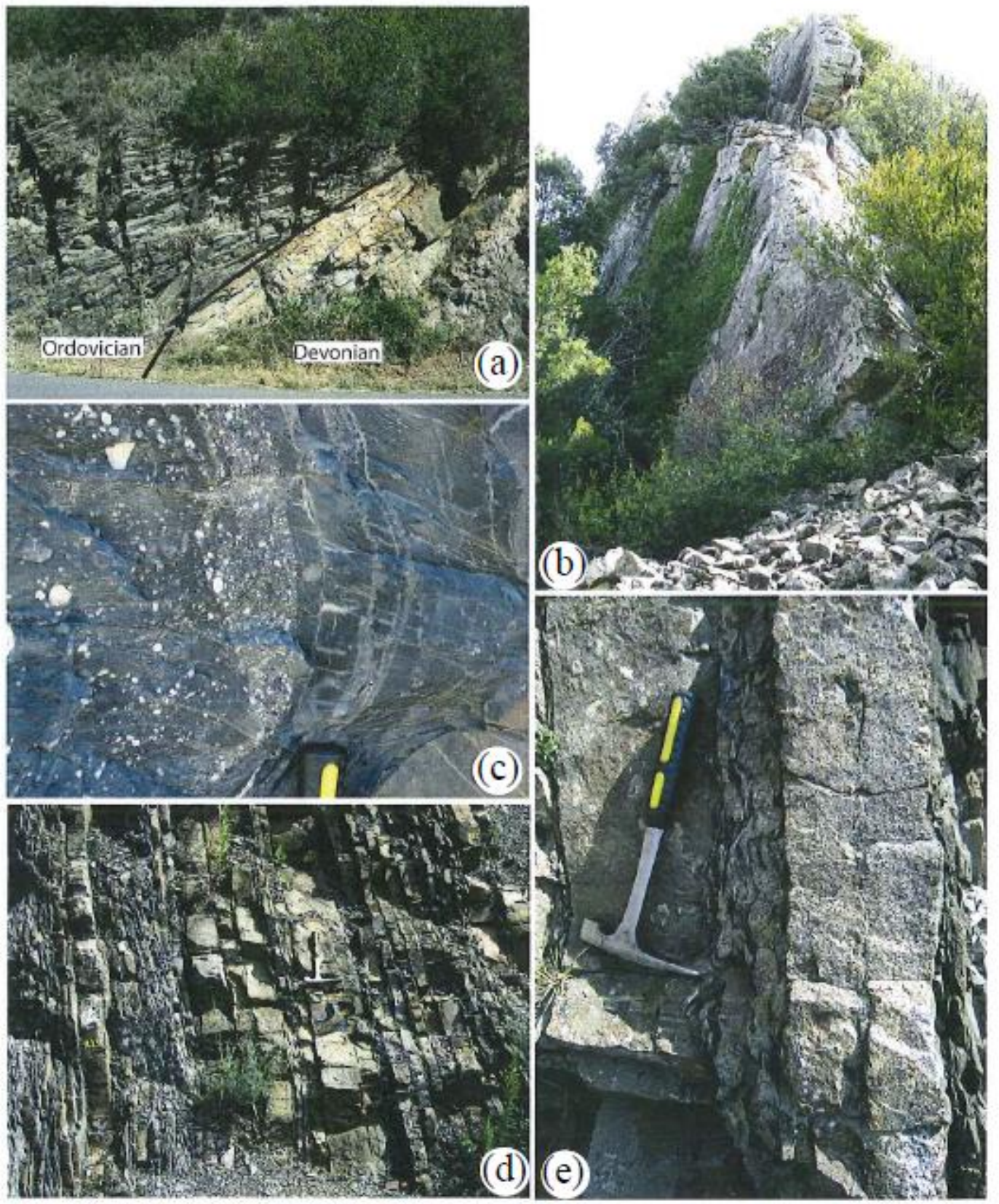

Fig. 4. Field picture of Devonian quartzite and Carboniferous turbidite. (a). Devonian unconformity overlying Early Ordovician sandstone (due to the Carboniferous tectonics, the unconformity is upside down); (b). Early Devonian massive white quartzite (Mur quartzeux), sample MO 18; (c).Visean-Serpukhovian turbidite with intraformational matrix-supported conglomerate; (d). General view of the Visean-Serpukhovian turbidite; (e). Close-up of D showing intraformational conglomerate with white quartz, radiolarian chert, and black siltite clasts (sample MO 17). 


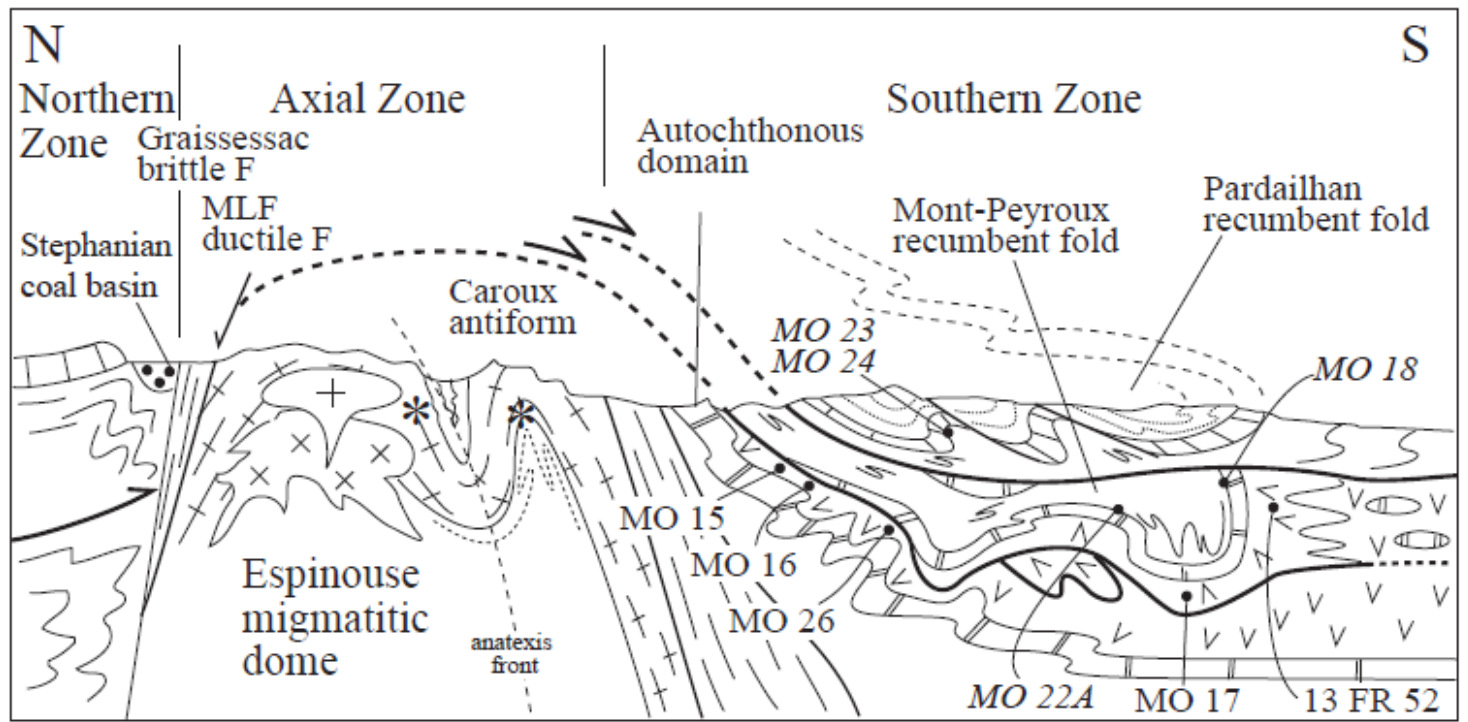

Axial Zone

Southern and Northern Zones

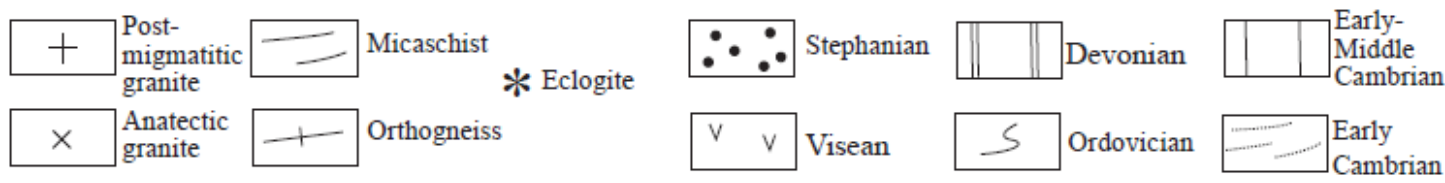

Fig. 5. Schematic cross section of Montagne Noire with sample location; italics: Devonian sandstone, plain: Visean-Serpukhovian sandstone. (Modified from Faure et al., 2014).

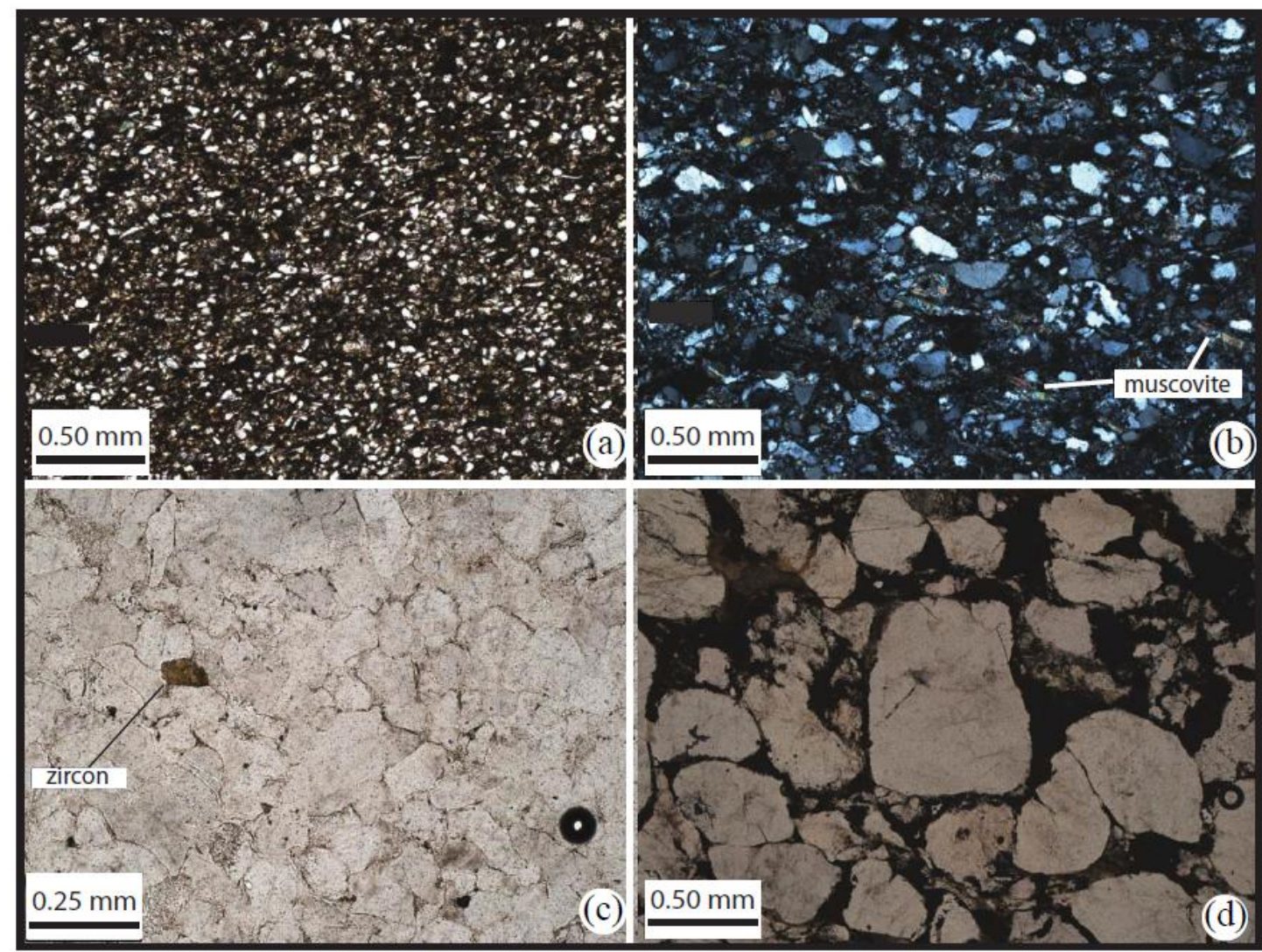

Fig. 6. Microscope thin sections representative of dated rocks. Late Visean-Serpukhovian rocks, (a). Fine grained sandstone (13FR 52), (b). Coarse grained sandstone (MO 15) with detrital muscovite. Early Devonian rocks, (c). White quartzite with detrital zircon (MO 18), (d). Black microconglomerate (MO 24). 


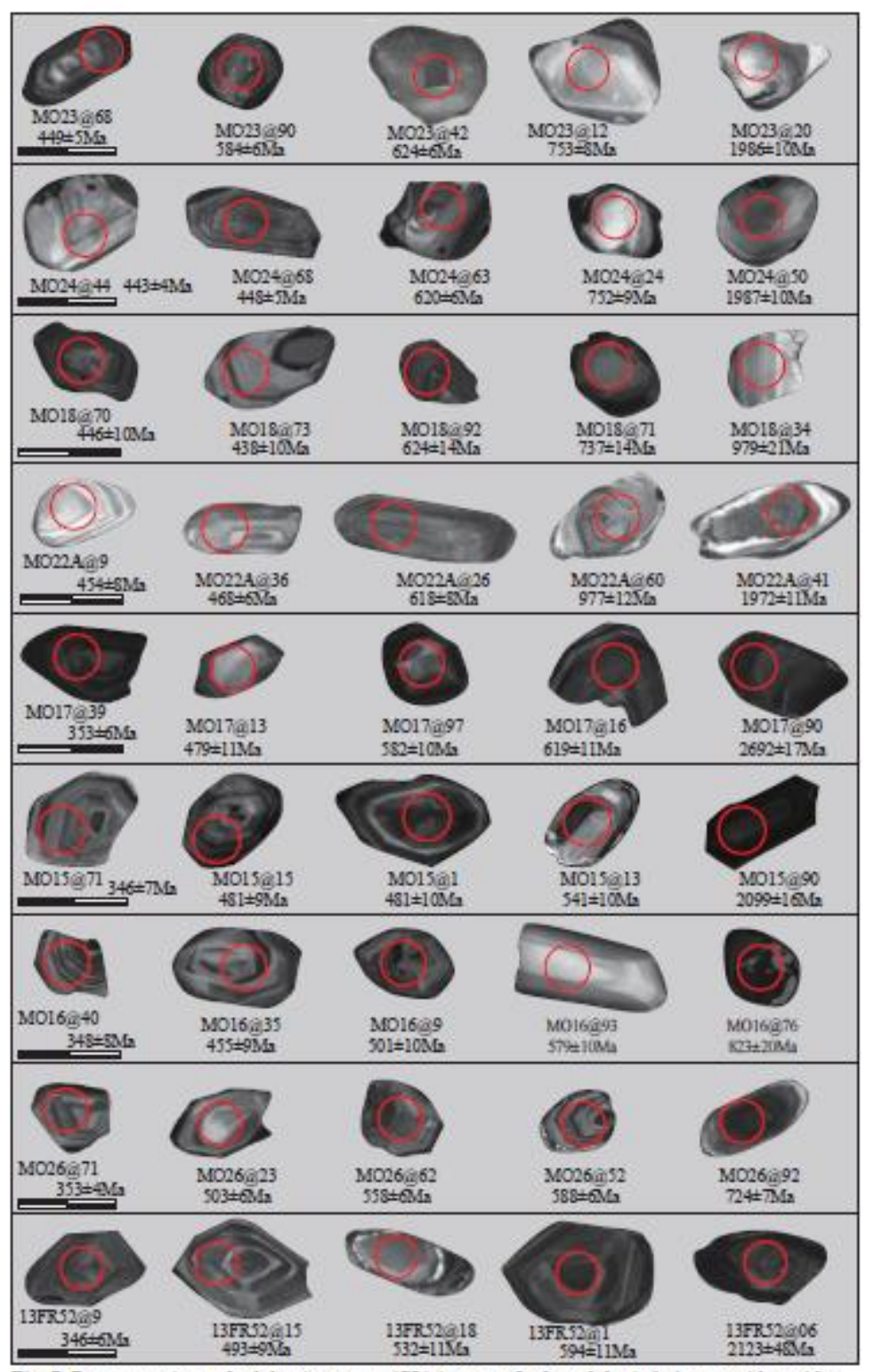

Fig. 7. Representative cathodoluminescence (CL) images of selected detrital zircons with a wide range in size and morphology. Most of the analyzed grains exhibit an inherited core surrounded by several recrystallization rims. The circles represent U-Pb analytical sites; analytical mumbers and ages presented below; the scale length is $100 \mathrm{pm}$. 

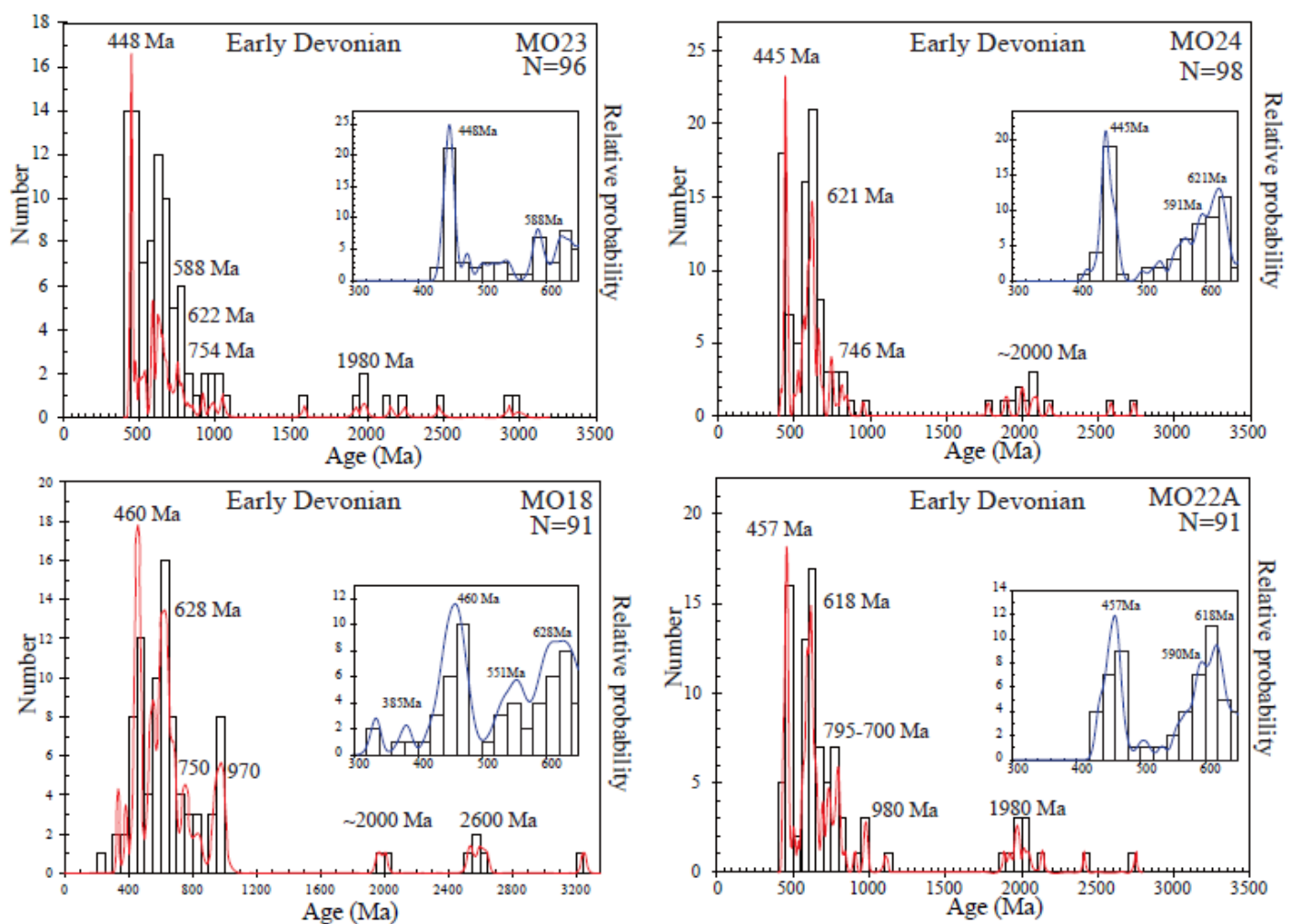

Fig. 8. Cumulative probability plots of detrital zircon U-Pb ages in Early Devonian sandstone, see Figs 2 and 5 , and Table 1 for location. 

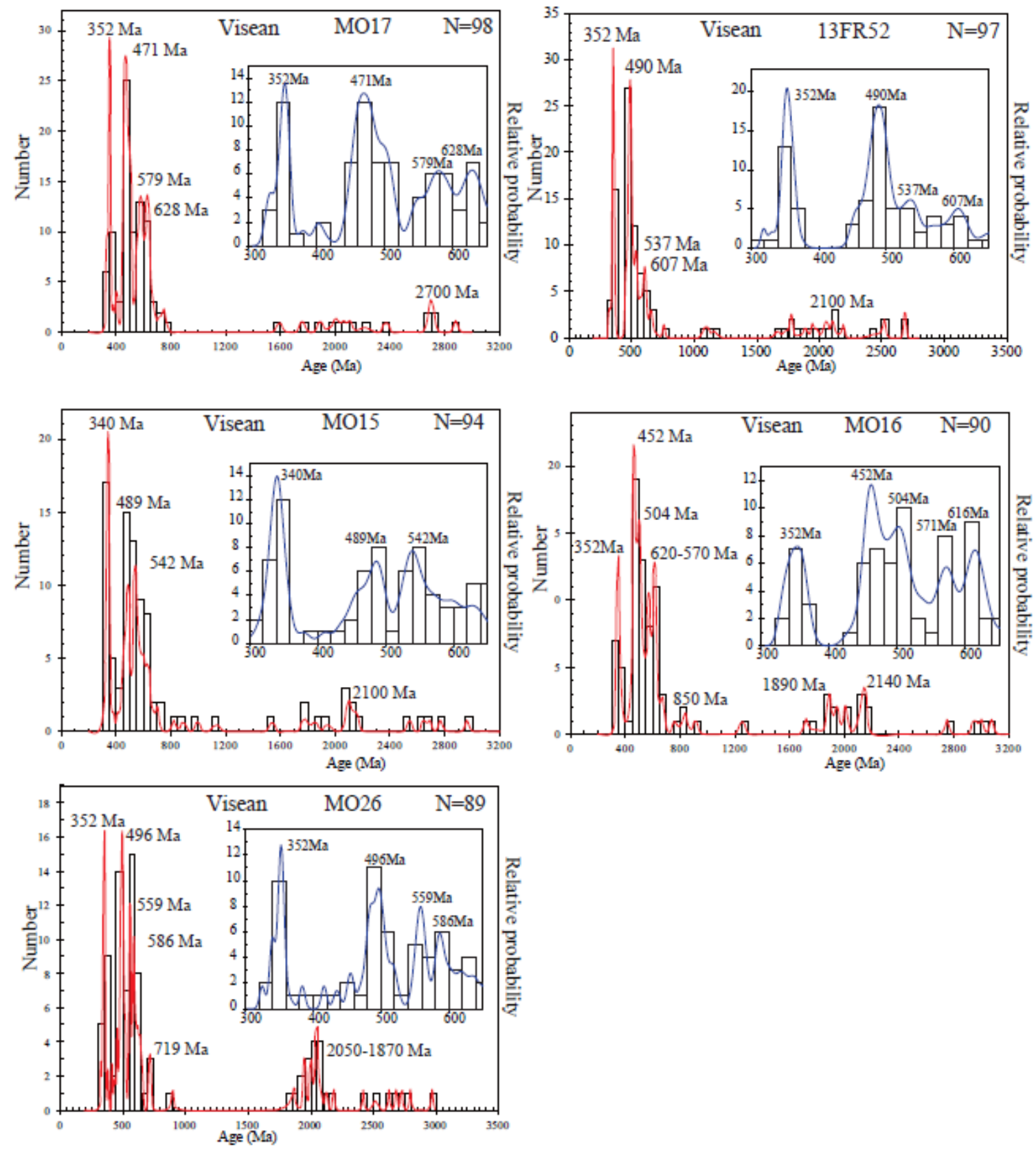

Fig. 9. Cumulative probability plots of detrital zircon U-Pb ages in Late Visean-Early Serpukhovian sandstone, see Figs 2 and 5, and Table 1 for location. 

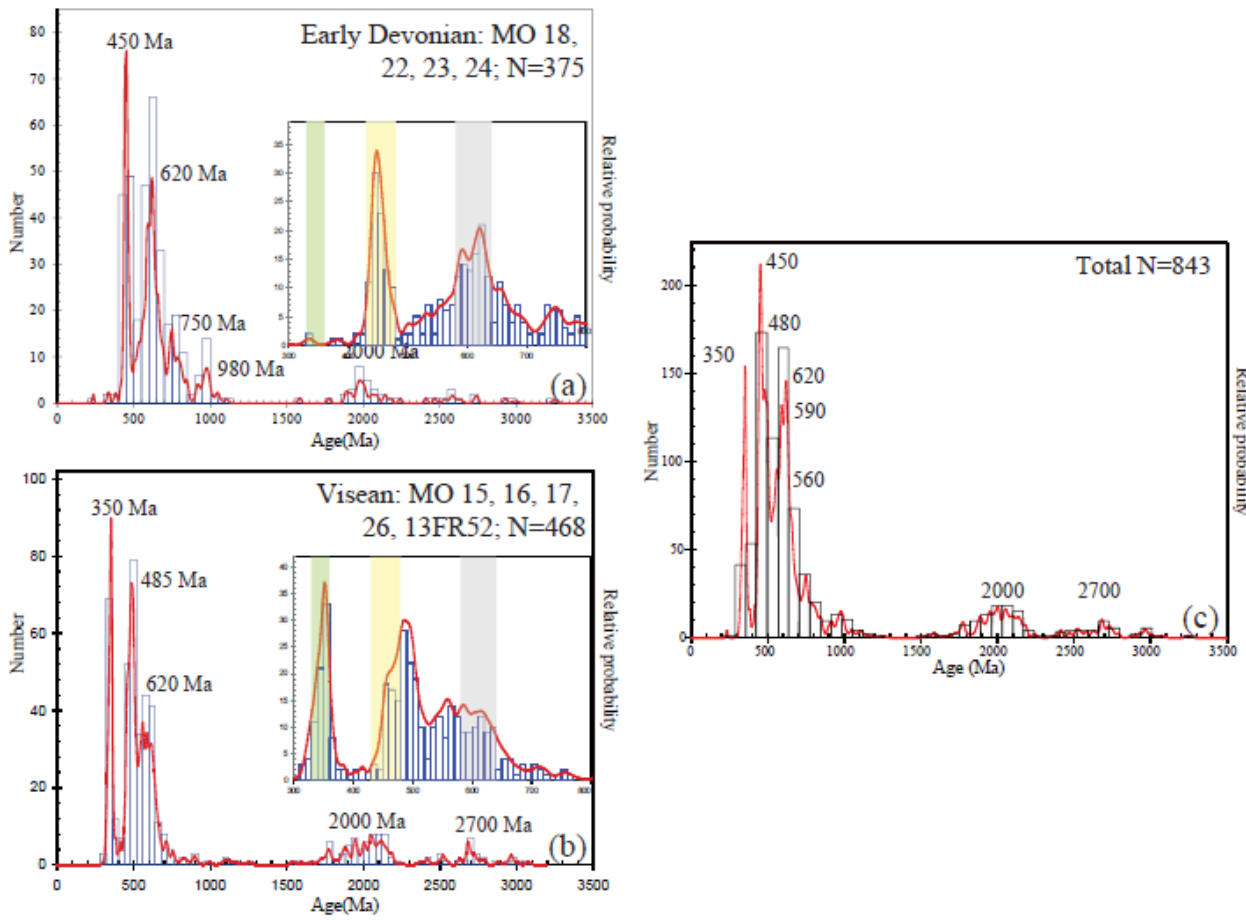

Fig. 10 Synthetic and comparison of the cumulative probability plots of detrital zircon U-Pb ages from the (a). Early Devonian sandstone, (b). Late Visean-Early Serpukhovian sandstone, and (c). Histograms of all the concordant detrital zircon ages obtained in this study. 

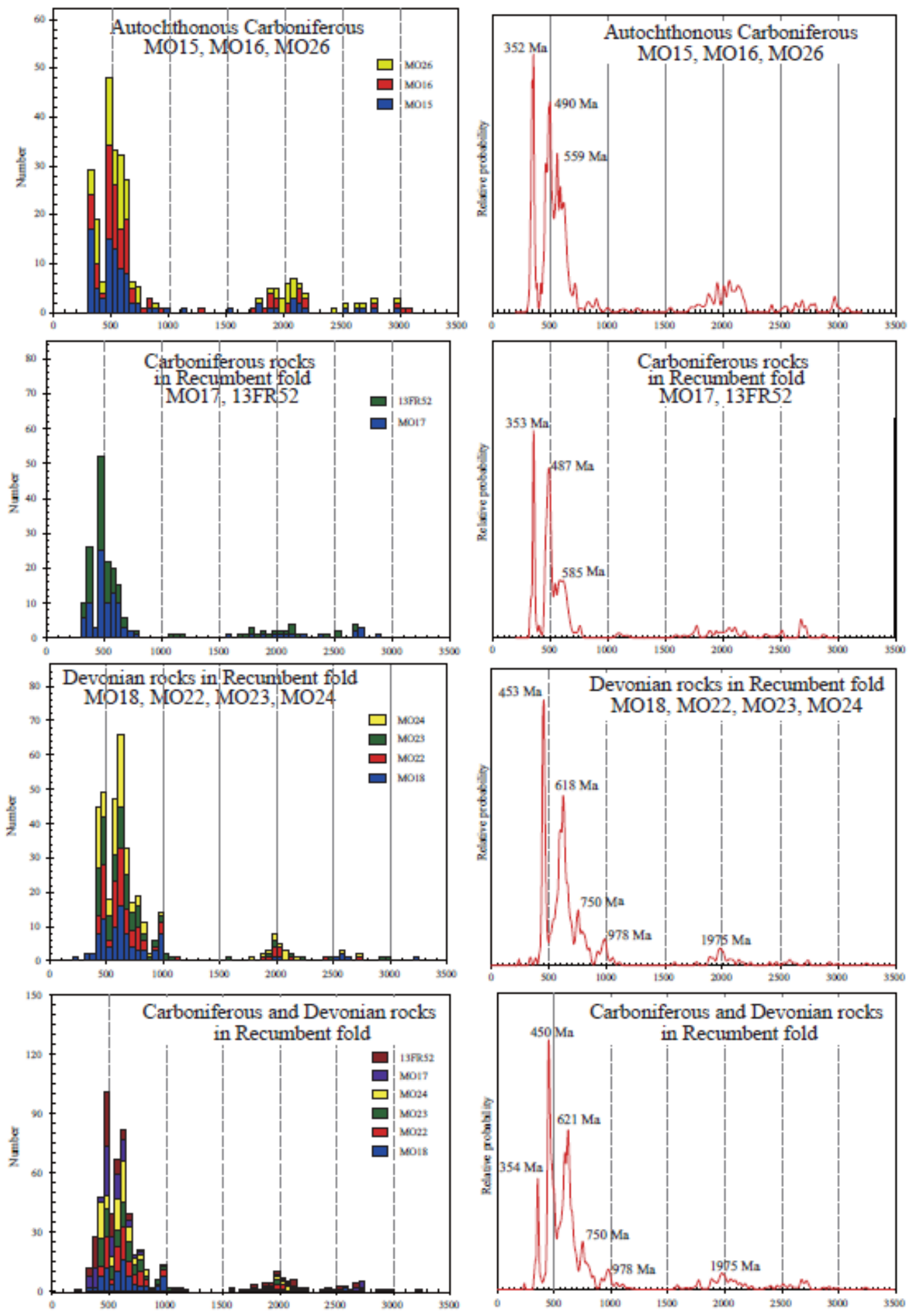

Fig. 11. Synthetic cumulative probability plots of detrital zircon U-Pb ages from the Carboniferous autochthonous foreland basin and the Carboniferous and Devonian from the recumbent folds. 
NEO - PROTEROZOIC

CAMBRIAN (ca $550 \mathrm{Ma}$ )

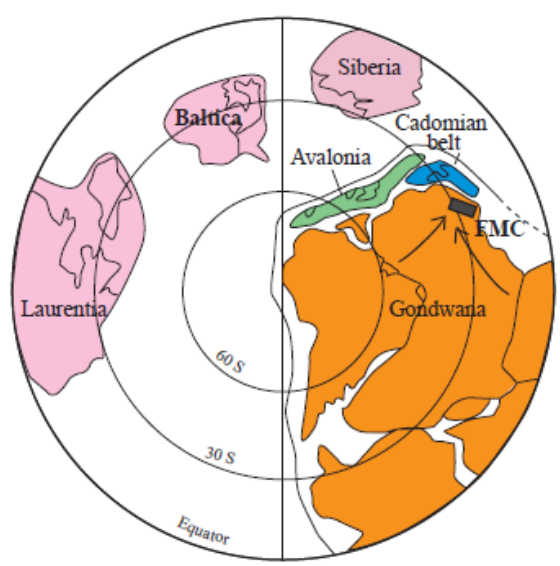

EARLY ORDOVICIEN

ca $480 \mathrm{Ma}$

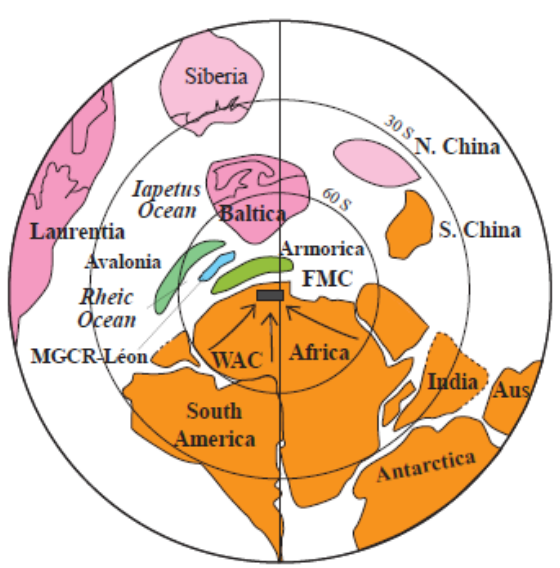

Fig. 12. Schematic paleogeographic map of the main continents at $550 \mathrm{Ma}-490 \mathrm{Ma}$ showing the situation of the French Massif Central (FMC) in the northern margin of Gondwana, and the Avalonia, Mid-German Crystalline Rise (MGCR) and Armorica microcontinents separated from Gondwana in Early Ordovician. Arrows indicate the possible source areas for the detrital zircons. In the Neoproterozoic-Early Cambrian, both northem (from the Cadomian belt), and southern (from the West African craton) sources are possible. In Ordovician due to the rifting, only a southern (i.e. Gondwanian) source was allowed. Note that after the Eovariscan tectonics, responsible for the rewelding of Armorica with Gondwana, the northern source was again possible (modified from Matte 2001). 


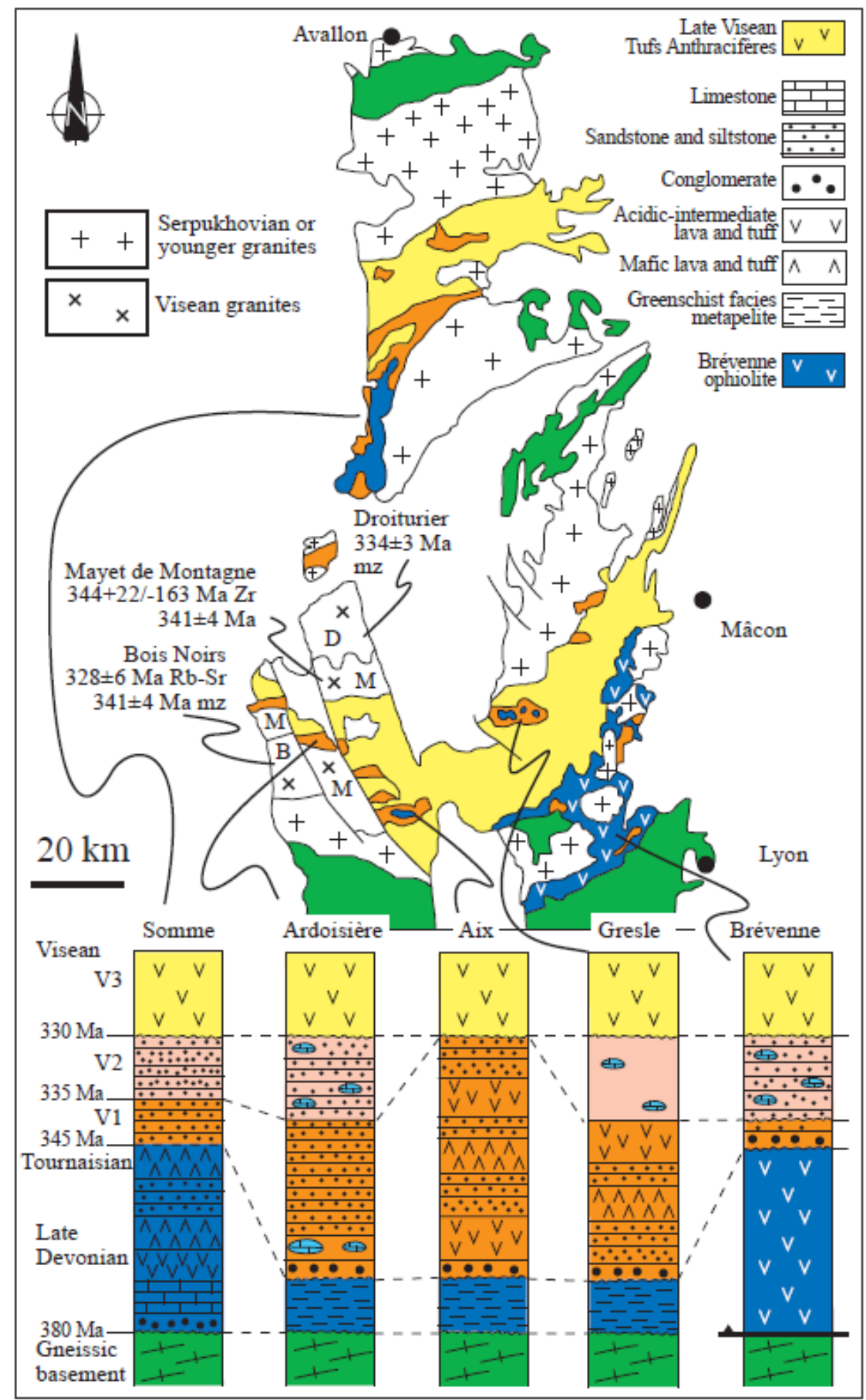

Fig. 13. Schematic geological map and lithostratigraphic logs of Devonian-Visean series recognized in NE Massif Central (modified from Leistel and Gagny, 1984; Binon and Pin, 1989; Delfour, 1989; Leloix et al., 1999). Ages of Visean plutons are from Binon and Pin (1989) for zircon ages, and A. Cocherie (personal communication) for monazite. $\mathrm{Zr}$ : zircon, $\mathrm{mz}$ : monazite. In the map, due to their limited extension the middle Visean series (V2) have been grouped with the early Visean (V1) ones. The late Visean (V3) "Tufs Anthraciferes" series unconformably covers all previous series. 


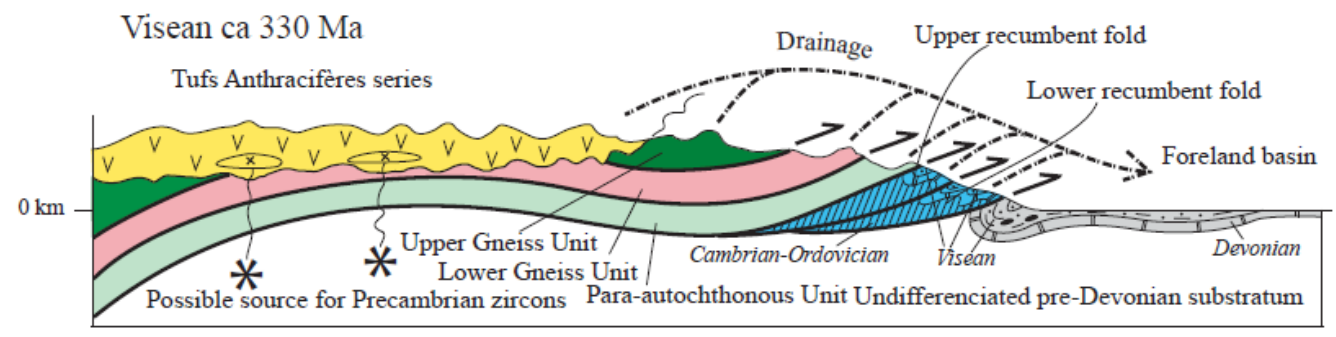

Fig. 14. Schematic topographic reconstruction of the French Massif Central during the Visean and the possible sources for the detrital zircons of the Foreland basin. 


\begin{tabular}{|c|c|c|c|c|}
\hline Stratigraphic Age & Sample $\mathrm{n}^{\circ}$ & GPS location & Petrography & Tectonic position \\
\hline \multirow[t]{3}{*}{ Serpukhovian } & MO 15 & $43^{\circ} 32^{\prime} 24.41^{\prime \prime}$ & Sandstone & Autochthonous \\
\hline & Cabrerolles & $03^{\circ} 07^{\prime} 47.30^{\prime \prime}$ & & \\
\hline & MO16 & $43^{\circ} 31^{\prime} 55.33^{\prime \prime}$ & Sandstone & Autochthonous \\
\hline \multirow[t]{3}{*}{ to } & Lenthéric & $03^{\circ} 08^{\prime} 58.50^{\prime \prime}$ & & \\
\hline & MO 26 & $43^{\circ} 31^{\prime} 38.54 "$ & Sands & Autochthonous \\
\hline & Roquebrun & $03^{\circ} 02^{\prime} 19.52^{\prime \prime}$ & & \\
\hline \multirow[t]{4}{*}{ Visean } & MO 17 & $43^{\circ} 29^{\prime} 54.72 "$ & Microconglomerate & Lower recumbent fold \\
\hline & Barrac & $03^{\circ} 06^{\prime} 57.02^{\prime \prime}$ & & (Mt-Peyroux) \\
\hline & 13 FR 52 & $43^{\circ} 28^{\prime} 37.18^{\prime \prime}$ & Sandstone & Lower recumbent fold \\
\hline & Landeyran & $03^{\circ} 04^{\prime} 03.63^{\prime \prime}$ & & (Mt-Peyroux) \\
\hline \multirow[t]{8}{*}{ Devonian } & MO 18 & $43^{\circ} 28^{\prime} 56.58^{\prime \prime}$ & Quartzite & Lower recumbent fold \\
\hline & Landeyran & $03^{\circ} 03^{\prime} 51.42^{\prime \prime}$ & & (Mt-Peyroux) \\
\hline & MO 22A & $43^{\circ} 29^{\prime} 58.8^{\prime \prime}$ & Quartzite & Lower recumbent fold \\
\hline & Roquebrun & $03^{\circ} 02^{\prime} 56.4^{\prime \prime}$ & & (Mt-Peyroux) \\
\hline & MO 23 & $43^{\circ} 32^{\prime} 31.70^{\prime \prime}$ & Microconglomerate & Upper recumbent fold \\
\hline & Malviès & $02^{\circ} 55^{\prime} 50.03^{\prime \prime}$ & & (Pardailhan) \\
\hline & MO 24 & $43^{\circ} 32^{\prime} 43.58^{\prime \prime}$ & Microconglomerate & Upper recumbent fold \\
\hline & Fenouillède & $02^{\circ} 56^{\prime} 24.13^{\prime \prime}$ & & (Pardailhan) \\
\hline
\end{tabular}




\begin{tabular}{|c|c|c|c|c|c|c|c|c|c|c|c|c|c|c|c|c|c|c|}
\hline $\mathrm{Sp}$ & $\mathrm{T}$ & $\mathrm{U}$ & $\mathrm{T}$ & & & Rat & & & & & & ges ( & Мa) & & & Dis & B & \pm \\
\hline ot & $\mathrm{h}$ & ( & $\mathrm{h}$ & 207 & \pm & 207 & \pm & 206 & \pm & 207 & \pm & 207 & \pm & 206 & \pm & con & $\mathrm{e}$ & \\
\hline No & ( & $\mathrm{p}$ & I & $\mathrm{Pb} /$ & 1 & $\mathrm{~Pb} /$ & 1 & $\mathrm{~Pb} /$ & 1 & $\mathrm{~Pb} /$ & & $\mathrm{Pb} /$ & & $\mathrm{Pb} /$ & & cor & st & 1 \\
\hline & $\mathrm{p}$ & $\mathrm{p}$ & $\mathrm{U}$ & 206 & $\sigma$ & 235 & $\sigma$ & 238 & $\sigma$ & 206 & 1 & 235 & 1 & 238 & 1 & - & A & $\sigma$ \\
\hline & $\mathrm{p}$ & $\mathrm{m}$ & & $\mathrm{Pb}$ & & $\mathrm{U}$ & & $\mathrm{U}$ & & $\mathrm{Pb}$ & $\sigma$ & & $\sigma$ & $\mathrm{U}$ & $\sigma$ & dan & $\mathrm{g}$ & \\
\hline & $\mathrm{m}$ & ) & & & & & & & & & & & & & & $\begin{array}{c}\mathrm{ce} \\
(\%)\end{array}$ & $\begin{array}{l}\mathrm{e} \\
\mathrm{s}\end{array}$ & \\
\hline $\mathrm{M}$ & 8 & 3 & 0. & 0.06 & 0. & 0.0 & 0. & 0.8 & 0. & 652 & 1 & 605 & 1 & 615 & 9 & 1.6 & 6 & 1 \\
\hline O1 & 8 & 1 & 2 & 135 & 00 & 983 & 00 & 321 & 01 & & 9 & & 1 & & & $5 \%$ & 0 & 1 \\
\hline 8- & & 1 & 8 & & 13 & 3 & 19 & 8 & 66 & & & & & & & & 5 & \\
\hline 01 & & & & & 4 & & 2 & & 5 & & & & & & & & & \\
\hline $\mathrm{M}$ & 1 & 2 & 0. & 0.07 & 0. & 0.0 & 0. & 0.8 & 0. & 875 & 1 & 494 & 1 & 568 & 2 & 14. & & \\
\hline O1 & 2 & 2 & 5 & 501 & 00 & 803 & 00 & 314 & 02 & & 2 & & 1 & & 3 & 98 & & \\
\hline 8- & 3 & 9 & 4 & & 20 & 6 & 17 & 4 & 05 & & 0 & & & & & $\%$ & & \\
\hline 02 & & & & & 6 & & 2 & & 4 & & & & & & & & & \\
\hline $\mathrm{M}$ & 1 & 3 & 0. & 0.06 & 0. & 0.1 & 0. & 1.1 & 0. & 724 & 8 & 763 & 1 & 753 & 1 & - & 7 & 1 \\
\hline O1 & 3 & 6 & 3 & 669 & 00 & 261 & 00 & 605 & 02 & & 5 & & 4 & & 8 & 1.3 & 6 & 4 \\
\hline 8- & 6 & 1 & 8 & & 12 & 5 & 24 & 6 & 02 & & & & & & & $1 \%$ & 3 & \\
\hline 03 & & & & & 8 & & 0 & & 4 & & & & & & & & & \\
\hline $\mathrm{M}$ & 9 & 9 & 0. & 0.07 & 0. & 0.1 & 0. & 1.6 & 0. & 104 & 2 & 973 & 2 & 996 & 1 & 2.3 & 9 & 2 \\
\hline $\mathrm{O} 1$ & 7 & 8 & 9 & 417 & 00 & 629 & 00 & 666 & 04 & 6 & 4 & & 0 & & 7 & $6 \%$ & 7 & 0 \\
\hline $8-$ & & & 9 & & 21 & 1 & $\begin{array}{c}36 \\
5\end{array}$ & 6 & 36 & & & & & & & & 3 & \\
\hline $\mathrm{M}$ & 4 & 1 & 0. & 0.05 & 0. & 0.0 & 0. & 0.6 & 0. & 541 & 5 & 475 & 1 & 487 & 1 & 2.5 & 4 & 1 \\
\hline $\mathrm{O} 1$ & 1 & 1 & 3 & 830 & 00 & 765 & 00 & 153 & 02 & & 0 & & 2 & & 7 & $3 \%$ & 7 & 2 \\
\hline $8-$ & & 4 & 6 & & 27 & 2 & 20 & 8 & 62 & & & & & & & & 5 & \\
\hline$\frac{05}{M}$ & 1 & 1 & 0. & 0.06 & $\frac{0}{0 .}$ & 0.0 & $\frac{0}{0 .}$ & 0.7 & $\begin{array}{c}6 \\
0 .\end{array}$ & 694 & 2 & 561 & 1 & 588 & 1 & 4.8 & 5 & 1 \\
\hline $\mathrm{O} 1$ & 0 & 6 & 6 & 259 & 00 & 909 & 00 & 851 & 02 & & 9 & & 2 & & 3 & $1 \%$ & 6 & 2 \\
\hline 8- & 2 & 3 & 2 & & 19 & 4 & 20 & 6 & 29 & & & & & & & & 1 & \\
\hline 06 & & & & & 9 & & 1 & & 4 & & & & & & & & & \\
\hline $\mathrm{M}$ & 1 & 3 & 0. & 0.05 & 0. & 0.0 & 0. & 0.7 & 0. & 583 & 1 & 591 & 1 & 589 & 9 & - & 5 & 1 \\
\hline $\mathrm{O} 1$ & 2 & 9 & 3 & 942 & 00 & 960 & 00 & 869 & 01 & & 9 & & 1 & & & 0.3 & 9 & 1 \\
\hline 8- & 7 & 7 & 2 & & 12 & 0 & $\begin{array}{c}18 \\
5\end{array}$ & 0 & 53 & & & & & & & $4 \%$ & 1 & \\
\hline 07 & & & & & 7 & & 5 & & 4 & & & & & & & & & \\
\hline $\mathrm{M}$ & 1 & 1 & 0. & 0.06 & 0. & 0.1 & 0. & 0.9 & 0. & 693 & 2 & 640 & 1 & 652 & 1 & 1.8 & 6 & 1 \\
\hline $\mathrm{O} 1$ & 2 & 8 & 6 & 256 & 00 & 043 & 00 & 004 & 02 & & 3 & & 3 & & 2 & $8 \%$ & 4 & 3 \\
\hline $\begin{array}{l}8- \\
08\end{array}$ & 2 & 9 & 5 & & $\begin{array}{c}16 \\
6\end{array}$ & 5 & $\begin{array}{c}21 \\
8\end{array}$ & 8 & $\begin{array}{c}19 \\
0\end{array}$ & & & & & & & & 0 & \\
\hline $\mathrm{M}$ & 6 & 1 & 0. & 0.05 & 0. & 0.0 & 0 . & 0.5 & 0. & 433 & 3 & 450 & 1 & 447 & 1 & - & 4 & 1 \\
\hline O1 & 4 & 9 & 3 & 551 & 00 & 722 & 00 & 531 & 01 & & 5 & & 0 & & 2 & 0.6 & 5 & 0 \\
\hline $\begin{array}{l}8- \\
09\end{array}$ & & 2 & 3 & & $\begin{array}{c}19 \\
5\end{array}$ & 4 & $\begin{array}{c}16 \\
3\end{array}$ & 2 & $\begin{array}{c}79 \\
0\end{array}$ & & & & & & & $7 \%$ & 0 & \\
\hline $\mathrm{M}$ & 5 & 2 & 0. & 0.05 & 0. & 0.0 & 0. & 0.6 & 0. & 595 & 2 & 470 & 1 & 492 & 1 & 4.6 & 4 & 1 \\
\hline $\mathrm{O} 1$ & 7 & 0 & 2 & 976 & 00 & 756 & 00 & 235 & 01 & & 8 & & 0 & & 1 & $8 \%$ & 7 & 0 \\
\hline 8- & & 3 & 8 & & 18 & 4 & 16 & 3 & 76 & & & & & & & & 0 & \\
\hline 10 & 1 & 4 & 0 & 007 & $\begin{array}{l}4 \\
0\end{array}$ & 00 & $\frac{4}{0}$ & 08 & 0 & 641 & & 551 & 1 & & & & & \\
\hline M & $\begin{array}{l}1 \\
3\end{array}$ & 4 & 0. & $\begin{array}{l}0.07 \\
101\end{array}$ & 0. & 0.0 & 0. & 0.8 & 0. & 041 & 2 & 551 & 1 & 569 & 4 & 3.2 & 5 & 1 \\
\hline $\begin{array}{l}01 \\
8-\end{array}$ & 3 & 1 & $\begin{array}{l}3 \\
2\end{array}$ & 196 & $\begin{array}{l}00 \\
47\end{array}$ & $\begin{array}{c}904 \\
5\end{array}$ & $\begin{array}{l}00 \\
31\end{array}$ & $\begin{array}{c}977 \\
2\end{array}$ & $\begin{array}{l}05 \\
38\end{array}$ & & $\begin{array}{l}3 \\
0\end{array}$ & & 8 & & 4 & $7 \%$ & 5 & 8 \\
\hline & & & & & & & & & & & & & & & & & 1 & \\
\hline
\end{tabular}




\begin{tabular}{|c|c|c|c|c|c|c|c|c|c|c|c|c|c|c|c|c|c|c|}
\hline 11 & & & & & 0 & & 0 & & 4 & & & & & & & & & \\
\hline $\mathrm{M}$ & 1 & 1 & 0 & 0.06 & 0 & 0.1 & 0. & 0.8 & 0 & \multirow{4}{*}{692} & 2 & \multirow[t]{4}{*}{625} & 1 & \multirow[t]{4}{*}{640} & \multirow{4}{*}{$\begin{array}{l}1 \\
2\end{array}$} & \multirow{4}{*}{$\begin{array}{l}2.4 \\
0 \%\end{array}$} & 6 & \multirow{4}{*}{$\begin{array}{l}1 \\
3\end{array}$} \\
\hline $\mathrm{O} 1$ & 3 & 9 & 6 & \multirow[t]{3}{*}{253} & 00 & 017 & 00 & 778 & 02 & & 5 & & 3 & & & & 2 & \\
\hline 8- & 2 & 6 & 8 & & 17 & 9 & 21 & 7 & 24 & & & & & & & & 5 & \\
\hline 12 & & & & & 5 & & 5 & & 7 & & & & & & & & & \\
\hline $\mathrm{M}$ & 4 & 1 & 0. & 0.06 & 0. & 0.1 & 0. & 1.0 & 0. & \multirow[t]{4}{*}{768} & 2 & \multirow[t]{4}{*}{689} & 1 & \multirow[t]{4}{*}{708} & \multirow{4}{*}{$\begin{array}{l}1 \\
3\end{array}$} & \multirow{4}{*}{$\begin{array}{l}2.7 \\
6 \%\end{array}$} & 6 & \multirow{4}{*}{$\begin{array}{l}1 \\
4\end{array}$} \\
\hline $\mathrm{O} 1$ & 6 & 6 & 2 & 480 & 00 & 128 & 00 & 081 & 02 & & 4 & & 4 & & & & 8 & \\
\hline 8- & & 4 & 8 & & 17 & 0 & 24 & 8 & 53 & & & & & & & & 9 & \\
\hline 13 & & & & & 8 & & 0 & & 8 & & & & & & & & & \\
\hline $\mathrm{M}$ & 8 & 1 & 0. & 0.06 & 0. & 0.1 & 0. & 1.2 & 0. & 918 & 2 & 816 & 1 & 844 & 1 & 3.4 & 8 & 1 \\
\hline $\mathrm{O} 1$ & 2 & 1 & 7 & 964 & 00 & 350 & 00 & 968 & 03 & & 6 & & 7 & & 6 & $3 \%$ & 1 & 7 \\
\hline $\begin{array}{l}8- \\
14\end{array}$ & & 5 & 1 & & $\begin{array}{c}20 \\
9\end{array}$ & 1 & 30 & 5 & $\begin{array}{c}56 \\
3\end{array}$ & & & & & & & & 6 & \\
\hline$\frac{14}{M}$ & 6 & 9 & 0 & & 9 & 0.1 & $\frac{1}{0}$ & 1.6 & $\begin{array}{l}3 \\
0\end{array}$ & 985 & 2 & 101 & 2 & 100 & 1 & & 9 & 2 \\
\hline O1 & $\begin{array}{l}0 \\
7\end{array}$ & 0 & $\begin{array}{l}0 . \\
7\end{array}$ & $\begin{array}{l}0.07 \\
196\end{array}$ & $\begin{array}{c}0 . \\
00\end{array}$ & $\begin{array}{l}0.1 \\
706\end{array}$ & 00 & $\begin{array}{l}1.0 \\
940\end{array}$ & $\begin{array}{l}0 . \\
04\end{array}$ & & 5 & 6 & 1 & 6 & $\begin{array}{l}1 \\
7\end{array}$ & 3.0 & 8 & 5 \\
\hline 8- & & & 4 & & 21 & 6 & 38 & 0 & 56 & & & & & & & $5 \%$ & 5 & \\
\hline 15 & & & & & 1 & & 3 & & 0 & & & & & & & & & \\
\hline $\mathrm{M}$ & 9 & 1 & 0. & 0.06 & 0. & 0.1 & 0. & 0.8 & 0 & 700 & 3 & 638 & 1 & 652 & 1 & 2.1 & 6 & 1 \\
\hline $\mathrm{O} 1$ & 1 & 2 & 7 & 275 & 00 & 039 & 00 & 998 & 02 & & 2 & & 4 & & 5 & $9 \%$ & 3 & 4 \\
\hline 8- & & 6 & 2 & & 21 & 6 & 23 & 3 & 79 & & & & & & & & 8 & \\
\hline 16 & & & & & 2 & & 7 & & 7 & & & & & & & & & \\
\hline $\mathrm{M}$ & 6 & 3 & 0. & 0.06 & 0. & 0.0 & 0. & 0.7 & 0. & 516 & 8 & 551 & 1 & 544 & 1 & - & 5 & 1 \\
\hline $\mathrm{O} 1$ & 6 & 4 & 1 & 182 & 00 & 896 & 00 & 644 & 01 & & 6 & & 1 & & 4 & 1.2 & 5 & 1 \\
\hline 8- & & 6 & 9 & & 14 & 6 & 17 & 7 & 63 & & & & & & & $7 \%$ & 1 & \\
\hline 17 & & & & & 5 & & 9 & ) & 8 & & & & & & & & & \\
\hline $\mathrm{M}$ & 8 & 1 & 0. & 0.05 & 0. & 0.0 & 0. & 0.5 & 0. & 534 & 3 & 444 & 1 & 459 & 1 & 3.3 & 4 & 1 \\
\hline $\mathrm{O} 1$ & 9 & 8 & 4 & 810 & 00 & 712 & 00 & 709 & 01 & & 3 & & 0 & & 1 & $8 \%$ & 4 & 0 \\
\hline 8- & & 1 & 9 & & 19 & 4 & 15 & 3 & 76 & & & & & & & & 4 & \\
\hline 18 & & & & & 5 & & 9 & & 6 & & & & & & & & & \\
\hline $\mathrm{M}$ & 2 & 6 & 0. & 0.06 & 0. & 0.1 & 0. & 0.8 & 0. & 638 & 4 & 620 & 1 & 624 & 2 & 0.6 & 6 & 1 \\
\hline O1 & 0 & 5 & 3 & 096 & 00 & 009 & 00 & 489 & 03 & & 7 & & 6 & & 0 & $5 \%$ & 2 & 6 \\
\hline 8- & & & 0 & & 28 & 8 & 27 & 8 & 58 & & & & & & & & 0 & \\
\hline 19 & & & & & 0 & & 1 & & 7 & & & & & & & & & \\
\hline M & 2 & 5 & 0. & 0.06 & 0 & 0.0 & 0. & 0.9 & 0. & 866 & 1 & 605 & 1 & 663 & 8 & 9.5 & 6 & 1 \\
\hline $\mathrm{O} 1$ & 4 & 1 & 4 & 790 & 00 & 984 & 00 & 217 & 01 & & 7 & & 1 & & & $9 \%$ & 0 & 1 \\
\hline 8- & 9 & 8 & 8 & 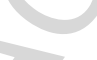 & 12 & 2 & 18 & 3 & 53 & & & & & & & & 5 & \\
\hline 20 & & & & 2 & 5 & & 6 & & 5 & & & & & & & & & \\
\hline $\mathrm{M}$ & 1 & 4 & 0 & 0.06 & 0 & 0.1 & 0. & 1.1 & 0 & 835 & 5 & 782 & 2 & 796 & 2 & 1.7 & 7 & 2 \\
\hline $\mathrm{O} 1$ & 9 & 6 & 4 & 692 & 00 & 289 & 00 & 900 & 05 & & 4 & & 1 & & 6 & $9 \%$ & 8 & 1 \\
\hline 8- & & & 1 & & 34 & 5 & 36 & 5 & 68 & & & & & & & & 2 & \\
\hline 21 & & & & & 4 & & 9 & & 9 & & & & & & & & & \\
\hline $\mathrm{M}$ & 2 & 5 & 0. & 0.07 & 0 & 0.1 & 0. & 1.0 & 0. & 977 & 4 & 644 & 1 & 723 & 2 & 12. & & \\
\hline $\mathrm{O} 1$ & 8 & 4 & 5 & 168 & 00 & 049 & 00 & 378 & 04 & & 2 & & 7 & & 1 & 27 & & \\
\hline 8- & & & 3 & & 32 & 8 & 29 & 2 & 25 & & & & & & & $\%$ & & \\
\hline 22 & & & & & 3 & & 1 & & 3 & & & & & & & & & \\
\hline $\mathrm{M}$ & 1 & 1 & 0. & 0.07 & 0. & 0.1 & 0. & 1.1 & 0. & 820 & 1 & 627 & 1 & 670 & 3 & 6.8 & 6 & 1 \\
\hline $\mathrm{O} 1$ & 4 & 5 & 9 & 909 & 00 & 037 & 00 & 320 & 02 & & 6 & & 4 & & 5 & $6 \%$ & 2 & 4 \\
\hline 8- & 6 & 9 & 2 & & 21 & 8 & 22 & 2 & 75 & & 1 & & & & & & 7 & \\
\hline 23 & & & & & 3 & & 4 & & 2 & & & & & & & & & \\
\hline $\mathrm{M}$ & 5 & 1 & 0. & 0.05 & 0. & 0.0 & 0. & 0.5 & 0. & 454 & 4 & 455 & 1 & 455 & 1 & 0.0 & 4 & 1 \\
\hline O1 & 7 & 2 & 4 & 604 & 00 & 731 & 00 & 653 & 02 & & 5 & & 1 & & 4 & $0 \%$ & 5 & 1 \\
\hline 8- & & 1 & 7 & & 23 & 4 & 18 & 1 & 19 & & & & & & & & 5 & \\
\hline 24 & & & & & 7 & & 2 & & 9 & & & & & & & & & \\
\hline $\mathrm{M}$ & 9 & 6 & 0. & 0.06 & 0. & 0.0 & 0. & 0.3 & 0. & 293 & 1 & 234 & 5 & 239 & 8 & 2.1 & 2 & 5 \\
\hline
\end{tabular}




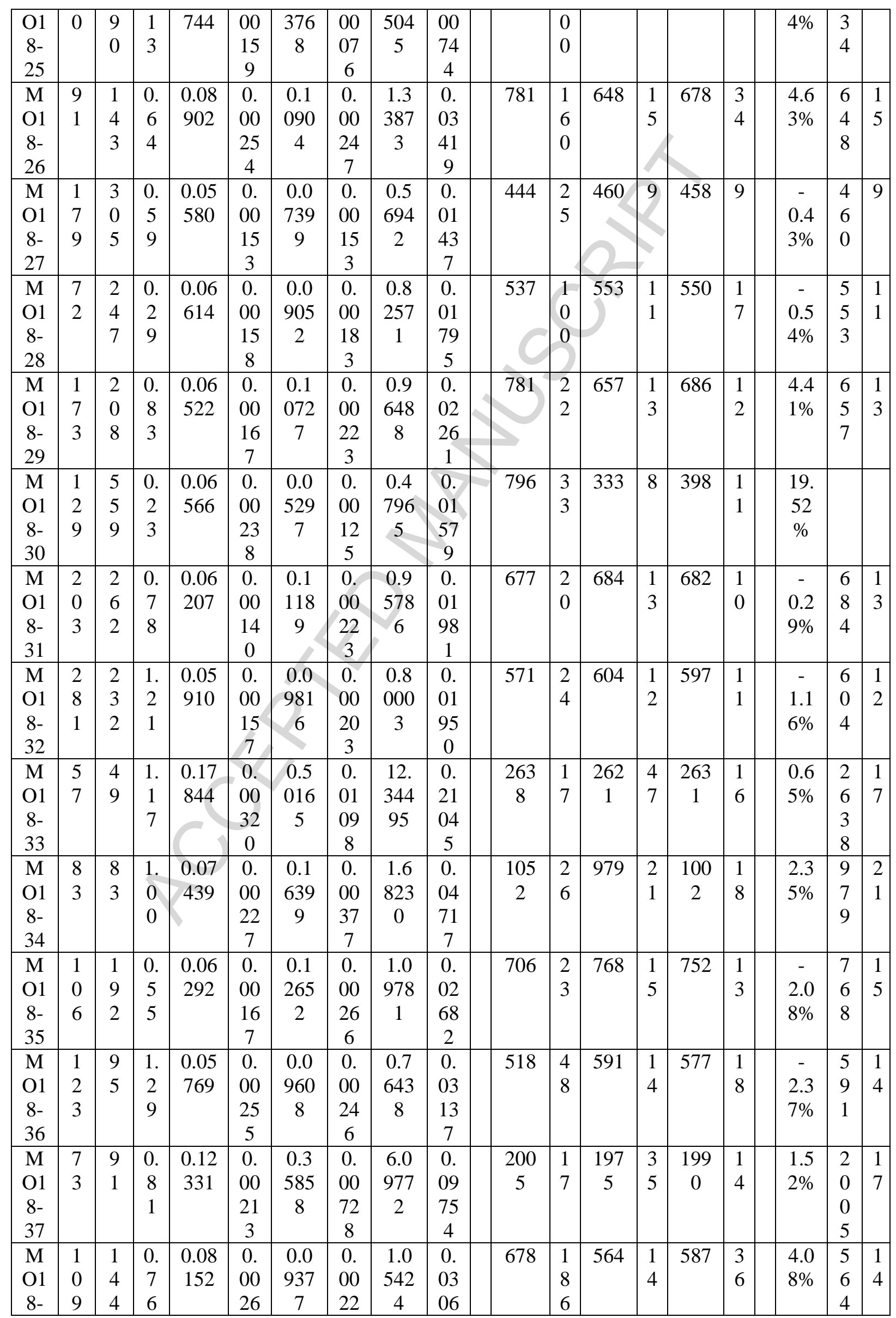




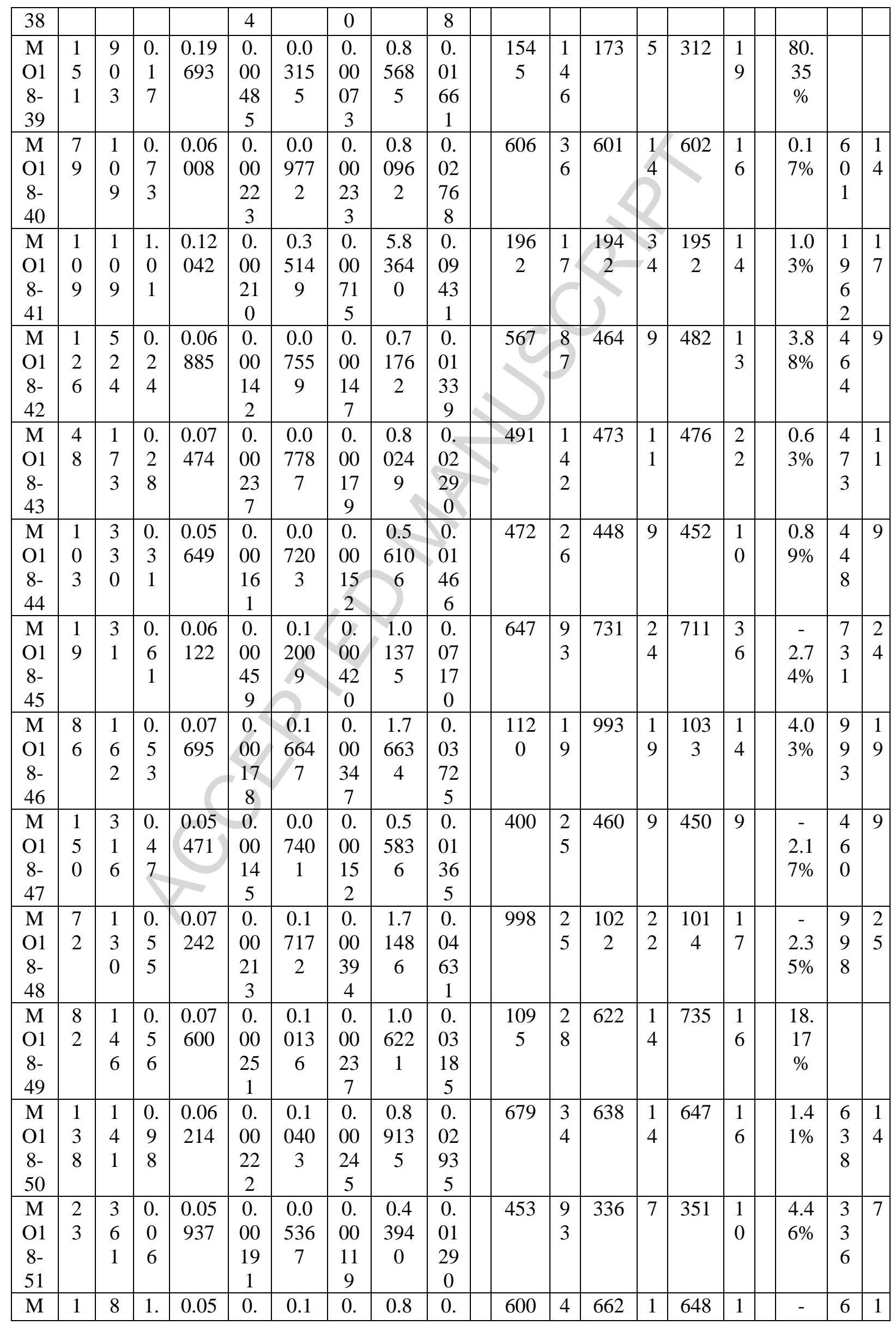




\begin{tabular}{|c|c|c|c|c|c|c|c|c|c|c|c|c|c|c|c|c|c|c|}
\hline $\begin{array}{l}\mathrm{O} 1 \\
8- \\
52\end{array}$ & $\begin{array}{l}0 \\
3\end{array}$ & 5 & $\begin{array}{l}2 \\
0\end{array}$ & 991 & $\begin{array}{c}00 \\
25 \\
9\end{array}$ & $\begin{array}{c}081 \\
0\end{array}$ & $\begin{array}{c}00 \\
28 \\
1\end{array}$ & $\begin{array}{c}930 \\
1\end{array}$ & $\begin{array}{c}03 \\
56 \\
3\end{array}$ & & 4 & & 6 & & 9 & $\begin{array}{l}2.1 \\
1 \%\end{array}$ & $\begin{array}{l}6 \\
2\end{array}$ & 6 \\
\hline $\begin{array}{c}\mathrm{M} \\
\mathrm{O} 1 \\
8- \\
53\end{array}$ & $\begin{array}{l}8 \\
9\end{array}$ & $\begin{array}{l}2 \\
3 \\
1\end{array}$ & $\begin{array}{l}0 . \\
3 \\
9\end{array}$ & $\begin{array}{c}0.06 \\
300\end{array}$ & $\begin{array}{c}0 . \\
00 \\
20 \\
3\end{array}$ & $\begin{array}{c}0.0 \\
740 \\
5\end{array}$ & $\begin{array}{c}0 . \\
00 \\
16 \\
7 \\
\end{array}$ & $\begin{array}{c}0.6 \\
433 \\
0\end{array}$ & $\begin{array}{c}0 . \\
01 \\
88 \\
7\end{array}$ & 708 & $\begin{array}{l}2 \\
9\end{array}$ & 461 & $\begin{array}{l}1 \\
0\end{array}$ & 504 & $\begin{array}{l}1 \\
2\end{array}$ & $\begin{array}{l}9.3 \\
3 \%\end{array}$ & $\begin{array}{l}4 \\
6 \\
1\end{array}$ & $\begin{array}{l}1 \\
0\end{array}$ \\
\hline $\begin{array}{c}\mathrm{M} \\
\mathrm{O} 1 \\
8- \\
54\end{array}$ & $\begin{array}{l}8 \\
4\end{array}$ & $\begin{array}{l}2 \\
5 \\
6\end{array}$ & $\begin{array}{l}0 . \\
3 \\
3\end{array}$ & $\begin{array}{l}0.05 \\
749\end{array}$ & $\begin{array}{c}0 . \\
00 \\
17 \\
1\end{array}$ & $\begin{array}{c}0.0 \\
858 \\
8\end{array}$ & $\begin{array}{c}0 . \\
00 \\
18 \\
5\end{array}$ & $\begin{array}{c}0.6 \\
808 \\
2\end{array}$ & $\begin{array}{c}0 . \\
01 \\
86 \\
7\end{array}$ & 510 & $\begin{array}{l}2 \\
8\end{array}$ & 531 & $\begin{array}{l}1 \\
1\end{array}$ & 527 & $\begin{array}{l}1 \\
1\end{array}$ & $\begin{array}{c}- \\
0.7 \\
5 \%\end{array}$ & $\begin{array}{l}5 \\
3 \\
1\end{array}$ & $\begin{array}{l}1 \\
1\end{array}$ \\
\hline $\begin{array}{l}\mathrm{M} \\
\mathrm{O} 1 \\
8- \\
55\end{array}$ & $\begin{array}{l}1 \\
2 \\
4\end{array}$ & $\begin{array}{l}1 \\
2 \\
1\end{array}$ & $\begin{array}{l}1 . \\
0 \\
3\end{array}$ & $\begin{array}{l}0.07 \\
210\end{array}$ & $\begin{array}{c}0 . \\
00 \\
20 \\
1 \\
\end{array}$ & $\begin{array}{c}0.1 \\
593 \\
3\end{array}$ & $\begin{array}{c}0 . \\
00 \\
35 \\
3\end{array}$ & $\begin{array}{c}1.5 \\
838 \\
3\end{array}$ & $\begin{array}{c}0 . \\
04 \\
05 \\
5\end{array}$ & 989 & $\begin{array}{l}2 \\
3\end{array}$ & 953 & $\begin{array}{l}2 \\
0\end{array}$ & 964 & $\begin{array}{l}1 \\
6\end{array}$ & $\begin{array}{l}1.1 \\
5 \%\end{array}$ & $\begin{array}{l}9 \\
5 \\
3\end{array}$ & $\begin{array}{l}2 \\
0\end{array}$ \\
\hline $\begin{array}{l}\mathrm{M} \\
\mathrm{O} 1 \\
8- \\
56 \\
\end{array}$ & $\begin{array}{l}9 \\
1\end{array}$ & $\begin{array}{l}2 \\
3 \\
7\end{array}$ & $\begin{array}{l}0 . \\
3 \\
8\end{array}$ & $\begin{array}{c}0.06 \\
646\end{array}$ & $\begin{array}{c}0 . \\
00 \\
31 \\
0\end{array}$ & $\begin{array}{c}0.0 \\
767 \\
5\end{array}$ & $\begin{array}{c}0 . \\
00 \\
21 \\
2 \\
\end{array}$ & $\begin{array}{c}0.7 \\
032 \\
8\end{array}$ & $\begin{array}{c}0 . \\
02 \\
98 \\
9\end{array}$ & 624 & $\begin{array}{l}1 \\
7 \\
9\end{array}$ & 473 & $\begin{array}{l}1 \\
3\end{array}$ & 500 & $\begin{array}{l}3 \\
0\end{array}$ & $\begin{array}{l}5.7 \\
1 \%\end{array}$ & $\begin{array}{l}4 \\
7 \\
3\end{array}$ & $\begin{array}{l}1 \\
3\end{array}$ \\
\hline $\begin{array}{l}\mathrm{M} \\
\mathrm{O} 1 \\
8- \\
57 \\
\end{array}$ & $\begin{array}{l}7 \\
7\end{array}$ & $\begin{array}{l}1 \\
3 \\
3\end{array}$ & $\begin{array}{l}0 . \\
5 \\
8\end{array}$ & $\begin{array}{l}0.04 \\
974\end{array}$ & $\begin{array}{c}0 . \\
00 \\
64 \\
6 \\
\end{array}$ & $\begin{array}{c}0.0 \\
761 \\
9\end{array}$ & $\begin{array}{c}0 . \\
00 \\
39 \\
4 \\
\end{array}$ & $\begin{array}{c}0.5 \\
225 \\
1\end{array}$ & $\begin{array}{c}0 . \\
06 \\
40 \\
0 \\
\end{array}$ & 183 & $\begin{array}{l}1 \\
8 \\
0\end{array}$ & 473 & $\begin{array}{l}2 \\
4\end{array}$ & 427 & $\begin{array}{l}4 \\
3\end{array}$ & $\begin{array}{c}- \\
9.7 \\
3 \%\end{array}$ & $\begin{array}{l}4 \\
7 \\
3\end{array}$ & $\begin{array}{l}2 \\
4\end{array}$ \\
\hline $\begin{array}{c}\mathrm{M} \\
\mathrm{O} 1 \\
8- \\
58\end{array}$ & $\begin{array}{l}9 \\
7\end{array}$ & $\begin{array}{l}1 \\
8 \\
9\end{array}$ & $\begin{array}{l}0 . \\
5 \\
1\end{array}$ & $\begin{array}{c}0.06 \\
120\end{array}$ & $\begin{array}{c}0 . \\
00 \\
19 \\
5\end{array}$ & $\begin{array}{c}0.1 \\
063 \\
8\end{array}$ & $\begin{array}{c}0 . \\
00 \\
23 \\
7\end{array}$ & $\begin{array}{c}0.8 \\
976 \\
0\end{array}$ & $\begin{array}{c}0 . \\
02 \\
63 \\
6\end{array}$ & 646 & $\begin{array}{l}3 \\
0\end{array}$ & 652 & $\begin{array}{l}1 \\
4\end{array}$ & 650 & $\begin{array}{l}1 \\
4\end{array}$ & $\begin{array}{l}0.3 \\
1 \%\end{array}$ & $\begin{array}{l}6 \\
5 \\
2\end{array}$ & $\begin{array}{l}1 \\
4\end{array}$ \\
\hline $\begin{array}{c}\mathrm{M} \\
\mathrm{O} 1 \\
8- \\
59\end{array}$ & $\begin{array}{l}5 \\
7\end{array}$ & $\begin{array}{l}3 \\
5 \\
3\end{array}$ & $\begin{array}{c}. \\
1 \\
6\end{array}$ & $\begin{array}{c}0.06 \\
607\end{array}$ & $\begin{array}{c}0 . \\
00 \\
15 \\
0\end{array}$ & $\begin{array}{c}0.1 \\
225 \\
0\end{array}$ & $\begin{array}{c}0 . \\
00 \\
24 \\
7\end{array}$ & $\begin{array}{c}1.1 \\
159 \\
3\end{array}$ & $\begin{array}{c}0 . \\
02 \\
33 \\
0\end{array}$ & 809 & $\begin{array}{l}2 \\
0\end{array}$ & 745 & $\begin{array}{l}1 \\
4\end{array}$ & 761 & $\begin{array}{l}1 \\
1\end{array}$ & $\begin{array}{l}2.1 \\
5 \%\end{array}$ & $\begin{array}{l}7 \\
4 \\
5\end{array}$ & $\begin{array}{l}1 \\
4\end{array}$ \\
\hline $\begin{array}{l}\mathrm{M} \\
\mathrm{O} 1 \\
8- \\
60\end{array}$ & $\begin{array}{l}4 \\
3\end{array}$ & $\begin{array}{l}1 \\
8 \\
4\end{array}$ & $\begin{array}{l}0 . \\
2 \\
3\end{array}$ & $\begin{array}{l}0.05 \\
896\end{array}$ & $\begin{array}{c}0 . \\
00 \\
19 \\
3\end{array}$ & $\begin{array}{c}0.1 \\
036 \\
8\end{array}$ & $\begin{array}{c}0 . \\
00 \\
23 \\
3\end{array}$ & $\begin{array}{c}0.8 \\
428 \\
0\end{array}$ & $\begin{array}{c}0 . \\
02 \\
55 \\
0\end{array}$ & 566 & $\begin{array}{l}3 \\
1\end{array}$ & 636 & $\begin{array}{l}1 \\
4\end{array}$ & 621 & $\begin{array}{l}1 \\
4\end{array}$ & $\begin{array}{c}- \\
2.3 \\
6 \%\end{array}$ & $\begin{array}{l}6 \\
3 \\
6\end{array}$ & $\begin{array}{l}1 \\
4\end{array}$ \\
\hline $\begin{array}{l}\mathrm{M} \\
\mathrm{O} 1 \\
8- \\
61 \\
\end{array}$ & $\begin{array}{l}6 \\
6\end{array}$ & $\begin{array}{l}2 \\
1 \\
0\end{array}$ & $\begin{array}{l}0 . \\
3 \\
1\end{array}$ & $\begin{array}{l}0.05 \\
809\end{array}$ & $\begin{array}{c}0 . \\
00 \\
19 \\
6\end{array}$ & $\begin{array}{c}0.0 \\
720 \\
8\end{array}$ & $\begin{array}{c}0 . \\
00 \\
16 \\
4\end{array}$ & $\begin{array}{c}0.5 \\
772 \\
1\end{array}$ & $\begin{array}{c}0 . \\
01 \\
78 \\
4 \\
\end{array}$ & 533 & $\begin{array}{l}3 \\
2\end{array}$ & 449 & $\begin{array}{l}1 \\
0\end{array}$ & 463 & $\begin{array}{l}1 \\
1\end{array}$ & $\begin{array}{l}3.1 \\
2 \%\end{array}$ & $\begin{array}{l}4 \\
4 \\
9\end{array}$ & $\begin{array}{l}1 \\
0\end{array}$ \\
\hline $\begin{array}{l}\mathrm{M} \\
\mathrm{O} 1 \\
8- \\
62 \\
\end{array}$ & $\begin{array}{l}3 \\
3\end{array}$ & $\begin{array}{l}7 \\
8\end{array}$ & $\begin{array}{l} \\
4 \\
2\end{array}$ & $\begin{array}{c}0.06 \\
003\end{array}$ & $\begin{array}{c}0 . \\
00 \\
43 \\
7 \\
\end{array}$ & $\begin{array}{c}0.1 \\
403 \\
5\end{array}$ & $\begin{array}{c}0 . \\
00 \\
45 \\
5 \\
\end{array}$ & $\begin{array}{c}1.1 \\
616 \\
1\end{array}$ & $\begin{array}{c}0 . \\
08 \\
06 \\
0 \\
\end{array}$ & 605 & $\begin{array}{l}9 \\
5\end{array}$ & 847 & $\begin{array}{l}2 \\
6\end{array}$ & 783 & $\begin{array}{l}3 \\
8\end{array}$ & $\begin{array}{c}- \\
7.5 \\
6 \%\end{array}$ & $\begin{array}{l}8 \\
4 \\
7\end{array}$ & $\begin{array}{l}2 \\
6\end{array}$ \\
\hline $\begin{array}{l}\mathrm{M} \\
\mathrm{O} 1 \\
8- \\
63\end{array}$ & $\begin{array}{l}1 \\
5 \\
4\end{array}$ & $\begin{array}{l}2 \\
2 \\
9\end{array}$ & $\begin{array}{l}0 . \\
6 \\
7\end{array}$ & $\begin{array}{c}0.05 \\
658\end{array}$ & $\begin{array}{c}0 . \\
00 \\
27 \\
1\end{array}$ & $\begin{array}{c}0.0 \\
947 \\
3\end{array}$ & $\begin{array}{c}0 . \\
00 \\
24 \\
4\end{array}$ & $\begin{array}{c}0.7 \\
389 \\
9\end{array}$ & $\begin{array}{c}0 . \\
03 \\
31 \\
9\end{array}$ & 475 & $\begin{array}{l}5 \\
5\end{array}$ & 583 & $\begin{array}{l}1 \\
4\end{array}$ & 562 & $\begin{array}{l}1 \\
9\end{array}$ & $\begin{array}{c}- \\
3.6 \\
0 \%\end{array}$ & $\begin{array}{l}5 \\
8 \\
3\end{array}$ & $\begin{array}{l}1 \\
4\end{array}$ \\
\hline $\begin{array}{l}\mathrm{M} \\
\mathrm{O} 1 \\
8- \\
64 \\
\end{array}$ & $\begin{array}{l}2 \\
5\end{array}$ & $\begin{array}{l}1 \\
5\end{array}$ & $\begin{array}{l}1 . \\
5 \\
9\end{array}$ & $\begin{array}{l}0.05 \\
785\end{array}$ & $\begin{array}{c}0 . \\
01 \\
22 \\
8\end{array}$ & $\begin{array}{c}0.1 \\
224 \\
6\end{array}$ & $\begin{array}{c}0 . \\
00 \\
89 \\
6\end{array}$ & $\begin{array}{c}0.9 \\
765 \\
6\end{array}$ & $\begin{array}{c}0 . \\
19 \\
89 \\
6 \\
\end{array}$ & 524 & $\begin{array}{l}3 \\
2 \\
2\end{array}$ & 745 & $\begin{array}{l}5 \\
1\end{array}$ & 692 & $\begin{array}{l}1 \\
0 \\
2\end{array}$ & $\begin{array}{c}- \\
7.1 \\
1 \%\end{array}$ & $\begin{array}{l}7 \\
4 \\
5\end{array}$ & $\begin{array}{l}5 \\
1\end{array}$ \\
\hline $\begin{array}{l}\text { M } \\
\text { O1 } \\
8-\end{array}$ & $\begin{array}{l}4 \\
4\end{array}$ & $\begin{array}{l}1 \\
8 \\
8 \\
\end{array}$ & $\begin{array}{l}0 . \\
2 \\
4\end{array}$ & $\begin{array}{l}0.05 \\
875\end{array}$ & $\begin{array}{c}0 . \\
00 \\
34 \\
\end{array}$ & $\begin{array}{c}0.0 \\
600 \\
8 \\
\end{array}$ & $\begin{array}{c}0 . \\
00 \\
17 \\
\end{array}$ & $\begin{array}{c}0.4 \\
866 \\
3 \\
\end{array}$ & $\begin{array}{c}0 . \\
02 \\
64 \\
\end{array}$ & 558 & $\begin{array}{l}6 \\
9\end{array}$ & 376 & $\begin{array}{l}1 \\
1\end{array}$ & 403 & $\begin{array}{l}1 \\
8\end{array}$ & $\begin{array}{l}7.1 \\
8 \%\end{array}$ & $\begin{array}{l}3 \\
7 \\
6 \\
\end{array}$ & $\begin{array}{l}1 \\
1\end{array}$ \\
\hline
\end{tabular}




\begin{tabular}{|c|c|c|c|c|c|c|c|c|c|c|c|c|c|c|c|c|c|c|}
\hline 65 & & & & & 3 & & 6 & & 2 & & & & & & & & & \\
\hline $\mathrm{M}$ & 2 & 4 & 0. & 0.06 & 0. & 0.1 & 0. & 0.9 & 0. & 731 & 2 & 686 & 1 & 697 & 1 & \begin{tabular}{|l|}
1.6 \\
\end{tabular} & 6 & 1 \\
\hline $\mathrm{O} 1$ & 2 & 0 & 0 & 368 & 00 & 123 & 00 & 859 & 02 & & 4 & & 4 & & 3 & $0 \%$ & 8 & 4 \\
\hline $8-$ & & 2 & 5 & & 17 & 1 & 24 & 9 & $\begin{array}{c}51 \\
5\end{array}$ & & & & & & & & 6 & \\
\hline $\mathrm{M}$ & 1 & 2 & 0. & 0.05 & 0. & 0.0 & 0. & 0.5 & 0. & 535 & 2 & 428 & 9 & 445 & 1 & 3.9 & 4 & 9 \\
\hline $\mathrm{O} 1$ & 0 & 1 & 5 & 814 & 00 & 686 & 00 & 501 & 01 & & 8 & & & & 0 & $7 \%$ & 2 & \\
\hline 8- & 5 & 0 & 0 & & 17 & 5 & 14 & 7 & 51 & & & & & & & & 8 & \\
\hline 67 & & & & & 4 & & 7 & & 7 & & & & & & & & & \\
\hline $\mathrm{M}$ & 4 & 3 & 0. & 0.05 & 0. & 0.0 & 0. & 0.4 & 0. & 509 & 2 & 384 & 8 & 403 & 8 & \begin{tabular}{|l|l}
4.9 \\
\end{tabular} & 3 & 8 \\
\hline $\mathrm{O} 1$ & 3 & 3 & 1 & 746 & 00 & 614 & 00 & 867 & 01 & & 4 & & & & & $5 \%$ & 8 & \\
\hline 8- & & 5 & 3 & & 15 & 5 & 12 & 9 & 18 & & 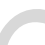 & & & & & & 4 & \\
\hline 68 & & & & & 3 & & 7 & & 7 & & & & & & & & & \\
\hline $\mathrm{M}$ & 1 & 1 & 0. & 0.06 & 0. & 0.0 & 0. & 0.7 & 0. & 606 & 2 & 530 & 1 & 544 & 1 & 2.6 & 5 & 1 \\
\hline $\mathrm{O} 1$ & 6 & 7 & 9 & 008 & 00 & 856 & 00 & 095 & 01 & & 6 & & 1 & & 1 & $4 \%$ & 3 & 1 \\
\hline 8- & 8 & 3 & 7 & & 17 & 7 & 18 & 6 & 87 & & & & & & & & 0 & \\
\hline 69 & & & & & 3 & & 5 & & 5 & & & & & & & & & \\
\hline $\mathrm{M}$ & 6 & 1 & 0 . & 0.05 & 0. & 0.0 & 0. & 0.5 & 0 . & 426 & 3 & 446 & 1 & 443 & 1 & - & 4 & 1 \\
\hline O1 & 6 & 8 & 3 & 535 & 00 & 716 & 00 & 465 & 01 & & 4 & & 0 & & 1 & 0.6 & 4 & 0 \\
\hline $\begin{array}{l}8- \\
70\end{array}$ & & 2 & 6 & & 18 & 3 & 16 & 8 & $\begin{array}{c}70 \\
5\end{array}$ & & & & & & & $7 \%$ & 6 & \\
\hline$\frac{70}{M}$ & 8 & 1 & 0 & 006 & $\begin{array}{r}7 \\
0\end{array}$ & 01 & $\begin{array}{c}1 \\
0\end{array}$ & 11 & $\frac{5}{0 .}$ & 839 & 2 & 737 & 1 & 763 & 1 & 35 & 7 & 1 \\
\hline $\mathrm{O} 1$ & 4 & 9 & 4 & $\begin{array}{l}0.00 \\
705\end{array}$ & 00 & $\begin{array}{l}0.1 \\
211\end{array}$ & 00 & 200 & 02 & & $\begin{array}{l}2 \\
0\end{array}$ & & $\begin{array}{l}1 \\
4\end{array}$ & & $\begin{array}{l}1 \\
1\end{array}$ & $\begin{array}{l}5.5 \\
3 \%\end{array}$ & 3 & $\begin{array}{l}1 \\
4\end{array}$ \\
\hline 8- & & 9 & 2 & & 15 & 9 & 24 & 5 & 38 & & & & & & & & & \\
\hline 71 & & & & & 6 & & 9 & 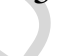 & 2 & & & & & & & & & \\
\hline $\mathrm{M}$ & 5 & 1 & 0. & 0.05 & 0 & 0.0 & 0. & 0.3 & 0 & 346 & 6 & 332 & 9 & 333 & 1 & \begin{tabular}{|l|l}
0.3 \\
\end{tabular} & 3 & 9 \\
\hline $\mathrm{O} 1$ & 7 & 2 & 4 & 340 & 00 & 528 & 00 & 886 & 02 & & 9 & & & & 5 & $0 \%$ & 3 & \\
\hline 8- & & 4 & 5 & & 29 & 0 & 14 & 7 & 00 & & & & & & & & 2 & \\
\hline 72 & & & & & 4 & 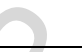 & 3 & & 5 & & & & & & & & & \\
\hline $\mathrm{M}$ & 3 & 1 & 0 . & 0.05 & 0 & 0.0 & 0. & 0.5 & 0. & 505 & 4 & 438 & 1 & 449 & 1 & 2.5 & 4 & 1 \\
\hline $\mathrm{O} 1$ & 9 & 1 & 3 & 735 & 00 & 703 & 00 & 559 & 02 & & 5 & & 0 & & 4 & $1 \%$ & 3 & 0 \\
\hline 8- & & 0 & 5 & & 24 & 2 & 17 & 1 & 18 & & & & & & & & 8 & \\
\hline 73 & & & & & 4 & & 4 & & 8 & & & & & & & & & \\
\hline $\mathrm{M}$ & 5 & 7 & 0 . & 0.06 & 0. & 0.1 & 0. & 1.0 & 0. & 889 & 7 & 670 & 2 & 723 & 3 & \begin{tabular}{|l|}
7.9 \\
\end{tabular} & 6 & 2 \\
\hline $\mathrm{O} 1$ & 4 & 5 & 7 & 867 & 00 & 095 & 00 & 371 & 06 & & 5 & & 1 & & 2 & $1 \%$ & 7 & 1 \\
\hline 8- & & & 2 & 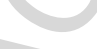 & 46 & 7 & 37 & 7 & 48 & & & & & & & & 0 & \\
\hline 74 & & & & 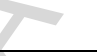 & 1 & & 0 & & 1 & & & & & & & & & \\
\hline $\mathrm{M}$ & 6 & 1 & 0 . & 0.07 & 0. & 0.1 & 0. & 1.6 & 0. & 948 & 2 & 101 & 2 & 990 & 1 & - & 9 & 2 \\
\hline $\mathrm{O} 1$ & 5 & 3 & 4 & 066 & 00 & 695 & 00 & 517 & 03 & & 1 & 0 & 0 & & 4 & 6.1 & 4 & 1 \\
\hline 8- & & 7 & 8 & & 17 & 9 & 35 & 2 & 70 & & & & & & & $4 \%$ & 8 & \\
\hline 75 & & & & & 2 & & 8 & & 0 & & & & & & & & & \\
\hline $\mathrm{M}$ & 5 & 1 & 0 . & 0.26 & 0. & 0.6 & 0. & 23. & 0 & 324 & 1 & 326 & 4 & 325 & 1 & - & 3 & 1 \\
\hline $\mathrm{O} 1$ & 9 & 4 & 4 & 046 & 00 & 586 & 01 & 646 & 27 & 9 & 6 & 2 & 9 & 4 & 1 & 0.4 & 2 & 6 \\
\hline 8- & & 3 & 1 & & 31 & 2 & 25 & 34 & 05 & & & & & & & $0 \%$ & 4 & \\
\hline 76 & & & & & 0 & & 9 & & 6 & & & & & & & & 9 & \\
\hline $\mathrm{M}$ & 2 & 2 & 1. & 0.06 & 0 & 0.1 & 0 & 1.4 & 0 & 904 & 1 & 926 & 1 & 919 & 1 & - & 9 & 1 \\
\hline $\mathrm{O} 1$ & 5 & 1 & 1 & 916 & 00 & 544 & 00 & 727 & 02 & & 8 & & 7 & & 2 & 0.7 & 2 & 7 \\
\hline 8- & 8 & 8 & 8 & & 14 & 8 & 30 & 5 & 80 & & & & & & & $6 \%$ & 6 & \\
\hline 77 & & & & & 3 & & 7 & & 7 & & & & & & & & & \\
\hline $\mathrm{M}$ & 5 & 3 & 0 . & 0.06 & 0. & 0.0 & 0. & 0.7 & 0. & 604 & 2 & 537 & 1 & 550 & 1 & \begin{tabular}{|l|}
2.4 \\
\end{tabular} & 5 & 1 \\
\hline $\mathrm{O} 1$ & 6 & 3 & 1 & 000 & 00 & 869 & 00 & 191 & 01 & & 2 & & 1 & & 0 & $2 \%$ & 3 & 1 \\
\hline 8- & & 8 & 7 & & 14 & 4 & 17 & 1 & 61 & & & & & & & & 7 & \\
\hline 78 & & & & & 7 & & 8 & & 6 & & & & & & & & & \\
\hline $\mathrm{M}$ & 1 & 3 & 0 . & 0.07 & 0. & 0.1 & 0. & 1.5 & 0. & 997 & 1 & 941 & 1 & 958 & 1 & \begin{tabular}{|l|}
1.8 \\
\end{tabular} & 9 & 1 \\
\hline
\end{tabular}




\begin{tabular}{|c|c|c|c|c|c|c|c|c|c|c|c|c|c|c|c|c|c|c|}
\hline $\begin{array}{l}\text { O1 } \\
8- \\
79\end{array}$ & $\begin{array}{l}6 \\
9\end{array}$ & $\begin{array}{l}0 \\
5\end{array}$ & $\begin{array}{l}5 \\
6\end{array}$ & 238 & $\begin{array}{c}00 \\
13 \\
3\end{array}$ & $\begin{array}{c}571 \\
9\end{array}$ & $\begin{array}{c}00 \\
30 \\
6\end{array}$ & $\begin{array}{c}682 \\
4\end{array}$ & $\begin{array}{c}02 \\
64 \\
3\end{array}$ & & 8 & & 7 & & 0 & $1 \%$ & $\begin{array}{l}4 \\
1\end{array}$ & 7 \\
\hline $\begin{array}{l}\mathrm{M} \\
\mathrm{O} 1 \\
8- \\
80\end{array}$ & $\begin{array}{l}1 \\
2 \\
2\end{array}$ & $\begin{array}{l}3 \\
9 \\
7\end{array}$ & $\begin{array}{l}0 . \\
3 \\
1\end{array}$ & $\begin{array}{l}0.05 \\
677\end{array}$ & $\begin{array}{c}0 . \\
00 \\
14 \\
2\end{array}$ & $\begin{array}{c}0.0 \\
701 \\
3\end{array}$ & $\begin{array}{c}0 . \\
00 \\
14 \\
3\end{array}$ & $\begin{array}{c}0.5 \\
487 \\
7\end{array}$ & $\begin{array}{c}0 . \\
01 \\
26 \\
2\end{array}$ & 483 & $\begin{array}{l}2 \\
3\end{array}$ & 437 & 9 & 444 & 8 & $\begin{array}{l}1.6 \\
0 \%\end{array}$ & $\begin{array}{l}4 \\
3 \\
7\end{array}$ & 9 \\
\hline $\begin{array}{l}\text { M } \\
\text { O1 } \\
8- \\
81\end{array}$ & $\begin{array}{l}2 \\
1 \\
1\end{array}$ & $\begin{array}{l}2 \\
4 \\
3\end{array}$ & $\begin{array}{c}0 . \\
8 \\
7\end{array}$ & $\begin{array}{l}0.11 \\
730\end{array}$ & $\begin{array}{c}0 . \\
00 \\
17 \\
8\end{array}$ & $\begin{array}{c}0.2 \\
543 \\
7\end{array}$ & $\begin{array}{c}0 . \\
00 \\
49 \\
3\end{array}$ & $\begin{array}{c}4.1 \\
128 \\
4\end{array}$ & $\begin{array}{c}0 . \\
05 \\
70 \\
1\end{array}$ & $\begin{array}{c}191 \\
5\end{array}$ & $\begin{array}{l}1 \\
7\end{array}$ & $\begin{array}{c}146 \\
1\end{array}$ & $\begin{array}{l}2 \\
5\end{array}$ & $\begin{array}{c}165 \\
7\end{array}$ & $\begin{array}{l}1 \\
1\end{array}$ & $\begin{array}{l}31 . \\
07 \\
\%\end{array}$ & & \\
\hline $\begin{array}{l}\mathrm{M} \\
\mathrm{O} 1 \\
8- \\
82\end{array}$ & $\begin{array}{l}3 \\
1 \\
8\end{array}$ & $\begin{array}{l}2 \\
5 \\
5\end{array}$ & $\begin{array}{l}1 . \\
2 \\
5\end{array}$ & $\begin{array}{c}0.06 \\
617\end{array}$ & $\begin{array}{c}0 . \\
00 \\
16 \\
0\end{array}$ & $\begin{array}{c}0.1 \\
024 \\
0\end{array}$ & $\begin{array}{c}0 . \\
00 \\
21 \\
2\end{array}$ & $\begin{array}{c}0.9 \\
339 \\
5\end{array}$ & $\begin{array}{c}0 . \\
02 \\
07 \\
1\end{array}$ & 812 & $\begin{array}{l}2 \\
1\end{array}$ & 628 & $\begin{array}{l}1 \\
2\end{array}$ & 670 & $\begin{array}{l}1 \\
1\end{array}$ & $\begin{array}{l}6.6 \\
9 \%\end{array}$ & $\begin{array}{l}6 \\
2 \\
8\end{array}$ & $\begin{array}{l}1 \\
2\end{array}$ \\
\hline $\begin{array}{l}\mathrm{M} \\
\mathrm{O} 1 \\
8- \\
83\end{array}$ & $\begin{array}{l}1 \\
1 \\
3\end{array}$ & $\begin{array}{l}2 \\
1 \\
9\end{array}$ & $\begin{array}{l}0 . \\
5 \\
2\end{array}$ & $\begin{array}{c}0.06 \\
132\end{array}$ & $\begin{array}{c}0 . \\
00 \\
17 \\
3\end{array}$ & $\begin{array}{c}0.0 \\
956 \\
1\end{array}$ & $\begin{array}{c}0 . \\
00 \\
20 \\
7\end{array}$ & $\begin{array}{c}0.8 \\
080 \\
7\end{array}$ & $\begin{array}{c}0 . \\
02 \\
08 \\
6\end{array}$ & 650 & $\begin{array}{l}2 \\
5\end{array}$ & 589 & $\begin{array}{l}1 \\
2\end{array}$ & 601 & $\begin{array}{l}1 \\
2\end{array}$ & $\begin{array}{l}2.0 \\
4 \%\end{array}$ & $\begin{array}{l}5 \\
8 \\
9\end{array}$ & $\begin{array}{l}1 \\
2\end{array}$ \\
\hline $\begin{array}{c}\text { M } \\
\text { O1 } \\
8- \\
84\end{array}$ & $\begin{array}{l}4 \\
2\end{array}$ & $\begin{array}{l}7 \\
1 \\
5\end{array}$ & $\begin{array}{l}0 . \\
0 \\
6\end{array}$ & $\begin{array}{l}0.11 \\
750\end{array}$ & $\begin{array}{c}0 . \\
00 \\
13 \\
8\end{array}$ & $\begin{array}{c}0.2 \\
575 \\
1\end{array}$ & $\begin{array}{c}0 . \\
00 \\
47 \\
0\end{array}$ & $\begin{array}{c}4.1 \\
705 \\
8\end{array}$ & $\begin{array}{c}0 . \\
04 \\
51 \\
1\end{array}$ & $\begin{array}{c}191 \\
9\end{array}$ & $\begin{array}{l}1 \\
8\end{array}$ & $\begin{array}{c}147 \\
7\end{array}$ & $\begin{array}{l}2 \\
4\end{array}$ & $\begin{array}{c}166 \\
8\end{array}$ & 9 & $\begin{array}{l}29 . \\
93 \\
\%\end{array}$ & & \\
\hline $\begin{array}{l}\mathrm{M} \\
\mathrm{O} 1 \\
8- \\
85\end{array}$ & $\begin{array}{l}9 \\
8\end{array}$ & $\begin{array}{l}9 \\
2\end{array}$ & $\begin{array}{l}1 . \\
0 \\
7\end{array}$ & $\begin{array}{l}0.06 \\
391\end{array}$ & $\begin{array}{c}0 . \\
00 \\
22 \\
8\end{array}$ & $\begin{array}{c}0.1 \\
402 \\
1\end{array}$ & $\begin{array}{c}0 . \\
00 \\
33 \\
6\end{array}$ & $\begin{array}{c}1.2 \\
351 \\
0\end{array}$ & $\begin{array}{c}0 . \\
04 \\
08 \\
2\end{array}$ & 739 & $\begin{array}{l}3 \\
4\end{array}$ & 846 & $\begin{array}{l}1 \\
9\end{array}$ & 817 & $\begin{array}{l}1 \\
9\end{array}$ & $\begin{array}{c}- \\
3.4 \\
3 \%\end{array}$ & $\begin{array}{l}8 \\
4 \\
6\end{array}$ & $\begin{array}{l}1 \\
9\end{array}$ \\
\hline $\begin{array}{l}\mathrm{M} \\
\mathrm{O} 1 \\
8- \\
86\end{array}$ & $\begin{array}{l}9 \\
3\end{array}$ & $\begin{array}{l}1 \\
4 \\
8\end{array}$ & $\begin{array}{l}0 . \\
6 \\
3\end{array}$ & $\begin{array}{l}0.05 \\
821\end{array}$ & $\begin{array}{c}0 . \\
00 \\
23 \\
5\end{array}$ & $\begin{array}{c}0.0 \\
763 \\
0\end{array}$ & $\begin{array}{c}0 . \\
00 \\
18 \\
8\end{array}$ & $\begin{array}{c}0.6 \\
121 \\
9\end{array}$ & $\begin{array}{c}0 . \\
02 \\
27 \\
8\end{array}$ & 538 & $\begin{array}{l}4 \\
1\end{array}$ & 474 & $\begin{array}{l}1 \\
1\end{array}$ & 485 & $\begin{array}{l}1 \\
4\end{array}$ & $\begin{array}{l}2.3 \\
2 \%\end{array}$ & $\begin{array}{l}4 \\
7 \\
4\end{array}$ & $\begin{array}{l}1 \\
1\end{array}$ \\
\hline $\begin{array}{l}\mathrm{M} \\
\mathrm{O} 1 \\
8- \\
87\end{array}$ & $\begin{array}{l}7 \\
0\end{array}$ & $\begin{array}{l}2 \\
8 \\
2\end{array}$ & $\begin{array}{l}0 . \\
2 \\
5\end{array}$ & $\begin{array}{l}0.05 \\
839\end{array}$ & $\begin{array}{c}0 . \\
00 \\
17 \\
6 \\
\end{array}$ & $\begin{array}{c}0.0 \\
666 \\
1\end{array}$ & $\begin{array}{c}0 . \\
00 \\
14 \\
5\end{array}$ & $\begin{array}{c}0.5 \\
360 \\
8\end{array}$ & $\begin{array}{c}0 . \\
01 \\
48 \\
7 \\
\end{array}$ & 544 & $\begin{array}{l}2 \\
8\end{array}$ & 416 & 9 & 436 & $\begin{array}{l}1 \\
0\end{array}$ & $\begin{array}{l}4.8 \\
1 \%\end{array}$ & $\begin{array}{l}4 \\
1 \\
6\end{array}$ & 9 \\
\hline $\begin{array}{c}\mathrm{M} \\
\mathrm{O} 1 \\
8- \\
88\end{array}$ & $\begin{array}{l}1 \\
6 \\
5\end{array}$ & $\begin{array}{l}2 \\
5 \\
0\end{array}$ & $\begin{array}{l}0 . \\
6 \\
6\end{array}$ & $\begin{array}{l}0.12 \\
209\end{array}$ & $\begin{array}{c}0 . \\
00 \\
19 \\
8\end{array}$ & $\begin{array}{c}0.2 \\
321 \\
8\end{array}$ & $\begin{array}{c}0 . \\
00 \\
45 \\
9\end{array}$ & $\begin{array}{c}3.9 \\
067 \\
4\end{array}$ & $\begin{array}{c}0 . \\
05 \\
71 \\
9\end{array}$ & $\begin{array}{c}153 \\
0\end{array}$ & $\begin{array}{l}8 \\
7\end{array}$ & $\begin{array}{c}130 \\
2\end{array}$ & $\begin{array}{l}2 \\
6\end{array}$ & $\begin{array}{c}139 \\
1\end{array}$ & $\begin{array}{l}3 \\
0\end{array}$ & $\begin{array}{l}17 . \\
51 \\
\%\end{array}$ & & \\
\hline $\begin{array}{c}\text { M } \\
\text { O1 } \\
8- \\
89\end{array}$ & $\begin{array}{l}2 \\
3 \\
0\end{array}$ & $\begin{array}{l}8 \\
4 \\
7\end{array}$ & $\begin{array}{l}0 . \\
2 \\
7\end{array}$ & $\begin{array}{c}0.21 \\
319\end{array}$ & $\begin{array}{c}0 . \\
00 \\
23 \\
0\end{array}$ & $\begin{array}{c}0.2 \\
923 \\
7\end{array}$ & $\begin{array}{c}0 . \\
00 \\
53 \\
1\end{array}$ & $\begin{array}{c}8.5 \\
906 \\
9\end{array}$ & $\begin{array}{c}0 . \\
08 \\
48 \\
3\end{array}$ & $\begin{array}{c}276 \\
1\end{array}$ & $\begin{array}{l}4 \\
0\end{array}$ & $\begin{array}{c}160 \\
6\end{array}$ & $\begin{array}{l}2 \\
7\end{array}$ & $\begin{array}{c}217 \\
3\end{array}$ & $\begin{array}{l}1 \\
3\end{array}$ & $\begin{array}{l}71 . \\
92 \\
\%\end{array}$ & & \\
\hline $\begin{array}{c}\text { M } \\
\text { O1 } \\
8- \\
90\end{array}$ & $\begin{array}{l}4 \\
0\end{array}$ & $\begin{array}{l}1 \\
0 \\
4\end{array}$ & $\begin{array}{l}0 . \\
3 \\
8\end{array}$ & $\begin{array}{l}0.16 \\
789\end{array}$ & $\begin{array}{c}0 . \\
00 \\
25 \\
8\end{array}$ & $\begin{array}{c}0.4 \\
872 \\
3\end{array}$ & $\begin{array}{c}0 . \\
00 \\
99 \\
5\end{array}$ & $\begin{array}{c}11 . \\
273 \\
86\end{array}$ & $\begin{array}{c}0 . \\
16 \\
35 \\
9\end{array}$ & $\begin{array}{c}253 \\
7\end{array}$ & $\begin{array}{l}1 \\
7\end{array}$ & $\begin{array}{c}255 \\
9\end{array}$ & $\begin{array}{l}4 \\
3\end{array}$ & $\begin{array}{c}254 \\
6\end{array}$ & $\begin{array}{l}1 \\
4\end{array}$ & $\begin{array}{c}- \\
0.8 \\
6 \%\end{array}$ & $\begin{array}{l}2 \\
5 \\
3 \\
7\end{array}$ & $\begin{array}{l}1 \\
7\end{array}$ \\
\hline $\begin{array}{l}\text { M } \\
\text { O1 } \\
8- \\
91\end{array}$ & $\begin{array}{l}9 \\
2\end{array}$ & $\begin{array}{l}3 \\
5 \\
9\end{array}$ & $\begin{array}{l}0 . \\
2 \\
6\end{array}$ & $\begin{array}{l}0.17 \\
226\end{array}$ & $\begin{array}{c}0 . \\
00 \\
21 \\
1\end{array}$ & $\begin{array}{c}0.3 \\
299 \\
6\end{array}$ & $\begin{array}{c}0 . \\
00 \\
61 \\
8\end{array}$ & $\begin{array}{c}7.8 \\
332 \\
7\end{array}$ & $\begin{array}{c}0 . \\
08 \\
80 \\
1\end{array}$ & $\begin{array}{c}256 \\
1\end{array}$ & $\begin{array}{l}4 \\
0\end{array}$ & $\begin{array}{c}183 \\
4\end{array}$ & $\begin{array}{l}3 \\
0\end{array}$ & $\begin{array}{c}220 \\
0\end{array}$ & $\begin{array}{l}1 \\
2\end{array}$ & $\begin{array}{l}39 . \\
64 \\
\%\end{array}$ & $\begin{array}{l}2 \\
5 \\
6 \\
1\end{array}$ & $\begin{array}{l}4 \\
0\end{array}$ \\
\hline $\begin{array}{l}\text { M } \\
\text { O1 } \\
8-\end{array}$ & $\begin{array}{l}1 \\
0 \\
2\end{array}$ & $\begin{array}{l}1 \\
8 \\
6\end{array}$ & $\begin{array}{l}0 . \\
5 \\
5\end{array}$ & $\begin{array}{l}0.05 \\
881\end{array}$ & $\begin{array}{c}0 . \\
00 \\
19\end{array}$ & $\begin{array}{c}0.1 \\
016 \\
2\end{array}$ & $\begin{array}{c}0 . \\
00 \\
23\end{array}$ & $\begin{array}{c}0.8 \\
235 \\
5\end{array}$ & $\begin{array}{c}0 . \\
02 \\
49\end{array}$ & 560 & $\begin{array}{l}3 \\
1\end{array}$ & 624 & $\begin{array}{l}1 \\
4\end{array}$ & 610 & $\begin{array}{l}1 \\
4\end{array}$ & $\begin{array}{c}- \\
2.2 \\
4 \%\end{array}$ & $\begin{array}{l}6 \\
2 \\
4\end{array}$ & $\begin{array}{l}1 \\
4\end{array}$ \\
\hline
\end{tabular}




\begin{tabular}{|c|c|c|c|c|c|c|c|c|c|c|c|c|c|c|c|c|c|c|}
\hline 92 & & & & & 3 & & 1 & & 9 & & & & & & & & & \\
\hline $\mathrm{M}$ & 1 & 1 & 0. & 0.17 & 0. & 0.4 & 0. & 11. & 0. & 259 & 1 & 260 & 4 & 260 & 1 & - & 2 & 1 \\
\hline O1 & 7 & 8 & 9 & 427 & 00 & 983 & 00 & 968 & 14 & 9 & 7 & 7 & 1 & 2 & 1 & 0.3 & 5 & 7 \\
\hline 8- & 8 & 1 & 8 & & 22 & 3 & 96 & 15 & 62 & & & & & & & $1 \%$ & 9 & \\
\hline$\frac{93}{M}$ & 4 & 1 & 0. & 006 & $\frac{5}{0}$ & 01 & $\frac{1}{0}$ & 09 & $\frac{2}{0}$ & 752 & 2 & 684 & 1 & 700 & 1 & 23 & $\begin{array}{l}9 \\
6\end{array}$ & 1 \\
\hline $\mathrm{O} 1$ & 1 & 5 & 2 & 431 & 00 & 118 & 00 & 916 & 02 & & 8 & & 5 & 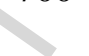 & 5 & $4 \%$ & 8 & 5 \\
\hline 8- & & 8 & 6 & & 20 & 9 & 25 & 5 & 88 & & & & & & & & 4 & \\
\hline 94 & & & & & 4 & & 4 & & 9 & & & & & & & & & \\
\hline $\mathrm{M}$ & 6 & 6 & 1. & 0.07 & 0. & 0.1 & 0. & 1.7 & 0. & 983 & 5 & 103 & 3 & 101 & 3 & - & 9 & 5 \\
\hline O1 & 9 & 6 & 0 & 190 & 00 & 740 & 00 & 240 & 08 & & 5 & 4 & 1 & 8 & 3 & 4.9 & 8 & 5 \\
\hline 8- & & & 4 & & 39 & 0 & 56 & 5 & 85 & & & & & & & $3 \%$ & 3 & \\
\hline 95 & & & & & 9 & & 5 & & 9 & & & & & & & & & \\
\hline $\mathrm{M}$ & 1 & 3 & 0. & 0.06 & 0. & 0.0 & 0. & 0.8 & 0. & 783 & 2 & 557 & 1 & 604 & 1 & 8.4 & 5 & 1 \\
\hline $\mathrm{O} 1$ & 9 & 1 & 6 & 526 & 00 & 903 & 00 & 124 & 01 & & 1 & & 1 & & 0 & $4 \%$ & 5 & 1 \\
\hline 8- & 3 & 2 & 2 & & 15 & 3 & 18 & 5 & 80 & & & & & & & & 7 & \\
\hline 96 & & & & & 9 & & 8 & & 5 & & & & & & & & & \\
\hline $\mathrm{M}$ & 1 & 2 & 0. & 0.05 & 0. & 0.0 & 0. & 0.8 & 0. & 594 & 2 & 610 & 1 & 607 & 1 & - & 6 & 1 \\
\hline $\mathrm{O} 1$ & 2 & 3 & 5 & 973 & 00 & 993 & 00 & 174 & 01 & & 3 & & 2 & & 1 & 0.4 & 1 & 2 \\
\hline 8- & 4 & 7 & 2 & & 15 & 2 & 20 & 9 & 95 & & & & & & & $9 \%$ & 0 & \\
\hline 97 & & & & & 6 & & 9 & & 7 & & & & & & & & & \\
\hline $\mathrm{M}$ & 1 & 3 & 0. & 0.06 & 0. & 0.1 & 0. & 1.5 & 0 . & 877 & 1 & 983 & 1 & 950 & 1 & - & 9 & 1 \\
\hline $\mathrm{O} 1$ & 1 & 5 & 3 & 827 & 00 & 646 & 00 & 491 & 02 & & 8 & & 7 & & 0 & 3.3 & 8 & 7 \\
\hline $\begin{array}{l}8- \\
08\end{array}$ & 9 & 1 & 4 & & 11 & 6 & 31 & 4 & 42 & & & & & & & $6 \%$ & 3 & \\
\hline $\begin{array}{l}90 \\
M\end{array}$ & 7 & 1 & 0. & 0.06 & $\begin{array}{l}0 \\
0 .\end{array}$ & 0.1 & 0. & 0.8 & 0. & 615 & 2 & 617 & 1 & 616 & 1 & - & 6 & 1 \\
\hline $\mathrm{O} 1$ & 8 & 7 & 4 & 031 & 00 & 004 & 00 & 348 & 02 & & 9 & & 3 & & 4 & 0.1 & 1 & 3 \\
\hline 8- & & 6 & 4 & & 19 & 5 & 22 & 8 & 44 & & & & & & & $6 \%$ & 7 & \\
\hline 99 & & & & & 1 & 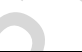 & 7 & & 0 & & & & & & & & & \\
\hline $\mathrm{M}$ & 5 & 1 & 0. & 0.05 & 0. & 0.0 & 0. & 0.6 & 0. & 451 & 4 & 514 & 1 & 502 & 1 & - & 5 & 1 \\
\hline O1 & 0 & 3 & 3 & 596 & 00 & 829 & 00 & 396 & 02 & & 3 & & 2 & & 5 & 2.3 & 1 & 2 \\
\hline 8- & & 7 & 6 & & 22 & 3 & 20 & 0 & 39 & & & & & & & $3 \%$ & 4 & \\
\hline $\begin{array}{c}10 \\
0\end{array}$ & & & & & 5 & & 1 & & 5 & & & & & & & & & \\
\hline & & & & & & & & & & & & & & & & & & \\
\hline M & 7 & 7 & 0. & 0.06 & 0. & 0.8 & 0. & 0.0 & 0. & 626 & 2 & 609 & 1 & 604 & 1 & 0.8 & 6 & 1 \\
\hline $\mathrm{O} 2$ & 0 & 9 & 8 & 064 & 00 & 213 & 02 & 982 & 00 & & 9 & & 2 & & 0 & $3 \%$ & 0 & 0 \\
\hline $2 \mathrm{~A}$ & & & 9 & & 17 & 0 & 14 & 5 & 16 & & & & & & & & 4 & \\
\hline- & & & & & 2 & & 0 & & 9 & & & & & & & & & \\
\hline $\mathrm{M}$ & 9 & 1 & 0. & 0.05 & 0. & 0.8 & 0. & 0.1 & 0. & 527 & 1 & 618 & 9 & 644 & 9 & - & 6 & 9 \\
\hline $\mathrm{O} 2$ & 1 & 5 & 6 & 792 & 00 & 383 & 01 & 050 & 00 & & 9 & & & & & 4.0 & 4 & \\
\hline $2 \mathrm{~A}$ & & 3 & 0 & & 11 & 8 & 54 & 1 & 14 & & & & & & & $4 \%$ & 4 & \\
\hline- & & & & & 5 & & 4 & & 8 & & & & & & & & & \\
\hline $\mathrm{M}$ & 3 & 3 & 0. & 0.12 & 0. & 6.2 & 0. & 0.3 & 0. & 204 & 1 & 201 & 1 & 198 & 2 & 3.0 & 2 & 1 \\
\hline $\mathrm{O} 2$ & 4 & 5 & 9 & 613 & 00 & 663 & 10 & 604 & 00 & 5 & 3 & 4 & 4 & 4 & 8 & $7 \%$ & 0 & 3 \\
\hline $2 \mathrm{~A}$ & & & 6 & & 22 & 9 & 14 & 0 & 58 & & & & & & & & 4 & \\
\hline- & & & & & 1 & & 0 & & 3 & & & & & & & & 5 & \\
\hline M & 6 & 8 & 0. & 0.11 & 0. & 6.0 & 0. & 0.3 & 0. & 195 & 1 & $\begin{array}{ll}198 \\
\end{array}$ & 1 & 200 & 2 & - & 1 & 1 \\
\hline $\mathrm{O} 2$ & 8 & 8 & 7 & 994 & 00 & 474 & 06 & 657 & 00 & 5 & 1 & 3 & 0 & 9 & 3 & 2.6 & 9 & 1 \\
\hline $2 \mathrm{~A}$ & & & 7 & & 14 & 3 & 66 & 3 & 48 & & & & & & & $9 \%$ & 5 & \\
\hline- & & & & & 0 & & 6 & & 3 & & & & & & & & 5 & \\
\hline
\end{tabular}




\begin{tabular}{|c|c|c|c|c|c|c|c|c|c|c|c|c|c|c|c|c|c|c|}
\hline 04 & & & & & & & & & & & & & & & & & & \\
\hline $\begin{array}{c}\mathrm{M} \\
\mathrm{O} 2 \\
2 \mathrm{~A} \\
- \\
05\end{array}$ & $\begin{array}{l}1 \\
2 \\
9\end{array}$ & $\begin{array}{l}1 \\
6 \\
6\end{array}$ & $\begin{array}{l}0 . \\
7 \\
8\end{array}$ & $\begin{array}{l}0.05 \\
772\end{array}$ & $\begin{array}{c}0 . \\
00 \\
11 \\
8\end{array}$ & $\begin{array}{c}0.7 \\
778 \\
0\end{array}$ & $\begin{array}{c}0 . \\
01 \\
47 \\
0\end{array}$ & $\begin{array}{c}0.0 \\
977 \\
5\end{array}$ & $\begin{array}{c}0 . \\
00 \\
13 \\
9\end{array}$ & 519 & $\begin{array}{l}2 \\
0\end{array}$ & 584 & 8 & 601 & 8 & $\begin{array}{c}- \\
2.8 \\
3 \%\end{array}$ & $\begin{array}{l}6 \\
0 \\
1\end{array}$ & 8 \\
\hline $\begin{array}{c}\mathrm{M} \\
\mathrm{O} 2 \\
2 \mathrm{~A} \\
- \\
06\end{array}$ & $\begin{array}{l}8 \\
9\end{array}$ & $\begin{array}{l}8 \\
8\end{array}$ & $\begin{array}{l}1 . \\
0 \\
1\end{array}$ & $\begin{array}{l}0.06 \\
263\end{array}$ & $\begin{array}{c}0 . \\
00 \\
14 \\
8\end{array}$ & $\begin{array}{c}1.0 \\
452 \\
3\end{array}$ & $\begin{array}{c}0 . \\
02 \\
26 \\
8\end{array}$ & $\begin{array}{c}0.1 \\
210 \\
5\end{array}$ & $\begin{array}{c}0 . \\
00 \\
18 \\
9\end{array}$ & 696 & $\begin{array}{l}2 \\
2\end{array}$ & 727 & $\begin{array}{l}1 \\
1\end{array}$ & 737 & $\begin{array}{l}1 \\
1\end{array}$ & $\begin{array}{c} \\
1.3 \\
6 \%\end{array}$ & $\begin{array}{l}7 \\
3 \\
7\end{array}$ & $\begin{array}{l}1 \\
1\end{array}$ \\
\hline $\begin{array}{c}\mathrm{M} \\
\mathrm{O} 2 \\
2 \mathrm{~A} \\
- \\
07\end{array}$ & $\begin{array}{l}8 \\
5\end{array}$ & \begin{tabular}{|l|}
3 \\
8 \\
9
\end{tabular} & $\begin{array}{l}0 . \\
2 \\
2\end{array}$ & $\begin{array}{l}0.05 \\
471\end{array}$ & $\begin{array}{c}0 . \\
00 \\
08 \\
8\end{array}$ & $\begin{array}{c}0.5 \\
605 \\
6\end{array}$ & $\begin{array}{c}0 . \\
00 \\
83 \\
6\end{array}$ & $\begin{array}{c}0.0 \\
743 \\
2\end{array}$ & $\begin{array}{c}0 . \\
00 \\
09 \\
5\end{array}$ & 400 & $\begin{array}{l}1 \\
5\end{array}$ & 452 & 5 & 462 & 6 & $\begin{array}{c}- \\
2.1 \\
6 \%\end{array}$ & $\begin{array}{l}4 \\
6 \\
2\end{array}$ & 6 \\
\hline $\begin{array}{c}\mathrm{M} \\
\mathrm{O} 2 \\
2 \mathrm{~A} \\
- \\
08\end{array}$ & $\begin{array}{l}6 \\
6\end{array}$ & $\begin{array}{l}9 \\
1\end{array}$ & $\begin{array}{l}0 . \\
7 \\
2\end{array}$ & $\begin{array}{c}0.06 \\
065\end{array}$ & $\begin{array}{c}0 . \\
00 \\
14 \\
4\end{array}$ & $\begin{array}{c}1.0 \\
028 \\
2\end{array}$ & $\begin{array}{c}0 . \\
02 \\
19 \\
9\end{array}$ & $\begin{array}{c}0.1 \\
199 \\
3\end{array}$ & $\begin{array}{c}0 . \\
00 \\
18 \\
7\end{array}$ & 627 & $\begin{array}{l}2 \\
3\end{array}$ & 705 & $\begin{array}{l}1 \\
1\end{array}$ & 730 & $\begin{array}{l}1 \\
1\end{array}$ & $\begin{array}{c}- \\
3.4 \\
2 \%\end{array}$ & $\begin{array}{l}7 \\
3 \\
0\end{array}$ & $\begin{array}{l}1 \\
1\end{array}$ \\
\hline $\begin{array}{c}\mathrm{M} \\
\mathrm{O} 2 \\
2 \mathrm{~A} \\
- \\
09\end{array}$ & $\begin{array}{l}9 \\
2\end{array}$ & $\begin{array}{l}1 \\
0 \\
5\end{array}$ & $\begin{array}{l}0 . \\
8 \\
8\end{array}$ & $\begin{array}{l}0.05 \\
728\end{array}$ & $\begin{array}{c}0 . \\
00 \\
17 \\
4\end{array}$ & $\begin{array}{c}0.5 \\
767 \\
9\end{array}$ & $\begin{array}{c}0 . \\
01 \\
60 \\
3\end{array}$ & $\begin{array}{c}0.0 \\
730 \\
4\end{array}$ & $\begin{array}{c}0 . \\
00 \\
12 \\
7\end{array}$ & 502 & $\begin{array}{l}3 \\
2\end{array}$ & 462 & $\begin{array}{l}1 \\
0\end{array}$ & 454 & 8 & \begin{tabular}{|l|}
1.7 \\
$6 \%$
\end{tabular} & $\begin{array}{l}4 \\
5 \\
4\end{array}$ & 8 \\
\hline $\begin{array}{c}\mathrm{M} \\
\mathrm{O} 2 \\
2 \mathrm{~A} \\
- \\
10\end{array}$ & $\begin{array}{l}5 \\
2\end{array}$ & \begin{tabular}{|l|}
7 \\
0
\end{tabular} & $\begin{array}{l}0 . \\
7 \\
5\end{array}$ & $\begin{array}{c}0.06 \\
146\end{array}$ & $\begin{array}{c}0 . \\
00 \\
18\end{array}$ & $\begin{array}{c}0.9 \\
014 \\
4\end{array}$ & $\begin{array}{c}0 . \\
02 \\
46 \\
1\end{array}$ & $\begin{array}{c}0.1 \\
063 \\
9\end{array}$ & $\begin{array}{c}0 . \\
00 \\
18 \\
8\end{array}$ & 655 & $\begin{array}{l}3 \\
0\end{array}$ & 652 & $\begin{array}{l}1 \\
3\end{array}$ & 652 & $\begin{array}{l}1 \\
1\end{array}$ & $\begin{array}{l}0.0 \\
0 \%\end{array}$ & $\begin{array}{l}6 \\
5 \\
2\end{array}$ & $\begin{array}{l}1 \\
1\end{array}$ \\
\hline $\begin{array}{c}\mathrm{M} \\
\mathrm{O} 2 \\
2 \mathrm{~A} \\
- \\
11\end{array}$ & $\begin{array}{l}2 \\
2 \\
5\end{array}$ & \begin{tabular}{|l|}
8 \\
4
\end{tabular} & $\begin{array}{l}2 . \\
6 \\
7\end{array}$ & $\begin{array}{l}0.15 \\
572\end{array}$ & $\begin{array}{c}0 . \\
00 \\
15 \\
7\end{array}$ & $\begin{array}{c}10 . \\
377 \\
83\end{array}$ & $\begin{array}{c}0 . \\
10 \\
11 \\
5\end{array}$ & $\begin{array}{c}0.4 \\
834 \\
1\end{array}$ & $\begin{array}{c}0 . \\
00 \\
62 \\
7\end{array}$ & $\begin{array}{c}241 \\
0\end{array}$ & $\begin{array}{l}1 \\
0\end{array}$ & $\begin{array}{c}246 \\
9\end{array}$ & 9 & $\begin{array}{c}254 \\
2\end{array}$ & $\begin{array}{l}2 \\
7\end{array}$ & $\begin{array}{c}- \\
5.1 \\
9 \%\end{array}$ & $\begin{array}{l}2 \\
4 \\
1 \\
0\end{array}$ & $\begin{array}{l}1 \\
0\end{array}$ \\
\hline $\begin{array}{c}\mathrm{M} \\
\mathrm{O} 2 \\
2 \mathrm{~A} \\
- \\
12\end{array}$ & $\begin{array}{l}3 \\
0\end{array}$ & \begin{tabular}{|l|}
1 \\
0 \\
3
\end{tabular} & $\begin{array}{l}0 . \\
2 \\
9\end{array}$ & $\begin{array}{c}0.06 \\
403\end{array}$ & $\begin{array}{c}0 . \\
00 \\
13 \\
4\end{array}$ & $\begin{array}{c}1.1 \\
629 \\
2\end{array}$ & $\begin{array}{c}0 . \\
02 \\
25 \\
1\end{array}$ & $\begin{array}{c}0.1 \\
317 \\
3\end{array}$ & $\begin{array}{c}0 . \\
00 \\
19 \\
5\end{array}$ & 743 & $\begin{array}{l}1 \\
9\end{array}$ & 783 & $\begin{array}{l}1 \\
1\end{array}$ & 798 & $\begin{array}{l}1 \\
1\end{array}$ & $\begin{array}{c}- \\
1.8 \\
8 \%\end{array}$ & $\begin{array}{l}7 \\
9 \\
8\end{array}$ & $\begin{array}{l}1 \\
1\end{array}$ \\
\hline $\begin{array}{c}\mathrm{M} \\
\mathrm{O} 2 \\
2 \mathrm{~A} \\
- \\
13\end{array}$ & $\begin{array}{l}3 \\
8 \\
5\end{array}$ & $\begin{array}{l}4 \\
7 \\
6\end{array}$ & $\begin{array}{l}0 . \\
8 \\
1\end{array}$ & $\begin{array}{l}0.07 \\
331\end{array}$ & $\begin{array}{c}0 . \\
00 \\
11 \\
3\end{array}$ & $\begin{array}{c}0.6 \\
092 \\
7\end{array}$ & $\begin{array}{c}0 . \\
00 \\
85 \\
5\end{array}$ & $\begin{array}{c}0.0 \\
602 \\
8\end{array}$ & $\begin{array}{c}0 . \\
00 \\
07 \\
9\end{array}$ & $\begin{array}{c}102 \\
3\end{array}$ & $\begin{array}{l}1 \\
3\end{array}$ & 483 & 5 & 377 & 5 & $\begin{array}{c}28 . \\
12 \\
\%\end{array}$ & & \\
\hline $\begin{array}{c}\mathrm{M} \\
\mathrm{O} 2 \\
2 \mathrm{~A} \\
- \\
14\end{array}$ & $\begin{array}{l}3 \\
1\end{array}$ & \begin{tabular}{|l|}
5 \\
0 \\
4
\end{tabular} & $\begin{array}{l}0 . \\
0 \\
6\end{array}$ & $\begin{array}{l}0.11 \\
760\end{array}$ & $\begin{array}{c}0 . \\
00 \\
07 \\
7\end{array}$ & $\begin{array}{c}5.5 \\
732 \\
1\end{array}$ & $\begin{array}{c}0 . \\
03 \\
66 \\
6\end{array}$ & $\begin{array}{c}0.3 \\
437 \\
4\end{array}$ & $\begin{array}{c}0 . \\
00 \\
38 \\
7\end{array}$ & $\begin{array}{c}192 \\
0\end{array}$ & $\begin{array}{l}1 \\
1\end{array}$ & $\begin{array}{c}191 \\
2\end{array}$ & 6 & $\begin{array}{c}190 \\
5\end{array}$ & $\begin{array}{l}1 \\
9\end{array}$ & \begin{tabular}{|l|}
0.7 \\
$9 \%$
\end{tabular} & $\begin{array}{l}1 \\
9 \\
2 \\
0\end{array}$ & $\begin{array}{l}1 \\
1\end{array}$ \\
\hline $\begin{array}{l}\mathrm{M} \\
\mathrm{O} 2 \\
2 \mathrm{~A}\end{array}$ & $\begin{array}{l}2 \\
6\end{array}$ & $\begin{array}{l}3 \\
4\end{array}$ & $\begin{array}{l}0 . \\
7 \\
5\end{array}$ & $\begin{array}{c}0.06 \\
540\end{array}$ & $\begin{array}{c}0 . \\
00 \\
22\end{array}$ & $\begin{array}{c}1.1 \\
538 \\
5\end{array}$ & $\begin{array}{c}0 . \\
03 \\
61\end{array}$ & $\begin{array}{c}0.1 \\
279 \\
8\end{array}$ & $\begin{array}{c}0 . \\
00 \\
25\end{array}$ & 787 & $\begin{array}{l}3 \\
4\end{array}$ & 779 & $\begin{array}{l}1 \\
7\end{array}$ & 776 & $\begin{array}{l}1 \\
5\end{array}$ & $\begin{array}{l}0.3 \\
9 \%\end{array}$ & $\begin{array}{l}7 \\
7 \\
6\end{array}$ & $\begin{array}{l}1 \\
5\end{array}$ \\
\hline
\end{tabular}




\begin{tabular}{|c|c|c|c|c|c|c|c|c|c|c|c|c|c|c|c|c|c|c|}
\hline $\begin{array}{l}- \\
15\end{array}$ & & & & & 4 & & 9 & & 5 & & & & & & & & & \\
\hline $\begin{array}{c}\mathrm{M} \\
\mathrm{O} 2 \\
2 \mathrm{~A} \\
- \\
16\end{array}$ & $\begin{array}{l}1 \\
7 \\
4\end{array}$ & $\begin{array}{l}2 \\
5 \\
0\end{array}$ & $\begin{array}{l}0 . \\
7 \\
0\end{array}$ & $\begin{array}{l}0.05 \\
538\end{array}$ & $\begin{array}{c}0 . \\
00 \\
11 \\
0\end{array}$ & $\begin{array}{c}0.5 \\
669 \\
3\end{array}$ & $\begin{array}{c}0 . \\
01 \\
04 \\
6\end{array}$ & $\begin{array}{c}0.0 \\
742 \\
6\end{array}$ & $\begin{array}{c}0 . \\
00 \\
10 \\
3\end{array}$ & 428 & $\begin{array}{l}1 \\
9\end{array}$ & 456 & 7 & 462 & 6 & $\begin{array}{c}- \\
1.3 \\
0 \%\end{array}$ & $\begin{array}{l}4 \\
6 \\
2\end{array}$ & 6 \\
\hline $\begin{array}{c}\mathrm{M} \\
\mathrm{O} 2 \\
2 \mathrm{~A} \\
- \\
17 \\
\end{array}$ & $\begin{array}{l}2 \\
0 \\
4\end{array}$ & $\begin{array}{l}2 \\
4 \\
0\end{array}$ & $\begin{array}{l}0 . \\
8 \\
5\end{array}$ & $\begin{array}{c}0.06 \\
170\end{array}$ & $\begin{array}{c}0 . \\
00 \\
10 \\
3\end{array}$ & $\begin{array}{c}0.8 \\
126 \\
0\end{array}$ & $\begin{array}{c}0 . \\
01 \\
24 \\
8\end{array}$ & $\begin{array}{c}0.0 \\
955 \\
3\end{array}$ & $\begin{array}{c}0 . \\
00 \\
12 \\
6\end{array}$ & 664 & $\begin{array}{l}1 \\
5\end{array}$ & 604 & 7 & 588 & 7 & \begin{tabular}{|l}
2.7 \\
$2 \%$
\end{tabular} & $\begin{array}{l}5 \\
8 \\
8\end{array}$ & 7 \\
\hline $\begin{array}{c}\mathrm{M} \\
\mathrm{O} 2 \\
2 \mathrm{~A} \\
- \\
18\end{array}$ & $\begin{array}{l}99 \\
1\end{array}$ & $\begin{array}{l}1 \\
7 \\
3\end{array}$ & $\begin{array}{l}0 . \\
5 \\
3\end{array}$ & $\begin{array}{l}0.19 \\
065\end{array}$ & $\begin{array}{c}0 . \\
00 \\
14 \\
0\end{array}$ & $\begin{array}{c}13 . \\
536 \\
17\end{array}$ & $\begin{array}{c}0 . \\
09 \\
92 \\
2\end{array}$ & $\begin{array}{c}0.5 \\
149 \\
9\end{array}$ & $\begin{array}{c}0 . \\
00 \\
60 \\
9\end{array}$ & $\begin{array}{c}274 \\
8\end{array}$ & $\begin{array}{l}1 \\
0\end{array}$ & $\begin{array}{c}271 \\
8\end{array}$ & 7 & $\begin{array}{c}267 \\
8\end{array}$ & $\begin{array}{l}2 \\
6\end{array}$ & $\begin{array}{l}2.6 \\
1 \%\end{array}$ & $\begin{array}{l}2 \\
7 \\
4 \\
8\end{array}$ & $\begin{array}{l}1 \\
0\end{array}$ \\
\hline $\begin{array}{c}\text { M } \\
\text { O2 } \\
2 \mathrm{~A} \\
- \\
19\end{array}$ & $\begin{array}{l}1 \\
1 \\
8\end{array}$ & $\begin{array}{l}1 \\
4 \\
5\end{array}$ & $\begin{array}{l}0 . \\
8 \\
1\end{array}$ & $\begin{array}{c}0.06 \\
429\end{array}$ & $\begin{array}{c}0 . \\
00 \\
14 \\
0\end{array}$ & $\begin{array}{c}0.8 \\
863 \\
1\end{array}$ & $\begin{array}{c}0 . \\
01 \\
77 \\
1\end{array}$ & $\begin{array}{c}0.0 \\
999 \\
9\end{array}$ & $\begin{array}{c}0 . \\
00 \\
15 \\
0\end{array}$ & 751 & $\begin{array}{l}2 \\
0\end{array}$ & 644 & $\begin{array}{l}1 \\
0\end{array}$ & 614 & 9 & $\begin{array}{l}4.8 \\
9 \%\end{array}$ & $\begin{array}{l}6 \\
1 \\
4\end{array}$ & 9 \\
\hline $\begin{array}{c}\mathrm{M} \\
\mathrm{O} 2 \\
2 \mathrm{~A} \\
- \\
20 \\
\end{array}$ & $\begin{array}{l}6 \\
9\end{array}$ & $\begin{array}{l}1 \\
1 \\
7\end{array}$ & $\begin{array}{l}0 . \\
5 \\
9\end{array}$ & $\begin{array}{l}0.06 \\
727\end{array}$ & $\begin{array}{c}0 . \\
00 \\
14 \\
8\end{array}$ & $\begin{array}{c}0.9 \\
582 \\
0\end{array}$ & $\begin{array}{c}0 . \\
01 \\
92 \\
7\end{array}$ & $\begin{array}{c}0.1 \\
033 \\
2\end{array}$ & $\begin{array}{c}0 \\
00 \\
15 \\
7\end{array}$ & 846 & $\begin{array}{l}2 \\
0\end{array}$ & 682 & $\begin{array}{l}1 \\
0\end{array}$ & 634 & 9 & $\begin{array}{l}7.5 \\
7 \%\end{array}$ & $\begin{array}{l}6 \\
3 \\
4\end{array}$ & 9 \\
\hline $\begin{array}{c}\mathrm{M} \\
\mathrm{O} 2 \\
2 \mathrm{~A} \\
- \\
21\end{array}$ & $\begin{array}{l}9 \\
7\end{array}$ & $\begin{array}{l}1 \\
7 \\
2\end{array}$ & $\begin{array}{l}0 . \\
5 \\
6\end{array}$ & $\begin{array}{c}0.06 \\
108\end{array}$ & $\begin{array}{c}0 . \\
00 \\
13 \\
4\end{array}$ & $\begin{array}{c}0.8 \\
973 \\
8\end{array}$ & $\begin{array}{c}0 . \\
01 \\
81 \\
9\end{array}$ & $\begin{array}{c}0.1 \\
065 \\
6\end{array}$ & $\begin{array}{c}0 . \\
00 \\
15 \\
7\end{array}$ & 642 & $\begin{array}{l}2 \\
1\end{array}$ & 650 & $\begin{array}{l}1 \\
0\end{array}$ & 653 & 9 & $\begin{array}{c}- \\
0.4 \\
6 \%\end{array}$ & $\begin{array}{l}6 \\
5 \\
3\end{array}$ & 9 \\
\hline $\begin{array}{c}\mathrm{M} \\
\mathrm{O} 2 \\
2 \mathrm{~A} \\
- \\
22\end{array}$ & $\begin{array}{l}5 \\
3 \\
9\end{array}$ & $\begin{array}{l}6 \\
6 \\
4\end{array}$ & $\begin{array}{l}0 . \\
8 \\
1\end{array}$ & $\begin{array}{l}0.07 \\
867 \\
\end{array}$ & $\begin{array}{c}0 . \\
00 \\
07 \\
0\end{array}$ & $\begin{array}{c}1.7 \\
630 \\
7\end{array}$ & $\begin{array}{c}0 . \\
01 \\
49 \\
9\end{array}$ & $\begin{array}{c}0.1 \\
625 \\
5\end{array}$ & $\begin{array}{c}0 . \\
00 \\
18 \\
8\end{array}$ & $\begin{array}{c}116 \\
4\end{array}$ & $\begin{array}{l}1 \\
1\end{array}$ & $\begin{array}{c}103 \\
2\end{array}$ & 6 & 971 & $\begin{array}{l}1 \\
0\end{array}$ & $\begin{array}{l}6.2 \\
8 \%\end{array}$ & $\begin{array}{l}9 \\
7 \\
1\end{array}$ & $\begin{array}{l}1 \\
0\end{array}$ \\
\hline $\begin{array}{c}\mathrm{M} \\
\mathrm{O} 2 \\
2 \mathrm{~A} \\
- \\
23\end{array}$ & $\begin{array}{l}9 \\
2\end{array}$ & $\begin{array}{l}1 \\
3 \\
2\end{array}$ & $\begin{array}{l}0 . \\
7 \\
0\end{array}$ & $\begin{array}{c}0.06 \\
590\end{array}$ & $\begin{array}{c}0 . \\
00 \\
13 \\
1\end{array}$ & $\begin{array}{c}1.1 \\
681 \\
7\end{array}$ & $\begin{array}{c}0 . \\
02 \\
13 \\
9\end{array}$ & $\begin{array}{c}0.1 \\
285 \\
7\end{array}$ & $\begin{array}{c}0 . \\
00 \\
18 \\
6\end{array}$ & 803 & $\begin{array}{l}1 \\
8\end{array}$ & 786 & $\begin{array}{l}1 \\
0\end{array}$ & 780 & $\begin{array}{l}1 \\
1\end{array}$ & $\begin{array}{l}0.7 \\
7 \%\end{array}$ & $\begin{array}{l}7 \\
8 \\
0\end{array}$ & $\begin{array}{l}1 \\
1\end{array}$ \\
\hline $\begin{array}{c}\mathrm{M} \\
\mathrm{O} 2 \\
2 \mathrm{~A} \\
- \\
24\end{array}$ & $\begin{array}{l}1 \\
2 \\
0\end{array}$ & $\begin{array}{l}2 \\
3 \\
7\end{array}$ & $\begin{array}{l}0 . \\
5 \\
1\end{array}$ & $\begin{array}{l}0.05 \\
639\end{array}$ & $\begin{array}{c}0 . \\
00 \\
10 \\
8\end{array}$ & $\begin{array}{c}0.5 \\
692 \\
9\end{array}$ & $\begin{array}{c}0 . \\
01 \\
01 \\
1\end{array}$ & $\begin{array}{c}0.0 \\
732 \\
3\end{array}$ & $\begin{array}{c}0 . \\
00 \\
10 \\
0\end{array}$ & 468 & $\begin{array}{l}1 \\
8\end{array}$ & 458 & 7 & 456 & 6 & $\begin{array}{l}0.4 \\
4 \%\end{array}$ & $\begin{array}{l}4 \\
5 \\
6\end{array}$ & 6 \\
\hline $\begin{array}{c}\mathrm{M} \\
\mathrm{O} 2 \\
2 \mathrm{~A} \\
- \\
25 \\
\end{array}$ & $\begin{array}{l}1 \\
7 \\
0\end{array}$ & $\begin{array}{l}3 \\
0 \\
5\end{array}$ & $\begin{array}{l}0 . \\
5 \\
6\end{array}$ & $\begin{array}{l}0.05 \\
619\end{array}$ & $\begin{array}{c}0 . \\
00 \\
11 \\
7\end{array}$ & $\begin{array}{c}0.6 \\
983 \\
5\end{array}$ & $\begin{array}{c}0 . \\
01 \\
35 \\
3\end{array}$ & $\begin{array}{c}0.0 \\
901 \\
5\end{array}$ & $\begin{array}{c}0 . \\
00 \\
12 \\
6\end{array}$ & 460 & $\begin{array}{l}2 \\
1\end{array}$ & 538 & 8 & 556 & 7 & $\begin{array}{c}- \\
3.2 \\
4 \%\end{array}$ & $\begin{array}{l}5 \\
5 \\
6\end{array}$ & 7 \\
\hline $\begin{array}{l}\mathrm{M} \\
\mathrm{O} 2\end{array}$ & $\begin{array}{l}1 \\
4\end{array}$ & $\begin{array}{l}1 \\
6\end{array}$ & $\begin{array}{l}0 . \\
8\end{array}$ & $\begin{array}{l}0.06 \\
266\end{array}$ & $\begin{array}{c}0 . \\
00\end{array}$ & $\begin{array}{l}0.8 \\
685\end{array}$ & $\begin{array}{c}0 . \\
01\end{array}$ & $\begin{array}{c}0.1 \\
005\end{array}$ & $\begin{array}{c}0 . \\
00\end{array}$ & 697 & $\begin{array}{l}1 \\
7\end{array}$ & 635 & 8 & 618 & 8 & $\begin{array}{l}2.7 \\
5 \%\end{array}$ & $\begin{array}{l}6 \\
1 \\
\end{array}$ & 8 \\
\hline
\end{tabular}




\begin{tabular}{|c|c|c|c|c|c|c|c|c|c|c|c|c|c|c|c|c|c|c|}
\hline $\begin{array}{c}2 \mathrm{~A} \\
- \\
26\end{array}$ & 1 & 8 & 4 & & $\begin{array}{c}12 \\
0\end{array}$ & 7 & $\begin{array}{c}53 \\
2\end{array}$ & 4 & \begin{tabular}{c|}
14 \\
1
\end{tabular} & & & & & & & & 8 & \\
\hline $\begin{array}{c}\mathrm{M} \\
\mathrm{O} 2 \\
2 \mathrm{~A} \\
- \\
27\end{array}$ & $\begin{array}{l}2 \\
2 \\
7\end{array}$ & $\begin{array}{l}2 \\
9 \\
9\end{array}$ & $\begin{array}{l}0 . \\
7 \\
6\end{array}$ & $\begin{array}{l}0.06 \\
345\end{array}$ & $\begin{array}{c}0 . \\
00 \\
09 \\
2\end{array}$ & $\begin{array}{c}1.0 \\
703 \\
0\end{array}$ & $\begin{array}{c}0 . \\
01 \\
44 \\
4\end{array}$ & $\begin{array}{c}0.1 \\
223 \\
4\end{array}$ & $\begin{array}{c}0 . \\
00 \\
15 \\
5\end{array}$ & 723 & $\begin{array}{l}1 \\
3\end{array}$ & 739 & 7 & 744 & 9 & $\begin{array}{c}- \\
0.6 \\
7 \%\end{array}$ & $\begin{array}{l}7 \\
4 \\
4\end{array}$ & 9 \\
\hline $\begin{array}{c}\mathrm{M} \\
\mathrm{O} 2 \\
2 \mathrm{~A} \\
- \\
28\end{array}$ & $\begin{array}{l}3 \\
5 \\
6\end{array}$ & $\begin{array}{l}2 \\
9 \\
4\end{array}$ & $\begin{array}{l}1 . \\
2 \\
1\end{array}$ & $\begin{array}{l}0.06 \\
056\end{array}$ & $\begin{array}{c}0 . \\
00 \\
09 \\
2\end{array}$ & $\begin{array}{c}0.8 \\
572 \\
0\end{array}$ & $\begin{array}{c}0 . \\
01 \\
21 \\
3\end{array}$ & $\begin{array}{c}0.1 \\
026 \\
6\end{array}$ & $\begin{array}{c}0 . \\
00 \\
13 \\
1\end{array}$ & 624 & $\begin{array}{l}1 \\
4\end{array}$ & 629 & 7 & 630 & 8 & $\begin{array}{c}- \\
0.1 \\
6 \%\end{array}$ & $\begin{array}{l}6 \\
3 \\
0\end{array}$ & 8 \\
\hline $\begin{array}{c}\mathrm{M} \\
\mathrm{O} 2 \\
2 \mathrm{~A} \\
- \\
29\end{array}$ & $\begin{array}{l}1 \\
9 \\
7\end{array}$ & $\begin{array}{l}2 \\
8 \\
6\end{array}$ & $\begin{array}{l}0 . \\
6 \\
9\end{array}$ & $\begin{array}{l}0.05 \\
577\end{array}$ & $\begin{array}{c}0 . \\
00 \\
10 \\
8\end{array}$ & $\begin{array}{c}0.5 \\
556 \\
3\end{array}$ & $\begin{array}{c}0 . \\
00 \\
99 \\
3\end{array}$ & $\begin{array}{c}0.0 \\
722 \\
7\end{array}$ & $\begin{array}{c}0 . \\
00 \\
09 \\
9\end{array}$ & 443 & $\begin{array}{l}1 \\
9\end{array}$ & 449 & 6 & 450 & 6 & $\begin{array}{c}- \\
0.2 \\
2 \%\end{array}$ & $\begin{array}{l}4 \\
5 \\
0\end{array}$ & 6 \\
\hline $\begin{array}{c}\mathrm{M} \\
\mathrm{O} 2 \\
2 \mathrm{~A} \\
- \\
30 \\
\end{array}$ & $\begin{array}{l}1 \\
8 \\
8\end{array}$ & $\begin{array}{l}2 \\
7 \\
2\end{array}$ & $\begin{array}{l}0 . \\
6 \\
9\end{array}$ & $\begin{array}{l}0.07 \\
225\end{array}$ & $\begin{array}{c}0 . \\
00 \\
08 \\
9\end{array}$ & $\begin{array}{c}1.6 \\
444 \\
5\end{array}$ & $\begin{array}{c}0 . \\
01 \\
89 \\
1\end{array}$ & $\begin{array}{c}0.1 \\
650 \\
8 \\
\end{array}$ & $\begin{array}{c}0 . \\
00 \\
20 \\
3\end{array}$ & 993 & $\begin{array}{l}1 \\
1\end{array}$ & 987 & 7 & 985 & $\begin{array}{l}1 \\
1\end{array}$ & $\begin{array}{l}0.2 \\
0 \%\end{array}$ & $\begin{array}{l}9 \\
8 \\
5\end{array}$ & $\begin{array}{l}1 \\
1\end{array}$ \\
\hline $\begin{array}{c}\mathrm{M} \\
\mathrm{O} 2 \\
2 \mathrm{~A} \\
- \\
31 \\
\end{array}$ & $\begin{array}{l}7 \\
6\end{array}$ & $\begin{array}{l}1 \\
0 \\
2\end{array}$ & $\begin{array}{l}0 . \\
7 \\
4\end{array}$ & $\begin{array}{l}0.06 \\
196\end{array}$ & $\begin{array}{c}0 . \\
00 \\
16 \\
8\end{array}$ & $\begin{array}{c}0.8 \\
249 \\
2\end{array}$ & $\begin{array}{c}0 . \\
02 \\
04 \\
8\end{array}$ & $\begin{array}{c}0.0 \\
965 \\
6\end{array}$ & $\begin{array}{c}0 . \\
00 \\
16 \\
1\end{array}$ & 673 & $\begin{array}{l}2 \\
7\end{array}$ & 611 & $\begin{array}{l}1 \\
1\end{array}$ & 594 & 9 & $\begin{array}{l}2.8 \\
6 \%\end{array}$ & $\begin{array}{l}5 \\
9 \\
4\end{array}$ & 9 \\
\hline $\begin{array}{c}\mathrm{M} \\
\mathrm{O} 2 \\
2 \mathrm{~A} \\
- \\
32\end{array}$ & $\begin{array}{l}1 \\
7 \\
4\end{array}$ & $\begin{array}{l}6 \\
0 \\
3\end{array}$ & $\begin{array}{l}0 . \\
2 \\
9\end{array}$ & $\begin{array}{l}0.06 \\
219\end{array}$ & $\begin{array}{c}0 . \\
00 \\
07 \\
3\end{array}$ & $\begin{array}{c}0.8 \\
712 \\
0\end{array}$ & $\begin{array}{c}0 . \\
00 \\
96 \\
1\end{array}$ & $\begin{array}{c}0.1 \\
016 \\
0\end{array}$ & $\begin{array}{c}0 . \\
00 \\
12 \\
1\end{array}$ & 681 & $\begin{array}{l}1 \\
1\end{array}$ & 636 & 5 & 624 & 7 & $\begin{array}{l}1.9 \\
2 \%\end{array}$ & $\begin{array}{l}6 \\
2 \\
4\end{array}$ & 7 \\
\hline $\begin{array}{c}\mathrm{M} \\
\mathrm{O} 2 \\
2 \mathrm{~A} \\
- \\
33\end{array}$ & $\begin{array}{l}6 \\
5\end{array}$ & $\begin{array}{l}2 \\
7 \\
2\end{array}$ & $\begin{array}{l}0 . \\
2 \\
4\end{array}$ & $\begin{array}{l}0.04 \\
605 \\
\end{array}$ & $\begin{array}{c}0 . \\
00 \\
65 \\
3\end{array}$ & $\begin{array}{c}0.4 \\
375 \\
2\end{array}$ & $\begin{array}{c}0 . \\
06 \\
16 \\
0\end{array}$ & $\begin{array}{c}0.0 \\
689 \\
1\end{array}$ & $\begin{array}{c}0 . \\
00 \\
12 \\
2\end{array}$ & & $\begin{array}{l}2 \\
5 \\
8\end{array}$ & 369 & $\begin{array}{l}4 \\
4\end{array}$ & 430 & 7 & $\begin{array}{c}- \\
14 . \\
19 \\
\%\end{array}$ & & \\
\hline $\begin{array}{c}\mathrm{M} \\
\mathrm{O} 2 \\
2 \mathrm{~A} \\
- \\
34 \\
\end{array}$ & $\begin{array}{l}7 \\
5\end{array}$ & $\begin{array}{l}1 \\
0 \\
5\end{array}$ & $\begin{array}{l}0 . \\
7 \\
1\end{array}$ & $\begin{array}{c}0.06 \\
118\end{array}$ & $\begin{array}{c}0 . \\
00 \\
14 \\
3\end{array}$ & $\begin{array}{c}0.8 \\
978 \\
5\end{array}$ & $\begin{array}{c}0 . \\
01 \\
93 \\
2\end{array}$ & $\begin{array}{c}0.1 \\
064 \\
3\end{array}$ & $\begin{array}{c}0 . \\
00 \\
16 \\
3\end{array}$ & 646 & $\begin{array}{l}2 \\
2\end{array}$ & 651 & $\begin{array}{l}1 \\
0\end{array}$ & 652 & 9 & $\begin{array}{c}- \\
0.1 \\
5 \%\end{array}$ & $\begin{array}{l}6 \\
5 \\
2\end{array}$ & 9 \\
\hline $\begin{array}{c}\mathrm{M} \\
\mathrm{O} 2 \\
2 \mathrm{~A} \\
- \\
35 \\
\end{array}$ & $\begin{array}{l}1 \\
1 \\
8\end{array}$ & $\begin{array}{l}2 \\
1 \\
9\end{array}$ & $\begin{array}{l}0 . \\
5 \\
4\end{array}$ & $\begin{array}{c}0.06 \\
179\end{array}$ & $\begin{array}{c}0 . \\
00 \\
11 \\
4\end{array}$ & $\begin{array}{c}0.8 \\
218 \\
6\end{array}$ & $\begin{array}{c}0 . \\
01 \\
39 \\
9\end{array}$ & $\begin{array}{c}0.0 \\
964 \\
7\end{array}$ & $\begin{array}{c}0 . \\
00 \\
13 \\
2\end{array}$ & 667 & $\begin{array}{l}1 \\
7\end{array}$ & 609 & 8 & 594 & 8 & $\begin{array}{l}2.5 \\
3 \%\end{array}$ & $\begin{array}{l}5 \\
9 \\
4\end{array}$ & 8 \\
\hline $\begin{array}{c}\mathrm{M} \\
\mathrm{O} 2 \\
2 \mathrm{~A} \\
- \\
36 \\
\end{array}$ & $\begin{array}{l}1 \\
4 \\
2\end{array}$ & \begin{tabular}{|l}
3 \\
6 \\
7
\end{tabular} & $\begin{array}{l}0 \\
3 \\
9\end{array}$ & $\begin{array}{l}0.05 \\
651\end{array}$ & $\begin{array}{c}0 . \\
00 \\
09 \\
7\end{array}$ & $\begin{array}{c}0.5 \\
861 \\
4\end{array}$ & $\begin{array}{c}0 . \\
00 \\
93 \\
2\end{array}$ & $\begin{array}{c}0.0 \\
752 \\
3\end{array}$ & $\begin{array}{c}0 . \\
00 \\
09 \\
9\end{array}$ & 472 & $\begin{array}{l}1 \\
6\end{array}$ & 468 & 6 & 468 & 6 & $\begin{array}{l}0.0 \\
0 \%\end{array}$ & $\begin{array}{l}4 \\
6 \\
8\end{array}$ & 6 \\
\hline $\mathrm{M}$ & 1 & 3 & 0. & 0.05 & 0. & 0.6 & 0. & 0.0 & 0. & 557 & 1 & 479 & 7 & 462 & 6 & 3.6 & 4 & 6 \\
\hline
\end{tabular}




\begin{tabular}{|c|c|c|c|c|c|c|c|c|c|c|c|c|c|c|c|c|c|c|}
\hline $\begin{array}{c}\mathrm{O} 2 \\
2 \mathrm{~A} \\
- \\
37\end{array}$ & $\begin{array}{l}6 \\
5\end{array}$ & $\begin{array}{l}0 \\
1\end{array}$ & $\begin{array}{l}5 \\
5\end{array}$ & 874 & $\begin{array}{c}00 \\
10 \\
9\end{array}$ & $\begin{array}{c}022 \\
3\end{array}$ & $\begin{array}{c}01 \\
03 \\
5\end{array}$ & $\begin{array}{c}743 \\
6\end{array}$ & $\begin{array}{c}00 \\
10 \\
1\end{array}$ & & 7 & & & & & $8 \%$ & $\begin{array}{l}6 \\
2\end{array}$ & \\
\hline $\begin{array}{c}\mathrm{M} \\
\mathrm{O} 2 \\
2 \mathrm{~A} \\
- \\
38\end{array}$ & $\begin{array}{l}6 \\
7\end{array}$ & $\begin{array}{l}2 \\
4 \\
6\end{array}$ & $\begin{array}{l}0 . \\
2 \\
7\end{array}$ & $\begin{array}{l}0.06 \\
529\end{array}$ & $\begin{array}{c}0 . \\
00 \\
09 \\
7\end{array}$ & $\begin{array}{c}1.0 \\
795 \\
0\end{array}$ & $\begin{array}{c}0 . \\
01 \\
49 \\
0\end{array}$ & $\begin{array}{c}0.1 \\
199 \\
3\end{array}$ & $\begin{array}{c}0 . \\
00 \\
15 \\
4\end{array}$ & 784 & $\begin{array}{l}1 \\
3\end{array}$ & 743 & 7 & 730 & 9 & $\begin{array}{l}1.7 \\
8 \%\end{array}$ & $\begin{array}{l}7 \\
3 \\
0\end{array}$ & 9 \\
\hline $\begin{array}{c}\mathrm{M} \\
\mathrm{O} 2 \\
2 \mathrm{~A} \\
- \\
39\end{array}$ & $\begin{array}{l}8 \\
7\end{array}$ & $\begin{array}{l}2 \\
4 \\
3\end{array}$ & $\begin{array}{l}0 . \\
3 \\
6\end{array}$ & $\begin{array}{l}0.05 \\
425\end{array}$ & $\begin{array}{c}0 . \\
00 \\
11 \\
5\end{array}$ & $\begin{array}{c}0.5 \\
577 \\
6\end{array}$ & $\begin{array}{c}0 . \\
01 \\
09 \\
6\end{array}$ & $\begin{array}{c}0.0 \\
745 \\
7\end{array}$ & $\begin{array}{c}0 . \\
00 \\
10 \\
5\end{array}$ & 381 & $\begin{array}{l}2 \\
1\end{array}$ & 450 & 7 & 464 & 6 & $\begin{array}{c}- \\
3.0 \\
2 \%\end{array}$ & $\begin{array}{l}4 \\
6 \\
4\end{array}$ & 6 \\
\hline $\begin{array}{c}\mathrm{M} \\
\mathrm{O} 2 \\
2 \mathrm{~A} \\
- \\
40\end{array}$ & $\begin{array}{l}1 \\
1 \\
4\end{array}$ & $\begin{array}{l}9 \\
8\end{array}$ & $\begin{array}{l}. \\
1 \\
6\end{array}$ & $\begin{array}{l}0.13 \\
283\end{array}$ & $\begin{array}{c}0 . \\
00 \\
15 \\
3\end{array}$ & $\begin{array}{c}7.0 \\
824 \\
2\end{array}$ & $\begin{array}{c}0 . \\
07 \\
73 \\
6\end{array}$ & $\begin{array}{c}0.3 \\
867 \\
1\end{array}$ & $\begin{array}{c}0 . \\
00 \\
51 \\
1\end{array}$ & $\begin{array}{c}213 \\
6\end{array}$ & $\begin{array}{l}1 \\
0\end{array}$ & $\begin{array}{c}212 \\
2\end{array}$ & $\begin{array}{l}1 \\
0\end{array}$ & $\begin{array}{c}210 \\
8\end{array}$ & $\begin{array}{l}2 \\
4\end{array}$ & $\begin{array}{l}1.3 \\
3 \%\end{array}$ & $\begin{array}{l}2 \\
1 \\
3 \\
6\end{array}$ & $\begin{array}{l}1 \\
0\end{array}$ \\
\hline $\begin{array}{c}\mathrm{M} \\
\mathrm{O} 2 \\
2 \mathrm{~A} \\
- \\
41\end{array}$ & $\begin{array}{l}2 \\
6 \\
3\end{array}$ & $\begin{array}{l}7 \\
4 \\
8\end{array}$ & $\begin{array}{l}0 . \\
3 \\
5\end{array}$ & $\begin{array}{c}0.12 \\
110\end{array}$ & $\begin{array}{c}0 . \\
00 \\
07 \\
5\end{array}$ & $\begin{array}{c}5.9 \\
907 \\
4\end{array}$ & $\begin{array}{c}0 . \\
03 \\
75 \\
8\end{array}$ & $\begin{array}{c}0.3 \\
587 \\
8\end{array}$ & $\begin{array}{c}0 . \\
00 \\
39 \\
7\end{array}$ & $\begin{array}{c}197 \\
2\end{array}$ & $\begin{array}{l}1 \\
1\end{array}$ & $\begin{array}{c}197 \\
5\end{array}$ & 5 & $\begin{array}{c}197 \\
6\end{array}$ & $\begin{array}{l}1 \\
9\end{array}$ & $\begin{array}{c}- \\
0.2 \\
0 \%\end{array}$ & $\begin{array}{l}1 \\
9 \\
7 \\
2\end{array}$ & $\begin{array}{l}1 \\
1\end{array}$ \\
\hline $\begin{array}{c}\mathrm{M} \\
\mathrm{O} 2 \\
2 \mathrm{~A} \\
- \\
42\end{array}$ & $\begin{array}{l}4 \\
4\end{array}$ & $\begin{array}{l}1 \\
5 \\
3\end{array}$ & $\begin{array}{l}0 . \\
2 \\
9\end{array}$ & $\begin{array}{l}0.05 \\
588\end{array}$ & $\begin{array}{c}0 . \\
00 \\
14 \\
1\end{array}$ & $\begin{array}{c}0.5 \\
723 \\
7 \\
\end{array}$ & $\begin{array}{c}0 . \\
01 \\
32 \\
5\end{array}$ & $\begin{array}{c}0.0 \\
743 \\
0\end{array}$ & $\begin{array}{c}0 . \\
00 \\
11 \\
5\end{array}$ & 448 & $\begin{array}{l}2 \\
6\end{array}$ & 460 & 9 & 462 & 7 & $\begin{array}{c}- \\
0.4 \\
3 \%\end{array}$ & $\begin{array}{l}4 \\
6 \\
2\end{array}$ & 7 \\
\hline $\begin{array}{c}\mathrm{M} \\
\mathrm{O} 2 \\
2 \mathrm{~A} \\
- \\
43 \\
\end{array}$ & $\begin{array}{l}4 \\
9\end{array}$ & $\begin{array}{l}3 \\
6\end{array}$ & $\begin{array}{l}1 . \\
3 \\
8\end{array}$ & $\begin{array}{l}0.06 \\
785\end{array}$ & $\begin{array}{c}0 . \\
00 \\
25 \\
9\end{array}$ & $\begin{array}{c}1.1 \\
943 \\
6\end{array}$ & $\begin{array}{c}0 . \\
04 \\
16 \\
8\end{array}$ & $\begin{array}{c}0.1 \\
276 \\
7\end{array}$ & $\begin{array}{c}0 . \\
00 \\
27 \\
6\end{array}$ & 864 & $\begin{array}{l}3 \\
8\end{array}$ & 798 & $\begin{array}{l}1 \\
9\end{array}$ & 775 & $\begin{array}{l}1 \\
6\end{array}$ & $\begin{array}{l}2.9 \\
7 \%\end{array}$ & $\begin{array}{l}7 \\
7 \\
5\end{array}$ & $\begin{array}{l}1 \\
6\end{array}$ \\
\hline $\begin{array}{c}M \\
\mathrm{O} 2 \\
2 \mathrm{~A} \\
- \\
44\end{array}$ & $\begin{array}{l}4 \\
5\end{array}$ & $\begin{array}{l}1 \\
3 \\
1\end{array}$ & $\begin{array}{l}0 . \\
3 \\
4\end{array}$ & $\begin{array}{l}0.05 \\
782\end{array}$ & $\begin{array}{c}0 . \\
00 \\
17 \\
3\end{array}$ & $\begin{array}{c}0.6 \\
051 \\
3\end{array}$ & $\begin{array}{c}0 . \\
01 \\
66 \\
0\end{array}$ & $\begin{array}{c}0.0 \\
759 \\
1\end{array}$ & $\begin{array}{c}0 . \\
00 \\
13 \\
0\end{array}$ & 523 & $\begin{array}{l}3 \\
2\end{array}$ & 480 & $\begin{array}{l}1 \\
1\end{array}$ & 472 & 8 & $\begin{array}{l}1.6 \\
9 \%\end{array}$ & $\begin{array}{l}4 \\
7 \\
2\end{array}$ & 8 \\
\hline $\begin{array}{c}\mathrm{M} \\
\mathrm{O} 2 \\
2 \mathrm{~A} \\
- \\
45\end{array}$ & $\begin{array}{l}1 \\
6 \\
3\end{array}$ & $\begin{array}{l}1 \\
5 \\
5\end{array}$ & $\begin{array}{l}1 . \\
0 \\
5\end{array}$ & $\begin{array}{c}0.05 \\
938\end{array}$ & $\begin{array}{c}0 . \\
00 \\
12 \\
6\end{array}$ & $\begin{array}{c}0.8 \\
213 \\
2\end{array}$ & $\begin{array}{c}0 . \\
01 \\
60 \\
9\end{array}$ & $\begin{array}{c}0.1 \\
003 \\
1\end{array}$ & $\begin{array}{c}0 . \\
00 \\
14 \\
5\end{array}$ & 581 & $\begin{array}{l}2 \\
0\end{array}$ & 609 & 9 & 616 & 8 & $\begin{array}{c}- \\
1.1 \\
4 \%\end{array}$ & $\begin{array}{l}6 \\
1 \\
6\end{array}$ & 8 \\
\hline $\begin{array}{c}\mathrm{M} \\
\mathrm{O} 2 \\
2 \mathrm{~A} \\
- \\
46\end{array}$ & $\begin{array}{l}9 \\
6\end{array}$ & $\begin{array}{l}2 \\
7 \\
7\end{array}$ & $\begin{array}{l}0 . \\
3 \\
5\end{array}$ & $\begin{array}{c}0.06 \\
151\end{array}$ & $\begin{array}{c}0 . \\
00 \\
09 \\
7\end{array}$ & $\begin{array}{c}0.8 \\
521 \\
0\end{array}$ & $\begin{array}{c}0 . \\
01 \\
24 \\
9\end{array}$ & $\begin{array}{c}0.1 \\
004 \\
8\end{array}$ & $\begin{array}{c}0 . \\
00 \\
13 \\
0\end{array}$ & 657 & $\begin{array}{l}1 \\
4\end{array}$ & 626 & 7 & 617 & 8 & $\begin{array}{l}1.4 \\
6 \%\end{array}$ & $\begin{array}{l}6 \\
1 \\
7\end{array}$ & 8 \\
\hline $\begin{array}{c}\mathrm{M} \\
\mathrm{O} 2 \\
2 \mathrm{~A} \\
- \\
47 \\
\end{array}$ & $\begin{array}{l}7 \\
6\end{array}$ & $\begin{array}{l}1 \\
6 \\
3\end{array}$ & $\begin{array}{l}0 . \\
4 \\
6\end{array}$ & $\begin{array}{c}0.84 \\
080\end{array}$ & $\begin{array}{c}0 . \\
00 \\
45 \\
2\end{array}$ & $\begin{array}{c}271 \\
.68 \\
826\end{array}$ & $\begin{array}{c}1 . \\
72 \\
71 \\
6\end{array}$ & $\begin{array}{c}2.3 \\
436 \\
4\end{array}$ & $\begin{array}{c}0 . \\
02 \\
67 \\
3\end{array}$ & $\begin{array}{c}499 \\
2\end{array}$ & 9 & $\begin{array}{c}569 \\
5\end{array}$ & 6 & $\begin{array}{c}778 \\
1\end{array}$ & $\begin{array}{l}5 \\
2\end{array}$ & $\begin{array}{c}- \\
35 \\
84 \\
\%\end{array}$ & & \\
\hline
\end{tabular}




\begin{tabular}{|c|c|c|c|c|c|c|c|c|c|c|c|c|c|c|c|c|c|c|}
\hline $\begin{array}{c}\mathrm{M} \\
\mathrm{O} 2 \\
2 \mathrm{~A} \\
- \\
48\end{array}$ & $\begin{array}{l}3 \\
2\end{array}$ & $\begin{array}{l}2 \\
5 \\
3\end{array}$ & $\begin{array}{c}0 . \\
1 \\
3\end{array}$ & $\begin{array}{c}0.05 \\
940\end{array}$ & $\begin{array}{c}0 . \\
00 \\
11 \\
1\end{array}$ & $\begin{array}{c}0.6 \\
594 \\
8\end{array}$ & $\begin{array}{c}0 . \\
01 \\
13 \\
6\end{array}$ & $\begin{array}{c}0.0 \\
805 \\
2\end{array}$ & $\begin{array}{c}0 . \\
00 \\
10 \\
9\end{array}$ & 582 & $\begin{array}{l}1 \\
7\end{array}$ & 514 & 7 & 499 & 7 & $\begin{array}{l}3.0 \\
1 \%\end{array}$ & $\begin{array}{l}4 \\
9 \\
9\end{array}$ & 7 \\
\hline $\begin{array}{c}\mathrm{M} \\
\mathrm{O} 2 \\
2 \mathrm{~A} \\
- \\
49\end{array}$ & $\begin{array}{l}9 \\
4\end{array}$ & $\begin{array}{l}2 \\
6 \\
0\end{array}$ & $\begin{array}{l}0 . \\
3 \\
6\end{array}$ & $\begin{array}{l}0.05 \\
842\end{array}$ & $\begin{array}{c}0 . \\
00 \\
10 \\
5\end{array}$ & $\begin{array}{c}0.6 \\
938 \\
4\end{array}$ & $\begin{array}{c}0 . \\
01 \\
15 \\
6\end{array}$ & $\begin{array}{c}0.0 \\
861 \\
4\end{array}$ & $\begin{array}{c}0 . \\
00 \\
11 \\
5\end{array}$ & 546 & $\begin{array}{l}1 \\
7\end{array}$ & 535 & 7 & 533 & 7 & $\begin{array}{l}0.3 \\
8 \%\end{array}$ & $\begin{array}{l}5 \\
3 \\
3\end{array}$ & 7 \\
\hline $\begin{array}{c}\mathrm{M} \\
\mathrm{O} 2 \\
2 \mathrm{~A} \\
- \\
50\end{array}$ & $\begin{array}{l}1 \\
0 \\
4\end{array}$ & $\begin{array}{l}8 \\
8\end{array}$ & $\begin{array}{l}1 . \\
1 \\
9\end{array}$ & $\begin{array}{c}0.08 \\
403\end{array}$ & $\begin{array}{c}0 . \\
00 \\
37 \\
1\end{array}$ & $\begin{array}{c}1.9 \\
854 \\
7\end{array}$ & $\begin{array}{c}0 . \\
08 \\
12 \\
5\end{array}$ & $\begin{array}{c}0.1 \\
713 \\
6\end{array}$ & $\begin{array}{c}0 . \\
00 \\
28 \\
1\end{array}$ & $\begin{array}{c}129 \\
3\end{array}$ & $\begin{array}{l}8 \\
8\end{array}$ & $\begin{array}{c}111 \\
1\end{array}$ & $\begin{array}{l}2 \\
8\end{array}$ & $\begin{array}{c}102 \\
0\end{array}$ & $\begin{array}{l}1 \\
5\end{array}$ & $\begin{array}{l}26 . \\
76 \\
\%\end{array}$ & & \\
\hline $\begin{array}{c}\mathrm{M} \\
\mathrm{O} 2 \\
2 \mathrm{~A} \\
- \\
51\end{array}$ & $\begin{array}{l}1 \\
7\end{array}$ & $\begin{array}{l}3 \\
9 \\
5\end{array}$ & $\begin{array}{l}0 . \\
0 \\
4\end{array}$ & $\begin{array}{l}0.12 \\
394\end{array}$ & $\begin{array}{c}0 . \\
00 \\
08 \\
9\end{array}$ & $\begin{array}{c}6.5 \\
959 \\
8\end{array}$ & $\begin{array}{c}0 . \\
04 \\
71 \\
6\end{array}$ & $\begin{array}{c}0.3 \\
860 \\
0\end{array}$ & $\begin{array}{c}0 . \\
00 \\
43 \\
8\end{array}$ & $\begin{array}{c}201 \\
4\end{array}$ & $\begin{array}{l}1 \\
1\end{array}$ & $\begin{array}{c}205 \\
9\end{array}$ & 6 & $\begin{array}{c}210 \\
4\end{array}$ & $\begin{array}{l}2 \\
0\end{array}$ & $\begin{array}{c}- \\
4.2 \\
8 \%\end{array}$ & $\begin{array}{l}2 \\
0 \\
1 \\
4\end{array}$ & $\begin{array}{l}1 \\
1\end{array}$ \\
\hline $\begin{array}{c}\mathrm{M} \\
\mathrm{O} 2 \\
2 \mathrm{~A} \\
- \\
52\end{array}$ & $\begin{array}{l}1 \\
6 \\
3\end{array}$ & $\begin{array}{l}1 \\
8 \\
5\end{array}$ & $\begin{array}{l}0 . \\
8 \\
8\end{array}$ & $\begin{array}{c}0.06 \\
105\end{array}$ & $\begin{array}{c}0 . \\
00 \\
11 \\
9\end{array}$ & $\begin{array}{c}0.8 \\
588 \\
5\end{array}$ & $\begin{array}{c}0 . \\
01 \\
54 \\
5\end{array}$ & $\begin{array}{c}0.1 \\
020 \\
4\end{array}$ & $\begin{array}{c}0 . \\
00 \\
14 \\
2\end{array}$ & 641 & $\begin{array}{l}1 \\
8\end{array}$ & 629 & 8 & 626 & 8 & $\begin{array}{l}0.4 \\
8 \%\end{array}$ & $\begin{array}{l}6 \\
2 \\
6\end{array}$ & 8 \\
\hline $\begin{array}{c}\mathrm{M} \\
\mathrm{O} 2 \\
2 \mathrm{~A} \\
- \\
53\end{array}$ & $\begin{array}{l}9 \\
8\end{array}$ & $\begin{array}{l}2 \\
9 \\
7\end{array}$ & $\begin{array}{l}0 . \\
3 \\
3\end{array}$ & $\begin{array}{c}0.06 \\
034\end{array}$ & $\begin{array}{c}0 . \\
00 \\
11 \\
4\end{array}$ & $\begin{array}{c}0.6 \\
086 \\
7\end{array}$ & $\begin{array}{c}0 . \\
01 \\
05 \\
6\end{array}$ & $\begin{array}{c}0.0 \\
731 \\
7\end{array}$ & $\begin{array}{c}0 . \\
00 \\
10 \\
0\end{array}$ & 616 & $\begin{array}{l}1 \\
7\end{array}$ & 483 & 7 & 455 & 6 & $\begin{array}{l}6.1 \\
5 \%\end{array}$ & $\begin{array}{l}4 \\
5 \\
5\end{array}$ & 6 \\
\hline $\begin{array}{c}\mathrm{M} \\
\mathrm{O} 2 \\
2 \mathrm{~A} \\
- \\
54\end{array}$ & $\begin{array}{l}4 \\
0\end{array}$ & $\begin{array}{l}8 \\
1\end{array}$ & $\begin{array}{l}0 . \\
4 \\
9\end{array}$ & $\begin{array}{c}0.06 \\
044\end{array}$ & $\begin{array}{c}0 . \\
00 \\
17 \\
3\end{array}$ & $\begin{array}{c}0.8 \\
621 \\
8\end{array}$ & $\begin{array}{c}0 . \\
02 \\
27 \\
7\end{array}$ & $\begin{array}{c}0.1 \\
034 \\
7\end{array}$ & $\begin{array}{c}0 . \\
00 \\
17 \\
6\end{array}$ & 619 & $\begin{array}{l}2 \\
9\end{array}$ & 631 & $\begin{array}{l}1 \\
2\end{array}$ & 635 & $\begin{array}{l}1 \\
0\end{array}$ & $\begin{array}{c}- \\
0.6 \\
3 \%\end{array}$ & $\begin{array}{l}6 \\
3 \\
5\end{array}$ & $\begin{array}{l}1 \\
0\end{array}$ \\
\hline $\begin{array}{c}\mathrm{M} \\
\mathrm{O} 2 \\
2 \mathrm{~A} \\
- \\
55\end{array}$ & $\begin{array}{l}1 \\
4 \\
0\end{array}$ & $\begin{array}{l}2 \\
0 \\
5\end{array}$ & $\begin{array}{l}0 . \\
6 \\
8\end{array}$ & $\begin{array}{l}0.06 \\
829\end{array}$ & $\begin{array}{c}0 . \\
00 \\
10 \\
5\end{array}$ & $\begin{array}{c}1.3 \\
120 \\
6\end{array}$ & $\begin{array}{c}0 . \\
01 \\
87 \\
3\end{array}$ & $\begin{array}{c}0.1 \\
393 \\
6\end{array}$ & $\begin{array}{c}0 . \\
00 \\
18 \\
1\end{array}$ & 877 & $\begin{array}{l}1 \\
3\end{array}$ & 851 & 8 & 841 & $\begin{array}{l}1 \\
0\end{array}$ & $\begin{array}{l}1.1 \\
9 \%\end{array}$ & $\begin{array}{l}8 \\
4 \\
1\end{array}$ & $\begin{array}{l}1 \\
0\end{array}$ \\
\hline $\begin{array}{c}\mathrm{M} \\
\mathrm{O} 2 \\
2 \mathrm{~A} \\
- \\
56\end{array}$ & $\begin{array}{l}3 \\
5\end{array}$ & $\begin{array}{l}4 \\
4\end{array}$ & $\begin{array}{l}0 . \\
8 \\
0\end{array}$ & $\begin{array}{l}0.11 \\
796\end{array}$ & $\begin{array}{c}0 . \\
00 \\
28 \\
6\end{array}$ & $\begin{array}{c}2.9 \\
077 \\
6\end{array}$ & $\begin{array}{c}0 . \\
06 \\
20 \\
1\end{array}$ & $\begin{array}{c}0.1 \\
787 \\
9\end{array}$ & $\begin{array}{c}0 . \\
00 \\
32 \\
9\end{array}$ & $\begin{array}{c}192 \\
6\end{array}$ & $\begin{array}{l}1 \\
7\end{array}$ & $\begin{array}{c}138 \\
4\end{array}$ & $\begin{array}{l}1 \\
6\end{array}$ & $\begin{array}{c}106 \\
0\end{array}$ & $\begin{array}{l}1 \\
8\end{array}$ & $\begin{array}{l}81 . \\
70 \\
\%\end{array}$ & & \\
\hline $\begin{array}{c}\mathrm{M} \\
\mathrm{O} 2 \\
2 \mathrm{~A} \\
- \\
57\end{array}$ & $\begin{array}{l}8 \\
2\end{array}$ & $\begin{array}{l}2 \\
4 \\
9\end{array}$ & $\begin{array}{l}0 . \\
3 \\
3\end{array}$ & $\begin{array}{c}0.05 \\
595\end{array}$ & $\begin{array}{c}0 . \\
00 \\
13 \\
3\end{array}$ & $\begin{array}{c}0.5 \\
583 \\
1\end{array}$ & $\begin{array}{c}0 . \\
01 \\
21 \\
9\end{array}$ & $\begin{array}{c}0.0 \\
723 \\
8\end{array}$ & $\begin{array}{c}0 . \\
00 \\
10 \\
8\end{array}$ & 450 & $\begin{array}{l}2 \\
4\end{array}$ & 450 & 8 & 450 & 6 & $\begin{array}{l}0.0 \\
0 \%\end{array}$ & $\begin{array}{l}4 \\
5 \\
0\end{array}$ & 6 \\
\hline $\begin{array}{c}\mathrm{M} \\
\mathrm{O} 2 \\
2 \mathrm{~A} \\
-\end{array}$ & $\begin{array}{l}1 \\
3 \\
4\end{array}$ & $\begin{array}{l}2 \\
7 \\
9\end{array}$ & $\begin{array}{l}0 . \\
4 \\
8\end{array}$ & $\begin{array}{c}0.05 \\
848\end{array}$ & $\begin{array}{c}0 . \\
00 \\
18 \\
1 \\
\end{array}$ & $\begin{array}{c}0.5 \\
632 \\
4\end{array}$ & $\begin{array}{c}0 . \\
01 \\
59 \\
4\end{array}$ & $\begin{array}{c}0.0 \\
698 \\
5\end{array}$ & $\begin{array}{c}0 . \\
00 \\
12 \\
3 \\
\end{array}$ & 548 & $\begin{array}{l}3 \\
3\end{array}$ & 454 & $\begin{array}{l}1 \\
0\end{array}$ & 435 & 7 & $\begin{array}{l}4.3 \\
7 \%\end{array}$ & $\begin{array}{l}4 \\
3 \\
5\end{array}$ & 7 \\
\hline
\end{tabular}




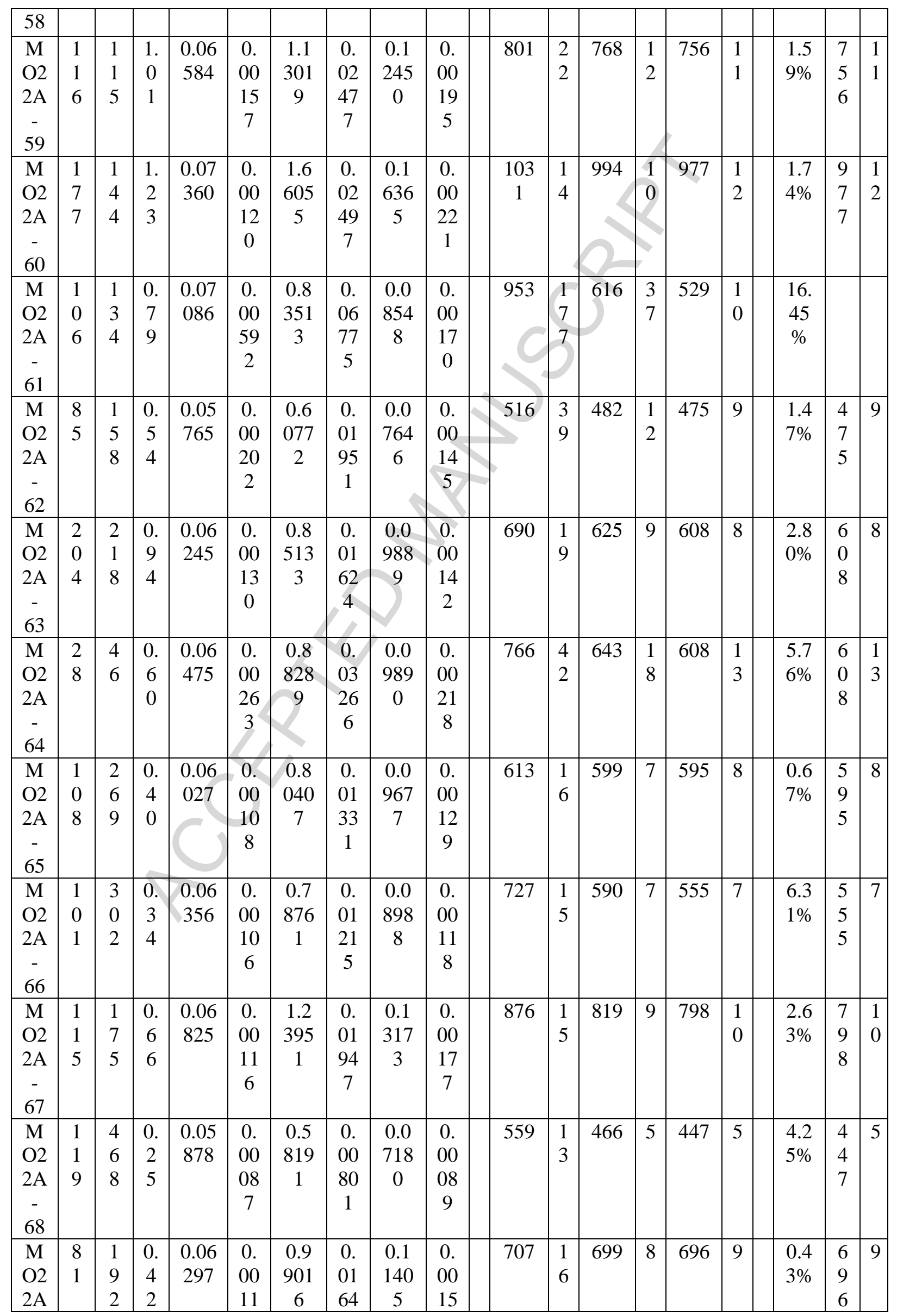




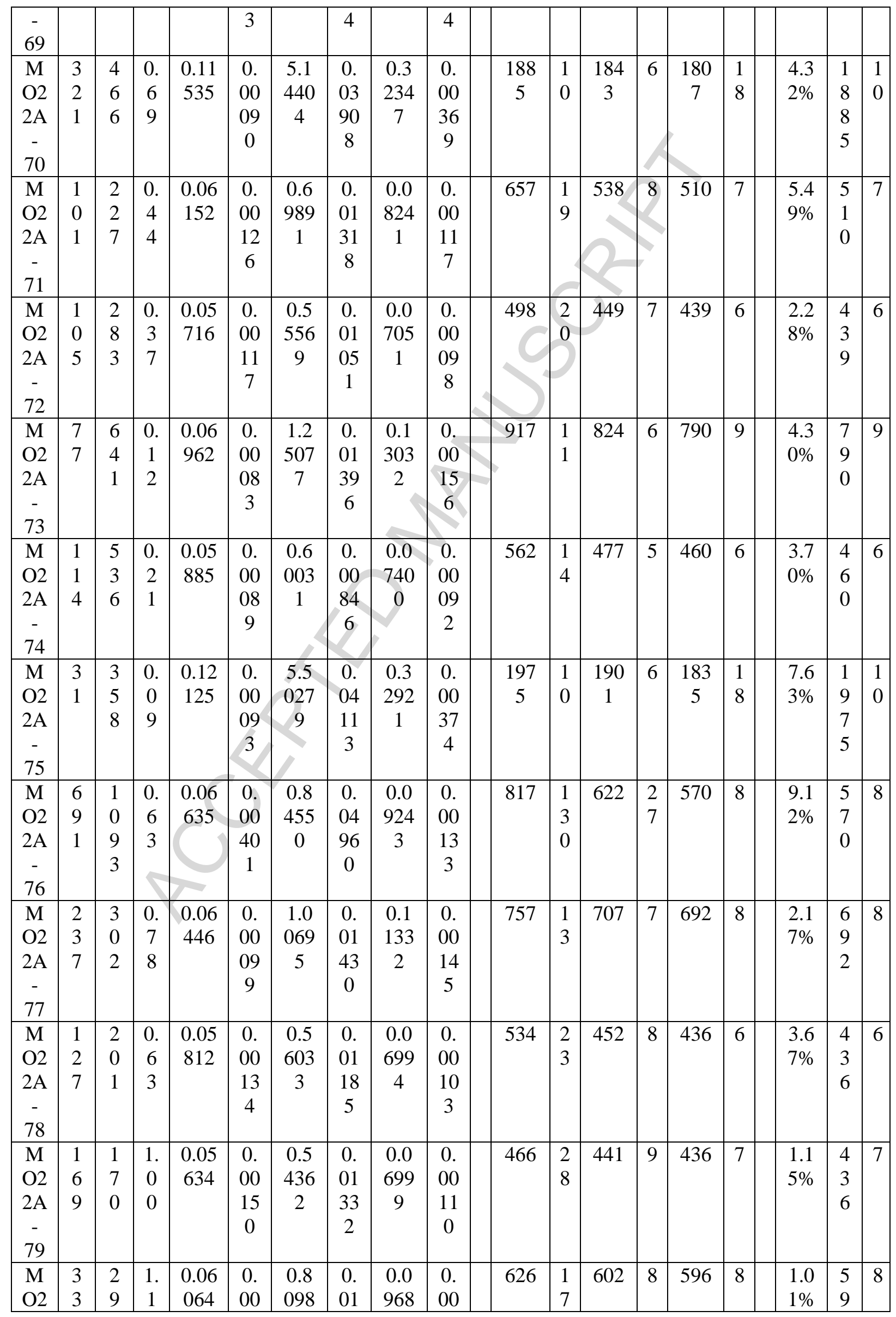




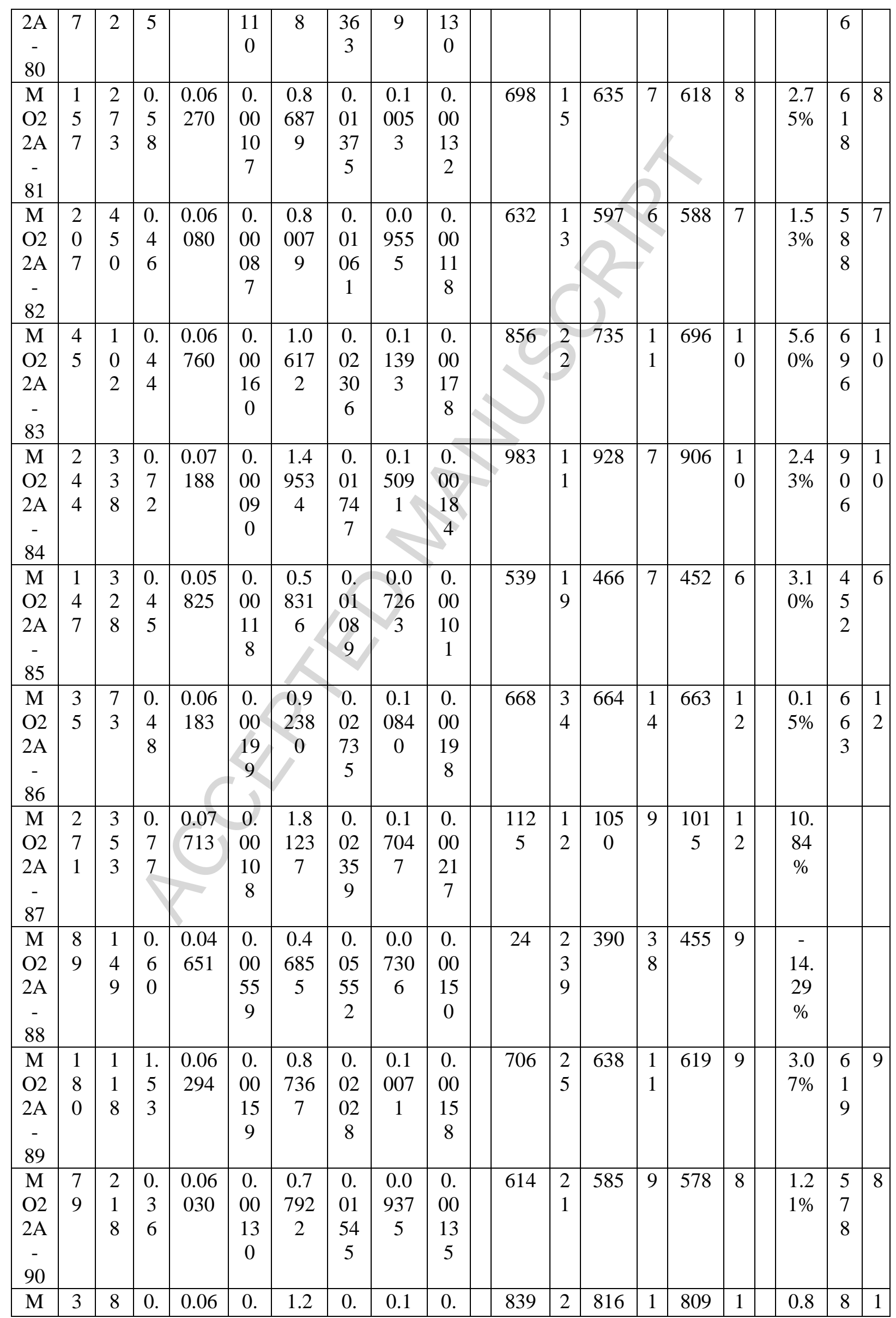




\begin{tabular}{|c|c|c|c|c|c|c|c|c|c|c|c|c|c|c|c|c|c|c|}
\hline $\begin{array}{c}\mathrm{O} 2 \\
2 \mathrm{~A} \\
- \\
91\end{array}$ & 3 & 8 & $\begin{array}{l}3 \\
7\end{array}$ & 703 & $\begin{array}{c}00 \\
16 \\
5\end{array}$ & $\begin{array}{c}347 \\
5\end{array}$ & $\begin{array}{c}02 \\
79 \\
7\end{array}$ & $\begin{array}{c}336 \\
4\end{array}$ & $\begin{array}{c}00 \\
21 \\
4\end{array}$ & & 3 & & 3 & & 2 & $7 \%$ & $\begin{array}{l}0 \\
9\end{array}$ & 2 \\
\hline $\begin{array}{c}\mathrm{M} \\
\mathrm{O} 2 \\
2 \mathrm{~A} \\
- \\
92\end{array}$ & $\begin{array}{l}3 \\
3 \\
8\end{array}$ & $\begin{array}{l}1 \\
1 \\
7\end{array}$ & $\begin{array}{l}2 . \\
9 \\
0\end{array}$ & $\begin{array}{c}0.06 \\
000\end{array}$ & $\begin{array}{c}0 . \\
00 \\
16 \\
2\end{array}$ & $\begin{array}{c}0.7 \\
923 \\
0\end{array}$ & $\begin{array}{c}0 . \\
01 \\
97 \\
4\end{array}$ & $\begin{array}{c}0.0 \\
958 \\
1\end{array}$ & $\begin{array}{c}0 . \\
00 \\
15 \\
5\end{array}$ & 604 & $\begin{array}{l}2 \\
8\end{array}$ & 592 & $\begin{array}{l}1 \\
1\end{array}$ & 590 & 9 & $\begin{array}{l}0.3 \\
4 \%\end{array}$ & $\begin{array}{l}5 \\
9 \\
0\end{array}$ & 9 \\
\hline $\begin{array}{c}\mathrm{M} \\
\mathrm{O} 2 \\
2 \mathrm{~A} \\
- \\
93\end{array}$ & $\begin{array}{l}1 \\
3 \\
3\end{array}$ & $\begin{array}{l}3 \\
0 \\
4\end{array}$ & $\begin{array}{l}0 . \\
4 \\
4\end{array}$ & $\begin{array}{c}0.06 \\
183\end{array}$ & $\begin{array}{c}0 . \\
00 \\
11 \\
0\end{array}$ & $\begin{array}{c}0.7 \\
869 \\
0\end{array}$ & $\begin{array}{c}0 . \\
01 \\
29 \\
4\end{array}$ & $\begin{array}{c}0.0 \\
923 \\
4\end{array}$ & $\begin{array}{c}0 . \\
00 \\
12 \\
3\end{array}$ & 668 & $\begin{array}{l}1 \\
6\end{array}$ & 589 & 7 & 569 & 7 & $\begin{array}{l}3.5 \\
1 \%\end{array}$ & $\begin{array}{l}5 \\
6 \\
9\end{array}$ & 7 \\
\hline $\begin{array}{c}\mathrm{M} \\
\mathrm{O} 2 \\
2 \mathrm{~A} \\
- \\
94\end{array}$ & $\begin{array}{l}1 \\
2 \\
9\end{array}$ & $\begin{array}{l}2 \\
8 \\
2\end{array}$ & $\begin{array}{l}0 . \\
4 \\
6\end{array}$ & $\begin{array}{l}0.06 \\
776\end{array}$ & $\begin{array}{c}0 . \\
00 \\
11 \\
4\end{array}$ & $\begin{array}{c}1.2 \\
388 \\
4\end{array}$ & $\begin{array}{c}0 . \\
01 \\
92 \\
4\end{array}$ & $\begin{array}{c}0.1 \\
326 \\
5\end{array}$ & $\begin{array}{c}0 . \\
00 \\
17 \\
6\end{array}$ & 861 & $\begin{array}{l}1 \\
4\end{array}$ & 818 & 9 & 803 & $\begin{array}{l}1 \\
0\end{array}$ & $\begin{array}{l}1.8 \\
7 \%\end{array}$ & $\begin{array}{l}8 \\
0 \\
3\end{array}$ & $\begin{array}{l}1 \\
0\end{array}$ \\
\hline $\begin{array}{c}\mathrm{M} \\
\mathrm{O} 2 \\
2 \mathrm{~A} \\
- \\
95 \\
\end{array}$ & $\begin{array}{l}2 \\
2 \\
4\end{array}$ & $\begin{array}{l}2 \\
2 \\
7\end{array}$ & $\begin{array}{l}0 . \\
9 \\
9\end{array}$ & $\begin{array}{c}0.06 \\
900\end{array}$ & $\begin{array}{c}0 . \\
00 \\
13 \\
0\end{array}$ & $\begin{array}{c}0.8 \\
583 \\
8\end{array}$ & $\begin{array}{c}0 . \\
01 \\
47 \\
7\end{array}$ & $\begin{array}{c}0.0 \\
902 \\
7\end{array}$ & $\begin{array}{c}0 . \\
00 \\
12 \\
5\end{array}$ & 899 & $\begin{array}{l}1 \\
6\end{array}$ & 629 & 8 & 557 & 7 & $\begin{array}{l}12 . \\
93 \\
\%\end{array}$ & & \\
\hline $\begin{array}{c}\mathrm{M} \\
\mathrm{O} 2 \\
2 \mathrm{~A} \\
- \\
96\end{array}$ & $\begin{array}{l}7 \\
4\end{array}$ & $\begin{array}{l}1 \\
3 \\
6\end{array}$ & $\begin{array}{l}0 . \\
5 \\
4\end{array}$ & $\begin{array}{c}0.06 \\
175\end{array}$ & $\begin{array}{c}0 . \\
00 \\
15 \\
3\end{array}$ & $\begin{array}{c}0.8 \\
479 \\
6\end{array}$ & $\begin{array}{c}0 . \\
01 \\
92 \\
8\end{array}$ & $\begin{array}{c}0.0 \\
996 \\
4\end{array}$ & $\begin{array}{c}0 . \\
00 \\
15 \\
5\end{array}$ & 665 & $\begin{array}{l}2 \\
4\end{array}$ & 624 & $\begin{array}{l}1 \\
1\end{array}$ & 612 & 9 & $\begin{array}{l}1.9 \\
6 \%\end{array}$ & $\begin{array}{l}6 \\
1 \\
2\end{array}$ & 9 \\
\hline $\begin{array}{c}\mathrm{M} \\
\mathrm{O} 2 \\
2 \mathrm{~A} \\
- \\
97\end{array}$ & $\begin{array}{l}1 \\
4 \\
2\end{array}$ & $\begin{array}{l}1 \\
9 \\
2\end{array}$ & $\begin{array}{l}0 . \\
7 \\
4\end{array}$ & $\begin{array}{c}0.06 \\
595\end{array}$ & $\begin{array}{c}0 . \\
00 \\
12 \\
9\end{array}$ & $\begin{array}{c}1.0 \\
760 \\
4\end{array}$ & $\begin{array}{c}0 . \\
01 \\
93 \\
5\end{array}$ & $\begin{array}{c}0.1 \\
183 \\
8\end{array}$ & $\begin{array}{c}0 . \\
00 \\
16 \\
6\end{array}$ & 805 & $\begin{array}{l}1 \\
7\end{array}$ & 742 & 9 & 721 & $\begin{array}{l}1 \\
0\end{array}$ & $\begin{array}{l}2.9 \\
1 \%\end{array}$ & $\begin{array}{l}7 \\
2 \\
1\end{array}$ & $\begin{array}{l}1 \\
0\end{array}$ \\
\hline $\begin{array}{c}\mathrm{M} \\
\mathrm{O} 2 \\
2 \mathrm{~A} \\
- \\
98\end{array}$ & $\begin{array}{l}3 \\
5 \\
8\end{array}$ & $\begin{array}{l}2 \\
1 \\
9\end{array}$ & $\begin{array}{l}1 . \\
6 \\
3\end{array}$ & $\begin{array}{l}0.07 \\
641\end{array}$ & $\begin{array}{c}0 . \\
00 \\
12 \\
2\end{array}$ & $\begin{array}{c}1.8 \\
068 \\
7\end{array}$ & $\begin{array}{c}0 . \\
02 \\
66 \\
2\end{array}$ & $\begin{array}{c}0.1 \\
715 \\
8\end{array}$ & $\begin{array}{c}0 . \\
00 \\
22 \\
9\end{array}$ & $\begin{array}{c}110 \\
6\end{array}$ & $\begin{array}{l}1 \\
3\end{array}$ & $\begin{array}{c}104 \\
8\end{array}$ & $\begin{array}{l}1 \\
0\end{array}$ & $\begin{array}{c}102 \\
1\end{array}$ & $\begin{array}{l}1 \\
3\end{array}$ & $\begin{array}{l}8.3 \\
3 \%\end{array}$ & $\begin{array}{l}1 \\
1 \\
0 \\
6\end{array}$ & $\begin{array}{l}1 \\
3\end{array}$ \\
\hline $\begin{array}{c}\mathrm{M} \\
\mathrm{O} 2 \\
2 \mathrm{~A} \\
- \\
99\end{array}$ & $\begin{array}{l}2 \\
2 \\
8\end{array}$ & $\begin{array}{l}2 \\
4 \\
2\end{array}$ & $\begin{array}{l}0 . \\
9 \\
4\end{array}$ & $\begin{array}{c}0.12 \\
560\end{array}$ & $\begin{array}{c}0 . \\
00 \\
34 \\
8\end{array}$ & $\begin{array}{c}6.5 \\
079 \\
4\end{array}$ & $\begin{array}{c}0 . \\
15 \\
72 \\
1\end{array}$ & $\begin{array}{c}0.3 \\
758 \\
1\end{array}$ & $\begin{array}{c}0 . \\
00 \\
51 \\
1\end{array}$ & $\begin{array}{c}203 \\
7\end{array}$ & $\begin{array}{l}5 \\
0\end{array}$ & $\begin{array}{c}204 \\
7\end{array}$ & $\begin{array}{l}2 \\
1\end{array}$ & $\begin{array}{c}205 \\
7\end{array}$ & $\begin{array}{l}2 \\
4\end{array}$ & $\begin{array}{c}- \\
0.9 \\
7 \%\end{array}$ & $\begin{array}{l}2 \\
0 \\
3 \\
7\end{array}$ & $\begin{array}{l}5 \\
0\end{array}$ \\
\hline $\begin{array}{c}\mathrm{M} \\
\mathrm{O} 2 \\
2 \mathrm{~A} \\
- \\
10 \\
0\end{array}$ & $\begin{array}{l}4 \\
9\end{array}$ & $\begin{array}{l}1 \\
0 \\
6\end{array}$ & $\begin{array}{l}0 . \\
4 \\
6\end{array}$ & $\begin{array}{c}0.06 \\
428\end{array}$ & $\begin{array}{c}0 . \\
00 \\
18 \\
8\end{array}$ & $\begin{array}{c}0.8 \\
219 \\
9\end{array}$ & $\begin{array}{c}0 . \\
02 \\
19 \\
8\end{array}$ & $\begin{array}{c}0.0 \\
927 \\
9\end{array}$ & $\begin{array}{c}0 . \\
00 \\
16 \\
0\end{array}$ & 751 & $\begin{array}{l}2 \\
9\end{array}$ & 609 & $\begin{array}{l}1 \\
2\end{array}$ & 572 & 9 & $\begin{array}{l}6.4 \\
7 \%\end{array}$ & $\begin{array}{l}5 \\
7 \\
2\end{array}$ & 9 \\
\hline $\begin{array}{l}\mathrm{M} \\
\mathrm{O} 2 \\
3-\end{array}$ & $\begin{array}{l}2 \\
3\end{array}$ & $\begin{array}{l}2 \\
8 \\
2\end{array}$ & $\begin{array}{c}0 . \\
0 \\
8\end{array}$ & $\begin{array}{l}0.05 \\
949\end{array}$ & $\begin{array}{c}0 . \\
00 \\
04\end{array}$ & $\begin{array}{c}0.8 \\
332 \\
6\end{array}$ & $\begin{array}{c}0 . \\
00 \\
64\end{array}$ & $\begin{array}{c}0.1 \\
015 \\
7\end{array}$ & $\begin{array}{c}0 . \\
00 \\
10\end{array}$ & 585 & $\begin{array}{l}1 \\
1\end{array}$ & 615 & 4 & 624 & 6 & $\begin{array}{c}- \\
1.4 \\
4 \%\end{array}$ & $\begin{array}{l}6 \\
2 \\
4\end{array}$ & 6 \\
\hline
\end{tabular}




\begin{tabular}{|c|c|c|c|c|c|c|c|c|c|c|c|c|c|c|c|c|c|c|}
\hline 01 & & & & & 8 & & 5 & & 9 & & & & & & & & & \\
\hline $\mathrm{M}$ & 2 & 3 & 0. & 0.05 & 0. & 0.5 & 0. & 0.0 & 0. & 407 & 1 & 452 & 3 & 460 & 5 & - & 4 & 5 \\
\hline $\mathrm{O} 2$ & 1 & 8 & 5 & 488 & 00 & 603 & 00 & 740 & 00 & & 2 & & & & & \begin{tabular}{|l|}
1.7 \\
\end{tabular} & 6 & \\
\hline $3-$ & 6 & 0 & 7 & & 04 & 2 & 44 & 4 & 08 & & & & & & & $4 \%$ & 0 & \\
\hline 02 & & & & & 5 & & 2 & & 0 & & & & & & & & & \\
\hline $\mathrm{M}$ & 6 & 5 & 0. & 0.12 & 0. & 5.9 & 0. & 0.3 & 0. & 196 & 1 & 196 & 6 & 196 & 1 & 0.2 & 1 & 1 \\
\hline $\mathrm{O} 2$ & 5 & 1 & 1 & 061 & 00 & 132 & 03 & 555 & 00 & 5 & 0 & 3 & & 1 & 8 & $0 \%$ & 9 & 0 \\
\hline $3-$ & & 9 & 3 & & 08 & 9 & 98 & 4 & 38 & & & & & & & & 6 & \\
\hline 03 & & & & & 3 & & 7 & & 8 & & & & & & & & 5 & \\
\hline $\mathrm{M}$ & 1 & 1 & 0. & 0.05 & 0. & 0.5 & 0. & 0.0 & 0. & 461 & 1 & 448 & 3 & 446 & 5 & \begin{tabular}{|l|}
0.4 \\
\end{tabular} & 4 & 5 \\
\hline $\mathrm{O} 2$ & 0 & 1 & 8 & 621 & 00 & 551 & 00 & 716 & 00 & & 1 & & & & & $5 \%$ & 4 & \\
\hline $3-$ & 2 & 9 & 6 & & 05 & 3 & 53 & 2 & 08 & & & & & & & & 6 & \\
\hline 04 & & & & & 8 & & 6 & & 0 & & & & & & & & & \\
\hline $\mathrm{M}$ & 1 & 3 & 0. & 0.05 & 0. & 0.5 & 0. & 0.0 & 0. & 357 & 1 & 440 & 3 & 456 & 5 & - & 4 & 5 \\
\hline $\mathrm{O} 2$ & 1 & 4 & 3 & 367 & 00 & 422 & 00 & 732 & 00 & & 2 & & & & & 3.5 & 5 & \\
\hline $3-$ & 0 & 7 & 2 & & 04 & 3 & 41 & 7 & 07 & & & & & & & $1 \%$ & 6 & \\
\hline 05 & & & & & 2 & & 0 & & 8 & & & & & & & & & \\
\hline $\mathrm{M}$ & 1 & 6 & 0. & 0.05 & 0. & 0.6 & 0. & 0.0 & 0 & 570 & 1 & 535 & 3 & 526 & 6 & \begin{tabular}{|l|}
1.7 \\
\end{tabular} & 5 & 6 \\
\hline $\mathrm{O} 2$ & 4 & 1 & 2 & 907 & 00 & 931 & 00 & 851 & 00 & & 1 & & & & & $1 \%$ & 2 & \\
\hline $3-$ & 4 & 3 & 4 & & 05 & 4 & 57 & 0 & 09 & & & & & & & & 6 & \\
\hline 06 & & & & & 2 & & 9 & & 3 & & & & & & & & & \\
\hline $\mathrm{M}$ & 1 & 3 & 0. & 0.05 & 0. & 0.5 & 0. & 0.0 & 0. & 439 & 1 & 451 & 3 & 453 & 5 & & 4 & 5 \\
\hline $\mathrm{O} 2$ & 7 & 7 & 4 & 567 & 00 & 584 & 00 & 727 & 00 & & 1 & & & & & 0.4 & 5 & \\
\hline $\begin{array}{l}3- \\
07\end{array}$ & 3 & 5 & 6 & & $\begin{array}{c}05 \\
4\end{array}$ & 4 & $\begin{array}{c}51 \\
4\end{array}$ & 5 & $\begin{array}{c}08 \\
0\end{array}$ & & & & & & & $4 \%$ & 3 & \\
\hline $\mathrm{M}$ & 5 & 3 & 0. & 0.11 & 0 . & 5.6 & 0 . & 0.3 & 0. & 192 & 1 & 192 & 5 & 192 & 1 & \begin{tabular}{|l|}
0.0 \\
\end{tabular} & 1 & 1 \\
\hline $\mathrm{O} 2$ & 4 & 0 & 1 & 772 & 00 & 345 & 03 & 471 & 00 & 2 & 1 & 1 & & 1 & 8 & $5 \%$ & 9 & 1 \\
\hline $3-$ & & 9 & 8 & & 07 & 7 & 41 & 1 & 37 & & & & & & & & 2 & \\
\hline 08 & & & & & 2 & 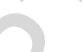 & 3 & & 0 & & & & & & & & 2 & \\
\hline $\mathrm{M}$ & 9 & 1 & 0. & 0.05 & 0. & 0.8 & 0. & 0.1 & 0. & 562 & 1 & 641 & 6 & 664 & 7 & - & 6 & 7 \\
\hline $\mathrm{O} 2$ & 3 & 9 & 4 & 885 & 00 & 805 & 01 & 085 & 00 & & 2 & & & & & 3.4 & 6 & \\
\hline 3- & & 7 & 7 & & 07 & 7 & 03 & 2 & 12 & & & & & & & $6 \%$ & 4 & \\
\hline 09 & & & & & 4 & & 2 & & 7 & & & & & & & & & \\
\hline $\mathrm{M}$ & 3 & 1 & 0. & 0.06 & 0. & 0.9 & 0. & 0.1 & 0. & 646 & 1 & 655 & 3 & 657 & 7 & - & 6 & 7 \\
\hline $\mathrm{O} 2$ & 6 & 1 & 3 & 118 & 00 & 057 & 00 & 073 & 00 & & 2 & & & & & 0.3 & 5 & \\
\hline $3-$ & & 4 & 2 & 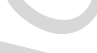 & 04 & 4 & 62 & 7 & 11 & & & & & & & $0 \%$ & 7 & \\
\hline 10 & & & . & 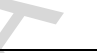 & 3 & & 2 & & 4 & & & & & & & & & \\
\hline $\mathrm{M}$ & 2 & 2 & 0. & 0.06 & 0. & 1.0 & 0. & 0.1 & 0. & 751 & 1 & 746 & 4 & 744 & 8 & \begin{tabular}{|l|}
0.2 \\
\end{tabular} & 7 & 8 \\
\hline $\mathrm{O} 2$ & 2 & 8 & 0 & 427 & 00 & 845 & 00 & 223 & 00 & & 1 & & & & & $7 \%$ & 4 & \\
\hline $3-$ & & 1 & 8 & & 05 & 5 & 85 & 8 & 13 & & & & & & & & 4 & \\
\hline 11 & & & & & 3 & & 5 & & 3 & & & & & & & & & \\
\hline $\mathrm{M}$ & 1 & 2 & 0. & 0.06 & 0. & 1.0 & 0. & 0.1 & 0. & 754 & 1 & 753 & 4 & 753 & 8 & 0.0 & 7 & 8 \\
\hline $\mathrm{O} 2$ & 7 & 7 & 6 & 439 & 00 & 997 & 00 & 238 & 00 & & 2 & & & & & $0 \%$ & 5 & \\
\hline $3-$ & 3 & 8 & 2 & & 04 & 1 & 76 & 6 & 13 & & & & & & & & 3 & \\
\hline 12 & & & & & 6 & & 2 & & 2 & & & & & & & & & \\
\hline $\mathrm{M}$ & 2 & 4 & 0. & 0.05 & 0. & 0.5 & 0. & 0.0 & 0. & 425 & 1 & 444 & 4 & 448 & 5 & - & 4 & 5 \\
\hline $\mathrm{O} 2$ & 5 & 8 & 5 & 531 & 00 & 489 & 00 & 719 & 00 & & 2 & & & & & 0.8 & 4 & \\
\hline $3-$ & 6 & 0 & 3 & & 06 & 6 & 63 & 8 & 08 & & & & & & & $9 \%$ & 8 & \\
\hline 13 & & & & & 8 & & 1 & & 3 & & & & & & & & & \\
\hline $\mathrm{M}$ & 1 & 2 & 0. & 0.07 & 0. & 1.6 & 0. & 0.1 & 0. & 969 & 1 & 998 & 5 & 101 & 1 & - & 9 & 1 \\
\hline $\mathrm{O} 2$ & 1 & 1 & 5 & 142 & 00 & 721 & 01 & 698 & 00 & & 1 & & & 1 & 0 & 4.1 & 6 & 1 \\
\hline $3-$ & 7 & 4 & 5 & & 06 & 7 & 43 & 0 & 18 & & & & & & & $5 \%$ & 9 & \\
\hline 14 & & & & & 5 & & 3 & & 9 & & & & & & & & & \\
\hline $\mathrm{M}$ & 1 & 1 & 0. & 0.05 & 0. & 0.5 & 0. & 0.0 & 0 . & 428 & 1 & 446 & 6 & 449 & 5 & - & 4 & 5 \\
\hline
\end{tabular}




\begin{tabular}{|c|c|c|c|c|c|c|c|c|c|c|c|c|c|c|c|c|c|c|}
\hline $\begin{array}{l}\mathrm{O} 2 \\
3- \\
15\end{array}$ & $\begin{array}{l}6 \\
2\end{array}$ & $\begin{array}{l}7 \\
5\end{array}$ & $\begin{array}{l}9 \\
2\end{array}$ & 539 & $\begin{array}{c}00 \\
09 \\
5\end{array}$ & $\begin{array}{c}512 \\
5\end{array}$ & $\begin{array}{c}00 \\
87 \\
9\end{array}$ & $\begin{array}{c}721 \\
8\end{array}$ & $\begin{array}{c}00 \\
09 \\
1\end{array}$ & & 6 & & & & & $\begin{array}{l}0.6 \\
7 \%\end{array}$ & $\begin{array}{l}4 \\
9\end{array}$ & \\
\hline $\begin{array}{l}\mathrm{M} \\
\mathrm{O} 2 \\
3- \\
16\end{array}$ & $\begin{array}{l}3 \\
4\end{array}$ & $\begin{array}{l}8 \\
6\end{array}$ & $\begin{array}{l}0 . \\
3 \\
9\end{array}$ & $\begin{array}{l}0.07 \\
414\end{array}$ & $\begin{array}{c}0 . \\
00 \\
14 \\
9\end{array}$ & $\begin{array}{c}1.8 \\
417 \\
1\end{array}$ & $\begin{array}{c}0 . \\
03 \\
06 \\
5\end{array}$ & $\begin{array}{c}0.1 \\
801 \\
7\end{array}$ & $\begin{array}{c}0 . \\
00 \\
20 \\
4\end{array}$ & $\begin{array}{c}104 \\
5\end{array}$ & $\begin{array}{l}4 \\
2\end{array}$ & $\begin{array}{c}106 \\
0\end{array}$ & $\begin{array}{l}1 \\
1\end{array}$ & $\begin{array}{c}106 \\
8\end{array}$ & $\begin{array}{l}1 \\
1\end{array}$ & $\begin{array}{l}- \\
2.1 \\
5 \%\end{array}$ & $\begin{array}{l}1 \\
0 \\
4 \\
5\end{array}$ & $\begin{array}{l}4 \\
2\end{array}$ \\
\hline $\begin{array}{c}\mathrm{M} \\
\mathrm{O} 2 \\
3- \\
17\end{array}$ & $\begin{array}{l}8 \\
8\end{array}$ & $\begin{array}{l}2 \\
2 \\
0\end{array}$ & $\begin{array}{l}0 . \\
4 \\
0\end{array}$ & $\begin{array}{l}0.06 \\
102\end{array}$ & $\begin{array}{c}0 . \\
00 \\
07 \\
5\end{array}$ & $\begin{array}{c}0.8 \\
781 \\
5\end{array}$ & $\begin{array}{c}0 . \\
01 \\
01 \\
4\end{array}$ & $\begin{array}{c}0.1 \\
043 \\
7\end{array}$ & $\begin{array}{c}0 . \\
00 \\
12 \\
2\end{array}$ & 640 & $\begin{array}{l}1 \\
1\end{array}$ & 640 & 5 & 640 & 7 & $\begin{array}{l}0.0 \\
0 \%\end{array}$ & $\begin{array}{l}6 \\
4 \\
0\end{array}$ & 7 \\
\hline $\begin{array}{c}\mathrm{M} \\
\mathrm{O} 2 \\
3- \\
18\end{array}$ & $\begin{array}{l}6 \\
9\end{array}$ & $\begin{array}{l}1 \\
1 \\
6\end{array}$ & $\begin{array}{c}0 . \\
6 \\
0\end{array}$ & $\begin{array}{c}0.06 \\
808\end{array}$ & $\begin{array}{c}0 . \\
00 \\
07 \\
3\end{array}$ & $\begin{array}{c}1.3 \\
236 \\
8\end{array}$ & $\begin{array}{c}0 . \\
01 \\
33 \\
2\end{array}$ & $\begin{array}{c}0.1 \\
410 \\
0\end{array}$ & $\begin{array}{c}0 . \\
00 \\
16 \\
2\end{array}$ & 871 & $\begin{array}{l}1 \\
1\end{array}$ & 856 & 6 & 850 & 9 & $\begin{array}{l}0.7 \\
1 \%\end{array}$ & $\begin{array}{l}8 \\
5 \\
0\end{array}$ & 9 \\
\hline $\begin{array}{c}\mathrm{M} \\
\mathrm{O} 2 \\
3- \\
19\end{array}$ & $\begin{array}{l}8 \\
5\end{array}$ & $\begin{array}{l}1 \\
1 \\
1\end{array}$ & $\begin{array}{l}0 . \\
7 \\
7\end{array}$ & $\begin{array}{l}0.05 \\
839\end{array}$ & $\begin{array}{c}0 . \\
00 \\
06 \\
6\end{array}$ & $\begin{array}{c}0.6 \\
148 \\
0\end{array}$ & $\begin{array}{c}0 . \\
00 \\
64 \\
8\end{array}$ & $\begin{array}{c}0.0 \\
763 \\
6\end{array}$ & $\begin{array}{c}0 . \\
00 \\
08 \\
7\end{array}$ & & $\begin{array}{l}1 \\
1\end{array}$ & 487 & 4 & 474 & 5 & $\begin{array}{l}2.7 \\
4 \%\end{array}$ & $\begin{array}{l}4 \\
7 \\
4\end{array}$ & 5 \\
\hline $\begin{array}{c}\mathrm{M} \\
\mathrm{O} 2 \\
3- \\
20 \\
\end{array}$ & $\begin{array}{l}1 \\
0 \\
0\end{array}$ & $\begin{array}{l}1 \\
9 \\
9\end{array}$ & $\begin{array}{l}0 . \\
5 \\
0\end{array}$ & $\begin{array}{l}0.12 \\
203\end{array}$ & $\begin{array}{c}0 . \\
00 \\
10 \\
6 \\
\end{array}$ & $\begin{array}{c}6.0 \\
702 \\
6\end{array}$ & $\begin{array}{c}0 . \\
05 \\
05 \\
6\end{array}$ & $\begin{array}{c}0.3 \\
607 \\
7 \\
\end{array}$ & $\begin{array}{c}0 . \\
00 \\
41 \\
7 \\
\end{array}$ & $\begin{array}{c}198 \\
6\end{array}$ & $\begin{array}{l}1 \\
0\end{array}$ & $\begin{array}{c}198 \\
6\end{array}$ & 7 & $\begin{array}{c}198 \\
6\end{array}$ & $\begin{array}{l}2 \\
0\end{array}$ & $\begin{array}{l}0.0 \\
0 \%\end{array}$ & $\begin{array}{l}1 \\
9 \\
8 \\
6\end{array}$ & $\begin{array}{l}1 \\
0\end{array}$ \\
\hline $\begin{array}{l}\mathrm{M} \\
\mathrm{O} 2 \\
3- \\
21\end{array}$ & $\begin{array}{l}2 \\
2\end{array}$ & $\begin{array}{l}2 \\
8 \\
1\end{array}$ & $\begin{array}{c}0 . \\
0 \\
8\end{array}$ & $\begin{array}{l}0.05 \\
994\end{array}$ & $\begin{array}{c}0 . \\
00 \\
06 \\
5\end{array}$ & $\begin{array}{c}0.7 \\
859 \\
9\end{array}$ & $\begin{array}{c}0 . \\
00 \\
79 \\
5\end{array}$ & $\begin{array}{c}0.0 \\
951 \\
1\end{array}$ & $\begin{array}{c}0 . \\
00 \\
10 \\
7\end{array}$ & 601 & $\begin{array}{l}1 \\
1\end{array}$ & 589 & 5 & 586 & 6 & $\begin{array}{l}0.5 \\
1 \%\end{array}$ & $\begin{array}{l}5 \\
8 \\
6\end{array}$ & 6 \\
\hline $\begin{array}{l}\mathrm{M} \\
\mathrm{O} 2 \\
3- \\
22\end{array}$ & $\begin{array}{l}1 \\
5 \\
2\end{array}$ & $\begin{array}{l}2 \\
1 \\
0\end{array}$ & $\begin{array}{l}0 . \\
7 \\
2\end{array}$ & $\begin{array}{c}0.06 \\
415\end{array}$ & $\begin{array}{c}0 . \\
00 \\
03 \\
9\end{array}$ & $\begin{array}{c}1.0 \\
230 \\
0\end{array}$ & $\begin{array}{c}0 . \\
00 \\
62 \\
3\end{array}$ & $\begin{array}{c}0.1 \\
156 \\
6\end{array}$ & $\begin{array}{c}0 . \\
00 \\
12 \\
2\end{array}$ & 747 & $\begin{array}{l}1 \\
2\end{array}$ & 715 & 3 & 706 & 7 & $\begin{array}{l}1.2 \\
7 \%\end{array}$ & $\begin{array}{l}7 \\
0 \\
6\end{array}$ & 7 \\
\hline $\begin{array}{l}\mathrm{M} \\
\mathrm{O} 2 \\
3- \\
23 \\
\end{array}$ & $\begin{array}{l}1 \\
6 \\
1\end{array}$ & $\begin{array}{l}9 \\
1 \\
7\end{array}$ & $\begin{array}{c}0 . \\
1 \\
8\end{array}$ & $\begin{array}{l}0.05 \\
942\end{array}$ & $\begin{array}{c}0 . \\
00 \\
06 \\
4\end{array}$ & $\begin{array}{c}0.6 \\
554 \\
7\end{array}$ & $\begin{array}{c}0 . \\
00 \\
65 \\
8 \\
\end{array}$ & $\begin{array}{c}0.0 \\
800 \\
0\end{array}$ & $\begin{array}{c}0 . \\
00 \\
09 \\
0 \\
\end{array}$ & 583 & $\begin{array}{l}1 \\
1\end{array}$ & 512 & 4 & 496 & 5 & $\begin{array}{l}3.2 \\
3 \%\end{array}$ & $\begin{array}{l}4 \\
9 \\
6\end{array}$ & 5 \\
\hline $\begin{array}{c}\mathrm{M} \\
\mathrm{O} 2 \\
3- \\
24\end{array}$ & $\begin{array}{l}6 \\
6\end{array}$ & $\begin{array}{l}2 \\
0 \\
7\end{array}$ & $\begin{array}{l}0 . \\
3 \\
2\end{array}$ & $\begin{array}{l}0.14 \\
124\end{array}$ & $\begin{array}{c}0 . \\
00 \\
07 \\
7\end{array}$ & $\begin{array}{c}7.7 \\
378 \\
6\end{array}$ & $\begin{array}{c}0 . \\
04 \\
26 \\
9\end{array}$ & $\begin{array}{c}0.3 \\
973 \\
4\end{array}$ & $\begin{array}{c}0 . \\
00 \\
41 \\
8\end{array}$ & $\begin{array}{c}224 \\
2\end{array}$ & $\begin{array}{l}1 \\
1\end{array}$ & $\begin{array}{c}220 \\
1\end{array}$ & 5 & $\begin{array}{c}215 \\
7\end{array}$ & $\begin{array}{l}1 \\
9\end{array}$ & $\begin{array}{l}3.9 \\
4 \%\end{array}$ & $\begin{array}{l}2 \\
2 \\
4 \\
2\end{array}$ & $\begin{array}{l}1 \\
1\end{array}$ \\
\hline $\begin{array}{c}\mathrm{M} \\
\mathrm{O} 2 \\
3- \\
25\end{array}$ & $\begin{array}{l}1 \\
3 \\
8\end{array}$ & $\begin{array}{l}3 \\
3 \\
9\end{array}$ & $\begin{array}{l}0 . \\
4 \\
1\end{array}$ & $\begin{array}{c}0.05 \\
618\end{array}$ & $\begin{array}{c}0 . \\
00 \\
05 \\
9\end{array}$ & $\begin{array}{c}0.5 \\
558 \\
0\end{array}$ & $\begin{array}{c}0 . \\
00 \\
54 \\
7\end{array}$ & $\begin{array}{c}0.0 \\
717 \\
5\end{array}$ & $\begin{array}{c}0 . \\
00 \\
08 \\
0\end{array}$ & 459 & $\begin{array}{l}1 \\
1\end{array}$ & 449 & 4 & 447 & 5 & $\begin{array}{l}0.4 \\
5 \%\end{array}$ & $\begin{array}{l}4 \\
4 \\
7\end{array}$ & 5 \\
\hline $\begin{array}{c}\mathrm{M} \\
\mathrm{O} 2 \\
3- \\
26\end{array}$ & $\begin{array}{l}1 \\
2 \\
1\end{array}$ & $\begin{array}{l}3 \\
2 \\
0\end{array}$ & $\begin{array}{l}0 . \\
3 \\
8\end{array}$ & $\begin{array}{c}0.05 \\
951\end{array}$ & $\begin{array}{c}0 . \\
00 \\
05 \\
5\end{array}$ & $\begin{array}{c}0.7 \\
239 \\
5\end{array}$ & $\begin{array}{c}0 . \\
00 \\
63 \\
5\end{array}$ & $\begin{array}{c}0.0 \\
882 \\
4\end{array}$ & $\begin{array}{c}0 . \\
00 \\
09 \\
7\end{array}$ & 586 & $\begin{array}{l}1 \\
1\end{array}$ & 553 & 4 & 545 & 6 & $\begin{array}{l}1.4 \\
7 \%\end{array}$ & $\begin{array}{l}5 \\
4 \\
5\end{array}$ & 6 \\
\hline $\begin{array}{l}\mathrm{M} \\
\mathrm{O} 2 \\
3- \\
27\end{array}$ & $\begin{array}{l}3 \\
3 \\
6\end{array}$ & $\begin{array}{l}2 \\
6 \\
9\end{array}$ & $\begin{array}{l}1 . \\
2 \\
5\end{array}$ & $\begin{array}{c}0.06 \\
640\end{array}$ & $\begin{array}{c}0 . \\
00 \\
12 \\
9\end{array}$ & $\begin{array}{c}1.0 \\
264 \\
2\end{array}$ & $\begin{array}{c}0 . \\
01 \\
83 \\
4\end{array}$ & $\begin{array}{c}0.1 \\
121 \\
2\end{array}$ & $\begin{array}{c}0 . \\
00 \\
15 \\
6\end{array}$ & 819 & $\begin{array}{l}1 \\
7\end{array}$ & 717 & 9 & 685 & 9 & $\begin{array}{l}4.6 \\
7 \%\end{array}$ & $\begin{array}{l}6 \\
8 \\
5\end{array}$ & 9 \\
\hline $\begin{array}{l}\mathrm{M} \\
\mathrm{O} 2 \\
3-\end{array}$ & $\begin{array}{l}4 \\
1\end{array}$ & $\begin{array}{l}5 \\
6\end{array}$ & $\begin{array}{l}0 . \\
7 \\
4\end{array}$ & $\begin{array}{l}0.06 \\
226\end{array}$ & $\begin{array}{c}0 . \\
00 \\
05\end{array}$ & $\begin{array}{c}0.9 \\
568 \\
2\end{array}$ & $\begin{array}{c}0 . \\
00 \\
81\end{array}$ & $\begin{array}{c}0.1 \\
114 \\
5\end{array}$ & $\begin{array}{c}0 . \\
00 \\
12\end{array}$ & 683 & $\begin{array}{l}1 \\
1\end{array}$ & 682 & 4 & 681 & 7 & $\begin{array}{l}0.1 \\
5 \%\end{array}$ & $\begin{array}{l}6 \\
8 \\
1\end{array}$ & 7 \\
\hline
\end{tabular}




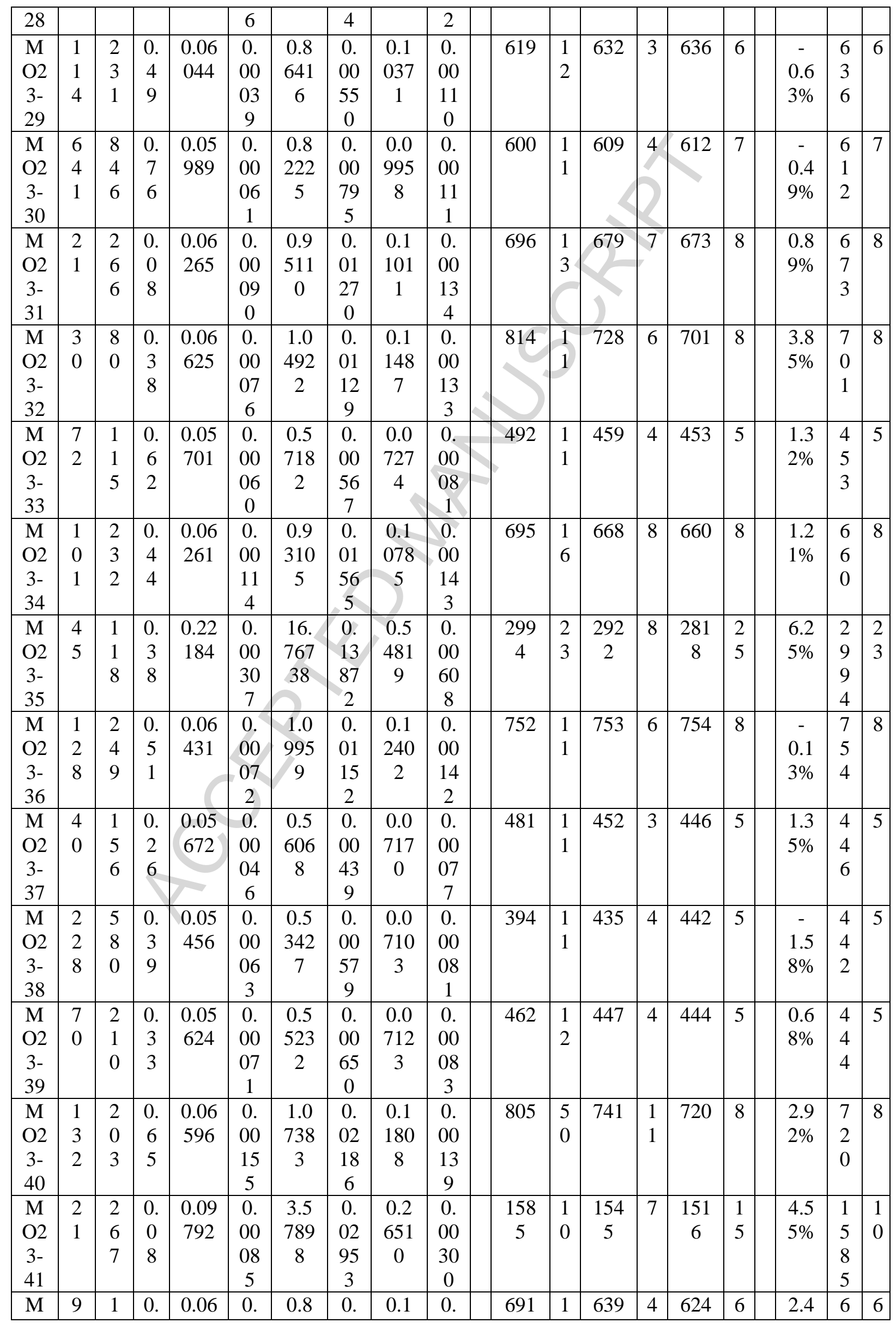




\begin{tabular}{|c|c|c|c|c|c|c|c|c|c|c|c|c|c|c|c|c|c|c|}
\hline $\begin{array}{l}\mathrm{O} 2 \\
3- \\
42\end{array}$ & 5 & $\begin{array}{l}8 \\
0\end{array}$ & $\begin{array}{l}5 \\
3\end{array}$ & 248 & $\begin{array}{c}00 \\
05 \\
2\end{array}$ & $\begin{array}{c}758 \\
9\end{array}$ & $\begin{array}{c}00 \\
69 \\
8 \\
\end{array}$ & $\begin{array}{c}016 \\
8\end{array}$ & $\begin{array}{c}00 \\
11 \\
1 \\
\end{array}$ & & 1 & & & & & $0 \%$ & $\begin{array}{l}2 \\
4\end{array}$ & \\
\hline $\begin{array}{c}\mathrm{M} \\
\mathrm{O} 2 \\
3- \\
43\end{array}$ & $\begin{array}{l}1 \\
3 \\
1\end{array}$ & $\begin{array}{l}3 \\
0 \\
8\end{array}$ & $\begin{array}{l}0 . \\
4 \\
3\end{array}$ & $\begin{array}{c}0.07 \\
056\end{array}$ & $\begin{array}{c}0 . \\
00 \\
11 \\
9\end{array}$ & $\begin{array}{c}1.4 \\
868 \\
4\end{array}$ & $\begin{array}{c}0 . \\
01 \\
92 \\
8\end{array}$ & $\begin{array}{c}0.1 \\
528 \\
3\end{array}$ & $\begin{array}{c}0 . \\
00 \\
16 \\
6\end{array}$ & 945 & $\begin{array}{l}3 \\
5\end{array}$ & 925 & 8 & 917 & 9 & \begin{tabular}{|l|}
0.8 \\
$7 \%$
\end{tabular} & $\begin{array}{l}9 \\
1 \\
7\end{array}$ & 9 \\
\hline $\begin{array}{c}\mathrm{M} \\
\mathrm{O} 2 \\
3- \\
44\end{array}$ & $\begin{array}{l}1 \\
1 \\
4\end{array}$ & $\begin{array}{l}3 \\
7 \\
2\end{array}$ & $\begin{array}{c}0 . \\
3 \\
1\end{array}$ & $\begin{array}{c}0.06 \\
175\end{array}$ & $\begin{array}{c}0 . \\
00 \\
07 \\
9\end{array}$ & $\begin{array}{c}0.8 \\
207 \\
4\end{array}$ & $\begin{array}{c}0 . \\
00 \\
97 \\
9\end{array}$ & $\begin{array}{c}0.0 \\
964 \\
1\end{array}$ & $\begin{array}{c}0 . \\
00 \\
11 \\
4\end{array}$ & 665 & $\begin{array}{l}1 \\
2\end{array}$ & 608 & 5 & 593 & 7 & \begin{tabular}{|l|}
2.5 \\
$3 \%$
\end{tabular} & $\begin{array}{l}5 \\
9 \\
3\end{array}$ & 7 \\
\hline $\begin{array}{c}\mathrm{M} \\
\mathrm{O} 2 \\
3- \\
45\end{array}$ & $\begin{array}{l}1 \\
7 \\
1\end{array}$ & $\begin{array}{l}1 \\
3 \\
1\end{array}$ & $\begin{array}{l}1 . \\
3 \\
0\end{array}$ & $\begin{array}{l}0.21 \\
296\end{array}$ & $\begin{array}{c}0 . \\
00 \\
14 \\
4\end{array}$ & $\begin{array}{c}16 . \\
234 \\
99\end{array}$ & $\begin{array}{c}0 . \\
10 \\
96 \\
1\end{array}$ & $\begin{array}{c}0.5 \\
529 \\
5\end{array}$ & $\begin{array}{c}0 . \\
00 \\
62 \\
0\end{array}$ & $\begin{array}{c}292 \\
8\end{array}$ & $\begin{array}{l}1 \\
0\end{array}$ & $\begin{array}{c}289 \\
1\end{array}$ & 6 & $\begin{array}{c}283 \\
7\end{array}$ & $\begin{array}{l}2 \\
6\end{array}$ & \begin{tabular}{|l|}
3.2 \\
$1 \%$
\end{tabular} & $\begin{array}{l}2 \\
9 \\
2 \\
8\end{array}$ & $\begin{array}{l}1 \\
0\end{array}$ \\
\hline $\begin{array}{c}\mathrm{M} \\
\mathrm{O} 2 \\
3- \\
46\end{array}$ & $\begin{array}{l}5 \\
7\end{array}$ & $\begin{array}{l}5 \\
9\end{array}$ & $\begin{array}{l}0 . \\
9 \\
6\end{array}$ & $\begin{array}{c}0.05 \\
947\end{array}$ & $\begin{array}{c}0 . \\
00 \\
05 \\
1\end{array}$ & $\begin{array}{c}0.7 \\
841 \\
2\end{array}$ & $\begin{array}{c}0 . \\
00 \\
64 \\
6\end{array}$ & $\begin{array}{c}0.0 \\
956 \\
4\end{array}$ & $\begin{array}{c}0 . \\
00 \\
10 \\
4\end{array}$ & & $\begin{array}{l}1 \\
1\end{array}$ & 588 & 4 & 589 & 6 & $\begin{array}{c}- \\
0.1 \\
7 \%\end{array}$ & $\begin{array}{l}5 \\
8 \\
9\end{array}$ & 6 \\
\hline $\begin{array}{c}\mathrm{M} \\
\mathrm{O} 2 \\
3- \\
47\end{array}$ & $\begin{array}{l}2 \\
4 \\
6\end{array}$ & $\begin{array}{l}3 \\
2 \\
6\end{array}$ & $\begin{array}{l}0 . \\
7 \\
5\end{array}$ & $\begin{array}{l}0.06 \\
249\end{array}$ & $\begin{array}{c}0 . \\
00 \\
07 \\
7\end{array}$ & $\begin{array}{c}0.8 \\
951 \\
3\end{array}$ & $\begin{array}{c}0 . \\
01 \\
02 \\
7\end{array}$ & $\begin{array}{c}0.1 \\
038 \\
9\end{array}$ & $\begin{array}{c}0 . \\
00 \\
12 \\
1\end{array}$ & 691 & $\begin{array}{l}1 \\
1\end{array}$ & 649 & 6 & 637 & 7 & $\begin{array}{l}1.8 \\
8 \%\end{array}$ & $\begin{array}{l}6 \\
3 \\
7\end{array}$ & 7 \\
\hline $\begin{array}{c}\mathrm{M} \\
\mathrm{O} 2 \\
3- \\
48\end{array}$ & $\begin{array}{l}8 \\
0\end{array}$ & $\begin{array}{l}1 \\
3 \\
9\end{array}$ & $\begin{array}{l}0 . \\
5 \\
8\end{array}$ & $\begin{array}{c}0.06 \\
119\end{array}$ & $\begin{array}{c}0 . \\
00 \\
07 \\
9\end{array}$ & $\begin{array}{c}0.7 \\
875 \\
3\end{array}$ & $\begin{array}{c}0 . \\
00 \\
94 \\
5\end{array}$ & $\begin{array}{c}0.0 \\
933 \\
5\end{array}$ & $\begin{array}{c}0 . \\
00 \\
11 \\
0\end{array}$ & 646 & $\begin{array}{l}1 \\
2\end{array}$ & 590 & 5 & 575 & 6 & $\begin{array}{l}2.6 \\
1 \%\end{array}$ & $\begin{array}{l}5 \\
7 \\
5\end{array}$ & 6 \\
\hline $\begin{array}{l}\mathrm{M} \\
\mathrm{O} 2 \\
3- \\
49\end{array}$ & $\begin{array}{l}1 \\
1 \\
9\end{array}$ & $\begin{array}{l}1 \\
0 \\
6\end{array}$ & $\begin{array}{l}1 . \\
1 \\
2\end{array}$ & $\begin{array}{c}0.06 \\
420\end{array}$ & $\begin{array}{c}0 . \\
00 \\
06 \\
8\end{array}$ & $\begin{array}{c}0.9 \\
027 \\
9\end{array}$ & $\begin{array}{c}0 . \\
00 \\
89 \\
8\end{array}$ & $\begin{array}{c}0.1 \\
020 \\
0\end{array}$ & $\begin{array}{c}0 . \\
00 \\
11 \\
6\end{array}$ & 748 & $\begin{array}{l}1 \\
1\end{array}$ & 653 & 5 & 626 & 7 & $\begin{array}{l}4.3 \\
1 \%\end{array}$ & $\begin{array}{l}6 \\
2 \\
6\end{array}$ & 7 \\
\hline $\begin{array}{c}\mathrm{M} \\
\mathrm{O} 2 \\
3- \\
50\end{array}$ & $\begin{array}{l}9 \\
1\end{array}$ & $\begin{array}{l}1 \\
6 \\
2\end{array}$ & $\begin{array}{l}0 . \\
5 \\
6\end{array}$ & $\begin{array}{l}0.06 \\
905\end{array}$ & $\begin{array}{c}0 . \\
00 \\
04 \\
7\end{array}$ & $\begin{array}{c}1.2 \\
966 \\
8\end{array}$ & $\begin{array}{c}0 . \\
00 \\
86 \\
0\end{array}$ & $\begin{array}{c}0.1 \\
362 \\
1\end{array}$ & $\begin{array}{c}0 . \\
00 \\
14 \\
5\end{array}$ & 900 & $\begin{array}{l}1 \\
2\end{array}$ & 844 & 4 & 823 & 8 & $\begin{array}{l}2.5 \\
5 \%\end{array}$ & $\begin{array}{l}8 \\
2 \\
3\end{array}$ & 8 \\
\hline $\begin{array}{c}\mathrm{M} \\
\mathrm{O} 2 \\
3- \\
51\end{array}$ & $\begin{array}{l}2 \\
0\end{array}$ & $\begin{array}{l}2 \\
5 \\
6\end{array}$ & $\begin{array}{l}0 . \\
0 \\
8\end{array}$ & $\begin{array}{l}0.18 \\
060\end{array}$ & $\begin{array}{c}0 . \\
00 \\
31 \\
5\end{array}$ & $\begin{array}{c}10 . \\
399 \\
57\end{array}$ & $\begin{array}{c}0 . \\
13 \\
41 \\
9\end{array}$ & $\begin{array}{c}0.4 \\
176 \\
3\end{array}$ & $\begin{array}{c}0 . \\
00 \\
49 \\
2\end{array}$ & $\begin{array}{c}265 \\
8\end{array}$ & $\begin{array}{l}3 \\
0\end{array}$ & $\begin{array}{c}247 \\
1\end{array}$ & $\begin{array}{l}1 \\
2\end{array}$ & $\begin{array}{c}225 \\
0\end{array}$ & $\begin{array}{l}2 \\
2\end{array}$ & $\begin{array}{l}18 . \\
13 \\
\%\end{array}$ & & \\
\hline $\begin{array}{c}\mathrm{M} \\
\mathrm{O} 2 \\
3- \\
52\end{array}$ & $\begin{array}{l}2 \\
6 \\
5\end{array}$ & $\begin{array}{l}2 \\
8 \\
0\end{array}$ & $\begin{array}{l}0 . \\
9 \\
5\end{array}$ & $\begin{array}{c}0.07 \\
574\end{array}$ & $\begin{array}{c}0 . \\
00 \\
06 \\
8\end{array}$ & $\begin{array}{c}1.7 \\
353 \\
6\end{array}$ & $\begin{array}{c}0 . \\
01 \\
47 \\
2\end{array}$ & $\begin{array}{c}0.1 \\
661 \\
8\end{array}$ & $\begin{array}{c}0 . \\
00 \\
18 \\
5\end{array}$ & $\begin{array}{c}108 \\
8\end{array}$ & $\begin{array}{l}1 \\
0\end{array}$ & $\begin{array}{c}102 \\
2\end{array}$ & 5 & 991 & 0 & $\begin{array}{l}3.1 \\
3 \%\end{array}$ & $\begin{array}{l}9 \\
9 \\
1\end{array}$ & $\begin{array}{l}1 \\
0\end{array}$ \\
\hline $\begin{array}{c}\mathrm{M} \\
\mathrm{O} 2 \\
3- \\
53\end{array}$ & $\begin{array}{l}3 \\
4\end{array}$ & $\begin{array}{l}1 \\
3 \\
0\end{array}$ & $\begin{array}{l}0 . \\
2 \\
6\end{array}$ & $\begin{array}{c}0.05 \\
683\end{array}$ & $\begin{array}{c}0 . \\
00 \\
07 \\
5\end{array}$ & $\begin{array}{c}0.5 \\
688 \\
1\end{array}$ & $\begin{array}{c}0 . \\
00 \\
70 \\
4\end{array}$ & $\begin{array}{c}0.0 \\
725 \\
9\end{array}$ & $\begin{array}{c}0 . \\
00 \\
08 \\
5\end{array}$ & 485 & $\begin{array}{l}1 \\
2\end{array}$ & 457 & 5 & 452 & 5 & $\begin{array}{l}1.1 \\
1 \%\end{array}$ & $\begin{array}{l}4 \\
5 \\
2\end{array}$ & 5 \\
\hline $\begin{array}{c}\mathrm{M} \\
\mathrm{O} 2 \\
3- \\
54\end{array}$ & $\begin{array}{l}8 \\
6\end{array}$ & $\begin{array}{l}1 \\
9 \\
5\end{array}$ & $\begin{array}{l}0 . \\
4 \\
4\end{array}$ & $\begin{array}{c}0.16 \\
113\end{array}$ & $\begin{array}{c}0 . \\
00 \\
11 \\
9\end{array}$ & $\begin{array}{c}10 . \\
283 \\
52\end{array}$ & $\begin{array}{c}0 . \\
07 \\
47 \\
7\end{array}$ & $\begin{array}{c}0.4 \\
629 \\
1\end{array}$ & $\begin{array}{c}0 . \\
00 \\
52 \\
4\end{array}$ & $\begin{array}{c}246 \\
8\end{array}$ & $\begin{array}{l}1 \\
0\end{array}$ & $\begin{array}{c}246 \\
1\end{array}$ & 7 & $\begin{array}{c}245 \\
2\end{array}$ & 3 & $\begin{array}{l}0.6 \\
5 \%\end{array}$ & $\begin{array}{l}2 \\
4 \\
6 \\
8\end{array}$ & $\begin{array}{l}1 \\
0\end{array}$ \\
\hline $\begin{array}{l}\mathrm{M} \\
\mathrm{O} 2 \\
3-\end{array}$ & $\begin{array}{l}6 \\
3\end{array}$ & $\begin{array}{l}6 \\
9\end{array}$ & $\begin{array}{l}0 . \\
9 \\
0\end{array}$ & $\begin{array}{c}0.06 \\
118\end{array}$ & $\begin{array}{c}0 . \\
00 \\
04\end{array}$ & $\begin{array}{c}0.8 \\
775 \\
1\end{array}$ & $\begin{array}{c}0 . \\
00 \\
64\end{array}$ & $\begin{array}{c}0.1 \\
040 \\
3\end{array}$ & $\begin{array}{c}0 . \\
00 \\
11\end{array}$ & 646 & $\begin{array}{l}1 \\
2\end{array}$ & 640 & 3 & 638 & 7 & \begin{tabular}{|l|}
0.3 \\
$1 \%$
\end{tabular} & $\begin{array}{l}6 \\
3 \\
8\end{array}$ & 7 \\
\hline
\end{tabular}




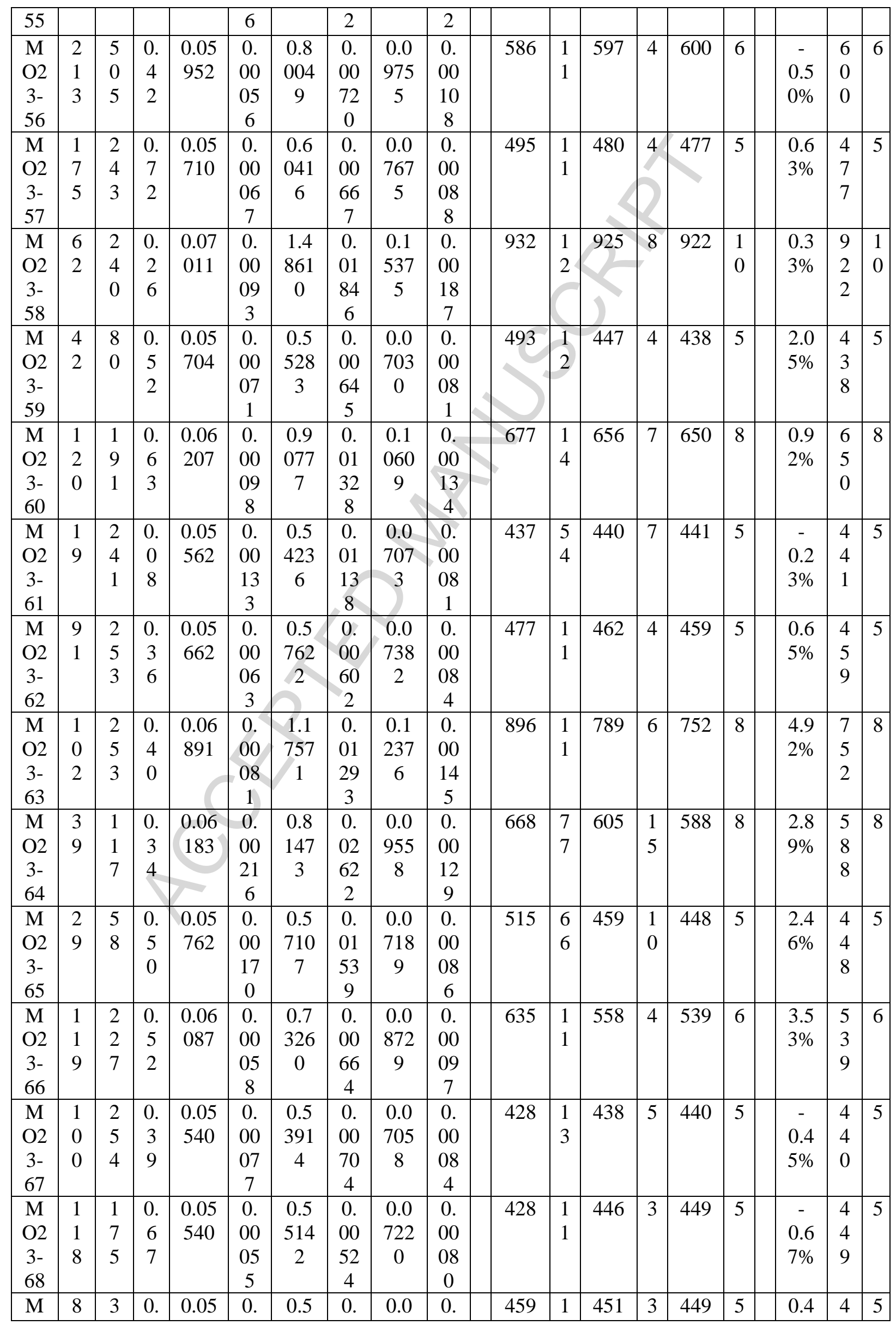




\begin{tabular}{|c|c|c|c|c|c|c|c|c|c|c|c|c|c|c|c|c|c|c|}
\hline $\begin{array}{l}\mathrm{O} 2 \\
3- \\
69\end{array}$ & 3 & $\begin{array}{l}2 \\
1\end{array}$ & $\begin{array}{l}2 \\
6\end{array}$ & 617 & $\begin{array}{c}00 \\
05 \\
4\end{array}$ & $\begin{array}{c}588 \\
0\end{array}$ & $\begin{array}{c}00 \\
51 \\
0 \\
\end{array}$ & $\begin{array}{c}721 \\
6\end{array}$ & $\begin{array}{c}00 \\
08 \\
0\end{array}$ & & 1 & & & & & $5 \%$ & $\begin{array}{l}4 \\
9\end{array}$ & \\
\hline $\begin{array}{l}\mathrm{M} \\
\mathrm{O} 2 \\
3- \\
70\end{array}$ & $\begin{array}{l}1 \\
9 \\
1\end{array}$ & $\begin{array}{l}3 \\
5 \\
7\end{array}$ & $\begin{array}{l}0 . \\
5 \\
3\end{array}$ & $\begin{array}{c}0.05 \\
640\end{array}$ & $\begin{array}{c}0 . \\
00 \\
08 \\
7\end{array}$ & $\begin{array}{c}0.5 \\
667 \\
5\end{array}$ & $\begin{array}{c}0 . \\
00 \\
81 \\
3\end{array}$ & $\begin{array}{c}0.0 \\
728 \\
8\end{array}$ & $\begin{array}{c}0 . \\
00 \\
08 \\
9\end{array}$ & 468 & $\begin{array}{l}1 \\
4\end{array}$ & 456 & 5 & 453 & 5 & $\begin{array}{l}0.6 \\
6 \%\end{array}$ & $\begin{array}{l}4 \\
5 \\
3\end{array}$ & 5 \\
\hline $\begin{array}{l}\mathrm{M} \\
\mathrm{O} 2 \\
3- \\
71\end{array}$ & $\begin{array}{l}1 \\
9\end{array}$ & $\begin{array}{l}2 \\
4 \\
1\end{array}$ & $\begin{array}{c}0 . \\
0 \\
8\end{array}$ & $\begin{array}{l}0.05 \\
601\end{array}$ & $\begin{array}{c}0 . \\
00 \\
05 \\
6\end{array}$ & $\begin{array}{c}0.5 \\
610 \\
2\end{array}$ & $\begin{array}{c}0 . \\
00 \\
52 \\
8\end{array}$ & $\begin{array}{c}0.0 \\
726 \\
6\end{array}$ & $\begin{array}{c}0 . \\
00 \\
08 \\
1\end{array}$ & 453 & $\begin{array}{l}1 \\
1\end{array}$ & 452 & 3 & 452 & 5 & \begin{tabular}{|l|}
0.0 \\
$0 \%$
\end{tabular} & $\begin{array}{l}4 \\
5 \\
2\end{array}$ & 5 \\
\hline $\begin{array}{l}\mathrm{M} \\
\mathrm{O} 2 \\
3- \\
72\end{array}$ & $\begin{array}{l}2 \\
1 \\
6\end{array}$ & $\begin{array}{l}3 \\
3 \\
8\end{array}$ & $\begin{array}{c}0 . \\
6 \\
4\end{array}$ & $\begin{array}{l}0.07 \\
412\end{array}$ & $\begin{array}{c}0 . \\
00 \\
05 \\
6\end{array}$ & $\begin{array}{c}1.7 \\
366 \\
7\end{array}$ & $\begin{array}{c}0 . \\
01 \\
27 \\
7\end{array}$ & $\begin{array}{c}0.1 \\
699 \\
4\end{array}$ & $\begin{array}{c}0 . \\
00 \\
18 \\
4\end{array}$ & $\begin{array}{c}104 \\
5\end{array}$ & $\begin{array}{l}1 \\
1\end{array}$ & $\begin{array}{c}102 \\
2\end{array}$ & 5 & $\begin{array}{c}101 \\
2\end{array}$ & $\begin{array}{l}1 \\
0\end{array}$ & \begin{tabular}{|l|}
3.2 \\
$6 \%$
\end{tabular} & $\begin{array}{l}1 \\
0 \\
4 \\
5 \\
\end{array}$ & $\begin{array}{l}1 \\
1\end{array}$ \\
\hline $\begin{array}{l}\mathrm{M} \\
\mathrm{O} 2 \\
3- \\
73\end{array}$ & $\begin{array}{l}3 \\
9 \\
7\end{array}$ & $\begin{array}{l}2 \\
9 \\
1\end{array}$ & $\begin{array}{l}1 . \\
3 \\
6\end{array}$ & $\begin{array}{c}0.06 \\
653\end{array}$ & $\begin{array}{c}0 . \\
00 \\
14 \\
0\end{array}$ & $\begin{array}{c}1.1 \\
821 \\
7\end{array}$ & $\begin{array}{c}0 . \\
02 \\
10 \\
0\end{array}$ & $\begin{array}{c}0.1 \\
288 \\
7\end{array}$ & $\begin{array}{c}0 . \\
00 \\
14 \\
5\end{array}$ & & $\begin{array}{l}4 \\
5\end{array}$ & 792 & $\begin{array}{l}1 \\
0\end{array}$ & 781 & 8 & \begin{tabular}{|l|}
1.4 \\
$1 \%$
\end{tabular} & $\begin{array}{l}7 \\
8 \\
1\end{array}$ & 8 \\
\hline $\begin{array}{l}\mathrm{M} \\
\mathrm{O} 2 \\
3- \\
74\end{array}$ & $\begin{array}{l}7 \\
5\end{array}$ & $\begin{array}{l}2 \\
2 \\
3\end{array}$ & $\begin{array}{l}0 . \\
3 \\
4\end{array}$ & $\begin{array}{l}0.13 \\
385\end{array}$ & $\begin{array}{c}0 . \\
00 \\
09 \\
5\end{array}$ & $\begin{array}{c}6.9 \\
461 \\
3\end{array}$ & $\begin{array}{c}0 . \\
04 \\
82 \\
5\end{array}$ & $\begin{array}{c}0.3 \\
764 \\
0 \\
\end{array}$ & $\begin{array}{c}0 . \\
00 \\
41 \\
6\end{array}$ & $\begin{array}{c}214 \\
9\end{array}$ & $\begin{array}{l}1 \\
0\end{array}$ & $\begin{array}{c}210 \\
5\end{array}$ & 6 & $\begin{array}{c}205 \\
9\end{array}$ & 9 & \begin{tabular}{|l|}
4.3 \\
$7 \%$
\end{tabular} & $\begin{array}{l}2 \\
1 \\
4 \\
9 \\
\end{array}$ & $\begin{array}{l}1 \\
0\end{array}$ \\
\hline $\begin{array}{l}\mathrm{M} \\
\mathrm{O} 2 \\
3- \\
75\end{array}$ & $\begin{array}{l}5 \\
4\end{array}$ & $\begin{array}{l}1 \\
0 \\
9\end{array}$ & $\begin{array}{l}0 . \\
4 \\
9\end{array}$ & $\begin{array}{c}0.07 \\
105\end{array}$ & $\begin{array}{c}0 . \\
00 \\
06 \\
0\end{array}$ & $\begin{array}{c}1.2 \\
604 \\
5\end{array}$ & $\begin{array}{c}0 . \\
01 \\
01 \\
1\end{array}$ & $\begin{array}{c}0.1 \\
286 \\
8\end{array}$ & $\begin{array}{c}0 . \\
00 \\
14 \\
1\end{array}$ & 959 & $\begin{array}{l}1 \\
1\end{array}$ & 828 & 5 & 780 & 8 & \begin{tabular}{|l|}
6.1 \\
$5 \%$
\end{tabular} & $\begin{array}{l}7 \\
8 \\
0\end{array}$ & 8 \\
\hline $\begin{array}{l}\mathrm{M} \\
\mathrm{O} 2 \\
3- \\
76\end{array}$ & $\begin{array}{l}4 \\
5\end{array}$ & $\begin{array}{l}1 \\
9 \\
8\end{array}$ & $\begin{array}{l}0 . \\
2 \\
3\end{array}$ & $\begin{array}{c}0.06 \\
157\end{array}$ & $\begin{array}{c}0 . \\
00 \\
18 \\
4\end{array}$ & $\begin{array}{c}0.8 \\
707 \\
3\end{array}$ & $\begin{array}{c}0 . \\
02 \\
37 \\
5\end{array}$ & $\begin{array}{c}0.1 \\
025 \\
6\end{array}$ & $\begin{array}{c}0 . \\
00 \\
12 \\
6\end{array}$ & 659 & $\begin{array}{l}6 \\
6\end{array}$ & 636 & $\begin{array}{l}1 \\
3\end{array}$ & 629 & 7 & $\begin{array}{l}1.1 \\
1 \%\end{array}$ & $\begin{array}{l}6 \\
2 \\
9\end{array}$ & 7 \\
\hline $\begin{array}{l}\mathrm{M} \\
\mathrm{O} 2 \\
3- \\
78 \\
\end{array}$ & $\begin{array}{l}7 \\
5\end{array}$ & $\begin{array}{l}1 \\
3 \\
6\end{array}$ & $\begin{array}{l}0 . \\
5 \\
5\end{array}$ & $\begin{array}{l}0.06 \\
172\end{array}$ & $\begin{array}{c}0 . \\
00 \\
04 \\
9\end{array}$ & $\begin{array}{c}0.8 \\
511 \\
2\end{array}$ & $\begin{array}{c}0 . \\
00 \\
65 \\
7\end{array}$ & $\begin{array}{c}0.1 \\
000 \\
2\end{array}$ & $\begin{array}{c}0 . \\
00 \\
10 \\
8\end{array}$ & 664 & $\begin{array}{l}1 \\
1\end{array}$ & 625 & 4 & 615 & 6 & $\begin{array}{l}1.6 \\
3 \%\end{array}$ & $\begin{array}{l}6 \\
1 \\
5\end{array}$ & 6 \\
\hline $\begin{array}{l}\mathrm{M} \\
\mathrm{O} 2 \\
3- \\
79\end{array}$ & $\begin{array}{l}3 \\
1 \\
3\end{array}$ & $\begin{array}{l}3 \\
9 \\
6\end{array}$ & $\begin{array}{l}0 . \\
7 \\
9\end{array}$ & $\begin{array}{l}0.07 \\
966\end{array}$ & $\begin{array}{c}0 . \\
00 \\
13 \\
8\end{array}$ & $\begin{array}{c}1.8 \\
943 \\
5\end{array}$ & $\begin{array}{c}0 . \\
02 \\
52 \\
7\end{array}$ & $\begin{array}{c}0.1 \\
724 \\
7\end{array}$ & $\begin{array}{c}0 . \\
00 \\
18 \\
9\end{array}$ & $\begin{array}{c}118 \\
9\end{array}$ & $\begin{array}{l}3 \\
5\end{array}$ & $\begin{array}{c}107 \\
9\end{array}$ & 9 & $\begin{array}{c}102 \\
6\end{array}$ & $\begin{array}{l}1 \\
0\end{array}$ & $\begin{array}{l}15 . \\
89 \\
\%\end{array}$ & & \\
\hline $\begin{array}{l}\mathrm{M} \\
\mathrm{O} 2 \\
3- \\
80\end{array}$ & $\begin{array}{l}1 \\
3 \\
1\end{array}$ & $\begin{array}{l}3 \\
3 \\
8\end{array}$ & $\begin{array}{l}0 . \\
3 \\
9\end{array}$ & $\begin{array}{c}0.06 \\
166\end{array}$ & $\begin{array}{c}0 . \\
00 \\
05 \\
9\end{array}$ & $\begin{array}{c}0.7 \\
346 \\
4\end{array}$ & $\begin{array}{c}0 . \\
00 \\
67 \\
0\end{array}$ & $\begin{array}{c}0.0 \\
864 \\
1\end{array}$ & $\begin{array}{c}0 . \\
00 \\
09 \\
6\end{array}$ & 662 & $\begin{array}{l}1 \\
1\end{array}$ & 559 & 4 & 534 & 6 & $\begin{array}{l}4.6 \\
8 \%\end{array}$ & $\begin{array}{l}5 \\
3 \\
4\end{array}$ & 6 \\
\hline $\begin{array}{l}\mathrm{M} \\
\mathrm{O} 2 \\
3- \\
81\end{array}$ & $\begin{array}{l}1 \\
8\end{array}$ & $\begin{array}{l}2 \\
3 \\
1\end{array}$ & $\begin{array}{l}0 . \\
0 \\
8\end{array}$ & $\begin{array}{l}0.06 \\
252\end{array}$ & $\begin{array}{c}0 . \\
00 \\
07 \\
2\end{array}$ & $\begin{array}{c}0.7 \\
123 \\
0\end{array}$ & $\begin{array}{c}0 . \\
00 \\
76 \\
9\end{array}$ & $\begin{array}{c}0.0 \\
826 \\
3\end{array}$ & $\begin{array}{c}0 . \\
00 \\
09 \\
5\end{array}$ & 692 & $\begin{array}{l}1 \\
1\end{array}$ & 546 & 5 & 512 & 6 & $\begin{array}{l}6.6 \\
4 \%\end{array}$ & $\begin{array}{l}5 \\
1 \\
2\end{array}$ & 6 \\
\hline $\begin{array}{l}\mathrm{M} \\
\mathrm{O} 2 \\
3- \\
82\end{array}$ & $\begin{array}{l}2 \\
4 \\
6\end{array}$ & $\begin{array}{l}2 \\
8 \\
9\end{array}$ & $\begin{array}{l}0 . \\
8 \\
5\end{array}$ & $\begin{array}{l}0.06 \\
299\end{array}$ & $\begin{array}{c}0 . \\
00 \\
05 \\
6\end{array}$ & $\begin{array}{c}0.9 \\
248 \\
8\end{array}$ & $\begin{array}{c}0 . \\
00 \\
78 \\
1\end{array}$ & $\begin{array}{c}0.1 \\
065 \\
0\end{array}$ & $\begin{array}{c}0 . \\
00 \\
11 \\
7\end{array}$ & 708 & $\begin{array}{l}1 \\
1\end{array}$ & 665 & 4 & 652 & 7 & \begin{tabular}{|l|}
1.9 \\
$9 \%$
\end{tabular} & $\begin{array}{l}6 \\
5 \\
2\end{array}$ & 7 \\
\hline $\begin{array}{l}\mathrm{M} \\
\mathrm{O} 2 \\
3-\end{array}$ & $\begin{array}{l}2 \\
2 \\
6\end{array}$ & $\begin{array}{l}2 \\
9 \\
5\end{array}$ & $\begin{array}{l}0 . \\
7 \\
7 \\
\end{array}$ & $\begin{array}{l}0.06 \\
290\end{array}$ & $\begin{array}{c}0 . \\
00 \\
05\end{array}$ & $\begin{array}{c}0.8 \\
752 \\
3\end{array}$ & $\begin{array}{c}0 . \\
00 \\
70\end{array}$ & $\begin{array}{c}0.1 \\
009 \\
2\end{array}$ & $\begin{array}{c}0 . \\
00 \\
11\end{array}$ & 705 & $\begin{array}{l}1 \\
1\end{array}$ & 638 & 4 & 620 & 6 & $\begin{array}{l}2.9 \\
0 \%\end{array}$ & $\begin{array}{l}6 \\
2 \\
0 \\
\end{array}$ & 6 \\
\hline
\end{tabular}




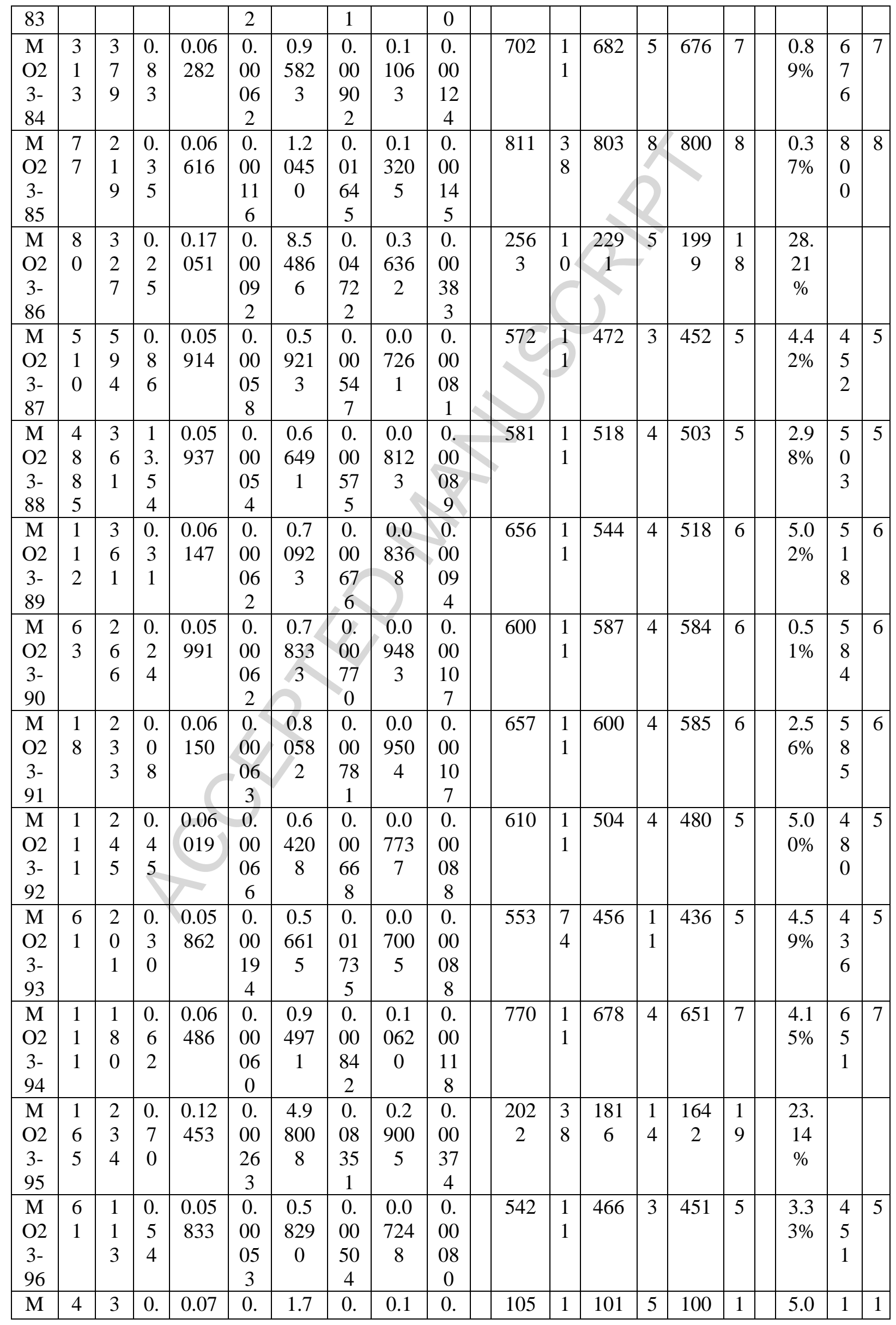




\begin{tabular}{|c|c|c|c|c|c|c|c|c|c|c|c|c|c|c|c|c|c|c|}
\hline $\begin{array}{l}\mathrm{O} 2 \\
3- \\
97\end{array}$ & 4 & $\begin{array}{l}8 \\
4\end{array}$ & $\begin{array}{l}1 \\
1\end{array}$ & 441 & $\begin{array}{c}00 \\
06 \\
0\end{array}$ & $\begin{array}{c}252 \\
8\end{array}$ & $\begin{array}{c}01 \\
34 \\
4\end{array}$ & $\begin{array}{c}681 \\
6\end{array}$ & $\begin{array}{c}00 \\
18 \\
4\end{array}$ & 3 & 1 & 8 & & 2 & 0 & $9 \%$ & $\begin{array}{l}0 \\
5 \\
3\end{array}$ & 1 \\
\hline $\begin{array}{l}\mathrm{M} \\
\mathrm{O} 2 \\
3- \\
98\end{array}$ & $\begin{array}{l}1 \\
5 \\
5\end{array}$ & $\begin{array}{l}2 \\
0 \\
9\end{array}$ & $\begin{array}{l}0 . \\
7 \\
4\end{array}$ & $\begin{array}{c}0.06 \\
813\end{array}$ & $\begin{array}{c}0 . \\
00 \\
07 \\
3\end{array}$ & $\begin{array}{c}1.1 \\
874 \\
9\end{array}$ & $\begin{array}{c}0 . \\
01 \\
20 \\
3\end{array}$ & $\begin{array}{c}0.1 \\
264 \\
2\end{array}$ & $\begin{array}{c}0 . \\
00 \\
14 \\
5\end{array}$ & 873 & $\begin{array}{l}1 \\
1\end{array}$ & 795 & 6 & 767 & 8 & $\begin{array}{l}3.6 \\
5 \%\end{array}$ & $\begin{array}{l}7 \\
6 \\
7\end{array}$ & 8 \\
\hline $\begin{array}{l}\mathrm{M} \\
\mathrm{O} 2 \\
3- \\
99\end{array}$ & $\begin{array}{l}1 \\
2 \\
8\end{array}$ & $\begin{array}{l}1 \\
4 \\
5\end{array}$ & $\begin{array}{l}0 . \\
8 \\
9\end{array}$ & $\begin{array}{l}0.06 \\
735\end{array}$ & $\begin{array}{c}0 . \\
00 \\
11 \\
3\end{array}$ & $\begin{array}{c}0.8 \\
905 \\
7\end{array}$ & $\begin{array}{c}0 . \\
01 \\
37 \\
8\end{array}$ & $\begin{array}{c}0.0 \\
959 \\
0\end{array}$ & $\begin{array}{c}0 . \\
00 \\
12 \\
5\end{array}$ & 849 & $\begin{array}{l}1 \\
4\end{array}$ & 647 & 7 & 590 & 7 & $\begin{array}{l}9.6 \\
6 \%\end{array}$ & $\begin{array}{l}5 \\
9 \\
0\end{array}$ & 7 \\
\hline $\begin{array}{c}\mathrm{M} \\
\mathrm{O} 2 \\
3- \\
10 \\
0\end{array}$ & $\begin{array}{l}9 \\
4\end{array}$ & $\begin{array}{l}7 \\
6\end{array}$ & $\begin{array}{l}1 . \\
2 \\
4\end{array}$ & $\begin{array}{c}0.06 \\
483\end{array}$ & $\begin{array}{c}0 . \\
00 \\
07 \\
4\end{array}$ & $\begin{array}{c}1.0 \\
726 \\
4\end{array}$ & $\begin{array}{c}0 . \\
01 \\
15 \\
6\end{array}$ & $\begin{array}{c}0.1 \\
199 \\
9\end{array}$ & $\begin{array}{c}0 . \\
00 \\
13 \\
9\end{array}$ & 769 & $\begin{array}{l}1 \\
1\end{array}$ & 740 & 6 & 731 & 8 & $\begin{array}{l}1.2 \\
3 \%\end{array}$ & $\begin{array}{l}7 \\
3 \\
1\end{array}$ & 8 \\
\hline $\begin{array}{c}\mathrm{M} \\
\mathrm{O} 2 \\
4- \\
01\end{array}$ & $\begin{array}{l}1 \\
8\end{array}$ & $\begin{array}{l}4 \\
3\end{array}$ & $\begin{array}{l}0 . \\
4 \\
1\end{array}$ & $\begin{array}{l}0.06 \\
934\end{array}$ & $\begin{array}{c}0 . \\
00 \\
15 \\
1\end{array}$ & $\begin{array}{c}1.3 \\
243 \\
5\end{array}$ & $\begin{array}{c}0 . \\
02 \\
66 \\
4\end{array}$ & $\begin{array}{c}0.1 \\
385 \\
1\end{array}$ & $\begin{array}{c}0 . \\
00 \\
20 \\
2\end{array}$ & 909 & $\begin{array}{l}2 \\
0\end{array}$ & 856 & $\begin{array}{l}1 \\
2\end{array}$ & 836 & $\begin{array}{l}1 \\
1\end{array}$ & $\begin{array}{l}2.3 \\
9 \%\end{array}$ & $\begin{array}{l}8 \\
3 \\
6\end{array}$ & $\begin{array}{l}1 \\
1\end{array}$ \\
\hline $\begin{array}{c}\mathrm{M} \\
\mathrm{O} 2 \\
4- \\
02\end{array}$ & $\begin{array}{l}6 \\
7\end{array}$ & $\begin{array}{l}1 \\
5 \\
2\end{array}$ & $\begin{array}{l}0 . \\
4 \\
4\end{array}$ & $\begin{array}{c}0.06 \\
038\end{array}$ & $\begin{array}{c}0 . \\
00 \\
08 \\
1\end{array}$ & $\begin{array}{c}0.7 \\
997 \\
5\end{array}$ & $\begin{array}{c}0 . \\
00 \\
99 \\
9\end{array}$ & $\begin{array}{c}0.0 \\
960 \\
6\end{array}$ & $\begin{array}{c}0 . \\
00 \\
11 \\
3\end{array}$ & 617 & $\begin{array}{l}1 \\
2\end{array}$ & 597 & 6 & 591 & 7 & $\begin{array}{l}1.0 \\
2 \%\end{array}$ & $\begin{array}{l}5 \\
9 \\
1\end{array}$ & 7 \\
\hline $\begin{array}{c}\mathrm{M} \\
\mathrm{O} 2 \\
4- \\
03\end{array}$ & $\begin{array}{l}1 \\
1 \\
0\end{array}$ & $\begin{array}{l}3 \\
6 \\
2\end{array}$ & $\begin{array}{l}0 . \\
3 \\
0\end{array}$ & $\begin{array}{c}0.06 \\
298\end{array}$ & $\begin{array}{c}0 . \\
00 \\
08 \\
2\end{array}$ & $\begin{array}{c}0.9 \\
678 \\
0 \\
\end{array}$ & $\begin{array}{c}0 . \\
01 \\
16 \\
9\end{array}$ & $\begin{array}{c}0.1 \\
114 \\
5\end{array}$ & $\begin{array}{c}0 . \\
00 \\
13 \\
2\end{array}$ & 708 & $\begin{array}{l}1 \\
2\end{array}$ & 687 & 6 & 681 & 8 & $\begin{array}{l}0.8 \\
8 \%\end{array}$ & $\begin{array}{l}6 \\
8 \\
1\end{array}$ & 8 \\
\hline $\begin{array}{c}\mathrm{M} \\
\mathrm{O} 2 \\
4- \\
04\end{array}$ & $\begin{array}{l}4 \\
0\end{array}$ & $\begin{array}{l}2 \\
6 \\
6\end{array}$ & $\begin{array}{c}0 . \\
1 \\
5\end{array}$ & $\begin{array}{l}0.05 \\
682\end{array}$ & $\begin{array}{c}0 . \\
00 \\
11 \\
2\end{array}$ & $\begin{array}{c}0.5 \\
877 \\
4\end{array}$ & $\begin{array}{c}0 . \\
00 \\
94 \\
3\end{array}$ & $\begin{array}{c}0.0 \\
750 \\
1\end{array}$ & $\begin{array}{c}0 . \\
00 \\
08 \\
6 \\
\end{array}$ & 485 & $\begin{array}{l}4 \\
5\end{array}$ & 469 & 6 & 466 & 5 & $\begin{array}{l}0.6 \\
4 \%\end{array}$ & $\begin{array}{l}4 \\
6 \\
6\end{array}$ & 5 \\
\hline $\begin{array}{c}\mathrm{M} \\
\mathrm{O} 2 \\
4- \\
05\end{array}$ & $\begin{array}{l}6 \\
1\end{array}$ & $\begin{array}{l}1 \\
4 \\
2\end{array}$ & $\begin{array}{l}0 . \\
4 \\
2\end{array}$ & $\begin{array}{l}0.06 \\
073 \\
\end{array}$ & $\begin{array}{c}0 . \\
00 \\
08 \\
8\end{array}$ & $\begin{array}{c}0.8 \\
718 \\
0\end{array}$ & $\begin{array}{c}0 . \\
01 \\
17 \\
3\end{array}$ & $\begin{array}{c}0.1 \\
041 \\
2\end{array}$ & $\begin{array}{c}0 . \\
00 \\
12 \\
7\end{array}$ & 630 & $\begin{array}{l}1 \\
3\end{array}$ & 637 & 6 & 639 & 7 & $\begin{array}{c}- \\
0.3 \\
1 \%\end{array}$ & $\begin{array}{l}6 \\
3 \\
9\end{array}$ & 7 \\
\hline $\begin{array}{c}\mathrm{M} \\
\mathrm{O} 2 \\
4- \\
06 \\
\end{array}$ & $\begin{array}{l}1 \\
0 \\
6\end{array}$ & $\begin{array}{l}1 \\
1 \\
9\end{array}$ & $\begin{array}{l}0 . \\
8 \\
9\end{array}$ & $\begin{array}{c}0.05 \\
618\end{array}$ & $\begin{array}{c}0 . \\
00 \\
10 \\
5\end{array}$ & $\begin{array}{c}0.5 \\
721 \\
5\end{array}$ & $\begin{array}{c}0 . \\
00 \\
99 \\
8 \\
\end{array}$ & $\begin{array}{c}0.0 \\
738 \\
6\end{array}$ & $\begin{array}{c}0 . \\
00 \\
09 \\
6 \\
\end{array}$ & 459 & $\begin{array}{l}1 \\
8\end{array}$ & 459 & 6 & 459 & 6 & $\begin{array}{l}0.0 \\
0 \%\end{array}$ & $\begin{array}{l}4 \\
5 \\
9\end{array}$ & 6 \\
\hline $\begin{array}{c}\mathrm{M} \\
\mathrm{O} 2 \\
4- \\
07\end{array}$ & $\begin{array}{l}3 \\
7 \\
0\end{array}$ & $\begin{array}{l}6 \\
8 \\
7\end{array}$ & $\begin{array}{l}0 . \\
5 \\
4\end{array}$ & $\begin{array}{l}0.07 \\
201\end{array}$ & $\begin{array}{c}0 . \\
00 \\
04 \\
6\end{array}$ & $\begin{array}{c}1.5 \\
856 \\
3\end{array}$ & $\begin{array}{c}0 . \\
01 \\
00 \\
4\end{array}$ & $\begin{array}{c}0.1 \\
597 \\
0\end{array}$ & $\begin{array}{c}0 . \\
00 \\
16 \\
9\end{array}$ & 986 & $\begin{array}{l}1 \\
2\end{array}$ & 965 & 4 & 955 & 9 & $\begin{array}{l}1.0 \\
5 \%\end{array}$ & $\begin{array}{l}9 \\
5 \\
5\end{array}$ & 9 \\
\hline $\begin{array}{c}\mathrm{M} \\
\mathrm{O} 2 \\
4- \\
08 \\
\end{array}$ & $\begin{array}{l}2 \\
3 \\
3\end{array}$ & $\begin{array}{l}2 \\
9 \\
5\end{array}$ & $\begin{array}{l}0 . \\
7 \\
9\end{array}$ & $\begin{array}{l}0.06 \\
657\end{array}$ & $\begin{array}{c}0 . \\
00 \\
06 \\
7\end{array}$ & $\begin{array}{c}1.1 \\
181 \\
7\end{array}$ & $\begin{array}{c}0 . \\
01 \\
05 \\
8\end{array}$ & $\begin{array}{c}0.1 \\
218 \\
3\end{array}$ & $\begin{array}{c}0 . \\
00 \\
13 \\
7 \\
\end{array}$ & 824 & $\begin{array}{l}1 \\
1\end{array}$ & 762 & 5 & 741 & 8 & $\begin{array}{l}2.8 \\
3 \%\end{array}$ & $\begin{array}{l}7 \\
4 \\
1\end{array}$ & 8 \\
\hline $\begin{array}{c}\mathrm{M} \\
\mathrm{O} 2 \\
4- \\
09\end{array}$ & $\begin{array}{l}2 \\
7 \\
9\end{array}$ & $\begin{array}{l}2 \\
5 \\
6\end{array}$ & $\begin{array}{l}1 . \\
0 \\
9\end{array}$ & $\begin{array}{l}0.06 \\
256\end{array}$ & $\begin{array}{c}0 . \\
00 \\
06 \\
5\end{array}$ & $\begin{array}{c}0.8 \\
546 \\
1\end{array}$ & $\begin{array}{c}0 . \\
00 \\
83 \\
5\end{array}$ & $\begin{array}{c}0.0 \\
990 \\
7\end{array}$ & $\begin{array}{c}0 . \\
00 \\
11 \\
1\end{array}$ & 693 & $\begin{array}{l}1 \\
1\end{array}$ & 627 & 5 & 609 & 7 & $\begin{array}{l}2.9 \\
6 \%\end{array}$ & $\begin{array}{l}6 \\
0 \\
9\end{array}$ & 7 \\
\hline $\mathrm{M}$ & 2 & 2 & 1. & 0.06 & 0. & 0.8 & 0. & 0.0 & 0. & 674 & 1 & 622 & 5 & 608 & 7 & 2.3 & 6 & 7 \\
\hline
\end{tabular}




\begin{tabular}{|c|c|c|c|c|c|c|c|c|c|c|c|c|c|c|c|c|c|c|}
\hline $\begin{array}{l}\mathrm{O} 2 \\
4- \\
10\end{array}$ & $\begin{array}{l}4 \\
7\end{array}$ & $\begin{array}{l}0 \\
7\end{array}$ & $\begin{array}{l}1 \\
9\end{array}$ & 200 & $\begin{array}{c}00 \\
07 \\
2\end{array}$ & $\begin{array}{c}459 \\
2\end{array}$ & $\begin{array}{c}00 \\
92 \\
2\end{array}$ & $\begin{array}{c}989 \\
5\end{array}$ & $\begin{array}{c}00 \\
11 \\
4\end{array}$ & & 1 & & & & & $0 \%$ & $\begin{array}{l}0 \\
8\end{array}$ & \\
\hline $\begin{array}{l}\mathrm{M} \\
\mathrm{O} 2 \\
4- \\
11\end{array}$ & $\begin{array}{l}5 \\
4\end{array}$ & $\begin{array}{l}2 \\
5 \\
5\end{array}$ & $\begin{array}{l}0 . \\
2 \\
1\end{array}$ & $\begin{array}{c}0.13 \\
619\end{array}$ & $\begin{array}{c}0 . \\
00 \\
08 \\
0\end{array}$ & $\begin{array}{c}7.5 \\
318 \\
2\end{array}$ & $\begin{array}{c}0 . \\
04 \\
41 \\
1\end{array}$ & $\begin{array}{c}0.4 \\
011 \\
0\end{array}$ & $\begin{array}{c}0 . \\
00 \\
42 \\
5\end{array}$ & $\begin{array}{c}217 \\
9\end{array}$ & $\begin{array}{l}1 \\
1\end{array}$ & $\begin{array}{c}217 \\
7\end{array}$ & 5 & $\begin{array}{c}217 \\
4\end{array}$ & $\begin{array}{l}2 \\
0\end{array}$ & $\begin{array}{l}0.2 \\
3 \%\end{array}$ & $\begin{array}{l}2 \\
1 \\
7 \\
9\end{array}$ & $\begin{array}{l}1 \\
1\end{array}$ \\
\hline $\begin{array}{c}\mathrm{M} \\
\mathrm{O} 2 \\
4- \\
12\end{array}$ & $\begin{array}{l}1 \\
9 \\
0\end{array}$ & $\begin{array}{l}1 \\
7 \\
9\end{array}$ & $\begin{array}{l}1 . \\
0 \\
6\end{array}$ & $\begin{array}{c}0.06 \\
067\end{array}$ & $\begin{array}{c}0 . \\
00 \\
07 \\
0\end{array}$ & $\begin{array}{c}0.9 \\
014 \\
6\end{array}$ & $\begin{array}{c}0 . \\
00 \\
97 \\
1\end{array}$ & $\begin{array}{c}0.1 \\
077 \\
7\end{array}$ & $\begin{array}{c}0 . \\
00 \\
12 \\
3\end{array}$ & 628 & $\begin{array}{l}1 \\
1\end{array}$ & 653 & 5 & 660 & 7 & $\begin{array}{c}- \\
1.0 \\
6 \%\end{array}$ & $\begin{array}{l}6 \\
6 \\
0\end{array}$ & 7 \\
\hline $\begin{array}{c}\mathrm{M} \\
\mathrm{O} 2 \\
4- \\
13\end{array}$ & $\begin{array}{l}7 \\
4\end{array}$ & $\begin{array}{l}1 \\
7 \\
6\end{array}$ & $\begin{array}{l}0 . \\
4 \\
2\end{array}$ & $\begin{array}{c}0.06 \\
481\end{array}$ & $\begin{array}{c}0 . \\
01 \\
97 \\
5\end{array}$ & $\begin{array}{c}0.6 \\
482 \\
9\end{array}$ & $\begin{array}{c}0 . \\
19 \\
68 \\
2\end{array}$ & $\begin{array}{c}0.0 \\
725 \\
5\end{array}$ & $\begin{array}{c}0 . \\
00 \\
19 \\
8\end{array}$ & 768 & $\begin{array}{l}6 \\
1\end{array}$ & 507 & $\begin{array}{l}1 \\
2 \\
1\end{array}$ & 452 & $\begin{array}{l}1 \\
2\end{array}$ & $\begin{array}{l}12 . \\
17 \\
\%\end{array}$ & & \\
\hline $\begin{array}{l}\mathrm{M} \\
\mathrm{O} 2 \\
4- \\
14\end{array}$ & $\begin{array}{l}2 \\
8 \\
5\end{array}$ & $\begin{array}{l}4 \\
4 \\
0\end{array}$ & $\begin{array}{l}0 . \\
6 \\
5\end{array}$ & $\begin{array}{c}0.06 \\
620\end{array}$ & $\begin{array}{c}0 . \\
00 \\
04 \\
9\end{array}$ & $\begin{array}{c}1.2 \\
320 \\
6\end{array}$ & $\begin{array}{c}0 . \\
00 \\
87 \\
9\end{array}$ & $\begin{array}{c}0.1 \\
349 \\
7\end{array}$ & $\begin{array}{c}0 . \\
00 \\
14 \\
4\end{array}$ & 813 & $\begin{array}{l}1 \\
1\end{array}$ & 815 & 4 & 816 & 8 & $\begin{array}{c}- \\
0.1 \\
2 \%\end{array}$ & $\begin{array}{l}8 \\
1 \\
6\end{array}$ & 8 \\
\hline $\begin{array}{c}\mathrm{M} \\
\mathrm{O} 2 \\
4- \\
15\end{array}$ & $\begin{array}{l}3 \\
4 \\
3\end{array}$ & $\begin{array}{l}2 \\
2 \\
3\end{array}$ & $\begin{array}{l}1 . \\
5 \\
4\end{array}$ & $\begin{array}{c}0.05 \\
963\end{array}$ & $\begin{array}{c}0 . \\
00 \\
06 \\
4\end{array}$ & $\begin{array}{c}0.8 \\
153 \\
9\end{array}$ & $\begin{array}{c}0 . \\
00 \\
81 \\
7\end{array}$ & $\begin{array}{c}0.0 \\
991 \\
8\end{array}$ & $\begin{array}{c}0 . \\
00 \\
11 \\
2\end{array}$ & 590 & $\begin{array}{l}1 \\
1\end{array}$ & 605 & 5 & 610 & 7 & $\begin{array}{c}- \\
0.8 \\
2 \%\end{array}$ & $\begin{array}{l}6 \\
1 \\
0\end{array}$ & 7 \\
\hline $\begin{array}{c}\mathrm{M} \\
\mathrm{O} 2 \\
4- \\
16\end{array}$ & $\begin{array}{l}1 \\
0 \\
7\end{array}$ & $\begin{array}{l}1 \\
0 \\
0\end{array}$ & $\begin{array}{l}1 . \\
0 \\
7\end{array}$ & $\begin{array}{c}0.06 \\
176\end{array}$ & $\begin{array}{c}0 . \\
00 \\
09 \\
7\end{array}$ & $\begin{array}{c}0.9 \\
035 \\
4\end{array}$ & $\begin{array}{c}0 . \\
01 \\
31 \\
5\end{array}$ & $\begin{array}{c}0.1 \\
061 \\
1\end{array}$ & $\begin{array}{c}0 . \\
00 \\
13 \\
2\end{array}$ & 666 & $\begin{array}{l}1 \\
4\end{array}$ & 654 & 7 & 650 & 8 & \begin{tabular}{|l|}
0.6 \\
$2 \%$
\end{tabular} & $\begin{array}{l}6 \\
5 \\
0\end{array}$ & 8 \\
\hline $\begin{array}{l}\mathrm{M} \\
\mathrm{O} 2 \\
4- \\
17\end{array}$ & $\begin{array}{l}1 \\
9 \\
3\end{array}$ & $\begin{array}{l}3 \\
0 \\
3\end{array}$ & $\begin{array}{l}0 . \\
6 \\
4\end{array}$ & $\begin{array}{c}0.06 \\
131\end{array}$ & $\begin{array}{c}0 . \\
00 \\
06 \\
2\end{array}$ & $\begin{array}{c}0.9 \\
144 \\
2\end{array}$ & $\begin{array}{c}0 . \\
00 \\
87 \\
8\end{array}$ & $\begin{array}{c}0.1 \\
081 \\
7\end{array}$ & $\begin{array}{c}0 . \\
00 \\
12 \\
1\end{array}$ & 650 & $\begin{array}{l}1 \\
1\end{array}$ & 659 & 5 & 662 & 7 & $\begin{array}{c} \\
0.4 \\
5 \%\end{array}$ & $\begin{array}{l}6 \\
6 \\
2\end{array}$ & 7 \\
\hline $\begin{array}{c}\mathrm{M} \\
\mathrm{O} 2 \\
4- \\
18\end{array}$ & $\begin{array}{l}9 \\
4\end{array}$ & $\begin{array}{l}1 \\
5 \\
9\end{array}$ & $\begin{array}{l}0 . \\
5 \\
9\end{array}$ & $\begin{array}{l}0.05 \\
579\end{array}$ & $\begin{array}{c}0 . \\
00 \\
08 \\
6\end{array}$ & $\begin{array}{c}0.5 \\
614 \\
5\end{array}$ & $\begin{array}{c}0 . \\
00 \\
80 \\
5\end{array}$ & $\begin{array}{c}0.0 \\
729 \\
9\end{array}$ & $\begin{array}{c}0 . \\
00 \\
08 \\
9\end{array}$ & 444 & $\begin{array}{l}1 \\
4\end{array}$ & 452 & 5 & 454 & 5 & $\begin{array}{c}- \\
0.4 \\
4 \%\end{array}$ & $\begin{array}{l}4 \\
5 \\
4\end{array}$ & 5 \\
\hline $\begin{array}{c}\mathrm{M} \\
\mathrm{O} 2 \\
4- \\
19\end{array}$ & $\begin{array}{l}1 \\
2 \\
9\end{array}$ & $\begin{array}{l}2 \\
0 \\
6\end{array}$ & $\begin{array}{l}0 . \\
6 \\
2\end{array}$ & $\begin{array}{l}0.10 \\
861\end{array}$ & $\begin{array}{c}0 . \\
00 \\
07 \\
4\end{array}$ & $\begin{array}{c}4.8 \\
845 \\
5\end{array}$ & $\begin{array}{c}0 . \\
03 \\
24 \\
4\end{array}$ & $\begin{array}{c}0.3 \\
261 \\
9\end{array}$ & $\begin{array}{c}0 . \\
00 \\
35 \\
2\end{array}$ & $\begin{array}{c}177 \\
6\end{array}$ & $\begin{array}{l}1 \\
0\end{array}$ & $\begin{array}{c}180 \\
0\end{array}$ & 6 & $\begin{array}{c}182 \\
0\end{array}$ & $\begin{array}{l}1 \\
7\end{array}$ & $\begin{array}{c}- \\
2.4 \\
2 \%\end{array}$ & $\begin{array}{l}1 \\
7 \\
7 \\
6\end{array}$ & $\begin{array}{l}1 \\
0\end{array}$ \\
\hline $\begin{array}{c}\mathrm{M} \\
\mathrm{O} 2 \\
4- \\
20\end{array}$ & $\begin{array}{l}9 \\
3\end{array}$ & $\begin{array}{l}3 \\
0 \\
7\end{array}$ & $\begin{array}{l}0 . \\
3 \\
0\end{array}$ & $\begin{array}{c}0.05 \\
649\end{array}$ & $\begin{array}{c}0 . \\
00 \\
06 \\
0\end{array}$ & $\begin{array}{c}0.5 \\
585 \\
8\end{array}$ & $\begin{array}{c}0 . \\
00 \\
56 \\
0\end{array}$ & $\begin{array}{c}0.0 \\
717 \\
1\end{array}$ & $\begin{array}{c}0 . \\
00 \\
08 \\
0\end{array}$ & 472 & $\begin{array}{l}1 \\
1\end{array}$ & 451 & 4 & 446 & 5 & $\begin{array}{l}1.1 \\
2 \%\end{array}$ & $\begin{array}{l}4 \\
4 \\
6\end{array}$ & 5 \\
\hline $\begin{array}{c}\mathrm{M} \\
\mathrm{O} 2 \\
4- \\
21\end{array}$ & $\begin{array}{l}1 \\
1 \\
7\end{array}$ & $\begin{array}{l}2 \\
9 \\
5\end{array}$ & $\begin{array}{l}0 . \\
4 \\
0\end{array}$ & $\begin{array}{l}0.12 \\
729\end{array}$ & $\begin{array}{c}0 . \\
00 \\
07 \\
5\end{array}$ & $\begin{array}{c}6.5 \\
866 \\
8\end{array}$ & $\begin{array}{c}0 . \\
03 \\
89 \\
2\end{array}$ & $\begin{array}{c}0.3 \\
752 \\
9\end{array}$ & $\begin{array}{c}0 . \\
00 \\
39 \\
7\end{array}$ & $\begin{array}{c}206 \\
1\end{array}$ & $\begin{array}{l}1 \\
1\end{array}$ & $\begin{array}{c}205 \\
8\end{array}$ & 5 & $\begin{array}{c}205 \\
4\end{array}$ & $\begin{array}{l}1 \\
9\end{array}$ & $\begin{array}{l}0.3 \\
4 \%\end{array}$ & $\begin{array}{l}2 \\
0 \\
6 \\
1\end{array}$ & $\begin{array}{l}1 \\
1\end{array}$ \\
\hline $\begin{array}{c}\mathrm{M} \\
\mathrm{O} 2 \\
4- \\
22\end{array}$ & $\begin{array}{l}1 \\
4 \\
3\end{array}$ & $\begin{array}{l}1 \\
2 \\
1\end{array}$ & $\begin{array}{l}1 . \\
1 \\
9\end{array}$ & $\begin{array}{l}0.12 \\
357\end{array}$ & $\begin{array}{c}0 . \\
00 \\
09 \\
1\end{array}$ & $\begin{array}{c}6.2 \\
616 \\
1\end{array}$ & $\begin{array}{c}0 . \\
04 \\
49 \\
0\end{array}$ & $\begin{array}{c}0.3 \\
675 \\
3\end{array}$ & $\begin{array}{c}0 . \\
00 \\
40 \\
6\end{array}$ & $\begin{array}{c}200 \\
8\end{array}$ & $\begin{array}{l}1 \\
0\end{array}$ & $\begin{array}{c}201 \\
3\end{array}$ & 6 & $\begin{array}{c}201 \\
8\end{array}$ & $\begin{array}{l}1 \\
9\end{array}$ & $\begin{array}{c} \\
0.5 \\
0 \%\end{array}$ & $\begin{array}{l}2 \\
0 \\
0 \\
8\end{array}$ & $\begin{array}{l}1 \\
0\end{array}$ \\
\hline $\begin{array}{l}\mathrm{M} \\
\mathrm{O} 2 \\
4-\end{array}$ & $\begin{array}{l}1 \\
1 \\
2\end{array}$ & $\begin{array}{l}6 \\
1\end{array}$ & $\begin{array}{l}1 . \\
8 \\
4\end{array}$ & $\begin{array}{l}0.06 \\
770\end{array}$ & $\begin{array}{c}0 . \\
00 \\
12\end{array}$ & $\begin{array}{c}0.9 \\
416 \\
5\end{array}$ & $\begin{array}{c}0 . \\
01 \\
62\end{array}$ & $\begin{array}{c}0.1 \\
008 \\
8\end{array}$ & $\begin{array}{c}0 . \\
00 \\
13\end{array}$ & 859 & $\begin{array}{l}1 \\
7\end{array}$ & 674 & 8 & 620 & 8 & \begin{tabular}{|l|}
8.7 \\
$1 \%$
\end{tabular} & $\begin{array}{l}6 \\
2 \\
0\end{array}$ & 8 \\
\hline
\end{tabular}




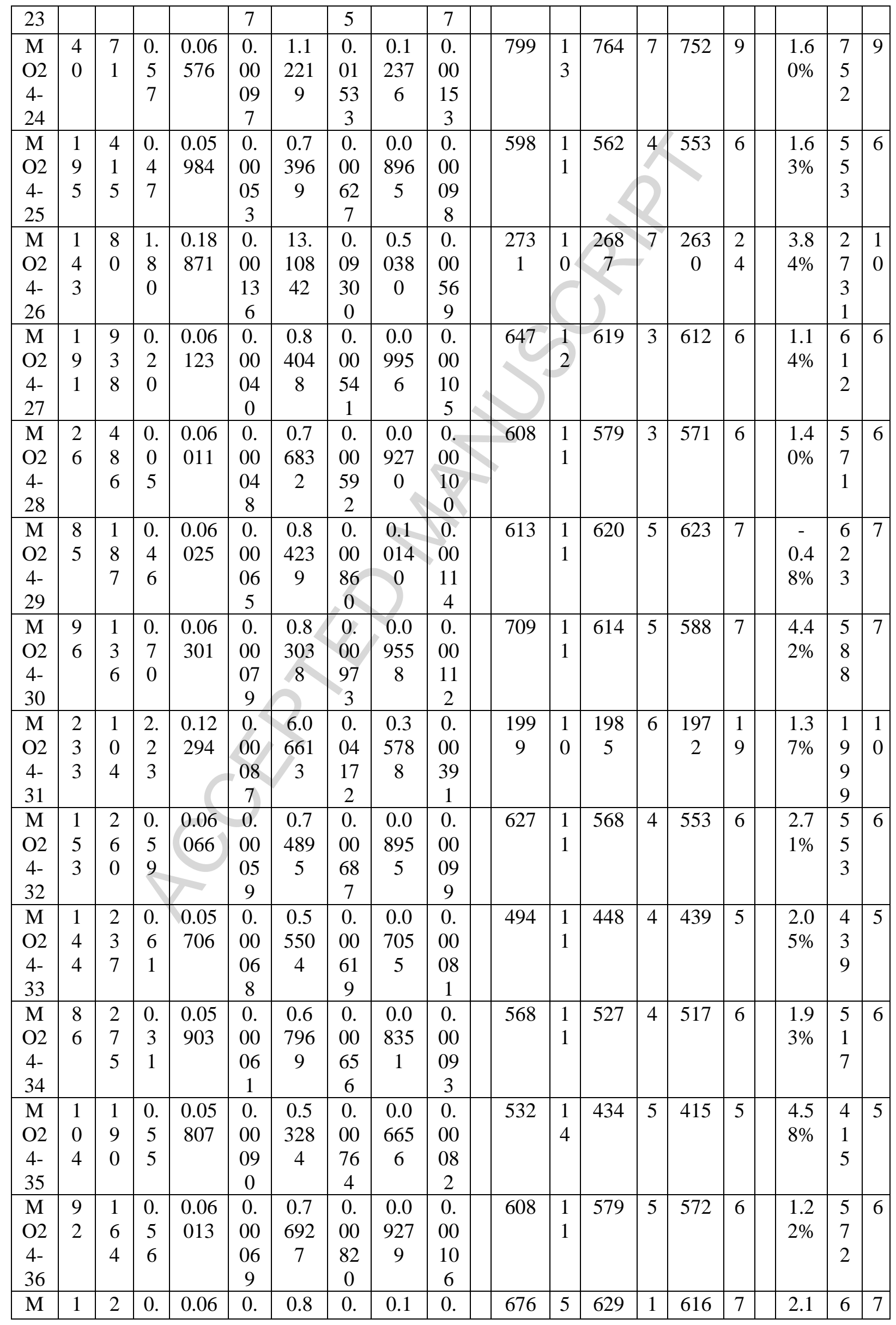




\begin{tabular}{|c|c|c|c|c|c|c|c|c|c|c|c|c|c|c|c|c|c|c|}
\hline $\begin{array}{l}\mathrm{O} 2 \\
4- \\
37\end{array}$ & $\begin{array}{l}4 \\
4\end{array}$ & $\begin{array}{l}5 \\
0\end{array}$ & $\begin{array}{l}5 \\
8\end{array}$ & 206 & $\begin{array}{c}00 \\
16 \\
2\end{array}$ & $\begin{array}{c}573 \\
3\end{array}$ & $\begin{array}{c}02 \\
01 \\
3\end{array}$ & $\begin{array}{c}001 \\
9\end{array}$ & $\begin{array}{c}00 \\
11 \\
5\end{array}$ & & 7 & & 1 & & & $1 \%$ & $\begin{array}{l}1 \\
6\end{array}$ & \\
\hline $\begin{array}{c}\mathrm{M} \\
\mathrm{O} 2 \\
4- \\
38\end{array}$ & $\begin{array}{l}9 \\
2\end{array}$ & $\begin{array}{l}2 \\
2 \\
5\end{array}$ & $\begin{array}{l}0 . \\
4 \\
1\end{array}$ & $\begin{array}{c}0.05 \\
530\end{array}$ & $\begin{array}{c}0 . \\
00 \\
06 \\
3\end{array}$ & $\begin{array}{c}0.5 \\
230 \\
7\end{array}$ & $\begin{array}{c}0 . \\
00 \\
55 \\
7\end{array}$ & $\begin{array}{c}0.0 \\
686 \\
0\end{array}$ & $\begin{array}{c}0 . \\
00 \\
07 \\
7\end{array}$ & 424 & $\begin{array}{l}1 \\
1\end{array}$ & 427 & 4 & 428 & 5 & $\begin{array}{c}- \\
0.2 \\
3 \%\end{array}$ & $\begin{array}{l}4 \\
2 \\
8\end{array}$ & 5 \\
\hline $\begin{array}{c}\mathrm{M} \\
\mathrm{O} 2 \\
4- \\
39\end{array}$ & $\begin{array}{l}1 \\
2 \\
1\end{array}$ & $\begin{array}{l}1 \\
2 \\
1\end{array}$ & $\begin{array}{l}1 . \\
0 \\
0\end{array}$ & $\begin{array}{l}0.05 \\
679\end{array}$ & $\begin{array}{c}0 . \\
00 \\
09 \\
3\end{array}$ & $\begin{array}{c}0.5 \\
510 \\
1\end{array}$ & $\begin{array}{c}0 . \\
00 \\
83 \\
7\end{array}$ & $\begin{array}{c}0.0 \\
703 \\
7\end{array}$ & $\begin{array}{c}0 . \\
00 \\
08 \\
7\end{array}$ & 483 & $\begin{array}{l}1 \\
5\end{array}$ & 446 & 5 & 438 & 5 & \begin{tabular}{|l|}
1.8 \\
$3 \%$
\end{tabular} & $\begin{array}{l}4 \\
3 \\
8\end{array}$ & 5 \\
\hline $\begin{array}{c}\mathrm{M} \\
\mathrm{O} 2 \\
4- \\
40\end{array}$ & $\begin{array}{l}2 \\
0 \\
6\end{array}$ & $\begin{array}{l}5 \\
8 \\
4\end{array}$ & $\begin{array}{l}0 . \\
3 \\
5\end{array}$ & $\begin{array}{c}0.05 \\
596\end{array}$ & $\begin{array}{c}0 . \\
00 \\
04 \\
6\end{array}$ & $\begin{array}{c}0.5 \\
558 \\
3\end{array}$ & $\begin{array}{c}0 . \\
00 \\
44 \\
1\end{array}$ & $\begin{array}{c}0.0 \\
720 \\
5\end{array}$ & $\begin{array}{c}0 . \\
00 \\
07 \\
7\end{array}$ & 451 & $\begin{array}{l}1 \\
1\end{array}$ & 449 & 3 & 448 & 5 & $\begin{array}{l}0.2 \\
2 \%\end{array}$ & $\begin{array}{l}4 \\
4 \\
8\end{array}$ & 5 \\
\hline $\begin{array}{l}\mathrm{M} \\
\mathrm{O} 2 \\
4- \\
41\end{array}$ & $\begin{array}{l}1 \\
9 \\
9\end{array}$ & $\begin{array}{l}1 \\
8 \\
7\end{array}$ & $\begin{array}{l}1 . \\
0 \\
6\end{array}$ & $\begin{array}{c}0.06 \\
094\end{array}$ & $\begin{array}{c}0 . \\
00 \\
06 \\
5\end{array}$ & $\begin{array}{c}0.8 \\
134 \\
5\end{array}$ & $\begin{array}{c}0 . \\
00 \\
81 \\
5\end{array}$ & $\begin{array}{c}0.0 \\
968 \\
2\end{array}$ & $\begin{array}{c}0 . \\
00 \\
10 \\
9\end{array}$ & 637 & $\begin{array}{l}1 \\
1\end{array}$ & 604 & 5 & 596 & 6 & $\begin{array}{l}1.3 \\
4 \%\end{array}$ & $\begin{array}{l}5 \\
9 \\
6\end{array}$ & 6 \\
\hline $\begin{array}{c}\mathrm{M} \\
\mathrm{O} 2 \\
4- \\
42\end{array}$ & $\begin{array}{l}1 \\
2 \\
0\end{array}$ & $\begin{array}{l}1 \\
2 \\
0\end{array}$ & $\begin{array}{l}1 . \\
0 \\
0\end{array}$ & $\begin{array}{l}0.06 \\
367\end{array}$ & $\begin{array}{c}0 . \\
00 \\
08 \\
4\end{array}$ & $\begin{array}{c}1.0 \\
642 \\
2\end{array}$ & $\begin{array}{c}0 . \\
01 \\
30 \\
2\end{array}$ & $\begin{array}{c}0.1 \\
212 \\
4\end{array}$ & $\begin{array}{c}0 . \\
00 \\
14 \\
4\end{array}$ & 731 & $\begin{array}{l}1 \\
2\end{array}$ & 736 & 6 & 738 & 8 & $\begin{array}{c}- \\
0.2 \\
7 \%\end{array}$ & $\begin{array}{l}7 \\
3 \\
8\end{array}$ & 8 \\
\hline $\begin{array}{c}\mathrm{M} \\
\mathrm{O} 2 \\
4- \\
43\end{array}$ & $\begin{array}{l}9 \\
2\end{array}$ & $\begin{array}{l}3 \\
9\end{array}$ & $\begin{array}{l}2 . \\
3 \\
7\end{array}$ & $\begin{array}{c}0.11 \\
643\end{array}$ & $\begin{array}{c}0 . \\
00 \\
11 \\
8\end{array}$ & $\begin{array}{c}5.4 \\
422 \\
7\end{array}$ & $\begin{array}{c}0 . \\
05 \\
21 \\
6\end{array}$ & $\begin{array}{c}0.3 \\
390 \\
4\end{array}$ & $\begin{array}{c}0 . \\
00 \\
40 \\
7\end{array}$ & $\begin{array}{c}190 \\
2\end{array}$ & $\begin{array}{l}1 \\
0\end{array}$ & $\begin{array}{c}189 \\
2\end{array}$ & 8 & $\begin{array}{c}188 \\
2\end{array}$ & $\begin{array}{l}2 \\
0\end{array}$ & $\begin{array}{l}1.0 \\
6 \%\end{array}$ & $\begin{array}{l}1 \\
9 \\
0 \\
2\end{array}$ & $\begin{array}{l}1 \\
0\end{array}$ \\
\hline $\begin{array}{l}\mathrm{M} \\
\mathrm{O} 2 \\
4- \\
44\end{array}$ & $\begin{array}{l}8 \\
6\end{array}$ & $\begin{array}{l}8 \\
6 \\
2\end{array}$ & $\begin{array}{c}0 . \\
1 \\
0\end{array}$ & $\begin{array}{l}0.05 \\
665\end{array}$ & $\begin{array}{c}0 . \\
00 \\
07 \\
8\end{array}$ & $\begin{array}{c}0.5 \\
554 \\
8\end{array}$ & $\begin{array}{c}0 . \\
00 \\
50 \\
1\end{array}$ & $\begin{array}{c}0.0 \\
711 \\
1\end{array}$ & $\begin{array}{c}0 . \\
00 \\
07 \\
4\end{array}$ & 478 & $\begin{array}{l}3 \\
1\end{array}$ & 449 & 3 & 443 & 4 & $\begin{array}{l}1.3 \\
5 \%\end{array}$ & $\begin{array}{l}4 \\
4 \\
3\end{array}$ & 4 \\
\hline $\begin{array}{c}\mathrm{M} \\
\mathrm{O} 2 \\
4- \\
45\end{array}$ & $\begin{array}{l}6 \\
3\end{array}$ & $\begin{array}{l}1 \\
9 \\
0\end{array}$ & $\begin{array}{l}0 . \\
3 \\
3\end{array}$ & $\begin{array}{l}0.05 \\
723\end{array}$ & $\begin{array}{c}0 . \\
00 \\
13 \\
8\end{array}$ & $\begin{array}{c}0.5 \\
696 \\
1\end{array}$ & $\begin{array}{c}0 . \\
01 \\
20 \\
1 \\
\end{array}$ & $\begin{array}{c}0.0 \\
721 \\
9\end{array}$ & $\begin{array}{c}0 . \\
00 \\
08 \\
4\end{array}$ & 500 & $\begin{array}{l}5 \\
4\end{array}$ & 458 & 8 & 449 & 5 & \begin{tabular}{|l|}
2.0 \\
$0 \%$
\end{tabular} & $\begin{array}{l}4 \\
4 \\
9\end{array}$ & 5 \\
\hline $\begin{array}{c}\mathrm{M} \\
\mathrm{O} 2 \\
4- \\
46\end{array}$ & $\begin{array}{l}3 \\
8 \\
3\end{array}$ & $\begin{array}{l}6 \\
9 \\
5\end{array}$ & $\begin{array}{l}0 . \\
5 \\
5\end{array}$ & $\begin{array}{l}0.06 \\
563\end{array}$ & $\begin{array}{c}0 . \\
00 \\
04 \\
5\end{array}$ & $\begin{array}{c}0.8 \\
990 \\
3\end{array}$ & $\begin{array}{c}0 . \\
00 \\
59 \\
7\end{array}$ & $\begin{array}{c}0.0 \\
993 \\
5\end{array}$ & $\begin{array}{c}0 . \\
00 \\
10 \\
5\end{array}$ & 795 & $\begin{array}{l}1 \\
2\end{array}$ & 651 & 3 & 611 & 6 & $\begin{array}{l}6.5 \\
5 \%\end{array}$ & $\begin{array}{l}6 \\
1 \\
1\end{array}$ & 6 \\
\hline $\begin{array}{c}\mathrm{M} \\
\mathrm{O} 2 \\
4- \\
47\end{array}$ & $\begin{array}{l}7 \\
9\end{array}$ & $\begin{array}{l}1 \\
2 \\
0\end{array}$ & $\begin{array}{c}0 . \\
6 \\
6\end{array}$ & $\begin{array}{l}0.05 \\
879\end{array}$ & $\begin{array}{c}0 . \\
00 \\
08 \\
5\end{array}$ & $\begin{array}{c}0.5 \\
774 \\
3\end{array}$ & $\begin{array}{c}0 . \\
00 \\
77 \\
4\end{array}$ & $\begin{array}{c}0.0 \\
712 \\
4\end{array}$ & $\begin{array}{c}0 . \\
00 \\
08 \\
5\end{array}$ & 559 & $\begin{array}{l}1 \\
3\end{array}$ & 463 & 5 & 444 & 5 & $\begin{array}{l}4.2 \\
8 \%\end{array}$ & $\begin{array}{l}4 \\
4 \\
4\end{array}$ & 5 \\
\hline $\begin{array}{c}\mathrm{M} \\
\mathrm{O} 2 \\
4- \\
48\end{array}$ & $\begin{array}{l}1 \\
3 \\
0\end{array}$ & $\begin{array}{l}1 \\
5 \\
4\end{array}$ & $\begin{array}{l}0 . \\
8 \\
4\end{array}$ & $\begin{array}{l}0.06 \\
742\end{array}$ & $\begin{array}{c}0 . \\
00 \\
06 \\
3\end{array}$ & $\begin{array}{c}1.2 \\
446 \\
7\end{array}$ & $\begin{array}{c}0 . \\
01 \\
09 \\
5\end{array}$ & $\begin{array}{c}0.1 \\
339 \\
1\end{array}$ & $\begin{array}{c}0 . \\
00 \\
14 \\
8\end{array}$ & 851 & $\begin{array}{l}1 \\
1\end{array}$ & 821 & 5 & 810 & 8 & $\begin{array}{l}1.3 \\
6 \%\end{array}$ & $\begin{array}{l}8 \\
1 \\
0\end{array}$ & 8 \\
\hline $\begin{array}{c}\mathrm{M} \\
\mathrm{O} 2 \\
4- \\
49\end{array}$ & $\begin{array}{l}1 \\
0 \\
3\end{array}$ & $\begin{array}{l}2 \\
4 \\
4\end{array}$ & $\begin{array}{l}0 . \\
4 \\
2\end{array}$ & $\begin{array}{c}0.05 \\
802\end{array}$ & $\begin{array}{c}0 . \\
00 \\
13 \\
5\end{array}$ & $\begin{array}{c}0.6 \\
868 \\
9\end{array}$ & $\begin{array}{c}0 . \\
01 \\
40 \\
3\end{array}$ & $\begin{array}{c}0.0 \\
858 \\
6\end{array}$ & $\begin{array}{c}0 . \\
00 \\
09 \\
6\end{array}$ & 531 & $\begin{array}{l}5 \\
2\end{array}$ & 531 & 8 & 531 & 6 & \begin{tabular}{|l|}
0.0 \\
$0 \%$
\end{tabular} & $\begin{array}{l}5 \\
3 \\
1\end{array}$ & 6 \\
\hline $\begin{array}{l}\mathrm{M} \\
\mathrm{O} 2 \\
4-\end{array}$ & $\begin{array}{l}7 \\
1\end{array}$ & $\begin{array}{l}8 \\
0\end{array}$ & $\begin{array}{l}0 . \\
8 \\
8\end{array}$ & $\begin{array}{l}0.12 \\
211\end{array}$ & $\begin{array}{c}0 . \\
00 \\
09\end{array}$ & $\begin{array}{c}6.0 \\
432 \\
0\end{array}$ & $\begin{array}{c}0 . \\
04 \\
51\end{array}$ & $\begin{array}{c}0.3 \\
589 \\
7\end{array}$ & $\begin{array}{c}0 . \\
00 \\
40\end{array}$ & $\begin{array}{c}198 \\
7\end{array}$ & $\begin{array}{l}1 \\
0\end{array}$ & $\begin{array}{c}198 \\
2\end{array}$ & 7 & $\begin{array}{c}197 \\
7\end{array}$ & 9 & $\begin{array}{l}0.5 \\
1 \%\end{array}$ & $\begin{array}{l}1 \\
9 \\
8\end{array}$ & $\begin{array}{l}1 \\
0\end{array}$ \\
\hline
\end{tabular}




\begin{tabular}{|c|c|c|c|c|c|c|c|c|c|c|c|c|c|c|c|c|c|c|}
\hline 50 & & & & & 5 & & 0 & & 0 & & & & & & & & 7 & \\
\hline $\mathrm{M}$ & 2 & 2 & 0. & 0.06 & 0. & 1.1 & 0. & 0.1 & 0. & 793 & 1 & 765 & 4 & 755 & 8 & 1.3 & 7 & 8 \\
\hline $\mathrm{O} 2$ & 0 & 5 & 8 & 559 & 00 & 243 & 00 & 243 & 00 & & 1 & & & & & $2 \%$ & 5 & \\
\hline $\begin{array}{l}4- \\
51\end{array}$ & 9 & 1 & 3 & & $\begin{array}{c}05 \\
5\end{array}$ & 5 & 89 & 4 & 13 & & & & & & & & 5 & \\
\hline $\begin{array}{l}51 \\
M\end{array}$ & 1 & 1 & 0. & 0.06 & 0 & 0.8 & 0 & 01 & $\begin{array}{l}4 \\
0\end{array}$ & 669 & 1 & 634 & 5 & 624 & 7 & 16 & 6 & 7 \\
\hline $\mathrm{O} 2$ & 1 & 4 & 8 & 185 & 00 & 670 & 00 & 016 & 00 & & 1 & & & & & $0 \%$ & 2 & \\
\hline 4- & 5 & 0 & 2 & & 07 & 3 & 94 & 7 & 11 & & & & & & & & 4 & \\
\hline 52 & & & & & 2 & & 2 & & 6 & & & & & & & & & \\
\hline $\mathrm{M}$ & 3 & 4 & 0. & 0.11 & 0. & 5.3 & 0. & 0.3 & 0. & 188 & 1 & 188 & 8 & 187 & 1 & 0.6 & 1 & 1 \\
\hline $\mathrm{O} 2$ & 0 & 9 & 6 & 546 & 00 & 736 & 05 & 375 & 00 & 7 & 0 & 1 & & 5 & 9 & $4 \%$ & 8 & 0 \\
\hline 4- & & & 2 & & 11 & 5 & 08 & 9 & 40 & & & & & & & & 8 & \\
\hline 53 & & & & & 6 & & 5 & & 2 & & & & & & & & 7 & \\
\hline $\mathrm{M}$ & 9 & 2 & 0. & 0.05 & 0. & 0.5 & 0. & 0.0 & 0. & 462 & 1 & 440 & 4 & 436 & 5 & 0.9 & 4 & 5 \\
\hline $\mathrm{O} 2$ & 8 & 0 & 4 & 625 & 00 & 428 & 00 & 699 & 00 & & 1 & & & & & $2 \%$ & 3 & \\
\hline 4- & & 6 & 8 & & 06 & 2 & 60 & 9 & 08 & & & & & & & & 6 & \\
\hline 54 & & & & & 7 & & 5 & & & & & & & & & & & \\
\hline $\mathrm{M}$ & 1 & 2 & 0. & 0.06 & 0. & 0.9 & 0. & 0.1 & 0. & 737 & 1 & 685 & 4 & 670 & 7 & 2.2 & 6 & 7 \\
\hline $\mathrm{O} 2$ & 0 & 2 & 4 & 385 & 00 & 636 & 00 & 094 & 00 & & 1 & & & & & $4 \%$ & 7 & \\
\hline 4- & 3 & 1 & 6 & & 05 & 6 & 82 & 8 & 12 & & & & & & & & 0 & \\
\hline 55 & & & & & 8 & & 6 & & 0 & & & & & & & & & \\
\hline $\mathrm{M}$ & 2 & 3 & 0. & 0.06 & 0. & 1.1 & 0. & 0.1 & 0. & 790 & 2 & 785 & 1 & 784 & 1 & 0.1 & 7 & 1 \\
\hline $\mathrm{O} 2$ & 4 & 2 & 7 & 549 & 00 & 673 & 02 & 292 & 00 & & 4 & & 2 & & 1 & $3 \%$ & 8 & 1 \\
\hline 4- & & & 5 & & 16 & 2 & 63 & 9 & 19 & & & & & & & & 4 & \\
\hline 56 & & & & & 0 & & 5 & , & 9 & & & & & & & & & \\
\hline $\mathrm{M}$ & 2 & 2 & 0. & 0.06 & 0. & 0.8 & 0. & 0.0 & 0. & 740 & 6 & 624 & 1 & 592 & 7 & 5.4 & 5 & 7 \\
\hline $\mathrm{O} 2$ & 0 & 7 & 7 & 396 & 00 & 488 & 02 & 962 & 00 & & 5 & & 3 & & & $1 \%$ & 9 & \\
\hline 4- & 0 & 1 & 4 & & 19 & 6 & 32 & 6 & 11 & & & & & & & & 2 & \\
\hline 57 & & & & & 1 & & 7 & & 3 & & & & & & & & & \\
\hline $\mathrm{M}$ & 9 & 2 & 0. & 0.05 & 0. & 0.7 & 0. & 0.0 & 0. & 599 & 4 & 569 & 8 & 562 & 6 & 1.2 & 5 & 6 \\
\hline $\mathrm{O} 2$ & 5 & 6 & 3 & 988 & 00 & 521 & 01 & 911 & 00 & & 7 & & & & & $5 \%$ & 6 & \\
\hline 4- & & 1 & 6 & & 12 & 4 & 35 & 0 & 10 & & & & & & & & 2 & \\
\hline 58 & & & & & 7 & & 5 & & 2 & & & & & & & & & \\
\hline $\mathrm{M}$ & 2 & 1 & 0. & 0.14 & 0. & 5.3 & 0. & 0.2 & 0. & 233 & 2 & 187 & 7 & 148 & 1 & 57. & & \\
\hline $\mathrm{O} 2$ & 9 & 9 & 1 & 922 & 00 & 302 & 04 & 590 & 00 & 7 & 4 & 4 & & 5 & 5 & 37 & & \\
\hline 4- & & 7 & 5 & 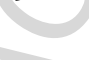 & 20 & 6 & 47 & 6 & 28 & & & & & & & $\%$ & & \\
\hline 59 & & & & & 6 & & 1 & & 4 & & & & & & & & & \\
\hline $\mathrm{M}$ & 8 & 1 & 0. & 0.05 & 0. & 0.5 & 0. & 0.0 & 0. & 521 & 1 & 454 & 5 & 441 & 5 & 2.9 & 4 & 5 \\
\hline $\mathrm{O} 2$ & 4 & 0 & 8 & 778 & 00 & 637 & 00 & 707 & 00 & & 4 & & & & & $5 \%$ & 4 & \\
\hline 4- & & 0 & 4 & & 09 & 9 & 81 & 7 & 08 & & & & & & & & 1 & \\
\hline 60 & & & & & 0 & & 6 & & 7 & & & & & & & & & \\
\hline $\mathrm{M}$ & 1 & 1 & 0. & 0.12 & 0. & 6.6 & 0. & 0.3 & 0. & 209 & 1 & 206 & 5 & 202 & 1 & 3.5 & 2 & 1 \\
\hline $\mathrm{O} 2$ & 2 & 8 & 6 & 985 & 00 & 081 & 03 & 691 & 00 & 6 & 0 & 0 & & 5 & 8 & $1 \%$ & 0 & 0 \\
\hline 4- & 4 & 3 & 8 & & 07 & 1 & 97 & 5 & 39 & & & & & & & & 9 & \\
\hline 61 & & & & & 9 & & 4 & & 1 & & & & & & & & 6 & \\
\hline $\mathrm{M}$ & 1 & 1 & 0. & 0.05 & 0. & 0.6 & 0. & 0.0 & 0. & 543 & 1 & 509 & 5 & 501 & 6 & 1.6 & 5 & 6 \\
\hline $\mathrm{O} 2$ & 6 & 1 & 1 & 835 & 00 & 501 & 00 & 808 & 00 & & 2 & & & & & $0 \%$ & 0 & \\
\hline 4- & & 0 & 5 & & 07 & 2 & 80 & 2 & 09 & & & & & & & & 1 & \\
\hline 62 & & & & & 7 & & 1 & & 5 & & & & & & & & & \\
\hline $\mathrm{M}$ & 1 & 2 & 0. & 0.06 & 0 & 0.8 & 0. & 0.1 & 0 & 617 & 1 & 619 & 4 & 620 & 6 & - & 6 & 6 \\
\hline $\mathrm{O} 2$ & 0 & 1 & 4 & 036 & 00 & 398 & 00 & 009 & 00 & & 1 & & & & & 0.1 & 2 & \\
\hline 4- & 3 & 0 & 9 & & 05 & 0 & 77 & 2 & 11 & & & & & & & $6 \%$ & 0 & \\
\hline 63 & & & & & 9 & & 5 & & 1 & & & & & & & & & \\
\hline $\mathrm{M}$ & 9 & 9 & 0. & 0.05 & 0. & 0.7 & 0. & 0.0 & 0. & 577 & 1 & 596 & 6 & 601 & 7 & - & 6 & 7 \\
\hline
\end{tabular}




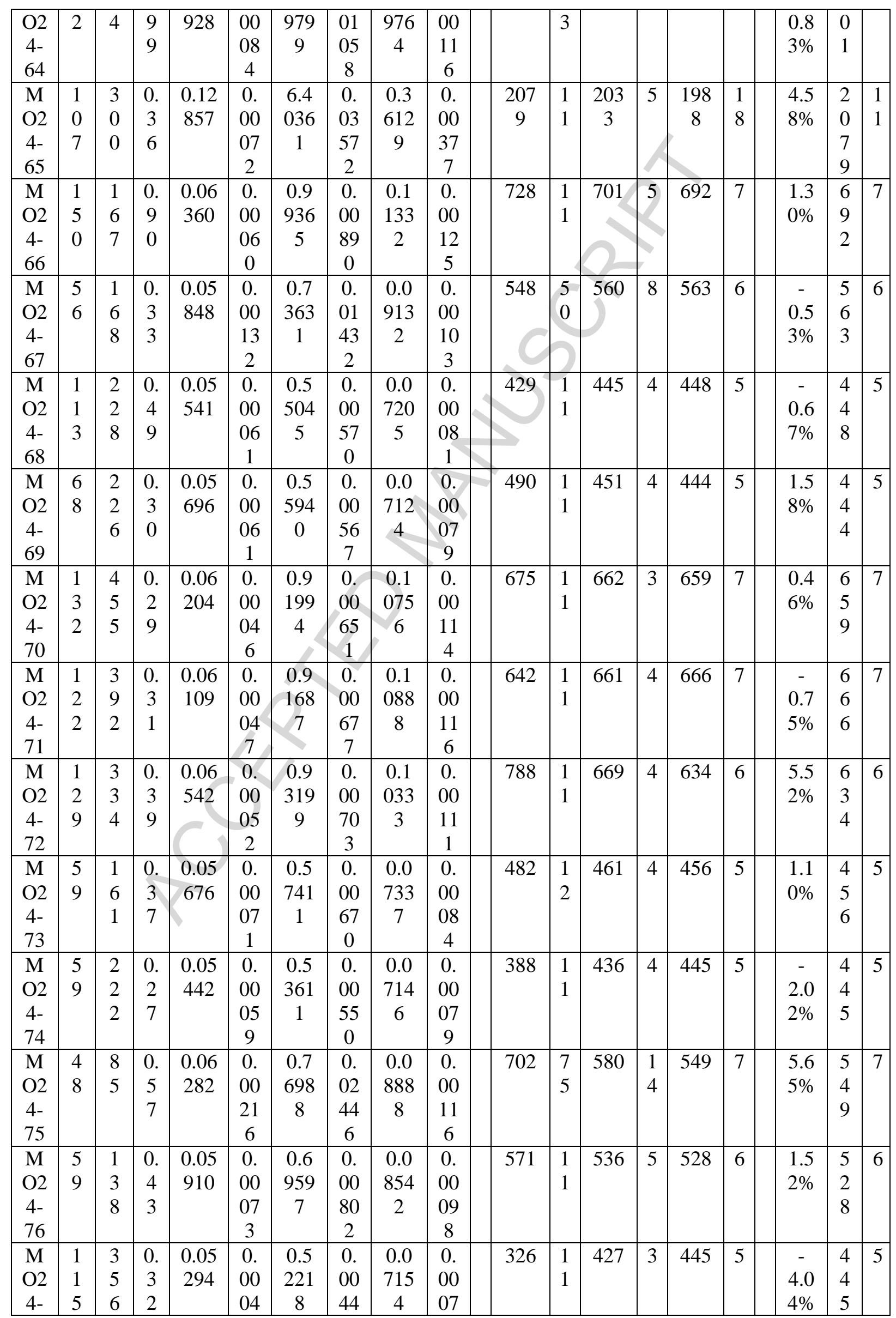




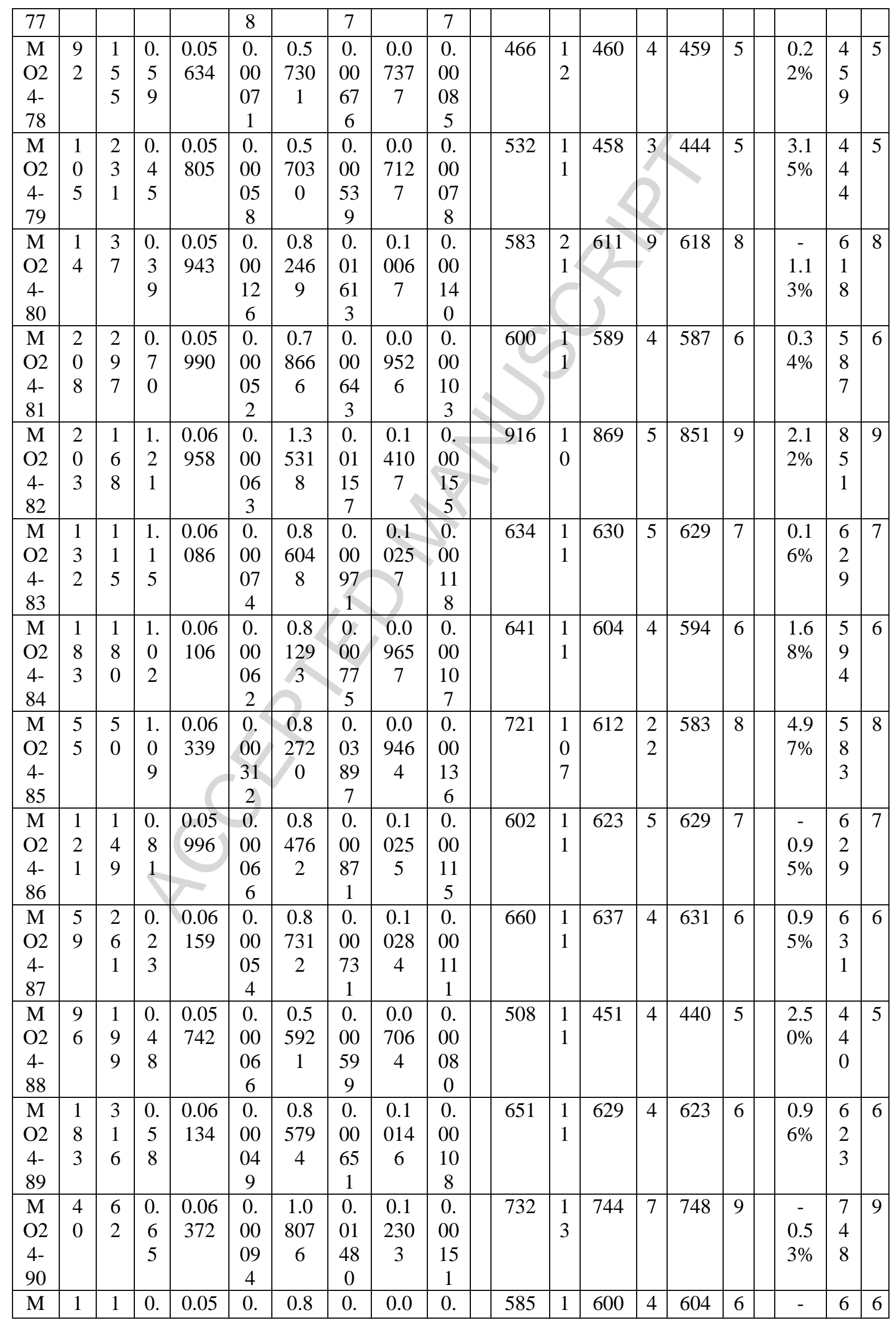




\begin{tabular}{|c|c|c|c|c|c|c|c|c|c|c|c|c|c|c|c|c|c|c|}
\hline $\begin{array}{l}\mathrm{O} 2 \\
4- \\
91\end{array}$ & $\begin{array}{l}4 \\
2\end{array}$ & $\begin{array}{l}7 \\
0\end{array}$ & $\begin{array}{l}8 \\
4\end{array}$ & 948 & $\begin{array}{c}00 \\
06 \\
1\end{array}$ & $\begin{array}{c}052 \\
3\end{array}$ & $\begin{array}{c}00 \\
78 \\
0\end{array}$ & $\begin{array}{c}982 \\
0\end{array}$ & $\begin{array}{c}00 \\
10 \\
9\end{array}$ & & 1 & & & & & $\begin{array}{l}0.6 \\
6 \%\end{array}$ & $\begin{array}{l}0 \\
4\end{array}$ & \\
\hline $\begin{array}{l}\mathrm{M} \\
\mathrm{O} 2 \\
4- \\
92\end{array}$ & $\begin{array}{l}7 \\
6\end{array}$ & $\begin{array}{l}2 \\
6 \\
5\end{array}$ & $\begin{array}{l}0 . \\
2 \\
9\end{array}$ & $\begin{array}{l}0.17 \\
231\end{array}$ & $\begin{array}{c}0 . \\
00 \\
10 \\
2\end{array}$ & $\begin{array}{c}10 . \\
841 \\
54\end{array}$ & $\begin{array}{c}0 . \\
06 \\
39 \\
9\end{array}$ & $\begin{array}{c}0.4 \\
564 \\
3\end{array}$ & $\begin{array}{c}0 . \\
00 \\
48 \\
4\end{array}$ & $\begin{array}{c}258 \\
0\end{array}$ & $\begin{array}{l}1 \\
0\end{array}$ & $\begin{array}{c}251 \\
0\end{array}$ & 5 & $\begin{array}{c}242 \\
4\end{array}$ & $\begin{array}{l}2 \\
1\end{array}$ & $\begin{array}{l}6.4 \\
4 \%\end{array}$ & $\begin{array}{l}2 \\
5 \\
8 \\
0\end{array}$ & $\begin{array}{l}1 \\
0\end{array}$ \\
\hline $\begin{array}{l}\mathrm{M} \\
\mathrm{O} 2 \\
4- \\
93 \\
\end{array}$ & $\begin{array}{l}2 \\
5 \\
7\end{array}$ & $\begin{array}{l}7 \\
0 \\
8\end{array}$ & $\begin{array}{l}0 . \\
3 \\
6\end{array}$ & $\begin{array}{l}0.05 \\
881\end{array}$ & $\begin{array}{c}0 . \\
00 \\
04 \\
0\end{array}$ & $\begin{array}{c}0.7 \\
463 \\
3\end{array}$ & $\begin{array}{c}0 . \\
00 \\
48 \\
8\end{array}$ & $\begin{array}{c}0.0 \\
920 \\
5\end{array}$ & $\begin{array}{c}0 . \\
00 \\
09 \\
6\end{array}$ & 560 & $\begin{array}{l}1 \\
2\end{array}$ & 566 & 3 & 568 & 6 & $\begin{array}{c}- \\
0.3 \\
5 \%\end{array}$ & $\begin{array}{l}5 \\
6 \\
8\end{array}$ & 6 \\
\hline $\begin{array}{l}\mathrm{M} \\
\mathrm{O} 2 \\
4- \\
94\end{array}$ & $\begin{array}{l}7 \\
6\end{array}$ & $\begin{array}{l}1 \\
8 \\
1\end{array}$ & $\begin{array}{l}0 . \\
4 \\
2\end{array}$ & $\begin{array}{l}0.05 \\
565\end{array}$ & $\begin{array}{c}0 . \\
00 \\
06 \\
6\end{array}$ & $\begin{array}{c}0.5 \\
663 \\
2\end{array}$ & $\begin{array}{c}0 . \\
00 \\
62 \\
8\end{array}$ & $\begin{array}{c}0.0 \\
738 \\
2\end{array}$ & $\begin{array}{c}0 . \\
00 \\
08 \\
3\end{array}$ & 438 & $\begin{array}{l}1 \\
1\end{array}$ & 456 & 4 & 459 & 5 & $\begin{array}{c}- \\
0.6 \\
5 \%\end{array}$ & $\begin{array}{l}4 \\
5 \\
9\end{array}$ & 5 \\
\hline $\begin{array}{l}\mathrm{M} \\
\mathrm{O} 2 \\
4- \\
95\end{array}$ & $\begin{array}{l}4 \\
6\end{array}$ & $\begin{array}{l}8 \\
0\end{array}$ & $\begin{array}{l}0 . \\
5 \\
8\end{array}$ & $\begin{array}{c}0.05 \\
978\end{array}$ & $\begin{array}{c}0 . \\
00 \\
08 \\
4\end{array}$ & $\begin{array}{c}0.7 \\
742 \\
3\end{array}$ & $\begin{array}{c}0 . \\
01 \\
01 \\
5\end{array}$ & $\begin{array}{c}0.0 \\
939 \\
6\end{array}$ & $\begin{array}{c}0 . \\
00 \\
11 \\
2\end{array}$ & 596 & $\begin{array}{l}1 \\
3\end{array}$ & 582 & 6 & 579 & 7 & $\begin{array}{l}0.5 \\
2 \%\end{array}$ & $\begin{array}{l}5 \\
7 \\
9\end{array}$ & 7 \\
\hline $\begin{array}{l}\mathrm{M} \\
\mathrm{O} 2 \\
4- \\
96\end{array}$ & $\begin{array}{l}3 \\
3\end{array}$ & $\begin{array}{l}9 \\
1\end{array}$ & $\begin{array}{l}0 . \\
3 \\
6\end{array}$ & $\begin{array}{c}0.06 \\
109\end{array}$ & $\begin{array}{c}0 . \\
00 \\
07 \\
7\end{array}$ & $\begin{array}{c}0.8 \\
569 \\
6\end{array}$ & $\begin{array}{c}0 . \\
01 \\
00 \\
7\end{array}$ & $\begin{array}{c}0.1 \\
017 \\
6\end{array}$ & $\begin{array}{c}0 . \\
00 \\
11 \\
8\end{array}$ & 642 & $\begin{array}{l}1 \\
1\end{array}$ & 628 & 6 & 625 & 7 & $\begin{array}{l}0.4 \\
8 \%\end{array}$ & $\begin{array}{l}6 \\
2 \\
5\end{array}$ & 7 \\
\hline $\begin{array}{l}\mathrm{M} \\
\mathrm{O} 2 \\
4- \\
97\end{array}$ & $\begin{array}{l}3 \\
6\end{array}$ & $\begin{array}{l}9 \\
5\end{array}$ & $\begin{array}{l}0 . \\
3 \\
7\end{array}$ & $\begin{array}{c}0.05 \\
980\end{array}$ & $\begin{array}{c}0 . \\
00 \\
07 \\
7\end{array}$ & $\begin{array}{c}0.8 \\
316 \\
4\end{array}$ & $\begin{array}{c}0 . \\
01 \\
00 \\
3\end{array}$ & $\begin{array}{c}0.1 \\
008 \\
8\end{array}$ & $\begin{array}{c}0 . \\
00 \\
11 \\
8\end{array}$ & 596 & $\begin{array}{l}1 \\
2\end{array}$ & 615 & 6 & 620 & 7 & $\begin{array}{l}0.8 \\
1 \%\end{array}$ & $\begin{array}{l}6 \\
2 \\
0\end{array}$ & 7 \\
\hline $\begin{array}{l}\mathrm{M} \\
\mathrm{O} 2 \\
4- \\
98\end{array}$ & $\begin{array}{l}4 \\
9\end{array}$ & $\begin{array}{l}8 \\
7\end{array}$ & $\begin{array}{l}0 . \\
5 \\
7\end{array}$ & $\begin{array}{l}0.05 \\
542\end{array}$ & $\begin{array}{c}0 . \\
00 \\
08 \\
9\end{array}$ & $\begin{array}{c}0.5 \\
632 \\
9\end{array}$ & $\begin{array}{c}0 . \\
00 \\
84 \\
3\end{array}$ & $\begin{array}{c}0.0 \\
737 \\
3\end{array}$ & $\begin{array}{c}0 . \\
00 \\
09 \\
1\end{array}$ & 429 & $\begin{array}{l}1 \\
5\end{array}$ & 454 & 5 & 459 & 5 & $\begin{array}{c}- \\
1.0 \\
9 \%\end{array}$ & $\begin{array}{l}4 \\
5 \\
9\end{array}$ & 5 \\
\hline $\begin{array}{l}\mathrm{M} \\
\mathrm{O} 2 \\
4- \\
99\end{array}$ & $\begin{array}{l}2 \\
3 \\
1\end{array}$ & $\begin{array}{l}3 \\
9 \\
9\end{array}$ & $\begin{array}{l}0 . \\
5 \\
8\end{array}$ & $\begin{array}{l}0.06 \\
054\end{array}$ & $\begin{array}{c}0 . \\
00 \\
04 \\
8\end{array}$ & $\begin{array}{c}0.8 \\
101 \\
2\end{array}$ & $\begin{array}{c}0 . \\
00 \\
61 \\
9\end{array}$ & $\begin{array}{c}0.0 \\
970 \\
8\end{array}$ & $\begin{array}{c}0 . \\
00 \\
10 \\
4\end{array}$ & 623 & $\begin{array}{l}1 \\
1\end{array}$ & 603 & 3 & 597 & 6 & $\begin{array}{l}1.0 \\
1 \%\end{array}$ & $\begin{array}{l}5 \\
9 \\
7\end{array}$ & 6 \\
\hline $\begin{array}{c}\mathrm{M} \\
\mathrm{O} 2 \\
4- \\
10 \\
0\end{array}$ & $\begin{array}{l}9 \\
3\end{array}$ & $\begin{array}{l}2 \\
6 \\
5\end{array}$ & $\begin{array}{l}0 . \\
3 \\
5\end{array}$ & $\begin{array}{l}0.05 \\
713\end{array}$ & $\begin{array}{c}0 . \\
00 \\
05 \\
4\end{array}$ & $\begin{array}{c}0.5 \\
667 \\
9\end{array}$ & $\begin{array}{c}0 . \\
00 \\
50 \\
3\end{array}$ & $\begin{array}{c}0.0 \\
719 \\
7\end{array}$ & $\begin{array}{c}0 . \\
00 \\
07 \\
8\end{array}$ & 497 & $\begin{array}{l}1 \\
1\end{array}$ & 456 & 3 & 448 & 5 & $\begin{array}{l}1.7 \\
9 \%\end{array}$ & $\begin{array}{l}4 \\
4 \\
8\end{array}$ & 5 \\
\hline
\end{tabular}




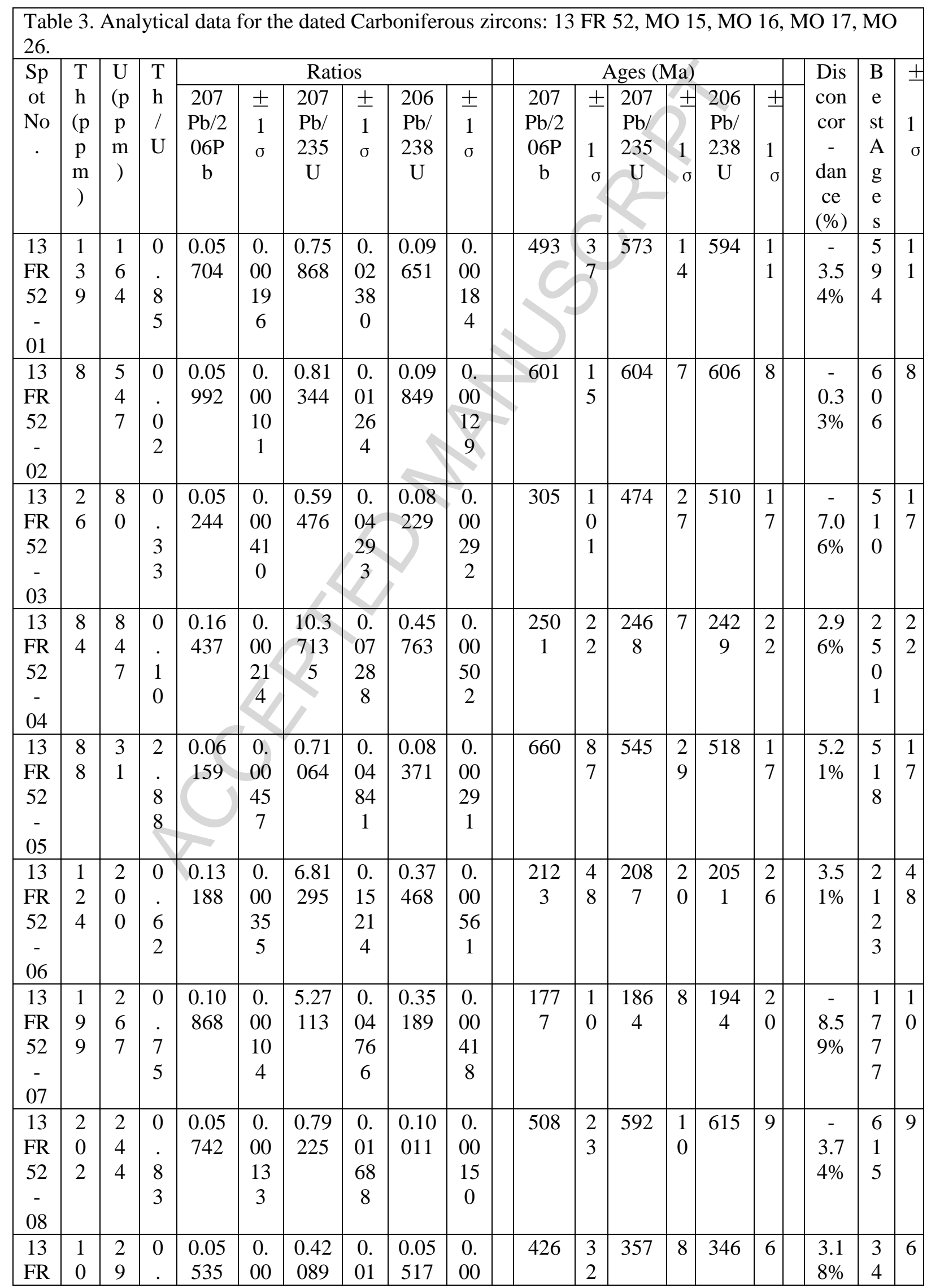




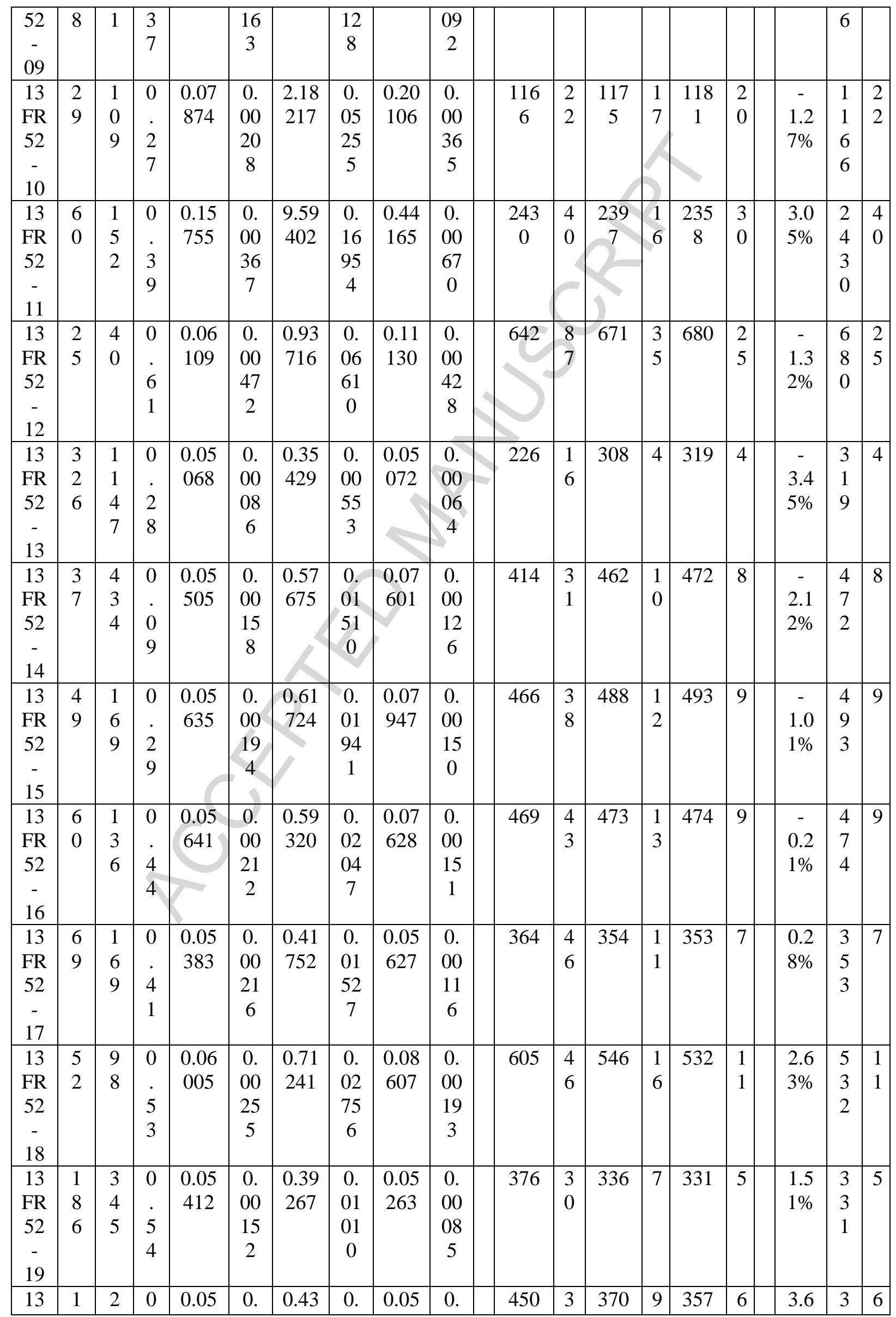




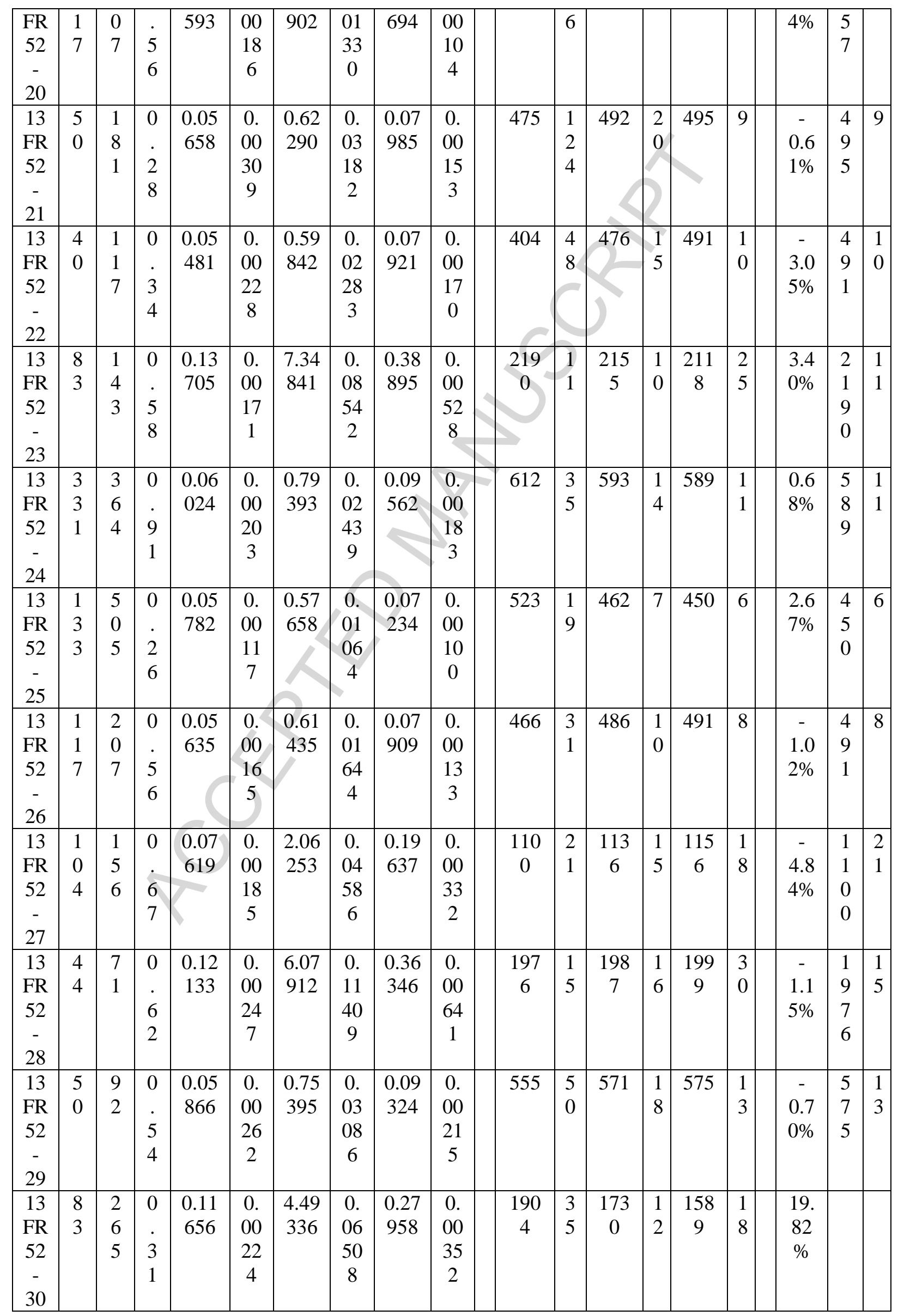




\begin{tabular}{|c|c|c|c|c|c|c|c|c|c|c|c|c|c|c|c|c|c|c|}
\hline $\begin{array}{c}13 \\
\text { FR } \\
52 \\
- \\
31\end{array}$ & $\begin{array}{l}8 \\
1\end{array}$ & $\begin{array}{l}5 \\
0 \\
5\end{array}$ & $\begin{array}{l}1 \\
6\end{array}$ & $\begin{array}{c}0.11 \\
914\end{array}$ & $\begin{array}{c}0 . \\
00 \\
09 \\
8\end{array}$ & $\begin{array}{c}5.88 \\
898\end{array}$ & $\begin{array}{c}0 . \\
04 \\
59 \\
8\end{array}$ & $\begin{array}{l}0.35 \\
855\end{array}$ & $\begin{array}{c}0 . \\
00 \\
40 \\
9\end{array}$ & $\begin{array}{c}194 \\
3\end{array}$ & $\begin{array}{l}1 \\
0\end{array}$ & $\begin{array}{c}196 \\
0\end{array}$ & 7 & $\begin{array}{c}197 \\
5\end{array}$ & $\begin{array}{l}1 \\
9\end{array}$ & $\begin{array}{c}- \\
1.6 \\
2 \%\end{array}$ & $\begin{array}{l}1 \\
9 \\
4 \\
3\end{array}$ & $\begin{array}{l}1 \\
0\end{array}$ \\
\hline $\begin{array}{c}13 \\
\text { FR } \\
52 \\
- \\
32\end{array}$ & $\begin{array}{l}1 \\
3 \\
2\end{array}$ & $\begin{array}{l}1 \\
7 \\
6\end{array}$ & $\begin{array}{l}0 \\
. \\
7 \\
5\end{array}$ & $\begin{array}{l}0.06 \\
137\end{array}$ & $\begin{array}{c}0 . \\
00 \\
16 \\
8\end{array}$ & $\begin{array}{l}0.91 \\
299\end{array}$ & $\begin{array}{c}0 . \\
02 \\
28 \\
1\end{array}$ & $\begin{array}{l}0.10 \\
792\end{array}$ & $\begin{array}{c}0 . \\
00 \\
18 \\
1\end{array}$ & 652 & $\begin{array}{l}2 \\
7\end{array}$ & 659 & $\begin{array}{l}1 \\
2\end{array}$ & 661 & $\begin{array}{l}1 \\
1\end{array}$ & $\begin{array}{c}- \\
0.3 \\
0 \%\end{array}$ & $\begin{array}{l}6 \\
6 \\
1\end{array}$ & $\begin{array}{l}1 \\
1\end{array}$ \\
\hline $\begin{array}{c}13 \\
\text { FR } \\
52 \\
- \\
33\end{array}$ & $\begin{array}{l}1 \\
2 \\
3\end{array}$ & $\begin{array}{l}2 \\
9 \\
3\end{array}$ & $\begin{array}{l}0 \\
4 \\
2\end{array}$ & $\begin{array}{l}0.05 \\
297\end{array}$ & $\begin{array}{c}0 . \\
00 \\
15 \\
6\end{array}$ & $\begin{array}{c}0.40 \\
650\end{array}$ & $\begin{array}{c}0 . \\
01 \\
09 \\
9\end{array}$ & $\begin{array}{l}0.05 \\
566\end{array}$ & $\begin{array}{c}0 . \\
00 \\
09 \\
2\end{array}$ & 328 & $\begin{array}{l}3 \\
3\end{array}$ & 46 & 8 & 349 & 6 & $\begin{array}{c}- \\
0.8 \\
6 \%\end{array}$ & $\begin{array}{l}3 \\
4 \\
9\end{array}$ & 6 \\
\hline $\begin{array}{c}13 \\
\text { FR } \\
52 \\
- \\
34\end{array}$ & $\begin{array}{l}9 \\
4\end{array}$ & $\begin{array}{l}1 \\
6 \\
4\end{array}$ & $\begin{array}{l}5 \\
7\end{array}$ & $\begin{array}{l}0.05 \\
709\end{array}$ & $\begin{array}{c}0 . \\
00 \\
19 \\
6\end{array}$ & $\begin{array}{l}0.57 \\
761\end{array}$ & $\begin{array}{c}0 . \\
01 \\
81 \\
5\end{array}$ & $\begin{array}{l}0.07 \\
339\end{array}$ & $\begin{array}{c}0 . \\
00 \\
13 \\
7\end{array}$ & 495 & $\begin{array}{l}3 \\
8\end{array}$ & 463 & $\begin{array}{l}1 \\
2\end{array}$ & 457 & 8 & $\begin{array}{l}1.3 \\
1 \%\end{array}$ & $\begin{array}{l}4 \\
5 \\
7\end{array}$ & 8 \\
\hline $\begin{array}{c}13 \\
\text { FR } \\
52 \\
- \\
35\end{array}$ & $\begin{array}{l}5 \\
2\end{array}$ & $\begin{array}{l}1 \\
1 \\
0\end{array}$ & $\begin{array}{l}0 \\
4 \\
7\end{array}$ & $\begin{array}{l}0.05 \\
722\end{array}$ & $\begin{array}{c}0 . \\
00 \\
20 \\
6\end{array}$ & $\begin{array}{l}0.68 \\
220\end{array}$ & $\begin{array}{c}0 . \\
02 \\
24 \\
3\end{array}$ & $\begin{array}{l}0.08 \\
649\end{array}$ & $\begin{array}{c}0 . \\
00 \\
17 \\
0\end{array}$ & 500 & $\begin{array}{l}3 \\
9\end{array}$ & 528 & $\begin{array}{l}1 \\
4\end{array}$ & 535 & $\begin{array}{l}1 \\
0\end{array}$ & $\begin{array}{c}- \\
1.3 \\
1 \%\end{array}$ & $\begin{array}{l}5 \\
3 \\
5\end{array}$ & $\begin{array}{l}1 \\
0\end{array}$ \\
\hline $\begin{array}{c}13 \\
\text { FR } \\
52 \\
- \\
36\end{array}$ & $\begin{array}{l}5 \\
7\end{array}$ & $\begin{array}{l}1 \\
8 \\
7\end{array}$ & $\begin{array}{l}0 \\
3 \\
1\end{array}$ & $\begin{array}{l}0.05 \\
607\end{array}$ & $\begin{array}{c}0 . \\
00 \\
17 \\
6\end{array}$ & $\begin{array}{l}0.59 \\
928\end{array}$ & $\begin{array}{c}0 . \\
01 \\
72 \\
0\end{array}$ & $\begin{array}{l}0.07 \\
753\end{array}$ & $\begin{array}{c}0 . \\
00 \\
13 \\
7\end{array}$ & 455 & $\begin{array}{l}3 \\
4\end{array}$ & 477 & $\begin{array}{l}1 \\
1\end{array}$ & 481 & 8 & $\begin{array}{c}- \\
0.8 \\
3 \%\end{array}$ & $\begin{array}{l}4 \\
8 \\
1\end{array}$ & 8 \\
\hline $\begin{array}{c}13 \\
\text { FR } \\
52 \\
- \\
37\end{array}$ & $\begin{array}{l}6 \\
6\end{array}$ & $\begin{array}{l}1 \\
0 \\
8\end{array}$ & $\begin{array}{l}6 \\
1\end{array}$ & $\begin{array}{c}0.13 \\
059\end{array}$ & $\begin{array}{c}0 . \\
00 \\
19 \\
9\end{array}$ & $\begin{array}{l}7.02 \\
280\end{array}$ & $\begin{array}{c}0 . \\
09 \\
92 \\
4\end{array}$ & $\begin{array}{c}0.39 \\
008\end{array}$ & $\begin{array}{c}0 . \\
00 \\
58 \\
3\end{array}$ & $\begin{array}{c}210 \\
6\end{array}$ & $\begin{array}{l}1 \\
2\end{array}$ & $\begin{array}{c}211 \\
4\end{array}$ & $\begin{array}{l}1 \\
3\end{array}$ & $\begin{array}{c}212 \\
3\end{array}$ & $\begin{array}{l}2 \\
7\end{array}$ & $\begin{array}{c}- \\
0.8 \\
0 \%\end{array}$ & $\begin{array}{l}2 \\
1 \\
0 \\
6\end{array}$ & $\begin{array}{l}1 \\
2\end{array}$ \\
\hline $\begin{array}{c}13 \\
\text { FR } \\
52 \\
- \\
38\end{array}$ & $\begin{array}{l}1 \\
2 \\
6\end{array}$ & $\begin{array}{l}2 \\
8 \\
6\end{array}$ & $\begin{array}{l}0 \\
4 \\
4\end{array}$ & $\begin{array}{l}0.05 \\
215\end{array}$ & $\begin{array}{c}0 . \\
00 \\
16 \\
2\end{array}$ & $\begin{array}{c}0.40 \\
570\end{array}$ & $\begin{array}{c}0 . \\
01 \\
15 \\
7\end{array}$ & $\begin{array}{l}0.05 \\
643\end{array}$ & $\begin{array}{c}0 . \\
00 \\
09 \\
6\end{array}$ & 292 & $\begin{array}{l}3 \\
5\end{array}$ & 346 & 8 & 354 & 6 & $\begin{array}{c}- \\
2.2 \\
6 \%\end{array}$ & $\begin{array}{l}3 \\
5 \\
4\end{array}$ & 6 \\
\hline $\begin{array}{c}13 \\
\text { FR } \\
52 \\
- \\
39\end{array}$ & $\begin{array}{l}1 \\
1 \\
8\end{array}$ & $\begin{array}{l}1 \\
3 \\
5\end{array}$ & $\begin{array}{l}0 \\
. \\
8 \\
7\end{array}$ & $\begin{array}{c}0.05 \\
970\end{array}$ & $\begin{array}{c}0 . \\
00 \\
21 \\
3\end{array}$ & $\begin{array}{l}0.74 \\
753\end{array}$ & $\begin{array}{c}0 . \\
02 \\
43 \\
6\end{array}$ & $\begin{array}{c}0.09 \\
082\end{array}$ & $\begin{array}{c}0 . \\
00 \\
17 \\
9\end{array}$ & 593 & $\begin{array}{l}3 \\
8\end{array}$ & 567 & $\begin{array}{l}1 \\
4\end{array}$ & 560 & $\begin{array}{l}1 \\
1\end{array}$ & $\begin{array}{l}1.2 \\
5 \%\end{array}$ & $\begin{array}{l}5 \\
6 \\
0\end{array}$ & $\begin{array}{l}1 \\
1\end{array}$ \\
\hline $\begin{array}{c}13 \\
\text { FR } \\
52 \\
- \\
40\end{array}$ & $\begin{array}{l}8 \\
0\end{array}$ & $\begin{array}{l}9 \\
9\end{array}$ & $\begin{array}{l}0 \\
. \\
8 \\
1\end{array}$ & $\begin{array}{l}0.05 \\
669\end{array}$ & $\begin{array}{c}0 . \\
00 \\
26 \\
8\end{array}$ & $\begin{array}{c}0.81 \\
034\end{array}$ & $\begin{array}{c}0 . \\
03 \\
50 \\
5\end{array}$ & $\begin{array}{l}0.10 \\
369\end{array}$ & $\begin{array}{c}0 . \\
00 \\
25 \\
3\end{array}$ & 479 & $\begin{array}{l}5 \\
4\end{array}$ & 603 & $\begin{array}{l}2 \\
0\end{array}$ & 636 & $\begin{array}{l}1 \\
5\end{array}$ & $\begin{array}{c}- \\
5.1 \\
9 \%\end{array}$ & $\begin{array}{l}6 \\
3 \\
6\end{array}$ & $\begin{array}{l}1 \\
5\end{array}$ \\
\hline $\begin{array}{c}13 \\
\text { FR } \\
52 \\
- \\
\end{array}$ & $\begin{array}{l}5 \\
8\end{array}$ & $\begin{array}{l}2 \\
3 \\
7\end{array}$ & $\begin{array}{l}0 \\
2 \\
5 \\
\end{array}$ & $\begin{array}{l}0.05 \\
093\end{array}$ & $\begin{array}{c}0 . \\
00 \\
17 \\
3 \\
\end{array}$ & $\begin{array}{c}0.40 \\
340\end{array}$ & $\begin{array}{c}0 . \\
01 \\
25 \\
9 \\
\end{array}$ & $\begin{array}{l}0.05 \\
745\end{array}$ & $\begin{array}{c}0 . \\
00 \\
10 \\
2 \\
\end{array}$ & 238 & $\begin{array}{l}4 \\
0\end{array}$ & 344 & 9 & 360 & 6 & $\begin{array}{c}- \\
4.4 \\
4 \%\end{array}$ & $\begin{array}{l}3 \\
6 \\
0\end{array}$ & 6 \\
\hline
\end{tabular}




\begin{tabular}{|c|c|c|c|c|c|c|c|c|c|c|c|c|c|c|c|c|c|c|}
\hline 41 & & & & & & & & & & & & & & & & & & \\
\hline $\begin{array}{c}13 \\
\text { FR } \\
52 \\
- \\
42\end{array}$ & $\begin{array}{l}6 \\
2\end{array}$ & $\begin{array}{l}1 \\
5 \\
0\end{array}$ & $\begin{array}{l}0 \\
. \\
4 \\
2\end{array}$ & $\begin{array}{l}0.05 \\
711\end{array}$ & $\begin{array}{c}0 . \\
00 \\
20 \\
2\end{array}$ & $\begin{array}{l}0.63 \\
727\end{array}$ & $\begin{array}{c}0 . \\
02 \\
05 \\
3\end{array}$ & $\begin{array}{l}0.08 \\
093\end{array}$ & $\begin{array}{c}0 . \\
00 \\
15 \\
6\end{array}$ & 496 & $\begin{array}{l}3 \\
9\end{array}$ & 501 & $\begin{array}{l}1 \\
3\end{array}$ & 502 & 9 & $\begin{array}{c}- \\
0.2 \\
0 \%\end{array}$ & $\begin{array}{l}5 \\
0 \\
2\end{array}$ & 9 \\
\hline $\begin{array}{c}13 \\
\text { FR } \\
52 \\
- \\
43\end{array}$ & $\begin{array}{l}7 \\
5\end{array}$ & $\begin{array}{l}1 \\
2 \\
6\end{array}$ & $\begin{array}{l}0 \\
6 \\
6 \\
0\end{array}$ & $\begin{array}{l}0.05 \\
921\end{array}$ & $\begin{array}{c}0 . \\
00 \\
21 \\
0\end{array}$ & $\begin{array}{l}0.65 \\
145\end{array}$ & $\begin{array}{c}0 . \\
02 \\
11 \\
0\end{array}$ & $\begin{array}{l}0.07 \\
981\end{array}$ & $\begin{array}{c}0 . \\
00 \\
15 \\
4\end{array}$ & 575 & $\begin{array}{l}3 \\
8\end{array}$ & 509 & $\begin{array}{l}1 \\
3\end{array}$ & 495 & 9 & $\begin{array}{l}2.8 \\
3 \%\end{array}$ & $\begin{array}{l}4 \\
9 \\
5\end{array}$ & 9 \\
\hline $\begin{array}{c}13 \\
\text { FR } \\
52 \\
- \\
44\end{array}$ & $\begin{array}{l}4 \\
4\end{array}$ & $\begin{array}{l}1 \\
2 \\
4\end{array}$ & $\begin{array}{l}3 \\
6\end{array}$ & $\begin{array}{c}0.05 \\
697\end{array}$ & $\begin{array}{c}0 . \\
00 \\
20 \\
5\end{array}$ & $\begin{array}{c}0.60 \\
429\end{array}$ & $\begin{array}{c}0 . \\
01 \\
98 \\
9\end{array}$ & $\begin{array}{r}0.07 \\
694\end{array}$ & $\begin{array}{c}0 . \\
00 \\
15 \\
0\end{array}$ & 490 & $\begin{array}{l}4 \\
0\end{array}$ & 480 & $\begin{array}{l}1 \\
3\end{array}$ & 478 & 9 & $\begin{array}{l}0.4 \\
2 \%\end{array}$ & $\begin{array}{l}4 \\
7 \\
8\end{array}$ & 9 \\
\hline $\begin{array}{c}13 \\
\text { FR } \\
52 \\
- \\
45\end{array}$ & $\begin{array}{l}3 \\
7\end{array}$ & $\begin{array}{l}3 \\
8\end{array}$ & $\begin{array}{l}0 \\
9 \\
9 \\
8\end{array}$ & $\begin{array}{c}0.10 \\
582\end{array}$ & $\begin{array}{c}0 . \\
00 \\
28 \\
4\end{array}$ & $\begin{array}{l}4.26 \\
257\end{array}$ & $\begin{array}{c}0 . \\
10 \\
43 \\
2\end{array}$ & $\begin{array}{l}0.29 \\
217\end{array}$ & $\begin{array}{c}0 . \\
00 \\
59 \\
2\end{array}$ & $\begin{array}{c}172 \\
9\end{array}$ & $\begin{array}{l}2 \\
0\end{array}$ & $\begin{array}{c}168 \\
6\end{array}$ & $\begin{array}{l}2 \\
0\end{array}$ & $\begin{array}{c}165 \\
2\end{array}$ & $\begin{array}{l}3 \\
0\end{array}$ & $\begin{array}{l}4.6 \\
6 \%\end{array}$ & $\begin{array}{l}1 \\
7 \\
2 \\
9\end{array}$ & $\begin{array}{l}2 \\
0\end{array}$ \\
\hline $\begin{array}{c}13 \\
\text { FR } \\
52 \\
- \\
46\end{array}$ & $\begin{array}{l}6 \\
1\end{array}$ & $\begin{array}{l}4 \\
5\end{array}$ & $\begin{array}{l}1 \\
3 \\
3 \\
5\end{array}$ & $\begin{array}{c}0.11 \\
561\end{array}$ & $\begin{array}{c}0 . \\
00 \\
26 \\
9\end{array}$ & $\begin{array}{l}5.29 \\
397\end{array}$ & $\begin{array}{c}0 . \\
11 \\
28 \\
2\end{array}$ & $\begin{array}{l}0.33 \\
214\end{array}$ & $\begin{array}{c}0 . \\
00 \\
62 \\
7\end{array}$ & $\begin{array}{c}188 \\
9\end{array}$ & $\begin{array}{l}1 \\
7\end{array}$ & $\begin{array}{c}186 \\
8\end{array}$ & $\begin{array}{l}1 \\
8\end{array}$ & $\begin{array}{c}184 \\
9\end{array}$ & $\begin{array}{l}3 \\
0\end{array}$ & $\begin{array}{l}2.1 \\
6 \%\end{array}$ & $\begin{array}{l}1 \\
8 \\
8 \\
9\end{array}$ & $\begin{array}{l}1 \\
7\end{array}$ \\
\hline $\begin{array}{c}13 \\
\text { FR } \\
52 \\
- \\
47 \\
\end{array}$ & $\begin{array}{l}1 \\
1 \\
4\end{array}$ & $\begin{array}{l}2 \\
3 \\
0\end{array}$ & $\begin{array}{l}0 \\
5 \\
5 \\
0\end{array}$ & $\begin{array}{l}0.05 \\
192\end{array}$ & $\begin{array}{c}0 . \\
00 \\
17 \\
6\end{array}$ & $\begin{array}{c}0.40 \\
109\end{array}$ & $\begin{array}{c}0 . \\
01 \\
24 \\
6\end{array}$ & $\begin{array}{l}0.05 \\
603\end{array}$ & $\begin{array}{c}0 . \\
00 \\
10 \\
0\end{array}$ & 282 & $\begin{array}{l}4 \\
0\end{array}$ & 342 & 9 & 351 & 6 & $\begin{array}{c}- \\
2.5 \\
6 \%\end{array}$ & $\begin{array}{l}3 \\
5 \\
1\end{array}$ & 6 \\
\hline $\begin{array}{c}13 \\
\text { FR } \\
52 \\
- \\
48\end{array}$ & $\begin{array}{l}3 \\
0\end{array}$ & $\begin{array}{l}1 \\
2 \\
2\end{array}$ & $\begin{array}{l}0 \\
2 \\
5\end{array}$ & $\begin{array}{l}0.05 \\
355 \\
\end{array}$ & $\begin{array}{c}0 . \\
00 \\
24 \\
6\end{array}$ & $\begin{array}{l}0.43 \\
772\end{array}$ & $\begin{array}{c}0 . \\
01 \\
84 \\
4\end{array}$ & $\begin{array}{l}0.05 \\
929\end{array}$ & $\begin{array}{c}0 . \\
00 \\
13 \\
3\end{array}$ & 352 & $\begin{array}{l}5 \\
6\end{array}$ & 369 & $\begin{array}{l}1 \\
3\end{array}$ & 371 & 8 & $\begin{array}{c}- \\
0.5 \\
4 \%\end{array}$ & $\begin{array}{l}3 \\
7 \\
1\end{array}$ & 8 \\
\hline $\begin{array}{c}13 \\
\text { FR } \\
52 \\
- \\
49\end{array}$ & $\begin{array}{l}1 \\
3 \\
8\end{array}$ & $\begin{array}{l}2 \\
4 \\
2\end{array}$ & $\begin{array}{l}0 \\
5 \\
5 \\
7\end{array}$ & $\begin{array}{c}0.04 \\
989\end{array}$ & $\begin{array}{c}0 . \\
00 \\
19 \\
6\end{array}$ & $\begin{array}{c}0.38 \\
533\end{array}$ & $\begin{array}{c}0 . \\
01 \\
39 \\
5\end{array}$ & $\begin{array}{l}0.05 \\
602\end{array}$ & $\begin{array}{c}0 . \\
00 \\
11 \\
1\end{array}$ & 190 & $\begin{array}{l}4 \\
8\end{array}$ & 331 & $\begin{array}{l}1 \\
0\end{array}$ & 351 & 7 & $\begin{array}{c}- \\
5.7 \\
0 \%\end{array}$ & $\begin{array}{l}3 \\
5 \\
1\end{array}$ & 7 \\
\hline $\begin{array}{c}13 \\
\text { FR } \\
52 \\
- \\
50 \\
\end{array}$ & $\begin{array}{l}1 \\
4 \\
0\end{array}$ & $\begin{array}{l}3 \\
1 \\
1\end{array}$ & $\begin{array}{l}0 \\
\dot{4} \\
5\end{array}$ & $\begin{array}{l}0.05 \\
355\end{array}$ & $\begin{array}{c}0 . \\
00 \\
15 \\
2\end{array}$ & $\begin{array}{l}0.41 \\
736\end{array}$ & $\begin{array}{c}0 . \\
01 \\
08 \\
6\end{array}$ & $\begin{array}{l}0.05 \\
654\end{array}$ & $\begin{array}{c}0 . \\
00 \\
09 \\
1\end{array}$ & 352 & $\begin{array}{l}3 \\
1\end{array}$ & 354 & 8 & 355 & 6 & $\begin{array}{c}- \\
0.2 \\
8 \%\end{array}$ & $\begin{array}{l}3 \\
5 \\
5\end{array}$ & 6 \\
\hline $\begin{array}{c}13 \\
\text { FR } \\
52 \\
- \\
51\end{array}$ & $\begin{array}{l}3 \\
5\end{array}$ & $\begin{array}{l}1 \\
0 \\
4\end{array}$ & $\begin{array}{l}0 \\
3 \\
4\end{array}$ & $\begin{array}{c}0.05 \\
550\end{array}$ & $\begin{array}{c}0 . \\
00 \\
24 \\
3\end{array}$ & $\begin{array}{l}0.60 \\
256\end{array}$ & $\begin{array}{c}0 . \\
02 \\
42 \\
4\end{array}$ & $\begin{array}{l}0.07 \\
875\end{array}$ & $\begin{array}{c}0 . \\
00 \\
17 \\
4\end{array}$ & 432 & $\begin{array}{l}5 \\
1\end{array}$ & 479 & $\begin{array}{l}1 \\
5\end{array}$ & 489 & $\begin{array}{l}1 \\
0\end{array}$ & $\begin{array}{c}- \\
2.0 \\
4 \%\end{array}$ & $\begin{array}{l}4 \\
8 \\
9\end{array}$ & $\begin{array}{l}1 \\
0\end{array}$ \\
\hline $\begin{array}{c}13 \\
\text { FR } \\
52\end{array}$ & $\begin{array}{l}5 \\
3\end{array}$ & $\begin{array}{l}7 \\
3\end{array}$ & 0 & $\begin{array}{l}0.10 \\
832\end{array}$ & $\begin{array}{c}0 . \\
00 \\
20\end{array}$ & $\begin{array}{l}5.18 \\
906\end{array}$ & $\begin{array}{c}0 . \\
08 \\
97\end{array}$ & $\begin{array}{c}0.34 \\
748\end{array}$ & $\begin{array}{c}0 . \\
00 \\
55\end{array}$ & $\begin{array}{c}177 \\
1\end{array}$ & $\begin{array}{l}1 \\
4\end{array}$ & $\begin{array}{c}185 \\
1\end{array}$ & $\begin{array}{l}1 \\
5\end{array}$ & $\begin{array}{c}192 \\
3\end{array}$ & $\begin{array}{l}2 \\
7\end{array}$ & $\begin{array}{c}- \\
7.9 \\
0 \%\end{array}$ & $\begin{array}{l}1 \\
7 \\
7\end{array}$ & $\begin{array}{l}1 \\
4\end{array}$ \\
\hline
\end{tabular}




\begin{tabular}{|c|c|c|c|c|c|c|c|c|c|c|c|c|c|c|c|c|c|c|}
\hline $\begin{array}{c}- \\
52\end{array}$ & & & 2 & & 2 & & 0 & & 9 & & & & & & & & 1 & \\
\hline $\begin{array}{c}13 \\
\text { FR } \\
52 \\
- \\
53\end{array}$ & $\begin{array}{l}6 \\
1\end{array}$ & $\begin{array}{l}1 \\
0 \\
3\end{array}$ & $\begin{array}{l}0 \\
. \\
5 \\
9\end{array}$ & $\begin{array}{l}0.05 \\
908\end{array}$ & $\begin{array}{c}0 . \\
00 \\
22 \\
3\end{array}$ & $\begin{array}{l}0.75 \\
208\end{array}$ & $\begin{array}{c}0 . \\
02 \\
59 \\
1\end{array}$ & $\begin{array}{l}0.09 \\
233\end{array}$ & $\begin{array}{c}0 . \\
00 \\
18 \\
8\end{array}$ & 570 & $\begin{array}{l}4 \\
1\end{array}$ & 569 & $\begin{array}{l}1 \\
5\end{array}$ & 569 & $\begin{array}{l}1 \\
1\end{array}$ & $\begin{array}{l}0.0 \\
0 \%\end{array}$ & $\begin{array}{l}5 \\
6 \\
9\end{array}$ & $\begin{array}{l}1 \\
1\end{array}$ \\
\hline $\begin{array}{c}13 \\
\text { FR } \\
52 \\
- \\
54\end{array}$ & $\begin{array}{l}5 \\
0\end{array}$ & $\begin{array}{l}5 \\
5\end{array}$ & $\begin{array}{l}0 \\
. \\
9 \\
1\end{array}$ & $\begin{array}{l}0.12 \\
706\end{array}$ & $\begin{array}{c}0 . \\
00 \\
25 \\
0\end{array}$ & $\begin{array}{l}6.53 \\
296\end{array}$ & $\begin{array}{c}0 . \\
11 \\
85 \\
7\end{array}$ & $\begin{array}{l}0.37 \\
293\end{array}$ & $\begin{array}{c}0 . \\
00 \\
64 \\
7\end{array}$ & $\begin{array}{c}205 \\
8\end{array}$ & $\begin{array}{l}1 \\
4\end{array}$ & $\begin{array}{c}205 \\
0\end{array}$ & $\begin{array}{l}1 \\
6\end{array}$ & $\begin{array}{c}204 \\
3\end{array}$ & $\begin{array}{l}3 \\
0\end{array}$ & $\begin{array}{l}0.7 \\
3 \%\end{array}$ & $\begin{array}{l}2 \\
0 \\
5 \\
8\end{array}$ & $\begin{array}{l}1 \\
4\end{array}$ \\
\hline $\begin{array}{c}13 \\
\text { FR } \\
52 \\
- \\
55\end{array}$ & $\begin{array}{l}5 \\
2\end{array}$ & $\begin{array}{l}9 \\
8\end{array}$ & $\begin{array}{l}0 \\
\dot{5} \\
3\end{array}$ & $\begin{array}{l}0.06 \\
054\end{array}$ & $\begin{array}{c}0 . \\
00 \\
28 \\
8\end{array}$ & $\begin{array}{l}0.70 \\
646\end{array}$ & $\begin{array}{c}0 . \\
03 \\
06 \\
1\end{array}$ & $\begin{array}{l}0.08 \\
464\end{array}$ & $\begin{array}{c}0 . \\
00 \\
20 \\
6\end{array}$ & 623 & 5 & 543 & $\begin{array}{l}1 \\
8\end{array}$ & 524 & $\begin{array}{l}1 \\
2\end{array}$ & $\begin{array}{l}3.6 \\
3 \%\end{array}$ & $\begin{array}{l}5 \\
2 \\
4\end{array}$ & $\begin{array}{l}1 \\
2\end{array}$ \\
\hline $\begin{array}{c}13 \\
\text { FR } \\
52 \\
- \\
56\end{array}$ & $\begin{array}{l}5 \\
3\end{array}$ & $\begin{array}{l}1 \\
4 \\
5\end{array}$ & $\begin{array}{l}0 \\
3 \\
3\end{array}$ & $\begin{array}{l}0.05 \\
711\end{array}$ & $\begin{array}{c}0 . \\
00 \\
28 \\
0\end{array}$ & $\begin{array}{c}0.63 \\
658\end{array}$ & $\begin{array}{c}0 . \\
02 \\
85 \\
0\end{array}$ & $\begin{array}{l}0.08 \\
084\end{array}$ & $\begin{array}{c}0 . \\
00 \\
20 \\
0\end{array}$ & 496 & $\begin{array}{l}5 \\
6\end{array}$ & 500 & $\begin{array}{l}1 \\
8\end{array}$ & 501 & $\begin{array}{l}1 \\
2\end{array}$ & $\begin{array}{c}- \\
0.2 \\
0 \%\end{array}$ & $\begin{array}{l}5 \\
0 \\
1\end{array}$ & $\begin{array}{l}1 \\
2\end{array}$ \\
\hline $\begin{array}{c}13 \\
\text { FR } \\
52 \\
- \\
57\end{array}$ & $\begin{array}{l}5 \\
1\end{array}$ & $\begin{array}{l}3 \\
7 \\
6\end{array}$ & $\begin{array}{l}1 \\
4\end{array}$ & $\begin{array}{c}0.05 \\
618\end{array}$ & $\begin{array}{c}0 . \\
00 \\
12 \\
8\end{array}$ & $\begin{array}{c}0.58 \\
414\end{array}$ & $\begin{array}{c}0 . \\
01 \\
22 \\
2\end{array}$ & $\begin{array}{l}0.07 \\
541\end{array}$ & $\begin{array}{c}0 . \\
00 \\
11 \\
0\end{array}$ & 459 & $\begin{array}{l}2 \\
3\end{array}$ & 467 & 8 & 469 & 7 & $\begin{array}{c}- \\
0.4 \\
3 \%\end{array}$ & $\begin{array}{l}4 \\
6 \\
9\end{array}$ & 7 \\
\hline $\begin{array}{c}13 \\
\text { FR } \\
52 \\
- \\
58\end{array}$ & $\begin{array}{l}6 \\
1\end{array}$ & $\begin{array}{l}8 \\
2\end{array}$ & $\begin{array}{l}0 \\
. \\
7 \\
4\end{array}$ & $\begin{array}{l}0.05 \\
777\end{array}$ & $\begin{array}{c}0 . \\
00 \\
24 \\
2\end{array}$ & $\begin{array}{c}0.64 \\
958\end{array}$ & $\begin{array}{c}0 . \\
02 \\
48 \\
8\end{array}$ & $\begin{array}{c}0.08 \\
156\end{array}$ & $\begin{array}{c}0 . \\
00 \\
17 \\
7\end{array}$ & 521 & $\begin{array}{l}4 \\
7\end{array}$ & 508 & $\begin{array}{l}1 \\
5\end{array}$ & 505 & $\begin{array}{l}1 \\
1\end{array}$ & $\begin{array}{l}0.5 \\
9 \%\end{array}$ & $\begin{array}{l}5 \\
0 \\
5\end{array}$ & $\begin{array}{l}1 \\
1\end{array}$ \\
\hline $\begin{array}{c}13 \\
\text { FR } \\
52 \\
- \\
59\end{array}$ & $\begin{array}{l}7 \\
2\end{array}$ & $\begin{array}{l}1 \\
7 \\
9\end{array}$ & $\begin{array}{l}0 \\
. \\
4 \\
0\end{array}$ & $\begin{array}{r}0.05 \\
672 \\
\end{array}$ & $\begin{array}{c}0 . \\
00 \\
19 \\
3\end{array}$ & $\begin{array}{l}0.61 \\
827\end{array}$ & $\begin{array}{c}0 . \\
01 \\
92 \\
5\end{array}$ & $\begin{array}{l}0.07 \\
906\end{array}$ & $\begin{array}{c}0 . \\
00 \\
14 \\
8\end{array}$ & 481 & $\begin{array}{l}3 \\
7\end{array}$ & 489 & $\begin{array}{l}1 \\
2\end{array}$ & 491 & 9 & $\begin{array}{c}- \\
0.4 \\
1 \%\end{array}$ & $\begin{array}{l}4 \\
9 \\
1\end{array}$ & 9 \\
\hline $\begin{array}{c}13 \\
\text { FR } \\
52 \\
- \\
60 \\
\end{array}$ & $\begin{array}{l}1 \\
5 \\
4\end{array}$ & $\begin{array}{l}3 \\
3 \\
8\end{array}$ & \begin{tabular}{|l}
0 \\
$\dot{4}$ \\
5
\end{tabular} & $\begin{array}{c}0.06 \\
581\end{array}$ & $\begin{array}{c}0 . \\
00 \\
12 \\
0\end{array}$ & $\begin{array}{l}1.13 \\
152\end{array}$ & $\begin{array}{c}0 . \\
01 \\
89 \\
5\end{array}$ & $\begin{array}{l}0.12 \\
470\end{array}$ & $\begin{array}{c}0 . \\
00 \\
17 \\
0\end{array}$ & 800 & $\begin{array}{l}1 \\
6\end{array}$ & 768 & 9 & 758 & $\begin{array}{l}1 \\
0\end{array}$ & $\begin{array}{l}1.3 \\
2 \%\end{array}$ & $\begin{array}{l}7 \\
5 \\
8\end{array}$ & $\begin{array}{l}1 \\
0\end{array}$ \\
\hline $\begin{array}{c}13 \\
\text { FR } \\
52 \\
- \\
61 \\
\end{array}$ & $\begin{array}{l}7 \\
5\end{array}$ & $\begin{array}{l}1 \\
6 \\
5\end{array}$ & $\begin{array}{l}0 \\
\dot{4} \\
5\end{array}$ & $\begin{array}{l}0.11 \\
251\end{array}$ & $\begin{array}{c}0 . \\
00 \\
34 \\
3\end{array}$ & $\begin{array}{l}4.87 \\
586\end{array}$ & $\begin{array}{c}0 . \\
12 \\
61 \\
4\end{array}$ & $\begin{array}{l}0.31 \\
431\end{array}$ & $\begin{array}{c}0 . \\
00 \\
50 \\
9\end{array}$ & $\begin{array}{c}184 \\
0\end{array}$ & $\begin{array}{l}5 \\
7\end{array}$ & $\begin{array}{c}179 \\
8\end{array}$ & $\begin{array}{l}2 \\
2\end{array}$ & $\begin{array}{c}176 \\
2\end{array}$ & $\begin{array}{l}2 \\
5\end{array}$ & $\begin{array}{l}4.4 \\
3 \%\end{array}$ & $\begin{array}{l}1 \\
8 \\
4 \\
0\end{array}$ & $\begin{array}{l}5 \\
7\end{array}$ \\
\hline $\begin{array}{c}13 \\
\text { FR } \\
52 \\
- \\
62\end{array}$ & $\begin{array}{l}6 \\
5\end{array}$ & $\begin{array}{l}9 \\
7\end{array}$ & $\begin{array}{l}0 \\
\dot{6} \\
7\end{array}$ & $\begin{array}{l}0.07 \\
585\end{array}$ & $\begin{array}{c}0 . \\
00 \\
20 \\
9\end{array}$ & $\begin{array}{l}1.95 \\
739\end{array}$ & $\begin{array}{c}0 . \\
04 \\
91 \\
3\end{array}$ & $\begin{array}{l}0.18 \\
717\end{array}$ & $\begin{array}{c}0 . \\
00 \\
34 \\
0\end{array}$ & $\begin{array}{c}109 \\
1\end{array}$ & $\begin{array}{l}2 \\
4\end{array}$ & $\begin{array}{c}110 \\
1\end{array}$ & $\begin{array}{l}1 \\
7\end{array}$ & $\begin{array}{c}110 \\
6\end{array}$ & $\begin{array}{l}1 \\
8\end{array}$ & $\begin{array}{c}- \\
1.3 \\
6 \%\end{array}$ & $\begin{array}{l}1 \\
0 \\
9 \\
1\end{array}$ & $\begin{array}{l}2 \\
4\end{array}$ \\
\hline $\begin{array}{c}13 \\
\text { FR }\end{array}$ & $\begin{array}{l}7 \\
2\end{array}$ & $\begin{array}{l}1 \\
8\end{array}$ & 0 & $\begin{array}{l}0.11 \\
374\end{array}$ & $\begin{array}{c}0 . \\
00\end{array}$ & $\begin{array}{l}2.63 \\
787\end{array}$ & $\begin{array}{c}0 . \\
06\end{array}$ & $\begin{array}{l}0.16 \\
821\end{array}$ & $\begin{array}{c}0 . \\
00\end{array}$ & $\begin{array}{c}186 \\
0\end{array}$ & $\begin{array}{l}5 \\
8\end{array}$ & $\begin{array}{c}131 \\
1\end{array}$ & $\begin{array}{l}1 \\
9\end{array}$ & $\begin{array}{c}100 \\
2\end{array}$ & $\begin{array}{l}1 \\
6\end{array}$ & $\begin{array}{c}85 \\
63\end{array}$ & & \\
\hline
\end{tabular}




\begin{tabular}{|c|c|c|c|c|c|c|c|c|c|c|c|c|c|c|c|c|c|c|}
\hline $\begin{array}{c}52 \\
- \\
63\end{array}$ & & 9 & $\begin{array}{l}3 \\
8\end{array}$ & & $\begin{array}{c}35 \\
5\end{array}$ & & $\begin{array}{c}94 \\
6\end{array}$ & & $\begin{array}{c}28 \\
3\end{array}$ & & & & & & & $\%$ & & \\
\hline $\begin{array}{c}13 \\
\text { FR } \\
52 \\
- \\
64\end{array}$ & $\begin{array}{l}3 \\
7\end{array}$ & $\begin{array}{l}6 \\
6\end{array}$ & $\begin{array}{l}0 \\
5 \\
6\end{array}$ & $\begin{array}{l}0.05 \\
769\end{array}$ & $\begin{array}{c}0 . \\
00 \\
31 \\
9\end{array}$ & $\begin{array}{c}0.62 \\
441\end{array}$ & $\begin{array}{c}0 . \\
03 \\
14 \\
9\end{array}$ & $\begin{array}{l}0.07 \\
850\end{array}$ & $\begin{array}{c}0 . \\
00 \\
21 \\
4\end{array}$ & 518 & $\begin{array}{l}6 \\
4\end{array}$ & 493 & $\begin{array}{l}2 \\
0\end{array}$ & 487 & $\begin{array}{l}1 \\
3\end{array}$ & $\begin{array}{l}1.2 \\
3 \%\end{array}$ & $\begin{array}{l}4 \\
8 \\
7\end{array}$ & $\begin{array}{l}1 \\
3\end{array}$ \\
\hline $\begin{array}{c}13 \\
\text { FR } \\
52 \\
- \\
65\end{array}$ & $\begin{array}{l}7 \\
7\end{array}$ & $\begin{array}{l}2 \\
5 \\
9\end{array}$ & $\begin{array}{l}0 \\
3 \\
3 \\
0\end{array}$ & $\begin{array}{l}0.05 \\
875\end{array}$ & $\begin{array}{c}0 . \\
00 \\
14 \\
9\end{array}$ & $\begin{array}{l}0.70 \\
221\end{array}$ & $\begin{array}{c}0 . \\
01 \\
63 \\
1\end{array}$ & $\begin{array}{c}0.08 \\
670\end{array}$ & $\begin{array}{c}0 . \\
00 \\
13 \\
6\end{array}$ & 558 & $\begin{array}{l}2 \\
5\end{array}$ & 540 & $\begin{array}{l}1 \\
0\end{array}$ & 536 & 8 & $\begin{array}{l}0.7 \\
5 \%\end{array}$ & $\begin{array}{l}5 \\
3 \\
6\end{array}$ & 8 \\
\hline $\begin{array}{c}13 \\
\text { FR } \\
52 \\
- \\
66\end{array}$ & $\begin{array}{l}5 \\
4\end{array}$ & $\begin{array}{l}1 \\
7 \\
2\end{array}$ & $\begin{array}{l}0 \\
3 \\
2\end{array}$ & $\begin{array}{l}0.05 \\
771\end{array}$ & $\begin{array}{c}0 . \\
00 \\
17 \\
5\end{array}$ & $\begin{array}{c}0.63 \\
639\end{array}$ & $\begin{array}{c}0 . \\
01 \\
76 \\
2\end{array}$ & $\begin{array}{l}0.07 \\
998\end{array}$ & $\begin{array}{c}0 . \\
00 \\
13 \\
8\end{array}$ & 519 & $\begin{array}{l}3 \\
2\end{array}$ & 500 & $\begin{array}{l}1 \\
1\end{array}$ & 496 & 8 & $\begin{array}{l}0.8 \\
1 \%\end{array}$ & $\begin{array}{l}4 \\
9 \\
6\end{array}$ & 8 \\
\hline $\begin{array}{c}13 \\
\text { FR } \\
52 \\
- \\
67\end{array}$ & $\begin{array}{l}1 \\
0 \\
0\end{array}$ & $\begin{array}{l}3 \\
6 \\
6\end{array}$ & $\begin{array}{l}0 \\
2 \\
7\end{array}$ & $\begin{array}{c}0.05 \\
819\end{array}$ & $\begin{array}{c}0 . \\
00 \\
11 \\
9\end{array}$ & $\begin{array}{c}0.79 \\
005\end{array}$ & $\begin{array}{c}0 . \\
01 \\
48 \\
3\end{array}$ & $\begin{array}{l}0.09 \\
848\end{array}$ & $\begin{array}{c}0 . \\
00 \\
13 \\
7\end{array}$ & 537 & $\begin{array}{l}2 \\
0\end{array}$ & 591 & 8 & 605 & 8 & $\begin{array}{c}- \\
2.3 \\
1 \%\end{array}$ & $\begin{array}{l}6 \\
0 \\
5\end{array}$ & 8 \\
\hline $\begin{array}{c}13 \\
\text { FR } \\
52 \\
- \\
68\end{array}$ & $\begin{array}{l}1 \\
0 \\
5\end{array}$ & $\begin{array}{l}4 \\
6\end{array}$ & $\begin{array}{l}2 \\
2 \\
6\end{array}$ & $\begin{array}{l}0.05 \\
772\end{array}$ & $\begin{array}{c}0 . \\
00 \\
40 \\
8\end{array}$ & $\begin{array}{l}0.62 \\
251\end{array}$ & $\begin{array}{c}0 . \\
04 \\
06 \\
4\end{array}$ & $\begin{array}{l}0.07 \\
823\end{array}$ & $\begin{array}{c}0 . \\
00 \\
25 \\
0\end{array}$ & 519 & $\begin{array}{l}8 \\
8\end{array}$ & 491 & $\begin{array}{l}2 \\
5\end{array}$ & 486 & \begin{tabular}{l|}
1 \\
5
\end{tabular} & $\begin{array}{l}1.0 \\
3 \%\end{array}$ & $\begin{array}{l}4 \\
8 \\
6\end{array}$ & $\begin{array}{l}1 \\
5\end{array}$ \\
\hline $\begin{array}{c}13 \\
\text { FR } \\
52 \\
- \\
69\end{array}$ & $\begin{array}{l}8 \\
7\end{array}$ & $\begin{array}{l}4 \\
0 \\
7\end{array}$ & $\begin{array}{l}0 \\
2 \\
1\end{array}$ & $\begin{array}{l}0.16 \\
615\end{array}$ & $\begin{array}{c}0 . \\
00 \\
20 \\
0\end{array}$ & $\begin{array}{l}9.91 \\
445\end{array}$ & $\begin{array}{c}0 . \\
11 \\
14 \\
6\end{array}$ & $\begin{array}{l}0.43 \\
278\end{array}$ & $\begin{array}{c}0 . \\
00 \\
59 \\
0\end{array}$ & $\begin{array}{c}251 \\
9\end{array}$ & $\begin{array}{l}1 \\
0\end{array}$ & $\begin{array}{c}242 \\
7\end{array}$ & $\begin{array}{l}1 \\
0\end{array}$ & $\begin{array}{c}231 \\
8\end{array}$ & $\begin{array}{l}2 \\
7\end{array}$ & $\begin{array}{l}8.6 \\
7 \%\end{array}$ & $\begin{array}{l}2 \\
5 \\
1 \\
9\end{array}$ & $\begin{array}{l}1 \\
0\end{array}$ \\
\hline $\begin{array}{c}13 \\
\text { FR } \\
52 \\
- \\
70\end{array}$ & $\begin{array}{l}7 \\
8\end{array}$ & $\begin{array}{l}2 \\
8 \\
1\end{array}$ & $\begin{array}{l}2 \\
8\end{array}$ & $\begin{array}{l}0.05 \\
613 \\
\end{array}$ & $\begin{array}{c}0 . \\
00 \\
15 \\
9\end{array}$ & $\begin{array}{c}0.61 \\
896\end{array}$ & $\begin{array}{c}0 . \\
01 \\
61 \\
1\end{array}$ & $\begin{array}{l}0.07 \\
998\end{array}$ & $\begin{array}{c}0 . \\
00 \\
13 \\
2\end{array}$ & 458 & $\begin{array}{l}3 \\
0\end{array}$ & 489 & $\begin{array}{l}1 \\
0\end{array}$ & 496 & 8 & $\begin{array}{c}- \\
1.4 \\
1 \%\end{array}$ & $\begin{array}{l}4 \\
9 \\
6\end{array}$ & 8 \\
\hline $\begin{array}{c}13 \\
\text { FR } \\
52 \\
- \\
71\end{array}$ & $\begin{array}{l}1 \\
3 \\
7\end{array}$ & $\begin{array}{l}1 \\
0 \\
9\end{array}$ & $\begin{array}{l}2 \\
6\end{array}$ & $\begin{array}{l}0.18 \\
298\end{array}$ & $\begin{array}{c}0 . \\
00 \\
21 \\
3\end{array}$ & $\begin{array}{c}13.1 \\
198 \\
9\end{array}$ & $\begin{array}{c}0 . \\
14 \\
60 \\
1\end{array}$ & $\begin{array}{c}0.52 \\
002\end{array}$ & $\begin{array}{c}0 . \\
00 \\
71 \\
6\end{array}$ & $\begin{array}{c}268 \\
0\end{array}$ & $\begin{array}{l}1 \\
0\end{array}$ & $\begin{array}{c}268 \\
8\end{array}$ & $\begin{array}{l}1 \\
0\end{array}$ & $\begin{array}{c}269 \\
9\end{array}$ & $\begin{array}{l}3 \\
0\end{array}$ & $\begin{array}{c}- \\
0.7 \\
0 \%\end{array}$ & $\begin{array}{l}2 \\
6 \\
8 \\
0\end{array}$ & $\begin{array}{l}1 \\
0\end{array}$ \\
\hline $\begin{array}{c}13 \\
\text { FR } \\
52 \\
- \\
72\end{array}$ & $\begin{array}{l}3 \\
5\end{array}$ & $\begin{array}{l}7 \\
2\end{array}$ & $\begin{array}{l}0 \\
5 \\
4 \\
9\end{array}$ & $\begin{array}{c}0.10 \\
218\end{array}$ & $\begin{array}{c}0 . \\
00 \\
27 \\
6\end{array}$ & $\begin{array}{l}4.02 \\
474\end{array}$ & $\begin{array}{c}0 . \\
09 \\
91 \\
8\end{array}$ & $\begin{array}{l}0.28 \\
566\end{array}$ & $\begin{array}{c}0 . \\
00 \\
57 \\
4\end{array}$ & $\begin{array}{c}166 \\
4\end{array}$ & $\begin{array}{l}2 \\
1\end{array}$ & $\begin{array}{c}163 \\
9\end{array}$ & $\begin{array}{l}2 \\
0\end{array}$ & $\begin{array}{c}162 \\
0\end{array}$ & $\begin{array}{l}2 \\
9\end{array}$ & $\begin{array}{l}2.7 \\
2 \%\end{array}$ & $\begin{array}{l}1 \\
6 \\
6 \\
4\end{array}$ & $\begin{array}{l}2 \\
1\end{array}$ \\
\hline $\begin{array}{c}13 \\
\text { FR } \\
52 \\
- \\
73 \\
\end{array}$ & 7 & $\begin{array}{l}2 \\
7\end{array}$ & $\begin{array}{l}0 \\
2 \\
6\end{array}$ & $\begin{array}{c}0.06 \\
077\end{array}$ & $\begin{array}{c}0 . \\
00 \\
93 \\
1\end{array}$ & $\begin{array}{c}0.63 \\
096\end{array}$ & $\begin{array}{c}0 . \\
09 \\
19 \\
5\end{array}$ & $\begin{array}{l}0.07 \\
530\end{array}$ & $\begin{array}{c}0 . \\
00 \\
35 \\
4\end{array}$ & 631 & $\begin{array}{l}3 \\
4 \\
8\end{array}$ & 497 & $\begin{array}{l}5 \\
7\end{array}$ & 468 & \begin{tabular}{l|}
2 \\
1
\end{tabular} & $\begin{array}{l}6.2 \\
0 \%\end{array}$ & $\begin{array}{l}4 \\
6 \\
8\end{array}$ & $\begin{array}{l}2 \\
1\end{array}$ \\
\hline 13 & 5 & 1 & 0 & 0.05 & 0. & 0.64 & 0. & 0.08 & 0. & 425 & 3 & 506 & 1 & 524 & 9 & - & 5 & 9 \\
\hline
\end{tabular}




\begin{tabular}{|c|c|c|c|c|c|c|c|c|c|c|c|c|c|c|c|c|c|c|}
\hline $\begin{array}{c}\text { FR } \\
52 \\
- \\
74\end{array}$ & 7 & $\begin{array}{l}8 \\
7\end{array}$ & $\begin{array}{l}3 \\
0\end{array}$ & 531 & $\begin{array}{c}00 \\
16 \\
8\end{array}$ & 644 & $\begin{array}{c}01 \\
80 \\
7\end{array}$ & 476 & $\begin{array}{c}00 \\
14 \\
5\end{array}$ & & 3 & & 1 & & & $\begin{array}{l}3.4 \\
4 \%\end{array}$ & $\begin{array}{l}2 \\
4\end{array}$ & \\
\hline $\begin{array}{c}13 \\
\text { FR } \\
52 \\
- \\
75\end{array}$ & $\begin{array}{l}2 \\
9\end{array}$ & $\begin{array}{l}9 \\
6\end{array}$ & $\begin{array}{l}0 \\
\dot{3} \\
0\end{array}$ & $\begin{array}{l}0.05 \\
399\end{array}$ & $\begin{array}{c}0 . \\
00 \\
39 \\
2\end{array}$ & $\begin{array}{c}0.59 \\
671\end{array}$ & $\begin{array}{c}0 . \\
03 \\
98 \\
0\end{array}$ & $\begin{array}{l}0.08 \\
016\end{array}$ & $\begin{array}{c}0 . \\
00 \\
27 \\
2\end{array}$ & 371 & $\begin{array}{l}9 \\
0\end{array}$ & 475 & $\begin{array}{l}2 \\
5\end{array}$ & $\begin{array}{l}497 \\
\end{array}$ & $\begin{array}{l}1 \\
6\end{array}$ & $\begin{array}{c}- \\
4.4 \\
3 \%\end{array}$ & $\begin{array}{l}4 \\
9 \\
7\end{array}$ & $\begin{array}{l}1 \\
6\end{array}$ \\
\hline $\begin{array}{c}13 \\
\text { FR } \\
52 \\
- \\
76\end{array}$ & $\begin{array}{l}7 \\
2\end{array}$ & $\begin{array}{l}3 \\
0 \\
5\end{array}$ & $\begin{array}{l}0 \\
2 \\
4\end{array}$ & $\begin{array}{l}0.05 \\
632\end{array}$ & $\begin{array}{c}0 . \\
00 \\
12 \\
5\end{array}$ & $\begin{array}{c}0.60 \\
399\end{array}$ & $\begin{array}{c}0 . \\
01 \\
23 \\
4\end{array}$ & $\begin{array}{l}0.07 \\
778\end{array}$ & $\begin{array}{c}0 . \\
00 \\
11 \\
2\end{array}$ & 465 & $\begin{array}{l}2 \\
2\end{array}$ & 480 & 8 & 483 & 7 & $\begin{array}{c}- \\
0.6 \\
2 \%\end{array}$ & $\begin{array}{l}4 \\
8 \\
3\end{array}$ & 7 \\
\hline $\begin{array}{c}13 \\
\text { FR } \\
52 \\
- \\
77\end{array}$ & $\begin{array}{l}1 \\
1 \\
8\end{array}$ & $\begin{array}{l}1 \\
4 \\
9\end{array}$ & $\begin{array}{l}0 \\
\dot{7} \\
9\end{array}$ & $\begin{array}{l}0.06 \\
077\end{array}$ & $\begin{array}{c}0 . \\
00 \\
16 \\
9\end{array}$ & $\begin{array}{c}0.81 \\
512\end{array}$ & $\begin{array}{c}0 . \\
02 \\
06 \\
5\end{array}$ & $\begin{array}{l}0.09 \\
728\end{array}$ & $\begin{array}{c}0 . \\
00 \\
16 \\
2\end{array}$ & 631 & $\begin{array}{l}2 \\
8\end{array}$ & 605 & $\begin{array}{l}1 \\
2\end{array}$ & 598 & $\begin{array}{l}1 \\
0\end{array}$ & $\begin{array}{l}1.1 \\
7 \%\end{array}$ & $\begin{array}{l}5 \\
9 \\
8\end{array}$ & $\begin{array}{l}1 \\
0\end{array}$ \\
\hline $\begin{array}{c}13 \\
\text { FR } \\
52 \\
- \\
78\end{array}$ & $\begin{array}{l}6 \\
6\end{array}$ & $\begin{array}{l}1 \\
0 \\
6\end{array}$ & $\begin{array}{l}0 \\
\dot{6} \\
3\end{array}$ & $\begin{array}{l}0.05 \\
850\end{array}$ & $\begin{array}{c}0 . \\
00 \\
22 \\
9\end{array}$ & $\begin{array}{c}0.62 \\
942\end{array}$ & $\begin{array}{c}0 . \\
02 \\
24 \\
3\end{array}$ & $\begin{array}{l}0.07 \\
804 \\
\end{array}$ & $\begin{array}{c}0 . \\
00 \\
16 \\
2\end{array}$ & 549 & $\begin{array}{l}4 \\
3\end{array}$ & 496 & $\begin{array}{l}1 \\
4\end{array}$ & 484 & $\begin{array}{l}1 \\
0\end{array}$ & $\begin{array}{l}2.4 \\
8 \%\end{array}$ & $\begin{array}{l}4 \\
8 \\
4\end{array}$ & $\begin{array}{l}1 \\
0\end{array}$ \\
\hline $\begin{array}{c}13 \\
\text { FR } \\
52 \\
- \\
79\end{array}$ & $\begin{array}{l}1 \\
5 \\
3\end{array}$ & $\begin{array}{l}3 \\
7 \\
2\end{array}$ & $\begin{array}{l}0 \\
\dot{4} \\
1\end{array}$ & $\begin{array}{l}0.05 \\
253\end{array}$ & $\begin{array}{c}0 . \\
00 \\
17 \\
2\end{array}$ & $\begin{array}{c}0.41 \\
809\end{array}$ & $\begin{array}{c}0 . \\
01 \\
25 \\
6\end{array}$ & $\begin{array}{l}0.05 \\
772\end{array}$ & $\begin{array}{c}0 . \\
00 \\
10 \\
2\end{array}$ & 309 & $\begin{array}{l}3 \\
8\end{array}$ & 355 & 9 & 362 & 6 & $\begin{array}{c}- \\
1.9 \\
3 \%\end{array}$ & $\begin{array}{l}3 \\
6 \\
2\end{array}$ & 6 \\
\hline $\begin{array}{c}13 \\
\text { FR } \\
52 \\
- \\
80 \\
\end{array}$ & $\begin{array}{l}1 \\
9 \\
9\end{array}$ & $\begin{array}{l}4 \\
4 \\
1\end{array}$ & $\begin{array}{l}0 \\
4 \\
5\end{array}$ & $\begin{array}{c}0.05 \\
508\end{array}$ & $\begin{array}{c}0 . \\
00 \\
12 \\
7\end{array}$ & $\begin{array}{l}0.42 \\
857\end{array}$ & $\begin{array}{c}0 . \\
00 \\
90 \\
2\end{array}$ & $\begin{array}{l}0.05 \\
643\end{array}$ & $\begin{array}{c}0 . \\
00 \\
08 \\
1\end{array}$ & 415 & $\begin{array}{l}2 \\
3\end{array}$ & 362 & 6 & 354 & 5 & $\begin{array}{l}2.2 \\
6 \%\end{array}$ & $\begin{array}{l}3 \\
5 \\
4\end{array}$ & 5 \\
\hline $\begin{array}{c}13 \\
\text { FR } \\
52 \\
- \\
81\end{array}$ & $\begin{array}{l}5 \\
5\end{array}$ & $\begin{array}{l}1 \\
0 \\
4\end{array}$ & $\begin{array}{l}0 \\
5 \\
5 \\
3\end{array}$ & $\begin{array}{l}0.06 \\
376\end{array}$ & $\begin{array}{c}0 . \\
00 \\
21 \\
6\end{array}$ & $\begin{array}{c}0.93 \\
269\end{array}$ & $\begin{array}{c}0 . \\
02 \\
88 \\
2\end{array}$ & $\begin{array}{l}0.10 \\
609\end{array}$ & $\begin{array}{c}0 . \\
00 \\
20 \\
5\end{array}$ & 734 & $\begin{array}{l}3 \\
4\end{array}$ & 669 & $\begin{array}{l}1 \\
5\end{array}$ & 650 & $\begin{array}{l}1 \\
2\end{array}$ & $\begin{array}{l}2.9 \\
2 \%\end{array}$ & $\begin{array}{l}6 \\
5 \\
0\end{array}$ & $\begin{array}{l}1 \\
2\end{array}$ \\
\hline $\begin{array}{c}13 \\
\text { FR } \\
52 \\
- \\
82\end{array}$ & $\begin{array}{l}4 \\
5\end{array}$ & $\begin{array}{l}1 \\
0 \\
4\end{array}$ & $\begin{array}{l}0 \\
4 \\
4\end{array}$ & $\begin{array}{l}0.08 \\
274\end{array}$ & $\begin{array}{c}0 . \\
00 \\
21 \\
5\end{array}$ & $\begin{array}{l}1.59 \\
118\end{array}$ & $\begin{array}{c}0 . \\
03 \\
71 \\
4\end{array}$ & $\begin{array}{l}0.13 \\
947\end{array}$ & $\begin{array}{c}0 . \\
00 \\
24 \\
4\end{array}$ & $\begin{array}{c}126 \\
3\end{array}$ & $\begin{array}{l}2 \\
2\end{array}$ & 967 & $\begin{array}{l}1 \\
5\end{array}$ & 842 & $\begin{array}{l}1 \\
4\end{array}$ & $\begin{array}{l}14 . \\
85 \\
\%\end{array}$ & & \\
\hline $\begin{array}{c}13 \\
\text { FR } \\
52 \\
- \\
83\end{array}$ & $\begin{array}{l}2 \\
0\end{array}$ & $\begin{array}{l}3 \\
5\end{array}$ & $\begin{array}{l}0 \\
\dot{5} \\
7\end{array}$ & $\begin{array}{l}0.12 \\
562\end{array}$ & $\begin{array}{c}0 . \\
00 \\
32 \\
0\end{array}$ & $\begin{array}{c}6.27 \\
592\end{array}$ & $\begin{array}{c}0 . \\
14 \\
68 \\
1\end{array}$ & $\begin{array}{l}0.36 \\
234\end{array}$ & $\begin{array}{c}0 . \\
00 \\
75 \\
0\end{array}$ & $\begin{array}{c}203 \\
8\end{array}$ & $\begin{array}{l}1 \\
8\end{array}$ & $\begin{array}{c}201 \\
5\end{array}$ & $\begin{array}{l}2 \\
0\end{array}$ & $\begin{array}{c}199 \\
3\end{array}$ & $\begin{array}{l}3 \\
5\end{array}$ & $\begin{array}{l}2.2 \\
6 \%\end{array}$ & $\begin{array}{l}2 \\
0 \\
3 \\
8\end{array}$ & $\begin{array}{l}1 \\
8\end{array}$ \\
\hline $\begin{array}{c}13 \\
\text { FR } \\
52 \\
- \\
84\end{array}$ & $\begin{array}{l}4 \\
3\end{array}$ & $\begin{array}{l}4 \\
1 \\
5\end{array}$ & $\begin{array}{l}0 \\
\dot{1} \\
0\end{array}$ & $\begin{array}{l}0.05 \\
895\end{array}$ & $\begin{array}{c}0 . \\
00 \\
11 \\
2\end{array}$ & $\begin{array}{c}0.71 \\
530\end{array}$ & $\begin{array}{c}0 . \\
01 \\
24 \\
3\end{array}$ & $\begin{array}{c}0.08 \\
800\end{array}$ & $\begin{array}{c}0 . \\
00 \\
11 \\
8\end{array}$ & 565 & $\begin{array}{l}1 \\
8\end{array}$ & 548 & 7 & 544 & 7 & $\begin{array}{l}0.7 \\
4 \%\end{array}$ & $\begin{array}{l}5 \\
4 \\
4\end{array}$ & 7 \\
\hline
\end{tabular}




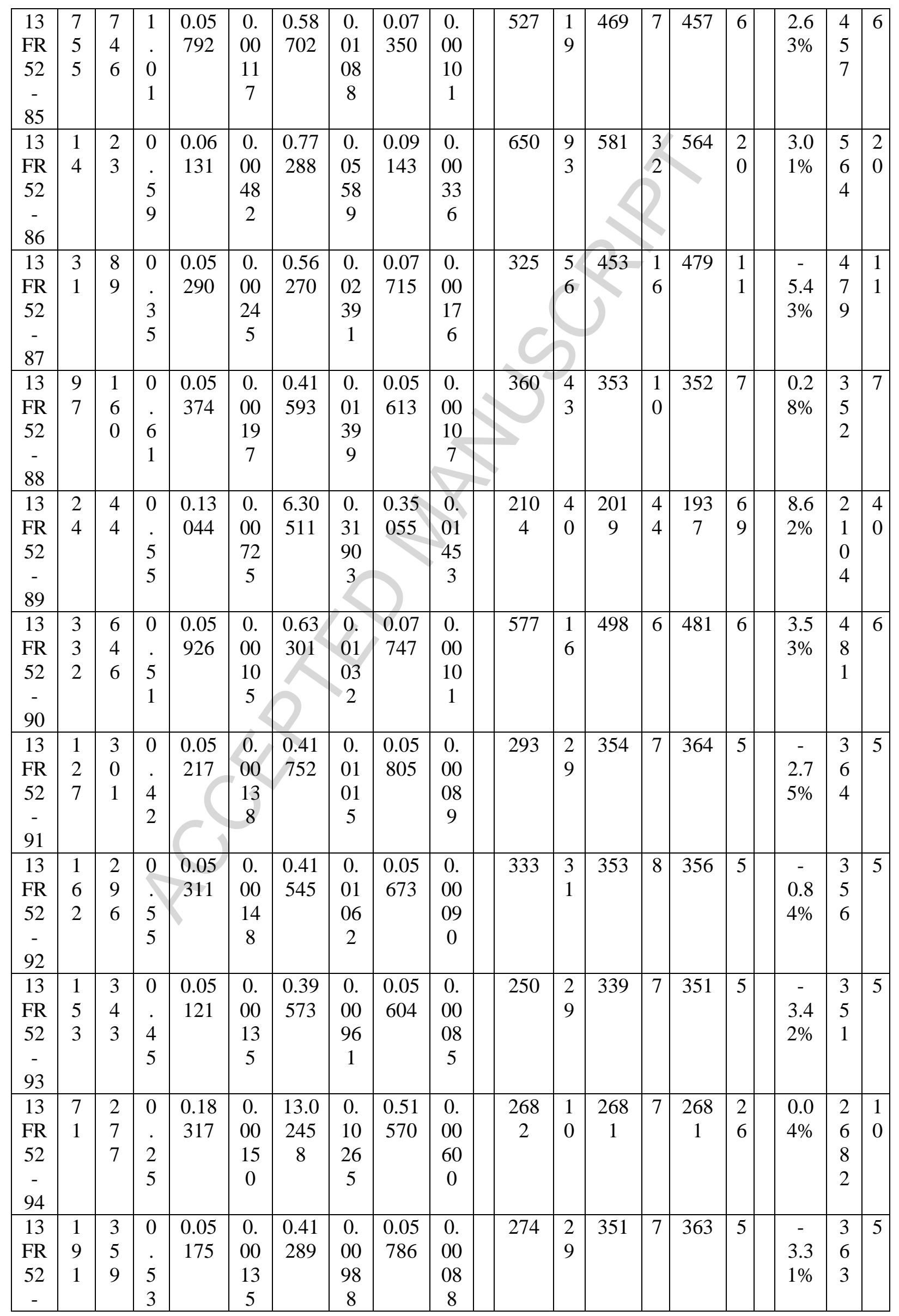




\begin{tabular}{|c|c|c|c|c|c|c|c|c|c|c|c|c|c|c|c|c|c|c|}
\hline 95 & & & & & & & & & & & & & & & & & & \\
\hline $\begin{array}{c}13 \\
\text { FR } \\
52 \\
- \\
96\end{array}$ & $\begin{array}{l}3 \\
3\end{array}$ & $\begin{array}{l}3 \\
6\end{array}$ & $\begin{array}{l}9 \\
2\end{array}$ & $\begin{array}{l}0.05 \\
905\end{array}$ & $\begin{array}{c}0 . \\
00 \\
40 \\
5\end{array}$ & $\begin{array}{c}0.64 \\
556\end{array}$ & $\begin{array}{c}0 . \\
04 \\
06 \\
2\end{array}$ & $\begin{array}{l}0.07 \\
929\end{array}$ & $\begin{array}{c}0 . \\
00 \\
25 \\
4\end{array}$ & 569 & $\begin{array}{l}8 \\
2\end{array}$ & 506 & $\begin{array}{l}2 \\
5\end{array}$ & 492 & $\begin{array}{l}1 \\
5\end{array}$ & $\begin{array}{l}2.8 \\
5 \%\end{array}$ & $\begin{array}{l}4 \\
9 \\
2\end{array}$ & $\begin{array}{l}1 \\
5\end{array}$ \\
\hline $\begin{array}{c}13 \\
\text { FR } \\
52 \\
- \\
97\end{array}$ & $\begin{array}{l}5 \\
2\end{array}$ & $\begin{array}{l}9 \\
1\end{array}$ & $\begin{array}{l}0 \\
5 \\
5 \\
7\end{array}$ & $\begin{array}{l}0.05 \\
897\end{array}$ & $\begin{array}{c}0 . \\
00 \\
23 \\
4\end{array}$ & $\begin{array}{c}0.71 \\
042\end{array}$ & $\begin{array}{c}0 . \\
02 \\
57 \\
1\end{array}$ & $\begin{array}{l}0.08 \\
737\end{array}$ & $\begin{array}{c}0 . \\
00 \\
18 \\
4\end{array}$ & 566 & $\begin{array}{l}4 \\
4\end{array}$ & 545 & $\begin{array}{l}1 \\
5\end{array}$ & 540 & $\begin{array}{l}1 \\
1\end{array}$ & $\begin{array}{l}0.9 \\
3 \%\end{array}$ & $\begin{array}{l}5 \\
4 \\
0\end{array}$ & $\begin{array}{l}1 \\
1\end{array}$ \\
\hline $\begin{array}{c}13 \\
\text { FR } \\
52 \\
- \\
98\end{array}$ & $\begin{array}{l}1 \\
2 \\
1\end{array}$ & $\begin{array}{l}2 \\
9 \\
6\end{array}$ & $\begin{array}{l}0 \\
4 \\
1\end{array}$ & $\begin{array}{l}0.05 \\
462\end{array}$ & $\begin{array}{c}0 . \\
00 \\
13 \\
8\end{array}$ & $\begin{array}{l}0.41 \\
969\end{array}$ & $\begin{array}{c}0 . \\
00 \\
97 \\
3\end{array}$ & $\begin{array}{l}0.05 \\
572\end{array}$ & $\begin{array}{c}0 . \\
00 \\
08 \\
4\end{array}$ & 397 & $\begin{array}{l}2 \\
7\end{array}$ & 356 & 7 & 350 & 5 & $\begin{array}{l}1.7 \\
1 \%\end{array}$ & $\begin{array}{l}3 \\
5 \\
0\end{array}$ & 5 \\
\hline $\begin{array}{c}13 \\
\text { FR } \\
52 \\
- \\
99\end{array}$ & $\begin{array}{l}4 \\
6\end{array}$ & $\begin{array}{l}1 \\
5 \\
1\end{array}$ & $\begin{array}{l}0 \\
3 \\
3 \\
1\end{array}$ & $\begin{array}{l}0.05 \\
744\end{array}$ & $\begin{array}{c}0 . \\
00 \\
16 \\
4\end{array}$ & $\begin{array}{l}0.62 \\
237\end{array}$ & $\begin{array}{c}0 . \\
01 \\
63 \\
1\end{array}$ & $\begin{array}{l}0.07 \\
857\end{array}$ & $\begin{array}{c}0 . \\
00 \\
13 \\
0\end{array}$ & 508 & $\begin{array}{l}3 \\
0\end{array}$ & 491 & $\begin{array}{l}1 \\
0\end{array}$ & 488 & 8 & $\begin{array}{l}0.6 \\
1 \%\end{array}$ & $\begin{array}{l}4 \\
8 \\
8\end{array}$ & 8 \\
\hline $\begin{array}{c}13 \\
\text { FR } \\
52 \\
- \\
10 \\
0\end{array}$ & $\begin{array}{l}5 \\
0\end{array}$ & $\begin{array}{l}2 \\
2 \\
8\end{array}$ & $\begin{array}{l}0 \\
2 \\
2\end{array}$ & $\begin{array}{l}0.05 \\
889\end{array}$ & $\begin{array}{c}0 . \\
00 \\
12 \\
5\end{array}$ & $\begin{array}{c}0.81 \\
588\end{array}$ & $\begin{array}{c}0 . \\
01 \\
59\end{array}$ & $\begin{array}{l}0.10 \\
048\end{array}$ & $\begin{array}{c}0 . \\
00 \\
14 \\
3\end{array}$ & 563 & $\begin{array}{l}2 \\
0\end{array}$ & 606 & 9 & 617 & 8 & $\begin{array}{c}- \\
1.7 \\
8 \%\end{array}$ & $\begin{array}{l}6 \\
1 \\
7\end{array}$ & 8 \\
\hline & & & & & & 8 & & & & & & & & & & & & \\
\hline $\begin{array}{c}\mathrm{M} \\
\mathrm{O} 1 \\
5- \\
01 \\
\end{array}$ & $\begin{array}{l}6 \\
4\end{array}$ & $\begin{array}{l}2 \\
1 \\
7\end{array}$ & $\begin{array}{l}0 \\
3 \\
0 \\
\end{array}$ & $\begin{array}{l}0.05 \\
993\end{array}$ & $\begin{array}{c}0 . \\
00 \\
17 \\
3 \\
\end{array}$ & $\begin{array}{l}0.07 \\
745\end{array}$ & $\begin{array}{c}0 . \\
00 \\
15 \\
9 \\
\end{array}$ & $\begin{array}{c}0.64 \\
010\end{array}$ & $\begin{array}{c}0 . \\
01 \\
69 \\
1 \\
\end{array}$ & 601 & $\begin{array}{l}2 \\
6\end{array}$ & 481 & $\begin{array}{l}1 \\
0\end{array}$ & 502 & $\begin{array}{l}1 \\
0\end{array}$ & $\begin{array}{l}4.3 \\
7 \%\end{array}$ & $\begin{array}{l}4 \\
8 \\
1\end{array}$ & $\begin{array}{l}1 \\
0\end{array}$ \\
\hline $\begin{array}{c}\mathrm{M} \\
\mathrm{O} 1 \\
5- \\
02\end{array}$ & $\begin{array}{l}2 \\
2 \\
8\end{array}$ & $\begin{array}{l}2 \\
4 \\
9\end{array}$ & $\begin{array}{l}0 \\
. \\
9 \\
2\end{array}$ & $\begin{array}{l}0.06 \\
350\end{array}$ & $\begin{array}{c}0 . \\
00 \\
13 \\
7 \\
\end{array}$ & $\begin{array}{l}0.09 \\
760\end{array}$ & $\begin{array}{c}0 . \\
00 \\
18 \\
5\end{array}$ & $\begin{array}{l}0.85 \\
453\end{array}$ & $\begin{array}{c}0 . \\
01 \\
67 \\
4\end{array}$ & 725 & $\begin{array}{l}1 \\
9\end{array}$ & 600 & $\begin{array}{l}1 \\
1\end{array}$ & 627 & 9 & $\begin{array}{l}4.5 \\
0 \%\end{array}$ & $\begin{array}{l}6 \\
0 \\
0\end{array}$ & $\begin{array}{l}1 \\
1\end{array}$ \\
\hline $\begin{array}{c}\mathrm{M} \\
\mathrm{O} 1 \\
5- \\
03\end{array}$ & $\begin{array}{l}1 \\
2 \\
1\end{array}$ & $\begin{array}{l}1 \\
8 \\
4\end{array}$ & $\begin{array}{l}0 \\
6 \\
6\end{array}$ & $\begin{array}{c}0.13 \\
145\end{array}$ & $\begin{array}{c}0 . \\
00 \\
19 \\
6\end{array}$ & $\begin{array}{l}0.38 \\
275\end{array}$ & $\begin{array}{c}0 . \\
00 \\
71 \\
4\end{array}$ & $\begin{array}{l}6.93 \\
720\end{array}$ & $\begin{array}{c}0 . \\
09 \\
47 \\
0\end{array}$ & $\begin{array}{c}211 \\
7\end{array}$ & $\begin{array}{l}1 \\
6\end{array}$ & $\begin{array}{c}208 \\
9\end{array}$ & $\begin{array}{l}3 \\
3\end{array}$ & $\begin{array}{c}210 \\
3\end{array}$ & $\begin{array}{l}1 \\
2\end{array}$ & $\begin{array}{l}1.3 \\
4 \%\end{array}$ & $\begin{array}{l}2 \\
1 \\
1 \\
7\end{array}$ & $\begin{array}{l}1 \\
6\end{array}$ \\
\hline $\begin{array}{c}\mathrm{M} \\
\mathrm{O} 1 \\
5- \\
04\end{array}$ & $\begin{array}{l}1 \\
1 \\
4\end{array}$ & $\begin{array}{l}3 \\
3 \\
9\end{array}$ & $\begin{array}{l}0 \\
3 \\
4\end{array}$ & $\begin{array}{c}0.05 \\
501\end{array}$ & $\begin{array}{c}0 . \\
00 \\
23 \\
9\end{array}$ & $\begin{array}{l}0.05 \\
384\end{array}$ & $\begin{array}{c}0 . \\
00 \\
13 \\
3\end{array}$ & $\begin{array}{l}0.40 \\
839\end{array}$ & $\begin{array}{c}0 . \\
01 \\
62 \\
1\end{array}$ & 413 & $\begin{array}{l}4 \\
7\end{array}$ & 338 & 8 & 348 & $\begin{array}{l}1 \\
2\end{array}$ & $\begin{array}{l}2.9 \\
6 \%\end{array}$ & $\begin{array}{l}3 \\
3 \\
8\end{array}$ & 8 \\
\hline $\begin{array}{c}\mathrm{M} \\
\mathrm{O} 1 \\
5- \\
05\end{array}$ & $\begin{array}{l}4 \\
1\end{array}$ & $\begin{array}{l}8 \\
0\end{array}$ & $\begin{array}{l}0 \\
5 \\
5 \\
2\end{array}$ & $\begin{array}{l}0.05 \\
973\end{array}$ & $\begin{array}{c}0 . \\
00 \\
34 \\
1\end{array}$ & $\begin{array}{c}0.08 \\
700\end{array}$ & $\begin{array}{c}0 . \\
00 \\
26 \\
3\end{array}$ & $\begin{array}{l}0.71 \\
657\end{array}$ & $\begin{array}{c}0 . \\
03 \\
76 \\
2\end{array}$ & 594 & $\begin{array}{l}6 \\
3\end{array}$ & 538 & $\begin{array}{l}1 \\
6\end{array}$ & 549 & $\begin{array}{l}2 \\
2\end{array}$ & $\begin{array}{l}2.0 \\
4 \%\end{array}$ & $\begin{array}{l}5 \\
3 \\
8\end{array}$ & $\begin{array}{l}1 \\
6\end{array}$ \\
\hline $\begin{array}{c}\mathrm{M} \\
\mathrm{O} 1 \\
5- \\
06\end{array}$ & $\begin{array}{l}1 \\
2 \\
5\end{array}$ & $\begin{array}{l}3 \\
7 \\
0\end{array}$ & $\begin{array}{l}0 \\
3 \\
4\end{array}$ & $\begin{array}{l}0.06 \\
385\end{array}$ & $\begin{array}{c}0 . \\
00 \\
14 \\
4\end{array}$ & $\begin{array}{c}0.10 \\
185\end{array}$ & $\begin{array}{c}0 . \\
00 \\
19 \\
6\end{array}$ & $\begin{array}{c}0.89 \\
669\end{array}$ & $\begin{array}{c}0 . \\
01 \\
83 \\
2\end{array}$ & 737 & $\begin{array}{l}1 \\
9\end{array}$ & 625 & $\begin{array}{l}1 \\
1\end{array}$ & 650 & $\begin{array}{l}1 \\
0\end{array}$ & $\begin{array}{l}4.0 \\
0 \%\end{array}$ & $\begin{array}{l}6 \\
2 \\
5\end{array}$ & $\begin{array}{l}1 \\
1\end{array}$ \\
\hline $\begin{array}{l}\mathrm{M} \\
\mathrm{O} 1\end{array}$ & $\begin{array}{l}3 \\
9 \\
\end{array}$ & $\begin{array}{l}8 \\
6\end{array}$ & 0 & $\begin{array}{c}0.06 \\
668\end{array}$ & $\begin{array}{c}0 . \\
00\end{array}$ & $\begin{array}{c}0.06 \\
594\end{array}$ & $\begin{array}{c}0 . \\
00\end{array}$ & $\begin{array}{l}0.60 \\
627\end{array}$ & $\begin{array}{c}0 . \\
02\end{array}$ & 828 & $\begin{array}{l}4 \\
6\end{array}$ & 412 & $\begin{array}{l}1 \\
1\end{array}$ & 481 & $\begin{array}{l}1 \\
6\end{array}$ & $\begin{array}{l}16 . \\
75\end{array}$ & & \\
\hline
\end{tabular}




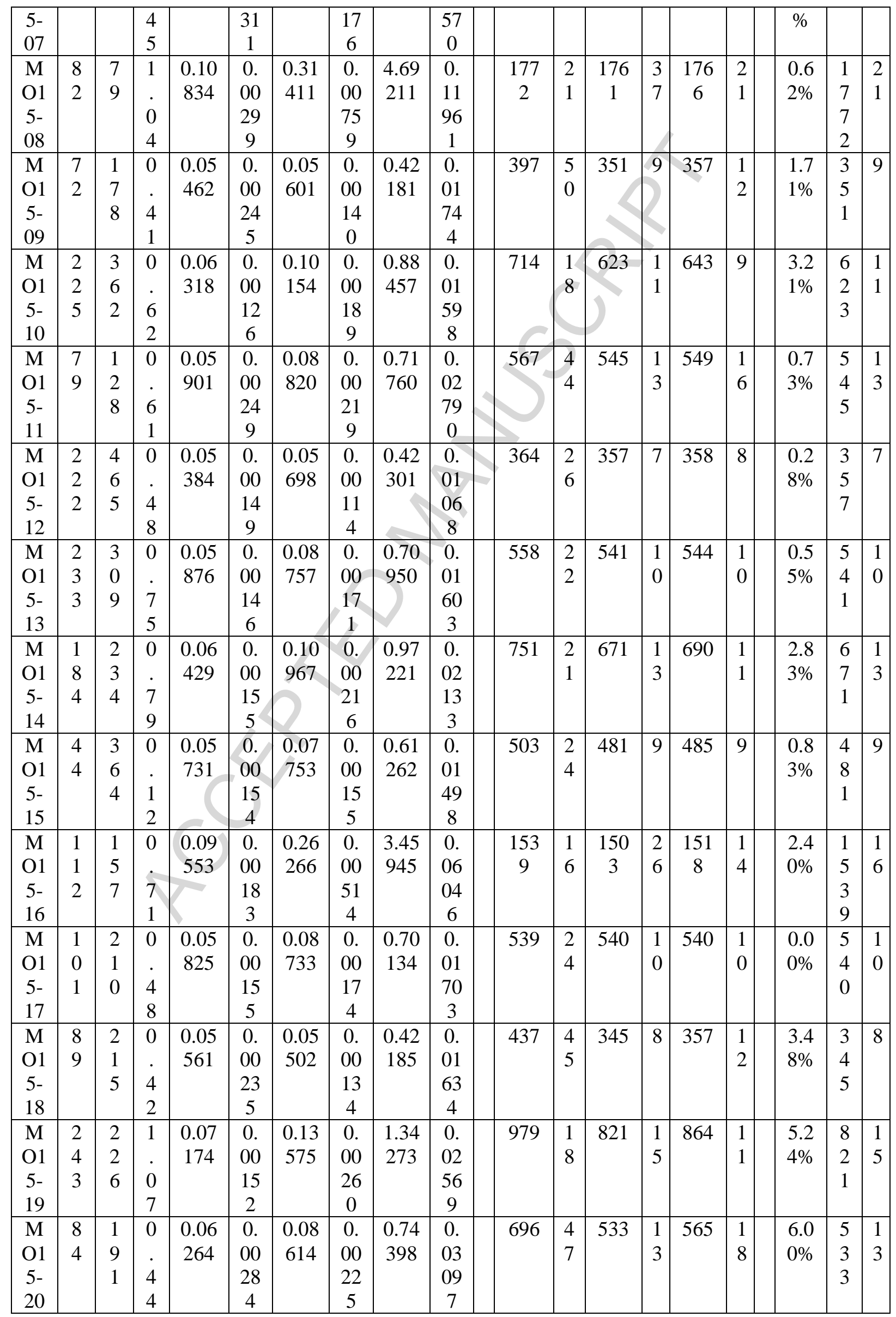




\begin{tabular}{|c|c|c|c|c|c|c|c|c|c|c|c|c|c|c|c|c|c|c|}
\hline $\begin{array}{c}\mathrm{M} \\
\mathrm{O} 1 \\
5- \\
21 \\
\end{array}$ & \begin{tabular}{|l|}
3 \\
4 \\
8
\end{tabular} & $\begin{array}{l}3 \\
8 \\
4\end{array}$ & $\begin{array}{l}9 \\
0 \\
\end{array}$ & $\begin{array}{c}0.06 \\
131\end{array}$ & $\begin{array}{c}0 . \\
00 \\
12 \\
1\end{array}$ & $\begin{array}{l}0.09 \\
229\end{array}$ & $\begin{array}{c}0 . \\
00 \\
17 \\
0\end{array}$ & $\begin{array}{c}0.78 \\
005\end{array}$ & $\begin{array}{c}0 . \\
01 \\
39 \\
8\end{array}$ & 650 & $\begin{array}{l}1 \\
8\end{array}$ & 569 & $\begin{array}{l}1 \\
0\end{array}$ & 586 & 8 & $\begin{array}{l}2.9 \\
9 \%\end{array}$ & $\begin{array}{l}5 \\
6 \\
9\end{array}$ & $\begin{array}{l}1 \\
0\end{array}$ \\
\hline $\begin{array}{c}\mathrm{M} \\
\mathrm{O} 1 \\
5- \\
22 \\
\end{array}$ & \begin{tabular}{l|}
3 \\
0
\end{tabular} & $\begin{array}{l}5 \\
0 \\
4\end{array}$ & $\begin{array}{l}0 \\
. \\
0 \\
6\end{array}$ & $\begin{array}{c}0.18 \\
408\end{array}$ & $\begin{array}{c}0 . \\
00 \\
23 \\
5\end{array}$ & $\begin{array}{l}0.51 \\
470\end{array}$ & $\begin{array}{c}0 . \\
00 \\
93 \\
5\end{array}$ & $\begin{array}{c}13.0 \\
622 \\
8\end{array}$ & $\begin{array}{c}0 . \\
15 \\
34 \\
8\end{array}$ & $\begin{array}{c}269 \\
0\end{array}$ & $\begin{array}{l}1 \\
5\end{array}$ & $\begin{array}{c}267 \\
7\end{array}$ & 4 & $\begin{array}{c}268 \\
4\end{array}$ & $\begin{array}{l}1 \\
1\end{array}$ & $\begin{array}{l}0.4 \\
9 \%\end{array}$ & $\begin{array}{l}2 \\
6 \\
9 \\
0 \\
\end{array}$ & $\begin{array}{l}1 \\
5\end{array}$ \\
\hline $\begin{array}{c}\mathrm{M} \\
\mathrm{O} 1 \\
5- \\
23\end{array}$ & \begin{tabular}{l|}
1 \\
2 \\
9
\end{tabular} & $\begin{array}{l}2 \\
8 \\
3\end{array}$ & $\begin{array}{l}0 \\
4 \\
6\end{array}$ & $\begin{array}{l}0.05 \\
395\end{array}$ & $\begin{array}{c}0 . \\
00 \\
19 \\
4\end{array}$ & $\begin{array}{l}0.05 \\
300\end{array}$ & $\begin{array}{c}0 . \\
00 \\
11 \\
7\end{array}$ & $\begin{array}{l}0.39 \\
421\end{array}$ & $\begin{array}{c}0 . \\
01 \\
30 \\
5\end{array}$ & 369 & $\begin{array}{l}3 \\
8\end{array}$ & & 7 & 337 & $\begin{array}{l}1 \\
0\end{array}$ & $\begin{array}{l}1.2 \\
0 \%\end{array}$ & $\begin{array}{l}3 \\
3 \\
3\end{array}$ & 7 \\
\hline $\begin{array}{c}\mathrm{M} \\
\mathrm{O} 1 \\
5- \\
24 \\
\end{array}$ & $\begin{array}{l}1 \\
1 \\
9\end{array}$ & $\begin{array}{l}3 \\
6 \\
0\end{array}$ & $\begin{array}{l}3 \\
3 \\
\end{array}$ & $\begin{array}{c}0.06 \\
138\end{array}$ & $\begin{array}{c}0 . \\
00 \\
14 \\
4 \\
\end{array}$ & $\begin{array}{c}0.10 \\
423\end{array}$ & $\begin{array}{c}0 . \\
00 \\
20 \\
2 \\
\end{array}$ & $\begin{array}{l}0.88 \\
205\end{array}$ & $\begin{array}{c}0 . \\
01 \\
87 \\
8 \\
\end{array}$ & 653 & $\begin{array}{l}2 \\
0\end{array}$ & 639 & $\begin{array}{l}1 \\
2\end{array}$ & 642 & $\begin{array}{l}1 \\
0\end{array}$ & $\begin{array}{l}0.4 \\
7 \%\end{array}$ & $\begin{array}{l}6 \\
3 \\
9\end{array}$ & $\begin{array}{l}1 \\
2\end{array}$ \\
\hline $\begin{array}{l}\mathrm{M} \\
\mathrm{O} 1 \\
5- \\
25\end{array}$ & $\begin{array}{l}1 \\
2 \\
2\end{array}$ & $\begin{array}{l}2 \\
5 \\
3\end{array}$ & $\begin{array}{l}0 \\
4 \\
8 \\
\end{array}$ & $\begin{array}{c}0.06 \\
045\end{array}$ & $\begin{array}{c}0 . \\
00 \\
16 \\
3 \\
\end{array}$ & $\begin{array}{r}0.09 \\
657\end{array}$ & $\begin{array}{c}0 . \\
00 \\
19 \\
6\end{array}$ & $\begin{array}{c}0.80 \\
480\end{array}$ & $\begin{array}{c}0 . \\
01 \\
98 \\
4\end{array}$ & 620 & $\begin{array}{l}2 \\
4\end{array}$ & 594 & $\begin{array}{l}1 \\
2\end{array}$ & 600 & $\begin{array}{l}1 \\
1\end{array}$ & $\begin{array}{l}1.0 \\
1 \%\end{array}$ & $\begin{array}{l}5 \\
9 \\
4\end{array}$ & $\begin{array}{l}1 \\
2\end{array}$ \\
\hline $\begin{array}{c}\mathrm{M} \\
\mathrm{O} 1 \\
5- \\
26\end{array}$ & $\begin{array}{l}1 \\
6 \\
3\end{array}$ & $\begin{array}{l}2 \\
5 \\
1\end{array}$ & $\begin{array}{l}6 \\
5\end{array}$ & $\begin{array}{l}0.05 \\
389\end{array}$ & $\begin{array}{c}0 . \\
00 \\
20 \\
0\end{array}$ & $\begin{array}{l}0.05 \\
467\end{array}$ & $\begin{array}{c}0 . \\
00 \\
12 \\
3\end{array}$ & $\begin{array}{c}0.40 \\
615\end{array}$ & $\begin{array}{c}0 . \\
01 \\
38 \\
1\end{array}$ & 366 & $\begin{array}{l}3 \\
9\end{array}$ & 343 & 8 & 346 & $\begin{array}{l}1 \\
0\end{array}$ & $\begin{array}{l}0.8 \\
7 \%\end{array}$ & $\begin{array}{l}3 \\
4 \\
3\end{array}$ & 8 \\
\hline $\begin{array}{c}\mathrm{M} \\
\mathrm{O} 1 \\
5- \\
27 \\
\end{array}$ & $\begin{array}{l}5 \\
6\end{array}$ & $\begin{array}{l}8 \\
5\end{array}$ & $\begin{array}{l}0 \\
6 \\
5 \\
\end{array}$ & $\begin{array}{c}0.10 \\
961\end{array}$ & $\begin{array}{c}0 . \\
00 \\
40 \\
8 \\
\end{array}$ & $\begin{array}{l}0.31 \\
582\end{array}$ & $\begin{array}{c}0 . \\
00 \\
93 \\
5\end{array}$ & $\begin{array}{r}4.77 \\
225\end{array}$ & $\begin{array}{c}0 . \\
16 \\
46 \\
9 \\
\end{array}$ & $\begin{array}{c}179 \\
3\end{array}$ & $\begin{array}{l}2 \\
8\end{array}$ & $\begin{array}{c}176 \\
9\end{array}$ & $\begin{array}{l}4 \\
6\end{array}$ & $\begin{array}{c}178 \\
0\end{array}$ & $\begin{array}{l}2 \\
9\end{array}$ & $\begin{array}{l}1.3 \\
6 \%\end{array}$ & $\begin{array}{l}1 \\
7 \\
9 \\
3 \\
\end{array}$ & $\begin{array}{l}2 \\
8\end{array}$ \\
\hline $\begin{array}{c}\mathrm{M} \\
\mathrm{O} 1 \\
5- \\
28\end{array}$ & $\begin{array}{l}1 \\
7 \\
3\end{array}$ & $\begin{array}{l}3 \\
4 \\
1\end{array}$ & $\begin{array}{l}0 \\
\dot{5} \\
1\end{array}$ & $\begin{array}{c}0.05 \\
537\end{array}$ & $\begin{array}{c}0 . \\
00 \\
19 \\
3\end{array}$ & $\begin{array}{l}0.05 \\
264\end{array}$ & $\begin{array}{c}0 . \\
00 \\
11 \\
6\end{array}$ & $\begin{array}{c}0.40 \\
186\end{array}$ & $\begin{array}{c}0 . \\
01 \\
28 \\
4\end{array}$ & 427 & $\begin{array}{l}3 \\
5\end{array}$ & 331 & 7 & 343 & 9 & $\begin{array}{l}3.6 \\
3 \%\end{array}$ & $\begin{array}{l}3 \\
3 \\
1\end{array}$ & 7 \\
\hline $\begin{array}{c}\text { M } \\
\text { O1 } \\
5- \\
29\end{array}$ & $\begin{array}{l}2 \\
2 \\
0\end{array}$ & $\begin{array}{l}4 \\
6 \\
2\end{array}$ & $\begin{array}{l}4 \\
8\end{array}$ & $\begin{array}{l}0.05 \\
600\end{array}$ & $\begin{array}{c}0 . \\
00 \\
39 \\
3\end{array}$ & $\begin{array}{c}0.05 \\
080\end{array}$ & $\begin{array}{c}0 . \\
00 \\
17 \\
7\end{array}$ & $\begin{array}{l}0.39 \\
223\end{array}$ & $\begin{array}{c}0 . \\
02 \\
51 \\
5\end{array}$ & 452 & $\begin{array}{l}8 \\
2\end{array}$ & 319 & 1 & 336 & $\begin{array}{l}1 \\
8\end{array}$ & $\begin{array}{l}5.3 \\
3 \%\end{array}$ & $\begin{array}{l}3 \\
1 \\
9\end{array}$ & $\begin{array}{l}1 \\
1\end{array}$ \\
\hline $\begin{array}{c}\mathrm{M} \\
\mathrm{O} 1 \\
5- \\
30\end{array}$ & $\begin{array}{l}1 \\
1 \\
8\end{array}$ & $\begin{array}{l}9 \\
7\end{array}$ & $\begin{array}{l}1 \\
2 \\
2\end{array}$ & $\begin{array}{l}0.11 \\
749\end{array}$ & $\begin{array}{c}0 . \\
00 \\
22 \\
2\end{array}$ & $\begin{array}{l}0.25 \\
953\end{array}$ & $\begin{array}{c}0 . \\
00 \\
51 \\
4\end{array}$ & $\begin{array}{l}4.20 \\
363\end{array}$ & $\begin{array}{c}0 . \\
07 \\
13 \\
6\end{array}$ & $\begin{array}{c}183 \\
7\end{array}$ & $\begin{array}{l}5 \\
8\end{array}$ & $\begin{array}{c}147 \\
8\end{array}$ & $\begin{array}{l}2 \\
7\end{array}$ & $\begin{array}{c}163 \\
2\end{array}$ & $\begin{array}{l}2 \\
0\end{array}$ & $\begin{array}{l}24 . \\
29 \\
\%\end{array}$ & & \\
\hline $\begin{array}{c}\mathrm{M} \\
\mathrm{O} 1 \\
5- \\
31\end{array}$ & $\begin{array}{l}8 \\
4\end{array}$ & $\begin{array}{l}2 \\
2 \\
2\end{array}$ & $\begin{array}{l}3 \\
8 \\
\end{array}$ & $\begin{array}{l}0.05 \\
970\end{array}$ & $\begin{array}{c}0 . \\
00 \\
16 \\
5\end{array}$ & $\begin{array}{l}0.07 \\
905\end{array}$ & $\begin{array}{c}0 . \\
00 \\
16 \\
1\end{array}$ & $\begin{array}{l}0.65 \\
064\end{array}$ & $\begin{array}{c}0 . \\
01 \\
63 \\
4\end{array}$ & 593 & $\begin{array}{l}2 \\
5\end{array}$ & 490 & $\begin{array}{l}1 \\
0\end{array}$ & 509 & $\begin{array}{l}1 \\
0\end{array}$ & $\begin{array}{l}3.8 \\
8 \%\end{array}$ & $\begin{array}{l}4 \\
9 \\
0\end{array}$ & $\begin{array}{l}1 \\
0\end{array}$ \\
\hline $\begin{array}{c}\mathrm{M} \\
\mathrm{O} 1 \\
5- \\
32 \\
\end{array}$ & $\begin{array}{l}1 \\
7 \\
0\end{array}$ & $\begin{array}{l}4 \\
5 \\
6\end{array}$ & $\begin{array}{l}0 \\
3 \\
7 \\
\end{array}$ & $\begin{array}{l}0.05 \\
202\end{array}$ & $\begin{array}{c}0 . \\
00 \\
13 \\
4 \\
\end{array}$ & $\begin{array}{l}0.05 \\
452\end{array}$ & $\begin{array}{c}0 . \\
00 \\
10 \\
5\end{array}$ & $\begin{array}{l}0.39 \\
095\end{array}$ & $\begin{array}{c}0 . \\
00 \\
91 \\
9 \\
\end{array}$ & 286 & $\begin{array}{l}2 \\
4\end{array}$ & 342 & 6 & 335 & 7 & $\begin{array}{c}- \\
2.0 \\
5 \%\end{array}$ & $\begin{array}{l}3 \\
4 \\
2\end{array}$ & 6 \\
\hline $\begin{array}{c}\mathrm{M} \\
\mathrm{O} 1 \\
5- \\
33\end{array}$ & $\begin{array}{l}5 \\
4\end{array}$ & $\begin{array}{l}1 \\
9 \\
4\end{array}$ & $\begin{array}{l}0 \\
2 \\
8\end{array}$ & $\begin{array}{l}0.05 \\
759\end{array}$ & $\begin{array}{c}0 . \\
00 \\
20 \\
2\end{array}$ & $\begin{array}{l}0.08 \\
624\end{array}$ & $\begin{array}{c}0 . \\
00 \\
19 \\
4\end{array}$ & $\begin{array}{l}0.68 \\
467\end{array}$ & $\begin{array}{c}0 . \\
02 \\
19 \\
9\end{array}$ & 514 & $\begin{array}{l}3 \\
5\end{array}$ & 533 & $\begin{array}{l}1 \\
2\end{array}$ & 530 & $\begin{array}{l}1 \\
3\end{array}$ & $\begin{array}{c}- \\
0.5 \\
6 \%\end{array}$ & $\begin{array}{l}5 \\
3 \\
3\end{array}$ & $\begin{array}{l}1 \\
2\end{array}$ \\
\hline $\begin{array}{l}\mathrm{M} \\
\mathrm{O} 1 \\
\end{array}$ & $\begin{array}{l}1 \\
0 \\
\end{array}$ & $\begin{array}{l}2 \\
3 \\
\end{array}$ & 0 & $\begin{array}{l}0.05 \\
673 \\
\end{array}$ & $\begin{array}{c}0 . \\
00\end{array}$ & $\begin{array}{l}0.05 \\
423 \\
\end{array}$ & $\begin{array}{c}0 . \\
00\end{array}$ & $\begin{array}{c}0.42 \\
407 \\
\end{array}$ & $\begin{array}{c}0 . \\
01\end{array}$ & 481 & $\begin{array}{l}3 \\
3 \\
\end{array}$ & 340 & 7 & 359 & 9 & $\begin{array}{l}5.5 \\
9 \%\end{array}$ & $\begin{array}{l}3 \\
4 \\
\end{array}$ & 7 \\
\hline
\end{tabular}




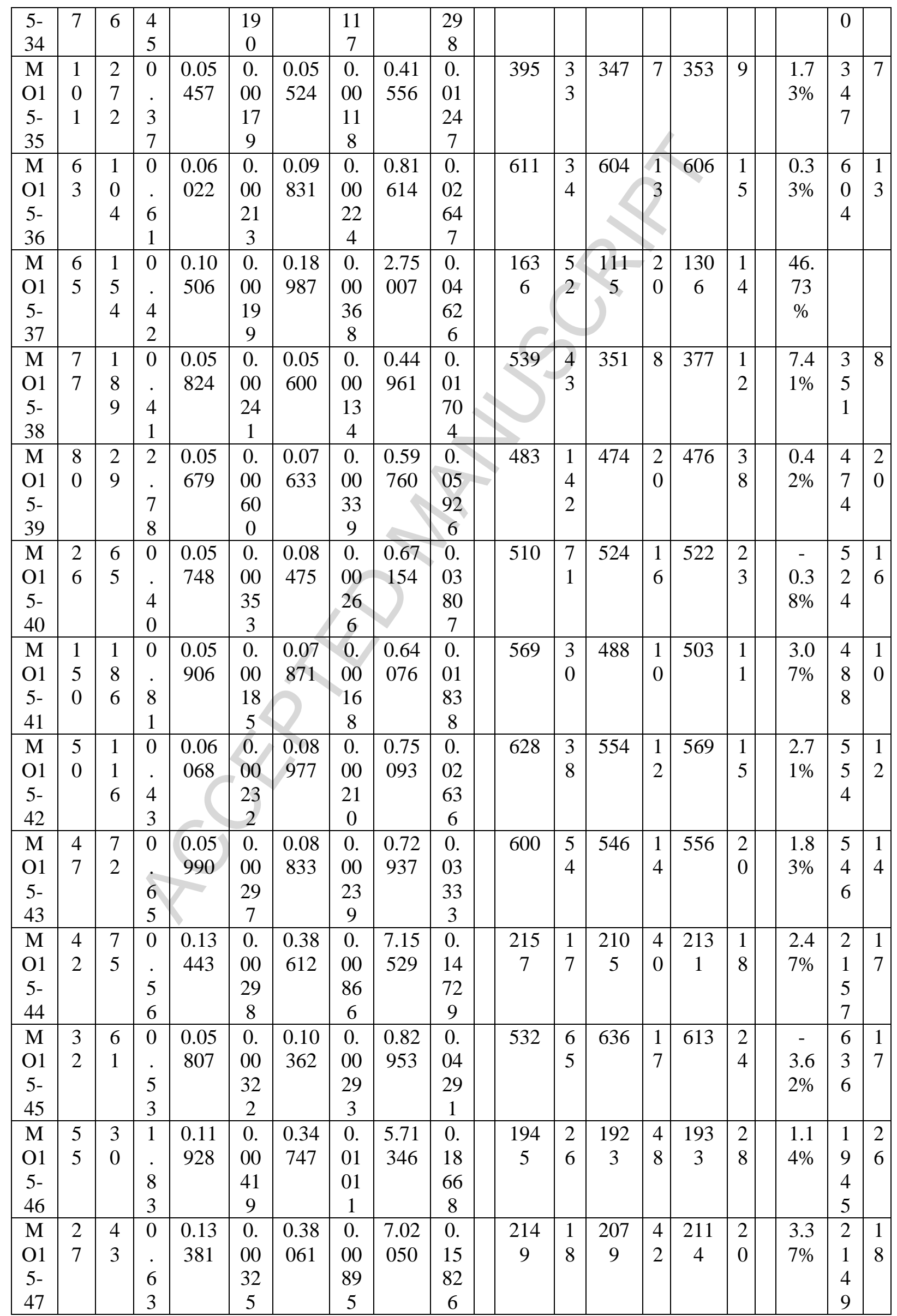




\begin{tabular}{|c|c|c|c|c|c|c|c|c|c|c|c|c|c|c|c|c|c|c|}
\hline $\begin{array}{c}\mathrm{M} \\
\mathrm{O} 1 \\
5- \\
48\end{array}$ & $\begin{array}{l}1 \\
3 \\
8\end{array}$ & $\begin{array}{l}2 \\
6 \\
7\end{array}$ & $\begin{array}{l}5 \\
2\end{array}$ & $\begin{array}{l}0.05 \\
720\end{array}$ & $\begin{array}{c}0 . \\
00 \\
21 \\
4\end{array}$ & $\begin{array}{l}0.07 \\
486\end{array}$ & $\begin{array}{c}0 . \\
00 \\
17 \\
2\end{array}$ & $\begin{array}{c}0.59 \\
030\end{array}$ & $\begin{array}{c}0 . \\
02 \\
02 \\
9\end{array}$ & 499 & $\begin{array}{l}3 \\
8\end{array}$ & 465 & $\begin{array}{l}1 \\
0\end{array}$ & 471 & $\begin{array}{l}1 \\
3\end{array}$ & $\begin{array}{l}1.2 \\
9 \%\end{array}$ & $\begin{array}{l}4 \\
6 \\
5\end{array}$ & $\begin{array}{l}1 \\
0\end{array}$ \\
\hline $\begin{array}{c}\mathrm{M} \\
\mathrm{O} 1 \\
5- \\
49\end{array}$ & $\begin{array}{l}4 \\
1\end{array}$ & $\begin{array}{l}1 \\
0 \\
4\end{array}$ & $\begin{array}{l}0 \\
. \\
3 \\
9\end{array}$ & $\begin{array}{c}0.06 \\
861\end{array}$ & $\begin{array}{c}0 . \\
00 \\
29 \\
1 \\
\end{array}$ & $\begin{array}{l}0.07 \\
342\end{array}$ & $\begin{array}{c}0 . \\
00 \\
18 \\
9 \\
\end{array}$ & $\begin{array}{l}0.69 \\
446\end{array}$ & $\begin{array}{c}0 . \\
02 \\
66 \\
2 \\
\end{array}$ & 887 & $\begin{array}{l}4 \\
0\end{array}$ & 457 & $\begin{array}{l}1 \\
1\end{array}$ & 535 & $\begin{array}{l}1 \\
6\end{array}$ & $\begin{array}{l}17 . \\
07 \\
\%\end{array}$ & & \\
\hline $\begin{array}{c}\mathrm{M} \\
\mathrm{O} 1 \\
5- \\
50\end{array}$ & $\begin{array}{l}1 \\
4 \\
0\end{array}$ & $\begin{array}{l}1 \\
9 \\
0\end{array}$ & $\begin{array}{l}0 \\
. \\
7 \\
4\end{array}$ & $\begin{array}{l}0.05 \\
426\end{array}$ & $\begin{array}{c}0 . \\
00 \\
23 \\
1\end{array}$ & $\begin{array}{c}0.06 \\
067\end{array}$ & $\begin{array}{c}0 . \\
00 \\
14 \\
5\end{array}$ & $\begin{array}{l}0.45 \\
383\end{array}$ & $\begin{array}{c}0 . \\
01 \\
78 \\
6\end{array}$ & 382 & $\begin{array}{l}4 \\
8\end{array}$ & & 9 & 380 & $\begin{array}{l}1 \\
2\end{array}$ & $\begin{array}{l}0.0 \\
0 \%\end{array}$ & $\begin{array}{l}3 \\
8 \\
0\end{array}$ & 9 \\
\hline $\begin{array}{c}\mathrm{M} \\
\mathrm{O} 1 \\
5- \\
51 \\
\end{array}$ & $\begin{array}{l}5 \\
7\end{array}$ & $\begin{array}{l}7 \\
0\end{array}$ & $\begin{array}{l}8 \\
1 \\
\end{array}$ & $\begin{array}{l}0.05 \\
964\end{array}$ & $\begin{array}{c}0 . \\
00 \\
38 \\
9 \\
\end{array}$ & $\begin{array}{l}0.09 \\
243\end{array}$ & $\begin{array}{c}0 . \\
00 \\
31 \\
4 \\
\end{array}$ & $\begin{array}{l}0.75 \\
991\end{array}$ & $\begin{array}{c}0 . \\
04 \\
54 \\
8 \\
\end{array}$ & 591 & $\begin{array}{l}7 \\
3\end{array}$ & 570 & $\begin{array}{l}1 \\
9\end{array}$ & 574 & $\begin{array}{l}2 \\
6\end{array}$ & $\begin{array}{l}0.7 \\
0 \%\end{array}$ & $\begin{array}{l}5 \\
7 \\
0\end{array}$ & $\begin{array}{l}1 \\
9\end{array}$ \\
\hline $\begin{array}{l}\mathrm{M} \\
\mathrm{O} 1 \\
5- \\
52\end{array}$ & $\begin{array}{l}3 \\
3\end{array}$ & $\begin{array}{l}6 \\
5\end{array}$ & $\begin{array}{l}0 \\
5 \\
5 \\
1\end{array}$ & $\begin{array}{c}0.13 \\
010\end{array}$ & $\begin{array}{c}0 . \\
00 \\
27 \\
4\end{array}$ & $\begin{array}{l}0.37 \\
915\end{array}$ & $\begin{array}{c}0 . \\
00 \\
82 \\
2\end{array}$ & $\begin{array}{l}6.79 \\
973\end{array}$ & $\begin{array}{c}0 . \\
13 \\
23 \\
6\end{array}$ & $\begin{array}{c}209 \\
9\end{array}$ & $\begin{array}{l}1 \\
7\end{array}$ & $\begin{array}{c}207 \\
2\end{array}$ & $\begin{array}{l}3 \\
8\end{array}$ & $\begin{array}{c}208 \\
6\end{array}$ & $\begin{array}{l}1 \\
7\end{array}$ & $\begin{array}{l}1.3 \\
0 \%\end{array}$ & $\begin{array}{l}2 \\
0 \\
9 \\
9 \\
\end{array}$ & $\begin{array}{l}1 \\
7\end{array}$ \\
\hline $\begin{array}{c}\mathrm{M} \\
\mathrm{O} 1 \\
5- \\
53\end{array}$ & $\begin{array}{l}7 \\
7\end{array}$ & $\begin{array}{l}1 \\
7 \\
5\end{array}$ & $\begin{array}{l}4 \\
4\end{array}$ & $\begin{array}{c}0.06 \\
013\end{array}$ & $\begin{array}{c}0 . \\
00 \\
21 \\
9\end{array}$ & $\begin{array}{r}0.07 \\
655\end{array}$ & $\begin{array}{c}0 . \\
00 \\
17 \\
5\end{array}$ & $\begin{array}{l}0.63 \\
453\end{array}$ & $\begin{array}{c}0 . \\
02 \\
11 \\
9\end{array}$ & 608 & $\begin{array}{l}3 \\
6\end{array}$ & 475 & $\begin{array}{l}1 \\
0\end{array}$ & 499 & $\begin{array}{l}1 \\
3\end{array}$ & $\begin{array}{l}5.0 \\
5 \%\end{array}$ & $\begin{array}{l}4 \\
7 \\
5\end{array}$ & $\begin{array}{l}1 \\
0\end{array}$ \\
\hline $\begin{array}{c}\mathrm{M} \\
\mathrm{O} 1 \\
5- \\
54\end{array}$ & $\begin{array}{l}1 \\
7 \\
5\end{array}$ & $\begin{array}{l}2 \\
3 \\
5\end{array}$ & $\begin{array}{l}0 \\
. \\
7 \\
5\end{array}$ & $\begin{array}{l}0.05 \\
953\end{array}$ & $\begin{array}{c}0 . \\
00 \\
19 \\
9 \\
\end{array}$ & $\begin{array}{l}0.09 \\
346\end{array}$ & $\begin{array}{c}0 . \\
00 \\
20 \\
8\end{array}$ & $\begin{array}{l}0.76 \\
695\end{array}$ & $\begin{array}{c}0 . \\
02 \\
34 \\
0 \\
\end{array}$ & 587 & $\begin{array}{l}3 \\
2\end{array}$ & 576 & $\begin{array}{l}1 \\
2\end{array}$ & 578 & $\begin{array}{l}1 \\
3\end{array}$ & $\begin{array}{l}0.3 \\
5 \%\end{array}$ & $\begin{array}{l}5 \\
7 \\
6\end{array}$ & $\begin{array}{l}1 \\
2\end{array}$ \\
\hline $\begin{array}{c}\mathrm{M} \\
\mathrm{O} 1 \\
5- \\
55\end{array}$ & $\begin{array}{l}5 \\
4\end{array}$ & $\begin{array}{l}1 \\
4 \\
0\end{array}$ & $\begin{array}{l}0 \\
3 \\
9\end{array}$ & $\begin{array}{l}0.05 \\
964\end{array}$ & $\begin{array}{c}0 . \\
00 \\
24 \\
2\end{array}$ & $\begin{array}{l}0.09 \\
595\end{array}$ & $\begin{array}{c}0 . \\
00 \\
23 \\
6\end{array}$ & $\begin{array}{l}0.78 \\
875\end{array}$ & $\begin{array}{c}0 . \\
02 \\
94 \\
0\end{array}$ & 591 & $\begin{array}{l}4 \\
1\end{array}$ & 591 & $\begin{array}{l}1 \\
4\end{array}$ & 590 & $\begin{array}{l}1 \\
7\end{array}$ & $\begin{array}{c}- \\
0.1 \\
7 \%\end{array}$ & $\begin{array}{l}5 \\
9 \\
1\end{array}$ & $\begin{array}{l}1 \\
4\end{array}$ \\
\hline $\begin{array}{c}\mathrm{M} \\
\mathrm{O} 1 \\
5- \\
56 \\
\end{array}$ & $\begin{array}{l}4 \\
7\end{array}$ & $\begin{array}{l}1 \\
5 \\
0\end{array}$ & $\begin{array}{l}3 \\
2 \\
\end{array}$ & $\begin{array}{l}0.05 \\
740 \\
\end{array}$ & $\begin{array}{c}0 . \\
00 \\
25 \\
9 \\
\end{array}$ & $\begin{array}{l}0.07 \\
816\end{array}$ & $\begin{array}{c}0 . \\
00 \\
20 \\
1 \\
\end{array}$ & $\begin{array}{l}0.61 \\
844\end{array}$ & $\begin{array}{c}0 . \\
02 \\
56 \\
0 \\
\end{array}$ & 507 & $\begin{array}{l}4 \\
8\end{array}$ & 485 & $\begin{array}{l}1 \\
2\end{array}$ & 489 & $\begin{array}{l}1 \\
6\end{array}$ & $\begin{array}{l}0.8 \\
2 \%\end{array}$ & $\begin{array}{l}4 \\
8 \\
5\end{array}$ & $\begin{array}{l}1 \\
2\end{array}$ \\
\hline $\begin{array}{c}\mathrm{M} \\
\mathrm{O} 1 \\
5- \\
57\end{array}$ & $\begin{array}{l}5 \\
0\end{array}$ & $\begin{array}{l}1 \\
7 \\
6\end{array}$ & $\begin{array}{l}0 \\
2 \\
8\end{array}$ & $\begin{array}{l}0.06 \\
743\end{array}$ & $\begin{array}{c}0 . \\
00 \\
22 \\
7 \\
\end{array}$ & $\begin{array}{c}0.11 \\
514\end{array}$ & $\begin{array}{c}0 . \\
00 \\
26 \\
5\end{array}$ & $\begin{array}{l}1.07 \\
025\end{array}$ & $\begin{array}{c}0 . \\
03 \\
27 \\
5\end{array}$ & 851 & $\begin{array}{l}3 \\
0\end{array}$ & 703 & $\begin{array}{l}1 \\
5\end{array}$ & 739 & $\begin{array}{l}1 \\
6\end{array}$ & $\begin{array}{l}5.1 \\
2 \%\end{array}$ & $\begin{array}{l}7 \\
0 \\
3\end{array}$ & $\begin{array}{l}1 \\
5\end{array}$ \\
\hline $\begin{array}{c}\mathrm{M} \\
\mathrm{O} 1 \\
5- \\
58 \\
\end{array}$ & $\begin{array}{l}7 \\
1\end{array}$ & $\begin{array}{l}1 \\
4 \\
9\end{array}$ & $\begin{array}{l}0 \\
4 \\
4 \\
7\end{array}$ & $\begin{array}{l}0.05 \\
773\end{array}$ & $\begin{array}{c}0 . \\
00 \\
35 \\
3 \\
\end{array}$ & $\begin{array}{c}0.05 \\
601\end{array}$ & $\begin{array}{c}0 . \\
00 \\
17 \\
2 \\
\end{array}$ & $\begin{array}{l}0.44 \\
568\end{array}$ & $\begin{array}{c}0 . \\
02 \\
50 \\
8 \\
\end{array}$ & 520 & $\begin{array}{l}7 \\
1\end{array}$ & 351 & $\begin{array}{l}1 \\
0\end{array}$ & 374 & $\begin{array}{l}1 \\
8\end{array}$ & $\begin{array}{l}6.5 \\
5 \%\end{array}$ & $\begin{array}{l}3 \\
5 \\
1\end{array}$ & $\begin{array}{l}1 \\
0\end{array}$ \\
\hline $\begin{array}{c}\mathrm{M} \\
\mathrm{O} 1 \\
5- \\
59 \\
\end{array}$ & $\begin{array}{l}5 \\
5\end{array}$ & $\begin{array}{l}1 \\
4 \\
1\end{array}$ & $\begin{array}{l}0 \\
3 \\
3 \\
9\end{array}$ & $\begin{array}{l}0.05 \\
760\end{array}$ & $\begin{array}{c}0 . \\
00 \\
24 \\
2 \\
\end{array}$ & $\begin{array}{l}0.07 \\
402\end{array}$ & $\begin{array}{c}0 . \\
00 \\
17 \\
7 \\
\end{array}$ & $\begin{array}{l}0.58 \\
771\end{array}$ & $\begin{array}{c}0 . \\
02 \\
28 \\
7 \\
\end{array}$ & 515 & $\begin{array}{l}4 \\
6\end{array}$ & 460 & $\begin{array}{l}1 \\
1\end{array}$ & 469 & $\begin{array}{l}1 \\
5\end{array}$ & $\begin{array}{l}1.9 \\
6 \%\end{array}$ & $\begin{array}{l}4 \\
6 \\
0\end{array}$ & $\begin{array}{l}1 \\
1\end{array}$ \\
\hline $\begin{array}{c}\mathrm{M} \\
\mathrm{O} 1 \\
5- \\
60 \\
\end{array}$ & $\begin{array}{l}1 \\
3 \\
5\end{array}$ & $\begin{array}{l}2 \\
9 \\
4\end{array}$ & $\begin{array}{l}0 \\
4 \\
6 \\
\end{array}$ & $\begin{array}{l}0.05 \\
545\end{array}$ & $\begin{array}{c}0 . \\
00 \\
19 \\
6 \\
\end{array}$ & $\begin{array}{l}0.05 \\
289\end{array}$ & $\begin{array}{c}0 . \\
00 \\
11 \\
4 \\
\end{array}$ & $\begin{array}{l}0.40 \\
425\end{array}$ & $\begin{array}{c}0 . \\
01 \\
32 \\
3 \\
\end{array}$ & 430 & $\begin{array}{l}3 \\
7\end{array}$ & 332 & 7 & 345 & $\begin{array}{l}1 \\
0\end{array}$ & $\begin{array}{l}3.9 \\
2 \%\end{array}$ & $\begin{array}{l}3 \\
3 \\
2\end{array}$ & 7 \\
\hline $\begin{array}{l}\mathrm{M} \\
\mathrm{O} 1 \\
\end{array}$ & $\begin{array}{l}1 \\
6 \\
\end{array}$ & $\begin{array}{l}1 \\
8 \\
\end{array}$ & 0 & $\begin{array}{c}0.06 \\
105 \\
\end{array}$ & $\begin{array}{c}0 . \\
00 \\
\end{array}$ & $\begin{array}{c}0.10 \\
641 \\
\end{array}$ & $\begin{array}{c}0 . \\
00\end{array}$ & $\begin{array}{l}0.89 \\
551 \\
\end{array}$ & $\begin{array}{c}0 . \\
03\end{array}$ & 641 & $\begin{array}{l}3 \\
6 \\
\end{array}$ & 652 & $\begin{array}{l}1 \\
5 \\
\end{array}$ & 649 & $\begin{array}{l}1 \\
6 \\
\end{array}$ & $\begin{array}{c} \\
0.4 \\
\end{array}$ & $\begin{array}{l}6 \\
5 \\
\end{array}$ & $\begin{array}{l}1 \\
5 \\
\end{array}$ \\
\hline
\end{tabular}




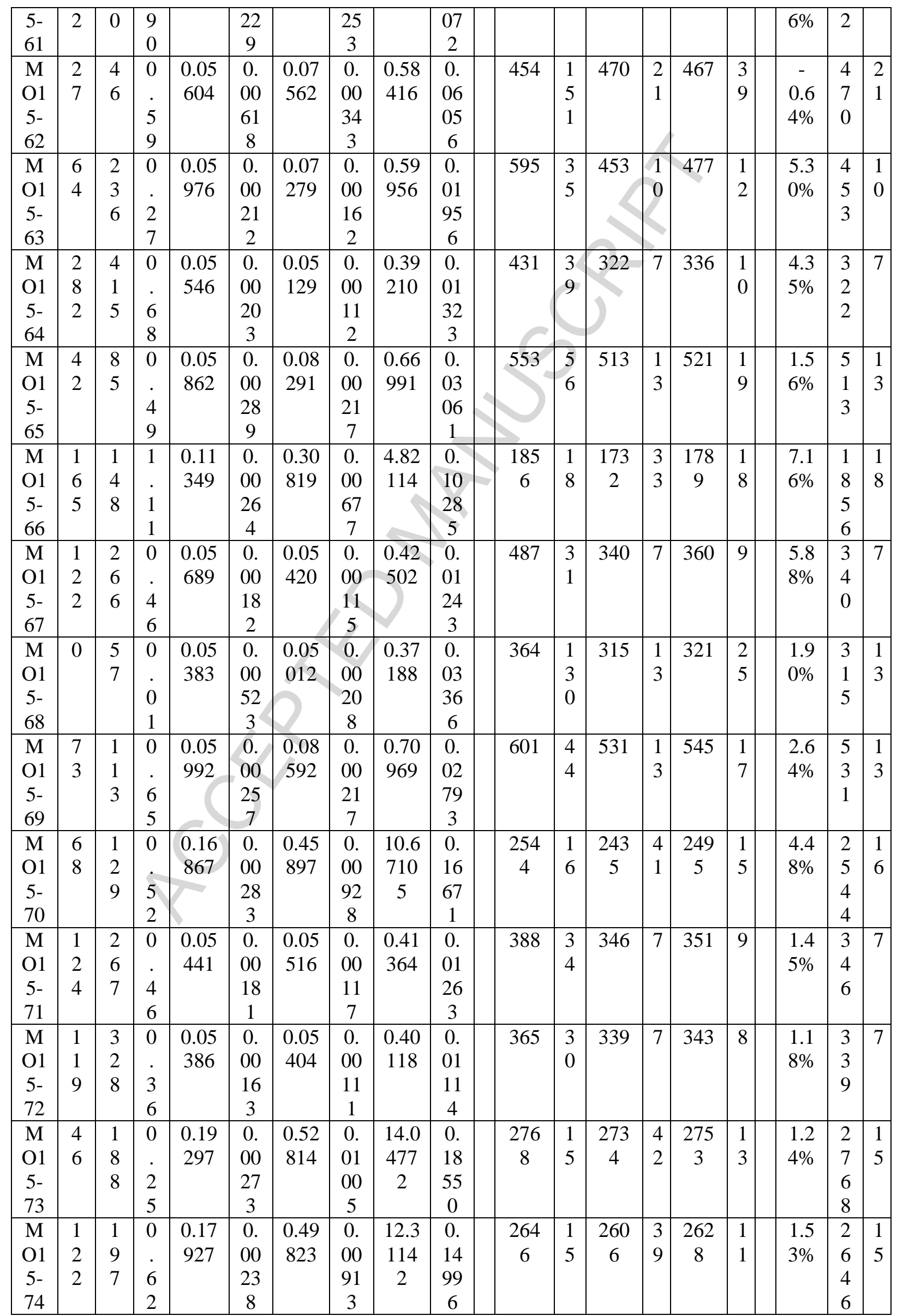




\begin{tabular}{|c|c|c|c|c|c|c|c|c|c|c|c|c|c|c|c|c|c|c|}
\hline $\begin{array}{l}M \\
\mathrm{O} 1 \\
5- \\
75\end{array}$ & $\begin{array}{l}8 \\
5\end{array}$ & $\begin{array}{l}2 \\
2 \\
7\end{array}$ & $\begin{array}{l}0 \\
. \\
3 \\
7\end{array}$ & $\begin{array}{c}0.05 \\
621\end{array}$ & $\begin{array}{c}0 . \\
00 \\
17 \\
6\end{array}$ & $\begin{array}{l}0.07 \\
392\end{array}$ & $\begin{array}{c}0 . \\
00 \\
15 \\
3\end{array}$ & $\begin{array}{l}0.57 \\
269\end{array}$ & $\begin{array}{c}0 . \\
01 \\
64 \\
9\end{array}$ & 461 & $\begin{array}{l}3 \\
1\end{array}$ & 460 & 9 & 460 & 1 & $\begin{array}{l}0.0 \\
0 \%\end{array}$ & $\begin{array}{l}4 \\
6 \\
0\end{array}$ & 9 \\
\hline $\begin{array}{c}M \\
\text { O1 } \\
5- \\
76\end{array}$ & $\begin{array}{l}8 \\
6\end{array}$ & $\begin{array}{l}3 \\
2 \\
6\end{array}$ & $\begin{array}{l}0 \\
. \\
2 \\
6\end{array}$ & $\begin{array}{l}0.05 \\
936\end{array}$ & $\begin{array}{c}0 . \\
00 \\
15 \\
2 \\
\end{array}$ & $\begin{array}{c}0.09 \\
433\end{array}$ & $\begin{array}{c}0 . \\
00 \\
18 \\
6 \\
\end{array}$ & $\begin{array}{l}0.77 \\
181\end{array}$ & $\begin{array}{c}0 . \\
01 \\
80 \\
6\end{array}$ & 580 & $\begin{array}{l}2 \\
3\end{array}$ & 581 & $\begin{array}{l}1 \\
1\end{array}$ & 581 & $\begin{array}{l}1 \\
0\end{array}$ & $\begin{array}{l}0.0 \\
0 \%\end{array}$ & $\begin{array}{l}5 \\
8 \\
1\end{array}$ & 1 \\
\hline $\begin{array}{c}\mathrm{M} \\
\mathrm{O} 1 \\
5- \\
77\end{array}$ & $\begin{array}{l}2 \\
0 \\
6\end{array}$ & $\begin{array}{l}6 \\
5 \\
5\end{array}$ & $\begin{array}{l}0 \\
\dot{3} \\
2\end{array}$ & $\begin{array}{l}0.05 \\
686\end{array}$ & $\begin{array}{c}0 . \\
00 \\
11 \\
2\end{array}$ & $\begin{array}{l}0.07 \\
880\end{array}$ & $\begin{array}{c}0 . \\
00 \\
14 \\
4\end{array}$ & $\begin{array}{l}0.61 \\
759\end{array}$ & $\begin{array}{c}0 . \\
01 \\
10 \\
6\end{array}$ & 486 & $\begin{array}{l}1 \\
8\end{array}$ & 89 & 9 & 488 & 7 & $\begin{array}{c}- \\
0.2 \\
0 \%\end{array}$ & $\begin{array}{l}4 \\
8 \\
9\end{array}$ & 9 \\
\hline $\begin{array}{c}\mathrm{M} \\
\mathrm{O} 1 \\
5- \\
78 \\
\end{array}$ & $\begin{array}{l}6 \\
9\end{array}$ & $\begin{array}{l}3 \\
5 \\
2\end{array}$ & $\begin{array}{l}0 \\
. \\
2 \\
0\end{array}$ & $\begin{array}{l}0.05 \\
831\end{array}$ & $\begin{array}{c}0 . \\
00 \\
14 \\
9 \\
\end{array}$ & $\begin{array}{l}0.07 \\
957\end{array}$ & $\begin{array}{c}0 . \\
00 \\
15 \\
7 \\
\end{array}$ & $\begin{array}{l}0.63 \\
949\end{array}$ & $\begin{array}{c}0 . \\
01 \\
49 \\
1 \\
\end{array}$ & 541 & $\begin{array}{l}2 \\
3\end{array}$ & 494 & 9 & 502 & 9 & $\begin{array}{l}1.6 \\
2 \%\end{array}$ & $\begin{array}{l}4 \\
9 \\
4\end{array}$ & 9 \\
\hline $\begin{array}{l}\text { M } \\
\text { O1 } \\
5- \\
79 \\
\end{array}$ & $\begin{array}{l}1 \\
1 \\
1\end{array}$ & $\begin{array}{l}2 \\
9 \\
2\end{array}$ & $\begin{array}{l}0 \\
. \\
3 \\
8\end{array}$ & $\begin{array}{c}0.05 \\
552\end{array}$ & $\begin{array}{c}0 . \\
00 \\
15 \\
8 \\
\end{array}$ & $\begin{array}{c}0.07 \\
181\end{array}$ & $\begin{array}{c}0 . \\
00 \\
14 \\
6\end{array}$ & $\begin{array}{l}0.54 \\
954\end{array}$ & $\begin{array}{c}0 . \\
01 \\
43 \\
0\end{array}$ & 433 & $\begin{array}{l}2 \\
7\end{array}$ & 447 & 9 & 445 & 9 & $\begin{array}{c}- \\
0.4 \\
5 \%\end{array}$ & $\begin{array}{l}4 \\
4 \\
7\end{array}$ & 9 \\
\hline $\begin{array}{c}\mathrm{M} \\
\mathrm{O} 1 \\
5- \\
80\end{array}$ & $\begin{array}{l}4 \\
7\end{array}$ & $\begin{array}{l}1 \\
0 \\
1\end{array}$ & $\begin{array}{l}0 \\
. \\
4 \\
7\end{array}$ & $\begin{array}{l}0.07 \\
097\end{array}$ & $\begin{array}{c}0 . \\
00 \\
21 \\
1 \\
\end{array}$ & $\begin{array}{l}0.14 \\
757\end{array}$ & $\begin{array}{c}0 . \\
00 \\
32 \\
3 \\
\end{array}$ & $\begin{array}{l}1.44 \\
360\end{array}$ & $\begin{array}{c}0 . \\
03 \\
91 \\
2 \\
\end{array}$ & 957 & $\begin{array}{l}2 \\
5\end{array}$ & 887 & $\begin{array}{l}1 \\
8\end{array}$ & 907 & $\begin{array}{l}1 \\
6\end{array}$ & $\begin{array}{l}2.2 \\
5 \%\end{array}$ & $\begin{array}{l}8 \\
8 \\
7\end{array}$ & $\begin{array}{l}1 \\
8\end{array}$ \\
\hline $\begin{array}{l}\text { M } \\
\text { O1 } \\
5- \\
81\end{array}$ & $\begin{array}{l}1 \\
0 \\
0\end{array}$ & $\begin{array}{l}2 \\
9 \\
8\end{array}$ & $\begin{array}{l}0 \\
3 \\
3 \\
3\end{array}$ & $\begin{array}{l}0.05 \\
475\end{array}$ & $\begin{array}{c}0 . \\
00 \\
17 \\
9\end{array}$ & $\begin{array}{l}0.05 \\
430\end{array}$ & $\begin{array}{c}0 . \\
00 \\
11 \\
5\end{array}$ & $\begin{array}{l}0.40 \\
975\end{array}$ & $\begin{array}{c}0 . \\
01 \\
23 \\
0\end{array}$ & 402 & $\begin{array}{l}3 \\
3\end{array}$ & 341 & 7 & 349 & 9 & $\begin{array}{l}2.3 \\
5 \%\end{array}$ & $\begin{array}{l}3 \\
4 \\
1\end{array}$ & 7 \\
\hline $\begin{array}{c}\mathrm{M} \\
\mathrm{O} 1 \\
5- \\
82\end{array}$ & $\begin{array}{l}4 \\
8\end{array}$ & $\begin{array}{l}1 \\
0 \\
9\end{array}$ & $\begin{array}{l}0 \\
. \\
4 \\
4\end{array}$ & $\begin{array}{l}0.21 \\
746\end{array}$ & $\begin{array}{c}0 . \\
00 \\
32 \\
5\end{array}$ & $\begin{array}{l}0.55 \\
919\end{array}$ & $\begin{array}{c}0 . \\
01 \\
10 \\
7\end{array}$ & $\begin{array}{c}16.7 \\
610 \\
0\end{array}$ & $\begin{array}{c}0 . \\
23 \\
66 \\
3\end{array}$ & $\begin{array}{c}296 \\
2\end{array}$ & $\begin{array}{l}1 \\
5\end{array}$ & $\begin{array}{c}286 \\
3\end{array}$ & $\begin{array}{l}44 \\
6\end{array}$ & $\begin{array}{c}292 \\
1\end{array}$ & $\begin{array}{l}1 \\
4\end{array}$ & $\begin{array}{l}3.4 \\
6 \%\end{array}$ & $\begin{array}{l}2 \\
9 \\
6 \\
2\end{array}$ & 1 \\
\hline $\begin{array}{c}\mathrm{M} \\
\mathrm{O} 1 \\
5- \\
83\end{array}$ & $\begin{array}{l}4 \\
6\end{array}$ & $\begin{array}{l}8 \\
3\end{array}$ & $\begin{array}{l}0 \\
. \\
5 \\
6\end{array}$ & $\begin{array}{l}0.07 \\
745\end{array}$ & $\begin{array}{c}0 . \\
00 \\
22 \\
7\end{array}$ & $\begin{array}{c}0.19 \\
582\end{array}$ & $\begin{array}{c}0 . \\
00 \\
43 \\
5\end{array}$ & $\begin{array}{l}2.09 \\
040\end{array}$ & $\begin{array}{c}0 . \\
05 \\
61 \\
3\end{array}$ & $\begin{array}{c}113 \\
3\end{array}$ & $\begin{array}{l}2 \\
4\end{array}$ & $\begin{array}{c}115 \\
3\end{array}$ & $\begin{array}{l}2 \\
3\end{array}$ & $\begin{array}{c}114 \\
6\end{array}$ & $\begin{array}{l}1 \\
8\end{array}$ & $\begin{array}{c}- \\
1.7 \\
3 \%\end{array}$ & $\begin{array}{l}1 \\
1 \\
3 \\
3\end{array}$ & $\begin{array}{l}2 \\
4\end{array}$ \\
\hline $\begin{array}{c}\mathrm{M} \\
\mathrm{O} 1 \\
5- \\
84 \\
\end{array}$ & $\begin{array}{l}3 \\
4\end{array}$ & $\begin{array}{l}4 \\
4\end{array}$ & $\begin{array}{l}0 \\
. \\
7 \\
6 \\
\end{array}$ & $\begin{array}{c}0.06 \\
173\end{array}$ & $\begin{array}{c}0 . \\
00 \\
43 \\
2 \\
\end{array}$ & $\begin{array}{c}0.08 \\
907\end{array}$ & $\begin{array}{c}0 . \\
00 \\
29 \\
8 \\
\end{array}$ & $\begin{array}{l}0.75 \\
793\end{array}$ & $\begin{array}{c}0 . \\
04 \\
93 \\
2 \\
\end{array}$ & 665 & $\begin{array}{l}8 \\
3\end{array}$ & 550 & $\begin{array}{l}1 \\
8\end{array}$ & 573 & $\begin{array}{l}2 \\
8\end{array}$ & $\begin{array}{l}4.1 \\
8 \%\end{array}$ & $\begin{array}{l}5 \\
5 \\
0\end{array}$ & $\begin{array}{l}1 \\
8\end{array}$ \\
\hline $\begin{array}{l}\mathrm{M} \\
\mathrm{O} 1 \\
5- \\
85\end{array}$ & $\begin{array}{l}3 \\
7\end{array}$ & $\begin{array}{l}2 \\
1 \\
9\end{array}$ & $\begin{array}{l}0 \\
. \\
1 \\
7\end{array}$ & $\begin{array}{l}0.05 \\
997\end{array}$ & $\begin{array}{c}0 . \\
00 \\
16 \\
0 \\
\end{array}$ & $\begin{array}{l}0.10 \\
374\end{array}$ & $\begin{array}{c}0 . \\
00 \\
20 \\
8 \\
\end{array}$ & $\begin{array}{l}0.85 \\
747\end{array}$ & $\begin{array}{c}0 . \\
02 \\
09 \\
3\end{array}$ & 602 & $\begin{array}{l}2 \\
4\end{array}$ & 636 & $\begin{array}{l}1 \\
2\end{array}$ & 629 & $\begin{array}{l}1 \\
1\end{array}$ & $\begin{array}{c}- \\
1.1 \\
0 \%\end{array}$ & $\begin{array}{l}6 \\
3 \\
6\end{array}$ & $\begin{array}{l}1 \\
2\end{array}$ \\
\hline $\begin{array}{c} \\
\text { O1 } \\
5- \\
86 \\
\end{array}$ & $\begin{array}{l}6 \\
0\end{array}$ & $\begin{array}{l}3 \\
1 \\
7\end{array}$ & $\begin{array}{l}0 \\
. \\
1 \\
9 \\
\end{array}$ & $\begin{array}{l}0.05 \\
809\end{array}$ & $\begin{array}{c}0 . \\
00 \\
14 \\
8 \\
\end{array}$ & $\begin{array}{l}0.07 \\
990\end{array}$ & $\begin{array}{c}0 . \\
00 \\
15 \\
7 \\
\end{array}$ & $\begin{array}{l}0.63 \\
972\end{array}$ & $\begin{array}{c}0 . \\
01 \\
48 \\
0 \\
\end{array}$ & 533 & $\begin{array}{l}2 \\
3\end{array}$ & 496 & 9 & 502 & 9 & $\begin{array}{l}1.2 \\
1 \%\end{array}$ & $\begin{array}{l}4 \\
9 \\
6\end{array}$ & 9 \\
\hline $\begin{array}{c}\mathrm{M} \\
\mathrm{O} 1 \\
5- \\
87 \\
\end{array}$ & $\begin{array}{l}1 \\
7\end{array}$ & $\begin{array}{l}1 \\
8 \\
7\end{array}$ & $\begin{array}{c}0 \\
. \\
0 \\
9 \\
\end{array}$ & $\begin{array}{c}0.06 \\
085\end{array}$ & $\begin{array}{c}0 . \\
00 \\
19 \\
3 \\
\end{array}$ & $\begin{array}{c}0.09 \\
130\end{array}$ & $\begin{array}{c}0 . \\
00 \\
19 \\
6 \\
\end{array}$ & $\begin{array}{c}0.76 \\
578\end{array}$ & $\begin{array}{c}0 . \\
02 \\
22 \\
7 \\
\end{array}$ & 634 & $\begin{array}{l}3 \\
0\end{array}$ & 563 & $\begin{array}{l}1 \\
2\end{array}$ & 577 & $\begin{array}{l}1 \\
3\end{array}$ & $\begin{array}{l}2.4 \\
9 \%\end{array}$ & $\begin{array}{l}5 \\
6 \\
3 \\
\end{array}$ & $\begin{array}{l}1 \\
2\end{array}$ \\
\hline $\begin{array}{l}\mathrm{M} \\
\mathrm{O} 1 \\
\end{array}$ & $\begin{array}{l}5 \\
8 \\
\end{array}$ & $\begin{array}{l}8 \\
3 \\
\end{array}$ & 0 & $\begin{array}{c}0.05 \\
523 \\
\end{array}$ & $\begin{array}{c}0 . \\
00 \\
\end{array}$ & $\begin{array}{l}0.05 \\
309 \\
\end{array}$ & $\begin{array}{c}0 . \\
00 \\
\end{array}$ & $\begin{array}{c}0.40 \\
417 \\
\end{array}$ & $\begin{array}{c}0 . \\
00 \\
\end{array}$ & 422 & $\begin{array}{l}1 \\
9 \\
\end{array}$ & 333 & 6 & 345 & 6 & $\begin{array}{l}3.6 \\
0 \% \\
\end{array}$ & $\begin{array}{l}3 \\
3 \\
\end{array}$ & 6 \\
\hline
\end{tabular}




\begin{tabular}{|c|c|c|c|c|c|c|c|c|c|c|c|c|c|c|c|c|c|c|}
\hline $\begin{array}{l}5- \\
88\end{array}$ & 7 & 1 & $\begin{array}{l}7 \\
1\end{array}$ & & $\begin{array}{c}11 \\
6\end{array}$ & & $\begin{array}{c}09 \\
8\end{array}$ & & $\begin{array}{c}76 \\
8\end{array}$ & & & & & & & & 3 & \\
\hline $\begin{array}{c}\mathrm{M} \\
\mathrm{O} 1 \\
5- \\
89\end{array}$ & $\begin{array}{l}3 \\
3\end{array}$ & $\begin{array}{l}2 \\
3 \\
2\end{array}$ & $\begin{array}{l}0 \\
1 \\
4\end{array}$ & $\begin{array}{c}0.06 \\
101\end{array}$ & $\begin{array}{c}0 . \\
00 \\
17 \\
6\end{array}$ & $\begin{array}{c}0.08 \\
631\end{array}$ & $\begin{array}{c}0 . \\
00 \\
17 \\
7\end{array}$ & $\begin{array}{c}0.72 \\
584\end{array}$ & $\begin{array}{c}0 . \\
01 \\
91 \\
9\end{array}$ & 640 & $\begin{array}{l}2 \\
6\end{array}$ & 534 & $\begin{array}{l}1 \\
1\end{array}$ & 554 & $\begin{array}{l}1 \\
1\end{array}$ & $\begin{array}{l}3.7 \\
5 \%\end{array}$ & $\begin{array}{l}5 \\
3 \\
4\end{array}$ & $\begin{array}{l}1 \\
1\end{array}$ \\
\hline $\begin{array}{c}\mathrm{M} \\
\mathrm{O} 1 \\
5- \\
90\end{array}$ & $\begin{array}{l}7 \\
2\end{array}$ & $\begin{array}{l}1 \\
1 \\
5\end{array}$ & $\begin{array}{l}0 \\
6 \\
6\end{array}$ & $\begin{array}{c}0.13 \\
005\end{array}$ & $\begin{array}{c}0 . \\
00 \\
23 \\
8\end{array}$ & $\begin{array}{c}0.38 \\
124\end{array}$ & $\begin{array}{c}0 . \\
00 \\
77 \\
1\end{array}$ & $\begin{array}{l}6.83 \\
418\end{array}$ & $\begin{array}{c}0 . \\
11 \\
53 \\
5\end{array}$ & $\begin{array}{c}209 \\
9\end{array}$ & $\begin{array}{l}1 \\
6\end{array}$ & $\begin{array}{c}208 \\
2\end{array}$ & $\begin{array}{l}3 \\
6\end{array}$ & $\begin{array}{c}209 \\
0\end{array}$ & $\begin{array}{l}1 \\
5\end{array}$ & $\begin{array}{l}0.8 \\
2 \%\end{array}$ & $\begin{array}{l}2 \\
0 \\
9 \\
9\end{array}$ & $\begin{array}{l}1 \\
6\end{array}$ \\
\hline $\begin{array}{c}\mathrm{M} \\
\mathrm{O} 1 \\
5- \\
91\end{array}$ & $\begin{array}{l}3 \\
7\end{array}$ & $\begin{array}{l}2 \\
0 \\
8\end{array}$ & $\begin{array}{l}0 \\
. \\
1 \\
8\end{array}$ & $\begin{array}{l}0.06 \\
375\end{array}$ & $\begin{array}{c}0 . \\
00 \\
16 \\
3\end{array}$ & $\begin{array}{l}0.11 \\
537\end{array}$ & $\begin{array}{c}0 . \\
00 \\
23 \\
2\end{array}$ & $\begin{array}{l}1.01 \\
378\end{array}$ & $\begin{array}{c}0 . \\
02 \\
36 \\
0\end{array}$ & 733 & $\begin{array}{l}2 \\
2\end{array}$ & 704 & $\begin{array}{l}1 \\
3\end{array}$ & 711 & $\begin{array}{l}1 \\
2\end{array}$ & $\begin{array}{l}0.9 \\
9 \%\end{array}$ & $\begin{array}{l}7 \\
0 \\
4\end{array}$ & $\begin{array}{l}1 \\
3\end{array}$ \\
\hline $\begin{array}{c}\mathrm{M} \\
\mathrm{O} 1 \\
5- \\
92\end{array}$ & $\begin{array}{l}6 \\
3\end{array}$ & $\begin{array}{l}4 \\
4 \\
2\end{array}$ & $\begin{array}{l}0 \\
. \\
4\end{array}$ & $\begin{array}{l}0.07 \\
277\end{array}$ & $\begin{array}{c}0 . \\
00 \\
11 \\
9\end{array}$ & $\begin{array}{c}0.16 \\
697\end{array}$ & $\begin{array}{c}0 . \\
00 \\
29 \\
9\end{array}$ & $\begin{array}{l}1.67 \\
479\end{array}$ & $\begin{array}{c}0 . \\
02 \\
45 \\
8\end{array}$ & $\begin{array}{c}100 \\
8\end{array}$ & $\begin{array}{l}1 \\
6\end{array}$ & 995 & $\begin{array}{l}1 \\
7\end{array}$ & 999 & 9 & $\begin{array}{l}0.4 \\
0 \%\end{array}$ & $\begin{array}{l}9 \\
9 \\
5\end{array}$ & $\begin{array}{l}1 \\
7\end{array}$ \\
\hline $\begin{array}{c}\mathrm{M} \\
\mathrm{O} 1 \\
5- \\
93\end{array}$ & $\begin{array}{l}1 \\
0 \\
1\end{array}$ & $\begin{array}{l}2 \\
1 \\
7\end{array}$ & $\begin{array}{l}0 \\
4 \\
6 \\
\end{array}$ & $\begin{array}{l}0.12 \\
855\end{array}$ & $\begin{array}{c}0 . \\
00 \\
20 \\
9\end{array}$ & $\begin{array}{c}0.34 \\
922\end{array}$ & $\begin{array}{c}0 . \\
00 \\
66 \\
7\end{array}$ & $\begin{array}{l}6.18 \\
800\end{array}$ & $\begin{array}{c}0 . \\
09 \\
15 \\
3\end{array}$ & $\begin{array}{c}207 \\
8\end{array}$ & $\begin{array}{l}1 \\
6\end{array}$ & $\begin{array}{c}193 \\
1\end{array}$ & $\begin{array}{l}3 \\
2\end{array}$ & $\begin{array}{c}200 \\
3\end{array}$ & $\begin{array}{l}1 \\
3\end{array}$ & $\begin{array}{l}7.6 \\
1 \%\end{array}$ & $\begin{array}{l}2 \\
0 \\
7 \\
8 \\
\end{array}$ & $\begin{array}{l}1 \\
6\end{array}$ \\
\hline $\begin{array}{c}\mathrm{M} \\
\mathrm{O} 1 \\
5- \\
94\end{array}$ & $\begin{array}{l}1 \\
2 \\
7\end{array}$ & $\begin{array}{l}8 \\
3\end{array}$ & $\begin{array}{l}5 \\
2\end{array}$ & $\begin{array}{l}0.06 \\
329\end{array}$ & $\begin{array}{c}0 . \\
00 \\
33 \\
0\end{array}$ & $\begin{array}{l}0.08 \\
799\end{array}$ & $\begin{array}{c}0 . \\
00 \\
25 \\
2\end{array}$ & $\begin{array}{c}0.76 \\
757\end{array}$ & $\begin{array}{c}0 . \\
03 \\
66 \\
8\end{array}$ & 718 & $\begin{array}{l}5 \\
5\end{array}$ & 544 & $\begin{array}{l}1 \\
5\end{array}$ & 578 & $\begin{array}{l}2 \\
1\end{array}$ & $\begin{array}{l}6.2 \\
5 \%\end{array}$ & $\begin{array}{l}5 \\
4 \\
4\end{array}$ & $\begin{array}{l}1 \\
5\end{array}$ \\
\hline $\begin{array}{c}\mathrm{M} \\
\mathrm{O} 1 \\
5- \\
95 \\
\end{array}$ & $\begin{array}{l}1 \\
2 \\
0\end{array}$ & $\begin{array}{l}5 \\
7 \\
0\end{array}$ & $\begin{array}{l}0 \\
2 \\
1\end{array}$ & $\begin{array}{l}0.05 \\
804\end{array}$ & $\begin{array}{c}0 . \\
00 \\
14 \\
5\end{array}$ & $\begin{array}{l}0.06 \\
947\end{array}$ & $\begin{array}{c}0 . \\
00 \\
13 \\
5\end{array}$ & $\begin{array}{l}0.55 \\
575\end{array}$ & $\begin{array}{c}0 . \\
01 \\
26 \\
2\end{array}$ & 531 & $\begin{array}{l}2 \\
2\end{array}$ & 433 & 8 & 449 & 8 & $\begin{array}{l}3.7 \\
0 \%\end{array}$ & $\begin{array}{l}4 \\
3 \\
3\end{array}$ & 8 \\
\hline $\begin{array}{c}\mathrm{M} \\
\mathrm{O} 1 \\
5- \\
96\end{array}$ & $\begin{array}{l}2 \\
5\end{array}$ & $\begin{array}{l}9 \\
2\end{array}$ & $\begin{array}{l}2 \\
8\end{array}$ & $\begin{array}{l}0.07 \\
048\end{array}$ & $\begin{array}{c}0 . \\
00 \\
42 \\
9\end{array}$ & $\begin{array}{l}0.07 \\
184\end{array}$ & $\begin{array}{c}0 . \\
00 \\
22 \\
5\end{array}$ & $\begin{array}{l}0.69 \\
791\end{array}$ & $\begin{array}{c}0 . \\
03 \\
90 \\
1\end{array}$ & 879 & $\begin{array}{l}1 \\
4 \\
0\end{array}$ & 446 & $\begin{array}{l}1 \\
3\end{array}$ & 524 & $\begin{array}{l}2 \\
4\end{array}$ & $\begin{array}{c}17 . \\
49 \\
\%\end{array}$ & & \\
\hline $\begin{array}{c}\mathrm{M} \\
\mathrm{O} 1 \\
5- \\
97\end{array}$ & $\begin{array}{l}6 \\
2\end{array}$ & $\begin{array}{l}3 \\
3 \\
7\end{array}$ & $\begin{array}{l}0 \\
1 \\
8\end{array}$ & $\begin{array}{l}0.07 \\
641\end{array}$ & $\begin{array}{c}0 . \\
00 \\
16 \\
5\end{array}$ & $\begin{array}{l}0.11 \\
533\end{array}$ & $\begin{array}{c}0 . \\
00 \\
22 \\
3\end{array}$ & $\begin{array}{l}1.21 \\
473\end{array}$ & $\begin{array}{c}0 . \\
02 \\
35 \\
3\end{array}$ & $\begin{array}{c}106 \\
5\end{array}$ & $\begin{array}{l}5 \\
7\end{array}$ & 702 & $\begin{array}{l}1 \\
3\end{array}$ & 795 & $\begin{array}{l}1 \\
1\end{array}$ & $\begin{array}{l}13 . \\
25 \\
\%\end{array}$ & & \\
\hline $\begin{array}{c}\text { M } \\
\text { O1 } \\
5- \\
98\end{array}$ & $\begin{array}{l}2 \\
3 \\
7\end{array}$ & $\begin{array}{l}3 \\
9 \\
4\end{array}$ & $\begin{array}{l}0 \\
6 \\
0\end{array}$ & $\begin{array}{l}0.06 \\
250\end{array}$ & $\begin{array}{c}0 . \\
00 \\
14 \\
5\end{array}$ & $\begin{array}{l}0.09 \\
864\end{array}$ & $\begin{array}{c}0 . \\
00 \\
19 \\
0\end{array}$ & $\begin{array}{l}0.84 \\
982\end{array}$ & $\begin{array}{c}0 . \\
01 \\
79 \\
5\end{array}$ & 691 & $\begin{array}{l}2 \\
0\end{array}$ & 606 & $\begin{array}{l}1 \\
1\end{array}$ & 625 & $\begin{array}{l}1 \\
0\end{array}$ & $\begin{array}{l}3.1 \\
4 \%\end{array}$ & $\begin{array}{l}6 \\
0 \\
6\end{array}$ & $\begin{array}{l}1 \\
1\end{array}$ \\
\hline $\begin{array}{c}\text { M } \\
\text { O1 } \\
5- \\
99\end{array}$ & $\begin{array}{l}5 \\
5\end{array}$ & $\begin{array}{l}8 \\
1\end{array}$ & $\begin{array}{l}6 \\
8\end{array}$ & $\begin{array}{l}0.06 \\
461\end{array}$ & $\begin{array}{c}0 . \\
00 \\
33 \\
3\end{array}$ & $\begin{array}{l}0.08 \\
771\end{array}$ & $\begin{array}{c}0 . \\
00 \\
24 \\
7\end{array}$ & $\begin{array}{c}0.78 \\
117\end{array}$ & $\begin{array}{c}0 . \\
03 \\
70 \\
4\end{array}$ & 762 & $\begin{array}{l}5 \\
4\end{array}$ & 542 & $\begin{array}{l}1 \\
5\end{array}$ & 586 & $\begin{array}{l}2 \\
1\end{array}$ & $\begin{array}{l}8.1 \\
2 \%\end{array}$ & $\begin{array}{l}5 \\
4 \\
2\end{array}$ & $\begin{array}{l}1 \\
5\end{array}$ \\
\hline $\begin{array}{c}\mathrm{M} \\
\mathrm{O} 1 \\
5- \\
10 \\
0\end{array}$ & $\begin{array}{l}2 \\
8 \\
9\end{array}$ & $\begin{array}{l}4 \\
0 \\
2\end{array}$ & $\begin{array}{l}0 \\
. \\
7 \\
2\end{array}$ & $\begin{array}{l}0.05 \\
848\end{array}$ & $\begin{array}{c}0 . \\
00 \\
22 \\
2\end{array}$ & $\begin{array}{c}0.06 \\
580\end{array}$ & $\begin{array}{c}0 . \\
00 \\
15 \\
4\end{array}$ & $\begin{array}{l}0.53 \\
043\end{array}$ & $\begin{array}{c}0 . \\
01 \\
83 \\
7\end{array}$ & 548 & $\begin{array}{l}3 \\
8\end{array}$ & 411 & 9 & 432 & $\begin{array}{l}1 \\
2\end{array}$ & $\begin{array}{l}5.1 \\
1 \%\end{array}$ & $\begin{array}{l}4 \\
1 \\
1\end{array}$ & 9 \\
\hline $\begin{array}{l}\mathrm{M} \\
\mathrm{O} 1\end{array}$ & $\begin{array}{l}3 \\
2\end{array}$ & $\begin{array}{l}8 \\
9\end{array}$ & 0 & $\begin{array}{c}0.05 \\
633\end{array}$ & $\begin{array}{c}0 . \\
00\end{array}$ & $\begin{array}{l}0.07 \\
724\end{array}$ & $\begin{array}{c}0 . \\
00\end{array}$ & $\begin{array}{l}0.60 \\
006\end{array}$ & $\begin{array}{c}0 . \\
01\end{array}$ & 465 & $\begin{array}{l}1 \\
9\end{array}$ & 480 & 9 & 477 & 7 & $\begin{array}{c}- \\
0.6\end{array}$ & $\begin{array}{l}4 \\
8\end{array}$ & 9 \\
\hline
\end{tabular}




\begin{tabular}{|c|c|c|c|c|c|c|c|c|c|c|c|c|c|c|c|c|c|c|}
\hline $\begin{array}{l}6- \\
01\end{array}$ & 7 & 2 & $\begin{array}{l}3 \\
7 \\
\end{array}$ & & $\begin{array}{c}11 \\
0\end{array}$ & & $\begin{array}{c}14 \\
8 \\
\end{array}$ & & $\begin{array}{c}07 \\
3 \\
\end{array}$ & & & & & & & $2 \%$ & 0 & \\
\hline $\mathrm{M}$ & 2 & 4 & 0 & 0.11 & 0. & 0.32 & 0. & 5.30 & 0. & 194 & 1 & 179 & 3 & 186 & 1 & 8.4 & 1 & 1 \\
\hline $\mathrm{O} 1$ & 4 & 4 & . & 950 & 00 & 161 & 00 & 018 & 08 & 9 & 7 & 8 & 1 & 9 & 3 & $0 \%$ & 9 & 7 \\
\hline 6- & 6 & 1 & 5 & & 19 & & 64 & & 15 & & & & & & & & 4 & \\
\hline 02 & & & 6 & & 9 & & 2 & & 6 & & & & & & & & 9 & \\
\hline M & 2 & 6 & 0 & 0.05 & 0 & 0.07 & 0. & 0.59 & 0. & 556 & 2 & 459 & 9 & 475 & 9 & 3.4 & 4 & 9 \\
\hline O1 & 9 & 3 & . & 871 & 00 & 375 & 00 & 712 & 01 & & 4 & & & & & $9 \%$ & 5 & \\
\hline 6- & 6 & 2 & 4 & & 15 & & 15 & & 47 & & & & & & & & 9 & \\
\hline 03 & & & 7 & & 8 & & 3 & & 9 & & & & & & & & & \\
\hline M & 3 & 2 & 1 & 0.11 & 0 & 0.31 & 0. & 4.96 & 0 & 189 & 1 & 174 & 3 & 181 & 1 & 8.5 & 1 & 1 \\
\hline $\mathrm{O} 1$ & 4 & 8 & . & 592 & 00 & 088 & 00 & 976 & 08 & 4 & 7 & 5 & 2 & 4 & 5 & $4 \%$ & 8 & 7 \\
\hline 6- & 8 & 0 & 2 & & 22 & & 65 & & 88 & & & & & & & & 9 & \\
\hline 04 & & & 4 & & 5 & & 4 & & 7 & & 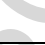 & & & & & & 4 & \\
\hline $\mathrm{M}$ & 5 & 1 & 0 & 0.05 & 0. & 0.07 & 0. & 0.61 & 0. & 466 & 4 & 492 & 1 & 488 & 1 & - & 4 & 1 \\
\hline $\mathrm{O} 1$ & 9 & 7 & . & 635 & 00 & 938 & 00 & 689 & 02 & & 5 & & 2 & & 5 & 0.8 & 9 & 2 \\
\hline 6- & & 9 & 3 & & 23 & & 19 & & 38 & & & & & & & $1 \%$ & 2 & \\
\hline 05 & & & 3 & & 6 & & 6 & & 9 & & & & & & & & & \\
\hline $\mathrm{M}$ & 4 & 8 & 0 & 0.12 & 0. & 0.25 & 0. & 4.56 & 0. & 204 & 6 & 148 & 3 & 173 & 2 & 37. & & \\
\hline $\mathrm{O} 1$ & 4 & 1 & . & 734 & 00 & 981 & 00 & 252 & 09 & 1 & 0 & 6 & 0 & 1 & 0 & 35 & & \\
\hline 6- & & & 5 & & 30 & & 60 & & 90 & & & & & & & $\%$ & & \\
\hline 06 & & & 3 & & 6 & & 0 & & 8 & & & & & & & & & \\
\hline $\mathrm{M}$ & 5 & 1 & 0 & 0.06 & 0. & 0.08 & 0. & 0.70 & 0. & 656 & 4 & 513 & 1 & 540 & 1 & 5.2 & 5 & 1 \\
\hline $\mathrm{O} 1$ & 0 & 5 & 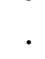 & 148 & 00 & 283 & 00 & 220 & 02 & & 7 & & 4 & & 8 & $6 \%$ & 1 & 4 \\
\hline 6- & & 0 & 3 & & 28 & & 22 & & 99 & & & & & & & & 3 & \\
\hline$\frac{07}{M}$ & 7 & & 4 & & $\frac{7}{0}$ & 005 & $\frac{8}{0 .}$ & 0.40 & $\begin{array}{r}7 \\
0\end{array}$ & 442 & 4 & 333 & 8 & 347 & 1 & 4.2 & 3 & 8 \\
\hline $\mathrm{O} 1$ & 0 & 0 & 0 & 573 & 00 & 304 & 00 & 768 & 01 & & 7 & & o & & 2 & $0 \%$ & 3 & \\
\hline $6-$ & & 2 & 2 & & 23 & & 12 & & 62 & & & & & & & & 3 & \\
\hline 08 & & & 3 & & 9 & & 9 & & 5 & & & & & & & & & \\
\hline $\mathrm{M}$ & 9 & 5 & 0 & 0.06 & 0. & 0.08 & 0. & 0.70 & 0. & 729 & 2 & 501 & 1 & 544 & 1 & 8.5 & 5 & 1 \\
\hline $\mathrm{O} 1$ & 7 & 5 & . & 362 & 00 & 080 & 00 & 888 & 01 & & 2 & & 0 & & 0 & $8 \%$ & 0 & 0 \\
\hline 6- & & 0 & 1 & & 15 & & 16 & & 61 & & & & & & & & 1 & \\
\hline 09 & & & 8 & 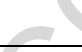 & 9 & & 7 & & 6 & & & & & & & & & \\
\hline $\mathrm{M}$ & 1 & 2 & 0 & 0.13 & 0. & 0.39 & 0. & 7.27 & 0. & 214 & 1 & 214 & 3 & 214 & 1 & - & 2 & 1 \\
\hline $\mathrm{O} 1$ & 6 & 5 & . & 354 & 00 & 523 & 00 & 841 & 11 & 5 & 7 & 7 & 8 & 6 & 5 & 0.0 & 1 & 7 \\
\hline 6- & 5 & 9 & 6 & 70 & 23 & & 81 & & 85 & & & & & & & $9 \%$ & 4 & \\
\hline 10 & & & 4 & & 3 & & 7 & & 1 & & & & & & & & 5 & \\
\hline $\mathrm{M}$ & 1 & 9 & 0 & 0.06 & 0. & 0.08 & 0. & 0.68 & 0. & 599 & 1 & 503 & 1 & 521 & 2 & 3.5 & 5 & 1 \\
\hline $\mathrm{O} 1$ & 5 & 6 & . & 111 & 00 & 135 & 00 & 551 & 03 & & 2 & & 4 & & 1 & $8 \%$ & 0 & 4 \\
\hline $6-$ & & & 1 & & 33 & & 23 & & 44 & & 8 & & & & & & 3 & \\
\hline 11 & & & 6 & & 1 & & 3 & & 3 & & & & & & & & & \\
\hline $\mathrm{M}$ & 1 & 6 & 0 & 0.05 & 0. & 0.05 & 0. & 0.38 & 0. & 338 & 2 & 330 & 7 & 331 & 7 & 0.3 & 3 & 7 \\
\hline $\mathrm{O} 1$ & 3 & 4 & . & 322 & 00 & 252 & 00 & 538 & 00 & & 5 & & & & & $0 \%$ & 3 & \\
\hline 6- & 9 & 1 & 2 & & 14 & & 10 & & 93 & & & & & & & & 0 & \\
\hline 12 & & & 2 & & 0 & & 7 & & 7 & & & & & & & & & \\
\hline M & 9 & 8 & 1 & 0.06 & 0 & 0.10 & 0. & 0.92 & 0. & 679 & 4 & 662 & 1 & 666 & 2 & 0.6 & 6 & 1 \\
\hline $\mathrm{O} 1$ & 0 & 8 & . & 215 & 00 & 817 & 00 & 705 & 03 & & 5 & & 7 & & 0 & $0 \%$ & 6 & 7 \\
\hline 6- & & & 0 & & 27 & & 29 & & 80 & & & & & & & & 2 & \\
\hline 13 & & & 3 & & 7 & & 0 & & 3 & & & & & & & & & \\
\hline M & 5 & 6 & 0 & 0.05 & 0 & 0.07 & 0. & 0.62 & 0 & 541 & 9 & 484 & 1 & 494 & 2 & 2.0 & 4 & 1 \\
\hline $\mathrm{O} 1$ & 0 & 8 & $\cdot$ & 831 & 00 & 793 & 00 & 670 & 04 & & 3 & & 5 & & 6 & $7 \%$ & 8 & 5 \\
\hline 6- & & & 7 & & 41 & & 24 & & 22 & & & & & & & & 4 & \\
\hline 14 & & & 4 & & 6 & & 4 & & 5 & & & & & & & & & \\
\hline
\end{tabular}




\begin{tabular}{|c|c|c|c|c|c|c|c|c|c|c|c|c|c|c|c|c|c|c|}
\hline $\begin{array}{c}\mathrm{M} \\
\mathrm{O} 1 \\
6- \\
15 \\
\end{array}$ & \begin{tabular}{l|}
1 \\
9 \\
8
\end{tabular} & $\begin{array}{l}4 \\
7 \\
7\end{array}$ & $\begin{array}{l}0 \\
4 \\
2 \\
\end{array}$ & $\begin{array}{c}0.05 \\
428\end{array}$ & $\begin{array}{c}0 . \\
00 \\
15 \\
3\end{array}$ & $\begin{array}{l}0.05 \\
790\end{array}$ & $\begin{array}{c}0 . \\
00 \\
12 \\
1\end{array}$ & $\begin{array}{l}0.43 \\
339\end{array}$ & $\begin{array}{c}0 . \\
01 \\
12 \\
7\end{array}$ & 383 & $\begin{array}{l}2 \\
7\end{array}$ & 363 & 7 & 366 & 8 & $\begin{array}{l}0.8 \\
3 \%\end{array}$ & $\begin{array}{l}3 \\
6 \\
3\end{array}$ & 7 \\
\hline $\begin{array}{c}\mathrm{M} \\
\mathrm{O} 1 \\
6- \\
16\end{array}$ & \begin{tabular}{l|}
5 \\
1
\end{tabular} & $\begin{array}{l}3 \\
3 \\
8\end{array}$ & $\begin{array}{l}0 \\
. \\
1 \\
5\end{array}$ & $\begin{array}{l}0.05 \\
985\end{array}$ & $\begin{array}{c}0 . \\
00 \\
14 \\
5\end{array}$ & $\begin{array}{l}0.08 \\
701\end{array}$ & $\begin{array}{c}0 . \\
00 \\
17 \\
7 \\
\end{array}$ & $\begin{array}{l}0.71 \\
816\end{array}$ & $\begin{array}{c}0 . \\
01 \\
60 \\
2 \\
\end{array}$ & 598 & $\begin{array}{l}2 \\
2\end{array}$ & 538 & $\begin{array}{l}1 \\
0\end{array}$ & 550 & 9 & $\begin{array}{l}2.2 \\
3 \%\end{array}$ & $\begin{array}{l}5 \\
3 \\
8\end{array}$ & $\begin{array}{l}1 \\
0\end{array}$ \\
\hline $\begin{array}{c}\mathrm{M} \\
\mathrm{O} 1 \\
6- \\
17\end{array}$ & \begin{tabular}{l|}
1 \\
0 \\
4
\end{tabular} & $\begin{array}{l}2 \\
7 \\
8\end{array}$ & $\begin{array}{l}3 \\
7\end{array}$ & $\begin{array}{l}0.06 \\
073\end{array}$ & $\begin{array}{c}0 . \\
00 \\
14 \\
7\end{array}$ & $\begin{array}{c}0.10 \\
131\end{array}$ & $\begin{array}{c}0 . \\
00 \\
20 \\
5\end{array}$ & $\begin{array}{l}0.84 \\
841\end{array}$ & $\begin{array}{c}0 . \\
01 \\
90 \\
1\end{array}$ & 630 & $\begin{array}{l}2 \\
2\end{array}$ & & $\begin{array}{l}1 \\
2\end{array}$ & 624 & $\begin{array}{l}1 \\
0\end{array}$ & $\begin{array}{l}0.3 \\
2 \%\end{array}$ & $\begin{array}{l}6 \\
2 \\
2\end{array}$ & $\begin{array}{l}1 \\
2\end{array}$ \\
\hline $\begin{array}{c}M \\
\text { O1 } \\
6- \\
18 \\
\end{array}$ & $\begin{array}{l}1 \\
3 \\
8\end{array}$ & $\begin{array}{l}1 \\
0 \\
5\end{array}$ & $\begin{array}{l}1 \\
3 \\
1 \\
\end{array}$ & $\begin{array}{l}0.06 \\
843\end{array}$ & $\begin{array}{c}0 . \\
00 \\
36 \\
8 \\
\end{array}$ & $\begin{array}{l}0.10 \\
915\end{array}$ & $\begin{array}{c}0 . \\
00 \\
33 \\
8\end{array}$ & $\begin{array}{l}1.02 \\
998\end{array}$ & $\begin{array}{c}0 . \\
05 \\
07 \\
4 \\
\end{array}$ & 882 & $\begin{array}{l}5 \\
4\end{array}$ & 668 & $\begin{array}{l}2 \\
0\end{array}$ & 719 & $\begin{array}{l}2 \\
5\end{array}$ & $\begin{array}{l}7.6 \\
3 \%\end{array}$ & $\begin{array}{l}6 \\
6 \\
8\end{array}$ & $\begin{array}{l}2 \\
0\end{array}$ \\
\hline $\begin{array}{c}\mathrm{M} \\
\mathrm{O} 1 \\
6- \\
19 \\
\end{array}$ & $\begin{array}{l}9 \\
5\end{array}$ & $\begin{array}{l}2 \\
5 \\
2\end{array}$ & $\begin{array}{l}3 \\
8 \\
\end{array}$ & $\begin{array}{l}0.08 \\
230\end{array}$ & $\begin{array}{c}0 . \\
00 \\
18 \\
6 \\
\end{array}$ & $\begin{array}{c}0.20 \\
533\end{array}$ & $\begin{array}{c}0 . \\
00 \\
43 \\
2\end{array}$ & $\begin{array}{l}2.33 \\
038\end{array}$ & $\begin{array}{c}0 . \\
04 \\
83 \\
6\end{array}$ & $\begin{array}{c}125 \\
3\end{array}$ & $\begin{array}{l}1 \\
9\end{array}$ & $\begin{array}{c}120 \\
4\end{array}$ & $\begin{array}{l}2 \\
3\end{array}$ & $\begin{array}{c}122 \\
2\end{array}$ & $\begin{array}{l}1 \\
5\end{array}$ & $\begin{array}{l}4.0 \\
7 \%\end{array}$ & $\begin{array}{l}1 \\
2 \\
5 \\
3 \\
\end{array}$ & $\begin{array}{l}1 \\
9\end{array}$ \\
\hline $\begin{array}{c}\mathrm{M} \\
\mathrm{O} 1 \\
6- \\
20 \\
\end{array}$ & $\begin{array}{l}7 \\
4\end{array}$ & $\begin{array}{l}2 \\
5 \\
2\end{array}$ & $\begin{array}{l}0 \\
2 \\
9\end{array}$ & $\begin{array}{c}0.06 \\
107\end{array}$ & $\begin{array}{c}0 . \\
00 \\
19 \\
6 \\
\end{array}$ & $\begin{array}{l}0.07 \\
425\end{array}$ & $\begin{array}{c}0 . \\
00 \\
16 \\
6 \\
\end{array}$ & $\begin{array}{l}0.62 \\
529\end{array}$ & $\begin{array}{c}0 . \\
01 \\
84 \\
0 \\
\end{array}$ & 642 & $\begin{array}{l}3 \\
0\end{array}$ & 462 & $\begin{array}{l}1 \\
0\end{array}$ & 493 & $\begin{array}{l}1 \\
1\end{array}$ & $\begin{array}{l}6.7 \\
1 \%\end{array}$ & $\begin{array}{l}4 \\
6 \\
2\end{array}$ & $\begin{array}{l}1 \\
0\end{array}$ \\
\hline $\begin{array}{c}\mathrm{M} \\
\mathrm{O} 1 \\
6- \\
21\end{array}$ & $\begin{array}{l}1 \\
7 \\
1\end{array}$ & $\begin{array}{l}2 \\
4 \\
4\end{array}$ & $\begin{array}{l}7 \\
0\end{array}$ & $\begin{array}{l}0.06 \\
012\end{array}$ & $\begin{array}{c}0 . \\
00 \\
22 \\
0\end{array}$ & $\begin{array}{l}0.07 \\
530\end{array}$ & $\begin{array}{c}0 . \\
00 \\
17 \\
7\end{array}$ & $\begin{array}{l}0.62 \\
421\end{array}$ & $\begin{array}{c}0 . \\
02 \\
10 \\
4\end{array}$ & 608 & $\begin{array}{l}3 \\
6\end{array}$ & 468 & $\begin{array}{l}1 \\
1\end{array}$ & 492 & $\begin{array}{l}1 \\
3\end{array}$ & $\begin{array}{l}5.1 \\
3 \%\end{array}$ & $\begin{array}{l}4 \\
6 \\
8\end{array}$ & $\begin{array}{l}1 \\
1\end{array}$ \\
\hline $\begin{array}{c}\mathrm{M} \\
\mathrm{O} 1 \\
6- \\
22\end{array}$ & \begin{tabular}{l|}
1 \\
1 \\
1
\end{tabular} & $\begin{array}{l}3 \\
7 \\
4\end{array}$ & $\begin{array}{l}3 \\
0\end{array}$ & $\begin{array}{l}0.05 \\
898\end{array}$ & $\begin{array}{c}0 . \\
00 \\
16 \\
2\end{array}$ & $\begin{array}{l}0.07 \\
748\end{array}$ & $\begin{array}{c}0 . \\
00 \\
16 \\
1\end{array}$ & $\begin{array}{c}0.63 \\
009\end{array}$ & $\begin{array}{c}0 . \\
01 \\
59 \\
9\end{array}$ & 566 & $\begin{array}{l}2 \\
5\end{array}$ & 481 & $\begin{array}{l}1 \\
0\end{array}$ & 496 & $\begin{array}{l}1 \\
0\end{array}$ & $\begin{array}{l}3.1 \\
2 \%\end{array}$ & $\begin{array}{l}4 \\
8 \\
1\end{array}$ & $\begin{array}{l}1 \\
0\end{array}$ \\
\hline $\begin{array}{c}\mathrm{M} \\
\mathrm{O} 1 \\
6- \\
23 \\
\end{array}$ & $\begin{array}{l}3 \\
8\end{array}$ & $\begin{array}{l}1 \\
2 \\
4\end{array}$ & $\begin{array}{l}0 \\
3 \\
1 \\
\end{array}$ & $\begin{array}{l}0.05 \\
660\end{array}$ & $\begin{array}{c}0 . \\
00 \\
26 \\
8 \\
\end{array}$ & $\begin{array}{c}0.08 \\
109\end{array}$ & $\begin{array}{c}0 . \\
00 \\
21 \\
1 \\
\end{array}$ & $\begin{array}{l}0.63 \\
282\end{array}$ & $\begin{array}{c}0 . \\
02 \\
79 \\
3 \\
\end{array}$ & 476 & $\begin{array}{l}5 \\
3\end{array}$ & 503 & $\begin{array}{l}1 \\
3\end{array}$ & 498 & $\begin{array}{l}1 \\
7\end{array}$ & $\begin{array}{c}- \\
0.9 \\
9 \%\end{array}$ & $\begin{array}{l}5 \\
0 \\
3\end{array}$ & $\begin{array}{l}1 \\
3\end{array}$ \\
\hline $\begin{array}{c}\mathrm{M} \\
\mathrm{O} 1 \\
6- \\
24\end{array}$ & $\begin{array}{l}1 \\
4 \\
9\end{array}$ & $\begin{array}{l}2 \\
2 \\
8\end{array}$ & $\begin{array}{l}0 \\
6 \\
5\end{array}$ & $\begin{array}{l}0.11 \\
913\end{array}$ & $\begin{array}{c}0 . \\
00 \\
19 \\
0\end{array}$ & $\begin{array}{c}0.33 \\
163\end{array}$ & $\begin{array}{c}0 . \\
00 \\
65 \\
0\end{array}$ & $\begin{array}{l}5.44 \\
770\end{array}$ & $\begin{array}{c}0 . \\
08 \\
03 \\
2\end{array}$ & $\begin{array}{c}194 \\
3\end{array}$ & $\begin{array}{l}1 \\
7\end{array}$ & $\begin{array}{c}184 \\
6\end{array}$ & $\begin{array}{l}3 \\
1\end{array}$ & $\begin{array}{c}189 \\
2\end{array}$ & $\begin{array}{l}1 \\
3\end{array}$ & $\begin{array}{l}5.2 \\
5 \%\end{array}$ & $\begin{array}{l}1 \\
9 \\
4 \\
3\end{array}$ & $\begin{array}{l}1 \\
7\end{array}$ \\
\hline $\begin{array}{c}\mathrm{M} \\
\mathrm{O} 1 \\
6- \\
25 \\
\end{array}$ & $\begin{array}{l}1 \\
5\end{array}$ & $\begin{array}{l}4 \\
1\end{array}$ & $\begin{array}{l}0 \\
3 \\
7 \\
\end{array}$ & $\begin{array}{l}0.05 \\
904\end{array}$ & $\begin{array}{c}0 . \\
00 \\
50 \\
1 \\
\end{array}$ & $\begin{array}{l}0.08 \\
152\end{array}$ & $\begin{array}{c}0 . \\
00 \\
28 \\
9 \\
\end{array}$ & $\begin{array}{l}0.66 \\
368\end{array}$ & $\begin{array}{c}0 . \\
05 \\
32 \\
7\end{array}$ & 569 & $\begin{array}{l}1 \\
1 \\
3\end{array}$ & 505 & $\begin{array}{l}1 \\
7\end{array}$ & 517 & $\begin{array}{l}3 \\
3\end{array}$ & $\begin{array}{l}2.3 \\
8 \%\end{array}$ & $\begin{array}{l}5 \\
0 \\
5\end{array}$ & $\begin{array}{l}1 \\
7\end{array}$ \\
\hline $\begin{array}{c}\mathrm{M} \\
\mathrm{O} 1 \\
6- \\
26 \\
\end{array}$ & $\begin{array}{l}4 \\
6\end{array}$ & $\begin{array}{l}6 \\
8\end{array}$ & $\begin{array}{l}0 \\
6 \\
8 \\
\end{array}$ & $\begin{array}{c}0.06 \\
485\end{array}$ & $\begin{array}{c}0 . \\
00 \\
40 \\
2 \\
\end{array}$ & $\begin{array}{c}0.09 \\
115\end{array}$ & $\begin{array}{c}0 . \\
00 \\
28 \\
4 \\
\end{array}$ & $\begin{array}{l}0.81 \\
511\end{array}$ & $\begin{array}{c}0 . \\
04 \\
69 \\
9 \\
\end{array}$ & 769 & $\begin{array}{l}7 \\
0\end{array}$ & 562 & $\begin{array}{l}1 \\
7\end{array}$ & 605 & $\begin{array}{l}2 \\
6\end{array}$ & $\begin{array}{l}7.6 \\
5 \%\end{array}$ & $\begin{array}{l}5 \\
6 \\
2\end{array}$ & $\begin{array}{l}1 \\
7\end{array}$ \\
\hline $\begin{array}{c}\mathrm{M} \\
\mathrm{O} 1 \\
6- \\
27 \\
\end{array}$ & $\begin{array}{l}6 \\
8\end{array}$ & $\begin{array}{l}1 \\
6 \\
5\end{array}$ & $\begin{array}{l}0 \\
4 \\
4 \\
1\end{array}$ & $\begin{array}{l}0.05 \\
977\end{array}$ & $\begin{array}{c}0 . \\
00 \\
25 \\
1 \\
\end{array}$ & $\begin{array}{l}0.07 \\
871\end{array}$ & $\begin{array}{c}0 . \\
00 \\
19 \\
7 \\
\end{array}$ & $\begin{array}{l}0.64 \\
865\end{array}$ & $\begin{array}{c}0 . \\
02 \\
51 \\
5 \\
\end{array}$ & 595 & $\begin{array}{l}4 \\
3\end{array}$ & 488 & $\begin{array}{l}1 \\
2\end{array}$ & 508 & $\begin{array}{l}1 \\
5\end{array}$ & $\begin{array}{l}4.1 \\
0 \%\end{array}$ & $\begin{array}{l}4 \\
8 \\
8\end{array}$ & $\begin{array}{l}1 \\
2\end{array}$ \\
\hline $\begin{array}{l}\mathrm{M} \\
\mathrm{O} 1 \\
\end{array}$ & $\begin{array}{l}1 \\
3 \\
\end{array}$ & $\begin{array}{l}2 \\
5 \\
\end{array}$ & 0 & $\begin{array}{c}0.06 \\
949 \\
\end{array}$ & $\begin{array}{c}0 . \\
00 \\
\end{array}$ & $\begin{array}{l}0.15 \\
214 \\
\end{array}$ & $\begin{array}{c}0 . \\
00 \\
\end{array}$ & $\begin{array}{l}1.45 \\
787 \\
\end{array}$ & $\begin{array}{c}0 . \\
03\end{array}$ & 913 & $\begin{array}{l}2 \\
2 \\
\end{array}$ & 913 & $\begin{array}{l}1 \\
8 \\
\end{array}$ & 913 & $\begin{array}{l}1 \\
4 \\
\end{array}$ & $\begin{array}{l}0.0 \\
0 \% \\
\end{array}$ & $\begin{array}{l}9 \\
1 \\
\end{array}$ & $\begin{array}{l}1 \\
8 \\
\end{array}$ \\
\hline
\end{tabular}




\begin{tabular}{|c|c|c|c|c|c|c|c|c|c|c|c|c|c|c|c|c|c|c|}
\hline $\begin{array}{l}6- \\
28\end{array}$ & 3 & 6 & $\begin{array}{l}5 \\
2\end{array}$ & & $\begin{array}{c}17 \\
8\end{array}$ & & $\begin{array}{c}32 \\
3\end{array}$ & & $\begin{array}{c}44 \\
4\end{array}$ & & & & & & & & 3 & \\
\hline $\mathrm{M}$ & 7 & 1 & 0 & 0.11 & 0. & 0.31 & 0. & 4.99 & 0. & 188 & 1 & 176 & 3 & 181 & 1 & 7.1 & 1 & 1 \\
\hline $\mathrm{O} 1$ & 1 & 1 & . & 540 & 00 & 383 & 00 & 341 & 10 & 6 & 8 & 0 & 5 & 8 & 8 & $6 \%$ & 8 & 8 \\
\hline $\begin{array}{l}6- \\
29\end{array}$ & & 1 & $\begin{array}{l}6 \\
4\end{array}$ & & $\begin{array}{c}26 \\
4\end{array}$ & & $\begin{array}{c}70 \\
6\end{array}$ & & $\begin{array}{c}53 \\
9\end{array}$ & & & & & & & & $\begin{array}{l}8 \\
6\end{array}$ & \\
\hline $\mathrm{M}$ & 2 & 1 & 1 & 0.06 & 0. & 0.10 & 0. & 0.86 & 0. & 689 & 3 & 619 & 1 & 635 & 1 & 2.5 & 6 & 1 \\
\hline O1 & 1 & 7 & . & 243 & 00 & 083 & 00 & 805 & 02 & & 3 & & 4 & & 5 & $8 \%$ & 1 & 4 \\
\hline 6- & 2 & 6 & 2 & & 21 & & 23 & & 77 & & & & & & & & 9 & \\
\hline 30 & & & 0 & & 6 & & 3 & & 6 & & & & & & & & & \\
\hline $\mathrm{M}$ & 7 & 2 & 0 & 0.06 & 0 & 0.09 & 0. & 0.78 & 0 & 696 & 3 & 564 & 1 & 591 & 1 & 4.7 & & \\
\hline $\mathrm{O} 1$ & 2 & 0 & . & 264 & 00 & 140 & 00 & 934 & 02 & & 3 & & 3 & & 5 & $9 \%$ & & \\
\hline 6- & & 1 & 3 & & 22 & & 21 & & 56 & & & & & & & & & \\
\hline 31 & & & 6 & & 1 & & 3 & & 4 & & . & & & & & & & \\
\hline $\mathrm{M}$ & 1 & 1 & 0 & 0.05 & 0. & 0.05 & 0. & 0.42 & 0. & 368 & 1 & 357 & 7 & 359 & 5 & 0.5 & 3 & 7 \\
\hline $\mathrm{O} 1$ & 9 & 5 & . & 393 & 00 & 697 & 00 & 360 & 00 & & 9 & & & & & $6 \%$ & 5 & \\
\hline $6-$ & 8 & 3 & 1 & & 10 & & 10 & & 73 & & & & & & & & 7 & \\
\hline 32 & & 7 & 3 & & 2 & & 7 & & 9 & & & & & & & & & \\
\hline $\mathrm{M}$ & 8 & 2 & 0 & 0.13 & 0. & 0.36 & 0. & 6.69 & 0. & 212 & 1 & 202 & 3 & 207 & 1 & 5.1 & 2 & 1 \\
\hline $\mathrm{O} 1$ & 7 & 5 & ${ }^{\circ}$ & 195 & 00 & 823 & 00 & 925 & 09 & 4 & 6 & 1 & 4 & 3 & 3 & $0 \%$ & 1 & 6 \\
\hline $6-$ & & 1 & 3 & & 20 & & 72 & & 74 & & & & & & & & 2 & \\
\hline 33 & & & 4 & & 7 & & 5 & & 8 & & & & & & & & 4 & \\
\hline $\mathrm{M}$ & 7 & 1 & 0 & 0.05 & 0. & 0.05 & 0. & 0.43 & 0. & 359 & 7 & 367 & 1 & 366 & 1 & - & 3 & 1 \\
\hline $\mathrm{O} 1$ & 7 & 6 & $\cdot$ & 372 & 00 & 861 & 00 & 408 & 02 & & 2 & & 0 & & 6 & 0.2 & 6 & 0 \\
\hline 6- & & 5 & 4 & & $\begin{array}{c}30 \\
5\end{array}$ & & 16 & & 31 & & & & & & & $7 \%$ & 7 & \\
\hline $\begin{array}{l}34 \\
\mathrm{M}\end{array}$ & 3 & 7 & $\begin{array}{l}7 \\
0\end{array}$ & 0.05 & $\begin{array}{r}5 \\
0 .\end{array}$ & 0.07 & $\frac{0}{0 .}$ & 0.56 & $\begin{array}{c}4 \\
0 .\end{array}$ & 450 & 2 & 455 & 9 & 454 & 1 & - & 4 & 9 \\
\hline $\mathrm{O} 1$ & 4 & 9 & 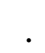 & 595 & 00 & 309 & 00 & 386 & 01 & & 7 & & & & 0 & 0.2 & 5 & \\
\hline $6-$ & 1 & 5 & 4 & & 16 & & 15 & & 49 & & & & & & & $2 \%$ & 5 & \\
\hline 35 & & & 3 & & 1 & & 4 & & 8 & & & & & & & & & \\
\hline $\mathrm{M}$ & 4 & 8 & 0 & 0.05 & 0. & 0.07 & 0. & 0.61 & 0. & 581 & 1 & 465 & 9 & 485 & 7 & 4.3 & 4 & 9 \\
\hline $\mathrm{O} 1$ & 0 & 7 & . & 938 & 00 & 475 & 00 & 199 & 01 & & 9 & & & & & $0 \%$ & 6 & \\
\hline $6-$ & 3 & 4 & 4 & & 12 & & 14 & & 13 & & & & & & & & 5 & \\
\hline 36 & & & 6 & 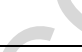 & 1 & & 4 & & 8 & & & & & & & & & \\
\hline $\mathrm{M}$ & 6 & 2 & 0 & 0.05 & 0. & 0.08 & 0. & 0.67 & 0. & 449 & 4 & 540 & 1 & 523 & 1 & - & 5 & 1 \\
\hline $\mathrm{O} 1$ & 7 & 1 & . & 591 & 00 & 742 & 00 & 395 & 02 & & 0 & & 2 & & 5 & 3.1 & 4 & 2 \\
\hline $6-$ & & 8 & 3 & 0 & 21 & & 20 & & 39 & & & & & & & $5 \%$ & 0 & \\
\hline 37 & & & 1 & & 5 & & 6 & & 9 & & & & & & & & & \\
\hline $\mathrm{M}$ & 6 & 1 & 0 & 0.06 & 0. & 0.08 & 0. & 0.75 & 0. & 652 & 3 & 553 & 1 & 573 & 1 & 3.6 & & \\
\hline $\mathrm{O} 1$ & 4 & 9 & . & 137 & 00 & 951 & 00 & 740 & 02 & & 9 & & 3 & & 6 & $2 \%$ & & \\
\hline 6- & & 5 & 3 & & 23 & & 21 & & 72 & & & & & & & & & \\
\hline 38 & & & 3 & & 9 & & 7 & & 3 & & & & & & & & & \\
\hline $\mathrm{M}$ & 7 & 1 & 0 & 0.05 & 0 & 0.05 & 0. & 0.40 & 0. & 378 & 6 & 341 & 9 & 346 & 1 & 1.4 & 3 & 9 \\
\hline O1 & 3 & 8 & . & 416 & 00 & 432 & 00 & 566 & 01 & & 2 & & & & 4 & $7 \%$ & 4 & \\
\hline 6- & & 5 & 3 & & 28 & & 14 & & 96 & & & & & & & & 1 & \\
\hline 39 & & & 9 & & 3 & & 7 & & 8 & & & & & & & & & \\
\hline $\mathrm{M}$ & 1 & 2 & 0 & 0.05 & 0 & 0.05 & 0. & 0.44 & 0. & 537 & 4 & 348 & 8 & 374 & 1 & 7.4 & 3 & 8 \\
\hline O1 & 2 & 4 & . & 820 & 00 & 549 & 00 & 529 & 01 & & 2 & & & & 2 & $7 \%$ & 4 & \\
\hline 6- & 5 & 3 & 5 & & 23 & & 13 & & 66 & & & & & & & & 8 & \\
\hline 40 & & & 1 & & 6 & & 4 & & 6 & & & & & & & & & \\
\hline $\mathrm{M}$ & 4 & 1 & 0 & 0.05 & 0 & 0.07 & 0. & 0.57 & 0 & 445 & 5 & 463 & 1 & 460 & 1 & - & 4 & 1 \\
\hline $\mathrm{O} 1$ & 3 & 8 & ${ }^{\circ}$ & 582 & 00 & 452 & 00 & 358 & 02 & & 3 & & 1 & & 6 & 0.6 & 6 & 1 \\
\hline $6-$ & & 1 & 2 & & 25 & & 18 & & 45 & & & & & & & $5 \%$ & 3 & \\
\hline 41 & & & 4 & & 6 & & 6 & & 7 & & & & & & & & & \\
\hline
\end{tabular}




\begin{tabular}{|c|c|c|c|c|c|c|c|c|c|c|c|c|c|c|c|c|c|c|}
\hline $\begin{array}{c}\mathrm{M} \\
\mathrm{O} 1 \\
6- \\
42\end{array}$ & \begin{tabular}{l|}
1 \\
1 \\
4
\end{tabular} & $\begin{array}{l}7 \\
5\end{array}$ & $\begin{array}{l}5 \\
1\end{array}$ & $\begin{array}{l}0.06 \\
745\end{array}$ & $\begin{array}{c}0 . \\
00 \\
46 \\
7\end{array}$ & $\begin{array}{c}0.06 \\
186\end{array}$ & $\begin{array}{c}0 . \\
00 \\
21 \\
1\end{array}$ & $\begin{array}{l}0.57 \\
530\end{array}$ & $\begin{array}{c}0 . \\
03 \\
66 \\
8\end{array}$ & 852 & $\begin{array}{l}7 \\
7\end{array}$ & 387 & $\begin{array}{l}1 \\
3\end{array}$ & 461 & $\begin{array}{l}2 \\
4\end{array}$ & $\begin{array}{l}19 . \\
12 \\
\%\end{array}$ & & \\
\hline $\begin{array}{c}\mathrm{M} \\
\mathrm{O} 1 \\
6- \\
43\end{array}$ & \begin{tabular}{l|}
1 \\
2 \\
3
\end{tabular} & $\begin{array}{l}2 \\
4 \\
0\end{array}$ & $\begin{array}{l}0 \\
5 \\
5 \\
1\end{array}$ & $\begin{array}{c}0.05 \\
518\end{array}$ & $\begin{array}{c}0 . \\
00 \\
22 \\
5\end{array}$ & $\begin{array}{l}0.05 \\
516\end{array}$ & $\begin{array}{c}0 . \\
00 \\
13 \\
1 \\
\end{array}$ & $\begin{array}{l}0.41 \\
969\end{array}$ & $\begin{array}{c}0 . \\
01 \\
58 \\
7\end{array}$ & 420 & $\begin{array}{l}4 \\
4\end{array}$ & 346 & 8 & 356 & $\begin{array}{l}1 \\
1\end{array}$ & $\begin{array}{l}2.8 \\
9 \%\end{array}$ & $\begin{array}{l}3 \\
4 \\
6\end{array}$ & 8 \\
\hline $\begin{array}{c}\mathrm{M} \\
\mathrm{O} 1 \\
6- \\
44\end{array}$ & $\begin{array}{l}3 \\
4\end{array}$ & $\begin{array}{l}2 \\
9 \\
2\end{array}$ & $\begin{array}{l}0 \\
1 \\
2\end{array}$ & $\begin{array}{c}0.15 \\
098\end{array}$ & $\begin{array}{c}0 . \\
00 \\
22 \\
4\end{array}$ & $\begin{array}{l}0.23 \\
855\end{array}$ & $\begin{array}{c}0 . \\
00 \\
45 \\
8\end{array}$ & $\begin{array}{l}4.96 \\
558\end{array}$ & $\begin{array}{c}0 . \\
06 \\
60 \\
2\end{array}$ & $\begin{array}{c}232 \\
6\end{array}$ & $\begin{array}{l}4 \\
0\end{array}$ & $\begin{array}{c}137 \\
4\end{array}$ & $\begin{array}{l}2 \\
3\end{array}$ & $\begin{array}{c}179 \\
5\end{array}$ & $\begin{array}{l}1 \\
1\end{array}$ & $\begin{array}{l}69 . \\
29 \\
\%\end{array}$ & & \\
\hline $\begin{array}{c}\mathrm{M} \\
\mathrm{O} 1 \\
6- \\
45\end{array}$ & $\begin{array}{l}5 \\
5\end{array}$ & $\begin{array}{l}2 \\
2 \\
6\end{array}$ & $\begin{array}{l}2 \\
4\end{array}$ & $\begin{array}{l}0.05 \\
834\end{array}$ & $\begin{array}{c}0 . \\
00 \\
18 \\
7\end{array}$ & $\begin{array}{l}0.07 \\
382\end{array}$ & $\begin{array}{c}0 . \\
00 \\
16 \\
3\end{array}$ & $\begin{array}{l}0.59 \\
378\end{array}$ & $\begin{array}{c}0 . \\
01 \\
75 \\
4\end{array}$ & 543 & $\begin{array}{l}3 \\
1\end{array}$ & 459 & $\begin{array}{l}1 \\
0\end{array}$ & 473 & $\begin{array}{l}1 \\
1\end{array}$ & $\begin{array}{l}3.0 \\
5 \%\end{array}$ & $\begin{array}{l}4 \\
5 \\
9\end{array}$ & $\begin{array}{l}1 \\
0\end{array}$ \\
\hline $\begin{array}{c}\mathrm{M} \\
\mathrm{O} 1 \\
6- \\
46\end{array}$ & $\begin{array}{l}1 \\
7 \\
4\end{array}$ & $\begin{array}{l}2 \\
9 \\
3\end{array}$ & $\begin{array}{l}5 \\
9 \\
\end{array}$ & $\begin{array}{l}0.06 \\
077\end{array}$ & $\begin{array}{c}0 . \\
00 \\
20 \\
6\end{array}$ & $\begin{array}{l}0.09 \\
244\end{array}$ & $\begin{array}{c}0 . \\
00 \\
21 \\
4\end{array}$ & $\begin{array}{l}0.77 \\
447\end{array}$ & $\begin{array}{c}0 . \\
02 \\
40 \\
5\end{array}$ & 631 & $\begin{array}{l}3 \\
2\end{array}$ & 570 & $\begin{array}{l}1 \\
3\end{array}$ & 582 & $\begin{array}{l}1 \\
4\end{array}$ & $\begin{array}{l}2.1 \\
1 \%\end{array}$ & $\begin{array}{l}5 \\
7 \\
0\end{array}$ & $\begin{array}{l}1 \\
3\end{array}$ \\
\hline $\begin{array}{c}\mathrm{M} \\
\mathrm{O} 1 \\
6- \\
47\end{array}$ & $\begin{array}{l}2 \\
0 \\
4\end{array}$ & $\begin{array}{l}2 \\
5 \\
6\end{array}$ & $\begin{array}{l}8 \\
0\end{array}$ & $\begin{array}{l}0.05 \\
971\end{array}$ & $\begin{array}{c}0 . \\
00 \\
15 \\
5\end{array}$ & $\begin{array}{l}0.09 \\
810\end{array}$ & $\begin{array}{c}0 . \\
00 \\
20 \\
3\end{array}$ & $\begin{array}{c}0.80 \\
762\end{array}$ & $\begin{array}{c}0 . \\
01 \\
92 \\
8\end{array}$ & 593 & $\begin{array}{l}2 \\
3\end{array}$ & 603 & $\begin{array}{l}1 \\
2\end{array}$ & 601 & $\begin{array}{l}1 \\
1\end{array}$ & $\begin{array}{c}- \\
0.3 \\
3 \%\end{array}$ & $\begin{array}{l}6 \\
0 \\
3\end{array}$ & $\begin{array}{l}1 \\
2\end{array}$ \\
\hline $\begin{array}{c}\mathrm{M} \\
\mathrm{O} 1 \\
6- \\
48 \\
\end{array}$ & $\begin{array}{l}1 \\
1 \\
1\end{array}$ & $\begin{array}{l}1 \\
2 \\
9\end{array}$ & $\begin{array}{l}0 \\
8 \\
8 \\
6\end{array}$ & $\begin{array}{l}0.10 \\
533\end{array}$ & $\begin{array}{c}0 . \\
00 \\
18 \\
6 \\
\end{array}$ & $\begin{array}{l}0.27 \\
986\end{array}$ & $\begin{array}{c}0 . \\
00 \\
55 \\
7 \\
\end{array}$ & $\begin{array}{r}4.06 \\
427\end{array}$ & $\begin{array}{c}0 . \\
06 \\
59 \\
5\end{array}$ & $\begin{array}{c}172 \\
0\end{array}$ & $\begin{array}{l}1 \\
7\end{array}$ & $\begin{array}{c}159 \\
1\end{array}$ & $\begin{array}{l}2 \\
8\end{array}$ & $\begin{array}{c}164 \\
7\end{array}$ & $\begin{array}{l}1 \\
3\end{array}$ & $\begin{array}{l}8.1 \\
1 \%\end{array}$ & $\begin{array}{l}1 \\
7 \\
2 \\
0 \\
\end{array}$ & $\begin{array}{l}1 \\
7\end{array}$ \\
\hline $\begin{array}{c}\mathrm{M} \\
\mathrm{O} 1 \\
6- \\
49\end{array}$ & 0 & $\begin{array}{l}1 \\
4\end{array}$ & $\begin{array}{l}0 \\
0 \\
3\end{array}$ & $\begin{array}{c}0.06 \\
578\end{array}$ & $\begin{array}{c}0 . \\
01 \\
14 \\
7\end{array}$ & $\begin{array}{l}0.05 \\
356\end{array}$ & $\begin{array}{c}0 . \\
00 \\
31 \\
5\end{array}$ & $\begin{array}{c}0.48 \\
575\end{array}$ & $\begin{array}{c}0 . \\
08 \\
08 \\
7\end{array}$ & 799 & $\begin{array}{l}2 \\
5 \\
6\end{array}$ & 336 & $\begin{array}{l}1 \\
9\end{array}$ & 402 & $\begin{array}{l}5 \\
5\end{array}$ & $\begin{array}{l}19 . \\
64 \\
\%\end{array}$ & & \\
\hline $\begin{array}{c}\mathrm{M} \\
\mathrm{O} 1 \\
6- \\
50 \\
\end{array}$ & $\begin{array}{l}6 \\
9\end{array}$ & $\begin{array}{l}9 \\
0\end{array}$ & $\begin{array}{l}7 \\
7\end{array}$ & $\begin{array}{l}0.12 \\
530\end{array}$ & $\begin{array}{c}0 . \\
00 \\
33 \\
0 \\
\end{array}$ & $\begin{array}{l}0.30 \\
359\end{array}$ & $\begin{array}{c}0 . \\
00 \\
73 \\
9 \\
\end{array}$ & $\begin{array}{l}5.24 \\
459\end{array}$ & $\begin{array}{c}0 . \\
12 \\
63 \\
1 \\
\end{array}$ & $\begin{array}{c}203 \\
3\end{array}$ & $\begin{array}{l}2 \\
0\end{array}$ & $\begin{array}{c}170 \\
9\end{array}$ & $\begin{array}{l}3 \\
7\end{array}$ & $\begin{array}{c}186 \\
0\end{array}$ & $\begin{array}{l}2 \\
1\end{array}$ & $\begin{array}{l}18 . \\
96 \\
\%\end{array}$ & & \\
\hline $\begin{array}{c}\mathrm{M} \\
\mathrm{O} 1 \\
6- \\
51\end{array}$ & $\begin{array}{l}7 \\
0\end{array}$ & $\begin{array}{l}7 \\
6\end{array}$ & $\begin{array}{l}0 \\
5 \\
9 \\
\end{array}$ & $\begin{array}{c}0.13 \\
393\end{array}$ & $\begin{array}{c}0 . \\
00 \\
29 \\
8 \\
\end{array}$ & $\begin{array}{l}0.37 \\
241\end{array}$ & $\begin{array}{c}0 . \\
00 \\
85 \\
2 \\
\end{array}$ & $\begin{array}{l}6.87 \\
654\end{array}$ & $\begin{array}{c}0 . \\
14 \\
20 \\
5\end{array}$ & $\begin{array}{c}215 \\
0\end{array}$ & $\begin{array}{l}1 \\
8\end{array}$ & $\begin{array}{c}204 \\
1\end{array}$ & $\begin{array}{l}4 \\
0\end{array}$ & $\begin{array}{c}209 \\
6\end{array}$ & $\begin{array}{l}1 \\
8\end{array}$ & $\begin{array}{l}5.3 \\
4 \%\end{array}$ & $\begin{array}{l}2 \\
1 \\
5 \\
0 \\
\end{array}$ & $\begin{array}{l}1 \\
8\end{array}$ \\
\hline $\begin{array}{c}\mathrm{M} \\
\mathrm{O} 1 \\
6- \\
52\end{array}$ & $\begin{array}{l}1 \\
4 \\
0\end{array}$ & $\begin{array}{l}2 \\
6 \\
2\end{array}$ & $\begin{array}{l}5 \\
3 \\
\end{array}$ & $\begin{array}{l}0.05 \\
393\end{array}$ & $\begin{array}{c}0 . \\
00 \\
18 \\
8\end{array}$ & $\begin{array}{l}0.05 \\
414\end{array}$ & $\begin{array}{c}0 . \\
00 \\
12 \\
1\end{array}$ & $\begin{array}{l}0.40 \\
249\end{array}$ & $\begin{array}{c}0 . \\
01 \\
30 \\
0\end{array}$ & 368 & $\begin{array}{l}3 \\
6\end{array}$ & 340 & 7 & 343 & 9 & $\begin{array}{l}0.8 \\
8 \%\end{array}$ & $\begin{array}{l}3 \\
4 \\
0\end{array}$ & 7 \\
\hline $\begin{array}{c}\mathrm{M} \\
\mathrm{O} 1 \\
6- \\
53 \\
\end{array}$ & 1 & $\begin{array}{l}2 \\
4 \\
1\end{array}$ & $\begin{array}{l}0 \\
. \\
0 \\
0\end{array}$ & $\begin{array}{l}0.06 \\
237\end{array}$ & $\begin{array}{c}0 . \\
00 \\
17 \\
4 \\
\end{array}$ & $\begin{array}{c}0.11 \\
147\end{array}$ & $\begin{array}{c}0 . \\
00 \\
23 \\
9 \\
\end{array}$ & $\begin{array}{l}0.95 \\
846\end{array}$ & $\begin{array}{c}0 . \\
02 \\
44 \\
9 \\
\end{array}$ & 687 & $\begin{array}{l}2 \\
5\end{array}$ & 681 & $\begin{array}{l}1 \\
4\end{array}$ & 682 & $\begin{array}{l}1 \\
3\end{array}$ & $\begin{array}{l}0.1 \\
5 \%\end{array}$ & $\begin{array}{l}6 \\
8 \\
1\end{array}$ & $\begin{array}{l}1 \\
4\end{array}$ \\
\hline $\begin{array}{c}\mathrm{M} \\
\mathrm{O} 1 \\
6- \\
54 \\
\end{array}$ & $\begin{array}{l}4 \\
4\end{array}$ & $\begin{array}{l}1 \\
4 \\
6\end{array}$ & $\begin{array}{l}0 \\
3 \\
3 \\
0\end{array}$ & $\begin{array}{c}0.14 \\
032\end{array}$ & $\begin{array}{c}0 . \\
00 \\
24 \\
9 \\
\end{array}$ & $\begin{array}{l}0.24 \\
782\end{array}$ & $\begin{array}{c}0 . \\
00 \\
50 \\
2 \\
\end{array}$ & $\begin{array}{l}4.79 \\
411\end{array}$ & $\begin{array}{c}0 . \\
07 \\
63 \\
1 \\
\end{array}$ & $\begin{array}{c}222 \\
2\end{array}$ & $\begin{array}{l}4 \\
6\end{array}$ & $\begin{array}{c}142 \\
6\end{array}$ & $\begin{array}{l}2 \\
5\end{array}$ & $\begin{array}{c}177 \\
9\end{array}$ & $\begin{array}{l}1 \\
4\end{array}$ & $\begin{array}{l}55 . \\
82 \\
\%\end{array}$ & & \\
\hline $\begin{array}{l}\mathrm{M} \\
\mathrm{O} 1 \\
\end{array}$ & $\begin{array}{l}1 \\
0 \\
\end{array}$ & $\begin{array}{l}2 \\
0 \\
\end{array}$ & 0 & $\begin{array}{l}0.06 \\
238 \\
\end{array}$ & $\begin{array}{c}0 . \\
00 \\
\end{array}$ & $\begin{array}{l}0.09 \\
992 \\
\end{array}$ & $\begin{array}{c}0 . \\
00\end{array}$ & $\begin{array}{l}0.85 \\
929 \\
\end{array}$ & $\begin{array}{c}0 . \\
02 \\
\end{array}$ & 687 & $\begin{array}{l}3 \\
0 \\
\end{array}$ & 614 & $\begin{array}{l}1 \\
3 \\
\end{array}$ & 630 & $\begin{array}{l}1 \\
4 \\
\end{array}$ & $\begin{array}{l}2.6 \\
1 \% \\
\end{array}$ & $\begin{array}{l}6 \\
1 \\
\end{array}$ & $\begin{array}{l}1 \\
3 \\
\end{array}$ \\
\hline
\end{tabular}




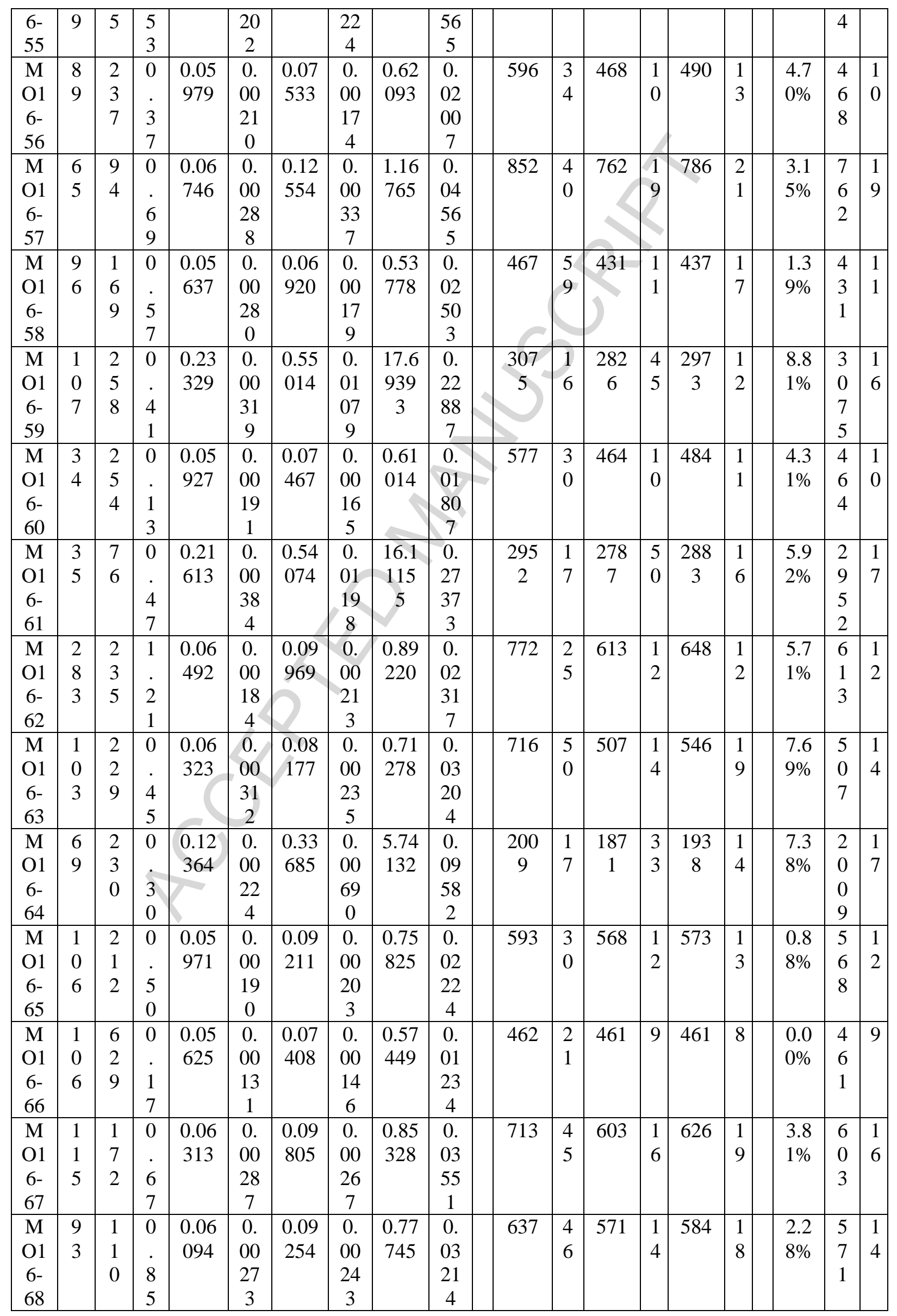




\begin{tabular}{|c|c|c|c|c|c|c|c|c|c|c|c|c|c|c|c|c|c|c|}
\hline $\begin{array}{c}\mathrm{M} \\
\mathrm{O} 1 \\
6- \\
69\end{array}$ & $\begin{array}{l}5 \\
7\end{array}$ & $\begin{array}{l}9 \\
1\end{array}$ & $\begin{array}{l}0 \\
6 \\
6 \\
3\end{array}$ & $\begin{array}{c}0.06 \\
001\end{array}$ & $\begin{array}{c}0 . \\
00 \\
32 \\
2\end{array}$ & $\begin{array}{c}0.10 \\
071\end{array}$ & $\begin{array}{c}0 . \\
00 \\
28 \\
9\end{array}$ & $\begin{array}{l}0.83 \\
316\end{array}$ & $\begin{array}{c}0 . \\
04 \\
16 \\
2\end{array}$ & 604 & $\begin{array}{l}6 \\
0\end{array}$ & 619 & $\begin{array}{l}1 \\
7\end{array}$ & 615 & $\begin{array}{l}2 \\
3\end{array}$ & $\begin{array}{c}- \\
0.6 \\
5 \%\end{array}$ & $\begin{array}{l}6 \\
1 \\
9\end{array}$ & $\begin{array}{l}1 \\
7\end{array}$ \\
\hline $\begin{array}{c}\mathrm{M} \\
\mathrm{O} 1 \\
6- \\
70\end{array}$ & $\begin{array}{l}2 \\
2 \\
1\end{array}$ & $\begin{array}{l}3 \\
4 \\
1\end{array}$ & $\begin{array}{l}0 \\
. \\
6 \\
5\end{array}$ & $\begin{array}{c}0.06 \\
078\end{array}$ & $\begin{array}{c}0 . \\
00 \\
13 \\
5\end{array}$ & $\begin{array}{l}0.10 \\
070\end{array}$ & $\begin{array}{c}0 . \\
00 \\
19 \\
8\end{array}$ & $\begin{array}{l}0.84 \\
380\end{array}$ & $\begin{array}{c}0 . \\
01 \\
72 \\
3\end{array}$ & 631 & $\begin{array}{l}2 \\
0\end{array}$ & 619 & $\begin{array}{l}1 \\
2\end{array}$ & 621 & 9 & $\begin{array}{l}0.3 \\
2 \%\end{array}$ & $\begin{array}{l}6 \\
1 \\
9\end{array}$ & $\begin{array}{l}1 \\
2\end{array}$ \\
\hline $\begin{array}{c}\mathrm{M} \\
\mathrm{O} 1 \\
6- \\
71\end{array}$ & \begin{tabular}{l|}
1 \\
4 \\
3
\end{tabular} & $\begin{array}{l}3 \\
3 \\
6\end{array}$ & $\begin{array}{l}4 \\
3\end{array}$ & $\begin{array}{l}0.06 \\
770\end{array}$ & $\begin{array}{c}0 . \\
00 \\
19 \\
4\end{array}$ & $\begin{array}{l}0.14 \\
030\end{array}$ & $\begin{array}{c}0 . \\
00 \\
30 \\
9\end{array}$ & $\begin{array}{l}1.30 \\
939\end{array}$ & $\begin{array}{c}0 . \\
03 \\
43 \\
1\end{array}$ & 859 & $\begin{array}{l}2 \\
4\end{array}$ & & $\begin{array}{l}1 \\
7\end{array}$ & 850 & $\begin{array}{l}1 \\
5\end{array}$ & $\begin{array}{l}0.4 \\
7 \%\end{array}$ & $\begin{array}{l}8 \\
4 \\
6\end{array}$ & $\begin{array}{l}1 \\
7\end{array}$ \\
\hline $\begin{array}{c}\mathrm{M} \\
\mathrm{O} 1 \\
6- \\
72 \\
\end{array}$ & $\begin{array}{l}1 \\
9 \\
9\end{array}$ & $\begin{array}{l}3 \\
3 \\
9\end{array}$ & $\begin{array}{l}0 \\
5 \\
5 \\
9\end{array}$ & $\begin{array}{l}0.11 \\
556\end{array}$ & $\begin{array}{c}0 . \\
00 \\
25 \\
7 \\
\end{array}$ & $\begin{array}{l}0.31 \\
445\end{array}$ & $\begin{array}{c}0 . \\
00 \\
69 \\
6 \\
\end{array}$ & $\begin{array}{l}5.00 \\
915\end{array}$ & $\begin{array}{c}0 . \\
10 \\
23 \\
6 \\
\end{array}$ & $\begin{array}{c}188 \\
9\end{array}$ & $\begin{array}{l}1 \\
8\end{array}$ & $\begin{array}{c}176 \\
3\end{array}$ & $\begin{array}{l}3 \\
4\end{array}$ & $\begin{array}{c}182 \\
1\end{array}$ & $\begin{array}{l}1 \\
7\end{array}$ & $\begin{array}{l}7.1 \\
5 \%\end{array}$ & $\begin{array}{l}1 \\
8 \\
8 \\
9 \\
\end{array}$ & $\begin{array}{l}1 \\
8\end{array}$ \\
\hline $\begin{array}{c}\mathrm{M} \\
\mathrm{O} 1 \\
6- \\
73\end{array}$ & $\begin{array}{l}7 \\
0\end{array}$ & $\begin{array}{l}7 \\
3\end{array}$ & $\begin{array}{l}9 \\
6 \\
\end{array}$ & $\begin{array}{c}0.13 \\
037\end{array}$ & $\begin{array}{c}0 . \\
00 \\
39 \\
7 \\
\end{array}$ & $\begin{array}{c}0.36 \\
174\end{array}$ & $\begin{array}{c}0 . \\
00 \\
99 \\
0\end{array}$ & $\begin{array}{c}6.50 \\
117\end{array}$ & $\begin{array}{c}0 . \\
18 \\
36 \\
1\end{array}$ & $\begin{array}{c}210 \\
3\end{array}$ & $\begin{array}{l}2 \\
2\end{array}$ & $\begin{array}{c}199 \\
0\end{array}$ & $\begin{array}{l}4 \\
7\end{array}$ & $\begin{array}{c}204 \\
6\end{array}$ & $\begin{array}{l}2 \\
5\end{array}$ & $\begin{array}{l}5.6 \\
8 \%\end{array}$ & $\begin{array}{l}2 \\
1 \\
0 \\
3 \\
\end{array}$ & $\begin{array}{l}2 \\
2\end{array}$ \\
\hline $\begin{array}{c}\mathrm{M} \\
\mathrm{O} 1 \\
6- \\
74\end{array}$ & $\begin{array}{l}5 \\
9\end{array}$ & $\begin{array}{l}9 \\
6\end{array}$ & $\begin{array}{l}0 \\
6 \\
6\end{array}$ & $\begin{array}{c}0.19 \\
092\end{array}$ & $\begin{array}{c}0 . \\
00 \\
29 \\
7\end{array}$ & $\begin{array}{l}0.51 \\
041\end{array}$ & $\begin{array}{c}0 . \\
01 \\
03 \\
9\end{array}$ & $\begin{array}{c}13.4 \\
334 \\
5\end{array}$ & $\begin{array}{c}0 . \\
19 \\
70 \\
9\end{array}$ & $\begin{array}{c}275 \\
0\end{array}$ & $\begin{array}{l}1 \\
6\end{array}$ & $\begin{array}{c}265 \\
8\end{array}$ & $\begin{array}{l}4 \\
4\end{array}$ & $\begin{array}{c}271 \\
1\end{array}$ & $\begin{array}{l}1 \\
4\end{array}$ & $\begin{array}{l}3.4 \\
6 \%\end{array}$ & $\begin{array}{l}2 \\
7 \\
5 \\
0\end{array}$ & $\begin{array}{l}1 \\
6\end{array}$ \\
\hline $\begin{array}{c}\mathrm{M} \\
\mathrm{O} 1 \\
6- \\
75\end{array}$ & $\begin{array}{l}8 \\
0\end{array}$ & $\begin{array}{l}1 \\
5 \\
0\end{array}$ & $\begin{array}{l}5 \\
3\end{array}$ & $\begin{array}{l}0.05 \\
825\end{array}$ & $\begin{array}{c}0 . \\
00 \\
26 \\
4\end{array}$ & $\begin{array}{l}0.09 \\
242\end{array}$ & $\begin{array}{c}0 . \\
00 \\
23 \\
1\end{array}$ & $\begin{array}{l}0.74 \\
213\end{array}$ & $\begin{array}{c}0 . \\
03 \\
14 \\
8\end{array}$ & 539 & $\begin{array}{l}5 \\
1\end{array}$ & 570 & $\begin{array}{l}1 \\
4\end{array}$ & 564 & $\begin{array}{l}1 \\
8\end{array}$ & $\begin{array}{c}- \\
1.0 \\
5 \%\end{array}$ & $\begin{array}{l}5 \\
7 \\
0\end{array}$ & $\begin{array}{l}1 \\
4\end{array}$ \\
\hline $\begin{array}{c}\mathrm{M} \\
\mathrm{O} 1 \\
6- \\
76\end{array}$ & $\begin{array}{l}5 \\
3\end{array}$ & $\begin{array}{l}9 \\
5\end{array}$ & $\begin{array}{l}5 \\
6\end{array}$ & $\begin{array}{c}0.06 \\
960\end{array}$ & $\begin{array}{c}0 . \\
00 \\
26 \\
7\end{array}$ & $\begin{array}{r}0.13 \\
616\end{array}$ & $\begin{array}{c}0 . \\
00 \\
34 \\
7\end{array}$ & $\begin{array}{l}1.30 \\
640\end{array}$ & $\begin{array}{c}0 . \\
04 \\
59 \\
5\end{array}$ & 917 & $\begin{array}{l}3 \\
5\end{array}$ & 823 & $\begin{array}{l}2 \\
0\end{array}$ & 849 & $\begin{array}{l}2 \\
0\end{array}$ & $\begin{array}{l}3.1 \\
6 \%\end{array}$ & $\begin{array}{l}8 \\
2 \\
3\end{array}$ & $\begin{array}{l}2 \\
0\end{array}$ \\
\hline $\begin{array}{c}\text { M } \\
\text { O1 } \\
6- \\
77\end{array}$ & $\begin{array}{l}3 \\
8\end{array}$ & $\begin{array}{l}1 \\
2 \\
2\end{array}$ & $\begin{array}{l}0 \\
3 \\
1\end{array}$ & $\begin{array}{l}0.06 \\
475 \\
\end{array}$ & $\begin{array}{c}0 . \\
00 \\
25 \\
9\end{array}$ & $\begin{array}{l}0.10 \\
202\end{array}$ & $\begin{array}{c}0 . \\
00 \\
25 \\
7\end{array}$ & $\begin{array}{l}0.91 \\
066\end{array}$ & $\begin{array}{c}0 . \\
03 \\
34 \\
1\end{array}$ & 766 & $\begin{array}{l}3 \\
8\end{array}$ & 626 & $\begin{array}{l}1 \\
5\end{array}$ & 657 & $\begin{array}{l}1 \\
8\end{array}$ & $\begin{array}{l}4.9 \\
5 \%\end{array}$ & $\begin{array}{l}6 \\
2 \\
6\end{array}$ & $\begin{array}{l}1 \\
5\end{array}$ \\
\hline $\begin{array}{c}\mathrm{M} \\
\mathrm{O} 1 \\
6- \\
78\end{array}$ & $\begin{array}{l}1 \\
0 \\
7\end{array}$ & $\begin{array}{l}2 \\
0 \\
0\end{array}$ & $\begin{array}{l}0 \\
5 \\
5 \\
4\end{array}$ & $\begin{array}{c}0.12 \\
710\end{array}$ & $\begin{array}{c}0 . \\
00 \\
21 \\
1 \\
\end{array}$ & $\begin{array}{l}0.25 \\
438\end{array}$ & $\begin{array}{c}0 . \\
00 \\
49 \\
9\end{array}$ & $\begin{array}{r}4.45 \\
685\end{array}$ & $\begin{array}{c}0 . \\
06 \\
67 \\
7\end{array}$ & $\begin{array}{c}200 \\
7\end{array}$ & $\begin{array}{l}4 \\
7\end{array}$ & $\begin{array}{c}145 \\
4\end{array}$ & $\begin{array}{l}2 \\
5\end{array}$ & $\begin{array}{c}169 \\
5\end{array}$ & $\begin{array}{l}1 \\
4\end{array}$ & $\begin{array}{c}38 . \\
03 \\
\%\end{array}$ & & \\
\hline $\begin{array}{c}\mathrm{M} \\
\mathrm{O} 1 \\
6- \\
79 \\
\end{array}$ & $\begin{array}{l}1 \\
1 \\
4\end{array}$ & $\begin{array}{l}3 \\
1 \\
0\end{array}$ & $\begin{array}{l}0 \\
3 \\
7 \\
\end{array}$ & $\begin{array}{c}0.05 \\
450\end{array}$ & $\begin{array}{c}0 . \\
00 \\
17 \\
2 \\
\end{array}$ & $\begin{array}{l}0.05 \\
695\end{array}$ & $\begin{array}{c}0 . \\
00 \\
12 \\
2 \\
\end{array}$ & $\begin{array}{l}0.42 \\
786\end{array}$ & $\begin{array}{c}0 . \\
01 \\
24 \\
4 \\
\end{array}$ & 392 & $\begin{array}{l}3 \\
1\end{array}$ & 357 & 7 & 362 & 9 & $\begin{array}{l}1.4 \\
0 \%\end{array}$ & $\begin{array}{l}3 \\
5 \\
7\end{array}$ & 7 \\
\hline $\begin{array}{c}\mathrm{M} \\
\mathrm{O} 1 \\
6- \\
80\end{array}$ & $\begin{array}{l}4 \\
1 \\
6\end{array}$ & $\begin{array}{l}5 \\
4 \\
3\end{array}$ & $\begin{array}{l}0 \\
. \\
7 \\
7\end{array}$ & $\begin{array}{c}0.05 \\
497\end{array}$ & $\begin{array}{c}0 . \\
00 \\
13 \\
6\end{array}$ & $\begin{array}{l}0.05 \\
563\end{array}$ & $\begin{array}{c}0 . \\
00 \\
11 \\
1\end{array}$ & $\begin{array}{c}0.42 \\
151\end{array}$ & $\begin{array}{c}0 . \\
00 \\
95 \\
5\end{array}$ & 411 & $\begin{array}{l}2 \\
3\end{array}$ & 349 & 7 & 357 & 7 & $\begin{array}{l}2.2 \\
9 \%\end{array}$ & $\begin{array}{l}3 \\
4 \\
9\end{array}$ & 7 \\
\hline $\begin{array}{c}\mathrm{M} \\
\mathrm{O} 1 \\
6- \\
81 \\
\end{array}$ & $\begin{array}{l}2 \\
7\end{array}$ & $\begin{array}{l}2 \\
7 \\
6\end{array}$ & $\begin{array}{l}0 \\
. \\
1 \\
0 \\
\end{array}$ & $\begin{array}{l}0.12 \\
366\end{array}$ & $\begin{array}{c}0 . \\
00 \\
19 \\
4 \\
\end{array}$ & $\begin{array}{l}0.30 \\
926\end{array}$ & $\begin{array}{c}0 . \\
00 \\
59 \\
8 \\
\end{array}$ & $\begin{array}{l}5.27 \\
170\end{array}$ & $\begin{array}{c}0 . \\
07 \\
57 \\
9 \\
\end{array}$ & $\begin{array}{c}199 \\
5\end{array}$ & $\begin{array}{l}4 \\
3\end{array}$ & $\begin{array}{c}173 \\
5\end{array}$ & $\begin{array}{l}2 \\
9\end{array}$ & $\begin{array}{c}185 \\
6\end{array}$ & $\begin{array}{l}1 \\
2\end{array}$ & $\begin{array}{l}14 . \\
99 \\
\%\end{array}$ & & \\
\hline $\begin{array}{l}\mathrm{M} \\
\mathrm{O} 1 \\
\end{array}$ & $\begin{array}{l}1 \\
7 \\
\end{array}$ & $\begin{array}{l}1 \\
3 \\
\end{array}$ & 1 & $\begin{array}{c}0.06 \\
321 \\
\end{array}$ & $\begin{array}{c}0 . \\
00 \\
\end{array}$ & $\begin{array}{l}0.09 \\
286 \\
\end{array}$ & $\begin{array}{c}0 . \\
00 \\
\end{array}$ & $\begin{array}{l}0.80 \\
905 \\
\end{array}$ & $\begin{array}{c}0 . \\
02\end{array}$ & 715 & $\begin{array}{l}3 \\
6 \\
\end{array}$ & 572 & $\begin{array}{l}1 \\
3 \\
\end{array}$ & 602 & $\begin{array}{l}1 \\
6 \\
\end{array}$ & $\begin{array}{l}5.2 \\
4 \% \\
\end{array}$ & $\begin{array}{l}5 \\
7 \\
\end{array}$ & $\begin{array}{l}1 \\
3 \\
\end{array}$ \\
\hline
\end{tabular}




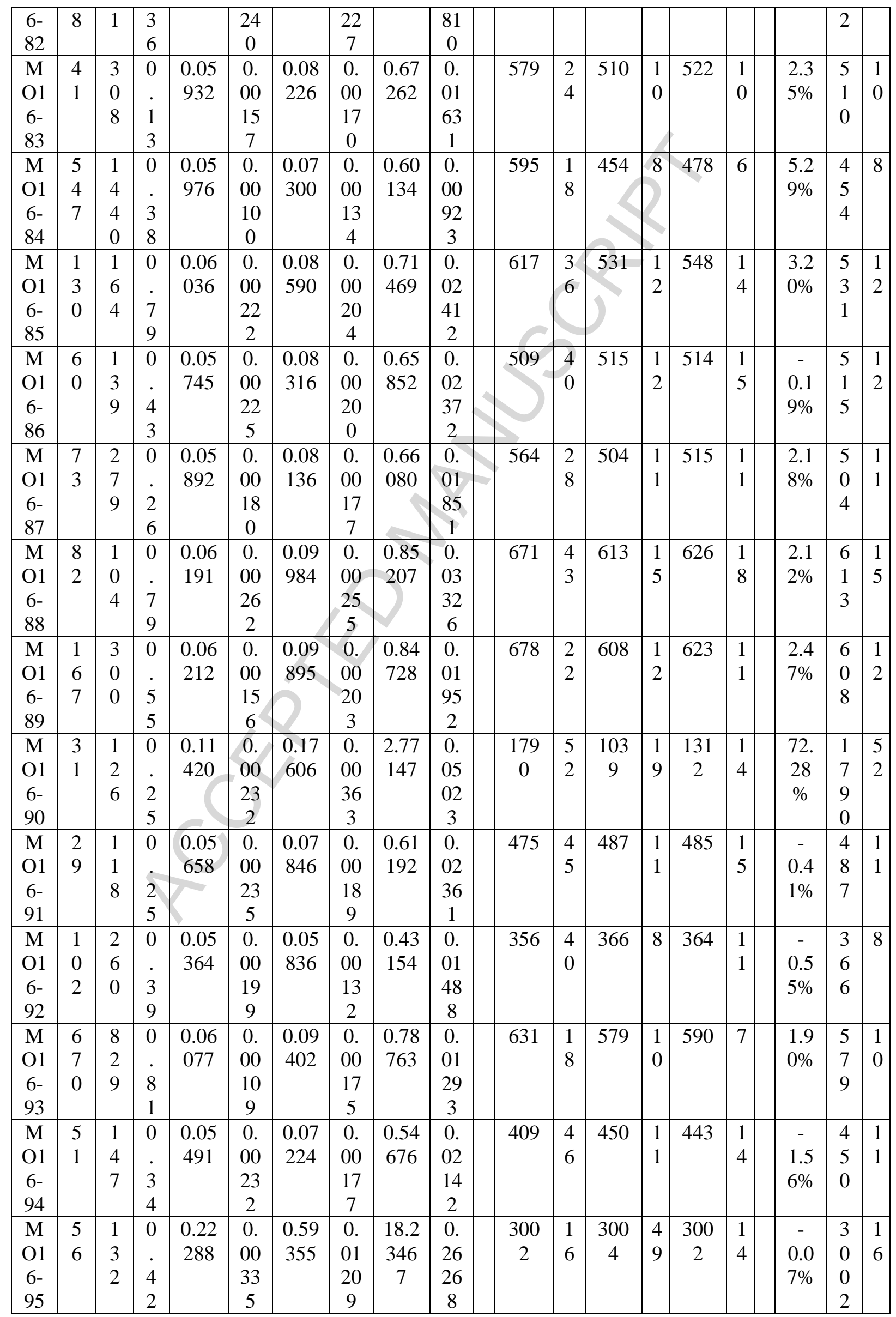




\begin{tabular}{|c|c|c|c|c|c|c|c|c|c|c|c|c|c|c|c|c|c|c|}
\hline $\begin{array}{c}\mathrm{M} \\
\mathrm{O} 1 \\
6- \\
96\end{array}$ & $\begin{array}{l}4 \\
3\end{array}$ & $\begin{array}{l}1 \\
4 \\
9\end{array}$ & $\begin{array}{l}2 \\
9\end{array}$ & $\begin{array}{l}0.05 \\
719\end{array}$ & $\begin{array}{c}0 . \\
00 \\
27 \\
5\end{array}$ & $\begin{array}{c}0.08 \\
155\end{array}$ & $\begin{array}{c}0 . \\
00 \\
21 \\
6\end{array}$ & $\begin{array}{l}0.64 \\
289\end{array}$ & $\begin{array}{c}0 . \\
02 \\
86 \\
2\end{array}$ & 499 & $\begin{array}{l}5 \\
3\end{array}$ & 505 & \begin{tabular}{l|}
1 \\
3
\end{tabular} & 504 & $\begin{array}{l}1 \\
8\end{array}$ & $\begin{array}{c}- \\
0.2 \\
0 \%\end{array}$ & $\begin{array}{l}5 \\
0 \\
5\end{array}$ & $\begin{array}{l}1 \\
3\end{array}$ \\
\hline $\begin{array}{c}\text { M } \\
\text { O1 } \\
6- \\
97\end{array}$ & $\begin{array}{l}4 \\
6\end{array}$ & $\begin{array}{l}1 \\
3 \\
6\end{array}$ & $\begin{array}{l}3 \\
4\end{array}$ & $\begin{array}{l}0.05 \\
715\end{array}$ & $\begin{array}{c}0 . \\
00 \\
33 \\
0\end{array}$ & $\begin{array}{l}0.07 \\
306\end{array}$ & $\begin{array}{c}0 . \\
00 \\
22 \\
5\end{array}$ & $\begin{array}{l}0.57 \\
550\end{array}$ & $\begin{array}{c}0 . \\
03 \\
04 \\
4\end{array}$ & 497 & $\begin{array}{l}6 \\
4\end{array}$ & 455 & $\begin{array}{l}1 \\
4\end{array}$ & 462 & $\begin{array}{l}2 \\
0\end{array}$ & $\begin{array}{l}1.5 \\
4 \%\end{array}$ & $\begin{array}{l}4 \\
5 \\
5\end{array}$ & $\begin{array}{l}1 \\
4\end{array}$ \\
\hline $\begin{array}{c}\mathrm{M} \\
\mathrm{O} 1 \\
6- \\
98\end{array}$ & $\begin{array}{l}4 \\
9\end{array}$ & $\begin{array}{l}2 \\
4 \\
8\end{array}$ & $\begin{array}{l}0 \\
\dot{2} \\
0\end{array}$ & $\begin{array}{l}0.12 \\
369\end{array}$ & $\begin{array}{c}0 . \\
00 \\
18 \\
2\end{array}$ & $\begin{array}{c}0.33 \\
533\end{array}$ & $\begin{array}{c}0 . \\
00 \\
63 \\
4\end{array}$ & $\begin{array}{c}5.71 \\
698\end{array}$ & $\begin{array}{c}0 . \\
07 \\
72 \\
8\end{array}$ & $\begin{array}{c}201 \\
0\end{array}$ & $\begin{array}{l}1 \\
6\end{array}$ & $\begin{array}{c}186 \\
4\end{array}$ & $\begin{array}{l}3 \\
1\end{array}$ & $\begin{array}{c}193 \\
4\end{array}$ & $\begin{array}{l}1 \\
2\end{array}$ & $\begin{array}{l}7.8 \\
3 \%\end{array}$ & $\begin{array}{l}2 \\
0 \\
1 \\
0\end{array}$ & $\begin{array}{l}1 \\
6\end{array}$ \\
\hline $\begin{array}{c}\text { M } \\
\text { O1 } \\
6- \\
99\end{array}$ & $\begin{array}{l}6 \\
8\end{array}$ & $\begin{array}{l}4 \\
8 \\
6\end{array}$ & $\begin{array}{l}1 \\
4\end{array}$ & $\begin{array}{l}0.13 \\
425\end{array}$ & $\begin{array}{c}0 . \\
00 \\
16 \\
9\end{array}$ & $\begin{array}{c}0.36 \\
671\end{array}$ & $\begin{array}{c}0 . \\
00 \\
66 \\
7\end{array}$ & $\begin{array}{c}6.78 \\
568\end{array}$ & $\begin{array}{c}0 . \\
07 \\
82 \\
8\end{array}$ & $\begin{array}{c}215 \\
4\end{array}$ & $\begin{array}{l}1 \\
7\end{array}$ & $\begin{array}{c}201 \\
4\end{array}$ & $\begin{array}{l}3 \\
1\end{array}$ & $\begin{array}{c}208 \\
4\end{array}$ & $\begin{array}{l}1 \\
0\end{array}$ & $\begin{array}{l}6.9 \\
5 \%\end{array}$ & $\begin{array}{l}2 \\
1 \\
5 \\
4\end{array}$ & $\begin{array}{l}1 \\
7\end{array}$ \\
\hline $\begin{array}{c}\mathrm{M} \\
\mathrm{O} 1 \\
6- \\
10 \\
0\end{array}$ & $\begin{array}{l}1 \\
5 \\
5\end{array}$ & $\begin{array}{l}1 \\
7 \\
4\end{array}$ & $\begin{array}{l}0 \\
\dot{8} \\
9\end{array}$ & $\begin{array}{c}0.06 \\
698\end{array}$ & $\begin{array}{c}0 . \\
00 \\
22 \\
5\end{array}$ & $\begin{array}{l}0.09 \\
276\end{array}$ & $\begin{array}{c}0 . \\
00 \\
21 \\
5\end{array}$ & $\begin{array}{l}0.85 \\
635\end{array}$ & $\begin{array}{c}0 . \\
02 \\
61 \\
2\end{array}$ & 686 & $\begin{array}{l}1 \\
0 \\
7\end{array}$ & 569 & $\begin{array}{l}1 \\
3\end{array}$ & 593 & $\begin{array}{l}1 \\
9\end{array}$ & $\begin{array}{l}4.2 \\
2 \%\end{array}$ & $\begin{array}{l}5 \\
6 \\
9\end{array}$ & $\begin{array}{l}1 \\
3\end{array}$ \\
\hline $\begin{array}{c}\text { M } \\
\text { O1 } \\
7- \\
01\end{array}$ & $\begin{array}{l}3 \\
6\end{array}$ & $\begin{array}{l}2 \\
1 \\
6\end{array}$ & $\begin{array}{l}0 \\
\dot{1} \\
6\end{array}$ & $\begin{array}{l}0.05 \\
786\end{array}$ & $\begin{array}{c}0 . \\
00 \\
09 \\
8\end{array}$ & $\begin{array}{l}0.07 \\
333\end{array}$ & $\begin{array}{c}0 . \\
00 \\
13 \\
6\end{array}$ & $\begin{array}{c}0.58 \\
501\end{array}$ & $\begin{array}{c}0 . \\
00 \\
91 \\
7\end{array}$ & 524 & $\begin{array}{l}1 \\
8\end{array}$ & 456 & 8 & 468 & 6 & $\begin{array}{l}2.6 \\
3 \%\end{array}$ & $\begin{array}{l}4 \\
5 \\
6\end{array}$ & 8 \\
\hline $\begin{array}{c}\mathrm{M} \\
\mathrm{O} 1 \\
7- \\
02\end{array}$ & $\begin{array}{l}9 \\
1\end{array}$ & $\begin{array}{l}3 \\
9 \\
2\end{array}$ & $\begin{array}{l}2 \\
3\end{array}$ & $\begin{array}{c}0.18 \\
762\end{array}$ & $\begin{array}{c}0 . \\
00 \\
18 \\
4\end{array}$ & $\begin{array}{l}0.51 \\
990\end{array}$ & $\begin{array}{c}0 . \\
00 \\
92 \\
2\end{array}$ & $\begin{array}{c}13.4 \\
482 \\
7\end{array}$ & $\begin{array}{c}0 . \\
12 \\
26 \\
0\end{array}$ & $\begin{array}{c}272 \\
1\end{array}$ & $\begin{array}{l}1 \\
7\end{array}$ & $\begin{array}{c}269 \\
9\end{array}$ & $\begin{array}{l}3 \\
9\end{array}$ & $\begin{array}{c}271 \\
2\end{array}$ & 9 & $\begin{array}{l}0.8 \\
2 \%\end{array}$ & $\begin{array}{l}2 \\
7 \\
2 \\
1\end{array}$ & $\begin{array}{l}1 \\
7\end{array}$ \\
\hline $\begin{array}{c}\mathrm{M} \\
\mathrm{O} 1 \\
7- \\
03\end{array}$ & $\begin{array}{l}3 \\
8\end{array}$ & $\begin{array}{l}2 \\
8 \\
8\end{array}$ & $\begin{array}{l}0 \\
\dot{1} \\
3\end{array}$ & $\begin{array}{c}0.06 \\
038\end{array}$ & $\begin{array}{c}0 . \\
00 \\
09 \\
0\end{array}$ & $\begin{array}{l}0.07 \\
882\end{array}$ & $\begin{array}{c}0 . \\
00 \\
14 \\
4\end{array}$ & $\begin{array}{l}0.65 \\
609\end{array}$ & $\begin{array}{c}0 . \\
00 \\
89 \\
6\end{array}$ & 617 & $\begin{array}{l}1 \\
9\end{array}$ & 489 & 9 & 512 & 5 & $\begin{array}{l}4.7 \\
0 \%\end{array}$ & $\begin{array}{l}4 \\
8 \\
9\end{array}$ & 9 \\
\hline $\begin{array}{c}\text { M } \\
\text { O1 } \\
7- \\
04 \\
\end{array}$ & $\begin{array}{l}5 \\
5\end{array}$ & $\begin{array}{l}5 \\
2\end{array}$ & $\begin{array}{l}1 \\
0 \\
6 \\
\end{array}$ & $\begin{array}{l}0.06 \\
203\end{array}$ & $\begin{array}{c}0 . \\
00 \\
18 \\
6 \\
\end{array}$ & $\begin{array}{c}0.10 \\
315\end{array}$ & $\begin{array}{c}0 . \\
00 \\
22 \\
3 \\
\end{array}$ & $\begin{array}{l}0.88 \\
219\end{array}$ & $\begin{array}{c}0 . \\
02 \\
45 \\
0 \\
\end{array}$ & 675 & $\begin{array}{l}2 \\
7\end{array}$ & 633 & $\begin{array}{l}1 \\
3\end{array}$ & 642 & $\begin{array}{l}1 \\
3\end{array}$ & $\begin{array}{l}1.4 \\
2 \%\end{array}$ & $\begin{array}{l}6 \\
3 \\
3\end{array}$ & $\begin{array}{l}1 \\
3\end{array}$ \\
\hline $\begin{array}{c}\mathrm{M} \\
\mathrm{O} 1 \\
7- \\
05\end{array}$ & $\begin{array}{l}8 \\
8\end{array}$ & $\begin{array}{l}3 \\
2 \\
4\end{array}$ & $\begin{array}{l}0 \\
\dot{2} \\
7\end{array}$ & $\begin{array}{c}0.06 \\
038\end{array}$ & $\begin{array}{c}0 . \\
00 \\
12 \\
0\end{array}$ & $\begin{array}{c}0.09 \\
313\end{array}$ & $\begin{array}{c}0 . \\
00 \\
18 \\
0\end{array}$ & $\begin{array}{l}0.77 \\
525\end{array}$ & $\begin{array}{c}0 . \\
01 \\
42 \\
1\end{array}$ & 617 & $\begin{array}{l}1 \\
9\end{array}$ & 574 & $\begin{array}{l}1 \\
1\end{array}$ & 583 & 8 & $\begin{array}{l}1.5 \\
7 \%\end{array}$ & $\begin{array}{l}5 \\
7 \\
4\end{array}$ & $\begin{array}{l}1 \\
1\end{array}$ \\
\hline $\begin{array}{c}\text { M } \\
\text { O1 } \\
7- \\
06\end{array}$ & $\begin{array}{l}1 \\
3\end{array}$ & $\begin{array}{l}2 \\
5\end{array}$ & $\begin{array}{l}0 \\
\dot{5} \\
2\end{array}$ & $\begin{array}{l}0.07 \\
247\end{array}$ & $\begin{array}{c}0 . \\
00 \\
26 \\
5\end{array}$ & $\begin{array}{c}0.12 \\
488\end{array}$ & $\begin{array}{c}0 . \\
00 \\
28 \\
7\end{array}$ & $\begin{array}{l}1.24 \\
773\end{array}$ & $\begin{array}{c}0 . \\
04 \\
27 \\
7\end{array}$ & 999 & $\begin{array}{l}3 \\
5\end{array}$ & 759 & $\begin{array}{l}1 \\
6\end{array}$ & 822 & $\begin{array}{l}1 \\
9\end{array}$ & $\begin{array}{l}8.3 \\
0 \%\end{array}$ & $\begin{array}{l}7 \\
5 \\
9\end{array}$ & $\begin{array}{l}1 \\
6\end{array}$ \\
\hline $\begin{array}{c}\text { M } \\
\text { O1 } \\
7- \\
07\end{array}$ & $\begin{array}{l}3 \\
9 \\
0\end{array}$ & $\begin{array}{l}3 \\
5 \\
8\end{array}$ & $\begin{array}{l}0 \\
9\end{array}$ & $\begin{array}{l}0.06 \\
273\end{array}$ & $\begin{array}{c}0 . \\
00 \\
08 \\
4\end{array}$ & $\begin{array}{c}0.10 \\
360\end{array}$ & $\begin{array}{c}0 . \\
00 \\
18 \\
8\end{array}$ & $\begin{array}{c}0.89 \\
600\end{array}$ & $\begin{array}{c}0 . \\
01 \\
09 \\
9\end{array}$ & 699 & $\begin{array}{l}1 \\
9\end{array}$ & 635 & $\begin{array}{l}1 \\
1\end{array}$ & 650 & 6 & $\begin{array}{l}2.3 \\
6 \%\end{array}$ & $\begin{array}{l}6 \\
3 \\
5\end{array}$ & $\begin{array}{l}1 \\
1\end{array}$ \\
\hline $\begin{array}{c}\mathrm{M} \\
\mathrm{O} 1 \\
7- \\
08\end{array}$ & $\begin{array}{l}6 \\
8\end{array}$ & $\begin{array}{l}3 \\
4 \\
1\end{array}$ & $\begin{array}{l}2 \\
0\end{array}$ & $\begin{array}{c}0.14 \\
083\end{array}$ & $\begin{array}{c}0 . \\
00 \\
14 \\
9\end{array}$ & $\begin{array}{c}0.38 \\
652\end{array}$ & $\begin{array}{c}0 . \\
00 \\
69 \\
3\end{array}$ & $\begin{array}{l}7.50 \\
483\end{array}$ & $\begin{array}{c}0 . \\
07 \\
36 \\
8\end{array}$ & $\begin{array}{c}221 \\
8\end{array}$ & $\begin{array}{l}3 \\
8\end{array}$ & $\begin{array}{c}210 \\
3\end{array}$ & $\begin{array}{l}3 \\
2\end{array}$ & $\begin{array}{c}216 \\
2\end{array}$ & $\begin{array}{l}1 \\
1\end{array}$ & $\begin{array}{l}5.4 \\
7 \%\end{array}$ & $\begin{array}{l}2 \\
2 \\
1 \\
8\end{array}$ & \begin{tabular}{|l}
3 \\
8
\end{tabular} \\
\hline
\end{tabular}




\begin{tabular}{|c|c|c|c|c|c|c|c|c|c|c|c|c|c|c|c|c|c|c|}
\hline $\begin{array}{l}\text { M } \\
\text { O1 } \\
7- \\
09\end{array}$ & $\begin{array}{l}1 \\
1 \\
4\end{array}$ & $\begin{array}{l}1 \\
5 \\
5\end{array}$ & $\begin{array}{l}0 \\
. \\
7 \\
3\end{array}$ & $\begin{array}{l}0.05 \\
834\end{array}$ & $\begin{array}{c}0 . \\
00 \\
18 \\
1\end{array}$ & $\begin{array}{r}0.09 \\
586\end{array}$ & $\begin{array}{c}0 . \\
00 \\
21 \\
1\end{array}$ & $\begin{array}{c}0.77 \\
102\end{array}$ & $\begin{array}{c}0 . \\
02 \\
21 \\
0\end{array}$ & 543 & $\begin{array}{l}2 \\
9\end{array}$ & 590 & $\begin{array}{l}1 \\
2\end{array}$ & 580 & 1 & $\begin{array}{c}- \\
1.6 \\
9 \%\end{array}$ & $\begin{array}{l}5 \\
9 \\
0\end{array}$ & $\begin{array}{l}1 \\
2\end{array}$ \\
\hline $\begin{array}{l}M \\
\text { O1 } \\
7- \\
10\end{array}$ & $\begin{array}{l}1 \\
6\end{array}$ & $\begin{array}{l}5 \\
0\end{array}$ & $\begin{array}{l}0 \\
3 \\
2\end{array}$ & $\begin{array}{l}0.05 \\
387\end{array}$ & $\begin{array}{c}0 . \\
00 \\
17 \\
7\end{array}$ & $\begin{array}{c}0.10 \\
013\end{array}$ & $\begin{array}{c}0 . \\
00 \\
20 \\
9\end{array}$ & $\begin{array}{l}0.74 \\
361\end{array}$ & $\begin{array}{c}0 . \\
02 \\
31 \\
4\end{array}$ & 366 & $\begin{array}{l}3 \\
5\end{array}$ & 615 & $\begin{array}{l}1 \\
2\end{array}$ & 565 & $\begin{array}{l}1 \\
3\end{array}$ & $\begin{array}{c}- \\
8.1 \\
3 \%\end{array}$ & $\begin{array}{l}6 \\
1 \\
5\end{array}$ & $\begin{array}{l}1 \\
2\end{array}$ \\
\hline $\begin{array}{l}\mathrm{M} \\
\mathrm{O} 1 \\
7- \\
11\end{array}$ & $\begin{array}{l}7 \\
0\end{array}$ & $\begin{array}{l}8 \\
0\end{array}$ & $\begin{array}{l}0 \\
\dot{8} \\
7\end{array}$ & $\begin{array}{l}0.10 \\
763\end{array}$ & $\begin{array}{c}0 . \\
00 \\
13 \\
9\end{array}$ & $\begin{array}{l}0.29 \\
890\end{array}$ & $\begin{array}{c}0 . \\
00 \\
55 \\
3\end{array}$ & $\begin{array}{l}4.43 \\
517\end{array}$ & $\begin{array}{c}0 . \\
05 \\
29 \\
1\end{array}$ & $\begin{array}{c}176 \\
0\end{array}$ & $\begin{array}{l}1 \\
7\end{array}$ & $\begin{array}{c}168 \\
6\end{array}$ & $\begin{array}{l}2 \\
7\end{array}$ & $\begin{array}{c}171 \\
9\end{array}$ & $\begin{array}{l}1 \\
0\end{array}$ & $\begin{array}{l}4.3 \\
9 \%\end{array}$ & $\begin{array}{l}1 \\
7 \\
6 \\
0\end{array}$ & $\begin{array}{l}1 \\
7\end{array}$ \\
\hline $\begin{array}{l}M \\
\text { O1 } \\
7- \\
12 \\
\end{array}$ & $\begin{array}{l}4 \\
3\end{array}$ & $\begin{array}{l}1 \\
5 \\
1\end{array}$ & $\begin{array}{l}0 \\
2 \\
8 \\
\end{array}$ & $\begin{array}{l}0.05 \\
861\end{array}$ & $\begin{array}{c}0 . \\
00 \\
11 \\
4 \\
\end{array}$ & $\begin{array}{c}0.08 \\
104\end{array}$ & $\begin{array}{c}0 . \\
00 \\
15 \\
4 \\
\end{array}$ & $\begin{array}{l}0.65 \\
492\end{array}$ & $\begin{array}{c}0 . \\
01 \\
17 \\
8 \\
\end{array}$ & 553 & $\begin{array}{l}1 \\
9\end{array}$ & 502 & 9 & 512 & 7 & $\begin{array}{l}1.9 \\
9 \%\end{array}$ & $\begin{array}{l}5 \\
0 \\
2\end{array}$ & 9 \\
\hline $\begin{array}{l}\mathrm{M} \\
\mathrm{O} 1 \\
7- \\
13 \\
\end{array}$ & $\begin{array}{l}2 \\
5\end{array}$ & $\begin{array}{l}3 \\
9\end{array}$ & $\begin{array}{l}6 \\
5\end{array}$ & $\begin{array}{l}0.05 \\
694\end{array}$ & $\begin{array}{c}0 . \\
00 \\
22 \\
7 \\
\end{array}$ & $\begin{array}{l}0.07 \\
707\end{array}$ & $\begin{array}{c}0 . \\
00 \\
17 \\
6\end{array}$ & $\begin{array}{l}0.60 \\
496\end{array}$ & $\begin{array}{c}0 . \\
02 \\
27 \\
5\end{array}$ & 489 & $\begin{array}{l}4 \\
5\end{array}$ & 479 & $\begin{array}{l}1 \\
1\end{array}$ & 480 & $\begin{array}{l}1 \\
4\end{array}$ & $\begin{array}{l}0.2 \\
1 \%\end{array}$ & $\begin{array}{l}4 \\
7 \\
9\end{array}$ & $\begin{array}{l}1 \\
1\end{array}$ \\
\hline $\begin{array}{l}M \\
\text { O1 } \\
7- \\
14\end{array}$ & $\begin{array}{l}2 \\
0 \\
5\end{array}$ & $\begin{array}{l}1 \\
9 \\
0\end{array}$ & $\begin{array}{l}1 \\
0 \\
8\end{array}$ & $\begin{array}{l}0.06 \\
213\end{array}$ & $\begin{array}{c}0 . \\
00 \\
09 \\
7\end{array}$ & $\begin{array}{c}0.08 \\
753\end{array}$ & $\begin{array}{c}0 . \\
00 \\
16 \\
2\end{array}$ & $\begin{array}{l}0.74 \\
974\end{array}$ & $\begin{array}{c}0 . \\
01 \\
07 \\
0\end{array}$ & 679 & $\begin{array}{l}1 \\
8\end{array}$ & 541 & $\begin{array}{l}1 \\
0\end{array}$ & 568 & 6 & $\begin{array}{l}4.9 \\
9 \%\end{array}$ & $\begin{array}{l}5 \\
4 \\
1\end{array}$ & $\begin{array}{l}1 \\
0\end{array}$ \\
\hline $\begin{array}{l}M \\
\text { O1 } \\
7- \\
15\end{array}$ & $\begin{array}{l}1 \\
0 \\
8\end{array}$ & $\begin{array}{l}3 \\
0 \\
1\end{array}$ & $\begin{array}{l}0 \\
3 \\
6\end{array}$ & $\begin{array}{l}0.05 \\
371\end{array}$ & $\begin{array}{c}0 . \\
00 \\
08 \\
8\end{array}$ & $\begin{array}{l}0.05 \\
713\end{array}$ & $\begin{array}{c}0 . \\
00 \\
10 \\
5\end{array}$ & $\begin{array}{c}0.42 \\
306\end{array}$ & $\begin{array}{c}0 . \\
00 \\
63 \\
6\end{array}$ & 359 & $\begin{array}{l}1 \\
9\end{array}$ & 358 & 6 & 358 & 5 & $\begin{array}{l}0.0 \\
0 \%\end{array}$ & $\begin{array}{l}3 \\
5 \\
8\end{array}$ & 6 \\
\hline $\begin{array}{l}M \\
\text { O1 } \\
7- \\
16\end{array}$ & $\begin{array}{l}3 \\
4 \\
9\end{array}$ & $\begin{array}{l}4 \\
1 \\
7\end{array}$ & $\begin{array}{l}0 \\
\dot{8} \\
4\end{array}$ & $\begin{array}{l}0.06 \\
245\end{array}$ & $\begin{array}{c}0 . \\
00 \\
07 \\
7\end{array}$ & $\begin{array}{l}0.10 \\
072\end{array}$ & $\begin{array}{c}0 . \\
00 \\
18 \\
1\end{array}$ & $\begin{array}{l}0.86 \\
729\end{array}$ & $\begin{array}{c}0 . \\
00 \\
98 \\
0\end{array}$ & 690 & $\begin{array}{l}2 \\
0\end{array}$ & 619 & $\begin{array}{l}1 \\
1\end{array}$ & 634 & 5 & $\begin{array}{l}2.4 \\
2 \%\end{array}$ & $\begin{array}{l}6 \\
1 \\
9\end{array}$ & $\begin{array}{l}1 \\
1\end{array}$ \\
\hline $\begin{array}{l}\mathrm{M} \\
\mathrm{O} 1 \\
7- \\
17\end{array}$ & $\begin{array}{l}1 \\
6 \\
5\end{array}$ & $\begin{array}{l}1 \\
8 \\
2\end{array}$ & $\begin{array}{l}0 \\
\dot{9} \\
1\end{array}$ & $\begin{array}{l}0.11 \\
572\end{array}$ & $\begin{array}{c}0 . \\
00 \\
12 \\
6\end{array}$ & $\begin{array}{c}0.32 \\
403\end{array}$ & $\begin{array}{c}0 . \\
00 \\
58 \\
2\end{array}$ & $\begin{array}{l}5.16 \\
981\end{array}$ & $\begin{array}{c}0 . \\
05 \\
21 \\
4\end{array}$ & $\begin{array}{c}189 \\
1\end{array}$ & $\begin{array}{l}1 \\
8\end{array}$ & $\begin{array}{c}180 \\
9\end{array}$ & $\begin{array}{l}2 \\
8\end{array}$ & $\begin{array}{c}184 \\
8\end{array}$ & 9 & $\begin{array}{l}4.5 \\
3 \%\end{array}$ & $\begin{array}{l}1 \\
8 \\
9 \\
1\end{array}$ & $\begin{array}{l}1 \\
8\end{array}$ \\
\hline $\begin{array}{l}\text { M } \\
\text { O1 } \\
7- \\
18 \\
\end{array}$ & $\begin{array}{l}2 \\
3\end{array}$ & $\begin{array}{l}3 \\
4\end{array}$ & $\begin{array}{l}0 \\
6 \\
8 \\
\end{array}$ & $\begin{array}{c}0.06 \\
141\end{array}$ & $\begin{array}{c}0 . \\
00 \\
23 \\
6\end{array}$ & $\begin{array}{c}0.10 \\
792\end{array}$ & $\begin{array}{c}0 . \\
00 \\
24 \\
6\end{array}$ & $\begin{array}{l}0.91 \\
365\end{array}$ & $\begin{array}{c}0 . \\
03 \\
30 \\
2\end{array}$ & 654 & $\begin{array}{l}4 \\
1\end{array}$ & 661 & $\begin{array}{l}1 \\
4\end{array}$ & 659 & $\begin{array}{l}1 \\
8\end{array}$ & $\begin{array}{c}- \\
0.3 \\
0 \%\end{array}$ & $\begin{array}{l}6 \\
6 \\
1\end{array}$ & $\begin{array}{l}1 \\
4\end{array}$ \\
\hline $\begin{array}{l}M \\
\text { O1 } \\
7- \\
19 \\
\end{array}$ & $\begin{array}{l}4 \\
8\end{array}$ & $\begin{array}{l}5 \\
4\end{array}$ & $\begin{array}{l}0 \\
8 \\
8 \\
9\end{array}$ & $\begin{array}{l}0.06 \\
212\end{array}$ & $\begin{array}{c}0 . \\
00 \\
16 \\
2 \\
\end{array}$ & $\begin{array}{c}0.10 \\
691\end{array}$ & $\begin{array}{c}0 . \\
00 \\
22 \\
0\end{array}$ & $\begin{array}{l}0.91 \\
559\end{array}$ & $\begin{array}{c}0 . \\
02 \\
21 \\
6 \\
\end{array}$ & 678 & $\begin{array}{l}2 \\
3\end{array}$ & 655 & $\begin{array}{l}1 \\
3\end{array}$ & 660 & $\begin{array}{l}1 \\
2\end{array}$ & $\begin{array}{l}0.7 \\
6 \%\end{array}$ & $\begin{array}{l}6 \\
5 \\
5\end{array}$ & $\begin{array}{l}1 \\
3\end{array}$ \\
\hline $\begin{array}{l}\text { M } \\
\text { O1 } \\
7- \\
20 \\
\end{array}$ & $\begin{array}{l}4 \\
3\end{array}$ & $\begin{array}{l}2 \\
4 \\
2\end{array}$ & $\begin{array}{l}0 \\
\dot{1} \\
8 \\
\end{array}$ & $\begin{array}{l}0.12 \\
334\end{array}$ & $\begin{array}{c}0 . \\
00 \\
13 \\
6 \\
\end{array}$ & $\begin{array}{l}0.33 \\
769\end{array}$ & $\begin{array}{c}0 . \\
00 \\
60 \\
8 \\
\end{array}$ & $\begin{array}{l}5.74 \\
215\end{array}$ & $\begin{array}{c}0 . \\
05 \\
85 \\
7 \\
\end{array}$ & $\begin{array}{c}197 \\
8\end{array}$ & $\begin{array}{l}4 \\
0\end{array}$ & $\begin{array}{c}187 \\
1\end{array}$ & $\begin{array}{l}2 \\
9\end{array}$ & $\begin{array}{c}192 \\
2\end{array}$ & $\begin{array}{l}1 \\
1\end{array}$ & $\begin{array}{l}5.7 \\
2 \%\end{array}$ & $\begin{array}{l}1 \\
9 \\
7 \\
8 \\
\end{array}$ & $\begin{array}{l}4 \\
0\end{array}$ \\
\hline $\begin{array}{l}\text { M } \\
\text { O1 } \\
7- \\
21 \\
\end{array}$ & $\begin{array}{l}1 \\
6 \\
3\end{array}$ & $\begin{array}{l}2 \\
8 \\
7\end{array}$ & $\begin{array}{l}0 \\
\dot{5} \\
7 \\
\end{array}$ & $\begin{array}{l}0.06 \\
515\end{array}$ & $\begin{array}{c}0 . \\
00 \\
09 \\
2 \\
\end{array}$ & $\begin{array}{c}0.10 \\
046\end{array}$ & $\begin{array}{c}0 . \\
00 \\
18 \\
3 \\
\end{array}$ & $\begin{array}{l}0.90 \\
233\end{array}$ & $\begin{array}{c}0 . \\
01 \\
17 \\
3 \\
\end{array}$ & 779 & $\begin{array}{l}1 \\
9\end{array}$ & 617 & $\begin{array}{l}1 \\
1\end{array}$ & 653 & 6 & $\begin{array}{l}5.8 \\
3 \%\end{array}$ & $\begin{array}{l}6 \\
1 \\
7\end{array}$ & $\begin{array}{l}1 \\
1\end{array}$ \\
\hline $\begin{array}{l}\mathrm{M} \\
\mathrm{O} 1 \\
\end{array}$ & $\begin{array}{l}1 \\
8 \\
\end{array}$ & $\begin{array}{l}1 \\
6 \\
\end{array}$ & 1 & $\begin{array}{r}0.05 \\
609 \\
\end{array}$ & $\begin{array}{c}0 . \\
00 \\
\end{array}$ & $\begin{array}{r}0.07 \\
511 \\
\end{array}$ & $\begin{array}{c}0 . \\
00 \\
\end{array}$ & $\begin{array}{l}0.58 \\
085 \\
\end{array}$ & $\begin{array}{c}0 . \\
01 \\
\end{array}$ & 456 & $\begin{array}{l}1 \\
9 \\
\end{array}$ & 467 & 9 & 465 & 7 & $\begin{array}{c}- \\
0.4 \\
\end{array}$ & $\begin{array}{l}4 \\
6 \\
\end{array}$ & 9 \\
\hline
\end{tabular}




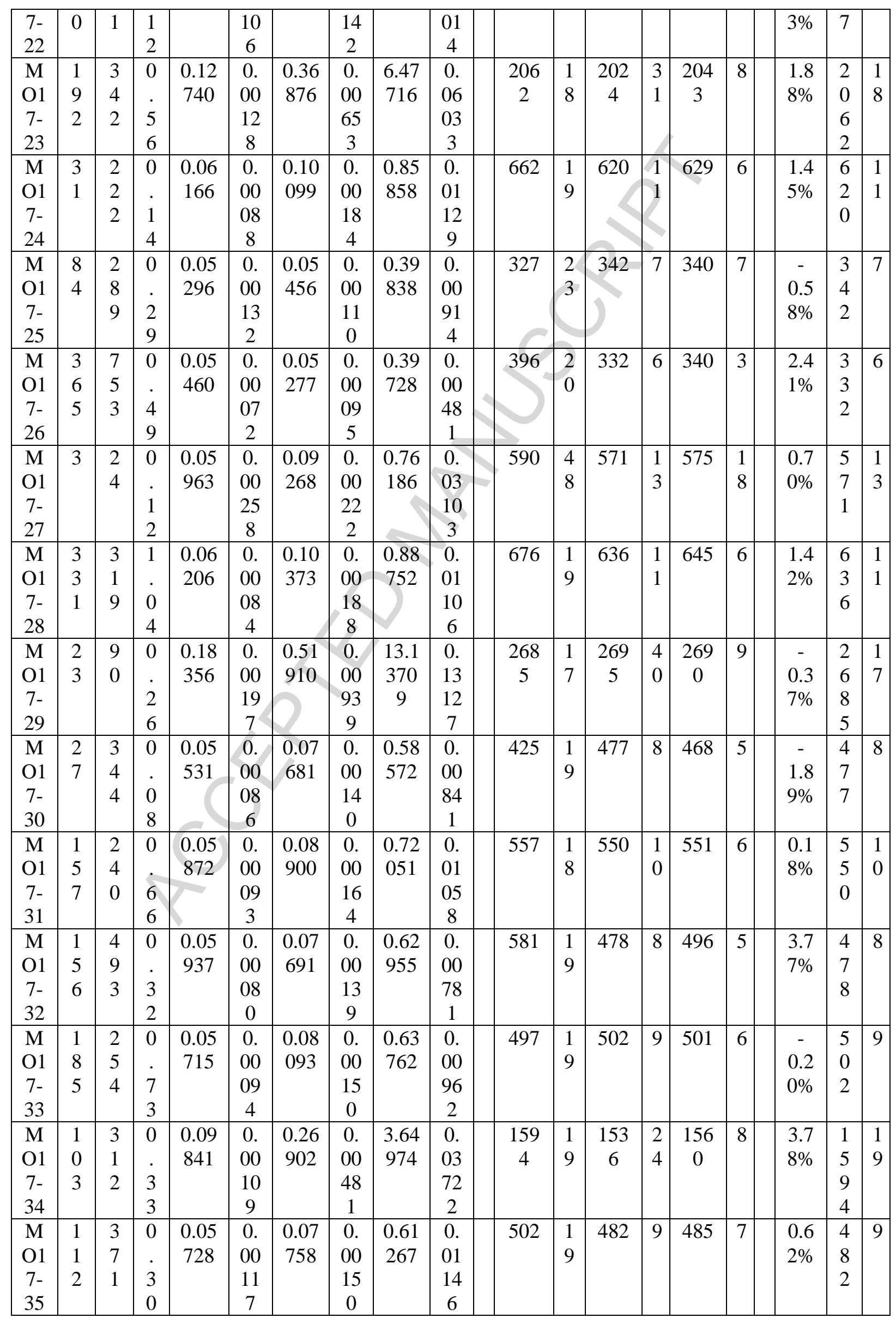




\begin{tabular}{|c|c|c|c|c|c|c|c|c|c|c|c|c|c|c|c|c|c|c|}
\hline $\begin{array}{c}\mathrm{M} \\
\mathrm{O} 1 \\
7- \\
36\end{array}$ & $\begin{array}{l}5 \\
2\end{array}$ & $\begin{array}{l}1 \\
9 \\
6\end{array}$ & $\begin{array}{l}2 \\
7\end{array}$ & $\begin{array}{c}0.05 \\
670\end{array}$ & $\begin{array}{c}0 . \\
00 \\
15 \\
0\end{array}$ & $\begin{array}{c}0.07 \\
568\end{array}$ & $\begin{array}{c}0 . \\
00 \\
15 \\
5\end{array}$ & $\begin{array}{c}0.59 \\
161\end{array}$ & $\begin{array}{c}0 . \\
01 \\
44 \\
5\end{array}$ & 480 & $\begin{array}{l}2 \\
4\end{array}$ & 470 & 9 & 472 & 9 & $\begin{array}{l}0.4 \\
3 \%\end{array}$ & $\begin{array}{l}4 \\
7 \\
0\end{array}$ & 9 \\
\hline $\begin{array}{c}\mathrm{M} \\
\mathrm{O} 1 \\
7- \\
37 \\
\end{array}$ & $\begin{array}{l}1 \\
3 \\
2\end{array}$ & $\begin{array}{l}3 \\
3 \\
5\end{array}$ & $\begin{array}{l}0 \\
. \\
3 \\
9\end{array}$ & $\begin{array}{l}0.05 \\
736\end{array}$ & $\begin{array}{c}0 . \\
00 \\
09 \\
3 \\
\end{array}$ & $\begin{array}{l}0.05 \\
655\end{array}$ & $\begin{array}{c}0 . \\
00 \\
10 \\
4\end{array}$ & $\begin{array}{l}0.44 \\
719\end{array}$ & $\begin{array}{c}0 . \\
00 \\
66 \\
6\end{array}$ & 505 & $\begin{array}{l}1 \\
8\end{array}$ & 355 & 6 & 375 & 5 & $\begin{array}{l}5.6 \\
3 \%\end{array}$ & $\begin{array}{l}3 \\
5 \\
5\end{array}$ & 6 \\
\hline $\begin{array}{c}\mathrm{M} \\
\mathrm{O} 1 \\
7- \\
38\end{array}$ & $\begin{array}{l}1 \\
0 \\
2\end{array}$ & $\begin{array}{l}2 \\
5 \\
6\end{array}$ & $\begin{array}{l}0 \\
4 \\
0\end{array}$ & $\begin{array}{l}0.05 \\
816\end{array}$ & $\begin{array}{c}0 . \\
00 \\
09 \\
1\end{array}$ & $\begin{array}{l}0.08 \\
242\end{array}$ & $\begin{array}{c}0 . \\
00 \\
15 \\
1\end{array}$ & $\begin{array}{c}0.66 \\
093\end{array}$ & $\begin{array}{c}0 . \\
00 \\
95 \\
2\end{array}$ & 536 & $\begin{array}{l}1 \\
8\end{array}$ & & 9 & 515 & 6 & $\begin{array}{l}0.7 \\
8 \%\end{array}$ & \begin{tabular}{l|}
5 \\
1 \\
1
\end{tabular} & 9 \\
\hline $\begin{array}{c}\mathrm{M} \\
\mathrm{O} 1 \\
7- \\
39\end{array}$ & $\begin{array}{l}1 \\
7 \\
8\end{array}$ & $\begin{array}{l}3 \\
8 \\
6\end{array}$ & $\begin{array}{l}4 \\
6\end{array}$ & $\begin{array}{c}0.05 \\
338\end{array}$ & $\begin{array}{c}0 . \\
00 \\
09 \\
0\end{array}$ & $\begin{array}{l}0.05 \\
623\end{array}$ & $\begin{array}{c}0 . \\
00 \\
10 \\
4\end{array}$ & $\begin{array}{l}0.41 \\
376\end{array}$ & $\begin{array}{c}0 . \\
00 \\
64 \\
7\end{array}$ & 345 & $\begin{array}{l}1 \\
9\end{array}$ & 353 & 6 & 352 & 5 & $\begin{array}{c}- \\
0.2 \\
8 \%\end{array}$ & $\begin{array}{l}3 \\
5 \\
3\end{array}$ & 6 \\
\hline $\begin{array}{c}\mathrm{M} \\
\mathrm{O} 1 \\
7- \\
40 \\
\end{array}$ & $\begin{array}{l}5 \\
0\end{array}$ & $\begin{array}{l}2 \\
1 \\
6\end{array}$ & $\begin{array}{l}0 \\
2 \\
3\end{array}$ & $\begin{array}{l}0.05 \\
547\end{array}$ & $\begin{array}{c}0 . \\
00 \\
09 \\
4\end{array}$ & $\begin{array}{l}0.08 \\
274\end{array}$ & $\begin{array}{c}0 . \\
00 \\
15 \\
3\end{array}$ & $\begin{array}{l}0.63 \\
274\end{array}$ & $\begin{array}{c}0 . \\
00 \\
99 \\
2\end{array}$ & 431 & $\begin{array}{l}1 \\
9\end{array}$ & 512 & 9 & 498 & 6 & $\begin{array}{c}- \\
2.7 \\
3 \%\end{array}$ & $\begin{array}{l}5 \\
1 \\
2\end{array}$ & 9 \\
\hline $\begin{array}{l}\mathrm{M} \\
\mathrm{O} 1 \\
7- \\
41\end{array}$ & $\begin{array}{l}3 \\
5\end{array}$ & $\begin{array}{l}2 \\
7 \\
6\end{array}$ & $\begin{array}{l}1 \\
3 \\
\end{array}$ & $\begin{array}{c}0.06 \\
419\end{array}$ & $\begin{array}{c}0 . \\
00 \\
12 \\
7\end{array}$ & $\begin{array}{l}0.09 \\
185\end{array}$ & $\begin{array}{c}0 . \\
00 \\
17 \\
8\end{array}$ & $\begin{array}{l}0.81 \\
281\end{array}$ & $\begin{array}{c}0 . \\
01 \\
46 \\
8\end{array}$ & 748 & $\begin{array}{l}1 \\
8\end{array}$ & 566 & $\begin{array}{l}1 \\
1\end{array}$ & 604 & 8 & $\begin{array}{l}6.7 \\
1 \%\end{array}$ & $\begin{array}{l}5 \\
6 \\
6\end{array}$ & $\begin{array}{l}1 \\
1\end{array}$ \\
\hline $\begin{array}{c}\text { M } \\
\text { O1 } \\
7- \\
42 \\
\end{array}$ & $\begin{array}{l}2 \\
2 \\
0\end{array}$ & $\begin{array}{l}4 \\
2 \\
0\end{array}$ & $\begin{array}{l}0 \\
5 \\
5 \\
2\end{array}$ & $\begin{array}{l}0.05 \\
749\end{array}$ & $\begin{array}{c}0 . \\
00 \\
10 \\
1 \\
\end{array}$ & $\begin{array}{l}0.05 \\
677\end{array}$ & $\begin{array}{c}0 . \\
00 \\
10 \\
6 \\
\end{array}$ & $\begin{array}{l}0.44 \\
999\end{array}$ & $\begin{array}{c}0 . \\
00 \\
72 \\
3 \\
\end{array}$ & 510 & $\begin{array}{l}1 \\
8\end{array}$ & 356 & 6 & 377 & 5 & $\begin{array}{l}5.9 \\
0 \%\end{array}$ & $\begin{array}{l}3 \\
5 \\
6\end{array}$ & 6 \\
\hline $\begin{array}{l}\mathrm{M} \\
\mathrm{O} 1 \\
7- \\
43\end{array}$ & $\begin{array}{l}7 \\
8\end{array}$ & $\begin{array}{l}2 \\
0 \\
6\end{array}$ & $\begin{array}{l}0 \\
3 \\
8\end{array}$ & $\begin{array}{l}0.05 \\
825\end{array}$ & $\begin{array}{c}0 . \\
00 \\
11 \\
1\end{array}$ & $\begin{array}{l}0.07 \\
236\end{array}$ & $\begin{array}{c}0 . \\
00 \\
13 \\
7\end{array}$ & $\begin{array}{c}0.58 \\
107\end{array}$ & $\begin{array}{c}0 . \\
01 \\
01 \\
8\end{array}$ & 539 & $\begin{array}{l}1 \\
9\end{array}$ & 450 & 8 & 465 & 7 & $\begin{array}{l}3.3 \\
3 \%\end{array}$ & $\begin{array}{l}4 \\
5 \\
0\end{array}$ & 8 \\
\hline $\begin{array}{c}\text { M } \\
\text { O1 } \\
7- \\
44\end{array}$ & $\begin{array}{l}2 \\
0 \\
0\end{array}$ & $\begin{array}{l}3 \\
7 \\
6\end{array}$ & $\begin{array}{l}5 \\
3\end{array}$ & $\begin{array}{l}0.12 \\
361\end{array}$ & $\begin{array}{c}0 . \\
00 \\
12 \\
6\end{array}$ & $\begin{array}{l}0.36 \\
984\end{array}$ & $\begin{array}{c}0 . \\
00 \\
65 \\
5\end{array}$ & $\begin{array}{l}6.30 \\
244\end{array}$ & $\begin{array}{c}0 . \\
05 \\
90 \\
4\end{array}$ & $\begin{array}{c}200 \\
9\end{array}$ & $\begin{array}{l}1 \\
8\end{array}$ & $\begin{array}{c}202 \\
9\end{array}$ & 3 & $\begin{array}{c}201 \\
9\end{array}$ & 8 & $\begin{array}{c}- \\
0.9 \\
9 \%\end{array}$ & $\begin{array}{l}2 \\
0 \\
0 \\
9\end{array}$ & $\begin{array}{l}1 \\
8\end{array}$ \\
\hline $\begin{array}{c}\mathrm{M} \\
\mathrm{O} 1 \\
7- \\
45\end{array}$ & $\begin{array}{l}6 \\
2\end{array}$ & $\begin{array}{l}3 \\
8 \\
1\end{array}$ & $\begin{array}{l}0 \\
1 \\
6\end{array}$ & $\begin{array}{l}0.05 \\
987\end{array}$ & $\begin{array}{c}0 . \\
00 \\
09 \\
2 \\
\end{array}$ & $\begin{array}{l}0.07 \\
782\end{array}$ & $\begin{array}{c}0 . \\
00 \\
14 \\
3\end{array}$ & $\begin{array}{l}0.64 \\
231\end{array}$ & $\begin{array}{c}0 . \\
00 \\
90 \\
9\end{array}$ & 533 & $\begin{array}{l}6 \\
2\end{array}$ & 482 & 8 & 491 & 8 & $\begin{array}{l}1.8 \\
7 \%\end{array}$ & $\begin{array}{l}4 \\
8 \\
2\end{array}$ & 8 \\
\hline $\begin{array}{l}\mathrm{M} \\
\mathrm{O} 1 \\
7- \\
46\end{array}$ & $\begin{array}{l}1 \\
0 \\
3\end{array}$ & $\begin{array}{l}2 \\
0 \\
0\end{array}$ & $\begin{array}{l}5 \\
2 \\
\end{array}$ & $\begin{array}{l}0.05 \\
954\end{array}$ & $\begin{array}{c}0 . \\
00 \\
10 \\
6\end{array}$ & $\begin{array}{l}0.09 \\
215\end{array}$ & $\begin{array}{c}0 . \\
00 \\
17 \\
3\end{array}$ & $\begin{array}{l}0.75 \\
636\end{array}$ & $\begin{array}{c}0 . \\
01 \\
23 \\
7\end{array}$ & 587 & $\begin{array}{l}1 \\
8\end{array}$ & 568 & $\begin{array}{l}1 \\
0\end{array}$ & 572 & 7 & $\begin{array}{l}0.7 \\
0 \%\end{array}$ & $\begin{array}{l}5 \\
6 \\
8\end{array}$ & $\begin{array}{l}1 \\
0\end{array}$ \\
\hline $\begin{array}{c}\text { M } \\
\text { O1 } \\
7- \\
47 \\
\end{array}$ & $\begin{array}{l}7 \\
5\end{array}$ & $\begin{array}{l}1 \\
8 \\
5\end{array}$ & $\begin{array}{l}0 \\
. \\
4 \\
1\end{array}$ & $\begin{array}{l}0.05 \\
625\end{array}$ & $\begin{array}{c}0 . \\
00 \\
12 \\
0 \\
\end{array}$ & $\begin{array}{l}0.05 \\
625\end{array}$ & $\begin{array}{c}0 . \\
00 \\
10 \\
9 \\
\end{array}$ & $\begin{array}{c}0.43 \\
619\end{array}$ & $\begin{array}{c}0 . \\
00 \\
85 \\
3 \\
\end{array}$ & 462 & $\begin{array}{l}2 \\
0\end{array}$ & 353 & 7 & 368 & 6 & $\begin{array}{l}4.2 \\
5 \%\end{array}$ & $\begin{array}{l}3 \\
5 \\
3\end{array}$ & 7 \\
\hline $\begin{array}{c}\mathrm{M} \\
\mathrm{O} 1 \\
7- \\
48 \\
\end{array}$ & $\begin{array}{l}1 \\
6 \\
0\end{array}$ & $\begin{array}{l}3 \\
0 \\
7\end{array}$ & $\begin{array}{l}0 \\
. \\
5 \\
2 \\
\end{array}$ & $\begin{array}{c}0.05 \\
533\end{array}$ & $\begin{array}{c}0 . \\
00 \\
09 \\
6 \\
\end{array}$ & $\begin{array}{l}0.05 \\
592\end{array}$ & $\begin{array}{c}0 . \\
00 \\
10 \\
4 \\
\end{array}$ & $\begin{array}{l}0.42 \\
659\end{array}$ & $\begin{array}{c}0 . \\
00 \\
67 \\
6 \\
\end{array}$ & 426 & $\begin{array}{l}1 \\
9\end{array}$ & 351 & 6 & 361 & 5 & $\begin{array}{l}2.8 \\
5 \%\end{array}$ & $\begin{array}{l}3 \\
5 \\
1\end{array}$ & 6 \\
\hline $\begin{array}{l}\mathrm{M} \\
\mathrm{O} 1 \\
\end{array}$ & $\begin{array}{l}1 \\
6 \\
\end{array}$ & $\begin{array}{l}7 \\
2 \\
\end{array}$ & 0 & \begin{tabular}{|l|}
0.05 \\
857 \\
\end{tabular} & $\begin{array}{c}0 . \\
00 \\
\end{array}$ & $\begin{array}{l}0.07 \\
123 \\
\end{array}$ & $\begin{array}{c}0 . \\
00\end{array}$ & $\begin{array}{l}0.57 \\
513 \\
\end{array}$ & $\begin{array}{c}0 . \\
00 \\
\end{array}$ & 551 & $\begin{array}{l}2 \\
0 \\
\end{array}$ & 444 & 8 & 461 & 4 & $\begin{array}{l}3.8 \\
3 \%\end{array}$ & $\begin{array}{l}4 \\
4\end{array}$ & 8 \\
\hline
\end{tabular}




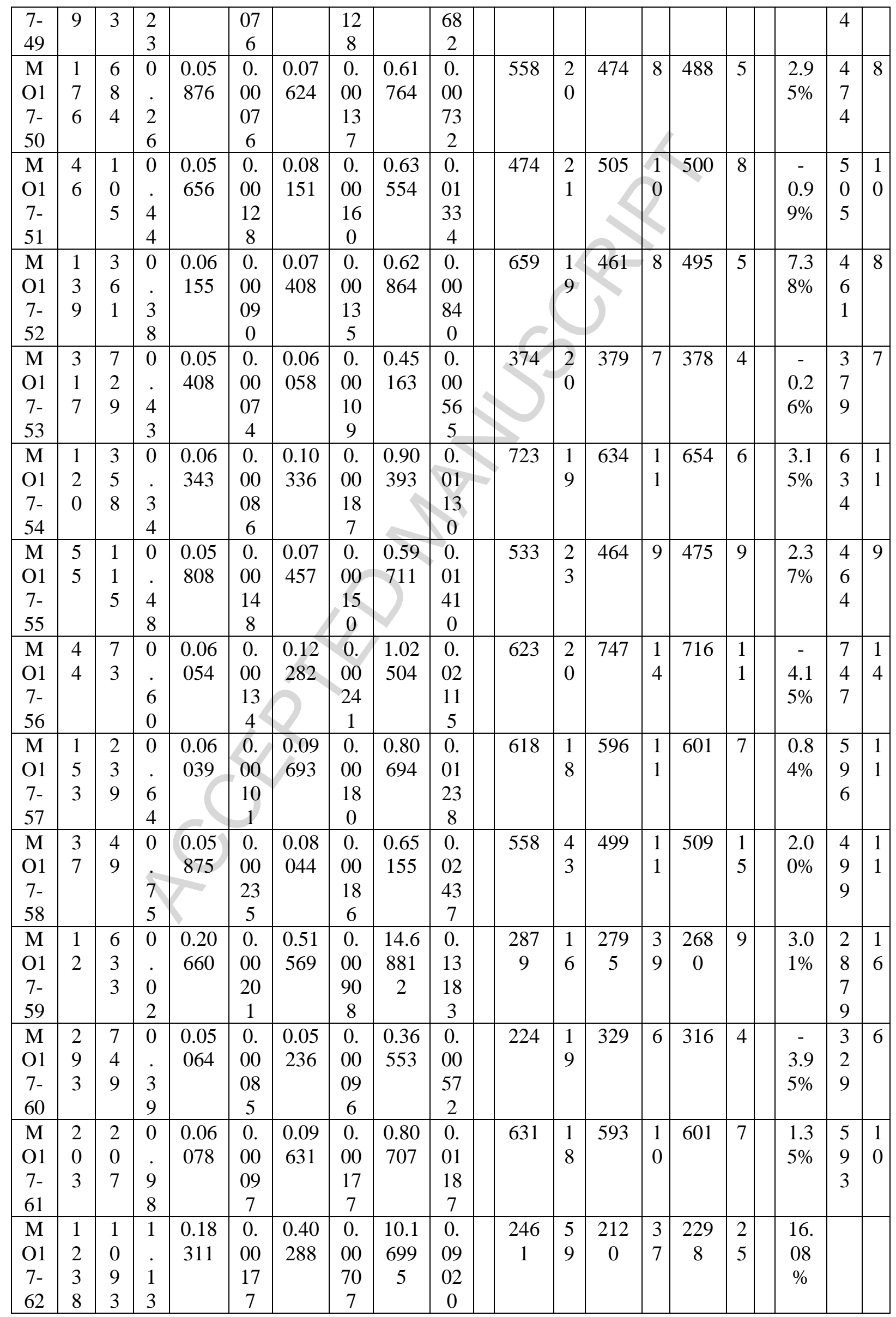




\begin{tabular}{|c|c|c|c|c|c|c|c|c|c|c|c|c|c|c|c|c|c|c|}
\hline $\begin{array}{c}\mathrm{M} \\
\mathrm{O} 1 \\
7- \\
63 \\
\end{array}$ & \begin{tabular}{l|}
5 \\
7
\end{tabular} & $\begin{array}{l}1 \\
4 \\
2\end{array}$ & $\begin{array}{l}0 \\
4 \\
0\end{array}$ & $\begin{array}{l}0.05 \\
303\end{array}$ & $\begin{array}{c}0 . \\
00 \\
18 \\
3\end{array}$ & $\begin{array}{l}0.05 \\
227\end{array}$ & $\begin{array}{c}0 . \\
00 \\
11 \\
1\end{array}$ & $\begin{array}{l}0.38 \\
215\end{array}$ & $\begin{array}{c}0 . \\
01 \\
23 \\
8\end{array}$ & 330 & $\begin{array}{l}3 \\
8\end{array}$ & 328 & 7 & 329 & 9 & $\begin{array}{l}0.3 \\
0 \%\end{array}$ & $\begin{array}{l}3 \\
2 \\
8\end{array}$ & 7 \\
\hline $\begin{array}{c}\mathrm{M} \\
\mathrm{O} 1 \\
7- \\
64\end{array}$ & $\begin{array}{l}4 \\
0\end{array}$ & $\begin{array}{l}6 \\
6 \\
1\end{array}$ & $\begin{array}{l}0 \\
. \\
0 \\
6\end{array}$ & $\begin{array}{l}0.05 \\
942\end{array}$ & $\begin{array}{c}0 . \\
00 \\
10 \\
1 \\
\end{array}$ & $\begin{array}{l}0.05 \\
499\end{array}$ & $\begin{array}{c}0 . \\
00 \\
10 \\
2 \\
\end{array}$ & $\begin{array}{l}0.45 \\
049\end{array}$ & $\begin{array}{c}0 . \\
00 \\
69 \\
8 \\
\end{array}$ & 583 & $\begin{array}{l}1 \\
8\end{array}$ & 345 & 6 & 378 & 5 & $\begin{array}{l}9.5 \\
7 \%\end{array}$ & $\begin{array}{l}3 \\
4 \\
5\end{array}$ & 6 \\
\hline $\begin{array}{l}\mathrm{M} \\
\mathrm{O} 1 \\
7- \\
65\end{array}$ & $\begin{array}{l}7 \\
4\end{array}$ & $\begin{array}{l}2 \\
6 \\
1\end{array}$ & $\begin{array}{l}2 \\
8\end{array}$ & $\begin{array}{c}0.06 \\
008\end{array}$ & $\begin{array}{c}0 . \\
00 \\
09 \\
4\end{array}$ & $\begin{array}{l}0.07 \\
574\end{array}$ & $\begin{array}{c}0 . \\
00 \\
13 \\
9\end{array}$ & $\begin{array}{l}0.62 \\
734\end{array}$ & $\begin{array}{c}0 . \\
00 \\
90 \\
2\end{array}$ & 606 & $\begin{array}{l}1 \\
8\end{array}$ & & 8 & 494 & 6 & $\begin{array}{l}4.8 \\
8 \%\end{array}$ & $\begin{array}{l}4 \\
7 \\
1\end{array}$ & 8 \\
\hline $\begin{array}{c}\mathrm{M} \\
\mathrm{O} 1 \\
7- \\
66 \\
\end{array}$ & $\begin{array}{l}6 \\
2\end{array}$ & $\begin{array}{l}4 \\
6 \\
6\end{array}$ & $\begin{array}{l}0 \\
1 \\
3 \\
\end{array}$ & $\begin{array}{l}0.07 \\
621\end{array}$ & $\begin{array}{c}0 . \\
00 \\
09 \\
2 \\
\end{array}$ & $\begin{array}{l}0.11 \\
261\end{array}$ & $\begin{array}{c}0 . \\
00 \\
20 \\
1 \\
\end{array}$ & $\begin{array}{l}1.18 \\
309\end{array}$ & $\begin{array}{c}0 . \\
01 \\
29 \\
9 \\
\end{array}$ & 843 & $\begin{array}{l}5 \\
3\end{array}$ & 680 & $\begin{array}{l}1 \\
2\end{array}$ & 719 & 9 & $\begin{array}{l}5.7 \\
4 \%\end{array}$ & $\begin{array}{l}6 \\
8 \\
0\end{array}$ & $\begin{array}{l}1 \\
2\end{array}$ \\
\hline $\begin{array}{l}\mathrm{M} \\
\mathrm{O} 1 \\
7- \\
67\end{array}$ & $\begin{array}{l}4 \\
9\end{array}$ & $\begin{array}{l}1 \\
2 \\
7\end{array}$ & $\begin{array}{l}0 \\
3 \\
9\end{array}$ & $\begin{array}{l}0.05 \\
334\end{array}$ & $\begin{array}{c}0 . \\
00 \\
15 \\
1 \\
\end{array}$ & $\begin{array}{c}0.05 \\
579\end{array}$ & $\begin{array}{c}0 . \\
00 \\
11 \\
3\end{array}$ & $\begin{array}{c}0.41 \\
024\end{array}$ & $\begin{array}{c}0 . \\
01 \\
08 \\
0\end{array}$ & 343 & $\begin{array}{l}2 \\
8\end{array}$ & 350 & 7 & 349 & 8 & $\begin{array}{c}- \\
0.2 \\
9 \%\end{array}$ & $\begin{array}{l}3 \\
5 \\
0\end{array}$ & 7 \\
\hline $\begin{array}{l}\mathrm{M} \\
\mathrm{O} 1 \\
7- \\
68 \\
\end{array}$ & $\begin{array}{l}5 \\
4\end{array}$ & $\begin{array}{l}1 \\
3 \\
7\end{array}$ & $\begin{array}{l}0 \\
3 \\
3 \\
9\end{array}$ & $\begin{array}{l}0.05 \\
866\end{array}$ & $\begin{array}{c}0 . \\
00 \\
13 \\
2 \\
\end{array}$ & $\begin{array}{l}0.07 \\
631\end{array}$ & $\begin{array}{c}0 . \\
00 \\
14 \\
8\end{array}$ & $\begin{array}{l}0.61 \\
708\end{array}$ & $\begin{array}{c}0 . \\
01 \\
29 \\
4\end{array}$ & 555 & $\begin{array}{l}2 \\
0\end{array}$ & 474 & 9 & 488 & 8 & $\begin{array}{l}2.9 \\
5 \%\end{array}$ & $\begin{array}{l}4 \\
7 \\
4\end{array}$ & 9 \\
\hline $\begin{array}{c}\text { M } \\
\text { O1 } \\
7- \\
69 \\
\end{array}$ & 9 & $\begin{array}{l}2 \\
8\end{array}$ & $\begin{array}{l}0 \\
3 \\
2 \\
\end{array}$ & $\begin{array}{l}0.05 \\
829\end{array}$ & $\begin{array}{c}0 . \\
00 \\
47 \\
7 \\
\end{array}$ & $\begin{array}{c}0.06 \\
531\end{array}$ & $\begin{array}{c}0 . \\
00 \\
18 \\
7 \\
\end{array}$ & $\begin{array}{l}0.52 \\
477\end{array}$ & $\begin{array}{c}0 . \\
04 \\
14 \\
8 \\
\end{array}$ & 541 & $\begin{array}{l}1 \\
2 \\
3\end{array}$ & 408 & $\begin{array}{l}1 \\
1\end{array}$ & 428 & $\begin{array}{l}2 \\
8\end{array}$ & $\begin{array}{l}4.9 \\
0 \%\end{array}$ & $\begin{array}{l}4 \\
0 \\
8\end{array}$ & $\begin{array}{l}1 \\
1\end{array}$ \\
\hline $\begin{array}{c}\mathrm{M} \\
\mathrm{O} 1 \\
7- \\
70\end{array}$ & $\begin{array}{l}1 \\
7\end{array}$ & $\begin{array}{l}2 \\
2\end{array}$ & $\begin{array}{l}0 \\
. \\
7 \\
9\end{array}$ & $\begin{array}{l}0.06 \\
276\end{array}$ & $\begin{array}{c}0 . \\
00 \\
41 \\
0\end{array}$ & $\begin{array}{l}0.10 \\
231\end{array}$ & $\begin{array}{c}0 . \\
00 \\
28 \\
8\end{array}$ & $\begin{array}{l}0.88 \\
515\end{array}$ & $\begin{array}{c}0 . \\
05 \\
52 \\
5\end{array}$ & 700 & $\begin{array}{l}8 \\
5\end{array}$ & 628 & $\begin{array}{l}1 \\
7\end{array}$ & 644 & $\begin{array}{l}3 \\
0\end{array}$ & $\begin{array}{l}2.5 \\
5 \%\end{array}$ & $\begin{array}{l}6 \\
2 \\
8\end{array}$ & $\begin{array}{l}1 \\
7\end{array}$ \\
\hline $\begin{array}{c}\text { M } \\
\text { O1 } \\
7- \\
71\end{array}$ & $\begin{array}{l}1 \\
8 \\
0\end{array}$ & $\begin{array}{l}1 \\
2 \\
5\end{array}$ & $\begin{array}{l}1 \\
4 \\
4\end{array}$ & $\begin{array}{l}0.06 \\
167 \\
\end{array}$ & $\begin{array}{c}0 . \\
00 \\
15 \\
1\end{array}$ & $\begin{array}{l}0.09 \\
248\end{array}$ & $\begin{array}{c}0 . \\
00 \\
18 \\
6\end{array}$ & $\begin{array}{c}0.78 \\
616\end{array}$ & $\begin{array}{c}0 . \\
01 \\
77 \\
2\end{array}$ & 663 & $\begin{array}{l}2 \\
2\end{array}$ & 570 & $\begin{array}{l}1 \\
1\end{array}$ & 589 & $\begin{array}{l}1 \\
0\end{array}$ & $\begin{array}{l}3.3 \\
3 \%\end{array}$ & $\begin{array}{l}5 \\
7 \\
0\end{array}$ & $\begin{array}{l}1 \\
1\end{array}$ \\
\hline $\begin{array}{c}\mathrm{M} \\
\mathrm{O} 1 \\
7- \\
72\end{array}$ & $\begin{array}{l}6 \\
4\end{array}$ & $\begin{array}{l}9 \\
1\end{array}$ & $\begin{array}{l}0 \\
7 \\
0\end{array}$ & $\begin{array}{l}0.05 \\
964\end{array}$ & $\begin{array}{c}0 . \\
00 \\
14 \\
4 \\
\end{array}$ & $\begin{array}{c}0.09 \\
697\end{array}$ & $\begin{array}{c}0 . \\
00 \\
19 \\
2 \\
\end{array}$ & $\begin{array}{l}0.79 \\
726\end{array}$ & $\begin{array}{c}0 . \\
01 \\
78 \\
9 \\
\end{array}$ & 591 & $\begin{array}{l}2 \\
2\end{array}$ & 597 & $\begin{array}{l}1 \\
1\end{array}$ & 595 & $\begin{array}{l}1 \\
0\end{array}$ & $\begin{array}{c}- \\
0.3 \\
4 \%\end{array}$ & $\begin{array}{l}5 \\
9 \\
7\end{array}$ & $\begin{array}{l}1 \\
1\end{array}$ \\
\hline $\begin{array}{c}\mathrm{M} \\
\mathrm{O} 1 \\
7- \\
73\end{array}$ & $\begin{array}{l}1 \\
0 \\
9\end{array}$ & $\begin{array}{l}1 \\
4 \\
6\end{array}$ & $\begin{array}{l}0 \\
5 \\
7 \\
5\end{array}$ & $\begin{array}{l}0.06 \\
208\end{array}$ & $\begin{array}{c}0 . \\
00 \\
10 \\
3 \\
\end{array}$ & $\begin{array}{l}0.10 \\
220\end{array}$ & $\begin{array}{c}0 . \\
00 \\
19 \\
0 \\
\end{array}$ & $\begin{array}{l}0.87 \\
458\end{array}$ & $\begin{array}{c}0 . \\
01 \\
33 \\
8 \\
\end{array}$ & 677 & $\begin{array}{l}1 \\
8\end{array}$ & 627 & $\begin{array}{l}1 \\
1\end{array}$ & 638 & 7 & $\begin{array}{l}1.7 \\
5 \%\end{array}$ & $\begin{array}{l}6 \\
2 \\
7\end{array}$ & $\begin{array}{l}1 \\
1\end{array}$ \\
\hline $\begin{array}{c}\text { M } \\
\text { O1 } \\
7- \\
74 \\
\end{array}$ & $\begin{array}{l}1 \\
8\end{array}$ & $\begin{array}{l}3 \\
5\end{array}$ & $\begin{array}{l}5 \\
1 \\
\end{array}$ & $\begin{array}{c}0.05 \\
610\end{array}$ & $\begin{array}{c}0 . \\
00 \\
34 \\
0 \\
\end{array}$ & $\begin{array}{l}0.07 \\
344\end{array}$ & $\begin{array}{c}0 . \\
00 \\
21 \\
4 \\
\end{array}$ & $\begin{array}{l}0.56 \\
793\end{array}$ & $\begin{array}{c}0 . \\
03 \\
23 \\
2 \\
\end{array}$ & 456 & $\begin{array}{l}7 \\
5\end{array}$ & 457 & $\begin{array}{l}1 \\
3\end{array}$ & 457 & $\begin{array}{l}2 \\
1\end{array}$ & $\begin{array}{l}0.0 \\
0 \%\end{array}$ & $\begin{array}{l}4 \\
5 \\
7\end{array}$ & $\begin{array}{l}1 \\
3\end{array}$ \\
\hline $\begin{array}{c}\mathrm{M} \\
\mathrm{O} 1 \\
7- \\
75 \\
\end{array}$ & $\begin{array}{l}4 \\
3\end{array}$ & $\begin{array}{l}4 \\
5\end{array}$ & $\begin{array}{l}9 \\
6 \\
\end{array}$ & $\begin{array}{c}0.13 \\
067\end{array}$ & $\begin{array}{c}0 . \\
00 \\
18 \\
8 \\
\end{array}$ & $\begin{array}{l}0.37 \\
661\end{array}$ & $\begin{array}{c}0 . \\
00 \\
72 \\
2 \\
\end{array}$ & $\begin{array}{l}6.78 \\
416\end{array}$ & $\begin{array}{c}0 . \\
09 \\
02 \\
7 \\
\end{array}$ & $\begin{array}{c}210 \\
7\end{array}$ & $\begin{array}{l}1 \\
7\end{array}$ & $\begin{array}{c}206 \\
0\end{array}$ & $\begin{array}{l}3 \\
4\end{array}$ & $\begin{array}{c}208 \\
4\end{array}$ & $\begin{array}{l}1 \\
2\end{array}$ & $\begin{array}{l}2.2 \\
8 \%\end{array}$ & $\begin{array}{l}2 \\
1 \\
0 \\
7\end{array}$ & $\begin{array}{l}1 \\
7\end{array}$ \\
\hline $\begin{array}{l}\mathrm{M} \\
\mathrm{O} 1 \\
\end{array}$ & $\begin{array}{l}4 \\
9 \\
\end{array}$ & $\begin{array}{l}1 \\
1 \\
\end{array}$ & 0 & $\begin{array}{c}0.18 \\
588\end{array}$ & $\begin{array}{c}0 . \\
00\end{array}$ & $\begin{array}{l}0.50 \\
302 \\
\end{array}$ & $\begin{array}{c}0 . \\
00\end{array}$ & $\begin{array}{l}12.8 \\
903 \\
\end{array}$ & $\begin{array}{l}0 . \\
12 \\
\end{array}$ & $\begin{array}{c}270 \\
6 \\
\end{array}$ & $\begin{array}{l}1 \\
7 \\
\end{array}$ & $\begin{array}{c}262 \\
7 \\
\end{array}$ & $\begin{array}{l}3 \\
9 \\
\end{array}$ & $\begin{array}{c}267 \\
2 \\
\end{array}$ & 9 & $\begin{array}{l}3.0 \\
1 \% \\
\end{array}$ & $\begin{array}{l}2 \\
7 \\
\end{array}$ & $\begin{array}{l}1 \\
7\end{array}$ \\
\hline
\end{tabular}




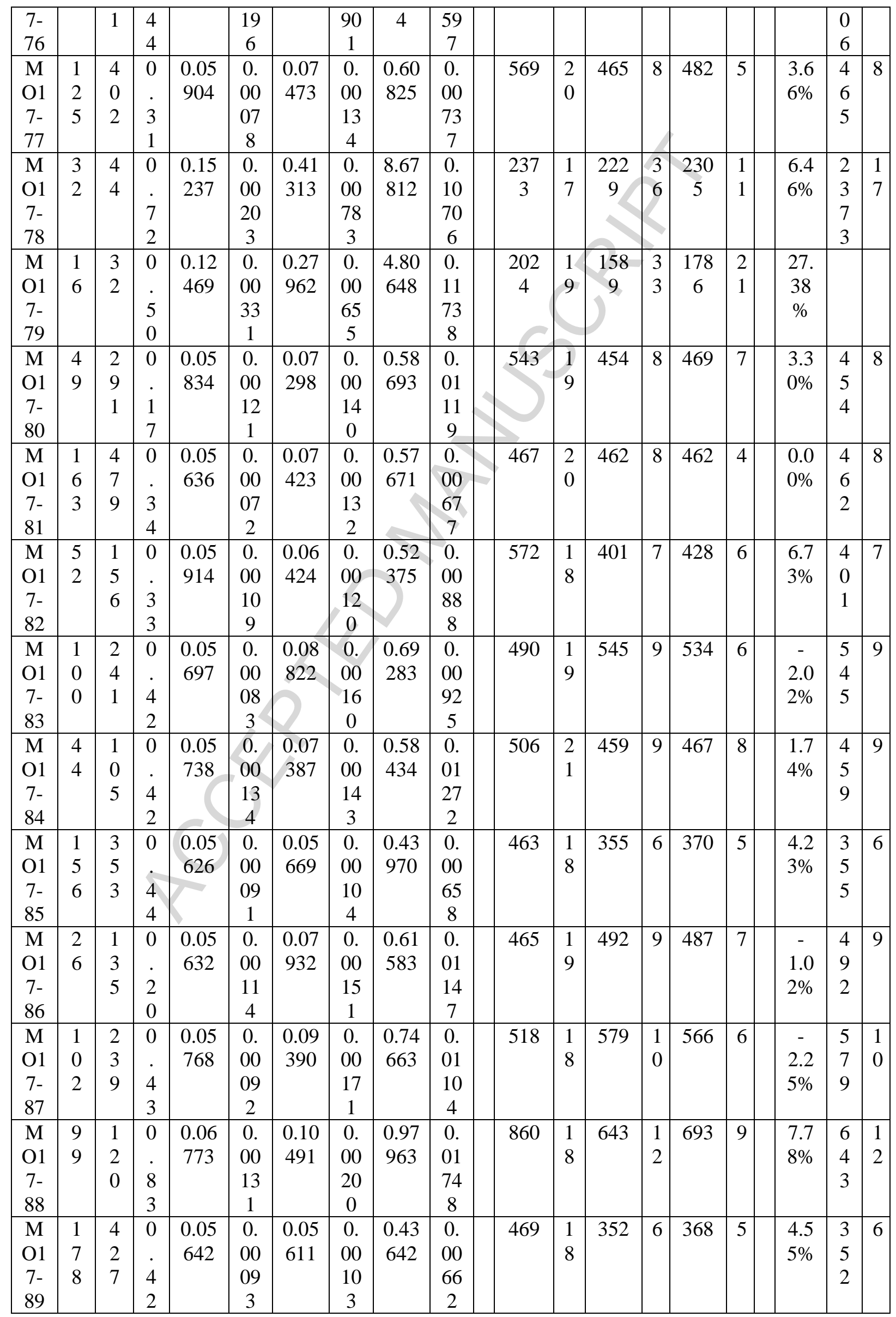




\begin{tabular}{|c|c|c|c|c|c|c|c|c|c|c|c|c|c|c|c|c|c|c|}
\hline $\begin{array}{c}\mathrm{M} \\
\mathrm{O} 1 \\
7- \\
90\end{array}$ & $\begin{array}{l}1 \\
0 \\
0\end{array}$ & $\begin{array}{l}2 \\
1 \\
3\end{array}$ & $\begin{array}{l}4 \\
7\end{array}$ & $\begin{array}{c}0.18 \\
429\end{array}$ & $\begin{array}{c}0 . \\
00 \\
19 \\
4\end{array}$ & $\begin{array}{c}0.50 \\
057\end{array}$ & $\begin{array}{c}0 . \\
00 \\
89 \\
5\end{array}$ & $\begin{array}{c}12.7 \\
174 \\
1\end{array}$ & $\begin{array}{c}0 . \\
12 \\
35 \\
9\end{array}$ & $\begin{array}{c}269 \\
2\end{array}$ & $\begin{array}{l}1 \\
7\end{array}$ & $\begin{array}{c}261 \\
6\end{array}$ & \begin{tabular}{l|}
3 \\
8
\end{tabular} & $\begin{array}{c}265 \\
9\end{array}$ & 9 & $\begin{array}{l}2.9 \\
1 \%\end{array}$ & $\begin{array}{l}2 \\
6 \\
9 \\
2\end{array}$ & $\begin{array}{l}1 \\
7\end{array}$ \\
\hline $\begin{array}{l}\mathrm{M} \\
\mathrm{O} 1 \\
7- \\
91\end{array}$ & $\begin{array}{l}4 \\
3\end{array}$ & $\begin{array}{l}1 \\
4 \\
6\end{array}$ & $\begin{array}{l}0 \\
2 \\
9 \\
\end{array}$ & \begin{tabular}{|l|}
0.05 \\
826
\end{tabular} & $\begin{array}{c}0 . \\
00 \\
13 \\
1 \\
\end{array}$ & $\begin{array}{l}0.07 \\
970\end{array}$ & $\begin{array}{c}0 . \\
00 \\
15 \\
6 \\
\end{array}$ & $\begin{array}{c}0.64 \\
019\end{array}$ & $\begin{array}{c}0 . \\
01 \\
32 \\
6\end{array}$ & 540 & $\begin{array}{l}2 \\
0\end{array}$ & 494 & 9 & 502 & 8 & $\begin{array}{l}1.6 \\
2 \%\end{array}$ & $\begin{array}{l}4 \\
9 \\
4\end{array}$ & 9 \\
\hline $\begin{array}{c}\mathrm{M} \\
\mathrm{O} 1 \\
7- \\
92\end{array}$ & $\begin{array}{l}1 \\
4 \\
7\end{array}$ & $\begin{array}{l}2 \\
6 \\
8\end{array}$ & $\begin{array}{l}5 \\
5\end{array}$ & $\begin{array}{l}0.05 \\
273\end{array}$ & $\begin{array}{c}0 . \\
00 \\
11 \\
3\end{array}$ & $\begin{array}{l}0.05 \\
551\end{array}$ & $\begin{array}{c}0 . \\
00 \\
10 \\
6\end{array}$ & $\begin{array}{l}0.40 \\
351\end{array}$ & $\begin{array}{c}0 . \\
00 \\
79 \\
9\end{array}$ & 317 & $\begin{array}{l}2 \\
0\end{array}$ & & 6 & 344 & 6 & $\begin{array}{c}- \\
1.1 \\
5 \%\end{array}$ & $\begin{array}{l}3 \\
4 \\
8\end{array}$ & 6 \\
\hline $\begin{array}{c}\mathrm{M} \\
\mathrm{O} 1 \\
7- \\
93 \\
\end{array}$ & $\begin{array}{l}4 \\
1\end{array}$ & $\begin{array}{l}4 \\
5 \\
2\end{array}$ & $\begin{array}{l}0 \\
9 \\
\end{array}$ & $\begin{array}{l}0.05 \\
752\end{array}$ & $\begin{array}{c}0 . \\
00 \\
08 \\
6 \\
\end{array}$ & $\begin{array}{c}0.08 \\
076\end{array}$ & $\begin{array}{c}0 . \\
00 \\
14 \\
7 \\
\end{array}$ & $\begin{array}{c}0.64 \\
041\end{array}$ & $\begin{array}{c}0 . \\
00 \\
87 \\
6 \\
\end{array}$ & 512 & $\begin{array}{l}1 \\
9\end{array}$ & 501 & 9 & 503 & 5 & $\begin{array}{l}0.4 \\
0 \%\end{array}$ & $\begin{array}{l}5 \\
0 \\
1\end{array}$ & 9 \\
\hline $\begin{array}{l}\text { M } \\
\text { O1 } \\
7- \\
94\end{array}$ & $\begin{array}{l}8 \\
9\end{array}$ & $\begin{array}{l}9 \\
0\end{array}$ & $\begin{array}{l}9 \\
9 \\
\end{array}$ & $\begin{array}{l}0.05 \\
707\end{array}$ & $\begin{array}{c}0 . \\
00 \\
16 \\
8 \\
\end{array}$ & $\begin{array}{c}0.09 \\
502\end{array}$ & $\begin{array}{c}0 . \\
00 \\
20 \\
0\end{array}$ & $\begin{array}{c}0.74 \\
760\end{array}$ & $\begin{array}{c}0 . \\
02 \\
04 \\
1\end{array}$ & 494 & $\begin{array}{l}2 \\
8\end{array}$ & 585 & $\begin{array}{l}1 \\
2\end{array}$ & 567 & $\begin{array}{l}1 \\
2\end{array}$ & $\begin{array}{c}- \\
3.0 \\
8 \%\end{array}$ & $\begin{array}{l}5 \\
8 \\
5\end{array}$ & $\begin{array}{l}1 \\
2\end{array}$ \\
\hline $\begin{array}{l}\mathrm{M} \\
\mathrm{O} 1 \\
7- \\
95\end{array}$ & $\begin{array}{l}3 \\
8\end{array}$ & $\begin{array}{l}3 \\
2 \\
2\end{array}$ & $\begin{array}{l}1 \\
2 \\
\end{array}$ & $\begin{array}{l}0.05 \\
634\end{array}$ & $\begin{array}{c}0 . \\
00 \\
09 \\
4\end{array}$ & $\begin{array}{l}0.07 \\
378\end{array}$ & $\begin{array}{c}0 . \\
00 \\
13 \\
5\end{array}$ & $\begin{array}{l}0.57 \\
301\end{array}$ & $\begin{array}{c}0 . \\
00 \\
88 \\
3\end{array}$ & 466 & $\begin{array}{l}1 \\
8\end{array}$ & 459 & 8 & 460 & 6 & $\begin{array}{l}0.2 \\
2 \%\end{array}$ & $\begin{array}{l}4 \\
5 \\
9\end{array}$ & 8 \\
\hline $\begin{array}{c}\text { M } \\
\text { O1 } \\
7- \\
96 \\
\end{array}$ & $\begin{array}{l}5 \\
2\end{array}$ & $\begin{array}{l}1 \\
8 \\
3\end{array}$ & $\begin{array}{l}0 \\
2 \\
8 \\
\end{array}$ & $\begin{array}{l}0.05 \\
771\end{array}$ & $\begin{array}{c}0 . \\
00 \\
10 \\
1 \\
\end{array}$ & $\begin{array}{l}0.07 \\
750\end{array}$ & $\begin{array}{c}0 . \\
00 \\
14 \\
4 \\
\end{array}$ & $\begin{array}{l}0.61 \\
655\end{array}$ & $\begin{array}{c}0 . \\
00 \\
99 \\
1 \\
\end{array}$ & 519 & $\begin{array}{l}1 \\
8\end{array}$ & 481 & 9 & 488 & 6 & $\begin{array}{l}1.4 \\
6 \%\end{array}$ & $\begin{array}{l}4 \\
8 \\
1\end{array}$ & 9 \\
\hline $\begin{array}{c}\mathrm{M} \\
\mathrm{O} 1 \\
7- \\
97\end{array}$ & $\begin{array}{l}6 \\
5\end{array}$ & $\begin{array}{l}2 \\
1 \\
5\end{array}$ & $\begin{array}{l}0 \\
3 \\
0\end{array}$ & $\begin{array}{l}0.05 \\
875\end{array}$ & $\begin{array}{c}0 . \\
00 \\
08 \\
9\end{array}$ & $\begin{array}{l}0.09 \\
446\end{array}$ & $\begin{array}{c}0 . \\
00 \\
17 \\
2\end{array}$ & $\begin{array}{c}0.76 \\
510\end{array}$ & $\begin{array}{c}0 . \\
01 \\
06 \\
5\end{array}$ & 558 & $\begin{array}{l}1 \\
9\end{array}$ & 582 & $\begin{array}{l}1 \\
0\end{array}$ & 577 & 6 & $\begin{array}{c}- \\
0.8 \\
6 \%\end{array}$ & $\begin{array}{l}5 \\
8 \\
2\end{array}$ & $\begin{array}{l}1 \\
0\end{array}$ \\
\hline $\begin{array}{c}\text { M } \\
\text { O1 } \\
7- \\
98\end{array}$ & $\begin{array}{l}6 \\
1\end{array}$ & $\begin{array}{l}1 \\
5 \\
5\end{array}$ & $\begin{array}{l}3 \\
9\end{array}$ & $\begin{array}{l}0.05 \\
598\end{array}$ & $\begin{array}{c}0 . \\
00 \\
11 \\
0\end{array}$ & $\begin{array}{c}0.08 \\
888\end{array}$ & $\begin{array}{c}0 . \\
00 \\
16 \\
8\end{array}$ & $\begin{array}{c}0.68 \\
590\end{array}$ & $\begin{array}{c}0 . \\
01 \\
24 \\
0\end{array}$ & 452 & $\begin{array}{l}1 \\
9\end{array}$ & 549 & $\begin{array}{l}1 \\
0\end{array}$ & 530 & 7 & $\begin{array}{c}- \\
3.4 \\
6 \%\end{array}$ & $\begin{array}{l}5 \\
4 \\
9\end{array}$ & $\begin{array}{l}1 \\
0\end{array}$ \\
\hline $\begin{array}{c}\text { M } \\
\text { O1 } \\
7- \\
99\end{array}$ & $\begin{array}{l}4 \\
7\end{array}$ & $\begin{array}{l}1 \\
6 \\
2\end{array}$ & $\begin{array}{l}0 \\
\dot{2} \\
9\end{array}$ & $\begin{array}{c}0.06 \\
149\end{array}$ & $\begin{array}{c}0 . \\
00 \\
11 \\
5\end{array}$ & $\begin{array}{l}0.11 \\
732\end{array}$ & $\begin{array}{c}0 . \\
00 \\
22 \\
3\end{array}$ & $\begin{array}{l}0.99 \\
441\end{array}$ & $\begin{array}{c}0 . \\
01 \\
71 \\
6\end{array}$ & 656 & $\begin{array}{l}1 \\
8\end{array}$ & 715 & $\begin{array}{l}1 \\
3\end{array}$ & 701 & 9 & $\begin{array}{c}- \\
1.9 \\
6 \%\end{array}$ & $\begin{array}{l}7 \\
1 \\
5\end{array}$ & $\begin{array}{l}1 \\
3\end{array}$ \\
\hline $\begin{array}{c}\mathrm{M} \\
\mathrm{O} 1 \\
7- \\
10 \\
0 \\
\end{array}$ & $\begin{array}{l}1 \\
9\end{array}$ & $\begin{array}{l}2 \\
4\end{array}$ & $\begin{array}{l}7 \\
8\end{array}$ & $\begin{array}{l}0.05 \\
537\end{array}$ & $\begin{array}{c}0 . \\
00 \\
39 \\
8\end{array}$ & $\begin{array}{c}0.08 \\
074\end{array}$ & $\begin{array}{c}0 . \\
00 \\
21 \\
4\end{array}$ & $\begin{array}{l}0.61 \\
629\end{array}$ & $\begin{array}{c}0 . \\
04 \\
28 \\
8\end{array}$ & 427 & $\begin{array}{l}1 \\
0 \\
8\end{array}$ & 501 & $\begin{array}{l}1 \\
3\end{array}$ & 488 & $\begin{array}{l}2 \\
7\end{array}$ & $\begin{array}{c}- \\
2.5 \\
9 \%\end{array}$ & $\begin{array}{l}5 \\
0 \\
1\end{array}$ & $\begin{array}{l}1 \\
3\end{array}$ \\
\hline $\begin{array}{c}\mathrm{M} \\
\mathrm{O} 2 \\
6- \\
01 \\
\end{array}$ & $\begin{array}{l}1 \\
4 \\
3\end{array}$ & $\begin{array}{l}1 \\
6 \\
0\end{array}$ & $\begin{array}{l}0 \\
. \\
9 \\
0 \\
\end{array}$ & $\begin{array}{c}0.18 \\
803\end{array}$ & $\begin{array}{c}0 . \\
00 \\
12 \\
6 \\
\end{array}$ & $\begin{array}{c}12.5 \\
928 \\
3\end{array}$ & $\begin{array}{c}0 . \\
08 \\
47 \\
8 \\
\end{array}$ & $\begin{array}{c}0.48 \\
569\end{array}$ & $\begin{array}{c}0 . \\
00 \\
54 \\
3 \\
\end{array}$ & $\begin{array}{c}272 \\
5\end{array}$ & $\begin{array}{l}1 \\
0\end{array}$ & $\begin{array}{c}265 \\
0\end{array}$ & 6 & $\begin{array}{c}255 \\
2\end{array}$ & $\begin{array}{l}2 \\
4\end{array}$ & $\begin{array}{l}6.7 \\
8 \%\end{array}$ & $\begin{array}{l}2 \\
7 \\
2 \\
5\end{array}$ & $\begin{array}{l}1 \\
0\end{array}$ \\
\hline $\begin{array}{c}\mathrm{M} \\
\mathrm{O} 2 \\
6- \\
02\end{array}$ & $\begin{array}{l}2 \\
2 \\
6\end{array}$ & $\begin{array}{l}5 \\
4 \\
9\end{array}$ & $\begin{array}{l}0 \\
4 \\
1\end{array}$ & $\begin{array}{l}0.05 \\
985\end{array}$ & $\begin{array}{c}0 . \\
00 \\
05 \\
2\end{array}$ & $\begin{array}{l}0.62 \\
440\end{array}$ & $\begin{array}{c}0 . \\
00 \\
52 \\
5\end{array}$ & $\begin{array}{l}0.07 \\
566\end{array}$ & $\begin{array}{c}0 . \\
00 \\
08 \\
3\end{array}$ & 598 & $\begin{array}{l}1 \\
1\end{array}$ & 493 & 3 & 470 & 5 & $\begin{array}{l}4.8 \\
9 \%\end{array}$ & $\begin{array}{l}4 \\
7 \\
0\end{array}$ & 5 \\
\hline
\end{tabular}




\begin{tabular}{|c|c|c|c|c|c|c|c|c|c|c|c|c|c|c|c|c|c|c|}
\hline $\begin{array}{c}\mathrm{M} \\
\mathrm{O} 2 \\
6- \\
03\end{array}$ & $\begin{array}{l}4 \\
7\end{array}$ & $\begin{array}{l}1 \\
0 \\
2 \\
2\end{array}$ & $\begin{array}{l}0 \\
. \\
0 \\
5\end{array}$ & $\begin{array}{l}0.17 \\
896\end{array}$ & $\begin{array}{c}0 . \\
00 \\
09 \\
5\end{array}$ & $\begin{array}{c}10.2 \\
238 \\
5\end{array}$ & $\begin{array}{c}0 . \\
05 \\
64 \\
9\end{array}$ & $\begin{array}{l}0.41 \\
432\end{array}$ & $\begin{array}{c}0 . \\
00 \\
43 \\
9\end{array}$ & $\begin{array}{c}264 \\
3\end{array}$ & $\begin{array}{l}1 \\
0\end{array}$ & $\begin{array}{c}245 \\
5\end{array}$ & 5 & $\begin{array}{c}223 \\
5\end{array}$ & $\begin{array}{l}2 \\
0\end{array}$ & $\begin{array}{l}18 . \\
26 \\
\%\end{array}$ & & \\
\hline $\begin{array}{c}\mathrm{M} \\
\mathrm{O} 2 \\
6- \\
04\end{array}$ & $\begin{array}{l}1 \\
4\end{array}$ & $\begin{array}{l}8 \\
0 \\
6\end{array}$ & $\begin{array}{l}0 \\
. \\
0 \\
2\end{array}$ & $\begin{array}{c}0.05 \\
562\end{array}$ & $\begin{array}{c}0 . \\
00 \\
04 \\
7 \\
\end{array}$ & $\begin{array}{c}0.51 \\
164\end{array}$ & $\begin{array}{c}0 . \\
00 \\
41 \\
7\end{array}$ & $\begin{array}{l}0.06 \\
672\end{array}$ & $\begin{array}{c}0 . \\
00 \\
07 \\
3 \\
\end{array}$ & 437 & $\begin{array}{l}1 \\
2\end{array}$ & 420 & 3 & 416 & 4 & $\begin{array}{l}0.9 \\
6 \%\end{array}$ & $\begin{array}{l}4 \\
1 \\
6\end{array}$ & 4 \\
\hline $\begin{array}{c}\mathrm{M} \\
\mathrm{O} 2 \\
6- \\
05\end{array}$ & $\begin{array}{l}5 \\
1\end{array}$ & $\begin{array}{l}5 \\
6 \\
0\end{array}$ & $\begin{array}{l}0 \\
9\end{array}$ & $\begin{array}{c}0.16 \\
104\end{array}$ & $\begin{array}{c}0 . \\
00 \\
08 \\
9\end{array}$ & $\begin{array}{l}8.32 \\
408\end{array}$ & $\begin{array}{c}0 . \\
04 \\
76 \\
4\end{array}$ & $\begin{array}{l}0.37 \\
488\end{array}$ & $\begin{array}{c}0 . \\
00 \\
40 \\
0\end{array}$ & $\begin{array}{c}246 \\
7\end{array}$ & $\begin{array}{l}1 \\
0\end{array}$ & 226 & 5 & $\begin{array}{c}205 \\
2\end{array}$ & $\begin{array}{l}1 \\
9\end{array}$ & $\begin{array}{l}20 . \\
22 \\
\%\end{array}$ & & \\
\hline $\begin{array}{c}\mathrm{M} \\
\mathrm{O} 2 \\
6- \\
06 \\
\end{array}$ & $\begin{array}{l}1 \\
1 \\
3\end{array}$ & $\begin{array}{l}2 \\
0 \\
8\end{array}$ & $\begin{array}{l}5 \\
4 \\
\end{array}$ & $\begin{array}{c}0.05 \\
603\end{array}$ & $\begin{array}{c}0 . \\
00 \\
07 \\
5 \\
\end{array}$ & $\begin{array}{c}0.43 \\
589\end{array}$ & $\begin{array}{c}0 . \\
00 \\
55 \\
0 \\
\end{array}$ & $\begin{array}{l}0.05 \\
642\end{array}$ & $\begin{array}{c}0 . \\
00 \\
06 \\
7 \\
\end{array}$ & 454 & $\begin{array}{l}1 \\
3\end{array}$ & 367 & 4 & 354 & 4 & $\begin{array}{l}3.6 \\
7 \%\end{array}$ & $\begin{array}{l}3 \\
5 \\
4\end{array}$ & 4 \\
\hline $\begin{array}{c}\mathrm{M} \\
\mathrm{O} 2 \\
6- \\
07\end{array}$ & $\begin{array}{l}2 \\
0\end{array}$ & $\begin{array}{l}3 \\
0\end{array}$ & $\begin{array}{l}6 \\
7 \\
\end{array}$ & $\begin{array}{l}0.07 \\
823\end{array}$ & $\begin{array}{c}0 . \\
00 \\
18 \\
6 \\
\end{array}$ & $\begin{array}{l}1.07 \\
548\end{array}$ & $\begin{array}{c}0 . \\
02 \\
30 \\
1\end{array}$ & $\begin{array}{l}0.09 \\
971\end{array}$ & $\begin{array}{c}0 . \\
00 \\
15 \\
9\end{array}$ & $\begin{array}{c}115 \\
3\end{array}$ & $\begin{array}{l}2 \\
0\end{array}$ & 741 & $\begin{array}{l}1 \\
1\end{array}$ & 613 & 9 & $\begin{array}{c}20 . \\
88 \\
\%\end{array}$ & & \\
\hline $\begin{array}{c}\mathrm{M} \\
\mathrm{O} 2 \\
6- \\
08 \\
\end{array}$ & $\begin{array}{l}5 \\
9\end{array}$ & $\begin{array}{l}3 \\
5\end{array}$ & $\begin{array}{l}1 \\
6 \\
8 \\
\end{array}$ & $\begin{array}{l}0.12 \\
294\end{array}$ & $\begin{array}{c}0 . \\
00 \\
16 \\
9 \\
\end{array}$ & $\begin{array}{l}5.86 \\
086\end{array}$ & $\begin{array}{c}0 . \\
07 \\
53 \\
2 \\
\end{array}$ & $\begin{array}{l}0.34 \\
574\end{array}$ & $\begin{array}{c}0 . \\
00 \\
47 \\
6\end{array}$ & $\begin{array}{c}199 \\
9\end{array}$ & $\begin{array}{l}1 \\
1\end{array}$ & $\begin{array}{c}195 \\
5\end{array}$ & $\begin{array}{l}1 \\
1\end{array}$ & $\begin{array}{c}191 \\
4\end{array}$ & $\begin{array}{l}2 \\
3\end{array}$ & $\begin{array}{l}4.4 \\
4 \%\end{array}$ & $\begin{array}{l}1 \\
9 \\
9 \\
9 \\
\end{array}$ & $\begin{array}{l}1 \\
1\end{array}$ \\
\hline $\begin{array}{c}\mathrm{M} \\
\mathrm{O} 2 \\
6- \\
09\end{array}$ & $\begin{array}{l}4 \\
3 \\
0\end{array}$ & $\begin{array}{l}5 \\
0 \\
9\end{array}$ & $\begin{array}{l}0 \\
. \\
8 \\
5\end{array}$ & $\begin{array}{l}0.05 \\
708\end{array}$ & $\begin{array}{c}0 . \\
00 \\
05 \\
6\end{array}$ & $\begin{array}{c}0.44 \\
116\end{array}$ & $\begin{array}{c}0 . \\
00 \\
41 \\
5\end{array}$ & $\begin{array}{l}0.05 \\
605\end{array}$ & $\begin{array}{c}0 . \\
00 \\
06 \\
3\end{array}$ & 495 & $\begin{array}{l}1 \\
1\end{array}$ & 371 & 3 & 352 & 4 & $\begin{array}{l}5.4 \\
0 \%\end{array}$ & $\begin{array}{l}3 \\
5 \\
2\end{array}$ & 4 \\
\hline $\begin{array}{c}\mathrm{M} \\
\mathrm{O} 2 \\
6- \\
10\end{array}$ & $\begin{array}{l}2 \\
1 \\
9\end{array}$ & $\begin{array}{l}5 \\
6 \\
8\end{array}$ & $\begin{array}{l}3 \\
9\end{array}$ & $\begin{array}{c}0.11 \\
633\end{array}$ & $\begin{array}{c}0 . \\
00 \\
18 \\
4\end{array}$ & $\begin{array}{l}3.65 \\
526\end{array}$ & $\begin{array}{c}0 . \\
04 \\
10 \\
7\end{array}$ & $\begin{array}{l}0.22 \\
789\end{array}$ & $\begin{array}{c}0 . \\
00 \\
25 \\
3\end{array}$ & $\begin{array}{c}190 \\
1\end{array}$ & $\begin{array}{l}2 \\
9\end{array}$ & $\begin{array}{c}156 \\
2\end{array}$ & 9 & $\begin{array}{c}132 \\
3\end{array}$ & $\begin{array}{l}1 \\
3\end{array}$ & $\begin{array}{c}43 . \\
69 \\
\%\end{array}$ & & \\
\hline $\begin{array}{c}\mathrm{M} \\
\mathrm{O} 2 \\
6- \\
11\end{array}$ & $\begin{array}{l}2 \\
7 \\
6\end{array}$ & $\begin{array}{l}5 \\
2 \\
4\end{array}$ & $\begin{array}{l}5 \\
3\end{array}$ & $\begin{array}{l}0.10 \\
794\end{array}$ & $\begin{array}{c}0 . \\
00 \\
07 \\
0\end{array}$ & $\begin{array}{l}3.75 \\
312\end{array}$ & $\begin{array}{c}0 . \\
02 \\
43 \\
7\end{array}$ & $\begin{array}{l}0.25 \\
217\end{array}$ & $\begin{array}{c}0 . \\
00 \\
27 \\
4\end{array}$ & $\begin{array}{c}176 \\
5\end{array}$ & $\begin{array}{l}1 \\
1\end{array}$ & $\begin{array}{c}158 \\
3\end{array}$ & 5 & $\begin{array}{c}145 \\
0\end{array}$ & $\begin{array}{l}1 \\
4\end{array}$ & $\begin{array}{l}21 . \\
72 \\
\%\end{array}$ & & \\
\hline $\begin{array}{c}\mathrm{M} \\
\mathrm{O} 2 \\
6- \\
12\end{array}$ & $\begin{array}{l}4 \\
0 \\
1\end{array}$ & $\begin{array}{l}5 \\
7 \\
3\end{array}$ & $\begin{array}{l}0 \\
7 \\
0\end{array}$ & $\begin{array}{c}0.06 \\
174\end{array}$ & $\begin{array}{c}0 . \\
00 \\
05 \\
0\end{array}$ & $\begin{array}{l}0.89 \\
951\end{array}$ & $\begin{array}{c}0 . \\
00 \\
71 \\
1 \\
\end{array}$ & $\begin{array}{c}0.10 \\
566\end{array}$ & $\begin{array}{c}0 . \\
00 \\
11 \\
6 \\
\end{array}$ & 665 & $\begin{array}{l}1 \\
1\end{array}$ & 651 & 4 & 647 & 7 & $\begin{array}{l}0.6 \\
2 \%\end{array}$ & $\begin{array}{l}6 \\
4 \\
7\end{array}$ & 7 \\
\hline $\begin{array}{c}\mathrm{M} \\
\mathrm{O} 2 \\
6- \\
13 \\
\end{array}$ & $\begin{array}{l}9 \\
5\end{array}$ & $\begin{array}{l}2 \\
9 \\
6\end{array}$ & $\begin{array}{l}0 \\
3 \\
2 \\
\end{array}$ & $\begin{array}{l}0.05 \\
768\end{array}$ & $\begin{array}{c}0 . \\
00 \\
06 \\
3 \\
\end{array}$ & $\begin{array}{c}0.66 \\
180\end{array}$ & $\begin{array}{c}0 . \\
00 \\
68 \\
7 \\
\end{array}$ & $\begin{array}{l}0.08 \\
321\end{array}$ & $\begin{array}{c}0 . \\
00 \\
09 \\
5\end{array}$ & 518 & $\begin{array}{l}1 \\
1\end{array}$ & 516 & 4 & 515 & 6 & $\begin{array}{l}0.1 \\
9 \%\end{array}$ & $\begin{array}{l}5 \\
1 \\
5\end{array}$ & 6 \\
\hline $\begin{array}{c}\mathrm{M} \\
\mathrm{O} 2 \\
6- \\
14 \\
\end{array}$ & $\begin{array}{l}2 \\
3 \\
1\end{array}$ & $\begin{array}{l}4 \\
0 \\
8\end{array}$ & $\begin{array}{l}5 \\
7 \\
\end{array}$ & $\begin{array}{l}0.05 \\
871\end{array}$ & $\begin{array}{c}0 . \\
00 \\
06 \\
6 \\
\end{array}$ & $\begin{array}{c}0.46 \\
021\end{array}$ & $\begin{array}{c}0 . \\
00 \\
48 \\
6 \\
\end{array}$ & $\begin{array}{l}0.05 \\
685\end{array}$ & $\begin{array}{c}0 . \\
00 \\
06 \\
5\end{array}$ & 556 & $\begin{array}{l}1 \\
1\end{array}$ & 384 & 3 & 356 & 4 & $\begin{array}{l}7.8 \\
7 \%\end{array}$ & $\begin{array}{l}3 \\
5 \\
6\end{array}$ & 4 \\
\hline $\begin{array}{c}\mathrm{M} \\
\mathrm{O} 2 \\
6- \\
15 \\
\end{array}$ & $\begin{array}{l}6 \\
3\end{array}$ & $\begin{array}{l}5 \\
9\end{array}$ & $\begin{array}{l}0 \\
7 \\
\end{array}$ & $\begin{array}{l}0.12 \\
664\end{array}$ & $\begin{array}{c}0 . \\
00 \\
12 \\
6 \\
\end{array}$ & $\begin{array}{l}6.17 \\
930\end{array}$ & $\begin{array}{c}0 . \\
05 \\
83 \\
9 \\
\end{array}$ & $\begin{array}{l}0.35 \\
387\end{array}$ & $\begin{array}{c}0 . \\
00 \\
42 \\
9 \\
\end{array}$ & $\begin{array}{c}205 \\
2\end{array}$ & $\begin{array}{l}1 \\
0\end{array}$ & $\begin{array}{c}200 \\
2\end{array}$ & 8 & $\begin{array}{c}195 \\
3\end{array}$ & $\begin{array}{l}2 \\
0\end{array}$ & $\begin{array}{l}5.0 \\
7 \%\end{array}$ & $\begin{array}{l}2 \\
0 \\
5 \\
2 \\
\end{array}$ & $\begin{array}{l}1 \\
0\end{array}$ \\
\hline $\begin{array}{l}\mathrm{M} \\
\mathrm{O} 2 \\
\end{array}$ & $\begin{array}{l}6 \\
7 \\
\end{array}$ & $\begin{array}{l}9 \\
3 \\
\end{array}$ & 0 & $\begin{array}{c}0.06 \\
357 \\
\end{array}$ & $\begin{array}{c}0 . \\
00 \\
\end{array}$ & $\begin{array}{c}0.88 \\
911 \\
\end{array}$ & $\begin{array}{c}0 . \\
01\end{array}$ & $\begin{array}{c}0.10 \\
143 \\
\end{array}$ & $\begin{array}{c}0 . \\
00 \\
\end{array}$ & 727 & $\begin{array}{l}1 \\
2 \\
\end{array}$ & 646 & 6 & 623 & 7 & $\begin{array}{l}3.6 \\
9 \% \\
\end{array}$ & $\begin{array}{l}6 \\
2 \\
\end{array}$ & 7 \\
\hline
\end{tabular}




\begin{tabular}{|c|c|c|c|c|c|c|c|c|c|c|c|c|c|c|c|c|c|c|}
\hline $\begin{array}{l}6- \\
16\end{array}$ & & & $\begin{array}{l}7 \\
3\end{array}$ & & $\begin{array}{c}08 \\
9\end{array}$ & & $\begin{array}{c}16 \\
5\end{array}$ & & $\begin{array}{c}12 \\
4\end{array}$ & & & & & & & & 3 & \\
\hline $\begin{array}{c}\mathrm{M} \\
\mathrm{O} 2 \\
6- \\
17\end{array}$ & $\begin{array}{l}8 \\
6\end{array}$ & $\begin{array}{l}1 \\
3 \\
0\end{array}$ & $\begin{array}{l}0 \\
6 \\
6\end{array}$ & $\begin{array}{c}0.05 \\
688\end{array}$ & $\begin{array}{c}0 . \\
00 \\
09 \\
9\end{array}$ & $\begin{array}{l}0.61 \\
797\end{array}$ & $\begin{array}{c}0 . \\
01 \\
00 \\
1\end{array}$ & $\begin{array}{l}0.07 \\
879\end{array}$ & $\begin{array}{c}0 . \\
00 \\
10 \\
2\end{array}$ & 487 & $\begin{array}{l}1 \\
6\end{array}$ & 489 & 6 & 489 & 6 & $\begin{array}{l}0.0 \\
0 \%\end{array}$ & $\begin{array}{l}4 \\
8 \\
9\end{array}$ & 6 \\
\hline $\begin{array}{c}\mathrm{M} \\
\mathrm{O} 2 \\
6- \\
18\end{array}$ & $\begin{array}{l}7 \\
5\end{array}$ & $\begin{array}{l}1 \\
2 \\
7\end{array}$ & $\begin{array}{l}0 \\
. \\
5 \\
9\end{array}$ & $\begin{array}{c}0.11 \\
173\end{array}$ & $\begin{array}{c}0 . \\
00 \\
23 \\
7\end{array}$ & $\begin{array}{l}4.71 \\
664\end{array}$ & $\begin{array}{c}0 . \\
08 \\
11 \\
6\end{array}$ & $\begin{array}{c}0.30 \\
618\end{array}$ & $\begin{array}{c}0 . \\
00 \\
38 \\
1\end{array}$ & $\begin{array}{c}182 \\
8\end{array}$ & $\begin{array}{l}3 \\
9\end{array}$ & $\begin{array}{c}177 \\
0\end{array}$ & 1 & $\begin{array}{c}172 \\
2\end{array}$ & $\begin{array}{l}1 \\
9\end{array}$ & $\begin{array}{l}6.1 \\
6 \%\end{array}$ & $\begin{array}{l}1 \\
8 \\
2 \\
8\end{array}$ & \begin{tabular}{|l|}
3 \\
9
\end{tabular} \\
\hline $\begin{array}{c}\mathrm{M} \\
\mathrm{O} 2 \\
6- \\
19\end{array}$ & $\begin{array}{l}1 \\
8 \\
1\end{array}$ & $\begin{array}{l}2 \\
8 \\
1\end{array}$ & $\begin{array}{l}0 \\
6 \\
4\end{array}$ & $\begin{array}{c}0.06 \\
074\end{array}$ & $\begin{array}{c}0 . \\
00 \\
06 \\
8\end{array}$ & $\begin{array}{l}0.86 \\
815\end{array}$ & $\begin{array}{c}0 . \\
00 \\
91 \\
9\end{array}$ & $\begin{array}{l}0.10 \\
366\end{array}$ & $\begin{array}{c}0 . \\
00 \\
11 \\
9\end{array}$ & 630 & $\begin{array}{l}1 \\
1\end{array}$ & 635 & 5 & 636 & 7 & $\begin{array}{c}- \\
0.1 \\
6 \%\end{array}$ & $\begin{array}{l}6 \\
3 \\
6\end{array}$ & 7 \\
\hline $\begin{array}{c}\mathrm{M} \\
\mathrm{O} 2 \\
6- \\
20\end{array}$ & $\begin{array}{l}2 \\
1 \\
9\end{array}$ & $\begin{array}{l}3 \\
3 \\
4\end{array}$ & $\begin{array}{l}0 \\
6 \\
6\end{array}$ & $\begin{array}{l}0.21 \\
807\end{array}$ & $\begin{array}{c}0 . \\
00 \\
11 \\
9\end{array}$ & $\begin{array}{c}16.8 \\
838 \\
8\end{array}$ & $\begin{array}{c}0 . \\
09 \\
55 \\
1\end{array}$ & $\begin{array}{c}0.56 \\
151\end{array}$ & $\begin{array}{c}0 . \\
00 \\
60 \\
0\end{array}$ & $\begin{array}{c}296 \\
6\end{array}$ & $\begin{array}{l}1 \\
0\end{array}$ & $\begin{array}{c}292 \\
8\end{array}$ & 5 & $\begin{array}{c}287 \\
3\end{array}$ & $\begin{array}{l}2 \\
5\end{array}$ & $\begin{array}{l}3.2 \\
4 \%\end{array}$ & $\begin{array}{l}2 \\
9 \\
6 \\
6\end{array}$ & $\begin{array}{l}1 \\
0\end{array}$ \\
\hline $\begin{array}{c}\mathrm{M} \\
\mathrm{O} 2 \\
6- \\
21\end{array}$ & $\begin{array}{l}7 \\
0\end{array}$ & $\begin{array}{l}2 \\
5 \\
1\end{array}$ & $\begin{array}{l}0 \\
2 \\
8\end{array}$ & $\begin{array}{c}0.05 \\
946\end{array}$ & $\begin{array}{c}0 . \\
00 \\
06 \\
7\end{array}$ & $\begin{array}{l}0.65 \\
702\end{array}$ & $\begin{array}{c}0 . \\
00 \\
69 \\
6\end{array}$ & $\begin{array}{l}0.08 \\
013\end{array}$ & $\begin{array}{c}0 . \\
00 \\
09 \\
2\end{array}$ & 584 & $\begin{array}{l}1 \\
1\end{array}$ & 513 & 4 & 497 & 5 & $\begin{array}{l}3.2 \\
2 \%\end{array}$ & $\begin{array}{l}4 \\
9 \\
7\end{array}$ & 5 \\
\hline $\begin{array}{c}\mathrm{M} \\
\mathrm{O} 2 \\
6- \\
22\end{array}$ & $\begin{array}{l}3 \\
1 \\
1\end{array}$ & $\begin{array}{l}7 \\
7 \\
1\end{array}$ & $\begin{array}{l}4 \\
0\end{array}$ & $\begin{array}{l}0.05 \\
902\end{array}$ & $\begin{array}{c}0 . \\
00 \\
04 \\
4\end{array}$ & $\begin{array}{l}0.67 \\
970\end{array}$ & $\begin{array}{c}0 . \\
00 \\
49 \\
9\end{array}$ & $\begin{array}{c}0.08 \\
353\end{array}$ & $\begin{array}{c}0 . \\
00 \\
09 \\
0\end{array}$ & 568 & $\begin{array}{l}1 \\
2\end{array}$ & 527 & 3 & 517 & 5 & $\begin{array}{l}1.9 \\
3 \%\end{array}$ & $\begin{array}{l}5 \\
1 \\
7\end{array}$ & 5 \\
\hline $\begin{array}{c}\mathrm{M} \\
\mathrm{O} 2 \\
6- \\
23\end{array}$ & $\begin{array}{l}8 \\
7\end{array}$ & $\begin{array}{l}1 \\
9 \\
8\end{array}$ & $\begin{array}{l}0 \\
4 \\
4 \\
\end{array}$ & $\begin{array}{l}0.05 \\
813\end{array}$ & $\begin{array}{c}0 . \\
00 \\
15 \\
5\end{array}$ & $\begin{array}{l}0.65 \\
030\end{array}$ & $\begin{array}{c}0 . \\
01 \\
55 \\
0\end{array}$ & $\begin{array}{c}0.08 \\
114\end{array}$ & $\begin{array}{c}0 . \\
00 \\
09 \\
8\end{array}$ & 535 & $\begin{array}{l}6 \\
0\end{array}$ & 509 & $\begin{array}{l}1 \\
0\end{array}$ & 503 & 6 & $\begin{array}{l}1.1 \\
9 \%\end{array}$ & $\begin{array}{l}5 \\
0 \\
3\end{array}$ & 6 \\
\hline $\begin{array}{c}\mathrm{M} \\
\mathrm{O} 2 \\
6- \\
24\end{array}$ & $\begin{array}{l}1 \\
7 \\
3\end{array}$ & $\begin{array}{l}3 \\
5 \\
3\end{array}$ & $\begin{array}{l}4 \\
9\end{array}$ & $\begin{array}{l}0.05 \\
745\end{array}$ & $\begin{array}{c}0 . \\
00 \\
07 \\
3\end{array}$ & $\begin{array}{l}0.64 \\
320\end{array}$ & $\begin{array}{c}0 . \\
00 \\
76 \\
5\end{array}$ & $\begin{array}{c}0.08 \\
119\end{array}$ & $\begin{array}{c}0 . \\
00 \\
09 \\
5\end{array}$ & 509 & $\begin{array}{l}1 \\
2\end{array}$ & 504 & 5 & 503 & 6 & $\begin{array}{l}0.2 \\
0 \%\end{array}$ & $\begin{array}{l}5 \\
0 \\
3\end{array}$ & 6 \\
\hline $\begin{array}{c}\mathrm{M} \\
\mathrm{O} 2 \\
6- \\
25 \\
\end{array}$ & $\begin{array}{l}1 \\
5 \\
8\end{array}$ & $\begin{array}{l}4 \\
2 \\
6\end{array}$ & $\begin{array}{l}0 \\
3 \\
7\end{array}$ & $\begin{array}{l}0.06 \\
018\end{array}$ & $\begin{array}{c}0 . \\
00 \\
05 \\
7 \\
\end{array}$ & $\begin{array}{l}0.67 \\
548\end{array}$ & $\begin{array}{c}0 . \\
00 \\
61 \\
5\end{array}$ & $\begin{array}{c}0.08 \\
140\end{array}$ & $\begin{array}{c}0 . \\
00 \\
09 \\
1\end{array}$ & 610 & $\begin{array}{l}1 \\
1\end{array}$ & 524 & 4 & 504 & 5 & $\begin{array}{l}3.9 \\
7 \%\end{array}$ & $\begin{array}{l}5 \\
0 \\
4\end{array}$ & 5 \\
\hline $\begin{array}{c}\mathrm{M} \\
\mathrm{O} 2 \\
6- \\
26 \\
\end{array}$ & $\begin{array}{l}1 \\
7 \\
8\end{array}$ & $\begin{array}{l}7 \\
3 \\
8\end{array}$ & $\begin{array}{l}0 \\
2 \\
4\end{array}$ & $\begin{array}{l}0.05 \\
942\end{array}$ & $\begin{array}{c}0 . \\
00 \\
04 \\
6 \\
\end{array}$ & $\begin{array}{c}0.65 \\
548\end{array}$ & $\begin{array}{c}0 . \\
00 \\
49 \\
4\end{array}$ & $\begin{array}{c}0.08 \\
000\end{array}$ & $\begin{array}{c}0 . \\
00 \\
08 \\
7\end{array}$ & 583 & $\begin{array}{l}1 \\
2\end{array}$ & 512 & 3 & 496 & 5 & $\begin{array}{l}3.2 \\
3 \%\end{array}$ & $\begin{array}{l}4 \\
9 \\
6\end{array}$ & 5 \\
\hline $\begin{array}{c}\mathrm{M} \\
\mathrm{O} 2 \\
6- \\
27\end{array}$ & $\begin{array}{l}1 \\
1 \\
0\end{array}$ & $\begin{array}{l}3 \\
1 \\
3\end{array}$ & $\begin{array}{l}0 \\
3 \\
5\end{array}$ & $\begin{array}{l}0.05 \\
981\end{array}$ & $\begin{array}{c}0 . \\
00 \\
07 \\
0\end{array}$ & $\begin{array}{c}0.66 \\
880\end{array}$ & $\begin{array}{c}0 . \\
00 \\
73 \\
9\end{array}$ & $\begin{array}{c}0.08 \\
109\end{array}$ & $\begin{array}{c}0 . \\
00 \\
09 \\
4\end{array}$ & 597 & $\begin{array}{l}1 \\
1\end{array}$ & 520 & 4 & 503 & 6 & $\begin{array}{l}3.3 \\
8 \%\end{array}$ & $\begin{array}{l}5 \\
0 \\
3\end{array}$ & 6 \\
\hline $\begin{array}{c}\mathrm{M} \\
\mathrm{O} 2 \\
6- \\
28 \\
\end{array}$ & $\begin{array}{l}8 \\
4\end{array}$ & $\begin{array}{l}1 \\
0 \\
2\end{array}$ & $\begin{array}{l}0 \\
. \\
8 \\
2\end{array}$ & $\begin{array}{l}0.05 \\
984\end{array}$ & $\begin{array}{c}0 . \\
00 \\
09 \\
0 \\
\end{array}$ & $\begin{array}{c}0.75 \\
093\end{array}$ & $\begin{array}{c}0 . \\
01 \\
05 \\
6 \\
\end{array}$ & $\begin{array}{c}0.09 \\
102\end{array}$ & $\begin{array}{c}0 . \\
00 \\
11 \\
3 \\
\end{array}$ & 598 & $\begin{array}{l}1 \\
4\end{array}$ & 569 & 6 & 562 & 7 & $\begin{array}{l}1.2 \\
5 \%\end{array}$ & $\begin{array}{l}5 \\
6 \\
2\end{array}$ & 7 \\
\hline $\begin{array}{c}\mathrm{M} \\
\mathrm{O} 2 \\
6- \\
29\end{array}$ & $\begin{array}{l}8 \\
1\end{array}$ & $\begin{array}{l}1 \\
3 \\
5\end{array}$ & $\begin{array}{l}0 \\
. \\
6 \\
0\end{array}$ & $\begin{array}{c}0.11 \\
996\end{array}$ & $\begin{array}{c}0 . \\
00 \\
08 \\
3\end{array}$ & $\begin{array}{l}5.71 \\
721\end{array}$ & $\begin{array}{c}0 . \\
03 \\
93 \\
6\end{array}$ & $\begin{array}{c}0.34 \\
566\end{array}$ & $\begin{array}{c}0 . \\
00 \\
38 \\
1\end{array}$ & $\begin{array}{c}195 \\
6\end{array}$ & $\begin{array}{l}1 \\
0\end{array}$ & $\begin{array}{c}193 \\
4\end{array}$ & 6 & $\begin{array}{c}191 \\
4\end{array}$ & $\begin{array}{l}1 \\
8\end{array}$ & $\begin{array}{l}2.1 \\
9 \%\end{array}$ & $\begin{array}{l}1 \\
9 \\
5 \\
6\end{array}$ & $\begin{array}{l}1 \\
0\end{array}$ \\
\hline
\end{tabular}




\begin{tabular}{|c|c|c|c|c|c|c|c|c|c|c|c|c|c|c|c|c|c|c|}
\hline $\begin{array}{c}\mathrm{M} \\
\mathrm{O} 2 \\
6- \\
30\end{array}$ & $\begin{array}{l}6 \\
9\end{array}$ & $\begin{array}{l}1 \\
8 \\
3\end{array}$ & $\begin{array}{l}3 \\
8\end{array}$ & $\begin{array}{l}0.11 \\
913\end{array}$ & $\begin{array}{c}0 . \\
00 \\
08 \\
3\end{array}$ & $\begin{array}{l}5.33 \\
682\end{array}$ & $\begin{array}{c}0 . \\
03 \\
67 \\
1\end{array}$ & $\begin{array}{c}0.32 \\
491\end{array}$ & $\begin{array}{c}0 . \\
00 \\
35 \\
8\end{array}$ & $\begin{array}{c}194 \\
3\end{array}$ & $\begin{array}{l}1 \\
0\end{array}$ & $\begin{array}{c}187 \\
5\end{array}$ & 6 & $\begin{array}{c}181 \\
4\end{array}$ & $\begin{array}{l}1 \\
7\end{array}$ & $\begin{array}{l}7.1 \\
1 \%\end{array}$ & $\begin{array}{l}1 \\
9 \\
4 \\
3\end{array}$ & $\begin{array}{l}1 \\
0\end{array}$ \\
\hline $\begin{array}{c}\mathrm{M} \\
\mathrm{O} 2 \\
6- \\
31\end{array}$ & $\begin{array}{l}7 \\
9\end{array}$ & $\begin{array}{l}2 \\
0 \\
1\end{array}$ & $\begin{array}{l}0 \\
3 \\
9 \\
\end{array}$ & $\begin{array}{c}0.06 \\
451\end{array}$ & $\begin{array}{c}0 . \\
00 \\
07 \\
3\end{array}$ & $\begin{array}{l}0.79 \\
743\end{array}$ & $\begin{array}{c}0 . \\
00 \\
84 \\
5\end{array}$ & $\begin{array}{c}0.08 \\
966\end{array}$ & $\begin{array}{c}0 . \\
00 \\
10 \\
4\end{array}$ & 758 & $\begin{array}{l}1 \\
1\end{array}$ & 595 & 5 & 554 & 6 & $\begin{array}{l}7.4 \\
0 \%\end{array}$ & $\begin{array}{l}5 \\
5 \\
4\end{array}$ & 6 \\
\hline $\begin{array}{c}\mathrm{M} \\
\mathrm{O} 2 \\
6- \\
32\end{array}$ & $\begin{array}{l}3 \\
7\end{array}$ & $\begin{array}{l}2 \\
2 \\
4\end{array}$ & $\begin{array}{l}0 \\
1 \\
6\end{array}$ & $\begin{array}{l}0.12 \\
291\end{array}$ & $\begin{array}{c}0 . \\
00 \\
07 \\
8\end{array}$ & $\begin{array}{l}5.99 \\
219\end{array}$ & $\begin{array}{c}0 . \\
03 \\
82 \\
6\end{array}$ & $\begin{array}{l}0.35 \\
357\end{array}$ & $\begin{array}{c}0 . \\
00 \\
38 \\
4\end{array}$ & $\begin{array}{c}199 \\
9\end{array}$ & $\begin{array}{l}1 \\
1\end{array}$ & 197 & 6 & $\begin{array}{c}195 \\
2\end{array}$ & $\begin{array}{l}1 \\
8\end{array}$ & $\begin{array}{l}2.4 \\
1 \%\end{array}$ & $\begin{array}{l}1 \\
9 \\
9 \\
9\end{array}$ & $\begin{array}{l}1 \\
1\end{array}$ \\
\hline $\begin{array}{c}\mathrm{M} \\
\mathrm{O} 2 \\
6- \\
33\end{array}$ & $\begin{array}{l}1 \\
4 \\
2\end{array}$ & $\begin{array}{l}1 \\
7 \\
5\end{array}$ & $\begin{array}{l}0 \\
8 \\
8 \\
1\end{array}$ & $\begin{array}{l}0.05 \\
759\end{array}$ & $\begin{array}{c}0 . \\
00 \\
07 \\
8\end{array}$ & $\begin{array}{l}0.61 \\
487\end{array}$ & $\begin{array}{c}0 . \\
00 \\
77 \\
7\end{array}$ & $\begin{array}{l}0.07 \\
743\end{array}$ & $\begin{array}{c}0 . \\
00 \\
09 \\
3\end{array}$ & 514 & $\begin{array}{l}1 \\
2\end{array}$ & 487 & 5 & 481 & 6 & $\begin{array}{l}1.2 \\
5 \%\end{array}$ & $\begin{array}{l}4 \\
8 \\
1\end{array}$ & 6 \\
\hline $\begin{array}{c}\mathrm{M} \\
\mathrm{O} 2 \\
6- \\
34\end{array}$ & $\begin{array}{l}1 \\
5 \\
9\end{array}$ & $\begin{array}{l}4 \\
3 \\
6\end{array}$ & $\begin{array}{l}0 \\
. \\
3 \\
7\end{array}$ & $\begin{array}{c}0.17 \\
675\end{array}$ & $\begin{array}{c}0 . \\
00 \\
09 \\
8\end{array}$ & $\begin{array}{c}12.0 \\
560 \\
0\end{array}$ & $\begin{array}{c}0 . \\
06 \\
90 \\
7\end{array}$ & $\begin{array}{l}0.49 \\
470\end{array}$ & $\begin{array}{c}0 . \\
00 \\
52 \\
9\end{array}$ & $\begin{array}{c}262 \\
3\end{array}$ & $\begin{array}{l}1 \\
0\end{array}$ & $\begin{array}{c}260 \\
9\end{array}$ & 5 & $\begin{array}{c}259 \\
1\end{array}$ & $\begin{array}{l}2 \\
3\end{array}$ & $\begin{array}{l}1.2 \\
4 \%\end{array}$ & $\begin{array}{l}2 \\
6 \\
2 \\
3\end{array}$ & $\begin{array}{l}1 \\
0\end{array}$ \\
\hline $\begin{array}{c}\mathrm{M} \\
\mathrm{O} 2 \\
6- \\
35\end{array}$ & $\begin{array}{l}1 \\
9 \\
4\end{array}$ & $\begin{array}{l}2 \\
7 \\
7\end{array}$ & $\begin{array}{l}0 \\
. \\
7 \\
0\end{array}$ & $\begin{array}{l}0.06 \\
370\end{array}$ & $\begin{array}{c}0 . \\
00 \\
05 \\
8\end{array}$ & $\begin{array}{c}0.94 \\
168\end{array}$ & $\begin{array}{c}0 . \\
00 \\
81 \\
6\end{array}$ & $\begin{array}{l}0.10 \\
722\end{array}$ & $\begin{array}{c}0 . \\
00 \\
11 \\
9\end{array}$ & 732 & $\begin{array}{l}1 \\
1\end{array}$ & 674 & 4 & 657 & 7 & $\begin{array}{l}2.5 \\
9 \%\end{array}$ & $\begin{array}{l}6 \\
5 \\
7\end{array}$ & 7 \\
\hline $\begin{array}{c}\mathrm{M} \\
\mathrm{O} 2 \\
6- \\
36\end{array}$ & $\begin{array}{l}2 \\
6 \\
0\end{array}$ & $\begin{array}{l}4 \\
6 \\
7\end{array}$ & $\begin{array}{l}5 \\
6\end{array}$ & $\begin{array}{c}0.05 \\
500\end{array}$ & $\begin{array}{c}0 . \\
00 \\
15 \\
1\end{array}$ & $\begin{array}{l}0.42 \\
849\end{array}$ & $\begin{array}{c}0 . \\
01 \\
06 \\
6\end{array}$ & $\begin{array}{l}0.05 \\
650\end{array}$ & $\begin{array}{c}0 . \\
00 \\
06 \\
6\end{array}$ & 412 & $\begin{array}{l}6 \\
3\end{array}$ & 362 & 8 & 354 & 4 & $\begin{array}{l}2.2 \\
6 \%\end{array}$ & $\begin{array}{l}3 \\
5 \\
4\end{array}$ & 4 \\
\hline $\begin{array}{c}\mathrm{M} \\
\mathrm{O} 2 \\
6- \\
37\end{array}$ & $\begin{array}{l}2 \\
0 \\
0\end{array}$ & $\begin{array}{l}3 \\
8 \\
7\end{array}$ & $\begin{array}{l}5 \\
2\end{array}$ & $\begin{array}{l}0.05 \\
782\end{array}$ & $\begin{array}{c}0 . \\
00 \\
06 \\
4\end{array}$ & $\begin{array}{l}0.44 \\
763\end{array}$ & $\begin{array}{c}0 . \\
00 \\
46 \\
5\end{array}$ & $\begin{array}{c}0.05 \\
614\end{array}$ & $\begin{array}{c}0 . \\
00 \\
06 \\
4\end{array}$ & 523 & $\begin{array}{l}1 \\
1\end{array}$ & 376 & 3 & 352 & 4 & $\begin{array}{l}6.8 \\
2 \%\end{array}$ & $\begin{array}{l}3 \\
5 \\
2\end{array}$ & 4 \\
\hline $\begin{array}{c}\mathrm{M} \\
\mathrm{O} 2 \\
6- \\
38 \\
\end{array}$ & $\begin{array}{l}4 \\
8\end{array}$ & $\begin{array}{l}6 \\
5\end{array}$ & $\begin{array}{l}7 \\
3 \\
\end{array}$ & $\begin{array}{l}0.19 \\
613 \\
\end{array}$ & $\begin{array}{c}0 . \\
00 \\
13 \\
9 \\
\end{array}$ & $\begin{array}{c}14.7 \\
264 \\
0\end{array}$ & $\begin{array}{c}0 . \\
10 \\
43 \\
0 \\
\end{array}$ & $\begin{array}{c}0.54 \\
457\end{array}$ & $\begin{array}{c}0 . \\
00 \\
62 \\
0 \\
\end{array}$ & $\begin{array}{c}279 \\
4\end{array}$ & $\begin{array}{l}1 \\
0\end{array}$ & $\begin{array}{c}279 \\
8\end{array}$ & 7 & $\begin{array}{c}280 \\
3\end{array}$ & $\begin{array}{l}2 \\
6\end{array}$ & $\begin{array}{c}- \\
0.3 \\
2 \%\end{array}$ & $\begin{array}{l}2 \\
7 \\
9 \\
4 \\
\end{array}$ & $\begin{array}{l}1 \\
0\end{array}$ \\
\hline $\begin{array}{c}\mathrm{M} \\
\mathrm{O} 2 \\
6- \\
39\end{array}$ & $\begin{array}{l}5 \\
6\end{array}$ & $\begin{array}{l}8 \\
3\end{array}$ & $\begin{array}{l}0 \\
6 \\
8\end{array}$ & $\begin{array}{l}0.10 \\
748\end{array}$ & $\begin{array}{c}0 . \\
00 \\
24 \\
2\end{array}$ & $\begin{array}{c}3.99 \\
429\end{array}$ & $\begin{array}{c}0 . \\
07 \\
52 \\
7\end{array}$ & $\begin{array}{c}0.26 \\
953\end{array}$ & $\begin{array}{c}0 . \\
00 \\
33 \\
3\end{array}$ & $\begin{array}{c}175 \\
7\end{array}$ & $\begin{array}{l}4 \\
2\end{array}$ & $\begin{array}{c}163 \\
3\end{array}$ & $\begin{array}{l}1 \\
5\end{array}$ & $\begin{array}{c}153 \\
8\end{array}$ & $\begin{array}{l}1 \\
7\end{array}$ & $\begin{array}{l}14 . \\
24 \\
\%\end{array}$ & & \\
\hline $\begin{array}{c}\mathrm{M} \\
\mathrm{O} 2 \\
6- \\
40\end{array}$ & $\begin{array}{l}5 \\
2\end{array}$ & $\begin{array}{l}8 \\
5\end{array}$ & $\begin{array}{l}0 \\
\dot{6} \\
1\end{array}$ & $\begin{array}{c}0.11 \\
910\end{array}$ & $\begin{array}{c}0 . \\
00 \\
08 \\
9\end{array}$ & $\begin{array}{l}5.27 \\
158\end{array}$ & $\begin{array}{c}0 . \\
03 \\
85 \\
6\end{array}$ & $\begin{array}{c}0.32 \\
102\end{array}$ & $\begin{array}{c}0 . \\
00 \\
35 \\
9\end{array}$ & $\begin{array}{c}194 \\
3\end{array}$ & $\begin{array}{l}1 \\
0\end{array}$ & $\begin{array}{c}186 \\
4\end{array}$ & 6 & $\begin{array}{c}179 \\
5\end{array}$ & $\begin{array}{l}1 \\
8\end{array}$ & $\begin{array}{l}8.2 \\
5 \%\end{array}$ & $\begin{array}{l}1 \\
9 \\
4 \\
3\end{array}$ & $\begin{array}{l}1 \\
0\end{array}$ \\
\hline $\begin{array}{c}\mathrm{M} \\
\mathrm{O} 2 \\
6- \\
41\end{array}$ & $\begin{array}{l}1 \\
9 \\
1\end{array}$ & $\begin{array}{l}3 \\
1 \\
0\end{array}$ & $\begin{array}{l}0 \\
6 \\
6\end{array}$ & $\begin{array}{c}0.06 \\
121\end{array}$ & $\begin{array}{c}0 . \\
00 \\
05 \\
9\end{array}$ & $\begin{array}{l}0.85 \\
247\end{array}$ & $\begin{array}{c}0 . \\
00 \\
78 \\
4\end{array}$ & $\begin{array}{c}0.10 \\
101\end{array}$ & $\begin{array}{c}0 . \\
00 \\
11 \\
3\end{array}$ & 647 & $\begin{array}{l}1 \\
1\end{array}$ & 626 & 4 & 620 & 7 & $\begin{array}{l}0.9 \\
7 \%\end{array}$ & $\begin{array}{l}6 \\
2 \\
0\end{array}$ & 7 \\
\hline $\begin{array}{c}\mathrm{M} \\
\mathrm{O} 2 \\
6- \\
42\end{array}$ & $\begin{array}{l}1 \\
2 \\
0 \\
1\end{array}$ & $\begin{array}{l}1 \\
0 \\
2 \\
5\end{array}$ & $\begin{array}{l}1 \\
1 \\
7\end{array}$ & $\begin{array}{l}0.05 \\
290\end{array}$ & $\begin{array}{c}0 . \\
00 \\
22 \\
2\end{array}$ & $\begin{array}{l}0.37 \\
755\end{array}$ & $\begin{array}{c}0 . \\
01 \\
51 \\
2\end{array}$ & $\begin{array}{l}0.05 \\
177\end{array}$ & $\begin{array}{c}0 . \\
00 \\
06 \\
4\end{array}$ & 324 & $\begin{array}{l}9 \\
8\end{array}$ & 325 & \begin{tabular}{l|}
1 \\
1
\end{tabular} & 325 & 4 & $\begin{array}{l}0.0 \\
0 \%\end{array}$ & $\begin{array}{l}3 \\
2 \\
5\end{array}$ & 4 \\
\hline $\begin{array}{l}\mathrm{M} \\
\mathrm{O} 2\end{array}$ & $\begin{array}{l}2 \\
3\end{array}$ & $\begin{array}{l}4 \\
1\end{array}$ & 0 & $\begin{array}{c}0.06 \\
178\end{array}$ & $\begin{array}{c}0 . \\
00\end{array}$ & $\begin{array}{c}0.83 \\
868\end{array}$ & $\begin{array}{c}0 . \\
00\end{array}$ & $\begin{array}{l}0.09 \\
846\end{array}$ & $\begin{array}{c}0 . \\
00\end{array}$ & 667 & $\begin{array}{l}1 \\
1\end{array}$ & 618 & 4 & 605 & 6 & $\begin{array}{l}2.1 \\
5 \%\end{array}$ & $\begin{array}{l}6 \\
0\end{array}$ & 6 \\
\hline
\end{tabular}




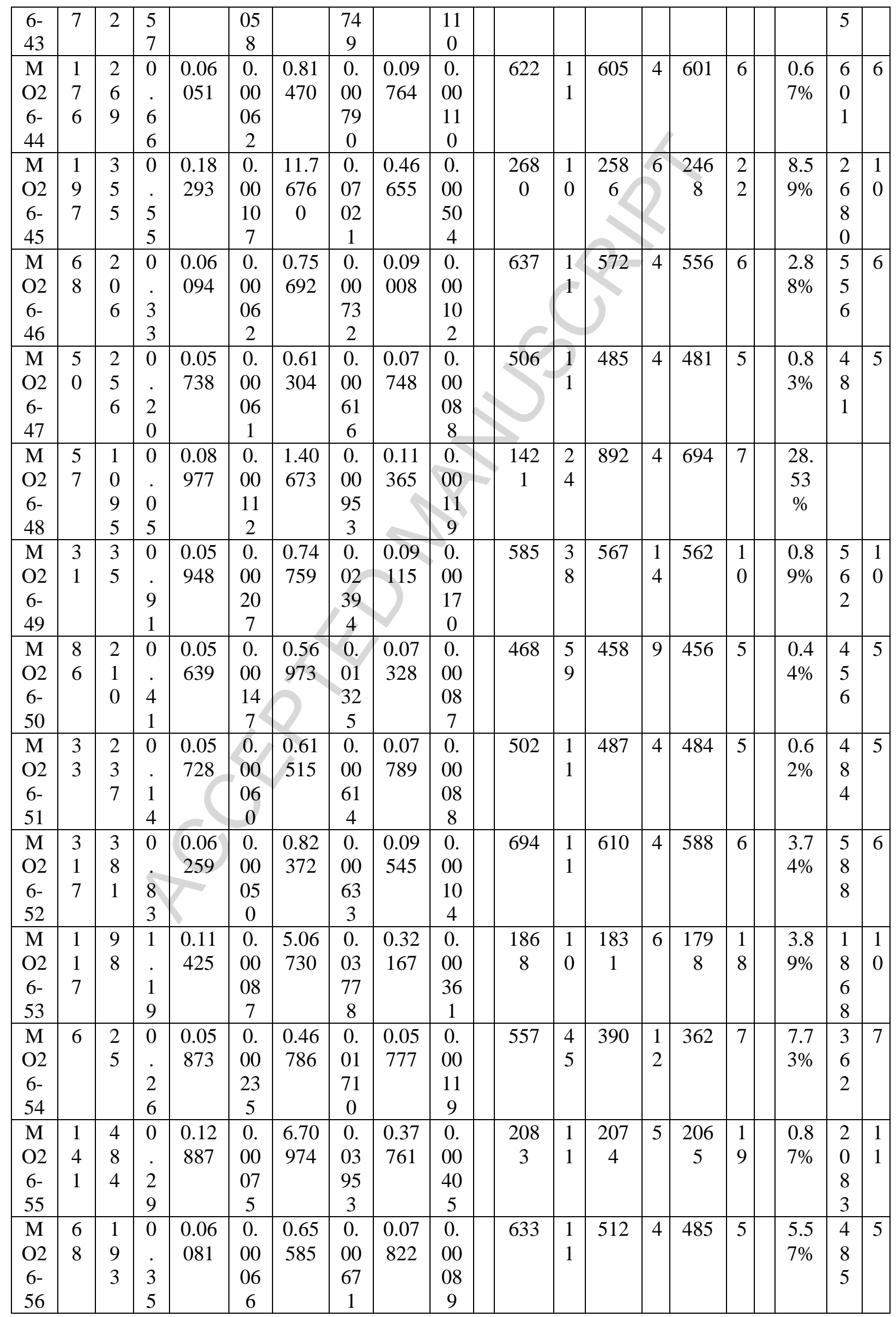




\begin{tabular}{|c|c|c|c|c|c|c|c|c|c|c|c|c|c|c|c|c|c|c|}
\hline $\begin{array}{c}\mathrm{M} \\
\mathrm{O} 2 \\
6- \\
57 \\
\end{array}$ & $\begin{array}{l}1 \\
1 \\
3\end{array}$ & $\begin{array}{l}3 \\
3 \\
9\end{array}$ & $\begin{array}{l}0 \\
3 \\
4 \\
\end{array}$ & $\begin{array}{l}0.05 \\
475\end{array}$ & $\begin{array}{c}0 . \\
00 \\
05 \\
6\end{array}$ & $\begin{array}{l}0.41 \\
882\end{array}$ & $\begin{array}{c}0 . \\
00 \\
40 \\
8\end{array}$ & $\begin{array}{l}0.05 \\
548\end{array}$ & $\begin{array}{c}0 . \\
00 \\
06 \\
2\end{array}$ & 402 & $\begin{array}{l}1 \\
1\end{array}$ & 355 & 3 & 348 & 4 & $\begin{array}{l}2.0 \\
1 \%\end{array}$ & $\begin{array}{l}3 \\
4 \\
8\end{array}$ & 4 \\
\hline $\begin{array}{c}\mathrm{M} \\
\mathrm{O} 2 \\
6- \\
58\end{array}$ & $\begin{array}{l}1 \\
2 \\
5\end{array}$ & $\begin{array}{l}4 \\
4 \\
1\end{array}$ & $\begin{array}{l}0 \\
2 \\
8 \\
\end{array}$ & $\begin{array}{l}0.07 \\
512\end{array}$ & $\begin{array}{c}0 . \\
00 \\
17 \\
8 \\
\end{array}$ & $\begin{array}{l}1.54 \\
637\end{array}$ & $\begin{array}{c}0 . \\
03 \\
22 \\
0\end{array}$ & $\begin{array}{l}0.14 \\
929\end{array}$ & $\begin{array}{c}0 . \\
00 \\
17 \\
1 \\
\end{array}$ & $\begin{array}{c}107 \\
2\end{array}$ & $\begin{array}{l}4 \\
9\end{array}$ & 949 & $\begin{array}{l}1 \\
3\end{array}$ & 897 & $\begin{array}{l}1 \\
0\end{array}$ & $\begin{array}{l}5.8 \\
0 \%\end{array}$ & $\begin{array}{l}8 \\
9 \\
7\end{array}$ & $\begin{array}{l}1 \\
0\end{array}$ \\
\hline $\begin{array}{c}\mathrm{M} \\
\mathrm{O} 2 \\
6- \\
59\end{array}$ & $\begin{array}{l}3 \\
2 \\
1\end{array}$ & $\begin{array}{l}5 \\
5 \\
5\end{array}$ & $\begin{array}{l}5 \\
8\end{array}$ & $\begin{array}{c}0.06 \\
185\end{array}$ & $\begin{array}{c}0 . \\
00 \\
04 \\
7\end{array}$ & $\begin{array}{l}0.88 \\
309\end{array}$ & $\begin{array}{c}0 . \\
00 \\
65 \\
8\end{array}$ & $\begin{array}{l}0.10 \\
355\end{array}$ & $\begin{array}{c}0 . \\
00 \\
11 \\
2\end{array}$ & 669 & $\begin{array}{l}1 \\
1\end{array}$ & & 4 & 635 & 7 & $\begin{array}{l}1.2 \\
6 \%\end{array}$ & $\begin{array}{l}6 \\
3 \\
5\end{array}$ & 7 \\
\hline $\begin{array}{c}\mathrm{M} \\
\mathrm{O} 2 \\
6- \\
60 \\
\end{array}$ & $\begin{array}{l}1 \\
5 \\
6\end{array}$ & $\begin{array}{l}4 \\
9 \\
0\end{array}$ & $\begin{array}{l}0 \\
3 \\
2 \\
\end{array}$ & $\begin{array}{l}0.07 \\
912\end{array}$ & $\begin{array}{c}0 . \\
00 \\
05 \\
2 \\
\end{array}$ & $\begin{array}{l}1.58 \\
083\end{array}$ & $\begin{array}{c}0 . \\
01 \\
02 \\
1 \\
\end{array}$ & $\begin{array}{l}0.14 \\
491\end{array}$ & $\begin{array}{c}0 . \\
00 \\
15 \\
6 \\
\end{array}$ & $\begin{array}{c}117 \\
5\end{array}$ & $\begin{array}{l}1 \\
2\end{array}$ & 963 & 4 & 872 & 9 & $\begin{array}{l}10 . \\
44 \\
\%\end{array}$ & & \\
\hline $\begin{array}{c}\mathrm{M} \\
\mathrm{O} 2 \\
6- \\
61\end{array}$ & $\begin{array}{l}2 \\
0 \\
3\end{array}$ & $\begin{array}{l}3 \\
1 \\
5\end{array}$ & $\begin{array}{l}0 \\
6 \\
5 \\
\end{array}$ & $\begin{array}{c}0.12 \\
772\end{array}$ & $\begin{array}{c}0 . \\
00 \\
07 \\
3\end{array}$ & $\begin{array}{l}5.35 \\
542\end{array}$ & $\begin{array}{c}0 . \\
03 \\
10 \\
2\end{array}$ & $\begin{array}{l}0.30 \\
412\end{array}$ & $\begin{array}{c}0 . \\
00 \\
32 \\
5\end{array}$ & $\begin{array}{c}206 \\
7\end{array}$ & $\begin{array}{l}1 \\
1\end{array}$ & $\begin{array}{c}187 \\
8\end{array}$ & 5 & $\begin{array}{c}171 \\
2\end{array}$ & $\begin{array}{l}1 \\
6\end{array}$ & $\begin{array}{c}20 . \\
74 \\
\%\end{array}$ & & \\
\hline $\begin{array}{c}\mathrm{M} \\
\mathrm{O} 2 \\
6- \\
62 \\
\end{array}$ & $\begin{array}{l}1 \\
3 \\
1\end{array}$ & $\begin{array}{l}2 \\
7 \\
0\end{array}$ & $\begin{array}{l}0 \\
4 \\
4 \\
9\end{array}$ & $\begin{array}{l}0.06 \\
320\end{array}$ & $\begin{array}{c}0 . \\
00 \\
06 \\
1 \\
\end{array}$ & $\begin{array}{l}0.78 \\
723\end{array}$ & $\begin{array}{c}0 . \\
00 \\
72 \\
2 \\
\end{array}$ & $\begin{array}{l}0.09 \\
034\end{array}$ & $\begin{array}{c}0 . \\
00 \\
10 \\
1 \\
\end{array}$ & 715 & $\begin{array}{l}1 \\
1\end{array}$ & 590 & 4 & 558 & 6 & $\begin{array}{l}5.7 \\
3 \%\end{array}$ & $\begin{array}{l}5 \\
5 \\
8\end{array}$ & 6 \\
\hline $\begin{array}{c}\mathrm{M} \\
\mathrm{O} 2 \\
6- \\
63\end{array}$ & $\begin{array}{l}3 \\
0 \\
8\end{array}$ & $\begin{array}{l}2 \\
1 \\
8\end{array}$ & $\begin{array}{l}1 \\
4 \\
1\end{array}$ & $\begin{array}{l}0.06 \\
280\end{array}$ & $\begin{array}{c}0 . \\
00 \\
05 \\
8\end{array}$ & $\begin{array}{l}0.83 \\
702\end{array}$ & $\begin{array}{c}0 . \\
00 \\
73 \\
1\end{array}$ & $\begin{array}{l}0.09 \\
667\end{array}$ & $\begin{array}{c}0 . \\
00 \\
10 \\
8\end{array}$ & 701 & $\begin{array}{l}1 \\
1\end{array}$ & 617 & 4 & 595 & 6 & $\begin{array}{l}3.7 \\
0 \%\end{array}$ & $\begin{array}{l}5 \\
9 \\
5\end{array}$ & 6 \\
\hline $\begin{array}{c}\mathrm{M} \\
\mathrm{O} 2 \\
6- \\
64\end{array}$ & $\begin{array}{l}4 \\
5 \\
7\end{array}$ & $\begin{array}{l}7 \\
7 \\
3\end{array}$ & $\begin{array}{l}5 \\
9\end{array}$ & $\begin{array}{l}0.05 \\
625\end{array}$ & $\begin{array}{c}0 . \\
00 \\
04 \\
2\end{array}$ & $\begin{array}{l}0.43 \\
381\end{array}$ & $\begin{array}{c}0 . \\
00 \\
31 \\
8\end{array}$ & $\begin{array}{l}0.05 \\
593\end{array}$ & $\begin{array}{c}0 . \\
00 \\
06 \\
0\end{array}$ & 462 & $\begin{array}{l}1 \\
2\end{array}$ & 366 & 2 & 351 & 4 & $\begin{array}{l}4.2 \\
7 \%\end{array}$ & $\begin{array}{l}3 \\
5 \\
1\end{array}$ & 4 \\
\hline $\begin{array}{c}\mathrm{M} \\
\mathrm{O} 2 \\
6- \\
65 \\
\end{array}$ & $\begin{array}{l}2 \\
3\end{array}$ & $\begin{array}{l}4 \\
8\end{array}$ & $\begin{array}{l}0 \\
4 \\
8 \\
\end{array}$ & $\begin{array}{l}0.12 \\
182\end{array}$ & $\begin{array}{c}0 . \\
00 \\
12 \\
5 \\
\end{array}$ & $\begin{array}{l}4.80 \\
734\end{array}$ & $\begin{array}{c}0 . \\
04 \\
63 \\
7 \\
\end{array}$ & $\begin{array}{l}0.28 \\
622\end{array}$ & $\begin{array}{c}0 . \\
00 \\
34 \\
7 \\
\end{array}$ & $\begin{array}{c}198 \\
3\end{array}$ & $\begin{array}{l}1 \\
0\end{array}$ & $\begin{array}{c}178 \\
6\end{array}$ & 8 & $\begin{array}{c}162 \\
3\end{array}$ & $\begin{array}{l}1 \\
7\end{array}$ & $\begin{array}{l}22 . \\
18 \\
\%\end{array}$ & & \\
\hline $\begin{array}{c}\mathrm{M} \\
\mathrm{O} 2 \\
6- \\
66\end{array}$ & $\begin{array}{l}2 \\
3 \\
1\end{array}$ & $\begin{array}{l}3 \\
8 \\
2\end{array}$ & $\begin{array}{l}6 \\
0\end{array}$ & $\begin{array}{l}0.05 \\
990\end{array}$ & $\begin{array}{c}0 . \\
00 \\
04 \\
8\end{array}$ & $\begin{array}{c}0.78 \\
158\end{array}$ & $\begin{array}{c}0 . \\
00 \\
60 \\
7\end{array}$ & $\begin{array}{l}0.09 \\
464\end{array}$ & $\begin{array}{c}0 . \\
00 \\
10 \\
3\end{array}$ & 600 & $\begin{array}{l}1 \\
1\end{array}$ & 586 & 3 & 583 & 6 & $\begin{array}{l}0.5 \\
1 \%\end{array}$ & $\begin{array}{l}5 \\
8 \\
3\end{array}$ & 6 \\
\hline $\begin{array}{c}\mathrm{M} \\
\mathrm{O} 2 \\
6- \\
67 \\
\end{array}$ & $\begin{array}{l}2 \\
5 \\
6\end{array}$ & $\begin{array}{l}8 \\
2 \\
4\end{array}$ & $\begin{array}{l}0 \\
3 \\
1 \\
\end{array}$ & $\begin{array}{l}0.05 \\
845\end{array}$ & $\begin{array}{c}0 . \\
00 \\
04 \\
0 \\
\end{array}$ & $\begin{array}{l}0.56 \\
272\end{array}$ & $\begin{array}{c}0 . \\
00 \\
38 \\
4 \\
\end{array}$ & $\begin{array}{l}0.06 \\
983\end{array}$ & $\begin{array}{c}0 . \\
00 \\
07 \\
5\end{array}$ & 547 & $\begin{array}{l}1 \\
2\end{array}$ & 453 & 2 & 435 & 5 & $\begin{array}{l}4.1 \\
4 \%\end{array}$ & $\begin{array}{l}4 \\
3 \\
5\end{array}$ & 5 \\
\hline $\begin{array}{c}\mathrm{M} \\
\mathrm{O} 2 \\
6- \\
68 \\
\end{array}$ & $\begin{array}{l}7 \\
0 \\
8\end{array}$ & $\begin{array}{l}7 \\
2 \\
3\end{array}$ & $\begin{array}{l}0 \\
. \\
9 \\
8 \\
\end{array}$ & $\begin{array}{c}0.06 \\
454\end{array}$ & $\begin{array}{c}0 . \\
00 \\
04 \\
9 \\
\end{array}$ & $\begin{array}{l}0.80 \\
889\end{array}$ & $\begin{array}{c}0 . \\
00 \\
59 \\
7 \\
\end{array}$ & $\begin{array}{l}0.09 \\
091\end{array}$ & $\begin{array}{c}0 . \\
00 \\
09 \\
9 \\
\end{array}$ & 759 & $\begin{array}{l}1 \\
2\end{array}$ & 602 & 3 & 561 & 6 & $\begin{array}{l}7.3 \\
1 \%\end{array}$ & $\begin{array}{l}5 \\
6 \\
1\end{array}$ & 6 \\
\hline $\begin{array}{c}\mathrm{M} \\
\mathrm{O} 2 \\
6- \\
69 \\
\end{array}$ & $\begin{array}{l}7 \\
3 \\
4\end{array}$ & $\begin{array}{l}1 \\
8 \\
9 \\
7 \\
\end{array}$ & $\begin{array}{l}3 \\
9 \\
\end{array}$ & $\begin{array}{c}0.06 \\
188\end{array}$ & $\begin{array}{c}0 . \\
00 \\
03 \\
9 \\
\end{array}$ & $\begin{array}{l}0.62 \\
287\end{array}$ & $\begin{array}{c}0 . \\
00 \\
38 \\
9 \\
\end{array}$ & $\begin{array}{l}0.07 \\
301\end{array}$ & $\begin{array}{c}0 . \\
00 \\
07 \\
8 \\
\end{array}$ & 670 & $\begin{array}{l}1 \\
3\end{array}$ & 492 & 2 & 454 & 5 & $\begin{array}{l}8.3 \\
7 \%\end{array}$ & $\begin{array}{l}4 \\
5 \\
4\end{array}$ & 5 \\
\hline $\begin{array}{l}\mathrm{M} \\
\mathrm{O} 2 \\
\end{array}$ & $\begin{array}{l}1 \\
6 \\
\end{array}$ & $\begin{array}{l}1 \\
8 \\
\end{array}$ & 0 & $\begin{array}{l}0.05 \\
963 \\
\end{array}$ & $\begin{array}{c}0 . \\
00 \\
\end{array}$ & $\begin{array}{c}0.78 \\
332 \\
\end{array}$ & $\begin{array}{c}0 . \\
00 \\
\end{array}$ & $\begin{array}{r}0.09 \\
527 \\
\end{array}$ & $\begin{array}{c}0 . \\
00 \\
\end{array}$ & 590 & $\begin{array}{l}1 \\
1 \\
\end{array}$ & 587 & 4 & 587 & 6 & $\begin{array}{l}0.0 \\
0 \% \\
\end{array}$ & $\begin{array}{l}5 \\
8 \\
\end{array}$ & 6 \\
\hline
\end{tabular}




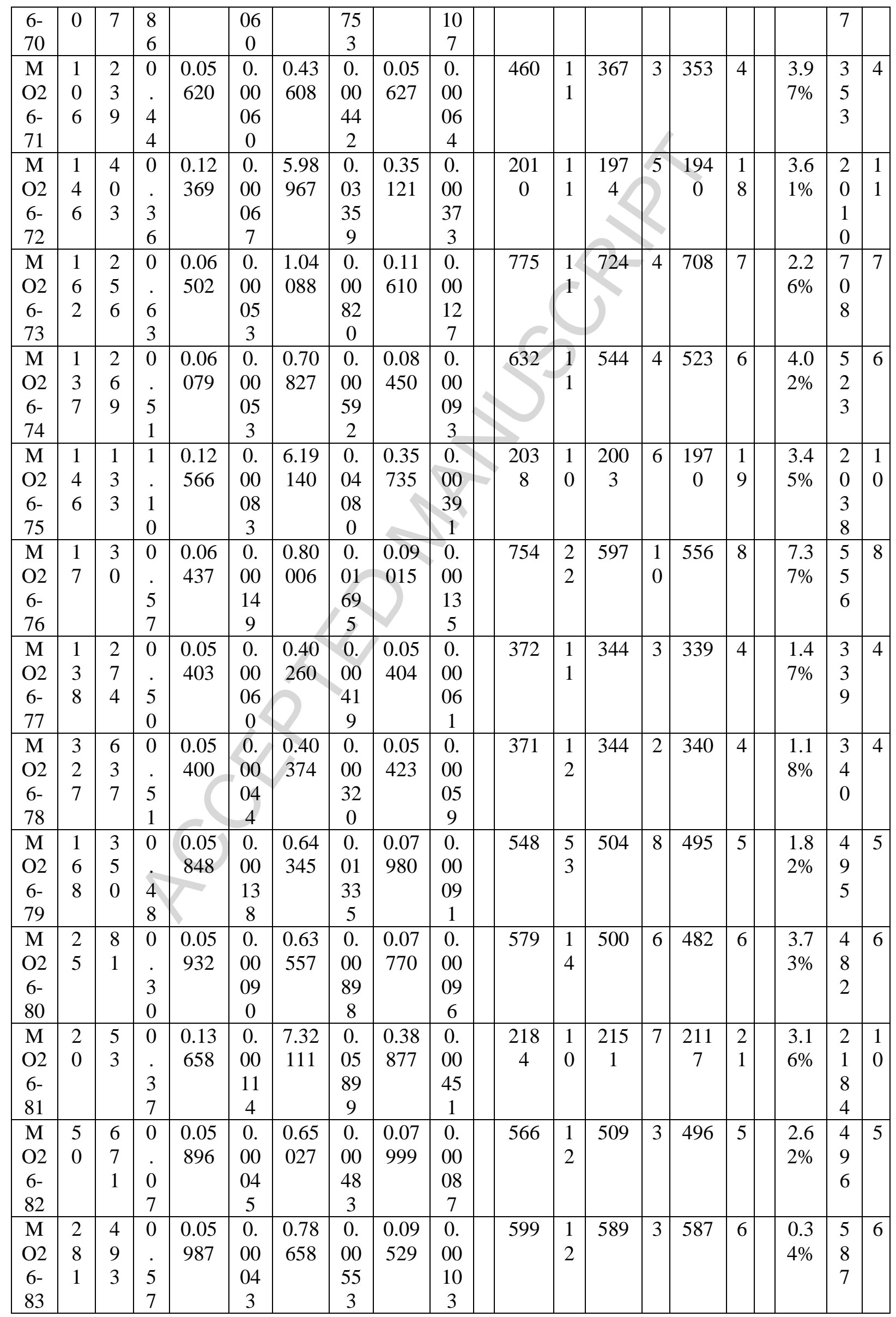




\begin{tabular}{|c|c|c|c|c|c|c|c|c|c|c|c|c|c|c|c|c|c|c|}
\hline $\begin{array}{c}\mathrm{M} \\
\mathrm{O} 2 \\
6- \\
84 \\
\end{array}$ & $\begin{array}{l}1 \\
1 \\
1\end{array}$ & $\begin{array}{l}2 \\
4 \\
9\end{array}$ & $\begin{array}{l}0 \\
5 \\
5 \\
\end{array}$ & $\begin{array}{l}0.05 \\
649\end{array}$ & $\begin{array}{c}0 . \\
00 \\
05 \\
6\end{array}$ & $\begin{array}{c}0.61 \\
555\end{array}$ & $\begin{array}{c}0 . \\
00 \\
58 \\
2\end{array}$ & $\begin{array}{l}0.07 \\
903\end{array}$ & $\begin{array}{c}0 . \\
00 \\
08 \\
9\end{array}$ & 472 & $\begin{array}{l}1 \\
1\end{array}$ & 487 & 4 & 490 & 5 & $\begin{array}{c}- \\
0.6 \\
1 \%\end{array}$ & $\begin{array}{l}4 \\
9 \\
0\end{array}$ & 5 \\
\hline $\begin{array}{c}\mathrm{M} \\
\mathrm{O} 2 \\
6- \\
85\end{array}$ & $\begin{array}{l}4 \\
8\end{array}$ & $\begin{array}{l}2 \\
6 \\
8\end{array}$ & $\begin{array}{l}0 \\
. \\
1 \\
8\end{array}$ & $\begin{array}{l}0.12 \\
717\end{array}$ & $\begin{array}{c}0 . \\
00 \\
09 \\
2 \\
\end{array}$ & $\begin{array}{l}6.05 \\
203\end{array}$ & $\begin{array}{c}0 . \\
04 \\
27 \\
6\end{array}$ & $\begin{array}{l}0.34 \\
516\end{array}$ & $\begin{array}{c}0 . \\
00 \\
38 \\
4\end{array}$ & $\begin{array}{c}205 \\
9\end{array}$ & $\begin{array}{l}1 \\
0\end{array}$ & $\begin{array}{c}198 \\
3\end{array}$ & 6 & $\begin{array}{c}191 \\
1\end{array}$ & $\begin{array}{l}1 \\
8\end{array}$ & $\begin{array}{l}7.7 \\
4 \%\end{array}$ & $\begin{array}{l}2 \\
0 \\
5 \\
9 \\
\end{array}$ & $\begin{array}{l}1 \\
0\end{array}$ \\
\hline $\begin{array}{c}\mathrm{M} \\
\mathrm{O} 2 \\
6- \\
86\end{array}$ & $\begin{array}{l}2 \\
0 \\
1\end{array}$ & $\begin{array}{l}4 \\
2 \\
6\end{array}$ & $\begin{array}{l}0 \\
4 \\
7\end{array}$ & $\begin{array}{l}0.05 \\
445\end{array}$ & $\begin{array}{c}0 . \\
00 \\
05 \\
1\end{array}$ & $\begin{array}{c}0.40 \\
660\end{array}$ & $\begin{array}{c}0 . \\
00 \\
36 \\
4\end{array}$ & $\begin{array}{l}0.05 \\
416\end{array}$ & $\begin{array}{c}0 . \\
00 \\
06 \\
0\end{array}$ & 390 & $\begin{array}{l}1 \\
1\end{array}$ & & 3 & 340 & 4 & $\begin{array}{l}1.7 \\
6 \%\end{array}$ & $\begin{array}{l}3 \\
4 \\
0\end{array}$ & 4 \\
\hline $\begin{array}{c}\mathrm{M} \\
\mathrm{O} 2 \\
6- \\
88 \\
\end{array}$ & $\begin{array}{l}2 \\
6 \\
4\end{array}$ & $\begin{array}{l}2 \\
9 \\
4\end{array}$ & $\begin{array}{l}0 \\
9 \\
9 \\
0\end{array}$ & $\begin{array}{l}0.05 \\
883\end{array}$ & $\begin{array}{c}0 . \\
00 \\
05 \\
5 \\
\end{array}$ & $\begin{array}{c}0.74 \\
037\end{array}$ & $\begin{array}{c}0 . \\
00 \\
65 \\
9 \\
\end{array}$ & $\begin{array}{c}0.09 \\
127\end{array}$ & $\begin{array}{c}0 . \\
00 \\
10 \\
1 \\
\end{array}$ & 561 & $\begin{array}{l}1 \\
1\end{array}$ & 563 & 4 & 563 & 6 & $\begin{array}{l}0.0 \\
0 \%\end{array}$ & $\begin{array}{l}5 \\
6 \\
3\end{array}$ & 6 \\
\hline $\begin{array}{c}\mathrm{M} \\
\mathrm{O} 2 \\
6- \\
89 \\
\end{array}$ & $\begin{array}{l}1 \\
6 \\
6\end{array}$ & $\begin{array}{l}2 \\
7 \\
4\end{array}$ & $\begin{array}{l}6 \\
1 \\
\end{array}$ & $\begin{array}{l}0.06 \\
061\end{array}$ & $\begin{array}{c}0 . \\
00 \\
07 \\
2 \\
\end{array}$ & $\begin{array}{c}0.78 \\
952\end{array}$ & $\begin{array}{c}0 . \\
00 \\
88 \\
4\end{array}$ & $\begin{array}{l}0.09 \\
448\end{array}$ & $\begin{array}{c}0 . \\
00 \\
11 \\
0\end{array}$ & 625 & $\begin{array}{l}1 \\
1\end{array}$ & 591 & 5 & 582 & 6 & $\begin{array}{l}1.5 \\
5 \%\end{array}$ & $\begin{array}{l}5 \\
8 \\
2\end{array}$ & 6 \\
\hline $\begin{array}{c}\mathrm{M} \\
\mathrm{O} 2 \\
6- \\
90 \\
\end{array}$ & $\begin{array}{l}9 \\
0\end{array}$ & $\begin{array}{l}7 \\
0\end{array}$ & $\begin{array}{l}1 \\
2 \\
9\end{array}$ & $\begin{array}{c}0.12 \\
580\end{array}$ & $\begin{array}{c}0 . \\
00 \\
10 \\
3 \\
\end{array}$ & $\begin{array}{l}6.44 \\
358\end{array}$ & $\begin{array}{c}0 . \\
05 \\
12 \\
3\end{array}$ & $\begin{array}{l}0.37 \\
149\end{array}$ & $\begin{array}{c}0 . \\
00 \\
42 \\
7\end{array}$ & $\begin{array}{c}204 \\
0\end{array}$ & $\begin{array}{l}1 \\
0\end{array}$ & $\begin{array}{c}203 \\
8\end{array}$ & 7 & $\begin{array}{c}203 \\
6\end{array}$ & $\begin{array}{l}2 \\
0\end{array}$ & $\begin{array}{l}0.2 \\
0 \%\end{array}$ & $\begin{array}{l}2 \\
0 \\
4 \\
0 \\
\end{array}$ & $\begin{array}{l}1 \\
0\end{array}$ \\
\hline $\begin{array}{c}\mathrm{M} \\
\mathrm{O} 2 \\
6- \\
91 \\
\end{array}$ & $\begin{array}{l}5 \\
5\end{array}$ & $\begin{array}{l}2 \\
7 \\
6\end{array}$ & $\begin{array}{l}0 \\
2 \\
0\end{array}$ & $\begin{array}{c}0.16 \\
575\end{array}$ & $\begin{array}{c}0 . \\
00 \\
21 \\
3 \\
\end{array}$ & $\begin{array}{c}10.0 \\
307 \\
4\end{array}$ & $\begin{array}{c}0 . \\
07 \\
05 \\
7 \\
\end{array}$ & $\begin{array}{l}0.43 \\
892\end{array}$ & $\begin{array}{c}0 . \\
00 \\
47 \\
1 \\
\end{array}$ & $\begin{array}{c}251 \\
5\end{array}$ & $\begin{array}{l}2 \\
2\end{array}$ & $\begin{array}{c}243 \\
8\end{array}$ & 6 & $\begin{array}{c}234 \\
6\end{array}$ & $\begin{array}{l}2 \\
1\end{array}$ & $\begin{array}{l}7.2 \\
0 \%\end{array}$ & $\begin{array}{l}2 \\
5 \\
1 \\
5 \\
\end{array}$ & $\begin{array}{l}2 \\
2\end{array}$ \\
\hline $\begin{array}{c}\mathrm{M} \\
\mathrm{O} 2 \\
6- \\
92\end{array}$ & $\begin{array}{l}5 \\
8\end{array}$ & $\begin{array}{l}6 \\
0 \\
0\end{array}$ & $\begin{array}{l}0 \\
1 \\
0\end{array}$ & $\begin{array}{l}0.06 \\
886\end{array}$ & $\begin{array}{c}0 . \\
00 \\
04 \\
3\end{array}$ & $\begin{array}{l}1.12 \\
881\end{array}$ & $\begin{array}{c}0 . \\
00 \\
71 \\
0\end{array}$ & $\begin{array}{l}0.11 \\
889\end{array}$ & $\begin{array}{c}0 . \\
00 \\
12 \\
7\end{array}$ & 895 & $\begin{array}{l}1 \\
2\end{array}$ & 767 & 3 & 724 & 7 & $\begin{array}{l}5.9 \\
4 \%\end{array}$ & $\begin{array}{l}7 \\
2 \\
4\end{array}$ & 7 \\
\hline $\begin{array}{c}\mathrm{M} \\
\mathrm{O} 2 \\
6- \\
93 \\
\end{array}$ & $\begin{array}{l}5 \\
0 \\
5\end{array}$ & $\begin{array}{l}8 \\
1 \\
9\end{array}$ & $\begin{array}{l}0 \\
6 \\
2 \\
\end{array}$ & $\begin{array}{l}0.06 \\
245\end{array}$ & $\begin{array}{c}0 . \\
00 \\
04 \\
2 \\
\end{array}$ & $\begin{array}{c}0.86 \\
075\end{array}$ & $\begin{array}{c}0 . \\
00 \\
56 \\
6 \\
\end{array}$ & $\begin{array}{l}0.09 \\
996\end{array}$ & $\begin{array}{c}0 . \\
00 \\
10 \\
7 \\
\end{array}$ & 690 & $\begin{array}{l}1 \\
2\end{array}$ & 631 & 3 & 614 & 6 & $\begin{array}{l}2.7 \\
7 \%\end{array}$ & $\begin{array}{l}6 \\
1 \\
4\end{array}$ & 6 \\
\hline $\begin{array}{c}\mathrm{M} \\
\mathrm{O} 2 \\
6- \\
94\end{array}$ & $\begin{array}{l}1 \\
3 \\
4\end{array}$ & $\begin{array}{l}1 \\
0 \\
1\end{array}$ & $\begin{array}{l}1 \\
3 \\
3\end{array}$ & $\begin{array}{c}0.12 \\
633\end{array}$ & $\begin{array}{c}0 . \\
00 \\
08 \\
3\end{array}$ & $\begin{array}{l}6.16 \\
925\end{array}$ & $\begin{array}{c}0 . \\
04 \\
03 \\
3\end{array}$ & $\begin{array}{l}0.35 \\
417\end{array}$ & $\begin{array}{c}0 . \\
00 \\
38 \\
7\end{array}$ & $\begin{array}{c}204 \\
8\end{array}$ & $\begin{array}{l}1 \\
0\end{array}$ & $\begin{array}{c}200 \\
0\end{array}$ & 6 & $\begin{array}{c}195 \\
4\end{array}$ & $\begin{array}{l}1 \\
8\end{array}$ & $\begin{array}{l}4.8 \\
1 \%\end{array}$ & $\begin{array}{l}2 \\
0 \\
4 \\
8\end{array}$ & $\begin{array}{l}1 \\
0\end{array}$ \\
\hline $\begin{array}{c}\mathrm{M} \\
\mathrm{O} 2 \\
6- \\
95 \\
\end{array}$ & $\begin{array}{l}1 \\
1 \\
9\end{array}$ & $\begin{array}{l}2 \\
4 \\
7\end{array}$ & $\begin{array}{l}0 \\
4 \\
8 \\
\end{array}$ & $\begin{array}{l}0.12 \\
748\end{array}$ & $\begin{array}{c}0 . \\
00 \\
07 \\
7 \\
\end{array}$ & $\begin{array}{l}6.16 \\
356\end{array}$ & $\begin{array}{c}0 . \\
03 \\
72 \\
4 \\
\end{array}$ & $\begin{array}{l}0.35 \\
065\end{array}$ & $\begin{array}{c}0 . \\
00 \\
37 \\
7 \\
\end{array}$ & $\begin{array}{c}206 \\
4\end{array}$ & $\begin{array}{l}1 \\
1\end{array}$ & $\begin{array}{c}199 \\
9\end{array}$ & 5 & $\begin{array}{c}193 \\
8\end{array}$ & $\begin{array}{l}1 \\
8\end{array}$ & $\begin{array}{l}6.5 \\
0 \%\end{array}$ & $\begin{array}{l}2 \\
0 \\
6 \\
4 \\
\end{array}$ & $\begin{array}{l}1 \\
1\end{array}$ \\
\hline $\begin{array}{c}\mathrm{M} \\
\mathrm{O} 2 \\
6- \\
96 \\
\end{array}$ & $\begin{array}{l}3 \\
3 \\
0\end{array}$ & $\begin{array}{l}9 \\
8 \\
1\end{array}$ & $\begin{array}{l}0 \\
3 \\
4 \\
\end{array}$ & $\begin{array}{c}0.05 \\
514\end{array}$ & $\begin{array}{c}0 . \\
00 \\
03 \\
8 \\
\end{array}$ & $\begin{array}{c}0.46 \\
639\end{array}$ & $\begin{array}{c}0 . \\
00 \\
31 \\
8\end{array}$ & $\begin{array}{l}0.06 \\
135\end{array}$ & $\begin{array}{c}0 . \\
00 \\
06 \\
6 \\
\end{array}$ & 418 & $\begin{array}{l}1 \\
3\end{array}$ & 389 & 2 & 384 & 4 & $\begin{array}{l}1.3 \\
0 \%\end{array}$ & $\begin{array}{l}3 \\
8 \\
4\end{array}$ & 4 \\
\hline $\begin{array}{c}\mathrm{M} \\
\mathrm{O} 2 \\
6- \\
97 \\
\end{array}$ & $\begin{array}{l}2 \\
7\end{array}$ & $\begin{array}{l}5 \\
2\end{array}$ & $\begin{array}{l}5 \\
1 \\
\end{array}$ & $\begin{array}{c}0.15 \\
649\end{array}$ & $\begin{array}{c}0 . \\
00 \\
11 \\
6 \\
\end{array}$ & $\begin{array}{l}9.15 \\
768\end{array}$ & $\begin{array}{c}0 . \\
06 \\
62 \\
8 \\
\end{array}$ & $\begin{array}{l}0.42 \\
443\end{array}$ & $\begin{array}{c}0 . \\
00 \\
48 \\
1 \\
\end{array}$ & $\begin{array}{c}241 \\
8\end{array}$ & $\begin{array}{l}1 \\
0\end{array}$ & $\begin{array}{c}235 \\
4\end{array}$ & 7 & $\begin{array}{c}228 \\
1\end{array}$ & $\begin{array}{l}2 \\
2\end{array}$ & $\begin{array}{l}6.0 \\
1 \%\end{array}$ & $\begin{array}{l}2 \\
4 \\
1 \\
8\end{array}$ & $\begin{array}{l}1 \\
0\end{array}$ \\
\hline $\begin{array}{l}\mathrm{M} \\
\mathrm{O} 2 \\
\end{array}$ & $\begin{array}{l}1 \\
3 \\
\end{array}$ & $\begin{array}{l}1 \\
9 \\
\end{array}$ & 0 & $\begin{array}{c}0.06 \\
311 \\
\end{array}$ & $\begin{array}{c}0 . \\
00 \\
\end{array}$ & $\begin{array}{l}1.02 \\
176 \\
\end{array}$ & $\begin{array}{c}0 . \\
00 \\
\end{array}$ & $\begin{array}{c}0.11 \\
743 \\
\end{array}$ & $\begin{array}{c}0 . \\
00 \\
\end{array}$ & 712 & $\begin{array}{l}1 \\
1\end{array}$ & 715 & 5 & 716 & 8 & $\begin{array}{c}- \\
0.1 \\
\end{array}$ & $\begin{array}{l}7 \\
1 \\
\end{array}$ & 8 \\
\hline
\end{tabular}




\begin{tabular}{|c|c|c|c|c|c|c|c|c|c|c|c|c|c|c|c|c|c|c|c|}
\hline $6-$ & 8 & 0 & 7 & & 06 & & 96 & & 13 & & & & & & & $4 \%$ & 6 & \\
98 & & & 3 & & 3 & & 5 & & 3 & & & & & & & & & \\
\hline $\mathrm{M}$ & 1 & 2 & 0 & 0.13 & 0. & 6.91 & 0. & 0.38 & 0. & 212 & 1 & 210 & 5 & 207 & 1 & 2.3 & 2 & 1 \\
$\mathrm{O} 2$ & 8 & 5 &. & 196 & 00 & 472 & 04 & 003 & 00 & 4 & 1 & 1 & & 6 & 9 & $1 \%$ & 1 & 1 \\
$6-$ & 1 & 5 & 7 & & 07 & & 00 & & 40 & & & & & & & & 2 & \\
99 & & & 1 & & 5 & & 8 & & 6 & & & & & & & & 4 & \\
\hline $\mathrm{M}$ & 4 & 6 & 0 & 0.06 & 0. & 0.74 & 0. & 0.08 & 0. & 614 & 1 & 563 & 8 & 550 & 7 & 2.3 & 5 & 7 \\
$\mathrm{O} 2$ & 0 & 1 & & 028 & 00 & 059 & 01 & 910 & 00 & & 9 & & & & & $6 \%$ & 5 & \\
$6-$ & & & 6 & & 12 & & 35 & & 12 & & & & & & & & & & 0 \\
10 & & & 5 & & 0 & & 8 & & 3 & & & & & & & & & \\
0 & & & & & & & & & & & & & & & & & & & \\
\hline
\end{tabular}


Graphical abstract
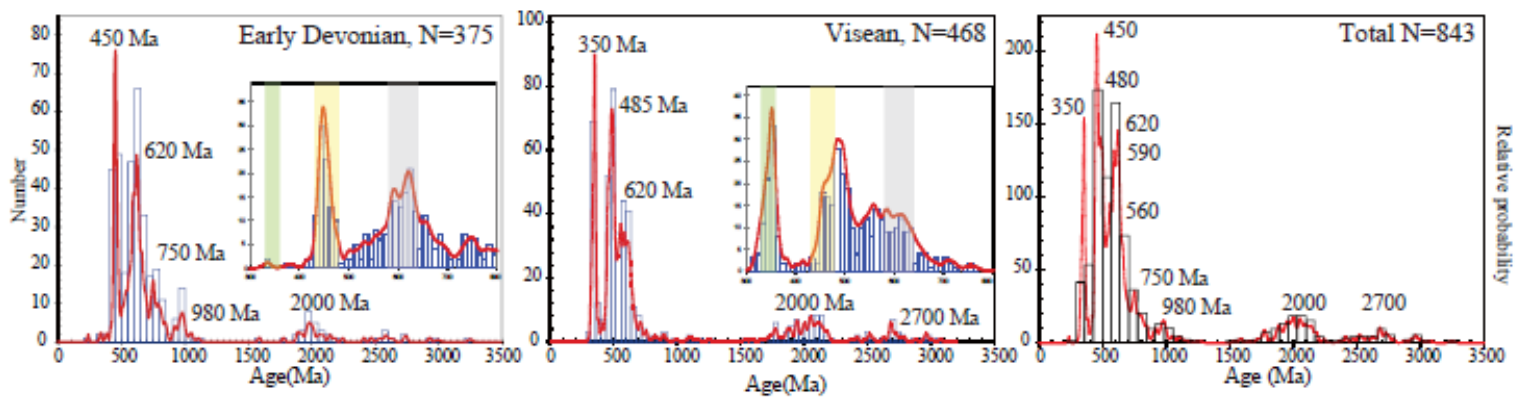

Visean ca $330 \mathrm{Ma}$

Tufs Anthracifères series

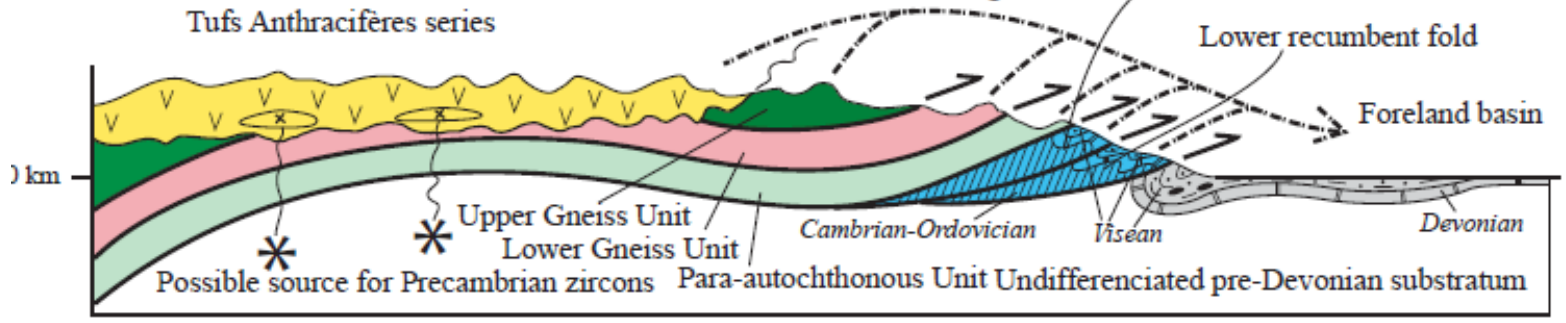

Schematic topographic reconstruction of the French Massif Central during the Visean and the possible sources for the detrital zircons of the Foreland basin with the cumulative probability plots of detrital zircon $\mathrm{U}-\mathrm{Pb}$ ages.

\section{Highlights}

Variscan Southern Massif Central Foreland basin helps to decipher tectonic events

Early Devonian and Carboniferous detrital zircon provenance is investigated

11 samples yielded U-Pb age spectra ranging from Neoarchean to Late Paleozoic

Rifting, volcanic, and plutonic events with a multi-recycling history are suggested 